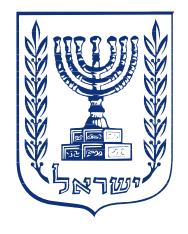

\title{
IMPLEMENTATION \\ OF THE SUSTAINABLE \\ DEVELOPMENT GOALS
}

National Review

ISRAEL 2019 


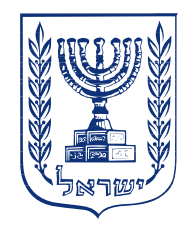

IMPLEMENTATION
OF THE SUSTAINABLE
DEVELOPMENT GOALS

National Review

ISRAEL 2019 


\section{ACKNOWLEDGMENTS}

Acknowledgments are due to representatives of government ministries and agencies as well as many others from a variety of organizations, for their essential contributions to each chapter of this book. Many of these bodies are specifically cited within the relevant parts of this report.

The inter-ministerial task force under the guidance of Ambassador Yacov Hadas-Handelsman, Israel's Special Envoy for Sustainability and Climate Change of the Ministry of Foreign Affairs, and Galit Cohen, Senior Deputy Director General for Planning, Policy and Strategy of the Ministry of Environmental Protection, provided invaluable input and support throughout the process.

Special thanks are due to Tzruya Calvão Chebach of Mentes Visíveis, Beth-Eden Kite of the Ministry of Foreign Affairs, Amit Yagur-Kroll of the Israel Central Bureau of Statistics, Ayelet Rosen of the Ministry of Environmental Protection and Shoshana Gabbay for compiling and editing this report and to Ziv Rotshtein of the Ministry of Environmental Protection for editorial assistance. 


\section{FOREWORD}

The international community is at a crossroads of historical proportions. The world is experiencing extreme challenges, not only climate change, but many social and economic upheavals to which only ambitious and concerted efforts by all countries can provide appropriate responses. The vision is clear. Our immediate and urgent obligation is to protect and improve our world for all its current inhabitants. We also have the responsibility as caretakers for future generations. Israel is fully committed to working in unison with others to turn the vision into a reality and the SDGs are our plan of action.

Israel's first Voluntary National Review is the result of a coordinated process by our two ministries with the contribution of many other bodies. The process has been significantly enriched by the expertise and experience of governmental agencies and non-governmental stakeholders.

This report surveys Israel's path toward the realization of Agenda 2030 and its seventeen Sustainable Development Goals by providing an upto-date view of where we are and, in many cases, how we plan to reach the 2030 Goals. It presents measures and activities carried out by various actors in Israel and elsewhere to implement the goals we collectively adopted in order to transform our world.

The report presents many accomplishments in fields such as education, water, health and the economy. However, just as importantly, the report highlights those areas where more needs to be done to improve the lives of our citizens, communities and families as well as our economy while, at the same time, contributing to the sustainable future of people everywhere. While the countries of the world are united in the common desire to achieve the SDGs, each country has a range of rich national characteristics and strengths which will make its path toward realization of the goals unique. Israel will continue to focus on innovative technologies and initiatives but through the prism of minimizing environmental degradation and enabling greater access to the benefits of technological advances and increased opportunities for all.

As a young country, we recently faced many of the difficulties currently encountered by many other countries. Moreover, our experience in overcoming resource scarcity is becoming more relevant to an ever-increasing circle of climate change affected areas of the world. Our cooperation with countries worldwide is given broad expression in our VNR, much of it carried out by Israel's International Development Agency (MASHAV) which is dedicated to furthering partnerships to increase the scope and effectivity of capacity building. Agenda 2030 has been the catalyst for a fruitful process in Israel which will continue to develop and progress over time. We look forward to the work that lies ahead, both at home and overseas, and to the harnessing of the creativity, enthusiasm, and optimism which characterizes Israel to the benefit of people everywhere.

Our vision is to harness our innovation to addressing global challenges while being mindful that the benefits must reach all sectors, for inequality within countries is as much of a challenge as between countries. The vision of building a society which mends the world - Tikun Olam - and which leaves no one behind has ancient roots in our tradition.

In describing the success and wealth of that wisest of sovereigns, King Solomon, the Bible describes, in Psalm 72, what is expected from the powerful in return for their good fortune.

"For he will deliver the needy when he cries, The poor also, and him who has no helper...

And will save the souls of the needy.

He will redeem their life from oppression and violence."

The SDGs give voice to the mutuality and interdependence of development. They highlight the need for concerted action on all three pillars - social, economic and environmental - by all countries at all stages of development and growth. Israel is proud to be an active player and contributor of this global undertaking.

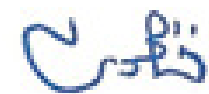

MK Israel Katz, Minister of Foreign Affairs, Minister of Transport and Minister of Intelligence

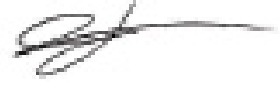

MK Zeev Elkin,

Minister of

Environmental

Protection and

Minister of Jerusalem Affairs and Heritage 


\section{TABLE OF CONTENTS}

\section{INTRODUCTION: ISRAEL'S VOLUNTARY NATIONAL REVIEW}

Methodology and process for the preparation of the review 8

Structure of Israel's Voluntary National Review 9

Policy and enabling environment 10

Partnering for a better world - Israel's international contribution toward Agenda 2030

\section{HARNESSING INNOVATION TO LEAVE NO ONE BEHIND}

A national priority and an opportunity to effect change in Israel and beyond 15

Broadening the discussion through the national process on Agenda $2030 \quad 17$

\section{GOALS AND TARGETS}

Goal 1: No Poverty 26

Goal 2: Zero Hunger 37

Goal 3: Good Health and Well-Being $\quad 54$

Goal 4: Quality Education $\quad 80$

Goal 5: Gender Equality 100

Goal 6: Clean Water and Sanitation $\quad 114$

Goal 7: Affordable and Clean Energy $\quad 134$

Goal 8: Decent Work and Economic Growth 145

Goal 9: Industry, Innovation and Infrastructure 165

Goal 10: Reduced Inequalities 183 
Goal 12: Responsible Consumption and Production 219

Goal 13: Climate Action 237

$\begin{array}{ll}\text { Goal 14: Life Below Water } & 248\end{array}$

Goal 15: Life on Land $\quad 262$

Goal 16: Peace, Justice and Strong Institutions 272

Goal 17: Partnerships for the Goals 293

4. STATISTICAL ANNEX: INDICATORS FOR SUSTAINABLE DEVELOPMENT GOALS IN ISRAEL 301

5. SDG RELATED NATIONAL STATISTICS: WELL-BEING INDICATORS IN ISRAEL

7. CONTRIBUTIONS FROM NON-GOVERNMENT STAKEHOLDERS 


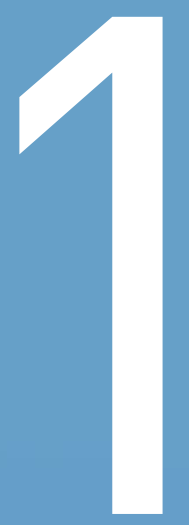

\section{INTRODUCTION}

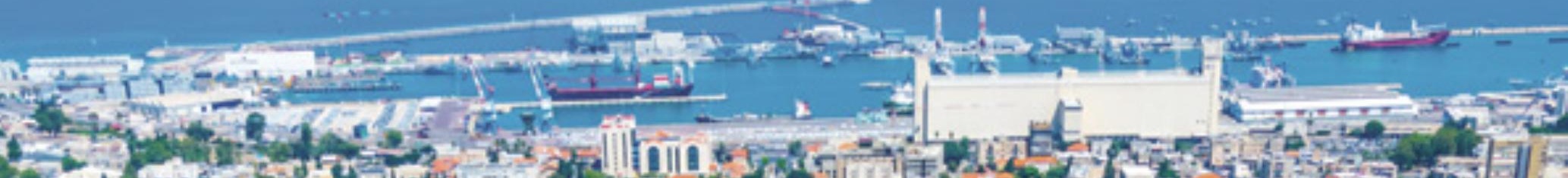

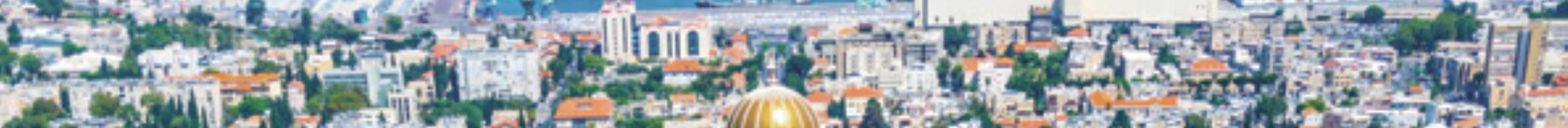

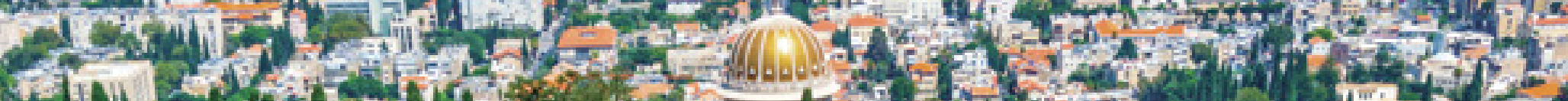

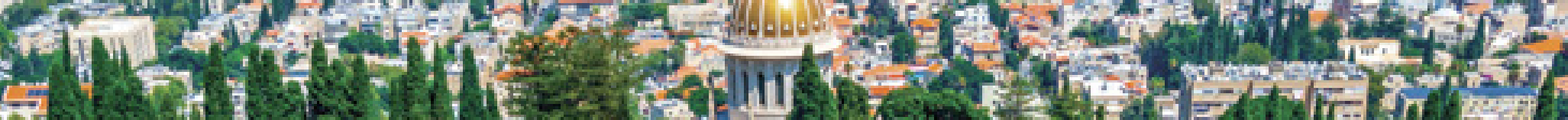

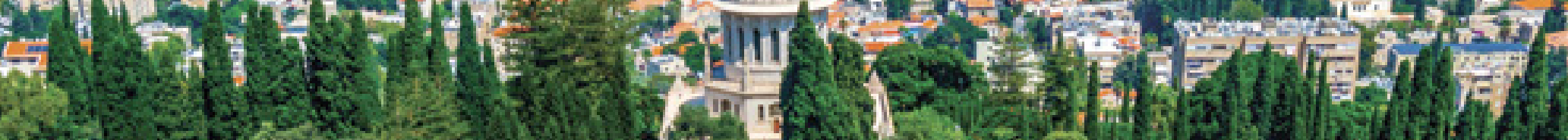
2akn Lit

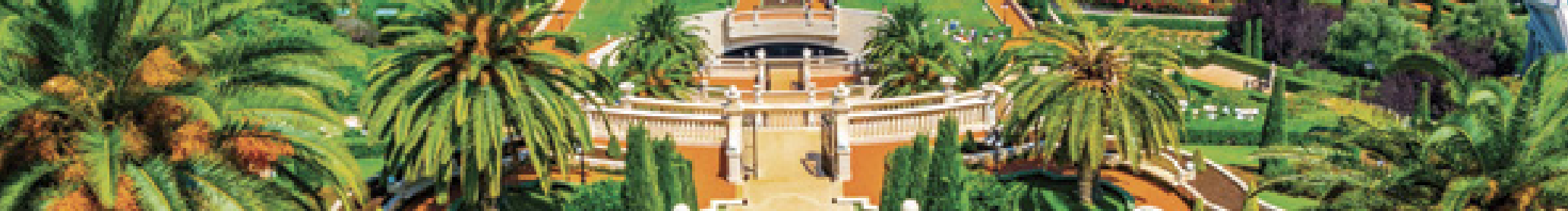

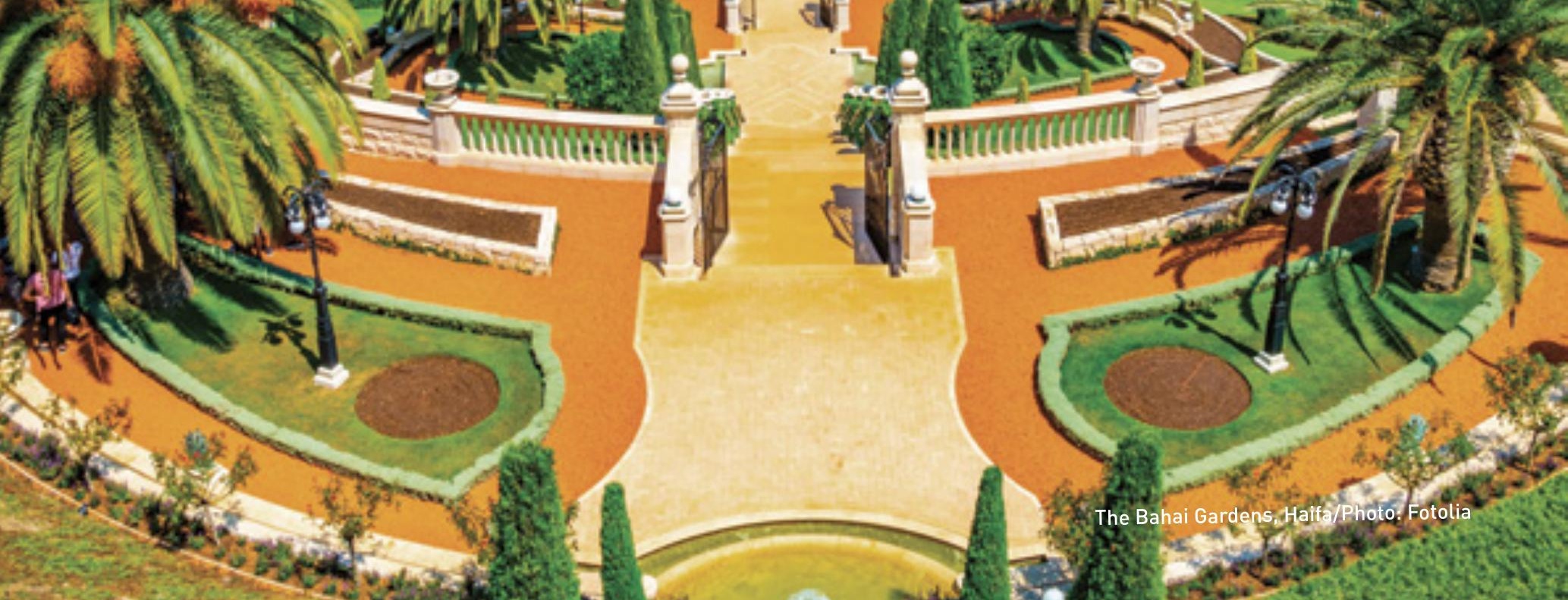




\title{
INTRODUCTION: ISRAEL'S VOLUNTARY NATIONAL REVIEW
}

\author{
Israel stands united with all UN member states in the support and \\ implementation of the 2030 Agenda for Sustainable Development, adopted
} at the UN Sustainable Development Summit on 25 September 2015. As an active partner in formulating the 2030 Agenda, we recognize that the greatest global challenge facing our world today is to eradicate the substantial gaps in access to resources - economic, social and environmental - and to adhere to the universal pledge to 'leave no one behind.'

\section{Methodology and process for the preparation of the review}

Under the guidance of the Director General of Israel's Ministry of Foreign Affairs, a governmental team headed by Israel's Special Envoy for Sustainability and Climate Change and the Deputy Director General for Strategy at the Ministry of Environment Protection was commissioned to compile Israel's first Voluntary National Review (VNR) in anticipation of the 2019 United Nations High-Level Political Forum, the framework for presenting the report.

One of the team's two key tasks was the preparation of a report including all relevant data and information related to sustainability and the implementation of the 2030 Agenda. The report presents a broad 'all of government' scoping and mapping review to best gauge Israel's status in progressing towards the 2030 Agenda, to provide for an informed basis for comprehensive and coherent policy decisions for the next stage of implementation, and to serve as a national baseline for future progress analysis in the next VNR report. An inter-ministerial committee was formed and consultations took place over the past 18 months. Government ministries were invited to submit their professional inputs on Israel's progress and activities towards meeting the targets and goals of Agenda 2030 under their responsibility. This preparation of Israel's first VNR also provided an opportunity to introduce Agenda 2030 across government ministries, authorities and organizations, often for the first time. Throughout the process, each ministry was introduced to the wealth of knowledge, experience, methodologies and best practices made available under Agenda 2030, and global cooperation and local relevance and opportunities were discussed.

Israel's urban density and scarcity of land resources highlight the centrality of Israel's urban sustainability to the country's successful implementation of Agenda 2030. A comprehensive multi-stakeholder process was led by the Ministry of Construction and Housing and some of the feedback by stakeholders is included as an annex to the report. As a result of this process, a new local indicator for urban renewal is under consideration which could provide the basis for a global indicator within SDG 11.

\section{Stakeholder involvement}

Although the report is a governmental one, Israel nevertheless engaged relevant stakeholders in the process. Representatives of civil society, the private sector and academia provided their contributions and took part in the discussions.

An examination of the means by which each sector can implement and use the goals to guide and further their work remains to be carried out, 
as well as the ongoing stakeholder engagement process with the government.

\section{Role of the Israel Central Bureau of} Statistics in implementing the 2030 Agenda

The Israel Central Bureau of Statistics (ICBS) is Israel's National Statistical Office (NSO) and serves as the national focal point for all processes connected to collecting and reporting data on SDG indicators. This also includes indicators that are not part of the official statistics produced by the ICBS and the National Statistical System (NSS). Such indicators mainly refer to policy measures and legislation related to the SDGs.

Prior to the preparation of Israel's VNR, the ICBS started a process of mapping available SDG indicators data within the national statistical office (NSO) and in other governmental agencies for the purpose of providing maximum international reporting of all relevant data. This mapping process was complex at times since relations between international organizations and national data producers and suppliers often did not involve the NSO. One of the main contributions of the governmental VNR process in this respect is that in creating an intergovernmental task force, improvements in the quality of information concerning availability, relevance and development needs for the future were facilitated. The initial ICBS mapping was submitted to all members for correction and completion. Members were also asked to send all past SDG reports to the ICBS and to coordinate all future reports with the ICBS so that after submitting Israel's first VNR no new data gaps will remain.

The data presented in this report are intended to serve as a basis for further development of SDG related statistics in the ICBS as well as a starting point for strengthening and improving relevant work of the NSS for the purpose of building a complete national knowledge platform for monitoring progress towards achieving the SDGs.

Currently there are 123 available indicators in Israel. 98 indicators are based on ICBS data and 25 indicators are based on data and information from other government ministries or other agencies. 31 indicators contain relevant disaggregation based on available data. Out of all the available indicators, 113 indicators are included in the statistical annex of this report. A few available indicators were not included in the statistical annex. These indicators require further development so that they can be computed and published. Some further development is also needed in other government ministries that are in charge of available indicators outside of the ICBS.

\section{Structure of Israel's Voluntary National Review}

\section{Goal by goal}

Israel's progress and activities on the national and international levels are detailed in the main core of this VNR in relation to each of the 17 SDGs. When describing the national and international aspects in the VNR, an effort was made to provide a coordinated and comprehensive mapping based on contributions from as many governmental sources in each of their respective areas of responsibility. However, the survey presented in this review does not encompass the full scope of nationally based activities nor the full extent of Israel's international contribution and should be considered as the first stage in a scoping and mapping process towards Israel's next VNR under the guidance of a national mechanism.

When describing the progress relevant to national implementation of the 17 SDGs, inputs were received from most government ministries and many other organizations in Israel and were complemented by statistical data and analysis performed by the ICBS, such as the well-being indicators report and the social survey. Much of the international review - Partnering for a Better World - highlights, among others, the extent and variety of Israel's cooperation with developing countries in projects which are aligned with the SDGs and carried out by Israel's Agency for International Development Cooperation (MASHAV), though not exclusively so. The international activities of 
government ministries, authorities, civil society, academia, and the private sector are also included, though to a lesser extent.

\section{Statistical Annex}

The statistical annex presents data on selected indicators showing the current state and trends with respect to measuring Israel's progress towards meeting the SDGs. The indicators in the report are based on available data in the ICBS, and available data in additional government agencies that are responsible for producing official statistics under the framework of the National Statistical System (NSS).

ICBS data for this report are based on two main types of data sources: surveys conducted on an ongoing basis by the ICBS and administrative sources such as the population register, social security and tax authority files, administrative files of the health and education systems and others.

Data for the selected indicators in this report include presentation of the current state at the national level and, wherever possible, the trend of at least the past ten years as well as international comparisons and any relevant disaggregation of the indicators (e.g., by sex, population group, age and more). The data presented also include statistics and indicators that do not precisely match the international UN SDG indicator definition but contain relevant data that can serve as proxies of information that help understand the state of the target and goal.

113 indicators are included in the statistical annex of this report. The process of data collection and publication is in accordance with the Israeli Statistical Ordinance. The Statistical Ordinance defines the role of the national statistician as responsible for all official statistics in Israel and, as such, responsible for all SDG indicators data reported by the ICBS or other members of the NSS.

SDG related national statistics: Well-being Indicators Annex

This annex presents a summary of the well-being indicators published in February 2019. To date, 70 indicators have been developed.

\section{Non-governmental Stakeholders Annex}

Submissions by a number of non-governmental stakeholders are included in the relevant annex to this report and were prepared by and are the sole responsibility of the relevant organizations.

Documents were provided by the following organizations:

- Coalition of Civil Society Organizations, a coalition of over 50 organizations from a wide range of social, environmental and economic non-governmental organizations interested in leveraging the SDGs within their organizations and ensuring their implementation in Israel;

- Maala, a Corporate Social Responsibility roof organization which sets and develops sustainability and responsible management standards among Israeli businesses, including implementation of the SDGs in that sector;

- Forum 15 - The Israeli Forum of Self-Government Cities, which represents Israel's largest cities and submitted a written response to the process carried out in preparing materials on Goal 11;

- Israel Urban Forum, a civil society, cross-sectoral and multi-disciplinary team, focusing on the role of civil society in shaping cities which submitted a document in response to work on Goal 11;

- Keren Kayemeth Lelsrael - Jewish National Fund (KKL-JNF), Israel's official afforestation administration which also engages in reforestation policy and the supply of ecosystem services in Israel and overseas to promote SDG implementation.

\section{Policy and enabling environment}

\section{Towards a national mechanism for 2030}

Establishment of a national SDG mechanism, based on a government decision, was the second major focus of the governmental VNR team.

Such an organ will oversee Israel's national and international sustainable development policies and strategies and will be entrusted with the authority and capabilities to follow up on relevant 


\section{WELL-BEING INDICATORS IN ISRAEL}

\section{A National Tool to Support Future Work on SDGs}

Economic growth is a central means of achieving improvements in people's quality of life, but it is not the sole end. Gross Domestic Product (GDP) is the most commonly used measure of a country's economic activity, but it was never designed to be an indicator of well-being. Decision-making processes today are largely based on measurements of outputs, deficits, government debt and unemployment rates. Yet these measurements of growth do not include expenditures associated with pollution, disease, war and crime; they do not include the economic benefits of social and volunteer activities; and they take no account of the distribution of output among different households or of damages to future generations. Recognition of the inadequacies associated with economic measurements alone has grown significantly in recent years. This can be attributed to a number of developments of recent times: to the global financial crisis, to the realization that the earth's resources are limited, to the publication in 2009 of the seminal report on the Measurement of Economic Performance and Social Progress by Joseph Stiglitz at the request of the French government, and to the launch of the OECD Better Life Initiative in 2011. In Israel, the combination of global developments, together with the social justice protest of 2011, underlined the need for a multiplicity of perspectives in deciding on government policy.

The project on developing well-being indicators for Israel began at the end of 2012 based on Government Decision 5255 of February 12, 2012. It was led by the Prime Minister's Office, the National Economic Council and the Ministry of Environmental Protection and involved multi-stakeholder discussions between different government ministries, academia, civil society and the private sector. The process of selecting the indicators also included public deliberations by means of online consultations and focus groups.

Eight indicators were selected for each domain, and the desired direction of change was defined for each indicator. Data on the indicators were taken from the ICBS databases and are based on administrative information and regular surveys conducted by the ICBS. The indicators present the trends from the beginning of the $21^{\text {st }}$ century and provide a basis for examining changes in well-being in Israel, comparing different population groups in Israel together with international comparisons.

Pursuant to a decision adopted by the government of Israel in April 2015 (Decision No. 2494), the ICBS is developing, reporting and updating indicators of well-being, sustainability, and national resilience in the following 11 domains: quality of employment; personal security; health; housing and infrastructure; education; higher education and skills; personal and social well-being; environment; civic engagement and governance; material standard of living; leisure, culture and community; and information technology.

As national non-SDG indicators built by a multi-stakeholder process, these indicators present a complementary and particularly relevant tool for scoping and gauging Israel's progress towards many different targets and goals in the 2030 Agenda. 
government decisions and coordinate between the various government ministries and agencies as well as between them and non-governmental stakeholders.

Israel has a robust and dedicated government mechanism for national strategic thinking the National Economic Council, which serves as a coordinating body for the Prime Minister on topics which require comprehensive and methodological economic thinking. Within this framework, the Council deals with issues relating to housing, health, encouraging capital investments, determining the State's share of national natural resources, developing financial tools to increase sources of funding for small and mediumsized businesses and R\&D oriented companies, formulating quality of life indices and formulating debt restructuring arrangements, all of which are essential for achieving the SDGs.
The following table highlights the connection between the SDGs and Israel's current strategic goals.

Like many other countries, Israel embarked on the SDG process while the institutional arrangements were being developed. Agenda 2030 was presented at the Senior Professional Forum for Strategic Planning. Furthermore, the Knesset (Israel's parliament) is examining ways in which SDG thinking and terminology can be incorporated in the process of preparing legislation by parliamentary committees. Finally, a key step towards the establishment of a national mechanism is a dedicated government decision. Such a decision is currently being prepared to continue the integration of the SDGs into the government's strategic planning and to realize the vision of leaving no one behind through Israeli innovation.

Table 1.1

Strategic issues for Israel, 2015, relevant for Israel's planned course on implementation of the SDGs Strategic issues for Israel

Relevant SDG goals

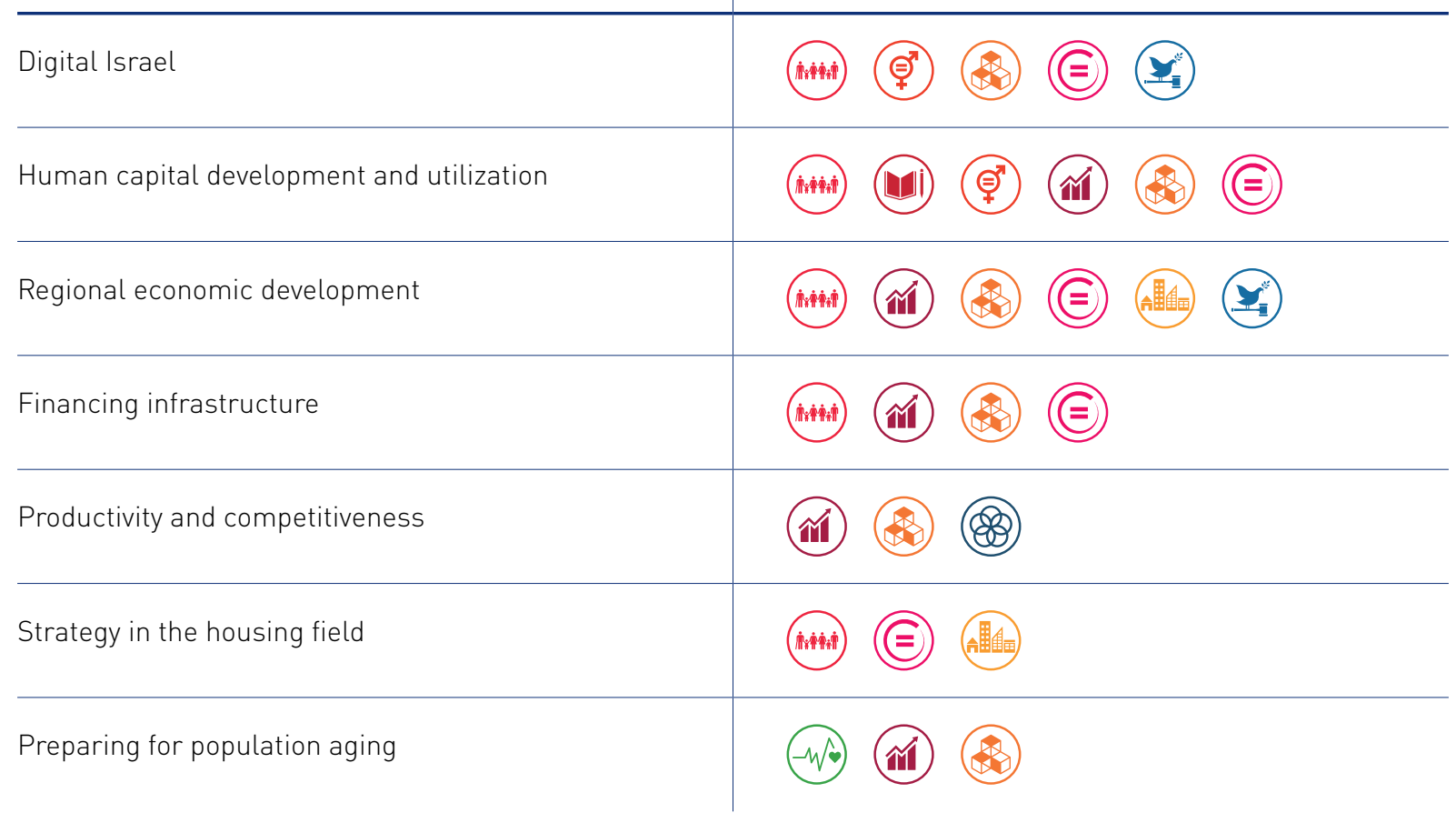




\section{Partnering for a Better World Israel's international contribution towards Agenda 2030}

Israel's international development cooperation program is committed to a people-centered strategy of Partnering for a Better World, embodying the theme of the High-Level Political Forum on Sustainable Development (HLPF) - Empowering people and ensuring inclusiveness and equality. This approach entails active engagement in multistakeholder initiatives designed to aid the extreme poor and most vulnerable in developing and least developed countries.

Israel is committed to promoting international development assistance within the framework of multilateral structures. This commitment is substantiated by its membership in the UN, Organization for Economic Cooperation and Development (OECD), World Bank Group (WBG), International Monetary Fund (IMF), and as a partner country in the Organization for Security Cooperation in Europe (OSCE).

Israel firmly believes in international development cooperation in order to succeed in securing a safer, more prosperous and just society for all people. In December 2017, MASHAV Israel's Agency for International Development Cooperation - marked its $60^{\text {th }}$ anniversary. Since its establishment in 1958, MASHAV has partnered with 140 countries, dispatching thousands of experts throughout the developing world, and hosting over 300,000 professionals in capacitybuilding, training and demonstration projects in Israel and abroad. MASHAV also plays a significant role in Israel's attempts to reach out to the Arab and Muslim worlds.

A year ago, on 23 July 2018, the Government of Israel decided to step-up its international development policy and approved a Government Decision on Advancing Israeli Activity in the Field of International Development (Decision No. 4021). To carry out the assignment, the government appointed the first Inter-ministerial Committee for International Development, tasked with formulating Israel's reformed international development strategy. Headed by the Director General of the Prime Minister's Office, the committee brings together delegates from government offices and directorates vital to the field of international development. The rationale for reforming Israel's strategy was based on acknowledging the country's global leadership in fields such as innovation, agriculture, water and health, and its potential to accelerate efforts to overcome global development challenges at a higher pace and on a greater scale than previously done.

When formulating the new strategy, the committee, whose work is still underway, is examining different approaches to Israel's international development policy. The committee's agenda includes mapping mechanisms to encourage Israeli innovation that meets the needs of the developing world, promoting more active involvement of the private sector in international development efforts, increasing financing of international development activities, and connecting with complementary efforts by the private sector, civil society and academia. 


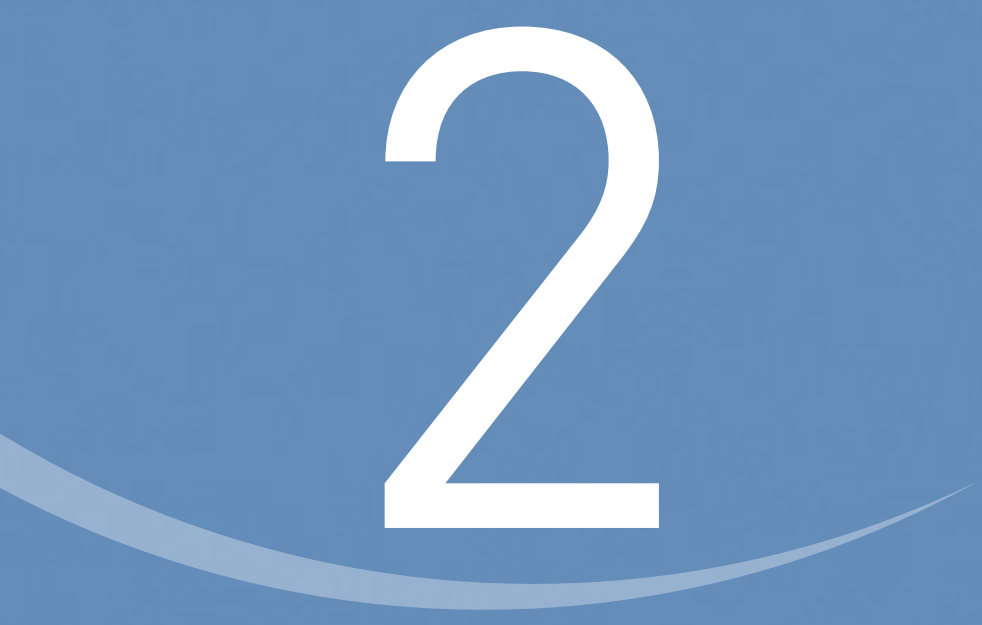

\section{HARNESSING INNOVATION TO LEAVE NO ONE BEHIND}




\section{HARNESSING INNOVATION TO LEAVE NO ONE BEHIND}

Israel believes that innovation is a key engine for economic growth and well-being. As such, all citizens, sectors, regions and spheres of life in Israel should be the beneficiaries. Making innovation inclusive and accessible to all is a national mission and a key driver for Israel's role and contribution to reaching the SDGs on a national and global level.

A national priority and an opportunity to effect change in Israel and beyond

The Israeli economy is remarkably resilient, and despite its youth, the country has made significant strides in terms of global impact. As a recent
OECD report shows, Israel's growth is strong, the average standard of living is improving and income inequality has fallen. Israeli innovation has attained great achievements in recent decades. The country is a global center with a vibrant innovation culture based on interdisciplinary capabilities, a spirit

Figure 2.1

Innovation has played a key role in Israel's unique development story over the years

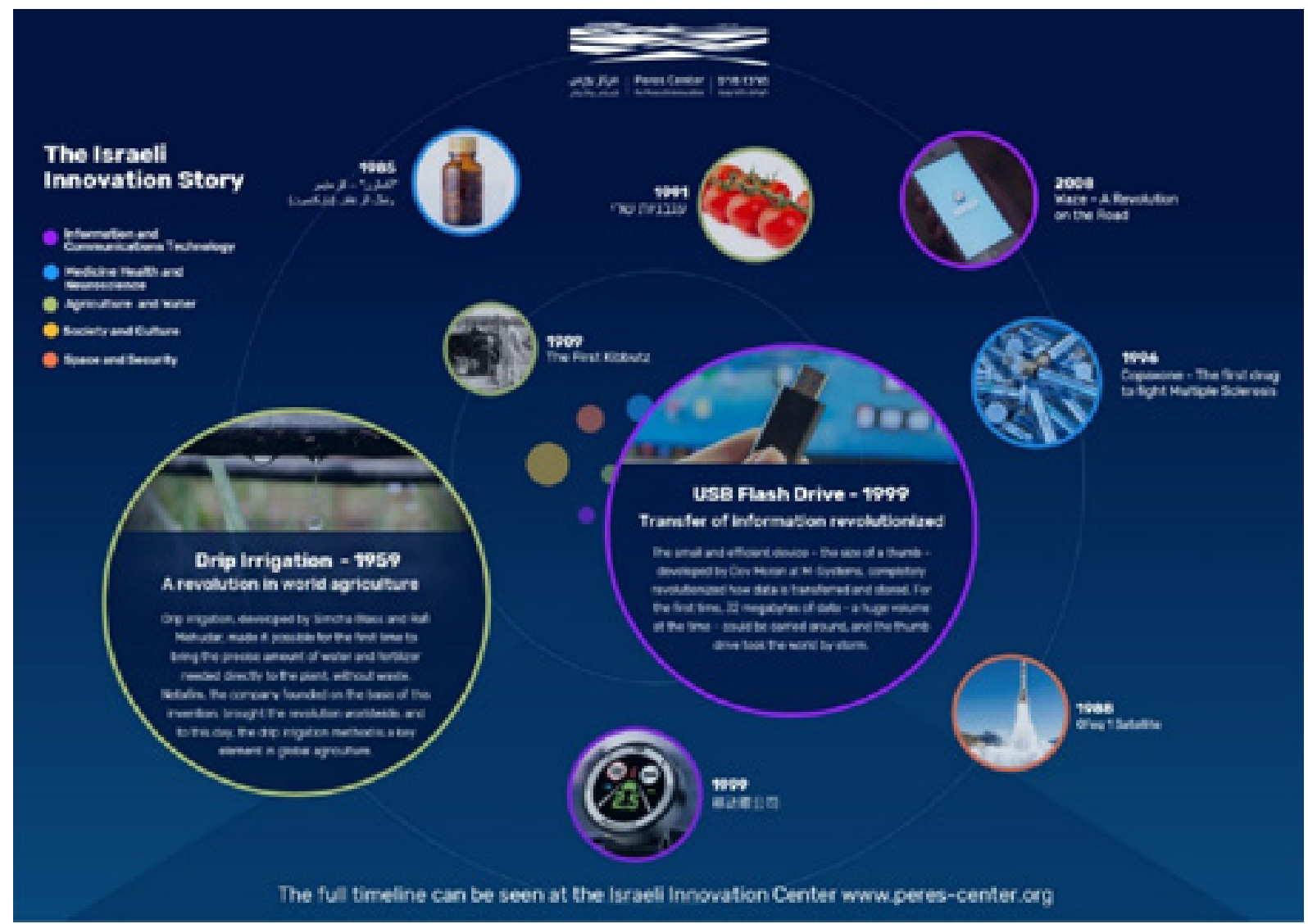

Source: The Peres Center for Peace and Innovation 
of entrepreneurship and strong technical skills. Recent years have witnessed a flourishing of Israeli hi-tech that is expressed by widespread activity of multinational corporations, making Israel a top destination for R\&D with over 330 multinational companies currently operating in Israel, in addition to innovative start-up companies, and Israeli growth companies. This spirit of innovation is multifaceted, engulfing technological innovation, social innovation and business model innovation. This same spirit enabled Israel's unique development story over the past few decades as a young country striving for progress while facing scarcity of resources. It has incentivized Israel to overcome major obstacles. From solutions to meet water scarcity which enable communities to decouple their economic growth from resource use and support more resilient and adaptive food systems, to critical solutions in biomed which enable access to state-of-the-art medical treatment, even from afar, Israel's innovations have made a major impact, both domestically and worldwide. They serve as a valuable driving force in Israel's international development assistance, allowing the country to share its own unique experience for the betterment of the global community.

Israeli innovation is defined by several dimensions. Technologically, the majority of innovation activity is focused on the ICT sector. From a geographical perspective, the dominant mass of activity is still concentrated in central Israel, with relatively lower activity in the periphery. From a demographic perspective, about half of all high-tech employees are up to 45-year-old non-Haredi (ultra-Orthodox) Jewish men. Moreover, technological innovation has yet to breach the bounds of the high-tech industry, with a shortage of skilled workers including engineers and programmers. As a result, the overwhelming majority of Israeli citizens do not always experience the benefits of technological innovations in areas that affect their daily lives, such as transportation, commerce, finance, and access to public services. The upshot is that the huge economic and social potential inherent in Israeli innovation remains largely unfulfilled.

Moreover, despite economic resilience, the percentage of working poor and the overall poverty rates, especially among children, remain high. Israel's share of low-paid workers is one of the OECD's highest. Disparities between different communities are evident in communities such as the Haredi and Israeli Arab communities and among women. Together, they comprise above 25\% of the general population although their weight among those living in poverty has reached some

Figure 2.2

Poverty risk by population group and religious lifestyle (Jews), 2016

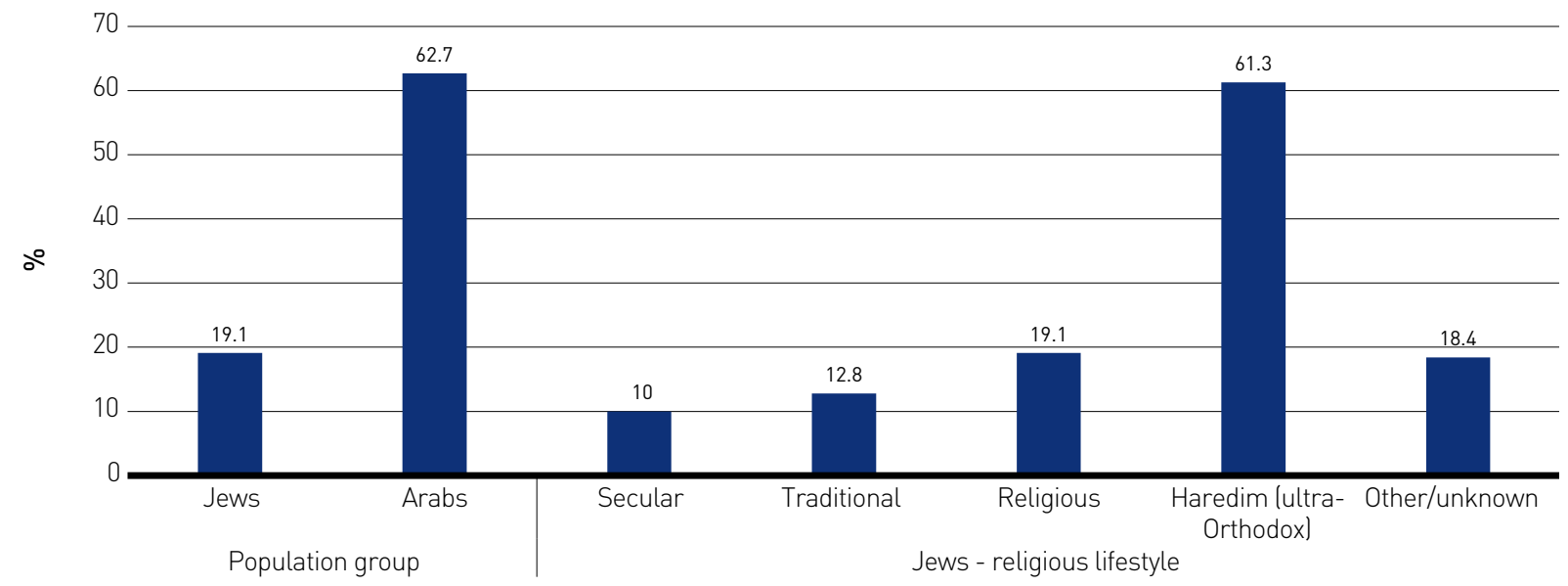

Source: ICBS, Society in Israel - Religion and Self-Definition of Level of Religiosity, 2018 
$60 \%$. Among Israelis of Ethiopian descent too, the poverty rate is high, standing at $13.6 \%$. In families headed by a self-employed parent, the poverty rate is $29.8 \%, 1.6$ times the rate of the general population. The challenge confronting Israel is to ensure that these and other groups are not left behind and that their potential can form a part of Israel's economic growth.

The challenge of leaving no one behind emerges in many sections of the VNR - from the identification of gaps in health profiles between different population groups in areas such as obesity and child injuries to the institution of programs such as universal child dental care (Goal 3) and from the identification of gaps in access to environmental services to dedicated programs which address the quality of environmental services in low-ranking socio-economic communities (Goal 12). Similarly, gaps need to be closed in other areas such as employment, accessibility, income disparity, vocational skills, literacy, and mathematical and ICT technology skills, or, in the case of women, in addressing disparities which express themselves in reduced personal security and higher poverty levels, among others.

\section{Broadening the discussion through the national process on Agenda 2030}

The national process on the 2030 Agenda for Sustainable Development further emphasized the mixed picture of Israeli reality created by two disparate trends - on the one hand, a small country playing a leadership role with impact on a global scale, while, on the one hand, various population groups not sharing this story of success. As demonstrated in the statistical and well-being annexes to the VNR and in the sections on Goals 5 and 10 , multiple initiatives are already in place to address the challenges.

The multi-stakeholder discussions on Agenda 2030 for Israel broadened the discussion and recognized additional opportunities for leaving no one behind that can best meet Israel's strengths as an innovation powerhouse. It was evident that as a society with such a broad cultural, ethnic and religious mix, the government's agenda has to continually include the search for creative and culturally sensitive ways to provide equal opportunities for all.

Israel's vision is to continue to nurture its culture of innovation and expand it to all people in need and to give practical expression to the value of leaving no one behind. This vision can leverage the experience and success of many different initiatives led by governmental and non-governmental sectors and benefit from a 'whole of society' approach based on collaboration and multi-actor knowhow and creativity. The vision will continue to play a central role in Israel's future work on Agenda 2030. Current activities and drivers for change that are already in place and enable facets of this vision to be realized can be demonstrated in multiple areas. For example, three key areas where such opportunities for bridging gaps and for collaboration stand out are: employment, advanced research and development (R\&D) to meet societal goals and enabling platforms for knowledge and information. As illustrated by the following examples, the commitment to leave no one behind must be based on collaborative efforts which build on the strengths of all sectors. While government remains a key player in many of the initiatives, only a multi-actor approach can ensure that by 2030, the sustainable development goals are met for everyone.

Providing employment for all population groups and in all regions of the country

While unemployment rates in Israel are low in comparison to many other OECD countries, lowproductivity sectors employ many Israeli-Arabs and Haredim in low-quality, low-wage jobs, with their low skill level further contributing to disproportional poverty in their communities. People living in poverty often experience a blow to their dignity and to their right to equality in numerous areas. Furthermore, in many situations, poverty is accompanied by social exclusion - a process whereby many groups are marginalized with no chance of fully participating in their community's social life. This predicament is reflected both by fewer opportunities (for education, services, 
employment) and by a lack of symbolic capital and social transparency.

The Well-being, Sustainability, and National Resilience Indicators (2017 data, published February 2019) illustrate an overall increase in employment rate, median gross income of households from work, and satisfaction with work as well as a decrease in the prolonged unemployment rate. Yet, alongside these welcome developments, an increase in gaps between the Jewish and Arab populations in employment rate and involuntary part-time employment is also noted. The employment rate among Arab women has been substantially lower than that of Jewish women over the years. In 2017, the employment rate of Arab women was $26.4 \%$, compared to $63.2 \%$ for Jewish women. Moreover, there is a large gap in employment rate between Jewish and Arab women. In addition, $9.7 \%$ of employees felt discrimination at work. In another area, the Survey of Adult Skills (PIAAC) (2014-2015) revealed that 27\% of persons in Israel had high-level problem-solving skills in a computerized environment. This percentage was lower than the average in OECD countries (31\%). The percentage of Jews with high-level skills in this area (33\%) was substantially greater than the percentage of Arabs (4\%).

Over the years, Israel has developed programs to encourage employment among populations suffering from particularly high poverty rates. Some programs are specifically designed to include disadvantaged communities in the high-tech sector and foster participation in thriving high skill sectors of the economy. Other incentive programs elaborate the scope of solutions provided by Israeli innovation to specifically target inclusion and access to services and address unique challenges faced by disability.

Opening and propelling a conceptual shift in the high-tech industry

The Societal Challenges Division at Israel's Innovation Authority is working to expand the supply of human capital skilled in high-tech professions by removing obstacles and developing

\section{CASE STUDY:}

\section{The Diverse Startups - Incentive Programs for Haredim and Minorities}

The incentive programs for the ultra-Orthodox and minorities are sub-programs of the Early Stage Incentive Program of the Israel Innovation Authority. They aim to create an incentive for investment in early stage companies owned by minority or Haredi entrepreneurs that constitute a critical factor in encouraging the growth and development of the Israeli high-tech industry. The programs are intended for Israeli companies in all of the industry's sectors, at the beginning of their activities, interested in developing or upgrading innovative products, improving local production processes and continuing operation and growth in Israel. The program offers a grant of $75 \%$ of the project's approved budget in the first year and $70 \%$ in the second year for companies in which at least 33\% of the shares are owned by an entrepreneur from the Haredi or minorities sector.

The program enables young startup companies to raise funds during the product development stage as well as supplementary financing for up to six months after the project's approval. It also provides an attractive funding model, as it assumes the risks associated with the company's development stage but does not participate in future profits. Companies undertake to repay the funding received to the Innovation Authority via royalty payments from sales, but only if the project succeeds in reaching commercialization stage. 
suitable infrastructure. Among other activities, it is advancing the integration of underrepresented populations in industry. There has been a $20 \%$ increase in the number of initiatives supported through the program in 2018 in comparison to 2017.

Another example is the Digital Talent pilot program which opens up career paths in high-tech, assuring that the training provided is the most up to date to meet industry's needs, by integrating potential employers in the curriculum design process.

Yet another major challenge is meeting culturally sensitive barriers to inclusivity in the employment market. One example is a creative arrangement for providing employment opportunities for Muslim women in a Bedouin community in the south of Israel.

\section{CASE STUDY:}

\section{Digital Talent Pilot Program - An Entry Ticket to High-Tech Professions}

Emerging digital fields such as SEO, PPC, Social Media, and Data Analysis create 3,000 new jobs in Israel each year. The Digital Talent program takes advantage of this demand to quickly train Israelis from the most economically vulnerable communities in digital professions. This creates an affordable, accessible path for disadvantaged Israelis to enter lucrative careers in a high-tech environment where they can continue to learn and advance. A secondary purpose of the program is to draw on untapped human resources from populations that are not well represented in the Israeli high-tech industry: Arabs, Israelis of Ethiopian descent, Haredim, and Israelis with disabilities. For example, in 2017, the mix of participants included $30 \%$ from the Arab community, $9 \%$ from the Haredi community, 20\% immigrants from Ethiopia and $9 \%$ people with disabilities. The students from diverse backgrounds bring new perspectives to digital industries, combining different worlds.

Digital Talent also improves the ways in which the Israeli government sponsors and certifies training for digital professions by formulating the training curriculum according to the needs of Israeli employers. The training is conducted in top-of-the-line vocational colleges, and some of the curriculum is taught by representatives of industry leaders. The program provides updated curricula, advanced teaching methods, quality labs and staff to draw students with employment and study abilities.

The program identifies digital professions in high demand, quickly develops training, and supplies industry with qualified and quality workers. Students are exposed to basic digital marketing terms, going on to specialize in one of several fields of study offered and acquiring tools that prepare them for the workforce. The three specialization tracks are Content and Social Media, Data Analysis and Search Engine Optimization (SEO).

Students also provided with the opportunity to experiment with the tools they acquired, putting together a portfolio of their digital work that serves as their entry ticket to the industry. At the end of the program help is provided in finding work.

The program provides a dual response - for employers who need professional staff and for participants seeking to enter a world that is largely out of bounds to many. A digital committee made up of five leading companies, along with government officials from the vocational training unit, meet to discuss emerging needs in the market. These companies build the curricula for training in the new professions that are then taught as part of the Digital Talent courses.

The government has committed to take over the funding for each professional course that completes two rounds and demonstrates successful participation and placement. 


\section{Innovative Culturally Sensitive Solutions to Enable Work Opportunities for Muslim Women}

The Bezeq telecommunications group, in cooperation with the Ministry of Economy and Industry runs a call center inside a mosque located in the Bedouin village of Hura. The call center, which provides assistance to Internet customers, was established by the Ministry of Economy and Industry in collaboration with the non-government organization JDC-Israel as part of the effort to enable work opportunities and address female unemployment in the Bedouin community in the south of Israel. The call center provides a solution for Hura's female residents, who seek to enter the job market without having to work outside the community. Integrating the Negev's Bedouin population into the workforce is a national goal of the highest importance.

A number of barriers prevent Bedouin women from entering the workforce, resulting in their lowest employment rate of all Israeli populations. These include conservative traditions which discourage women from working away from home, high costs of child-care and lack of basic skills in computers, math and in Hebrew and English.

In overcoming these barriers, a guiding principle was to assure that decisions and solutions are developed from within the community, acknowledging that the residents' understanding can best cater to the cultural sensitivities, needs and skills that are required. In addition to providing the necessary training. Bezeq located the call center inside a mosque, where workers use a separate entrance from the worshipers. Moreover, a technological solution diverts calls from the call center to another center during calls for prayer.

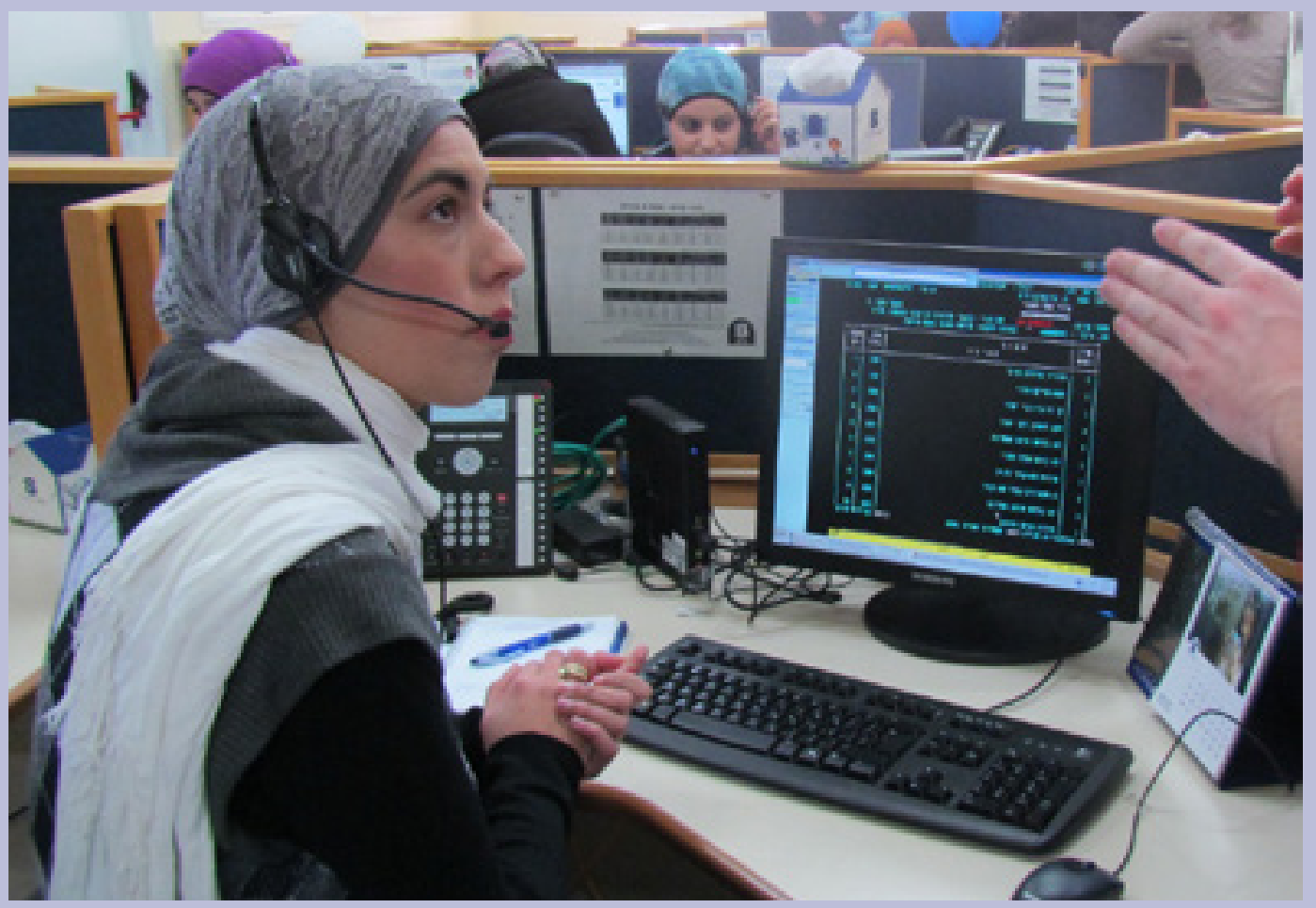

Bedouin women working at the call center/Photo: JDC-Israel 


\section{Encouraging R\&D to meet societal goals}

Israel encourages R\&D of assistive technologies for the disabled through a dedicated incentive program which contributes to their quality of life while easing their integration into society, the community and the labor market and resulting in significant financial saving on social and national benefits. The goal of the incentive program is to encourage $R \& D$ on industrial products that provide technological solutions for the disabled to enable them to integrate into society and the labor market. The program, the result of collaboration between the Innovation Authority and the National Insurance Funds, is intended for companies and non-profit organizations interested in developing technologies that serve groups with physical, mental or cognitive disabilities, who collectively represent up to $5 \%$ of the population. Under the terms of the program, an NGO project that is approved will receive $85 \%$ of the approved budget up to the maximum grant and is exempt from paying any future royalties. A commercial corporation's project that is approved will receive $65 \%$ of the approved budget up to the maximum grant if the corporation was established up to five years before submission of the request and $30 \%-50 \%$ of the approved budget if the corporation was established more than five years before submission of the request.
Enabling platforms for knowledge and information

Despite Israel's innovations and advanced technologies, the advantages and potential offered by the Innovation and the Digital Age have yet to permeate all parts of Israeli society and of the economy. The Digital Israel program (see Goal 10 ) is a key driver to addressing this digital divide and advancing equal opportunities and improved services to the public through the use of digital tools. A dedicated R\&D incentive program focusing on catalyzing the development of technological solutions catered for the public sector has been developed. It provides the opportunity to foster data driven innovation in the public sector, to improve digital infrastructure and the accessibility of state and local government services to the population, and to propel Israeli 'Smart Government' to the forefront.

Providing access to knowledge and information are central to successfully reaching the SDGs. Yet, there is a major gap globally in access to information on sustainability. Creative solutions that have been initiated in Israel include a dedicated science and environment news agency, providing journalists with free scientifically vetted stories, and penetrating and shaping the public discourse in Israel on crucial topics of sustainability and well-being.

\section{CASE STUDY:}

\section{Beit Issie Shapiro - Using Technological Innovation to Expand Opportunities for People with Disabilities}

Established in 1980, Beit Issie Shapiro (BIS) develops and provides innovative therapies and state-of-the-art services for the disabled, impacting on some half a million children and adults each year. Its vision is that every person with a disability has the right to equal opportunities and to active participation in society. Its mission is pioneering therapies and educational models of intervention aiming at improving well-being, independence and inclusion for children and adults across the entire range of disabilities. It promotes social change though a three-pronged approach: development and provision of cutting-edge services; advocacy; and research and training throughout Israel and internationally. 
The rapid advancement of technology in the last decade has opened up a world of opportunities for people with disabilities. Statistics show that only $10 \%$ of people with disabilities use technology, which can lead to exclusion and to the deepening of social gaps. In order to bridge these gaps, BIS focuses on two main tracks:

1. Enhancing entrepreneurship in the field of assistive technology (AT): BIS developed the first accelerator specializing in solutions for children and adults with various disabilities. Examples include: ATvisor - the first unbiased digital platform to aid rehabilitation professionals and people with disabilities in the sourcing and matching of AT, and Sesame Enable - an initiative that has developed hands-free control technologies for mobile devices, enabling people to use the smartphone with head movements only.

2. Establishing a Technology Center for the development and implementation of assistive technology models: In partnership with SAP Laboratories in Israel, five applications have been developed to date, including:

- IssieBoard - a customizable keyboard app developed for the iOS platform which allows for customization of the keyboard;

- IssieSign - an app for learning how to sign basic vocabulary, based on Israeli Sign Language (ISL) for expressing needs and ideas.

BIS has also established the Tech it Issie Blog in three languages - English, Hebrew and Arabic - in order to disseminate up-to-date and applied knowledge on the use of AT. AT innovations developed at BIS in the field of education and communication are now used by educators, therapists, clients and their parents across Israel and globally.

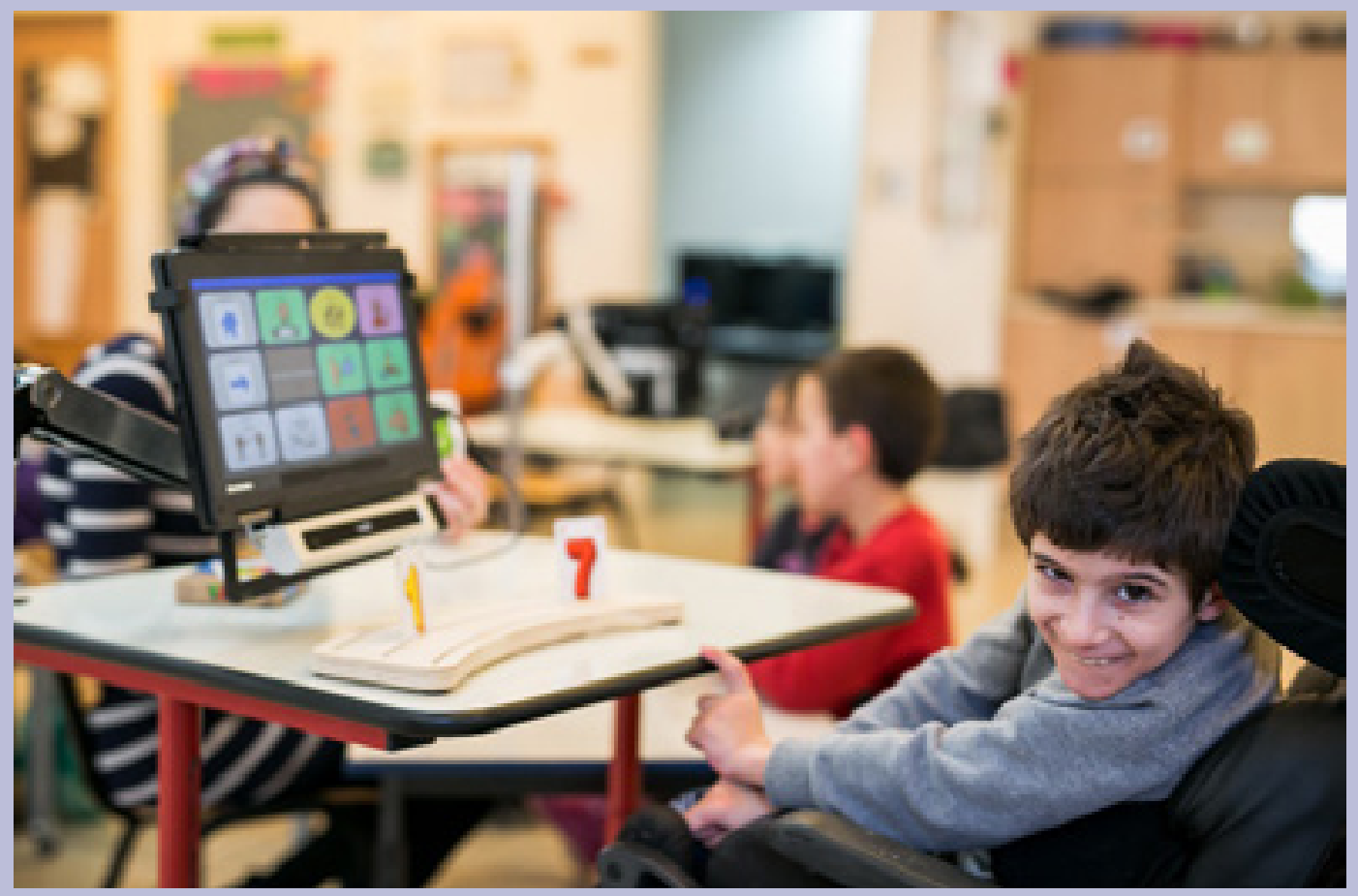

New opportunities for the disabled at Beit Issie Shapiro/Photo: Ronit Cohen 


\section{CASE STUDY:}

An Incentive Program for Digital Innovation in the Public Sector

The goal of the incentive program is to encourage entrepreneurs seeking to develop, manufacture, and commercialize products, services, and processes meet the challenges and needs of the public sector with innovative technological solutions. The program fosters:

- Improved public-government service for citizens;

- Streamlining of the public sector and public services sector;

- Improved public accessibility of information;

- Protection of public sector data systems.

The program offers participation in the risks involved in the development process and may allow a "field trial" on a large scale using national infrastructure. Success in proving the feasibility and selling to a government customer provides a springboard to other markets in Israel and the world. 


\section{CASE STUDY:}

\section{Zavit Israel's Science and Environment News Agency}

Zavit - Science and Environment News Agency is an online content platform providing media outlets and the public with cutting-edge Israeli environmental science news using simple and accessible language.

With more than 50 media stories per month, Zavit is a powerful tool that disseminates exclusive coverage of Israeli environmental science through various media outlets. Its online platform provides journalists with free access to exclusive stories, including scientific reports, interviews with scientists, videos, and interactive multimedia content, thus reaching over 400,000 readers per month worldwide.

To increase its impact, Zavit has launched Zavit in the Classroom, a pedagogical web-based platform that allows students and educators to access updated coverage of relevant scientific research, both in Hebrew and in Arabic. Zavit in the Classroom aims to enrich the existing curriculum with current, science-based content while increasing the environmental science literacy of teachers and students alike. Zavit's growing teachers' community includes more than 1,000 active teachers.

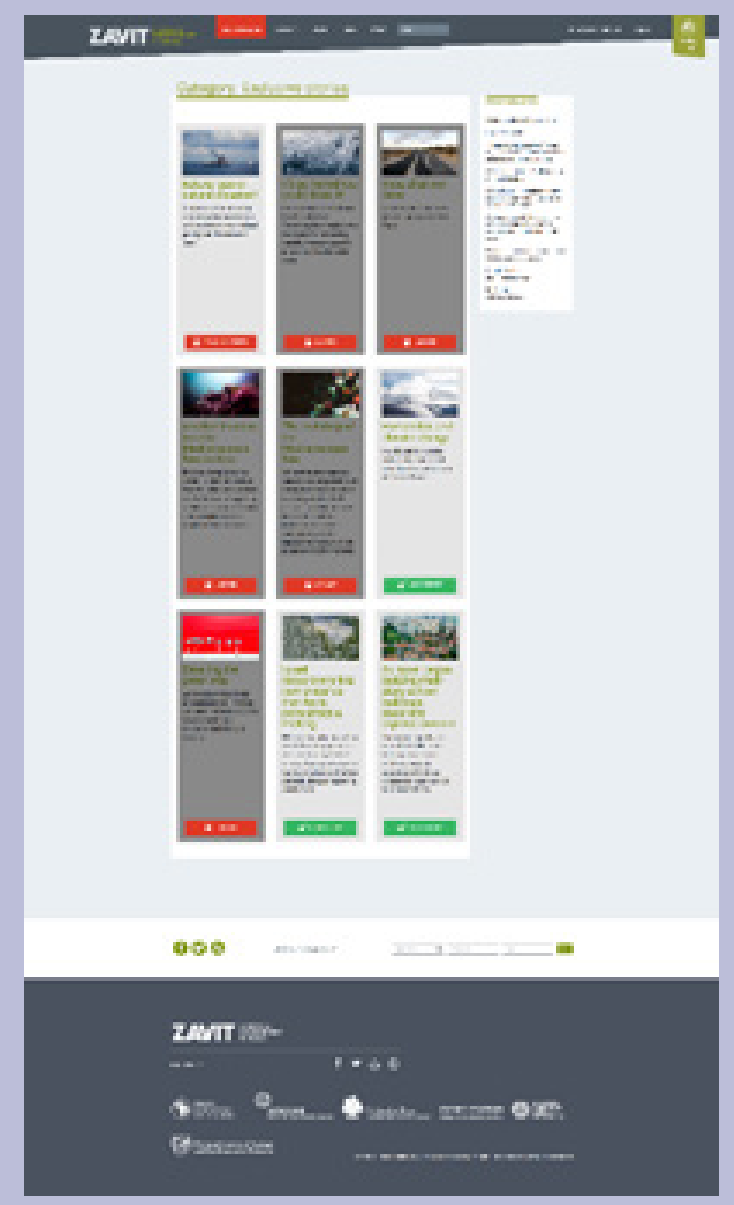

Zavit - Science and Environment News Agency/Photo: The Israel Society of Ecology and Environment 


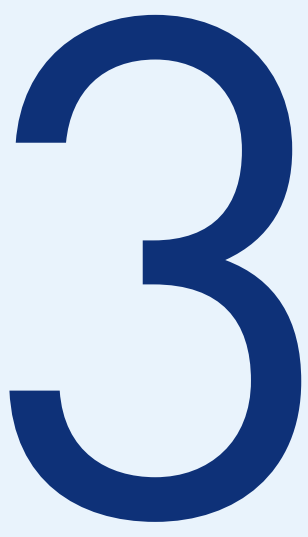

\section{GOALS AND TARGETS}

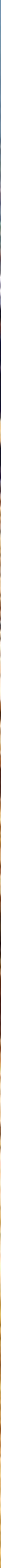




\section{GOAL}

NO POVERTY

Poverty in Israel

Access to financial services

mivinil

Earlier payment of the seniority supplement

Freezing of the plan to raise women's retirement age

Concessions regarding income tests for self-employed parents

Partnering for a Better World 


\section{END POVERTY IN ALL ITS FORMS EVERYWHERE}

Poverty is a multifaceted, complex social phenomenon with severe implications for all affected - individuals, families, communities and society as a whole. The United Nations, in its International Covenant on Economic, Social and Cultural Rights, stipulated that poverty is a human condition marked by an enduring or chronic dearth of necessary resources, capabilities, choices, security, and strengths that would enable a reasonable standard of living and additional civil, cultural, economic, political and social rights. Poverty may also prevent the full exercise of human rights. 


\section{( Poverty in Israel (SDG 1.2)}

In Israel, according to the current official definition of the poverty line, a person is defined as poor if their available income per household member falls below $50 \%$ of the median per capita disposable income in the country. However, in recent years, this definition has been an object of discussion and argument among economists, jurists, and policy experts in both Israel and other countries.
Israel has a relatively high rate of poverty and social inequality in comparison to other OECD countries. While the incidence of poverty lextent of poverty after transfer payments and taxes) per family decreased from $18.5 \%$ in 2016 to $18.4 \%$ in 2017 and 1,780,500 people (a decrease of 1.2\%) including 814,800 children (a decrease of $2.8 \%$ ) lived in poverty, in 2017, 466,400 families lived in poverty (a rise of $0.9 \%$ ) nevertheless.

Based on EU definitions of poverty and social exclusion, $27.8 \%$ of the population was at risk of

Figure 1.1

Persons below the national poverty line, 2005-2017

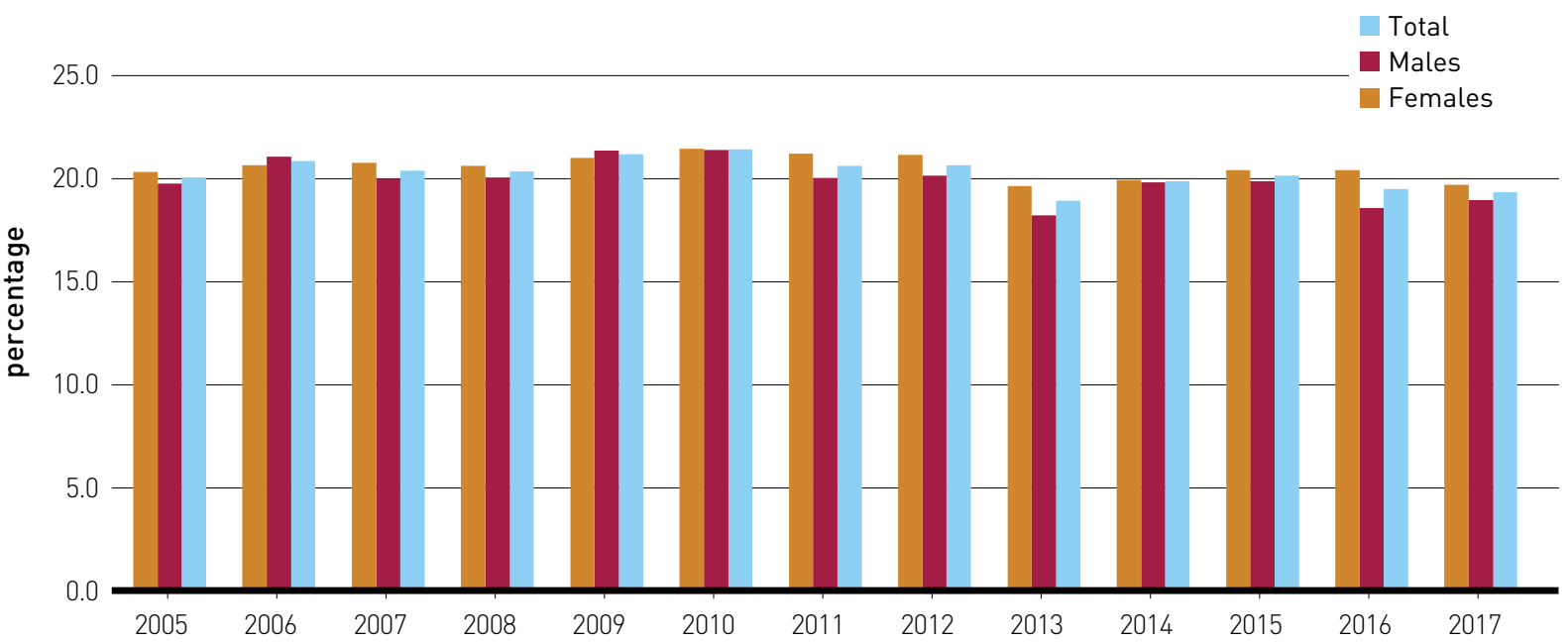

Figure 1.2

\section{Population at risk of poverty}

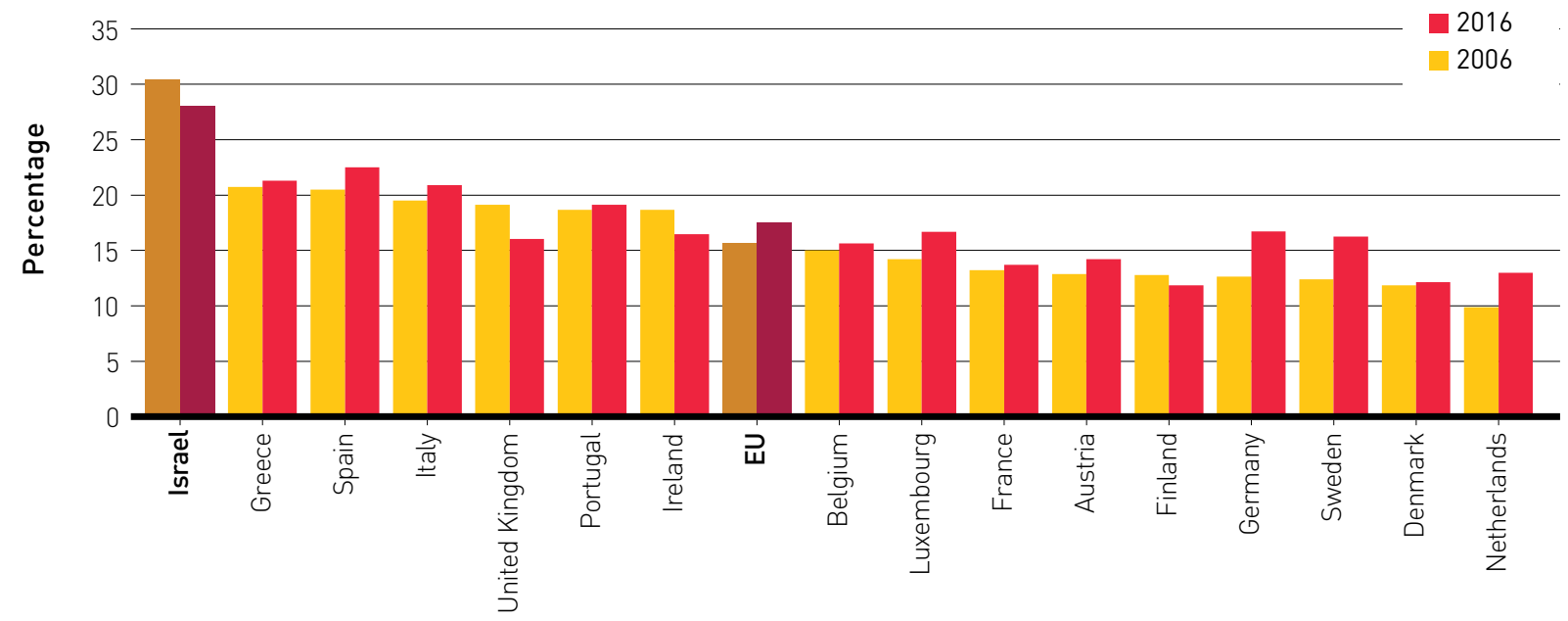


Figure 1.3

\section{Population at risk of poverty ages $0-17$ and $65+, 2016$}

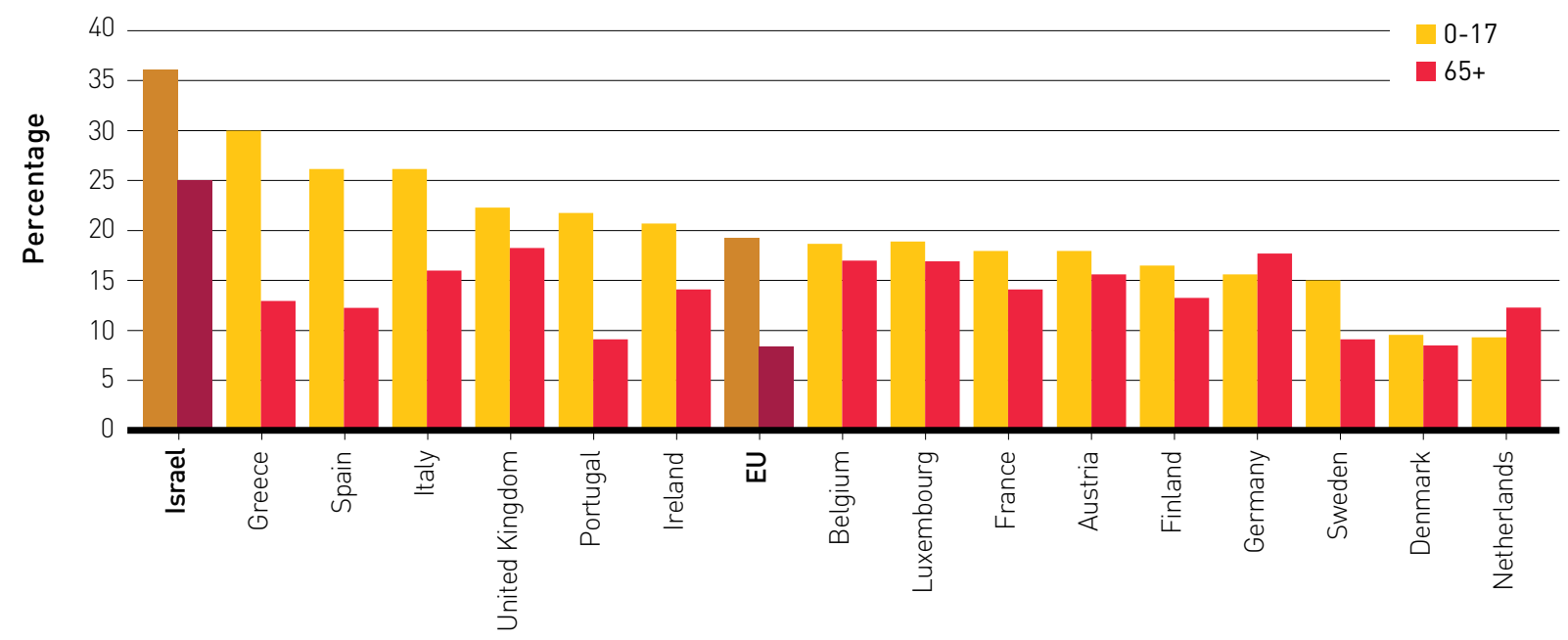

poverty in 2016, higher than the EU average (17.4\%). The net money income per standard person in the upper quintile was 7.3 times higher than the income in the lower quintile. This gap is higher than the average gap in the EU (Figures 1.2 and 1.3).

A number of bodies exercise authorities designed to aid in combatting poverty, each of which provides assistance in a different area:

- The National Insurance Institute (NII) is responsible for the payment of pensions and benefits. It plays a key role in the area of poverty as it defines both the poverty line and "the people living in poverty" who are eligible for assistance as stipulated by law. It is also responsible for regularly updating other ministries on eligibility criteria for a variety of benefits. Any delay or inaccuracy in the transfer of information can disrupt the continuity of entitlement and delay the receipt of benefits;

- The Ministry of Labor, Social Affairs and Social Services (MOLSA) deals with families in financial distress through its Service for Individual and Family Welfare, whose departments operate in local authorities throughout the country;

- The Ministry of Construction and Housing is responsible for public housing rentals and for housing allocations for those eligible;

- The Employment Service is responsible for labor market arrangements and assists the NII in locating candidates eligible for various benefits such as income support and unemployment insurance;

- The Ministry of Education provides assistance to children through the provision of study grants, funding of tests and scholarships, and rescheduling of parents' payments to schools and kindergartens;

- The Ministry of Justice offers free legal aid in civil cases that meet certain criteria;

- Reductions are granted by local authorities for city taxes, by the Israel Electric Corporation for electricity, and by the Ministry of Transport for public transport.

Over the years, Israel has developed programs to encourage employment among populations suffering from particularly high poverty rates, such as Haredim (ultra-Orthodox), Israeli Arabs, people on welfare and people with disabilities. These populations face various obstacles en route to the labor market, making it difficult for them to integrate into employment and leading to low income levels relative to the rest of the population. From the perspective of the past two decades, the poverty level affecting these populations has not been uniform, with severe pockets of poverty especially evident in the Haredi and Arab 
Figure 1.4

\section{Inequality and poverty remain high}

2016 or latest year available
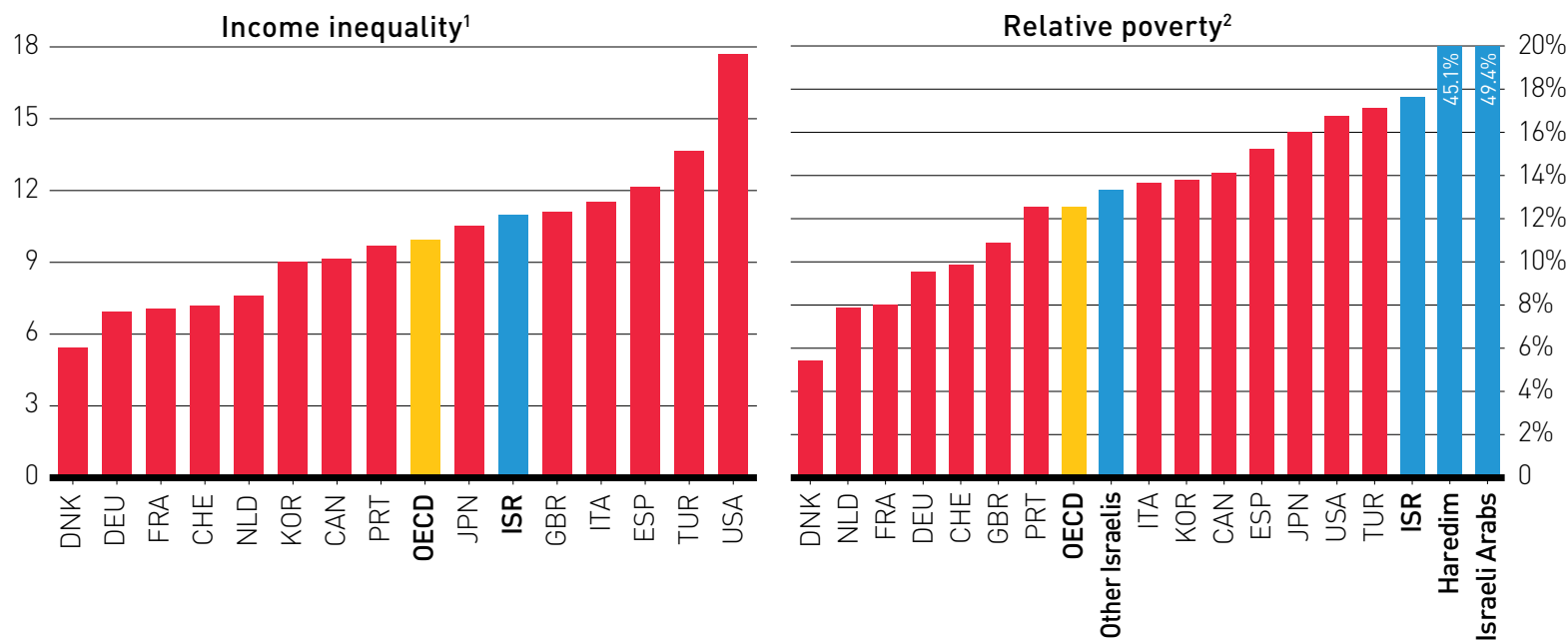

Defined as the S90/S10 disposable income share ratio, i.e., the share of all disposable income received by the top decile devided by the share of the bottom decile.

2 Poverty rate relative to threshold of $50 \%$ of median disposable income.

Source: OECD (2017), Income Distribution Database \& National Insurance Institute

populations. Together, the two comprise above $25 \%$ of the general population although their weight among those living in poverty has reached some $60 \%$. Among Israelis of Ethiopian descent too, the poverty rate is high, standing at $13.6 \%$. In families headed by a self-employed parent, the poverty rate is $29.8 \%, 1.6$ times the rate of the general population.

Despite growth in employment figures, which has also benefitted the income of the poor, the share of the working poor in Israel has continued to rise. According to the OECD Economic Survey of Israel for 2018, workers from such groups as Israeli Arabs and Haredim remain in low-paid jobs because of their weaker skill sets. They are often trapped in low quality jobs, leading to continued inequality and implications for child poverty in large families, which are common in these groups.

\section{Addressing poverty in Israel}

Over the years, diverse services have been developed and incorporated to help people cope with personal and family distress, exacerbated by poverty and exclusion. These included fostering of children, preventing and treating violence in the family, rehabilitating addicts, and more. Israel's policy has had major impact on how the social services perceived their role. In the past, poverty was considered a social problem outside of their purview and people living in poverty were not a specific target population. They focused, instead, on individual-family assistance. Most of the efforts to cope with the problem of poverty in Israel have been undertaken by the MOLSA and by the NII by changing the eligibility categories for benefits and by developing services to the poor.

In recent years, economic and social processes in Israel and around the world have led to the recognition that it is vital to address the phenomenon of poverty in its larger context, and it is necessary to develop practical programs to curtail it. Consequently, new measures and plans were adopted. In 2010, MOLSA formulated a policy that defined the population living in poverty as one of high priority for intervention. It undertook to formulate an overall policy to assist communities, families, and individuals living in poverty and, concomitantly, to strive to prevent their slide into an 
Figure 1.5

\section{The share of working poor is high and increasing}

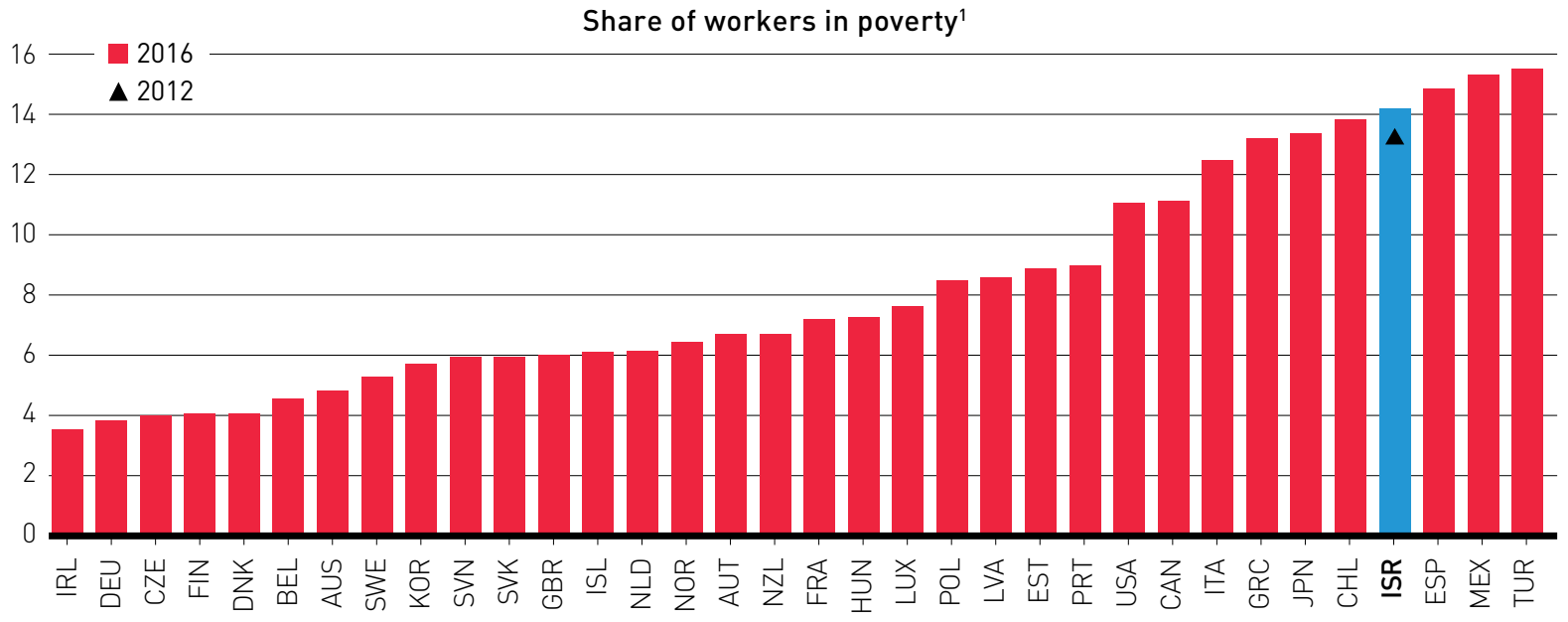

Those with income below the poverty line, living in households with a working-age head and at least one worker. Source: OECD (2017), Income Distribution Database

enduring dependency on social services by, among other things, integrating excluded populations, people with disabilities, and the newly unemployed. MOLSA began to develop responses resting on experience in the field, such as:

- Assistance centers developed by the Jerusalem Municipality and expanded to additional authorities with the help of the NII's Demonstration Projects;

- An innovative program for its time, known as "From Work to Well-being," that addresses the newly unemployed;

- The "Eshet Chayil" (Women of Valor) Program to empower Arab and immigrant women for employment.

In addition, MOLSA participated in developing and applying programs led by civil society and philanthropic organizations and social services, with a focus on promoting employment when the family is perceived as the unit of intervention, such as:

- "Our Responsibility - Their Independence," led by the "Shoulder to Shoulder" Association;

- The Hadijda Program to promote employment among Arab women in the city of Rahat;

- The MAPA program (Hebrew acronym for Family
Meets Opportunity), a program for students at Ben-Gurion University of the Negev in cooperation with the Beersheba Municipality, focusing on interventions in poverty-aware social work practice with families living in extreme poverty and profound distress.

In 2013, the Committee for the War on Poverty in Israel (the Alalouf Committee) was appointed by the Minister of Labor, Social Affairs and Social Services, with the participation of representatives of academia, civil society, the government and the Union of Local Authorities. It presented a comprehensive program to address poverty on different planes with numerous recommendations for the government as a whole and particularly MOLSA. At the cornerstone of the committee's work was the adoption of a broader concept of poverty that not only focuses on income but on the broader concept of multi-dimensional poverty. Simultaneously, there was growing recognition at MOLSA that although poverty is a social phenomenon, it had to be addressed by recruiting all government ministries and civil society and that the best way to lead significant change was through a government initiative to propel local authorities to address poverty. Consequently, it 
initiated the development of an array of services to intervene with people living in poverty and exclusion. The Social Service Departments of local authorities were identified as the natural choice to implement the new provision. Their location, authority, and knowledge qualified them to coordinate and connect all the important bodies operating in local authorities to advance people living in poverty and exclusion.

\section{"Noshmim Le-Revaha"}

The "Noshmim Le-Revaha" programs were launched in 2015 at MOLSA's initiative and funding. MOLSA harnessed two NGOs - JDC-Israel and the Rashi Foundation - to the process of program development, to a strategic partnership in the implementation of responses, and to participation in funding.

The goals of the flagship program include:

- To decrease the level of poverty and increase financial well-being;

- To improve family functioning and relations;

- To reduce dependency on social services and broaden natural support circles;

- To promote integration into the community.

An array of social services is provided through two integrated programs: "Noshmim Le-Revaha" and Otzma Center (Empowerment Center). Their role is to help people living in poverty and exclusion to cope with the realities of their lives, taking account of their needs, circumstances and resources. Characterized by a daily struggle to overcome multiple obstacles, these people have few opportunities to change their situation and are hard put to exercise their rights and utilize the resources of the community and the State.

The provision of services within the "Noshmim LeRevaha" at Otzma Center programs is designed for participants who, in the assessment of social workers, do not suffer from significant functional difficulties and are not in a state of crisis. Rather, these people are confronted with difficulties on various levels: service consumption and the exercise of rights, transient financial troubles, multidimensional, enduring poverty, loneliness or lack of a support group, housing problems, income problems, lack of basic necessities, food insecurity, management of the household budget, and more.

"Noshmim Le-Revaha" is a family intervention program, limited to two years, and based on the recognition that the family is the central axis in the process of change as it shapes the desired future and determines the ensuing aims. The program staff - a program social worker and a family support person - defines the expected outcomes together with the family and charts the intervention program targeting the change. The changes to be achieved must include at least two areas of intervention from a choice of six: acquisition of tools for the exercise of rights, improvement of employment status and income, improved efficiency of household management, better sense of individual and family capabilities, the making of a community that serves as a resource, and improvement of basic living conditions. In addition, the family is provided with a flexible basket of responses designed to help remove obstacles and acquire resources.

The array of services offered by "Noshmim LeRevaha" and Otzma Center includes:

- Specific resources to cope with diverse family needs: a family support person, a flexible basket of services consisting of material assistance aimed at removing obstacles and providing opportunities, and group and community programs;

\section{- Training and instruction aimed at instilling} program concepts and utilizing resources wisely: recognition of the family's unique characteristics, needs, and resources with regard to its financial situation, employment, exercise of rights, management of household budget, and integration into the community;

- Structured outcome-oriented models of intervention for different areas of the program: a model for the active exercise of rights, promotion of employment and utilization of the community as a resource.

Noshmim Le-Revaha at Otzma Centers currently operates in 94 local authorities countrywide, selected according to criteria defined by MOLSA. Of these local authorities, 45 are Jewish of which four have a Haredi 
population, 44 are Arab, and another six are mixed. Despite their scope, the services constitute a pilot program aimed at examining interventions with people living in poverty and exclusion who are registered with the social services. The results of the pilot will guide MOLSA in future processes of intervention.

\section{Benefits increased by income supplements}

As part of the recommendations of the Committee of the War on Poverty, the NII increased senior citizen benefits by the addition of an income supplement to bridge the poverty line gap. Following are some recent initiatives aimed at increasing benefits to populations at need:

- Benefits Adjustments: In addition to the income supplement, the benefits to individuals, families and couples were adjusted to the poverty line in December 2015, retaining the three distinct age groups $(-70,70-79,80+)$;

- Budget Arrangements Law 2017-18: According to the law, benefits increase gradually over two years by various sums according to family composition and age group. The Income Supplement Law was amended so that the table itemizing the benefit rates shows an increase of $7 \%$. The sum not included as income-from-work for the purposes of calculating benefits was increased by $1.5 \%$ of the average income to $21.5 \%$ for an individual, and $25.5 \%$ for others.

\section{( Expanding eligibility for a heating grant for recipients of income supplements (SDG 1.4)}

Since the winter of 2017-18, a uniform sum has been paid by the NII as a heating grant to all recipients of an old age and widow(er) benefit augmented by an income supplement, or to recipients of an extra benefit for disability. Until 2016, only recipients of an income supplement or extra benefit for a disability who lived in "cold areas," as defined by law, had been eligible for the heating grant. Since 2016, recipients not living in cold areas are eligible for a reduced grant.

\section{( Access to financial services (SDG 1.4, 8.10)}

The Knesset (Israel parliament) enacted the Control of Financial Services (Regulated Financial Services) Law in 2016. Its goal is to create "regulation that is as inclusive, comprehensive and coherent as possible, for the entire financial service provision market and the entities active in it." The law includes lenders and others providing financial services, which were not regulated previously, and is designed to increase competition, expand the market and ensure a fair interest rate. A process is also underway to manage the Gemachim, the social bodies that serve as the source of financial services and mutual support in the Haredi lultraOrthodox) communities in Israel.

\section{( Earlier payment of the seniority supplement (SDG 1.3)}

According to the Budget Arrangements Law 2017-18, the seniority supplement for recipients of benefits under the National Insurance Institute (NII) Law, which was formerly paid from the $11^{\text {th }}$ insurance year on, is now to be paid from the first insurance year. The change will take effect gradually over four years: in January 2017, the supplement was paid from the $10^{\text {th }}$ year on; in 2018 - from the ninth year; and in 2020 - it will be paid from the first year. According to the Budget Arrangements Law 2019, the two payments for 2018 and 2019 are to be merged so that the 2018 supplement will be paid from the fifth year on and in 2019 - from the first year.

\section{( Freezing of the plan to raise women's retirement age (SDG 1.b)}

Women's retirement age was to be raised from January 1, 2017 from 62 to 64, but the measure has been deferred. There is consensus that raising the retirement age is economically desirable but discussions are still taking place on provisions to assist those who may be adversely affected.

It was also decided that the eligibility of women 
for an old age pension (under the NII Law) would be the same as the retirement age under the Retirement Age Law.

\section{( Concessions regarding income tests for self-employed parents (SDG 1.b)}

In January 2017 the income tests determining eligibility for income supplements were eased. For self-employed mothers receiving a pension with an income supplement, the offset rate of income- from-work against a given sum was decreased from $60 \%$ to $25 \%$ for a gross salary of up to NIS 3,270 . Beyond this sum, the offset rate remained $60 \%$. The program is to operate as a temporary order for two years in order to examine its impact on the rate of employment and income. The women may receive an employment grant from the tax authorities only if the addition to their benefit following this change is lower than the employment grant. The difference is to be paid as an employment grant by the tax authorities.

\section{CASE STUDY}

\section{Smallholder Horticulture Project in Ethiopia}

The Smallholder Horticulture Project is a Joint Technical Cooperation Program between Israel, the United States and Ethiopia which is implemented by MASHAV, USAID and Ethiopia's Ministry of Agriculture. The project aims to promote economic growth in rural areas and improve smallholders' income and livelihoods by strengthening the commercialization of horticulture production through the competitive and sustainable development of the avocado sector. It is designed to access new domestic and avocado export markets, as well as to provide technical support to improve phytosanitary regulation for Ethiopian horticultural crops.

Implementation is based on demonstration, innovative technologies, training and capacity building, transfer of know-how and management, and application of a "training-of -trainers" approach. It includes:

- Introduction of new high-quality Israeli avocado varieties for the domestic and export market

- Provision of quality training and consultancies in Israel and Ethiopia

- Capacity building for nursery and tissue culture lab management and extension

- Establishment of tissue culture laboratories

- Establishment of new nurseries and demonstration plots for the propagation of avocado trees

- Elaboration of professional material

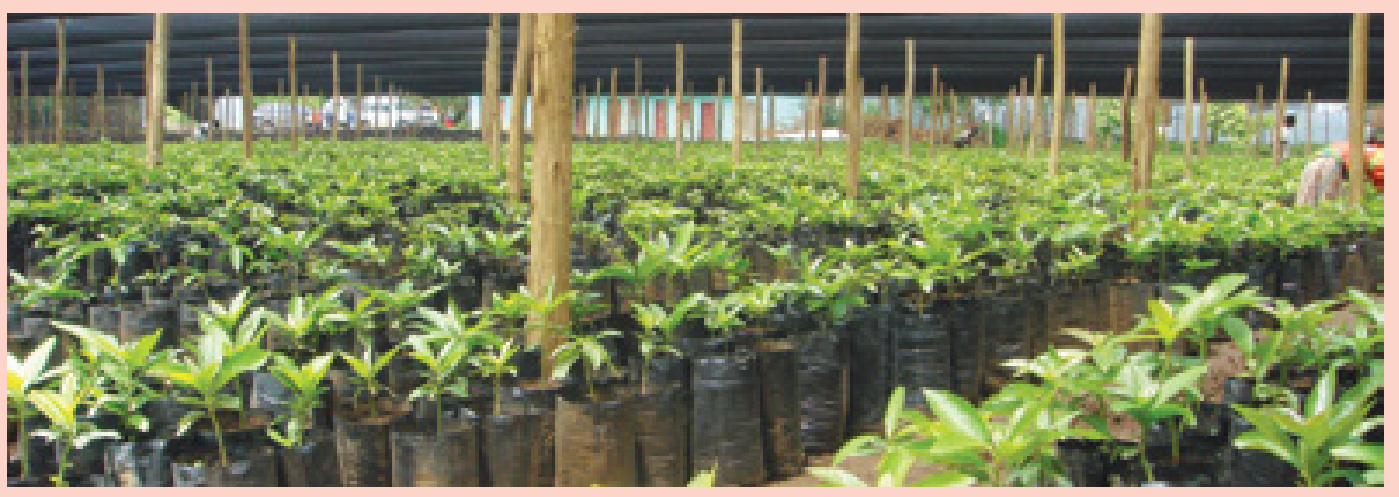

Source: MASHAV 


\section{PARTNERING FOR A BETTER WORLD}

When cooperating bilaterally with a partner country or region in an effort to work towards ending poverty, Israel's overseas development assistance bases its programming on the national development plans and policies of its cooperating partners. In developing countries, for example, national plans are designed to meet the criteria of international development banks, aid organizations, and global business communities in order to receive loans for priority projects. The donor community understands the benefits and is assured of the government's political will and commitment. A national development agenda also reduces wasteful overlap and competition through more effective coordination and transparency in the programs.

Overseas development assistance is a fundamental component in the global effort to end poverty in all its forms everywhere. Joining the consensus on the adoption of the UN Resolution on the Implementation of the Third United Nations Decade for the Eradication of Poverty (2018-2027), Israel reiterated its full commitment to the 2030 Agenda for Sustainable Development, considering it an historic opportunity to make a decisive push against poverty.

Eradicating poverty (SDG 1 ) is the greatest responsibility for all who value human rights. Alleviating the suffering of the extreme poor, the marginalized, the disenfranchised, and the most vulnerable is more than a global challenge - it is a universal moral imperative. Israel pledges to commit its resolve and resources to realize this Goal.

Israel has launched dozens of programs and cooperation agreements over the years that are ultimately aimed at ending poverty in all its forms everywhere. For example, the President of Liberia visited Israel (February 2019) accompanied by a delegation of Ministers including Finance and Development Planning, Agriculture, Health and Mines and Energy. Both countries decided to strengthen cooperation, based on the principles of the National Development Plan of Liberia - the Pro-Poor Agenda for Prosperity and Development
(PAPD) 2018-2023. The two countries agreed to enhance collaboration particularly in the fields of agriculture, health, capacity-building, energy, and infrastructure. (SDG 1.b, 17. 6)

All of Israel's international assistance activities, in fields such as food security, education, health, gender equality, and more, are designated to help alleviate poverty, and are therefore described in the following chapters. 


\section{CASE STUDY}

\section{Horticulture Center of Excellence in Rwanda}

As part of a bilateral initiative between the Government of Rwanda and the State of Israel, MASHAV and Rwanda's Ministry of Agriculture and Animal Resources, through the Rwanda Agricultural Board, officially inaugurated (April 2019) a project to establish a Rwanda-Israel Horticulture Center of Excellence at the Mulindi Expo Ground in Eastern Kigali. The Horticulture Center of Excellence was conceived as part of Rwanda's Economic Development and Poverty Reduction Strategy II, which identifies the agricultural sector as having great potential to reduce poverty and ensure inclusive growth. It will serve as a regional demonstration and capacitybuilding center to increase vegetable and fruit yields, enhance productivity and improve quality, with emphasis on building local capacities in agriculture and agricultural entrepreneurship.

Rapid agricultural growth requires the introduction of new technologies and the dissemination of agricultural knowhow, together with a strong emphasis on increased agricultural yields and crop diversification particularly by smallholder farmers. The Center, therefore, provides a suitable platform for the rapid transfer of knowledge on modern Israeli agro-technologies and agricultural best practices to farmers.

Implementation of the project is accompanied by a MASHAV long-term expert and includes the following:

- Professional training programs conducted both in Israel and Rwanda

- Introduction of improved quality varieties

- Production of vegetables in greenhouses, walk-in-tunnels, net house and open areas

- Postharvest techniques for improved quality and longer shelf life

- Utilization of modern irrigation and fertigation systems and their adaptation to local conditions and farmers requirements

- Provision of quality training and consultancies

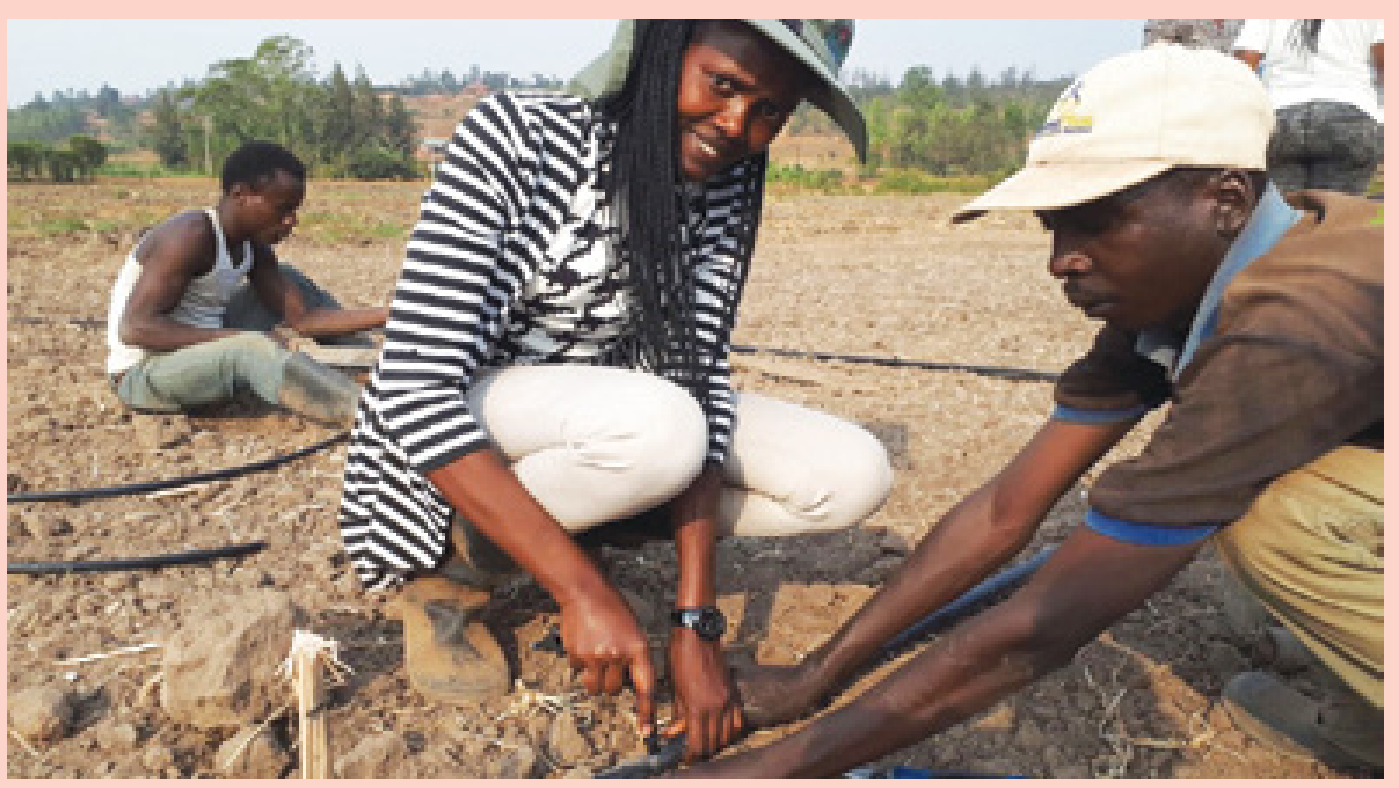

Source: MASHAV 


\section{GOAL}

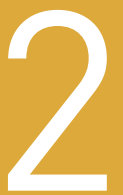

\section{ZERO HUNGER}

«s
Prevalence of food insecurity, based on the Food Insecurity Experience Scale

The National Project of Nutrition Security

Supporting agricultural productivity and sustainability

Maintaining the genetic diversity of seeds

Support mechanisms and export subsidies

Partnering for a Better World 


\section{END HUNGER, ACHIEVE FOOD SECURITY AND IMPROVED NUTRITION AND PROMOTE SUSTAINABLE AGRICULTURE}

Many developed countries today are confronted with the problem of inadequate nutrition due to economic difficulties. In contrast to developing countries, where food shortages may be severe and result in serious, lifethreatening malnutrition demanding emergency medical intervention, in developed countries - such as Israel - the problem is defined as "a lack of regular access to a sufficient quantity of nutritious food permitting a healthy, active life and the fulfillment of one's developmental potential." Nutrition insecurity is greatly affected by the dimensions of poverty and the trend of the poverty rate. The main obstacle to achieving food security in the Western world relates to a household's financial ability to purchase food. 
Chronic undernutrition is associated with impaired cognitive ability and reduced school and work performance. In addition, there is "hidden hunger" among overweight and obese children and adults, and this has emerged as a new and challenging issue. Eating behavior marked by a preference for ultra-processed foods over healthier ones also causes nutritional deficiencies in Israel. Worldwide, as in Israel, the proportion of children under age five who are overweight has increased dramatically.

The aim of current policies in Israel is to ensure that everyone everywhere has sufficient good quality food to lead a healthy life. Achieving this goal requires better access to food and the widespread promotion of sustainable agriculture.

Israel's vision for its agricultural sector is to maximize production and contribute to rural and economic development, while protecting scarce land and water resources, reducing pollution and waste and contributing to biodiversity and landscape conservation. Despite limited availability of land and water, agricultural productivity has grown dramatically since the early 1950s with ongoing resource efficiency gains per unit per cubic meter of water.

The agricultural sector is based on sciencelinked technology, with government agencies, academic institutions, industry and cooperative bodies working together to meet and overcome challenges. Agricultural research is translated into programs for the protection of open landscape, soil conservation, beneficial farm practices, efficient water use and a host of technological advances that have helped make Israel's agricultural production efficient and sustainable.

\section{Prevalence of food insecurity, based on the Food Insecurity Experience Scale (FIES) (SDG 2.1)}

The 2016 Food Security Survey is the third survey carried out in Israel by the Research and Planning Administration of the National Insurance Institute of Israel (NII). The findings show that in 2016 , $82.2 \%$ of families were food secure, and $17.8 \%$ experienced food insecurity. The 2016 survey reveals an improvement in food security in the population compared to the previous 2011-2012 survey. The trend of improvement among the population as a whole is supported by a drop between the two periods in the proportion of families and individuals living in poverty. This decline is well reflected among families with children, where the level of food insecurity dropped from rates of around $30 \%$ to around $20 \%$.

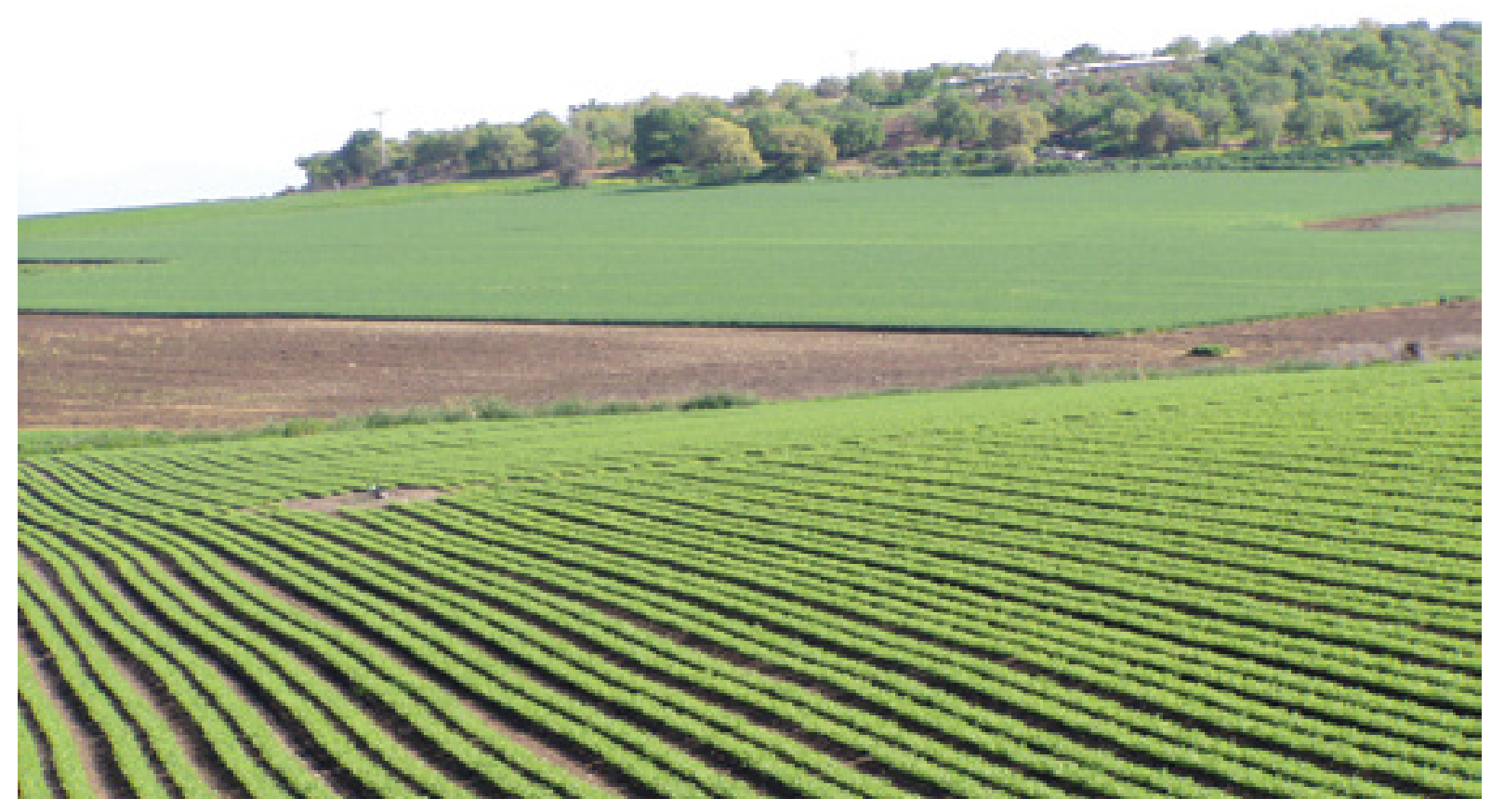

Field crops in the Jezreel Valley/Photo: Anat Lowengart 
Similarly to previous findings, significant differences were found among various groups. Among the Arab population and among recipients of subsistence benefits and single-parent families, the percentage of those with food insecurity was highest. As to the persistence of food insecurity, findings exhibit that most families (92.4\%) found to have food security five years earlier were food secure in 2016 as well. However, among those with food insecurity in 2011/12, slightly less than half (44.7\%) remained so in 2016. In the report, questions were added regarding food intake among those interviewed. The findings do not show significant differences in food patterns between families living in food security and those with food insecurity.

\section{The National Project of Nutrition Security (SDG 2.1)}

The National Project of Nutrition Security provides assistance to families suffering from severe nutrition insecurity through year-long financial support to purchase food. The families in the project enjoy regular assistance on a monthly basis by means of a rechargeable electronic card (not valid for cigarettes and alcohol), a package of fruits and vegetables and a package of dry goods. The baskets are delivered directly to a family's home. Nationwide, the project operates in 36 local authorities.

Receipt of assistance is dependent on meeting three criteria:

- The first means test: A family's eligibility is checked based on a household's total income against its "hard" expenses, such as rent/ mortgage payments, city rates, electricity, water, gas, daycare, medication, disability expenses (for recipients of a disability pension) and (defrayed) debts. After subtracting the hard expenses, the system checks whether the remaining sum is sufficient for the purchase of food, taking into account the family's composition.

- The second means test: The likelihood of continuing crisis, the level of earning capacity, receipt of a maintenance allowance, and various indices indicating the chances of continuing crisis are checked.
- The non-prevention test: The system checks whether a family's income is no higher than 1.5 times the poverty line. Many of the families in the project (some $40 \%$ ) are single-parent and a high percentage are Arab or Haredi (ultra-Orthodox) who are over-represented in the population living in poverty.

The duration of the assistance depends on a family's characteristics. Families characterized by ongoing deprivation (typical families requiring social services suffer from chronic distress) receive assistance for two years. At the end of that period, the data are checked again, the documentation is updated, and eligibility for continuing aid is examined. Families suffering from a temporary crisis la family that has to date managed on its own but needs assistance due to an acute crisis, with the expectation at intake that the crisis will come to an end), receive up to six months of assistance.

In the framework of the project, hands-on workshops are held to empower families and supplement what they lack to the extent possible. A change of atmosphere and the provision of "quality time" to families may grant them the strength to get ahead in the future. Family workshops include study/learning days on topics such as the exercise of rights, family and individual support, etc. Another goal emerging from the workshops is the consolidation of support groups for families accompanying the nutrition assistance. The workshops help ensure that every family receives the necessary information to consume healthy food, feed and provide all household members with proper nutrition until sated and reduce the morbidity prevalent among people suffering from severe food insecurity; to raise the families' awareness of wise consumerism and intelligent, optimal utilization of their resources, thereby safeguarding nutrition security as much as possible; and to ascertain that a family knows how to fully utilize its pantry products for continued benefit throughout the month.

The findings of a recent evaluation study showed that despite the relatively low level of assistance offered within the framework of the project, there was a statistically significant improvement in 


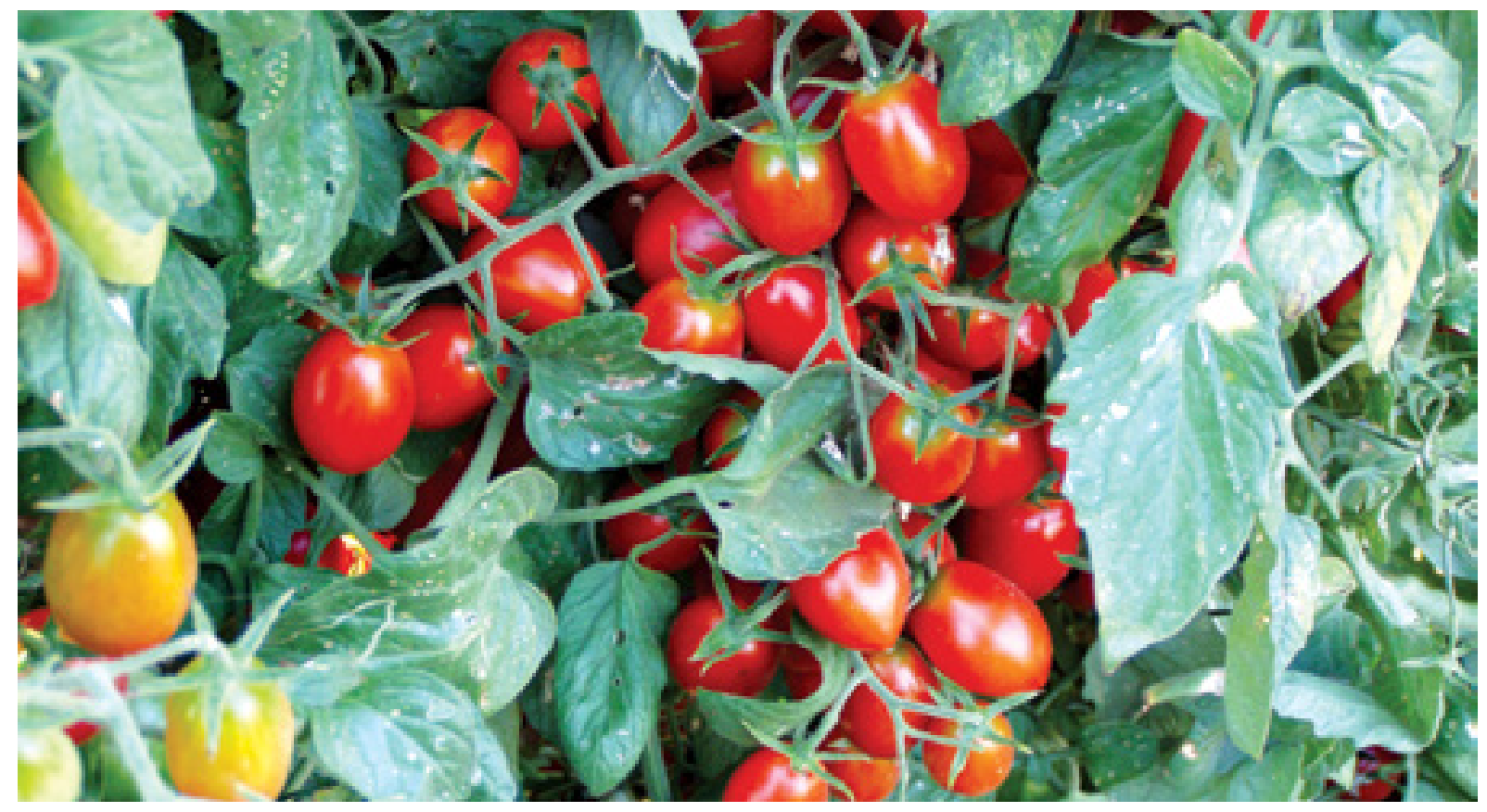

Cherry tomatoes/Photo: Anat Lowengart

nutrition and the dimensions of poverty diminished among participating families. At the same time, many families still suffer from nutrition insecurity in light of the fact that their points of departure were very low. Presumably, more profound and extensive improvement will be achieved by raising the assistance sums. The study also showed that the participants consumed few harmful foods, a finding related to their participation in the workshops and their high satisfaction. Beyond the financial aid, the project raised the participants' general level of satisfaction with life.

The project began as a pilot in 24 cities and local authorities in 2012-15. In February 2017 it was officially launched by the Minister of Labor and Social Affairs and his partners, and in October 2017, another 12 local authorities joined.

\section{( Supporting agricultural productivity and sustainability (SDG 2.3, 2.4)}

The government is supporting projects for increasing the productivity and sustainability of agriculture including the adoption of new technologies in the field of precision agriculture, soil conservation management, and regional projects of agriculture and environment. Various support measures are implemented to increase agricultural productivity by research funds, grants for investments, agricultural extension service and sector-specific reforms (e.g., the dairy sector).

The contribution of the agricultural sector to national greenhouse gas emissions is 3.2\% (based on a 2014 Israel Central Bureau of Statistics report). In 2017, the Investment Administration of the Ministry of Agriculture and Rural Development (MARD) continued to support and promote the agricultural sector to treat agricultural waste in order to mitigate the damages caused by the waste to the environment as well as to human health, and to transform the byproducts into usable recycled products or as raw material for renewable energy production. In addition, the Investment Administration provided support for the reduction of waste transportation to landfills and promotion of waste treatment as close to the source as possible. Support was provided for the establishment of animal waste treatment facilities (carcasses and excrement of poultry, fish, sheep and pigs), treatment of tree branches (pruning) to prevent the severe environmental hazards resulting from charcoal production, and construction of facilities for the treatment of dairy effluents in order to comply with sewage quality regulations. 


\section{CASE STUDY}

\section{Healthy Nutrition as a Right for All}

The Ministry of Health $(\mathrm{MOH})$ in cooperation with the National Food Security Council has approached all governmental and non-governmental organizations involved with food support (donations) to improve the quality of their food donations to meet the recommendations of the Mediterranean diet, by replacing unhealthy products with foods of higher nutritional value. A "Healthy Basket" was calculated, to meet the nutritional needs of recipients.

An additional inter-ministerial initiative of the $\mathrm{MOH}$ and the Ministry of Agriculture is dealing with the future planning of Israeli agriculture and aims to identify the type of agriculture to be encouraged for better health of the population. Two current programs include:

- Launching of a fruit and vegetable campaign - "Five Colors a Day."

- Promoting community and hospital vegetables gardens in cities and mental health hospitals. In order to effect change, a national program for an active and healthy life known as "EfshariBari" (Healthy is Possible) has been initiated.

Yet another issue relates to the need for food fortification, partly due to the very extensive use of desalinated water in Israel, which lacks magnesium, fluoride, iodine and other minerals. A study conducted in Israel showed that $60 \%$ of children and more than $80 \%$ of women suffer from iodine deficiency. A committee on the formulation of food fortification policy was established and its recommendations will be implemented shortly for vitamin D in milk, iodine in salt, magnesium and fluoride in water, and in the future for folic acid and vitamin B12 in flour.

Additional programs aimed at improving nutritional status and the nutritional quality of food served in institutions and to other captive sub-populations for preventing malnutrition and improving health and wellbeing include:

- Annual inspections in health care facilities to check on the amounts and types of food served;

- Development of guidelines for meal services in hospitals and other care settings lold age homes, sheltered housing, day care centers for special needs children), with an emphasis on nutritious food and reduction of food waste;

- Pilot program to improve health and nutrition status among residents of mental health hostels to postpone the onset of diabetes through better nutrition and physical activity;

- Promotion of legislation limiting sales of foods of poor nutritional quality in schools, at summer camps, and in hospital vending machines;

- Recommendation by a national committee of the MOH in 2018 for legislation banning advertisements of harmful foods that are aimed at children;

- Active involvement with the Ministry of Education to improve nutrition education in schools;

- Active participation in the ONCA (Optimal Nutrition Care for All) Pan European program, with emphasis on improving nutritional status among the undernourished elderly, in particular those institutionalized, including the development of a unified texture for institutional patients with chewing problems and the development of a teaching model for all first contact professionals at health care settings to detect risk for malnutrition; 
- Adaptation of the IDDSI (International Dysphagia Diet Standardization Initiative) to improve the suitability of food served in institutions and avoid dysphagia-related malnutrition and adverse events;

- Introduction of a new role - "Food Service Dietitian" - in hospitals to improve the nutritional quality of food, reduce hospital-induced malnutrition, and reduce food waste;

- Addition of dietitians to the multi-disciplinary staff at Well Baby Clinics to improve nutrition guidance given to mothers of infants;

- Involvement with "The Natural Step," an organization focused on reduction of food waste and increase in sustainability;

- Active involvement in the Israel Forum for Sustainable Nutrition;

- Research and programs on identifying needs within sub populations, including vulnerable populations, with particular focus on preparing culturally correct programs for the Arab population;

- A front of pack regulation for positive and negative labeling scheduled to be launched on January 1, 2020 for the purpose of promoting reformulation of packaged foods to help people choose healthier products at the point of purchase.

As part of the mission to improve food supply and enable better nutrition for all, dietary guidelines were recently published by the $\mathrm{MOH}$, which incorporate all elements of the Mediterranean diet. All current programs and future programs include this diet as an integral portion. The guidelines model includes four pillars: sustainability as well as social, economic and health components.

Measures to promote the adoption of new technologies in the field of "precision agriculture" le.g., information services which include interpreted satellite images, interpreted drone images or sensors which transmit data used for irrigation control, fertilization, spraying and growth) are being promoted. This online monitoring saves inputs and enables the sustainable use of natural resources as well as responding to changing climate conditions. Support is also directed to mechanization that encourages farmers to adopt soil conservation management.

\section{Climatic Data Quantifying}

An effort to quantify relevant climatic data for agriculture in Israel is currently underway in cooperation with the Israel Meteorological Services (IMS). The objective is to provide tools for future risk assessment with regard to climate change and necessary measures. The project includes more than 50 critical climatic indicators for the different production sectors in agriculture li.e., plant and animal production and sectors, as well as soil and water aspects), in 11 different agro-climatic zones in Israel. The project, which is scheduled for completion in 2019, consists of two components:

- Temperature, precipitation and relative humidity trends based on historic data (from 1951 to 2016);

- Forecasts based on an ensemble of models (2030-2050).

\section{Soil Conservation}

In Israel, as in many parts of the world, agricultural land is subject to depletion and increased runoff and erosion processes. Intensive long-lasting agricultural activity, allowing year-round production, imposes severe pressures on agricultural soil resource. A decade-old National Soil Study revealed that $60 \%$ of all agricultural land is subject to moderate to severe 


\section{CASE STUDY}

\section{Regional Projects of Agriculture and Environment}

In order to foster sustainable agriculture in farmers' fields in Israel, a budget was allocated for regional projects aimed at promoting agricultural practices that will decrease the negative impact of intensive agriculture on natural resources as well as supporting adaptation to climate change and positive externalities while engaging relevant stakeholders in the region (farmers, residents, environmental organizations, other ministries, etc.). The budget will be allocated to four regional councils in four different parts of the country (from north to south) during a five-year period (2017-2021). The projects will promote the reduction of pesticide and herbicide use, soil conservation management, and waste treatment, as well as the evaluation and management practices regarding damage caused to agricultural areas by wild animals. The aim being to minimize conflict between nature and agricultural production. The projects are based on cooperation with the farmers, extension service experts, experts from the Ministry of Agriculture, regional council representatives, residents, ecologists, economists and experts from the Israel Nature and Parks Authority.

erosion pressure. Predictions of climate change, which foresee a reduction in the amount of rain events, on the one hand, and an increase in their intensity, on the other hand, will further increase the potential for soil erosion of agricultural land and harm the soil's production capacity.

Soil conservation in agriculture promotes sustainable agriculture and minimizes soil loss from erosion. The Land Conservation and Drainage Division in the MARD has, for the past decade, offered support measures to encourage soil conservation. These include support for drainage engineering and agrotechnical activities for soil and water conservation, support for soil conservation measures such as minimum tillage and no-tillage of agricultural fields, and support for agricultural machinery that encourages sustainable soil cultivation which benefits the soil and reduces the effects of runoff and erosion.

In recent years there has been an increase in awareness and willingness to promote the field of soil and water conservation. In 2017, the Soil Conservation and Drainage Division allocated resources aimed at minimizing runoff and erosion processes.

In addition, soil conservation measures play a central role not only in preserving the soil in practice, but also in protecting urban areas in the lower part of the watershed from flooding and flood damage. Due to accelerated construction processes (which increase the contributing area for runoff) and the expected increase in rain intensity, agricultural fields can become a flood plain and a controlling factor in minimizing flood damages. For this purpose, support is provided to promote plans for runoff control in small streams in the upper parts of the watershed and in agricultural fields in the lower part of the drainage system for use as designated flood plains.

\section{( Maintaining the genetic diversity of seeds (SDG 2.5)}

Cultivating new agricultural varieties able to withstand extreme climate change is a challenge that requires focus beyond crop quantity or quality. Plant breeders today must emphasize resilience to extreme temperatures, short and intense rainfall regimes, and the ever-changing diseases caused by climate change. Wild plants and landraces are vast repositories of genetic diversity for crop improvement. Their genetic variety can improve the adaptability of crops to current and future environmental challenges. Israel contains some of the richest varieties of plant species in the world. 


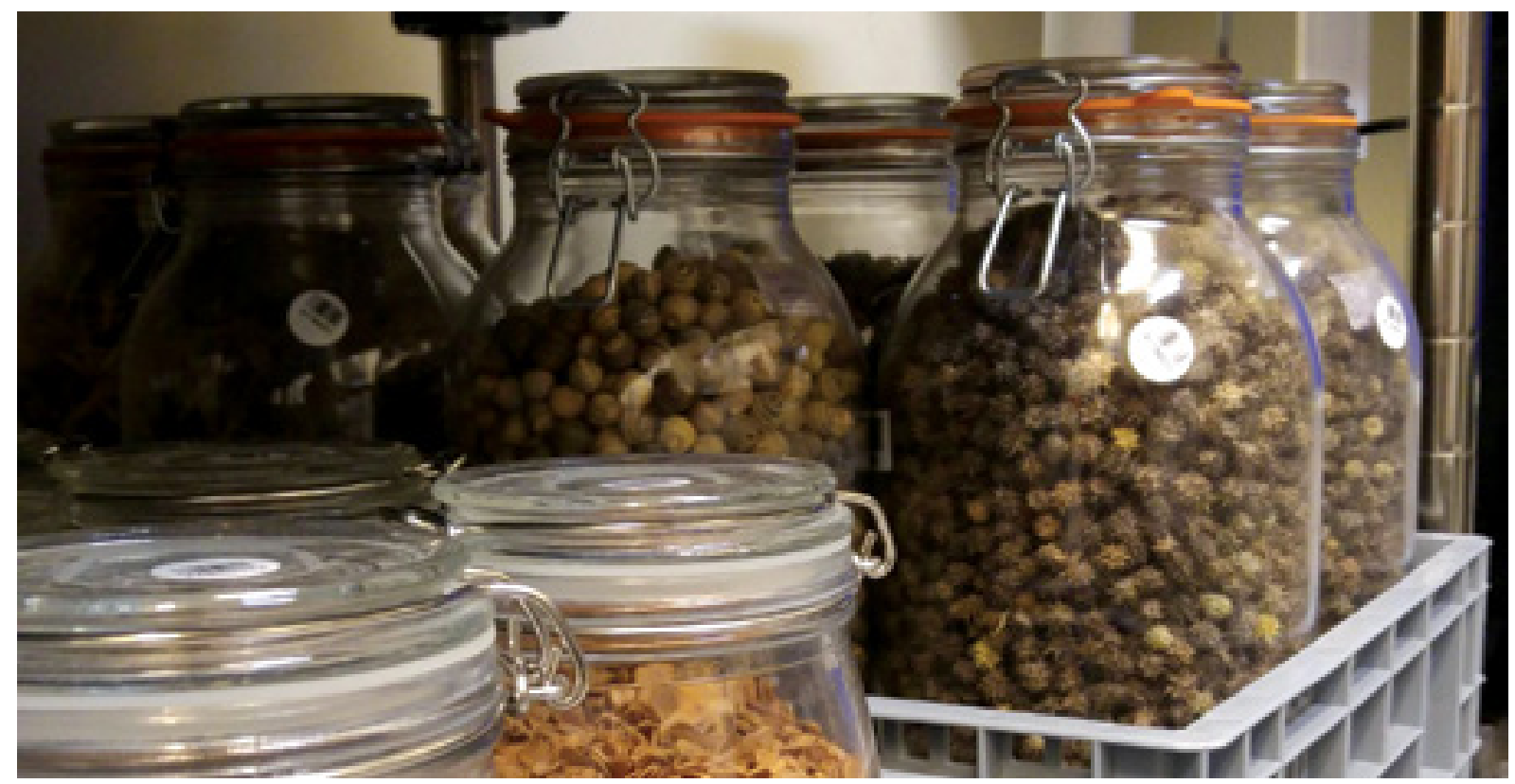

Israel Gene Bank collections/Photo: Einat Mayzlish-Gati

\section{The Israel Plant Gene Bank (IGB)}

Conserving plant resources for future agriculture, biotechnology and biomedical development is critical. Israel, with some key global genetic resources and advanced agricultural, genetic and biotechnology research centers, seeks to preserve its genetic resources to secure its own economic and scientific future and that of the world. The Israel Gene Bank (IGB) of the Agricultural Research Organization, the Volacani Center, serves as a physical repository for collected seeds for current and future development, including a program to collect and conserve wild plants and landraces seeds from natural habitats all over Israel. The IGB currently houses more than 30,000 different seed accessions and supplies samples for research, cultivation and ecological conservation. The Gene Bank promotes research to establish and maintain genetic pools, to identify important features that can benefit humankind and the environment, and to cooperate nationally and internationally on the operation of gene banks, the conservation of plant material and genetic variance. It is responsible for the collection and conservation of wild seeds of all plants in Israel, with an emphasis on crop wild relatives that may have future agriculturaleconomic or social-cultural potential. In recent years, the IGB has expanded its activities to include rare wild, endemic or endangered plants le.g., as part of the National Program for Biodiversity in Israel, 2010). The Gene Bank aims to create a genetic database of important plant traits found in Israeli flora. It also dedicates resources to conserve local landraces, the ancestors of ancient agricultural crops that contain an evolutionary record of traits (genes) that have developed over time.

\section{( Support mechanisms and export subsidies (SDG 2.a, 2.b, 2.c)}

Different programs exist to support the agricultural sector. Total budgetary support to agriculture totals about NIS 1 billion (about \$278 million) including crop insurance subsidies, investment support, and funding of agricultural research, among others. While small export subsidies in the agricultural sector still exist, Israel intends to eliminate all export subsidies by 2022, in line with the Nairobi Ministerial Decision on Export Competition. This is also in line with Israel's position on the need to eliminate export subsidies and represents another step in the current relatively-small export subsidies given by Israel, well below its WTO schedule commitments. MARD collects and publishes daily wholesale prices of fruit and vegetables. 


\section{CASE STUDY}

\section{Sustainable Solutions for Agricultural Waste}

To provide sustainable solutions for agricultural waste, support is provided for investments in technological solutions of treating and processing plastic, woody and green plant material, fruit and vegetable residues and animal carcasses (sheep, poultry, fish and swine).

Another direction is support for the treatment of woody waste. In order to regulate and apply sustainable administrative and technological solutions to treat and process uprooted orchards, pruned branches, trimmed foliage and more, an inter-ministerial effort is being implemented which includes the following measures: two legal orders (in force since January 15, 2017) regarding licenses for uprooting orchards, recruitment of human resources responsible for monitoring and imposing the orders, support for massive chopping machinery, and support for industrial processing units (lines) that use farm plantation residue for the production of usable products (e.g., wood pellets).

\section{PARTNERING FOR A BETTER WORLD}

Eradicating hunger and achieving food security, especially in Africa, have always been the cornerstone of Israel's overseas development assistance program, based on the country's own agriculture and rural development experience under semi-arid and arid climatic conditions. In this context, Israel initiated a UN Resolution on Agricultural Technology for Sustainable Development, adopted at the UN Second Committee (2017). The Resolution, first adopted in 2007, calls for promoting food security and the sustainability of food systems without sacrificing the resources of future generations. As Israel will join the Executive Board of the Food and Agriculture Organization (FAO) in 2020, Israel's Ministry of Foreign Affairs looks forward to expanding collaboration with the FAO towards implementing the mandate of the Organization. (SDG 2.a)

In many developing countries where poverty is pervasive, agriculture is the most important sector and the principle source of livelihood. However, this sector faces exposure to risks, including extreme climate conditions leading to repeated cycles of drought and desertification. Intensification of agriculture through the production of irrigated high-value crops, particularly in dry regions is a way to overcome the constraints of climate and soil while significantly increasing farmers' income. Given that 70-80\% of smallholder farmers are women, Israel's poverty reduction programs focus on capacity-building support, inclusiveness, and the institutionalization of local economic development as a framework through which partnerships are strengthened between national and local governments, the business sector, civil society and academia.

The eradication of hunger is critical in attaining sustainable development. In this context, a Memorandum of Understanding (MOU) was signed (2015) between FAO and MASHAV on multisectoral issues dealing with the scarcity of water in agricultural production, sustainable livestock feed development and breeding techniques in resource constrained situations in Africa. Within the framework of this MOU and promoting South-South and Triangular Cooperation (SSTC), MASHAV and FAO organized (2017) a capacitybuilding course in Israel on Sustainable Livestock Feed Development and Breeding Techniques in Resource-Constrained Situations for professionals 
from Anglophone countries in Africa. In 2018, a joint course was conducted in Israel targeting Francophone countries, within the framework of the African Regional Initiative: Building Resilience in Africa's Drylands. (SDG 2.a)

In 2017, MASHAV welcomed FAO representatives from 15 African countries on a study tour to Israel on Climate Change Adaptation in Arid Zones \& Green Growth. The mission focused on adapting, innovating and improving agricultural productivity, protected crops, water management and modern dairy farming, the linkage between research and development, extension and farmers, and policy, legal and institutional frameworks. (SDG 2.4)

The Tikkun Olam Ventures (TOV), the American Jewish Joint Distribution Committee's (JDC) new development program, aims at mitigating poverty among smallholder farmers in the developing world. To support TOV, JDC and Israel's Ministry of Economy and Industry signed an agreement committing to a $\$ 3.6$ million strategic partnership over five years. Other financial and funding partners include an Ethiopian commercial bank and individual philanthropists. The venture, launched in 2018, comprises a two-year pilot in rural communities in Ethiopia, with the goal to expand to additional countries. TOV helps smallholder farmers lift themselves out of poverty, empowers women and young people in rural communities, and bolsters economic growth. Smallholder farmers are provided with access to finance, to Israeli agricultural technology (AgTech), know-how, and entry into markets. Currently, 18 demonstration farming plots operate in Ethiopia's Southern Nations, Nationalities and Peoples Region aimed at reaching between 4,000-5,000 smallholder farmers and benefitting some 22,000 by the end of five years, catalyzing sustainable outcomes by encouraging relationships between smallholders, local banks and other AgTech companies. (SDG 2.3, 17.17)

Israel recognizes that young women and men are the world's future farmers, and that the challenge of meeting food demand in the upcoming decades, developing vibrant rural centers and promoting broad-based economic growth depend on the young. MASHAV is working with local governments, civil society and the private sector to enhance the role of women in the development process and gain access to economic resources through its Agribusiness as a Tool for the Empowerment and Inclusion of Rural Women Platform. (SDG 2.3, 5.5)

Israel remains steadfast in its long-standing commitment to development cooperation with Africa, giving priority to the sub-Saharan region. In 2017, Israel's Prime Minister took part in the 51st Economic Community of West African States (ECOWAS) Conference Heads of State and Government Summit in Liberia, where he reiterated the strategic importance of laying the foundation for a more vital development cooperation program with West Africa. Following the Summit, a high-level ECOWAS delegation representing 20 member states set in motion a cooperation program with MASHAV on the Linkages between Applied Research, Extension and the Farmer. (SDG 2.a)

The launching of the Israel-Africa Agricultural Innovation Center by the Agricultural Research Organization (ARO)/Nolcani Center and its non-profit partner, the Volcani International Partnerships, aims at harnessing Israel's capacity for agricultural innovation, knowledge and expertise for African agriculture development. The mission of the Center is to make Israeli agricultural research and development capacity, expertise and innovation easily accessible and holistically applied to maximum impact across the agricultural value chain in Africa. Together with African partners, the ARO will develop and provide comprehensive solutions, tailored to the unique challenges and opportunities facing Africa's smallholder farmers. Where relevant, it will help identify and adapt existing Israeli technologies to Africa's diverse agro-ecological conditions and implement capacitybuilding programs. (SDG 2.a)

MASHAV and the UN Development Program (UNDP) are collaborating on innovative irrigation and water management technologies in Central Asia as core components in agriculture strategies and policies.

\section{(SDG 2.4, 6.a)}

Israel is committed to meeting SDG 2.3 as it relates to agricultural productivity and incomes of 
small-scale producers, specifically among young people. MASHAV and the International Fund for Agriculture (IFAD) are cooperating on a program designed to provide young men and women in rural Africa sustainable employment opportunities through the implementation of profitable initiatives in agro-pastoral sectors. (SDG 2.3, 8.3)

To ensure sustainable food production systems under adverse climate conditions, MASHAV works together with the World Meteorological Organization (WMO) and Israel's Meteorological Service (IMS) to build national, regional and international frameworks dealing with extreme weather conditions, as well as conducting institutional capacity-building activities on the practical application of modern technologies and agrometeorological techniques. (SDG 2.4)

The development transformation envisaged in the 2030 Agenda can only occur with a coordinated approach among countries and aid actors. In this spirit, MASHAV has actively undertaken triangular initiatives supporting the Global Partnership for Sustainable Development including: (SDG 2.a, 17.16)

- Israel-Germany (BMZ/GIZ)-Cameroon are engaged in the Trilateral Cooperation in Agriculture: Improving the Market Value Chain Project to improve the mango value chain within the framework of the German-Israeli Initiative;

- Israel-Germany (BMZ/GIZ)-Ethiopia are cooperating to strengthen drought resilience among the pastoral and agro-pastoral population of the Afar region through the provision of training and capacity-building activities in the fields of irrigation and cross-production;

- Israel-United States (USAID)-Ethiopia Joint Technical Cooperation Program: The Smallholder Horticulture Project has resulted in the setting up of six fruit tree nurseries. The program is coordinated by an onsite long-term Israeli expert assigned to promote economic growth in rural areas, as the production of fruit trees is vital to Ethiopian agriculture as an additional source of income for local farmers;

- Israel-France (AFD)-Cameroon Initiative to establish three demonstration plots for growing vegetable and open field crops in the central and southern regions of Cameroon, focusing on sustainable soil, water management, and modern irrigation techniques;

- Israel-Netherlands-Ethiopia jointly implemented a Small-Scale and Micro-Irrigation Support Project providing capacity-building in the field of small-scale irrigation and horticulture;

- Israel-Canada (MEDA)-Ukraine are cooperating within the framework of the Ukrainian Horticulture Business Development Project to empower over 30,000 small and medium-size household farming entrepreneurs in the field of horticultural growing, safe storage and access to markets;

- Israel-Canada (Global Affairs Canada/MEDA)Ghana Trilateral Cooperation Project to establish in Ghana's northern region demonstration plots for growing soybeans during the dry season, using modern irrigation and fertilization technologies;

- Israel-Italy-United States (USAID) sponsored a special event at the 2018 Global Framework on Water Scarcity for Agriculture (WASAG) in Brasilia, focusing on best practices in using water in agriculture to ensure food security;

- Israel-USAID-Georgia are collaborating on Georgia's Strategy for Agricultural Development to modernize the agricultural sector and improve productivity by enhancing vegetable production along the entire value chain. The main goal is to boost sustainable economic development and decrease dependence on agricultural imports. The initiative will likewise strengthen the capacity of agricultural extension services through the introduction of modern agricultural technologies and innovative farming techniques. Collaboration takes place in connection with USAID's programs, with a strong emphasis on capacity-building, training and technology transfer;

- Israel-Canada (Global Affairs)-Myanmar (MEDA/ Mennonite Economic Development Associates) project to support rural development and food security in the Shan and Kayin States by building the agricultural and entrepreneurial capacities of smallholder farmers, focusing on rural women. Within this framework, MASHAV provides training and capacity-building in irrigation, agricultural 


\section{CASE STUDY}

\section{Improving the Mango Value Chain in Cameroon}

As part of a trilateral cooperation between Israel, Cameroon and Germany, MASHAV, the Ministry of Agriculture and Rural Development of Cameroon-MINADER, and the Deutsche Gesellschaft für Internationale Zusammenarbeit (GIZ) are carrying out a project to improve the mango value chain in Cameroon. The project aims to increase the productivity and income of smallholder farmers in Cameroon, introduce added-value crops, and generate greater employment opportunities through the improvement of the mango value chain.

The project is in alignment with the 2030 Agenda and is based on Israel's agricultural and rural experience and on Cameroon's national strategy for agricultural and rural development to reduce food insecurity, improve agricultural outputs and stimulate economic growth.

Implementation is based on introducing best practices related to the mango value-chain and Israeli innovative techniques, including:

- Introduction of new high-quality mango varieties (Israeli and local) for the export market and improvement of the supply of mango rootstocks

- Provision of quality training and consultancies for the management of orchards and nurseries on mango fruit production (irrigation, fertilization, fertigation, pruning, pest management)

- Professional training and consultancy along the entire value chain

- Establishment of new nurseries and demonstration plots

- Elaboration of professional material for orchard management and for the establishment and management of nurseries for mango fruit production including integrated pest management

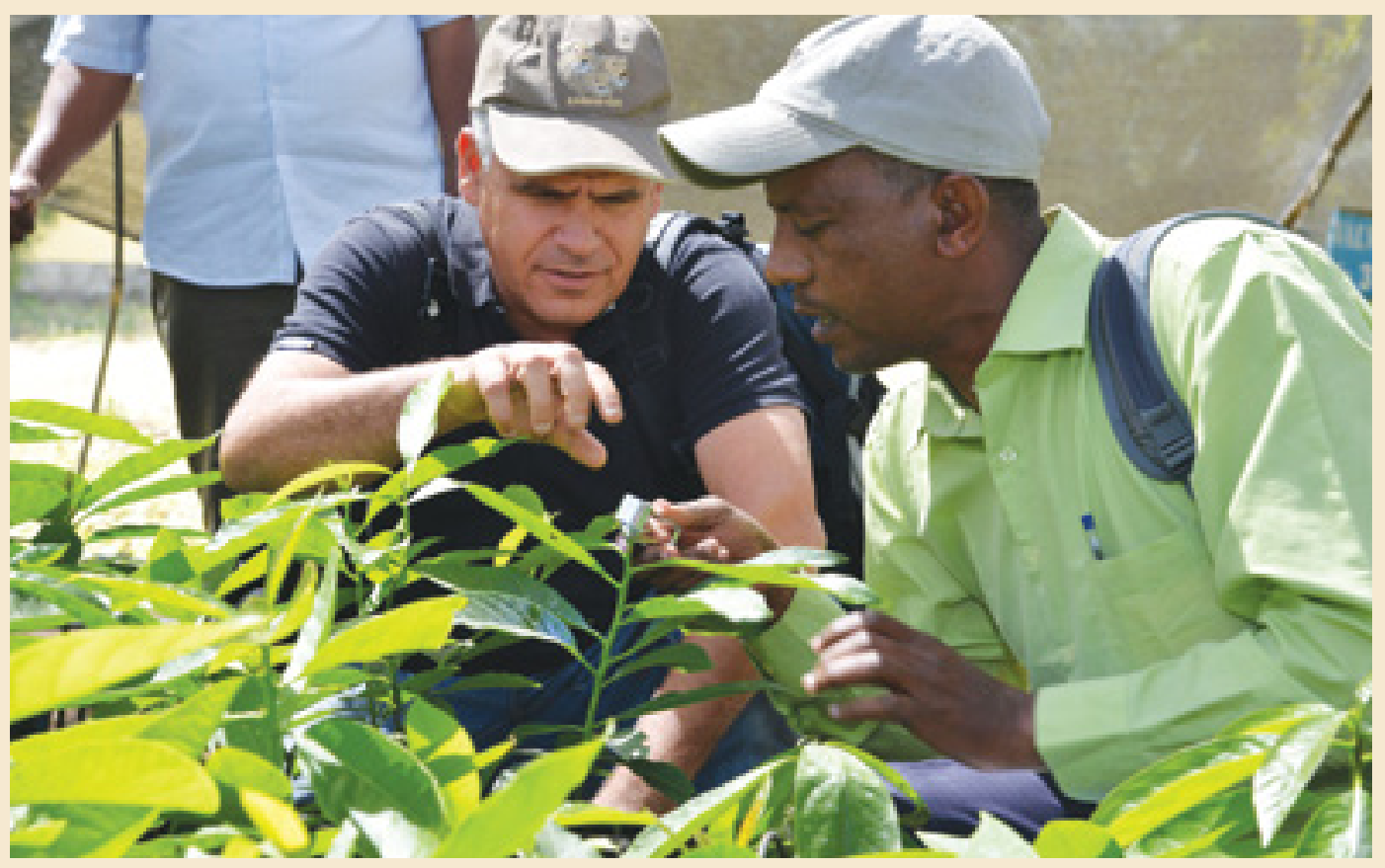

Photo: MASHAV 
production and entrepreneurial know-how, emphasizing environmental sustainability and gender equality strategies for sustainable agrobusiness development.

In addition, MASHAV also engages in bilateral partnerships with national and local governments by implementing agricultural projects benefitting the extreme poor and vulnerable, as follows:

(SDG 2.3, 2.4)

- The Prime Minister of Israel and the President of Mexico signed an MOU to facilitate cooperation in Latin America on issues relating to agriculture, water, entrepreneurship and innovation;

- During a state visit to Vietnam, the President of Israel and the First Lady visited an Israeliinitiated Dairy Demonstration Farm near Ho Chi Minh City, geared to support modern and intensive dairy farming and serve as a Center of Excellence for the dairy sector;

- In the Sino-Israel Demonstration Dairy Farm in Yongeldian, all activity including milk production and quality, herd health and herd fertility is monitored daily by a computer with advanced herd management software from Israel. Milk production was raised to the highest in China, and the farm serves as a training center for thousands of dairy producers;

- In the framework of the Indo-Israel Agricultural Cooperation Project, launched in 2008, 30 Centers of Excellence in various agricultural sectors have been or are in the process of being established in several states across India. A long-term Israeli expert provides onsite technical support to achieve sustainable agriculture for the benefit of small and large farmholders;

- KIDRAC - the Kenya-Israel Drought Resilience Agricultural Center was established to implement capacity-building programs on irrigation and water resource management for agricultural use;

- In accordance with Rwanda's national plan to modernize its agricultural sector, stimulate economic growth and alleviate rural poverty, a three-year Agreement for Development Cooperation was signed to establish a Horticulture Center of Excellence for agricultural sustainability near Kigali. The Center is creating self-sustaining agricultural production methods and focuses on applied research and development;

- An Israel-Kazakhstan Irrigation Demonstration Center was inaugurated in the Ushkonyr, Almaty region - the first of its kind in Central Asia - focusing on demonstration and innovative irrigation technologies adapted to local environmental conditions. The Center also provides guidance and support to agro-technology projects in the rural sector;

- An Israel-Paraguay Drip Irrigation Project assists 300 smallholder farmers in the San Pedro area;

- Within the Framework for Development Cooperation, Israel and Honduras are implementing a long-term agriculture capacitybuilding program under the guidance of an onsite Israeli expert;

- An MOU was entered into with the Ministry of Agriculture of Fiji on sustainable agriculture, water and food security;

- An ongoing dairy cattle project was established in partnership with Colombia in the northern region of the country. The project focuses on nutrition, milk production and quality, including capacity-building activities geared to achieve a greater and more efficient production value chain.

- Israel's ARO and Kazakhstan's National Agrarian Scientific-Educational Center (NPJSC) signed an MOU expressing their mutual interest in scientific-educational cooperation in agricultural and environmental research and development. Cooperation focuses on crop production, plant sciences, plant protection, post-harvest, soil and water, health, food and desert agriculture, including capacity-building activities. (SDG 2.5)

Israel views development cooperation with its Middle East neighbors as a fundamental value and national priority. Existing programs further professional dialogues on shared obstacles in the development process and foster a better understanding between people. For example, MASHAV and Japan's International Cooperation Agency (JICA) jointly implemented an agricultural program involving Israel, Jordan and the Palestinian Authority which includes onsite and regional 


\section{CASE STUDY}

\section{Enhancing Vegetable Production in Georgia}

In accordance with the 2030 Agenda for Sustainable Development and Georgia's Strategy for Agricultural Development, MASHAV and USAID have partnered with Georgia's Ministry of Agriculture to modernize the country's agricultural sector and improve agricultural productivity. The project aims to boost sustainable economic development and decrease dependence on agricultural imports by enhancing agricultural productivity and production quality, increasing competitiveness in the agro-food sector and ensuring food safety.

The focus of the project is on enhancing vegetable production along the entire value chain among smallholder farmers and rural enterprises, as well as strengthening the capacity of agricultural extension services through the introduction of modern agricultural technologies, innovative farming techniques and best practices. Collaboration takes place in connection with USAID's programs with a strong emphasis on capacity building, training and technology transfer.

Implementation is based on the transfer of best practices and know-how on open-field and protected vegetable production and irrigation along the entire value chain among smallholder farmers and rural enterprises. It includes:

- Introduction of Israeli inputs, modern agricultural technologies, innovative farming techniques and best practices

- Provision of quality training and consultancies to extend and expand capabilities

- Short-term consultancies and professional study tours

- Strengthening the capacity of agricultural extension services

- Improvement of production techniques and technology

- Building of post-harvest handling capabilities

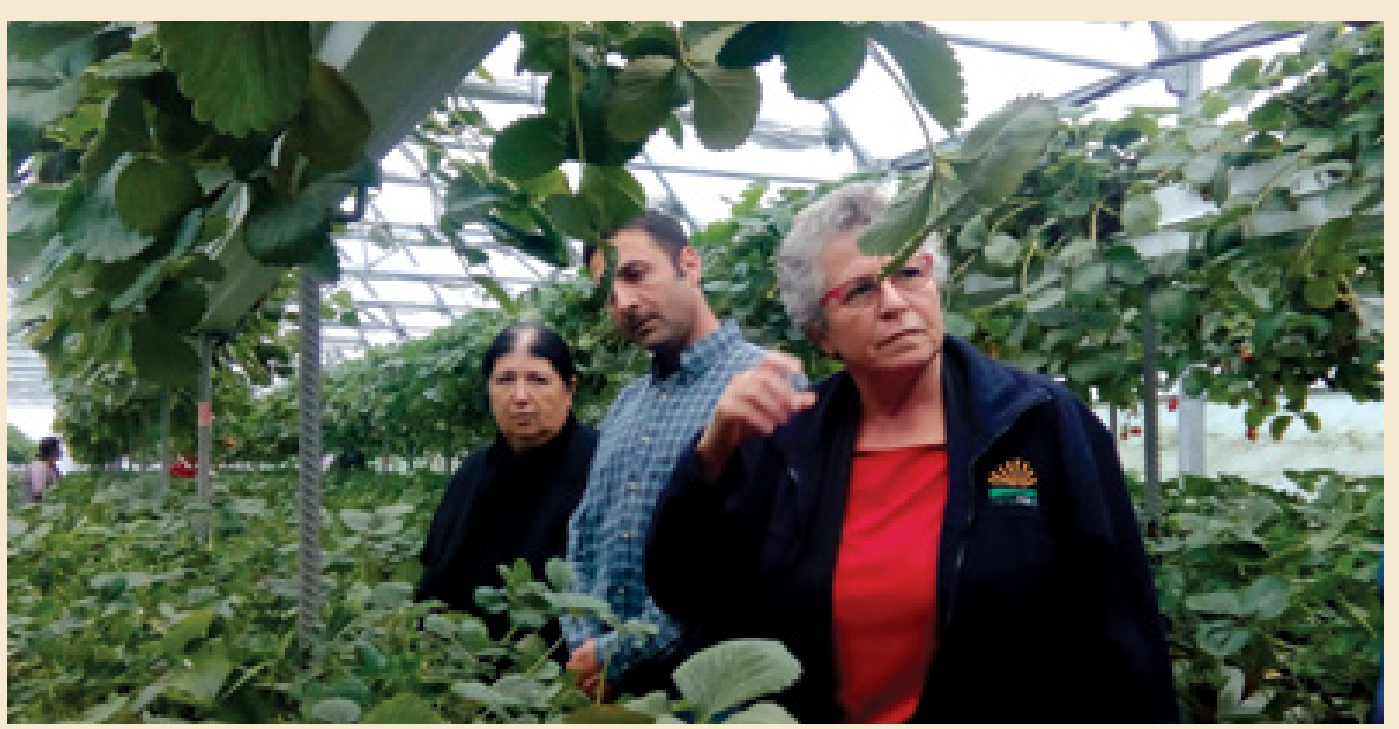

Photo: MASHAV 
training activities. Since 2008, the MASHAV-JICAJordan Agricultural Cooperation Program has also carried out professional training activities in the areas of irrigation, fertilization, organic agriculture and post-harvest care. (SDG 2.4)

As part of another regional initiative, Enhancing Livelihoods in the Middle East - Optimizing Irrigation and Olive Yields for Growth in Arid Areas, ARO researchers developed advanced protocols to optimize irrigation and maximize olive yields in arid areas. A partnership was formed between Israeli, Jordanian and Palestinian researchers to characterize superior varieties for the future.

\section{(SDG 2.4, 12.a)}

The Regional Developing Wheat for Climate Change Conditions Initiative (SDG 2.4, 13.2) confronts the impacts of climate change, increased temperatures, and drought on global wheat production. Israel has a wide genetic diversity of wheat wild relatives and landraces that possess unique characteristics to develop wheat better adapted to climate change. ARO scientists collected a wide range of local wild relatives and landraces, explored their adaptive strategies and utilized them for breeding, resulting in several commercial cultivars. Israeli, Palestinian and Jordanian scientists are collaborating on collecting, conserving and characterizing these genetic materials. ARO scientists also partner with specialized UN agencies, regional programs, as well as bilaterally to enhance Israel's National Gene Bank, sharing best practices and working to conserve the genetic resources of regional vegetation. (SDG 2.5)

In 2018, the ARO established a Sustainable Model Farm for the testing, development and demonstration of sustainable intensive agricultural practices to attain higher production levels and economically viable approaches that do not sacrifice long-term environmental health for short-term gain. The ARO hosts MASHAV's capacity-building programs for professionals from the developing world on topics including post-harvest and integrated pest management, improving production of animal husbandry, agricultural engineering technologies, and agriculture in arid and semi-arid areas. (SDG 2.4)
One way to enhance a country's agricultural productive capacity is through the stimulation of innovation in diverse fields of activity. Agritech Israel is one of the world's most important international exhibitions on agricultural technologies. In partnership with the private sector, Israel's overseas development assistance continues to share with the developing world groundbreaking products and modern approaches for a more efficient, productive and sustainable agriculture. (SDG 2.a)

In many developing countries a significant portion of the population suffers from malnourishment. Raising the quantity of indigenous crops with higher levels of nutritious protein is one strategy to address food security. However, even a sufficient supply of nutritious crop may not sustain the population if the safety of the food is compromised. Improperly treated crops and unsanitary handling threaten the food supply. In alignment with the UN Decade of Action on Nutrition 2016-2025 to eradicate hunger and prevent all forms of malnutrition, Israel's international development programs address hunger and under-nutrition within sustainable food systems as a key policy priority, especially among rural women smallholders. In addition, Israel cosponsored the Resolution adopted at the 72nd UN General Assembly on Global Health and Foreign Policy: A Healthier World through Better Nutrition. (SDG 2.2)

Healthy nutrition plays a critical role in the prevention and management of noncommunicable diseases. Israel's Ministry of Health worked with the WHO, FAO and other international organizations on numerous projects supporting improved nutritional guidelines. Israeli experts assisted in writing the report Tackling Food Marketing to Children in a Digital World: Trans-disciplinary Perspectives, and Israeli nutritionists participate in the Global Network for Front-of-Pack Labeling, working with WHO and FAO to build more efficient and clearer labeling of food products. (SDG 2.1)

Major changes have occurred in the global diet, resulting in an emerging trend towards overnutrition and chronic diseases. Nutrition transition has made fast foods the preferred menu for millions of individuals, especially children, and a growing 
percentage of populations in the developing world as more natural, locally-grown produce disappears. With better understanding of how diet affects global health, it is clear that significant dietary changes must be formulated at all levels of government. MASHAV addresses these issues in the context of the Nutrition in a Changing Global Environment Platform. (SDG 2.2)

In 2018, MASHAV's agriculture related programming included the implementation of 27 capacity-building and training activities in Israel, with the participation of 697 professionals from the developing world. At the request of partner countries, 37 on-thespot (OTS) courses were conducted, with the participation of 1,677 professionals, and 40 short-term consultancies carried out. In support of joint agricultural projects in Africa, Asia and Latin America, three long-term consultancies were conducted to further project development and extension services. (SDG 2.a) 
GOAL

3

GOOD HEALTH

\& WELL-BEING

$-W$
Maternal and child health

Tuberculosis and HIV elimination in Israel

Antibiotic resistance

Strengthen the prevention and treatment of substance abuse

Importation of wild poliovirus: Public health response, lessons learned, and implications for the future

Environmental health

Control of tobacco in Israel

National Program for Quality Indicators in Hospitals

Partnering for a Better World 


\section{ENSURE HEALTHY LIVES AND PROMOTE WELL-BEING FOR ALL AT ALL AGES}

Every Israeli citizen is entitled to health care services under the National Health Insurance Law. All residents have the right to register as members of a health maintenance organization ( $\mathrm{HMO}$ ) of their choice, free of any preconditions or limitations stemming from their age or the state of their health. All residents have a right to receive, via their $\mathrm{HMO}$, all the services included in the medical services basket, subject to medical discretion, and at a reasonable quality level, within a reasonable period and at a reasonable distance from their home. 


\section{( Maternal and child health (SDG 3.1, 3.2)}

Israel is a child-oriented society characterized by strong family ties, universal monthly child benefits, free education from age three through $12^{\text {th }}$ grade, universal rights to health services guaranteed by the National Health Insurance (NHI) Law, and strong community-based primary care with dedicated preventive care services. Infant and maternal mortality rates are below the OECD average (3.1/1,000 and 2.0/100,000 respectively). Israel has already exceeded the developed regions' target for the UN Sustainable Development Goals (SDGs) for the year 2030 for the following goals: maternal mortality ratios and neonatal, infant, and under-five mortality (UFM) in all population groups (Table 3.1). The annual decrease in the rate of UFM between the years 1990-2015 was 4.3\%, as compared to $3.7 \%$ for MDG developed regions and $3.0 \%$ for the world. In 2015, Israel's maternal mortality ratio was 2.0/100,000 and the stillbirth rate was 5.9/1,000 births. These rates exceed the SDG targets for reduction of child mortality and improvement of maternal health for the year 2030.

Israel's total fertility rate in 2015 of 3.1 is more than $30 \%$ greater than that of the next ranked country in the OECD. This high rate persists despite high rates of maternal education and ready access to modern contraception. More than $99 \%$ of births occur in hospitals, and all are attended to by skilled birth attendants. In 2016, 15.8\% of births were by caesarian section, placing Israel among the lowest-ranked countries in the OECD. The rate of low birthweight (LBW) births was $8.1 \%$ in 2013 , as compared to $6.6 \%$ in OECD countries. Preterm births accounted for $7.4 \%$ of live births in 2014 .

Israel has a state-of-the-art system for newborn metabolic testing for ten inborn errors of metabolism, hypothyroidism, and severe combined immunodeficiency disease. The national birth

Table 3.1

\section{Under-five, infant neonatal and maternal mortality rates for Israel in 2015, Millennium Development Goal regions and Sustainable Development Goals 2030}

\begin{tabular}{l|c|c|c|c} 
& $\begin{array}{c}\text { Neonatal } \\
\text { (per 1,000) } \\
2015\end{array}$ & $\begin{array}{c}\text { Infant } \\
\text { (per 1,000) } \\
2015\end{array}$ & $\begin{array}{c}\text { Under 5y\& } \\
\text { (per 1,000) } \\
2015\end{array}$ & $\begin{array}{c}\text { Maternal^ }^{\star} \\
\text { (per 100,000) } \\
2015\end{array}$ \\
\hline Israel & 2 & 3 & 4 & 2 \\
\hline MDG World estimate\# & 19 & 32 & 43 & $216^{*}$ \\
\hline MDG Developed Region\# & 3 & 5 & 6 & $12^{*}$ \\
\hline $\begin{array}{l}\text { Sustainable Development } \\
\text { Goal for 2030 }\end{array}$ & 12 & Not defined & 25 & 70 \\
\hline
\end{tabular}

$\sim$ Neonatal mortality rate is the number of neonates dying before reaching 28 days of age, per 1,000 live births in a given calendar year.

$\neq$ Infant mortality rate is the number of infants dying before reaching one year of age, per 1,000 live births in a given calendar year.

\& Under-five mortality rate is the probability per 1,000 that a newborn baby will die before reaching age five, if subject to age-specific mortality rates of the specified year.

^ Maternal mortality rate is the ratio of maternal deaths to the women-years of exposure for women aged 15-49 years.

\# UN Inter-agency Group for Child Mortality Estimation (UNICEF, WHO, World Bank, UN DESA Population Division) at www.childmortality.org

* Alkema L, Chou D, Hogan D, Zhang S, Moller AB, Gemmill A, Fat DM, Boerma T, Temmerman M, Mathers C, Say L; United Nations Maternal Mortality Estimation Inter-Agency Group collaborators and technical advisory group. Global, regional, and national levels and trends in maternal mortality between 1990 and 2015, with scenario-based projections to 2030: a systematic analysis by the UN Maternal Mortality Estimation Inter-Agency Group. Lancet. 2016 Jan 30;387(10017):462-74. doi: 10.1016/ S0140-6736(15)00838-7. PubMed PMID: 26584737. 
database enables verification of the receipt of blood samples from every infant born, with coverage of over $99.8 \%$. The majority of infants identified by the screen are in the first week of life when referred for diagnosis and treatment. Expansion of the program to include other disorders is now in process. The national neonatal hearing screen program has effectively reduced the age at which hearing loss is diagnosed and at which infants enter habilitation. The median age of cochlear implantation for children under age 6 dropped from 2.1 years in 2005 to 1.5 in 2014.

The breastfeeding initiation rate in Israel is $88 \%$. Concerted efforts to train all public health nurses in basic breastfeeding skills, increase the availability of certified lactation consultants, and require obstetric units to provide a certified nurse lactation specialist have resulted in improved duration of breastfeeding. In 2016 the rate of exclusive breastfeeding at 6 months was $17 \%$, and overall breastfeeding rates at 6 months were $51 \%$, as compared with $38 \%$ in 2002. No obstetric units are yet in compliance with the WHO Baby Friendly directives.

\section{Preventive care}

Israel provides free universal preventive pediatric care (PPC) from birth to age five. This includes immunizations, growth and developmental surveillance, anticipatory guidance, breastfeeding support, screening for postpartum depression (PPD), and identification of high-risk families and intra-familial violence. An electronic health record facilitates follow up as well as real time assessment of health indicators. National preventive care quality indicators promote high coverage and stimulate improvement over time. More than $77 \%$ of mothers are screened for PPD, permitting their needs to be addressed and generate referrals as necessary. 83\% of toddlers undergo developmental assessment which includes language and communication competencies. $75 \%$ of 18 -month old toddlers have received their fourth DTaP dose, and $60 \%$ of 13-month old toddlers have received their MMRV. Collecting this data adds another dimension to the overall high immunization coverage in Israel and promotes timeliness of vaccination.

\section{Dental health}

Israel is one of the few countries to include children's dental services in its basic health coverage. Thirty percent of the funds are spent on preventive services, including yearly in-school caries screening and dental hygiene instruction, while the remainder covers maintenance and restorative treatments. Inclusion of dental services for children has reduced disparities in care for children. $70 \%$ of children's dental visits are to the publicly funded service.

\section{Disparities}

The overall infant mortality rate (IMR) in Israel has consistently declined over the years (Figure 3.1). However, the overall Arab IMR has been more than two-fold greater than that of Jewish infants. In 2016, the overall IMR was 3.4 deaths per 1000 live births, with a rate of 2.4 for the Jewish population and 6.7 for the Arab population.

This difference can be attributed primarily to the almost four-fold excess of deaths due to congenital malformations and inherited diseases among Arab infants (the cause-specific IMR is 2.73 , versus 0.70 among Jewish infants) (Figure 3.2). Much of the excess mortality from congenital malformations and inherited diseases can be attributed to the 31\% rate of consanguinity among the Arab population. In addition, there is a 1.7-fold excess in infant mortality from preterm birth and other perinatal conditions. Public and professional advocacy led to the funding of a multi-disciplinary communitybased program to address this issue. This program has been active for over 15 years. Its goal is to reduce the IMR among Bedouin Arabs in the southern region of Israel, the population with the highest IMR in Israel.

Unintentional injuries constitute the leading cause of mortality among children aged 1-5 (23\%), the second leading cause of mortality among children aged 5-14 (20.3\%), and the leading cause of mortality among young persons aged 15-24 (61.4\%). Two population groups are at a particularly high risk of injury: Arab children aged 0-4 and all adolescent boys aged 15-17. These two groups 
together comprise only $14 \%$ of the total child and adolescent population but experience almost half (48\%) of injury-related mortality among children and adolescents in Israel. The $40 \%$ decline in mortality rates over the past decade, with a concomitant $14 \%$ decline in hospitalizations and 3\% decline in emergency department visits due to injury can be attributed to coordinated efforts of multiple governmental agencies and NGOs. Injury-related mortality rates among Arab children have declined less sharply than those among Jewish children. A government resolution to mandate a multi-year National Child Safety Action Plan to be led by the Ministry of Health $(\mathrm{MOH})$ in cooperation with 15 additional ministries and national institutions was funded in December 2016.

Israel is facing rising rates of obesity and its complications. Currently, almost one in four children in $7^{\text {th }}-12^{\text {th }}$ grades are overweight or obese. Rates are higher among Arab youth. There has been a striking $50 \%$ increase in obesity (BMI $>97 \%$-ilel among Arab youth over the past 12 years with current rates of $15 \%$ among males and $9.2 \%$ among females. The corresponding obesity rates for Jewish males are $8.4 \%$ and females 7.3\%. Municipality-level weight, height, and BMI measurements of first and seventh grade pupils are now routinely reported and available to the public online, providing a strong impetus for the development of local programs to address obesity and health lifestyle behaviors.

\section{Tuberculosis and HIV elimination in Israel (SDG 3.3)}

\section{Tuberculosis (TB)}

Israel is one of the few countries considered to be in a pre-elimination phase. Many of the WHO-End TB strategy components have been incorporated into the Israeli National TB Program (NTP). The following declines in TB incidence were noted between 2012 to 2015: a 46.8\% decline in the overall TB notification rate, from 6.2 to 3.3 cases per 100,000 and a decrease in TB incidence among all residents born abroad from 9.4 to 6.7.

Cumulative TB rates among new immigrants from Ethiopia and from the Former Soviet Union (FSU) were analyzed in a five-year cohort (1999-2013). The results demonstrated that TB incidence amongst Ethiopian immigrants fell from 310.0 to 51.7, while for immigrants from the FSU, TB rates fell from 151.1 to 27.5.

Figure 3.1

Infant mortality rates by religion and year of birth, 1955-2014

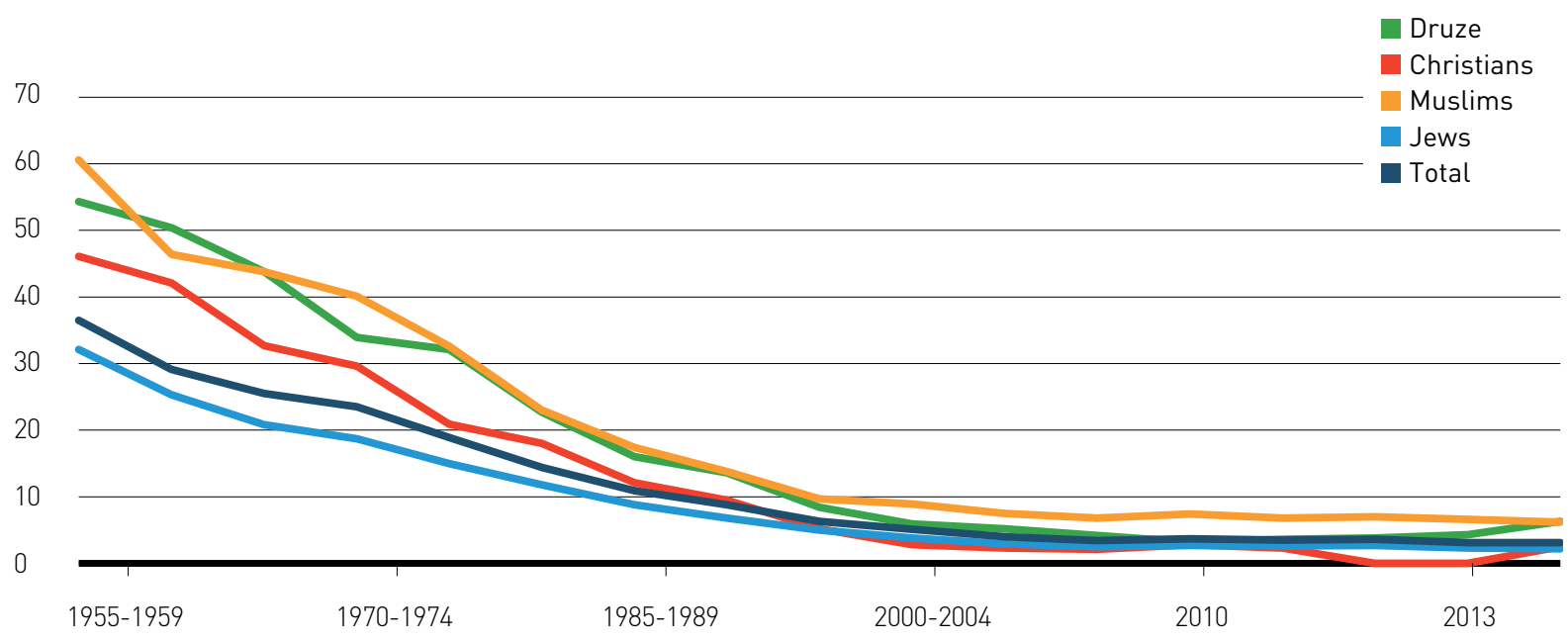


Figure 3.2

\section{Cause-specific infant mortality by population groups, 2009-2011}

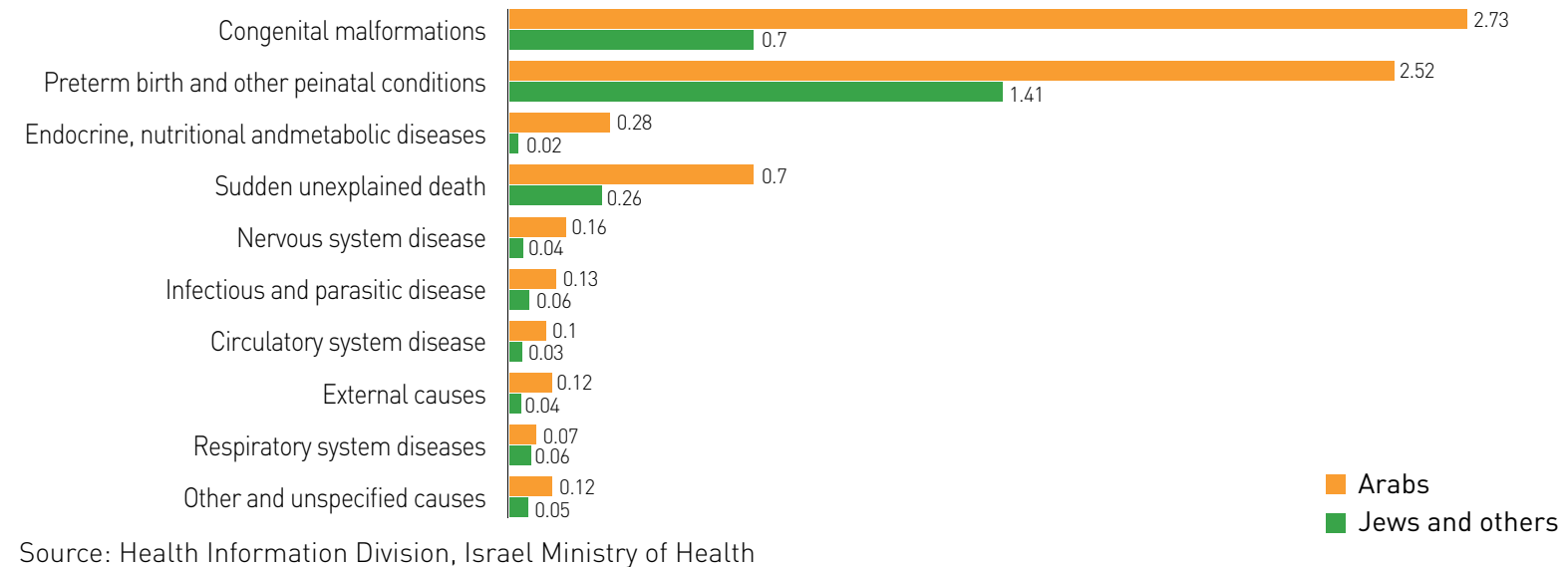

In order to reach TB elimination by 2035, it is imperative for Israel to continue to provide adequate resources for TB prevention and care, including the provision of new drug regimens. Eliminating TB among the foreign-born population is dependent upon migration patterns and continuing the integrity of the NTP despite a declining case-load.

\section{HIV/AIDS}

Israel is a country with a low HIV endemicity, but with specific characteristics related to migration from countries with intermediate and high HIV endemicity. In 2017, some $72.5 \%$ of people living with HIV (PLWHIV) were foreign-born, mostly originating from moderate and high endemicity countries. Between 1981-2017, 9,593 new cases of HIV/AIDS were reported. In 2017, there were 7,745 reported cases of PLWHIV in Israel, and an additional estimated 1,129 cases residing in Israel (total 8,874). Of Israeli adults and children currently reported as living with HIV in Israel, 87.7\% have received anti-retroviral therapy (ART). Undocumented migrants are eligible for gratuitous ART and HIV medical surveillance. Prevention interventions tailored for each high-risk group and for the general population are in place.

Israel adapted the ECDC HIV modeling estimation tools to gauge undiagnosed PLWHIV in Israel and is strengthening outreach activities for earlier detection in several high-risk groups. Its policy and practice of ART and CD4/VL at all HIV centers adhere to the latest international guidelines. Israel has also adapted its reporting system to prospectively monitor incident cases and has investigated the HIV cascade of care in Israel for the years 2011-2015 to promote achievement of the $90-90-90$ international goals $190 \%$ of people living with HIV diagnosed by $2020,90 \%$ of diagnosed people on antiretroviral treatment by $2020,90 \%$ of people in treatment with fully suppressed viral load by 2020).

\section{( Antibiotic resistance (SDG 3.3, 3.d)}

In 2006, Israel experienced a country-wide outbreak of infections caused by Carbapenem-Resistant Enterobacteriaceae (CRE). In response, the National Center for Infection Control (NCIC) was created within the $\mathrm{MOH}$, mandated to collect data and intervene in healthcare institutions to contain the spread of multi-drug resistant organisms (MDROs). The NCIC instituted major changes in the Israeli healthcare system, including: creation of standards for infection control Iguidelines on hand hygiene; cleaning, disinfection and sterilization; and prevention of device-associated infections); establishment of national surveillance systems for 
Table 3.2

\section{National antibiogram, Israel, 2017}

\begin{tabular}{|c|c|c|c|c|c|c|c|c|c|c|c|c|c|c|c|c|c|c|c|c|c|c|c|c|}
\hline 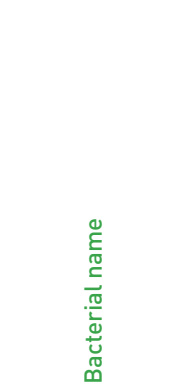 & 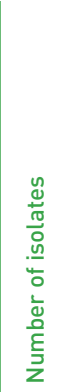 & 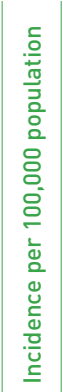 & 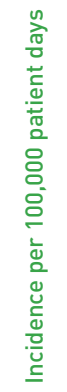 & 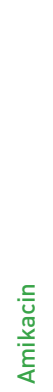 & 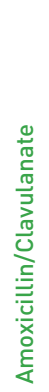 & 竞 & 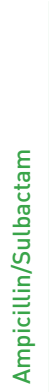 & 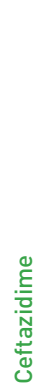 & 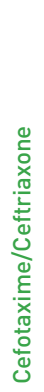 & 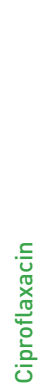 & 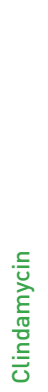 & 莺 & 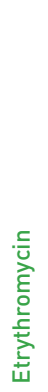 & $\begin{array}{l}. \frac{\mathrm{U}}{\mathrm{U}} \\
\frac{\mathrm{E}}{\mathbb{N}} \\
\frac{\mathrm{V}}{\mathrm{d}}\end{array}$ & 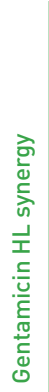 & 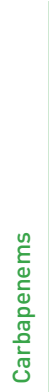 & 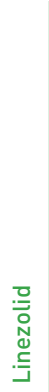 & 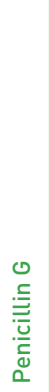 & 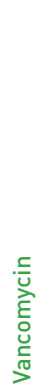 & 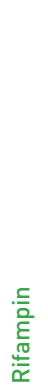 & 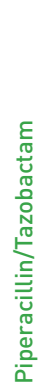 & 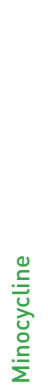 & 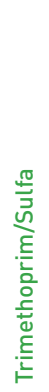 & 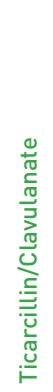 \\
\hline A. Baumannii & 595 & 6.8 & 11.7 & 65 & & & 67 & 83 & 3 & 83 & & ND & & 72 & & 78 & & & & & 83 & 22 & 70 & 23.0 \\
\hline E. faecalis & 1111 & 12.8 & 21.9 & & & 2.1 & & & & & & & 86 & & 49 & & 0 & 8 & 0.1 & & & & & \\
\hline E. faecium & 404 & 4.6 & 8.0 & & & 84 & & & & & & & 95 & & 45 & & 0 & 92 & 19 & & & & & \\
\hline E. coli & 5440 & 62.4 & 107.4 & 2.1 & 37 & 71 & 52 & 31 & 32 & 35 & & ND & & 16 & & 0.3 & & & & & 9 & & 38 & 11.0 \\
\hline K. pneumoniae & 2323 & 26.7 & 45.8 & 6 & 43 & & 60 & 50 & 51 & 37 & & ND & & 29 & & 4 & & & & & 25 & & 52 & 41.0 \\
\hline MRSA & 705 & 8.1 & 13.9 & & & & & & & 88 & 71 & & 70 & 35 & & & 0.2 & & 0 & 3 & & 0 & 3 & \\
\hline MSSA & 1602 & 18.4 & 31.6 & & & & & & & 5 & 26 & & 28 & 4 & & & 0 & ND & 0 & 0.4 & & 0.1 & 1.2 & \\
\hline P. aeruginosa & 1262 & 14.5 & 24.9 & 4 & & & & 13 & & 17 & & ND & & 13 & & 13 & & & & & 13 & & & 60.0 \\
\hline
\end{tabular}

Numbers in antibiotic columns are \% of isolates that are resistant

HL - High level; MRSA - methicillin-resistant S. aureus; MSSA - methicillin-susceptible S. aureaus, Sulfa - Sulfamethoxabole

antibiotic resistance, antibiotic use, and hospitalacquired infections; and enhancing the capabilities of microbiology laboratories.

Two indicators are used to track MDROs in Israel. The first is the percentage of sentinel bacterial species that are resistant to a given antibiotic. For example, at the height of the CRE outbreak, 22\% of $K$. pneumoniae blood isolates were resistant to carbapenems while in 2017, the percentage was $4 \%$, with the goal for 2019 set at 3.5\%. For comparison, among 30 European countries in 2017, the median percentage of K. pneumoniae blood isolates resistant to carbapenems was 1.8\%, ranging from $0 \%$ in Norway to $66 \%$ in Greece. The national antibiogram showing resistance among eight species is presented in Table 3.2.

The second indicator is the incidence of hospitalacquired infections caused by eight sentinel MDROs. For example, from 2014 to 2017, there was a $25 \%$ decrease in the number of bloodstream infections (BSI) caused by carbapenem-resistant $A$. baumannii and $P$. aeruginosa and a 10\% decrease in BSI caused by methicillin-resistant S. aureus. The goal for 2019 is less than 380 cases of BSI caused by carbapenem-resistant $A$. baumannii.
Several programs have been implemented by the $\mathrm{NCIC}$ to limit the spread of antibiotic resistance. Since 2007, policies have been in place to limit the spread of CRE, including screening for CRE carriage, isolation of colonized or infected patients and separation of nursing staff who care for CREpositive and negative patients. In 2019, a new program will be launched to control the spread of multidrug-resistant $A$. baumannii by applying the same measures used successfully to contain CRE.

A national antibiotic stewardship program, begun in 2012, established guidelines for responsible antibiotic use. Antibiotic use between 2012 and 2017 decreased by $11 \%$ in intensive care units, $16 \%$ in general surgery and internal medicine units in acute care hospitals, 38\% in post-acute care hospitals, and 4\% in ambulatory care.

Surveillance is crucial for identifying outbreaks or gradual increases in MDRO infections that require intervention. All acute-care hospitals report to the $\mathrm{NCIC}$ daily on all patients colonized or infected with CRE, and monthly on BSI caused by sentinel bacteria.

A national hand hygiene program is being launched in 2019 in collaboration with Hand Hygiene 
Figure 3.3

\section{Antibiotic consumption in acute care hospitals, Israel, 2012-2017}

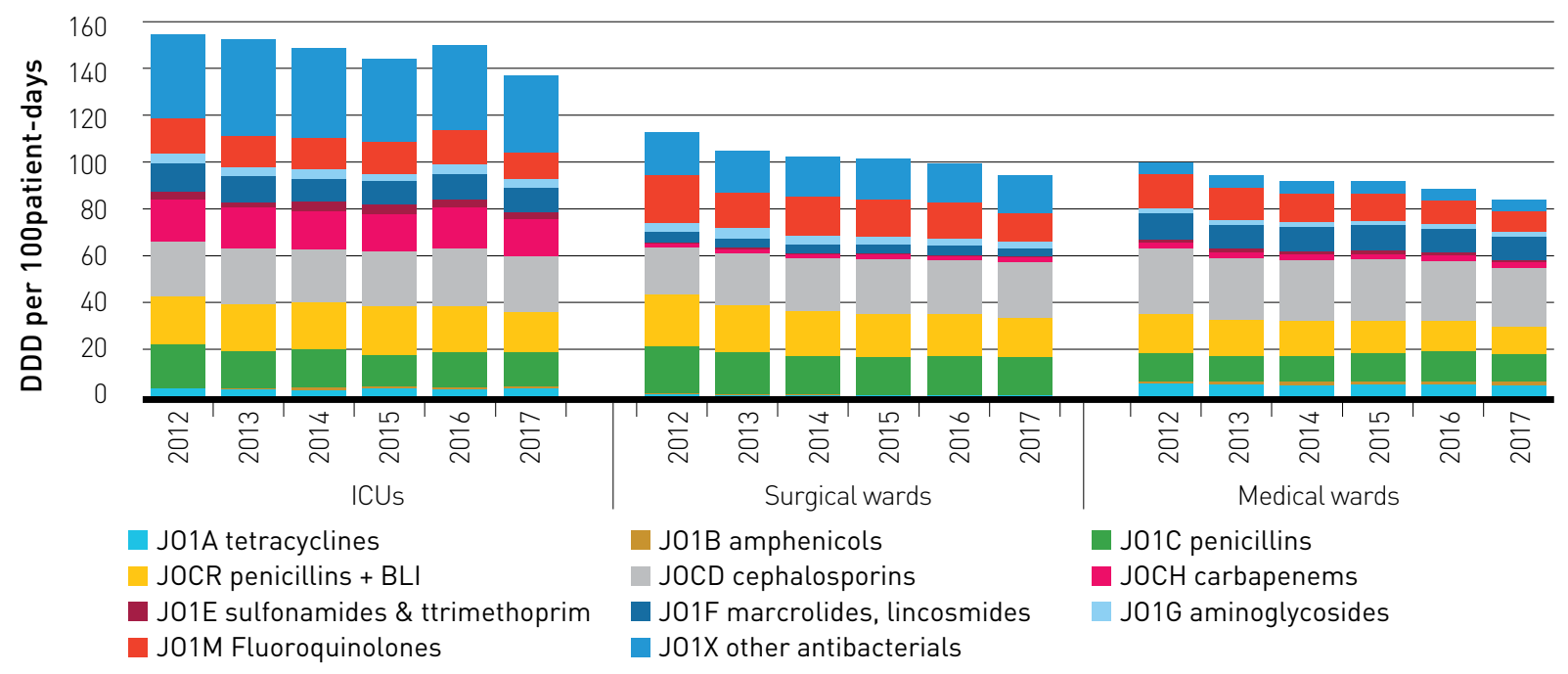

Sources: Dickstein Y et al. (2018) Trends in Antibiotic Resistance in Israel, 2014-2017. Dickstein Y et al. (2018) Trends in Antibiotic Consumption in Israel, 2012-2017 (both in peer review).

Australia. This program trains healthcare workers to conduct structured observations of hand hygiene in their hospitals and to provide feedback to staff.

A program in collaboration with the Ministry of Agriculture to monitor antibiotic use and antibiotic resistance in farm animals is scheduled to begin in 2019 as well.

Goals for 2030 include:

- Continued reduction of the spread of MDROs in healthcare settings, and specifically reduction of the number of hospital-acquired BSI caused by CRE and multidrug-resistant $A$. baumannii by $50 \%$ and the number of hospital-acquired BSI caused by MRSA by $20 \%$;

- Reduction of antibiotic use in ambulatory care, aimed at moving Israel from the middle tertile to the lower tertile of use among European countries;

- Establishment of an active "One Health" program that will integrate surveillance of antibiotic consumption and resistance, interventions to reduce antibiotic consumption and resistance in humans and animals.

\section{Investigation of Campylobacter - an emerging pathogen causing gastrointestinal infection in Israel}

Campylobacter has become the leading cause of foodborne infection in many EU countries despite extensive control efforts. Humans contract campylobacteriosis mainly by consumption or handling of contaminated meat as well as raw milk and unpasteurized dairy products or direct contact with food and pets.

Over the last decades, campylobacteriosis has increasingly been reported in Israel and, as a result, several epidemiological and microbiological studies by the Public Health Laboratories of the $\mathrm{MOH}$ have been conducted. Campylobacteriosis is a reportable disease in Israel. Microbiology laboratories countrywide refer human isolates from all sources to the National Campylobacter Reference Laboratory for confirmatory testing. New methods have been introduced to investigate campylobacteriosis and, in particular, molecular and genomic epidemiologic studies, including multi-locus sequence typing (MLST) and whole genome sequencing (WGS). 
In an initial study of 47,253 laboratory confirmed episodes of Campylobacterinfection over a 12-year period, the vast majority ( $>99 \%$ ) of episodes were due to $C$. jejuni and $C$. coli. Over this period, the annual incidence rate of all laboratory-confirmed Campylobacterinfection episodes increased from 31.04 to $90.99 / 100,000$ population. The linear annual increase in the incidence rate for the entire study period was $10.24 \%$ (95\% Cl 8.46-12.06) for all Campylobacter infection episodes, $10.07 \%$ 195\% Cl 8.42-11.74) for infection due to $C$. jejuni, and $10.73 \%(95 \% \mathrm{Cl} 8.19-13.33)$ for infection due to C. coli. A sharp rise in the annual increase rate from $8.22 \%(95 \% \mathrm{Cl} 4.88-11.68)$ to $18.97 \%$ (95\% Cl 12.95-25.31) was noted between 1999-2006 and 2007-2010.

Campylobacter incidence was 1.36 times higher in males than in females. The rapid increase and the high incidence of campylobacteriosis in Israel resembles that of New Zealand. While the food source of Campylobacterinfection in Israel has not been elucidated, during the study period poultry meat sales had markedly transitioned from mainly frozen to mainly fresh or chilled products.

The series of epidemiological and microbiological investigations taken by the Public Health Services have identified specific sub-populations at particular risk for emerging campylobacteriosis in Israel. Moreover, the investigations have delineated the clonal diversity of the organism in Israel, as compared to other countries and with respect to age and geography. Analyses support the assessment that poultry and cattle are likely food sources of $C$. jejuni infection in Israel with isolates able to persist over years. Campylobacteriosis is a well-known "One Health" challenge and the data generated by these studies should facilitate public health interventions across multiple sectors in order to mitigate the significant increase of campylobacteriosis in Israel over the last decade, aiming to significantly lower its health burden by 2030 .

\section{Strengthen the prevention and treatment of substance abuse (SDG 3.5)}

The field of substance abuse is covered by two government ministries: the Department for the Treatment of Substance Abuse in the $\mathrm{MOH}$ is responsible for medical prevention and treatment, including the training of health professionals, early detection in medical settings, medically assisted therapy (MAT) for opioid addicted patients and patients addicted to opioid pain-killers, detoxification in-patient centers, dual diagnosis treatment and rehabilitation, and the medical treatment of alcohol abuse. The Ministry of Labor, Social Affairs and Social Services (MOLSA) is

Figure 3.4

\section{Average annual kilograms of opioids seized per million inhabitants in OECD countries, 2012-2016}

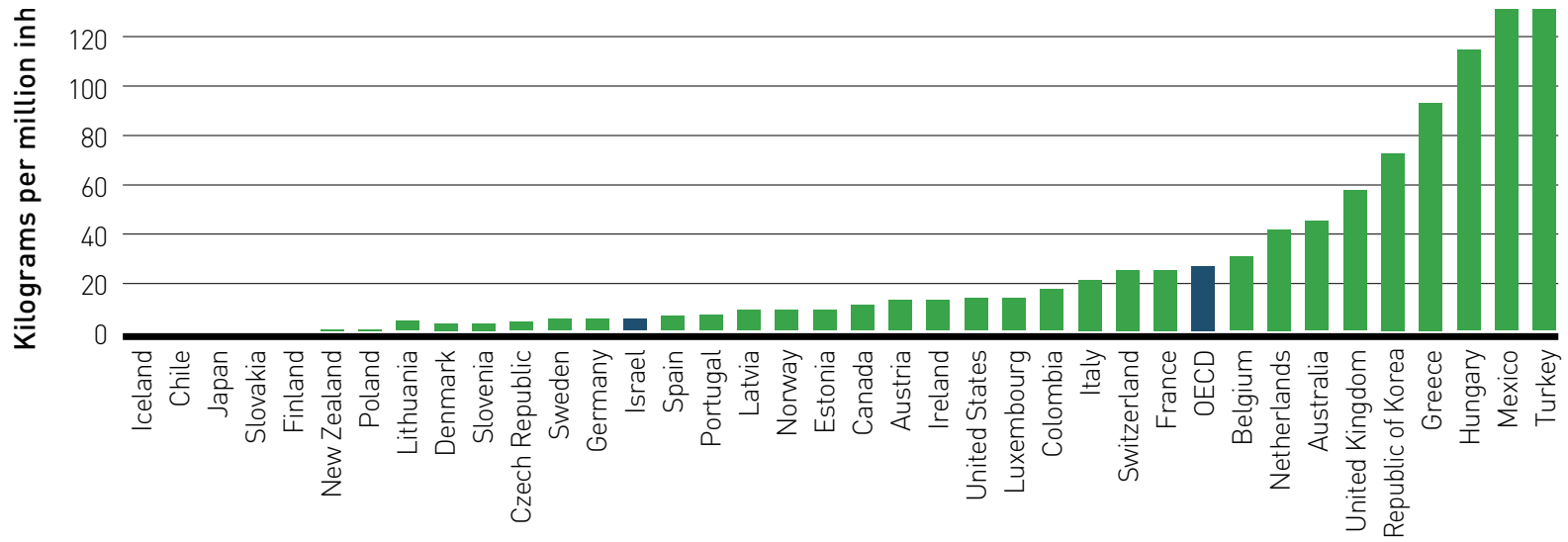

Source: OECD 
Figure 3.5

\section{Mean availability of analgesic opioids in OECD countries, 2011-13 and 2014-16 S-DDDs per million inhabitants per day}

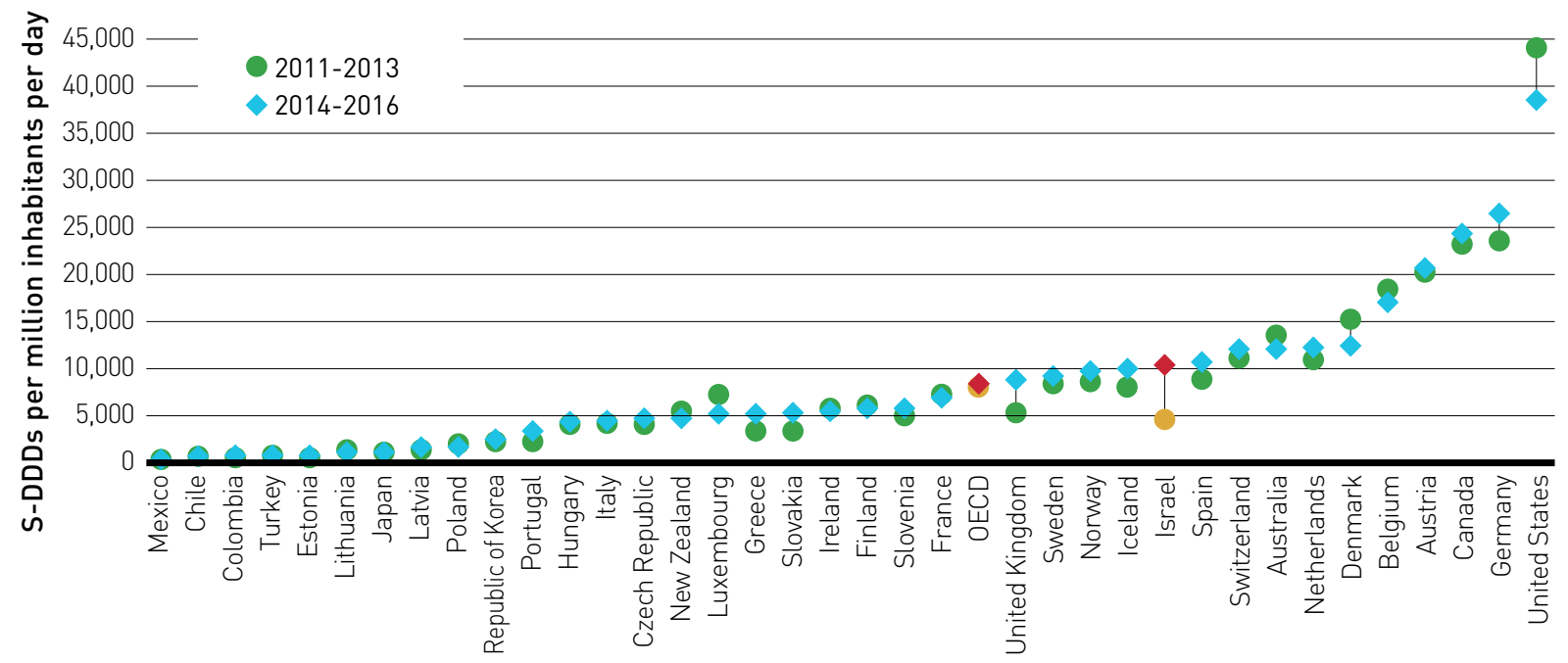

Source: OECD

responsible for psycho-social treatment and for the rehabilitation of substance use disorder patients who are substance free following detoxification.

\section{Opioids}

In 2017 4,066 patients underwent MAT treatment, of which $13.46 \%$ were females and about $20 \%$ were of Arab ethnicity. MAT treatment is a holistic treatment that includes both medical and psychosocial interventions for opioid addicted patients. Its main goal is to stabilize the patients and help them avoid street drugs while improving their medical condition and promoting their rehabilitation. The extent of each patient's copayment is a function of their use or non-use of street drugs.

One important indicator is the number of patients free from street drugs (72.69\%) and the number of patients who remain in treatment and adhere to it $(90 \%)$. Another indicator is the number of new patients per year. A decrease in the number of new heroin-addicted patients was noted in the last three years. This is likely due to changes in the profile of users in the local drug market, with more people using new psychoactive substances (NPS), stimulants, and cannabis. These individuals are more likely to be treated in detoxification centers or in ambulatory centers of the MOLSA, rather than in $\mathrm{MOH}$ centers.

Based on data obtained from three of the four HMOs, the number of people abusing opioid medications for chronic pain treatment in 20152017 has increased in Israel. The percentage of HMO patients increasing their doses of opioid medications over a period exceeding six months increased by $2.5-3.0 \%$ in 2016-2017 Irelative to 2014-2015)

As these patients are mostly normative and high functioning, they do not consider themselves part of the typical addicted population and consequently feel that attendance at MAT centers is inappropriate. Unique treatment opportunities have therefore been developed for this population including different treatment hours in existing centers as well as different treatment strategies and locations aimed at recruiting more patients into treatment.

The phenomenon of "doctor-shopping" in Israel has precipitated deliberations on the possibility of implementing an electronic monitoring system to connect pharmacies in both the HMOs and the private market. At this time, it has been decided 
that only electronic prescriptions for narcotics will be permitted. Implementation of this decision is underway and requires amending the Dangerous Drug Ordinance.

Another important indicator for monitoring opioid abuse is the number of deaths from opioid overdose. Data for this indicator show that the number is still relatively low in Israel. Due to the spread of Fentanyl on the black market, an increase of overdose deaths is expected in coming years. To prevent this and to increase patient awareness of possible dangerous sequelae and the possibility that addiction may develop while using opioids, the $\mathrm{MOH}$ has published and disseminated an alert document to be handed to each patient starting opioid treatment

Activities are also being promoted during National Overdose Awareness Day in all treatment centers. Plans are being advanced for additional quality indicators including the number of patients with chronic pain treated with high doses of opioids for long periods of time and the number of patients on chronic opioid use referred to addiction assessment or to psychiatric evaluation.

\section{Pharmacological treatment}

In 2013 buprenorphine/Naloxone (Suboxone) was added to the national health technology basket. This treatment is safer for patients than methadone and limits the risk of misuse. The number of patients with Suboxone treatment is still relatively low (around 700). To help patients shift from Methadone to Suboxone, a seminar on Motivational Enhancement Therapy was organized.
The number of patients who switched as well as the number of new patients who were prescribed Suboxone as initial treatment was monitored.

The Suboxone film formulation has recently been introduced, and it is expected that in 2019 Sublocade long-term injection will be included as a treatment both for high-functioning patients who work and wish to reduce lost work days, as well as for unstable patients.

\section{Hepatitis C}

$58 \%$ of MAT patients are hepatitis C-positive. Therefore, as part of the National Hepatitis C Strategy, they are referred to specialist direct antiviral agent (DAAS) treatment, which is included in the health basket. In 2017, 200 patients received treatment and had a complete recovery. Efforts are made to actively address this important issue and link each MAT center with a hepatologist. To check on progress, the following indicator will be used: number of positive HCV patients who received treatment and number of those who recovered. It should be noted that about $2.5 \%$ of drug users are HIV positive and receive treatment.

Following are some of the challenges that must still be addressed:

- The percentage of MAT patients over the age of 55 is around $20 \%$. As these patients undergo earlier mental and physical deterioration than their peers, special programs such as home-delivery and home treatment will be required as well as specific training of geriatricians and advocacy for nursing care coverage by the National Insurance Institute;

Table 3.3

Number of deaths from opioid overdose

\begin{tabular}{|c|c|c|c|c|c|c|c|}
\hline & 2009 & 2010 & 2011 & 2012 & 2013 & 2014 & 2015 \\
\hline $\begin{array}{l}\text { Opioid overdose as the } \\
\text { principal cause of death }\end{array}$ & 52 & 34 & 20 & 19 & 20 & 10 & 15 \\
\hline $\begin{array}{l}\text { Opioid abuse mentioned but } \\
\text { not as the principal cause }\end{array}$ & 4 & 2 & 5 & 0 & 5 & 1 & 4 \\
\hline Total & 56 & 36 & 25 & 19 & 25 & 11 & 19 \\
\hline
\end{tabular}


- Occupational rehabilitation is important, as about $60 \%$ of patients suffer from ADHD and sensory modulation disorder (SMD) and need to undergo a diagnostic and assessment process and referral to appropriate working places;

- Recruitment of more women into treatment is needed, with the aid of tailor-made programs that address motherhood, promote sexual health, baby-sitting in treatment centers, etc.

\section{Detoxification in-patient centers}

In 2017 2,502 people were admitted to detoxification centers, of which $15.5 \%$ were female. However, only $56.3 \%$ completed the entire detox program which lasts up to three weeks.

There are eight public detoxification centers in Israel with 170 beds. Two of them are for youths and young adults up to age 30 , one is for females and one is for males. There is a single center for mental dual diagnosis stabilized patients and one for physical dual diagnosis patients. Two centers are for alcohol detox. All are licensed by law and are audited on a monthly basis by the $\mathrm{MOH}$.

Most of the substances used by these patients are poly-substances lopiates, alcohol, benzodiazepines, cannabis, cocaine and stimulants, NPS, and medications). The number of patients who relapse and need more than one detoxification process is regularly monitored. As this number is high laround $50 \%$ ), staff are instructed to refer relapsing opioid use disorder patients to MAT treatment centers until they are stabilized.

Evidence-based practices have been introduced into all detoxification centers as well as continuity of care and door-to-door referral to the community treatment settings run by the MOLSA. Israel has also opened the first detox center for transgenders and sex-workers.

Despite the progress, challenges remain including:

- Culture-sensitive programs should be introduced for specific ethnic groups, including Bedouins, Muslim Arabs, Orthodox Jews, and Jews of Ethiopian descent. Early detection of these cases and their referral to specific culture-sensitive centers remains a challenge. As part of the National Ethiopian Program, awareness-raising seminars have been planned for professionals, and culture-based translation and mediation programs will be implemented in 2019;

- A dual diagnosis department for youth does not yet exist, but plans are on the table to open such a service in 2020;

- The number of dual diagnosis adult patients has been increasing, and currently around $45 \%$ of all mental hospitalizations are of dual diagnosis patients. Consequently, dual diagnosis departments will be opened in every mental health hospital in Israel by 2020.

\section{Alcohol}

In comparison with other European countries, Israel has a relatively low annual per capita consumption of pure alcohol (2.6 liters). Nevertheless, problem alcohol drinking and especially binge drinking is frequent among youths and young adults. During the years 2010-2017, the Israeli government launched the inter-ministerial National Strategy for the Reduction of Alcohol Use. As a result of this strategy, legislation limiting the sale and advertisement of alcohol beverages was enacted and succeeded in bringing about a reduction in alcohol-related violence. A pilot study based on the Screening, Brief Intervention and Referral to Treatment (SBIRT) model was conducted in the emergency room (ER) of a tertiary urban medical center in Tel Aviv, and the results confirmed the effectiveness of this intervention, especially among the young. Recommendations call for expanding this intervention to the ERs of medical centers throughout the country.

\section{Fetal alcohol spectrum disorders (FASD)}

In 2013, Israel joined other countries in adopting a National FASD day every September $9^{\text {th }}$ and has since organized an annual conference on the topic with the cooperation of the Israel Anti-Drug Authority (IADA) and medical specialists.

An outpatient center has been set up at the Schneider Children's Hospital as a specialist 
center to which HMOs may refer patients with the syndrome for diagnosis and counselling. In 2017. a question about alcohol consumption during pregnancy was included in the questionnaires administered to pregnant women by nurses in well-baby clinics (Tipat-Halav).

The syndrome has been recognized by the $\mathrm{MOH}$ as a complex somatic syndrome, with the right of patients to receive at least three free different treatments per week up to age 18 .

Challenges that still need to be addressed include:

- Developing evidence-based treatment programs for FASD children and training of child psychiatrists, psychologists and other relevant therapists;

- Collecting national data on the burden of FASD cases in Israel and following up on them.

\section{Drug-facilitated sexual assaults (DFSA)}

Victims of sexual assaults are preferably referred to acute rape centers situated in five medical centers in Israel. The link between substance use, both voluntary and involuntary, and sexual assaults has been identified, and awareness-raising activities targeted at medical and para-medical staff are conducted to help them detect the use of different substances.

A new regulation was recently issued instructing ERs and acute rape centers to store and refrigerate urine tests in order to allow drugs to be detected via uranalysis. Specific questions on rape-drugs were included in the general questionnaire for the staff of acute rape centers.

A pilot study involving analysis of hair is performed on victims of rape-drugs to identify the substances used. A study was conducted to assess the association between use of substances and mental disorders in sexual assaults victims attending the Wolfson Rape Center in Tel Aviv. The results showed that those hospitalized after a sexual assault together with the use of substances had been previously hospitalized in mental hospitals, and most suffered from schizophrenia, which worsened after the sexual assault with recurrent hospitalizations.

\section{Importation of wild poliovirus: Public health response, lessons learned, and implications for the future (SDG 3.8)}

Israel was certified as a polio-free country in 2002 along with the WHO European region. A routine childhood polio vaccine program has been in place in Israel since 1957. Since 2005, this program has included vaccination exclusively with IPV until the public health event described below.

On May 28, 2013, wild poliovirus type 1 (WPV-1) was detected in a sewage sample obtained during routine monthly environmental surveillance at a wastewater treatment plant in the Southern District. An emergency response team was appointed to manage the event consisting of virology experts, experts in infectious diseases, risk-communication specialists, and local health department officers. The team conferred with local and international experts on vaccine policy and infectious diseases and hosted onsite visits of a team of experts from the WHO and the US Centers for Disease Control and Prevention. The public health response to the event, which included a series of steps, proved highly successful (see box). In addition, it led to the formulation of several goals and interventions for 2030, including:

- Preventing the introduction and silent circulation of WPV/VDPV or Sabin strain;

- Ensuring control of potential PV sources, including continued environmental surveillance;

- Maintaining qualified laboratory and surveillance infrastructure;

- Verifying the presence of sufficiently trained human resources;

- Continuing the IPV vaccination only;

- Assuring preparedness and a rapid response plan in case of the re-introduction of WPV, VDPV or Sabin strain. 


\section{( Environmental health (SDG 3.9)}

A government resolution calling for the preparation of a national action plan on health and the environment was approved in March 2016. The resolution recognizes the direct link between environmental protection and health and calls on the Ministry of Environmental Protection (MoEP) and the $\mathrm{MOH}$ to formulate a national plan to prevent or minimize environmental conditions and hazards which may damage human health. The process is being led by an inter-ministerial task force.

\section{Ambient and household air pollution}

According to an OECD Environment Directorate report published in 2017, there were 2,240 deaths in Israel due to air pollution in 2015 , and there was an overall

\section{CASE STUDY}

\section{Public Health Response to the Detection of WPV-1}

The detection of wild poliovirus type 1 (WPV-1) at a wastewater treatment plant in Israel's Southern District in May 2013 led to a series of steps. Initially, population immunity levels against WPV-1 were determined retrospectively and prospectively. A seroprotective level of neutralizing antibodies was confirmed in $100 \%$ of the 104 children and in $98.2 \%$ of the 400 prospectively sampled individuals. A review of computerized records from well-baby clinics indicated that there were no pockets of under-vaccinated or unvaccinated individuals (i.e., <90\% coverage).

Simultaneously, rapid characterization of WPV-1 was performed. The virus found in these samples was closely related to polioviruses that had been circulating in polio-endemic Pakistan since 2012 that had been isolated from sewage samples in neighboring Egypt in December 2012.

Based on increased surveillance, the geographical viral transmission epicenter was identified showing WPV-1 positive sewage samples in the Southern District (Bedouin communities). A stool-survey analysis of 2,196 fecal samples collected from individuals residing within the identified epicenter was performed and the point prevalence established. WPV-1 was detected in $2.8 \%$, of which $90.2 \%$ were obtained from a Bedouin population and $96.7 \%$ were obtained from children under 10 years old. Hospital diagnoses were scrutinized on a weekly basis to detect cases of acute flaccid paralysis and aseptic meningitis in all age groups from June 2013 to December 2014.

To protect the population, especially children, from paralysis, an emergency response team recommended completion of a routine IPV childhood schedule of four doses for children up to age six throughout Israel, as well as for children up to age 18 and adults residing in the epicenter and its surrounding perimeter. An IPV booster was given to $31 \%$ of adults who had been previously vaccinated.

With the aim of breaking the chain of viral transmission, supplemental immunization with bivalent OPV (bOPV) polio vaccine, containing Sabin 1 and Sabin 3 polio vaccine strains, was recommended for all children younger than 10 years who had been removed from the routine vaccine program in 2005. This decision posed risk-communication challenges because reintroducing a live attenuated vaccine in an IPV-only country with nearly universal protection against paralytic disease and no clinical poliomyelitis cases identified was unprecedented. Two major campaigns were launched, media platforms were used to communicate the rationale for the decision, and a call-center was opened to address questions from the public. 
The bOPV SIA was initiated on August 5, 2013 in the Southern District and was expanded on Aug 18, 2013 to the rest of the country. An estimated 1.2 million individuals were vaccinated during the first two weeks. At the end of the bOPV SIA, national bOPV coverage reached $79 \%$ 1943,587 children younger than 10 years), and bOPV coverage for the entire target age group in the Southern District reached $90 \%$. Almost $100 \%$ coverage for the eligible target age group was reached in the southern Bedouin communities at the epicenter. All potential adverse reactions to bOPV were thoroughly investigated and reported to the public.

To determine the effectiveness of the intervention, vaccine strains in the sewage were monitored as a surrogate for vaccine compliance. Additionally, fecal samples were tested for WPV-1 among children at the transmission epicenter, with the last two positive samples detected in October 2013. On April 28, 2015, the WHO officially declared Israel a polio-free country.

The successful interruption of transmission may be attributed to several actions. The surveillance early warning system allowed public health officials to respond in a timely fashion to the public health threat. A risk communication strategy was critical in conveying the importance of vaccination with bOPV, enabling high vaccination coverage to be achieved in the targeted population. The absence of clinical cases was likely attributable to the high routine IPV vaccination coverage of children younger than 10 years at the epicenter and to the consistently high national routine childhood IPV coverage over the years.

To reduce the risk of re-emergence of WPV type 1, the emergency response team recommended vaccinating all children born after July 1, 2013 with a dose of bOPV at 6 months followed by a second dose at 18 months, in addition to the routine IPV-only schedule. This sustainable interim policy is expected to continue as long as the risk of importation of WPV exists.

increase in the mortality rate as a result of particulate matter (PM) air pollution between 2010 (238 per million) and 2015 (265 per million). The report estimated the cost of premature mortality due to air pollution in 2015 at $\$ 7.3$ billion. The mortality rate from PM in Israel in 2017 was low compared to Western Europe $(264$ per million compared to 349 per million).

The Clean Air Law was approved by the Knesset (Israel parliament) in July 2008 and entered into force in 2011. Its objectives are "to bring about an improvement of air quality and to prevent and reduce air pollution...to protect human life, the health and quality of life of human beings and to protect the environment...for the public and for future generations, and in consideration of their needs."

Among others, the law calls for the preparation of a national program for air pollution reduction that is to be updated every five years. In 2013, reduction requirements and implementation measures for the national plan were anchored in Government Decision 707 - National Plan for the Prevention and Reduction of Air Pollution in Israel. The program focuses on the prevention of air pollution from transportation, industry, energy and households.

The first update of the national program is currently being finalized. It establishes a multi-year plan for meeting pollutant reduction emission targets for the years 2023 and 2028. Reduction targets are measured according to emissions from seven pollutants (PM2.5, PM10, SOx, NOx, NMVOC, benzene and 1,3 butadiene). To date, significant reductions have been recorded and additional targets are expected to be met in the next decade.

Reducing pollution from diesel vehicles is high on Israel's priority list for air pollution reduction, based on data that show that diesel vehicles emit some $80 \%$ of fine respirable particulates emitted by cars, although they are responsible for only $20 \%$ 


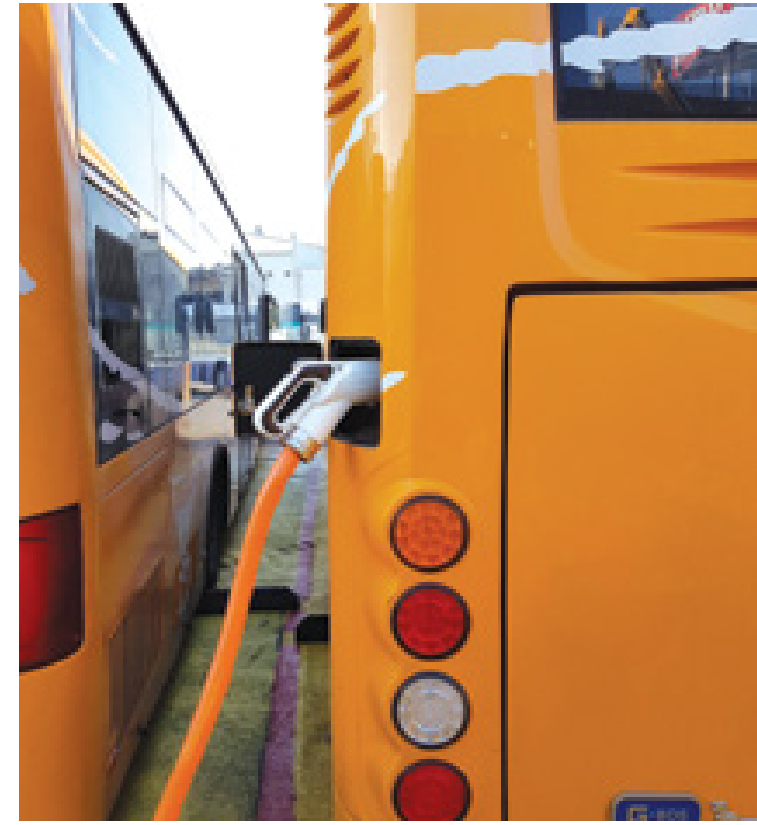

Electric bus charging/Photo: Amir Zalzberg

of the kilometers traveled. In recent years, several plans for pollution abatement in the transportation sector have been approved and implemented. They include:

- Government subsidies for the installation of particulate filters on all heavy diesel vehicles in the country. Licenses for old, heavy diesel vehicles will be renewed only if a filter has been installed;

- A hybrid taxi program to encourage taxi drivers to buy hybrid cars with a "green index";

- Addition of electric buses to Israel's public transportation fleet with government support;

- Financial assistance to local authorities for establishing electric car-sharing programs, with designated parking spots;

- Provision of government remuneration for the scrapping of old diesel vehicles;

- Establishment and operation of low emission zones (LEZ) in cities to which entry of polluting diesel vehicles will be prohibited.

Israel's national air monitoring system is composed of a network of 146 air quality monitoring stations across the country that measure criteria pollutants as well as additional sampling sites that measure non-criteria pollutants. In addition to the MoEP, the stations are operated by local authorities, the
Israel Electric Corporation, industry and others. A total of 21 pollutants are monitored. Since 2016 black carbon is monitored as well.

The Israel Air Monitoring Network Laboratories received accreditation for testing in 2013 , becoming the third system in the world to receive such accreditation.

\section{Drinking water quality}

Mortality from unsafe water, unsafe sanitation and lack of hygiene is extremely low in Israel. Reported outbreaks of legionella are rare and annual prevalence is $0.6 / 100,000$.

Based on 2015-2016 data, the main chemical contaminants in Israel's drinking water include atrazine lin $8.4 \%$ of the sources, concentrations between $0.08-0.67 \mu \mathrm{g} / \mathrm{L}$ ), simazine (in $13.4 \%$ of the sources, concentrations between 0.08-0.52 $\mu \mathrm{g} / \mathrm{L}$ ), trichloroethylene (in $13.7 \%$ of the sources, concentrations between 0.1-101.6 $\mu \mathrm{g} / \mathrm{L}$ ), and tetrachloroethylene (in $10.3 \%$ of the sources, concentrations between 0.1-176.8 $\mu \mathrm{g} / \mathrm{L}$ ). In light of the findings, simazine has been phased out and atrazine use has been restricted to critical uses only.

Data from 2014-2016 show that lead concentrations were below detection levels in $65 \%$ of the samples from municipal water systems. Lead concentrations exceeded the standard in $0.3 \%$ of the samples. Israel has adopted new requirements restricting lead use in products in contact with drinking water. As of 2018-2019, lead content in all products in contact with drinking water is limited to $0.25 \%$. This further strengthens regulatory requirements restricting migration of lead and other heavy metals from products in contact with drinking water.

Based on 2014-2016 data, 82\% of the samples were above the detection level for trihalomethanes. In three cities, the average weighted concentration was between $80-100 \%$ of the standard. In the wake of national policies in 2016 regarding disinfectant and supply systems, the dilution of surface water with groundwater or desalinated water, and the aeration of water reservoirs, trihalomethane levels decreased significantly in Israel's drinking water sources. 


\section{CASE STUDY}

\section{Air Pollution Reduction in Haifa Bay}

Haifa Bay has long been considered one of Israel's air pollution hotspots due, among others, to its large concentration of industrial plants and its topographical features and climate which impede pollutant dispersion. In September 2015, the government approved a National Plan for the Reduction of Pollution and Environmental Hazards in Haifa Bay, which was submitted by the Ministers of Environmental Protection, Finance, Transport and Health. Environmental and health concerns in the highly industrialized Haifa Bay region precipitated the government decision to significantly reduce pollutant emissions to the environment while expanding monitoring and increasing epidemiological research on pollution-related mortality and morbidity in the area.

The national plan includes the following components, among others:

- Reduction of air pollution from industry and transportation;

- Expansion of air quality monitoring and sampling;

- Increase in environmental and health surveys and research;

- Reduction of hazardous material risks;

- Transparency and accessibility of information to the public.

A low-emission zone (LEZ) has also begun operating in Haifa, promoted by the Ministry of Environmental Protection and the Haifa Municipality, based on examples from Europe. Polluting heavy diesel vehicles are no longer allowed in the center of the city, while buses and trucks are required to install a particulate filter in order to enter the restricted area. A subsidy program for operating electric buses in the city is in place.

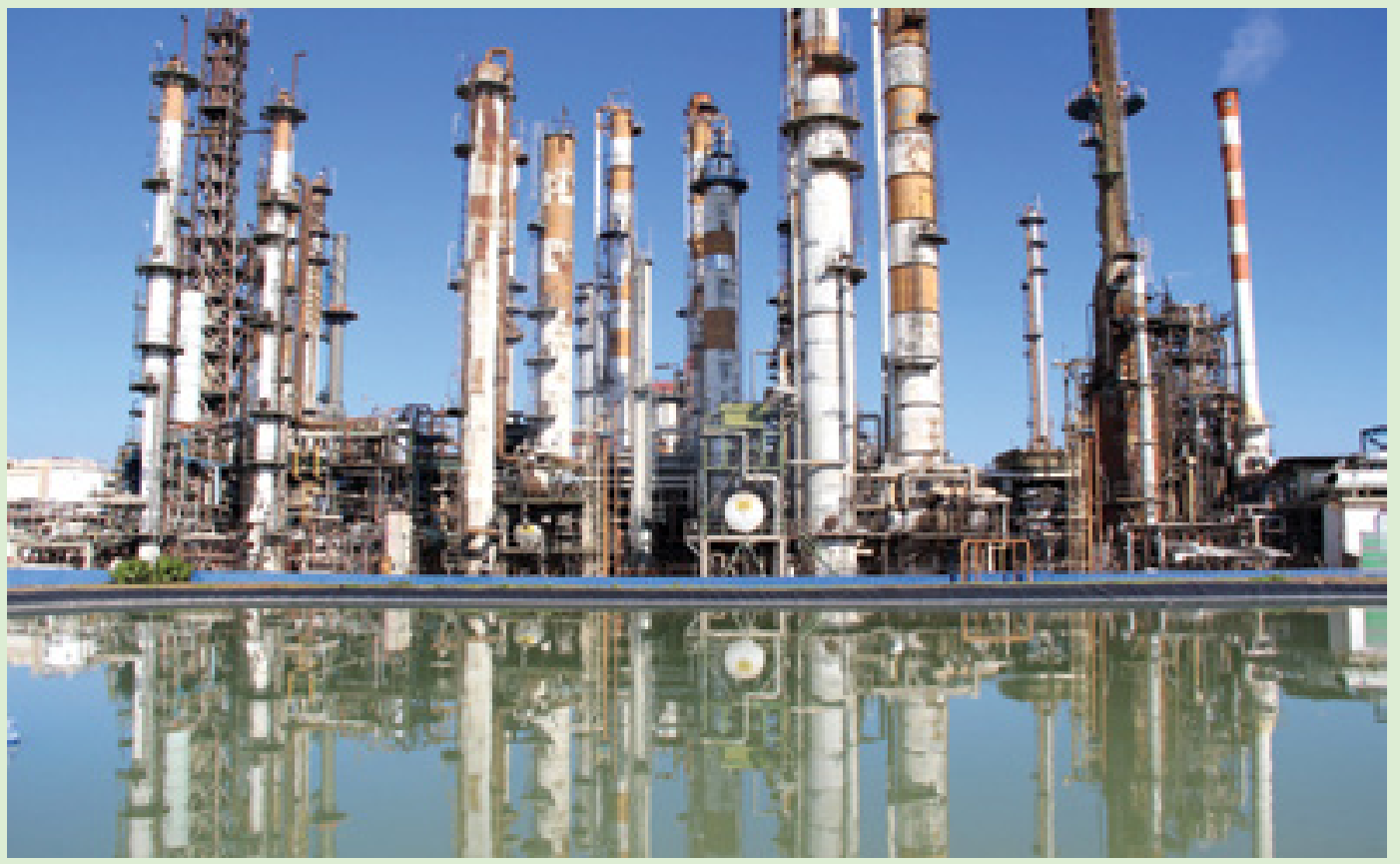

Industry in Haifa Bay/Photo: Ilan Malester 
As part of the national response, the $\mathrm{MOH}$ published Guidelines for Preventing Legionella in Water Systems (2016), a Guide for Preventing Legionella in Institutions and Businesses (2015), and a Guide for Epidemiologic Investigation of Legionnaire's Disease (2015).

It is expected that the following targets will be reached in 2030:

- $30 \%$ reduction in the percent of drinking water sources contaminated by atrazine or simazine;

- $30 \%$ reduction in the number of drinking water samples with detectable levels of lead (above $2 \mathrm{ppb}$ );

- $30 \%$ reduction in the number of drinking water samples with detectable levels of trihalomethanes.

\section{Poisonings and exposure to toxic chemicals}

Between 2015-2017, there were 5,966 emergency room visits among children (0-14 years), and two deaths from poisonings (in 2011-2015). Between 2008-2018, a total of 16 deaths from poisoning were reported, half in children under the age of four. Emergency visits related to poisonings are underreported.

Two children died in 2014 as a result of the illegal use of phosphine for domestic pest control in a residential building in Jerusalem. In 2013, a child died of liquid nicotine poisoning. In southern Israel, there were 43 cases of carbon monoxide poisoning in children between 2011-2015, and five cases of pediatric mortality. In 2012-2015, the mortality rate in adults for poisonings (undetermined intent) was 1.1 per 100,000 in people between the ages of 25-44.

According to data collected by the Israel Poison Information Center, there were 1,932 poisonings in 2014, of which only 27 resulted from occupational exposure. Most moderate or severe poisonings were attributed to exposure to organophosphates (OP) or carbamates. The total number of OP and carbamate poisonings in 2014 was 194. Data on emergency room visits reveals 18 visits in 2015 related to pesticide exposure, including seven cases involving children under the age of four. These data are partial and likely reflect underreporting by physicians.

The National Program for Preventing Childhood Injury includes programs to prevent childhood poisoning from chemicals and pharmaceuticals. Proposed legislation includes a requirement for the liquid component of electronic cigarettes to be marketed only in child-resistant containers. Israel Standard 4272 from 2009, which relates to corrosivity of oven-cleaning products, aims to prevent chemical burns. An additional standard (1346) relates to childproof packaging for pharmaceuticals.

Following findings of much higher concentrations of lead and cadmium in children's jewelry, the Standards Institution of Israel approved a new standard restricting lead and cadmium in children's jewelry in 2018, which is mandatory as of August 2018. In addition, following findings of high concentrations of lead in painted surfaces in playgrounds and in spray paints, the Standards Institution approved a new standard restricting lead in all paints, including industrial paints, to 90 ppm, as per the Model Law by the UNEP-WHO Global Alliance to Eliminate Lead in Paint.

Following the death of two children from phosphine poisoning, severe restrictions on the sale and use of phosphine have been implemented. Many organophosphate and carbamate pesticides have been phased out of agricultural use.

Targets for 2030 include:

- Zero pediatric mortality from pesticides and other hazardous chemicals;

- $30 \%$ reduction in emergency room visits by children from poisonings.

\section{Control of tobacco in Israel (SDG 3.a)}

The $\mathrm{MOH}$ leads the implementation of the National Tobacco Control Plan, which is based on the MPOWER (Monitor, Protect, Offer help, Warn, Enforce bans, Raise prices) and the WHO FCTC (Framework Convention on Tobacco Control) initiatives. Following are some highlights of the progress made:

- Biannual surveys to monitor smoking status in the general population and within specific groups, such as minorities, are conducted; 
- The prevalence of smoking in the general population aged $21+$ was $20.5 \%$ in 2017 , a decrease of $1.6 \%$ relative to the $23.1 \%$ prevalence in 2014:

- By law, the Minister of Health must submit an annual report to the Knesset on tobacco and tobacco control in Israel, including data on such relevant aspects as epidemiology, economics, legislation, enforcement, smoking cessation, tobacco industry expenditures, exposure to second-hand smoke, etc.:

- Since 2012, legislation that bans smoking in public places and potential exposure to second-hand smoke (passive or environmental smokingl has been upgraded. A complete ban on smoking is imposed in government buildings, municipalities, hospitals, schools, kindergartens and playgrounds, gymnastic areas, and in every gathering consisting of 50 or more people in a defined place. There is also a partial ban (designated smoking area or rooms are allowed) in public transportation stations, sport stadiums, pubs and restaurants;

- Smoking cessation counseling groups are offered free of charge by the four HMOs, and those receiving counseling are entitled to an $85 \%$ reimbursement for smoking cessation prescription drugs Varenicline (Champix) or Buproprion (Zyban). Patients also receive an $85 \%$ reimbursement on second line drugs such as NRT (nicotine replacement therapy). In two of the HMOs there is also a tobacco cessation program;

- During the 2018 FIFA world cup, a €630,000 campaign on smoking cessation that ran for a few weeks on TV and the Internet was initiated;

- During 2017, the MOH addressed the introduction of IQOS into the Israeli market assuring that IQOS is considered a tobacco product similar to cigarettes, with identical regulation and taxation;

- Based on WAP (weighted average price), the tax on cigarettes as a percent of the wholesale price, was $80.38 \%$ in 2015 . This percent has since decreased to $78 \%$ due to several price increases by the tobacco industry;
- The Knesset is in final stages of implementing most of the guidelines of article 11 and 13 of the FCTC, including plain packets for cigarettes and for electronic cigarettes and a total ban on flavors both in tobacco and e-liquids.

\section{National Program for Quality Indicators in Hospitals (SDG 3.8)}

The National Program for Quality Indicators in Hospitals, established in 2012, promotes quality healthcare in core areas of the Israeli health system by measuring the quality of clinical care throughout the human lifecycle. This program encompasses general, geriatric and mental health hospitals, well-baby clinics, and psychiatric rehabilitation and ambulance services. It supports consistent inspection of the quality of health services to advance prevention, diagnosis, clinical care, and rehabilitation. The program is accompanied by an advisory committee for quality indicators (QIs), which recommends indicators for service providers, defines the measurable variables for each indicator, analyzes the results, and generates conclusions and recommendations for improving quality. As of 2013, all Israeli hospitals report on five initial clinical quality indicators.

Qls are recommended after a long process of selection which includes public scrutiny. Complete transparency assists in accelerating improvements in the healthcare system and helps create a positive perceptual change among healthcare service providers.

The first Qls included the care and treatment of acute myocardial infarction and acute cerebral ischemic stroke and infection prevention. For each of these areas, the $\mathrm{MOH}$ raised care standards by defining and instituting a national battery of linked Qls that could be applied across all relevant facilities and contexts, with the ability to measure socio-economic and socio-geographic disparities. Following the implementation of the initial QIs, the percentage of patients with ST-Elevation Myocardial Infarction (STEMI) who received percutaneous coronary intervention (PCI) within 90 minutes of arrival at the hospital increased by $27 \%$, from $63 \%$ in 2013 to $90 \%$ in 2016. 
Yet another $\mathrm{Q}$ l relates to caesarean section (CS), one of the most common surgical procedures. Surgicalsite-infection (SSI) occurs in approximately 5-10\% of CS, and the benefit of prophylactic antibiotics for prevention of SSI has been demonstrated extensively in the literature. In January 2014, the $\mathrm{MOH}$ introduced a national quality measure that monitors the administration of prophylactic antibiotics in CS, leading to a significant improvement nationwide - from $78 \%$ compliance in 2014 to $93 \%$ in 2016 .

The QI Program has expanded to include 83 quality indicators, with plans for expansion into the following areas:

- Medical treatment outcomes, including treatment success, complications, and side effects;

- Medical procedures, including medical documentation, accuracy of diagnosis, medication, medical interventions, and compliance with regulations;

- Common medical diagnoses and test results;

- Level of investment in safety, including adverse event reporting, existence and functioning of oversight committees, conduction of investigations of unusual events, coping with exceptional events, investment in infrastructures and structure;

- Presence and use of medical infrastructure and personnel resources, including equipment, medical technologies, medical equipment and computers;

- Use of technology to improve service, including improvement of patient overall care and patient satisfaction.

Following five years of the QI Program, it may be concluded that there has been a significant and consistent improvement in most of the measured quality indicators, both nationally and institutionally, as well as a change in organizational culture within the Israeli healthcare system. The program has helped create processes that enhance the clinical continuity at the intersections between service providers.

\section{PARTNERING FOR A BETTER WORLD}

In supporting SDG 3, Israel champions the right of all people to live better and healthier lives, and remains committed to working in the spirit of global cooperation to create an enabling environment among the poor and marginalized for equitable access to quality health systems and services. Israel actively engages in cooperating with UN specialized agencies, regional organizations and partner countries on global public health issues. In 2018, during the 71st Session of the World Health Assembly (WHA), the Associate Director General of Israel's Ministry of Health was elected to the Executive Board of the World Health Organization (WHO) as a representative of the European Region. As a board member, Israel will advocate for such priority issues as emergency health programs, polio eradication, access to assistive technologies, technology and innovation for health, and reduction of noncommunicable diseases. (SDG 3.d)
Reducing infant and child mortality is central to Israel's development cooperation program, with special focus on neonatal care/maternal health in rural and underserved areas, as well as management of healthcare systems for rural women. The following are two examples of MASHAV's projects implemented to reduce maternal mortality in Africa and end preventable deaths of newborns and children: (SDG 3.1, 3.2, 3.c)

In 2006, MASHAV and the Millennium Cities Initiative (MCI) began a joint project to reduce neonatal and maternal mortality in Kumasi, Ghana. Following a fact-finding mission, MASHAV established (20082009) two Mother and Baby Kangaroo Mother Care Units for high-risk and moderately sick newborns and their mothers, in partnership with the Soroka University Medical Center. Throughout the years, an onsite training program for the medical staff of the units has continued. In addition, and in line with the Government of Ghana's development goals to 
reduce infant mortality and improve access to quality healthcare, MASHAV is working together with local authorities to enhance the professional capacities of hospitals and their medical staff to provide adequate neonatal care for sick and high-risk infants.

Within the multilateral framework, Israel spearheaded the UN Resolution on Supporting the Availability, Accessibility and Diversity of Scientific Evidence-Based Treatment and Care for Children and Young People with Substance Abuse Disorders, adopted at the $58^{\text {th }}$ Session of the UN Commission on Narcotic Drugs (CND) in Vienna. (SDG 3.5)

Furthermore, MASHAV, the UN Office on Drugs and Crime (UNODC) and Israel's Anti-Drug Authority (IADA) established a long-term relationship comprising the implementation of joint capacitybuilding courses in Israel for professionals from the developing world on A Comprehensive Drug Control Strategy - The Israeli Model. (SDG 3.5)

UNODC rolled out a workshop in Kenya based on the publication jointly written with the WHO on Treatment and Care for People with Drug Use Disorders in Contact with the Criminal Justice System - Alternatives to Conviction or Punishment. The workshop, supported by the European Union, Israel and Sweden, is part of a broader initiative exploring strategies and options to direct people with drug use disorders in contact with the criminal justice system to the health care system, in line with international drug control conventions, while addressing the specific needs of women with drug use disorders in Kenya. (SDG 3.5)

Israel's Embassy in Cameroon, in a multistakeholder partnership with the UN High Commissioner for Refugees (UNHCR), WHO, the local Ministry of Health, and the Pediatricians Association (CPA), organized (2016) the third mission of Operation Soleil - Israel, supporting Cameroon's National Vaccination Campaign. More than 4,000 polio vaccines and over 500 pediatric consultations were conducted for the benefit of local and refugee populations of Garoua Boulai and Gado-Badzere. Later that year, urgent assistance was provided to help fight the spread of the bird flu epidemic affecting the country. (SDG 3.b)
In alignment with SDG 3.9 to substantially reduce the number of deaths and illnesses from hazardous chemicals by 2030, Israel is working in cooperation with the Global Alliance to Eliminate Lead Paint. In 2016, Israel's Ministry of Health became a partner in the Global Alliance, and since 2018, the US Environmental Protection Agency (EPA) has provided Israel with technical advice on how to establish a strong legal limit for lead paint.

Israel has supported the important work of the European Environment and Health Ministerial Board (EHMB) since its establishment at the Parma Conference (2010) as the political face and driving force of international environment and health policies. The Task Force, the leading international body for implementation and monitoring of the European Environment and Health Process, includes leading officials from all member states in the WHO European region. From 2010 to 2017, the EHMB was chaired by 10 countries, including Israel. In 2015, Israel hosted the European Environment and Health Process Mid-Term Review and continues to serve on the European Environment and Health Task Force (EHTF). In 2018, the Task Force Bureau comprised eight member states, including Israel. (SDG 3.d)

As noncommunicable diseases (NCDs) are the main contributors to the global disease burden, Israel sponsored the first meeting of the WHO Global Coordination Mechanism (GCM) on the Prevention and Control of Noncommunicable Diseases (GMC/ NCD). In addition, during the Third High-Level Meeting (HLM3) of the UN General Assembly on NCDs (2018), Israel, China, Switzerland and the Czech Republic, in cooperation with the WHO and non-state actors, conducted a secondary event on The Power of Partnerships: The Realization of High-Level Commitments to Prevent and Control NCDs. (SDG 3.4)

The Clalit Research Institute in Israel was designated as a WHO Collaborating Center on Noncommunicable Diseases, Research, Prevention and Control, under the WHO European Region Division of Noncommunicable Diseases and LifeCourse. The Institute provides follow-up technical support and undertakes activities to contribute to 
policy development, prioritization and planning pertaining to the implementation of the Global Action Plan for the Prevention and Control of NCDs 2013-2020, the Action Plan for Implementation of the European Strategy for the Prevention and Control of Noncommunicable Diseases, and the Ashgabat Declaration on the Prevention and Control of NCDs in the Context of Health 2020. (SDG 3.4)

Israel joined the consensus on the adoption of the UN Resolution on Scope, Modalities, Format and Organization of the High-Level Meeting on the Fight against Tuberculosis (72/268) at the $72^{\text {nd }}$ General Assembly. Between the years 2016-2018, Israel's Ministry of Health built focused projects to tackle these issues internationally, including meeting the prerequisite requirements to become a WHO Consulting Center for TB Elimination. This past year, the National Tuberculosis Control Program (NTP) launched the First Tuberculosis Training Program for Consultancy in High TB Endemic Countries hosted by Israel, in cooperation with the WHO Global TB Program and other institutes, including the Braun School of Public Health at Hebrew University. The 17 participants represented low and middle-income countries, 15 of whom were from sub-Saharan Africa. Over the next two years, Israeli TB experts will join several of the participants in their home country for onsite monitoring and mentoring. In 2019, Israel will again host a training program for doctors from high TB endemic countries. (SDG 3.3)

In addition, Israel was selected to be a Core Member of the Working Group on the European Tuberculosis Research Initiative (ERI-TB), based in Copenhagen. Partnership in the Working Group is supplemented by participation in annual workshops throughout the WHO European region on TB and AIDs. Since 2016, Israel participated in four WHO workshops: Meeting of the National HIV Program Managers from Eastern Europe and Central Asia (EECA) and Non-EU/EEA Countries; First Global Ministerial Tuberculosis Conference: Ending TB in the Sustainable Development Era: A Multisector Response; Regional AIDS Surveillance Meeting; and Regional Workshop on HIV and Viral Hepatitis Testing and HIV Pre-Exposure Prophylaxis (PrEP) in
Non-EU/EEA Countries - European Region. Israeli experts also participated in the ECDC/UNAIDS European Meeting on HIV PrEP - Pre-Exposure Prophylaxis in the EU/EEA Setting, the Agence Nationale de Recherche Scientifique (ANRS) Medical Research Council (MRC) Expert Workshop on HIV Pre-Exposure Prophylaxis (PrEP) Trial Design in Europe, and workshops held in Austria and the Netherlands. (SDG 3.3)

Israel engages with both UN specialized agencies and bilaterally with partner countries in the field of disaster medicine, trauma care, and crisis management. Examples of its extensive programming include: (SDG 3.d)

- Israel hosted a delegation from the UN Peacekeeping Forces - Medical Services Division for a Life-Saving Treatment training activity, focusing on imparting basic medical techniques in situations of physical trauma. Guidelines based on Israel's methods were included in official UN procedures. Following the training, the UN Medical Director visited Israel and conducted a recruitment seminar aimed at Israeli medical professionals;

- Israel took part in the UN Buddy First-Aid Course in Uganda under the auspices of the UN Medical Director. Furthermore, an Israeli medical expert participated in the UN organized Triangular Partnership Project Seminar in Rwanda, focusing on training medical staff in UN Peacekeeping Forces. The fruitful collaboration with the UN resulted in a special invitation for Israel to serve as a Super Trainer, along with Japan and Great Britain, and to lead the UN course on "First-Aid Treatment" (Italy 2018);

- Since 2016, Israel has participated in, or led, over 30 capacity-building and evaluation missions in partnership with the WHO and other UN bodies. In the last two years, Israel participated in Joint External Evaluation (JEE) Missions to Namibia, Finland, Belgium, Thailand, Moldova, Switzerland and Liechtenstein, and took part in the JEE Leaders Course;

- In 2016 and 2018, Israel hosted the biennial International Conference on Preparedness and Response to Emergency Disasters (IPRED), 
with the participation of delegations from more than 35 countries. Topics included urban terrorism, consequences of prolonged conflicts, national, international and inter-organizational preparedness, and ethical aspects;

- Israel's Ministry of Health hosted (2018) highranking official delegations from Japan, Singapore, China, Germany and the European Union to exchange best practices and study the Israeli emergency medical preparedness model;

- A Workshop on Mass Casualty Management and Emergency Medical Teams was conducted (2017) in Israel in cooperation with the Ministry of Health and the WHO Regional Office for Europe. Topics included pre-hospital and hospital mass casualty management, cyber security for the health sector, and safety/security in a field hospital situation. Participating countries included Albania, Armenia, Azerbaijan, Estonia, Georgia, Kazakhstan, Kyrgyzstan, Moldova, Serbia, Tajikistan and Turkmenistan;

- Within the framework of the Government of Israel's decision to direct its overseas development assistance at upgrading the quality of healthcare services in Africa, MASHAV constructed emergency and trauma units in Guinea Conakry, Togo and Tanzania, supported by onsite capacity-building programs for community medical staff. In 2018, following the establishment of a new intensive care and trauma unit by Israel in the Atakpamé Hospital in Togo, MASHAV conducted its first regional training course in Togo on emergency preparedness for doctors and senior medical staff from Togo, Benin, Ghana, Burkina Faso, Cote d'Ivoire, Senegal, Cameroon, Liberia and Guinea Conakry;

- Building on its international reputation, the Department of Emergency and Disaster Management, Tel Aviv University Sackler Faculty of Medicine was designated (2018) as a WHO Collaborating Center for Disaster and Emergency Medicine Management and Research. Joint activities are carried out in partnership with Israel's National Center for Trauma and Emergency Medicine Research and Tel Aviv University;
- The Gertner Institute for Epidemiology and Health Policy Research was designated as a WHO Collaboration Center for Research on Trauma and Emergency Medicine and for Emergency and Disaster Management. Israel actively supports the work of the WHO Emergency Medical Teams (EMT) Initiative, assisting organizations and member states in building institutional capacities and strengthening health systems by coordinating the deployment of quality assured medical teams in emergencies.

Affirming SDG 3.c that an expertly trained health workforce is essential for local and national health systems to function more effectively, MASHAV's programming included:

- Cooperation with the International Master of Public Health (IMPH) Program conducted annually in Israel by The Braun School of Public Health \& Community Medicine. This one-year APHEA-accredited IMPH Program provides tools for examining public health challenges and formulating relevant institutional responses at the national and community levels. WHO designated (2007) the School as a Collaborating Center for Capacity-Building in Public Health;

- Capacity-building courses on Innovations in Women's Health and Modern Infectious Disease Epidemiology: Concepts, Methods and Public Health;

- Triangular collaboration between Israel, Italy, and Ethiopia within the framework of a Memorandum of Understanding (MOU) on Cooperation in the Fields of Health and Medicine, geared to train Ethiopian healthcare personnel in pediatric emergency medicine, public health and ICTs;

- Development cooperation in the Middle East region through capacity-building programs conducted on Management of Health Systems and Balance Control and Fall Prevention for professionals from the Palestinian Authority, in partnership with the Green Land Society for Health Development;

- Regional Workshop on Introduction to Motor Learning for Physical Therapists, in partnership with the Jordan Red Crescent; 
- Workshop for Syrian Children and Their Mothers, in cooperation with Ziv Hospital in northern Israel. Israel possesses the capability to mobilize humanitarian aid anywhere in the world. It is the only WHO certified Type 3 field hospital, equipped to dispatch teams of doctors, nurses, paramedics and search and rescue experts within 24 hours. Examples of Israel's humanitarian aid activities include:

- Syria: In response to the shortage of medical infrastructure, doctors, nurses and supplies due to the civil war in Syria, Israel is providing life-saving humanitarian aid as part of Operation Good Neighbor. Aid includes medical equipment, medicines, and treatment of the injured in Israeli hospitals;

- Brazil: Israel dispatched a delegation to Brazil following the devastating dam collapse in 2019;

- Nepal: Between the years 2015-2016, following a $7.8 \mathrm{M}_{\mathrm{w}}$ earthquake, Israel's mobile field hospital welcomed more than 250 medical experts to deliver on-the-ground assistance;

- Guatemala: A team of 10 medical experts was dispatched to treat victims of the Volcano de Fuego. The team brought a special enzyme (developed at the Soroka University Medical Center) to facilitate the speedy recovery of burn victims, reducing the need for surgery;

- Sierra Leone, Liberia: Within the framework of MASHAV's Operation Hospitals of Hope (2015), two mobile field clinics were set up in Sierra Leone and in Liberia to assist local medical professionals combat the spread of the Ebola epidemic;

- Sierra Leone: An Israeli medical mission dispatched by MASHAV constructed and installed the first dialysis unit in the country, supported by onsite training of medical personnel;

- Peru: In 2017, the unusual heavy rains powered by El Niño conditions drenched parts of Peru with 10 times more rainfall than normal, causing the worst flooding in the country in 20 years. MASHAV dispatched epidemiology and emergency medicine experts to assist in preventing health crises in the aftermath of the natural disaster;
- Laos: With the collapse of a dam in the Attapeu District causing flooding and the loss of human life, displacement of thousands, and possible waterborne diseases, MASHAV responded by contributing water purification systems to prevent the spread of disease and ensure access to safe drinking water;

- Cameroon: Medicine and equipment were donated to the Mother and Child Medical Center, and the Israeli NGO, Save a Child's Heart, brought a young girl to Israel for surgery and rehabilitation;

- Nepal: Israel's Embassy in Kathmandu organized a five-day health camp for 1,800 children and the elderly in Smikot, Humla. MASHAV also donated medical supplies.

To reduce global deaths and injuries from road traffic accidents, Israel joined the consensus on the UN Resolution on Improving Global Road Safety (72/271), adopted at the $72^{\text {nd }} U N$ General Assembly in 2018. The Resolution requested that the WHO and relevant bodies in the UN system continue supporting the implementation of the Decade of Action for Road Safety 2011-2020 and road safety related targets in the 2030 Agenda. (SDG 3.6)

Healthy and sustainable transport options help prevent the negative health effects of air pollution and physical inactivity. Among others, methods and tools to assess the health impact of transport should be developed and policies for sustainable transport and healthy transport modes should be promoted. Israel is a member of the Working Group on Transport and Health of the WHO/Europe, within the Transport, Health and Environment PanEuropean Program (PEP). (SDG 3.6) 


\section{CASE STUDY}

\section{Upgrading Medical and Health Care Services: Establishing an Emergency and Trauma Unit in Togo}

As part of the bilateral cooperation between Israel and Togo, MASHAV and Togo's Ministry of Health are carrying out a project aimed at creating a framework for upgrading peripheral medical and health care services through the introduction of a modern intensive care unit at the Regional Hospital Atakpamé.

The project, carried out in alignment with the 2030 Agenda, includes the establishment of a fully equipped trauma and emergency unit within the premises of the hospital. MASHAV is providing capacity building and training for the operation and maintenance of the unit as well as training of the local medical teams.

Implementation of the project includes the following:

- Provision of state-of-the-art intensive care medical equipment

- Construction and installation by Israeli experts

- Technical assistance regarding operation and maintenance

- Capacity building of the local and regional medical teams

- Training programs conducted in Togo and Israel

- Transfer of daily operation responsibility to local teams

- Regional training in emergency medicine

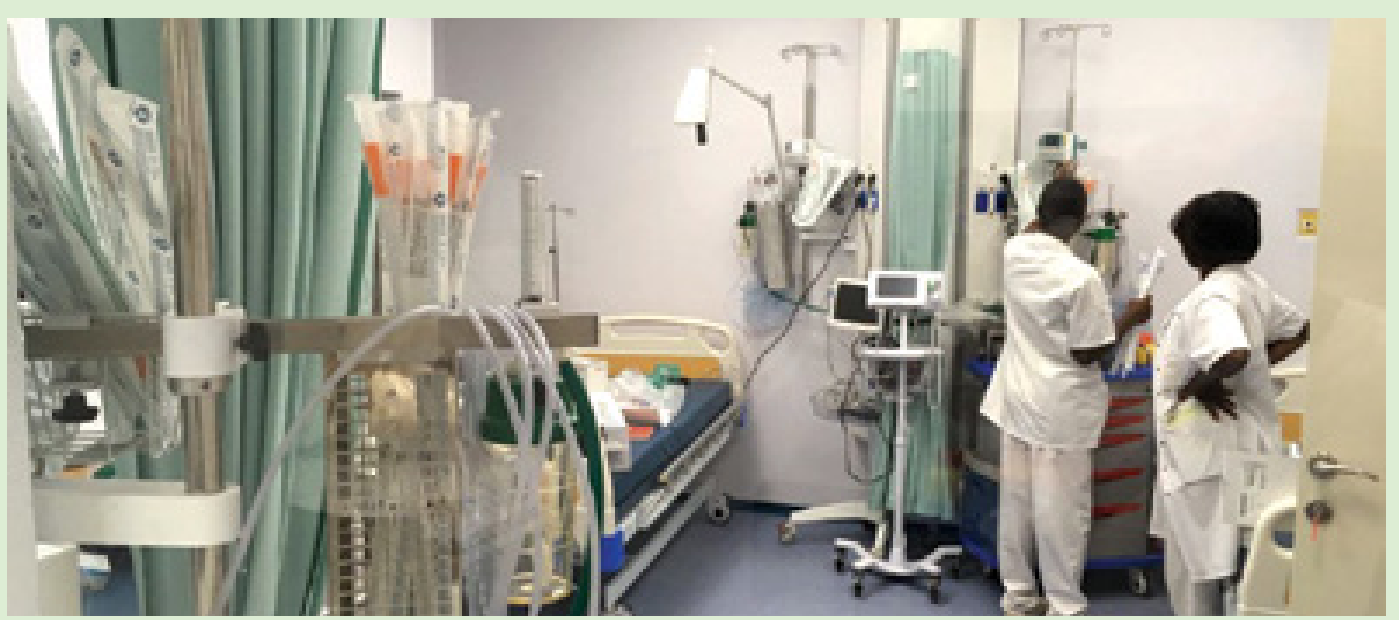

Photo: Mashav

\section{References for SDG 3}

Levels and Trends in Child Mortality Report 2015 Estimates developed by the UN Inter-agency Group for Child Mortality Estimation (UNICEF, WHO, World Bank, UN DESA Population Division) at www.childmortality.org

OECD. Society at a Glance 2014 Highlights: ISRAEL. OECD Social Indicators. OECD March, 2014; http://www.oecd- ilibrary.org/economics/oecd-factbook-2013/total-fertilityrates factbook-2013-table9-en laccessed May 20, 2015)

Delia Pergola S, The Israel Fertility Experience. United Nations Commission on Population and Development Forty-fourth session, Agenda Item 4: National Experience in Population Matters: Fertility, Reproductive Health and Development. New York: UN. 2011 http://www.un.org/esa/ population/cpd/cpd2011/countrystatements/agendaitem4/ 
israel.pdf laccessed June 30, 2015

Keidar N, Plotnick R, Afeq A. Israel's Health Care System Seen through the OECD Lens 2013. Report published July 2015. http://www.health.gov.il/PublicationsFiles/OECD2013a. pdf laccessed June 7, 2016)

Health at a Glance 2015, OECD Indicators. http://www. oecd-ilibrary.org/social-issues-migration-health/healthat-a-glance-2015 health glance-2015-en, DOI:10.1787/ health glance-2015-en

Almoshano S, Sack J, Sela B, Zlotogora J. The National Neonatal Metabolic Screening Program. Israeli Journal of Pediatrics 2008. 63:8-12

Data retrieved from the National Metabolic Screening Laboratory Database, Ministry of Health, 2014

Data retrieved from Ministry of Health National Hospitalization Data Bank, Information Division Ministry of Health, 2016

National Health and Nutrition Survey From birth until age 2 years 2009-2012, Israel Center for Disease Control, 2014. Publication 352. http://www.health.gov.il/PublicationsFiles/ mabat_352.pdf laccessed May 20, 2015

Preventive care quality indicators Israel, https://www. health.gov.il/Subjects/Patient Safety/

hospitals/National plan dimensions of quality/Pages/ Quality indicators in Israeli hospitals.aspx

Ashkenazi Y, Yankelevich A, Zusman S, Napatov L. Use and Experience with Children's Dental Health Service following the Reform in Israel - A research report. JDC MyersSmukler Institute, http://brookdaleheb.jdc.org.il/ Uploads/ PublicationsFiles/Hebrew-report-updated.pdf

Survey of Consanguinous Marriages, ICDC publication 340 , 2011, http://www.health.gov.il/PublicationsFiles/Relative ARB 2010.pdf laccessed May 21, 2015

Ivancovsky M., Weiss O., Kislev S. Child injuries in Israel. .Beterem National Report. Israel 2013. http://www.beterem. org laccessed July 5, 2015)

Beterem Childhood Mortality from Unintentional Injuries 2015 report , Publication Number 1097 http://www.beterem. org/download/files/00029046.pdf

National Youth Nutrition Survey 2015-2016. Israel Center for Disease Control. http://www.health.gov.il/PublicationsFiles/ mabat yong 2015 2016.pdf. (accessed January 26, 2017)

Lönnroth K, Migliori GB, Abubakar I, D'Ambrosio L, de Vries $G$. Diel $R$, et al. Towards tuberculosis elimination: an action framework for low-incidence countries. Eur Respir J 2015

DOI:10,1183/09031936.00214014

Chemtob D, Leventhal A, Berlowitz Y, Weiler-Ravell D. The new National Tuberculosis Control Programme in Israel, a country of high immigration. Int J Tuberc Lung Dis 2003;7(8):828-836

Chemtob D and Rosenberg E. Healthy Israel 2020:

Objectives, targets, and evidence-based strategies to preven tuberculosis and HIV infection in Israel. Int Public Health J 2010;2(3):289-97. ;

Chemtob D, Bendayan D, Rorman E, Levin Y, Harel N, Averick N, Grotto I. (2017). Ending Tuberculosis in Israel: current progress and future challenges. 48th World Conference on Lung Health, Guadalajara, Mexico, SOA-36912, October 2017

Mor Z, Weinstein R, Grotto I, Levin Y, Chemtob D. Thirty years of HIV in Israel: current epidemiology and future challenges. BMJ Open 2013:3:e003078.doi:10.1136/ bmjopen-2013-003078

Chemtob D. Overview of HIV/AIDS in Israel, by end 2015. WHO Meeting of the National HIV Programme Managers from Eastern Europe, Central Asia (EECA) and non-EU/EEA countries, Copenhagen, Denmark, September 2017 (Poster) Chemtob D. HIV HIV/AIDS in Israel: Periodic Epidemiological Report 1981-2017. Annual Report of the Department of Tuberculosis and AIDS, MOH, Israel, November 2018 Chowers M, Chemtob D, Mor O, Levi Y, Elbrit D, Elinav H, Rizenberg K, Lorberg M, Istomin V, Nemet S, Turner D. Cascade of HIV Care of Newly-Diagnosed Citizens in Israel 2011-2015: A Population-Based Cohort Study. IDWeek, San Francisco, USA, October 2018 (Poster)

Rokney A, Valinsky L, Moran-Gilad J, Vranckx K, Agmon V, Weinberger M. Genomic Epidemiology of Campylobacter jejuni Transmission in Israel. Front Microbiol. 2018 Oct 16:9:2432. doi: 10.3389/fmicb.2018.02432. eCollection 2018 Weinberger M, Moran-Gilad J, Rokney A, Davidov Y, Agmon V, Peretz C, Valinsky L. Molecular epidemiology of Campylobacter jejuni infection in Israel-a nationwide study. Clin Microbiol Infect. 2016 Dec;22(12):1005.e9-1005.e15. doi: 10.1016/j.cmi.2016.08.014. Epub 2016 Sep 9

Weinberger M, Lerner L, Valinsky L, Moran-Gilad J, Nissan I. Agmon V. Peretz C. Increased incidence of Campylobacter spp. infection and high rates among children, Israel. Emerg Infect Dis. 2013 Nov;19(11):1828-31. doi: 10.3201/eid19

Kaliner E, Kopel E, et al. The Israeli public health response to wild poliovirus importation. Lancet Infect Dis. 2015 Oct;15(10):1236-1242; Moran-Gilad J, Kaliner E, Gdalevich M, Grotto I. Public health response to the silent reintroduction of wild poliovirus to Israel, 2013-2014. Clin Microbiol Infect. 2016 Dec 1;22 Suppl 5:S140-S145

Kopel E, Kaliner E, Grotto I. Lessons from a public health emergency - importation of wild poliovirus to Israel. N Engl J Med. 2014 Sep 11;371(11):981-3

Kaliner E, Moran-Gilad J, Grotto I, Somekh E, Kopel E, Gdalevich M, Shimron E, Amikam Y, Leventhal A, Lev B, Gamzu R. Silent reintroduction of wild-type poliovirus to Israel, 2013 - risk communication challenges in an argumentative atmosphere. Euro Surveill. 2014 Feb 20;19(7)

Anis E, Kopel E, Singer SR, Kaliner E, Moerman L, MoranGilad J, Sofer D, Manor Y, Shulman LM, Mendelson E, Gdalevich M, Lev B, Gamzu R, Grotto I. Insidious reintroduction of wild poliovirus into Israel, 2013. Euro Surveill. 2013 Sep 19:18(38)

Awawdi, K., Abu-Rakia, R., Zidan, D., \& Tarabeih, M. (2018). Implementing Quality Care Indicators in Israeli Stroke Care: main barriers and the role of regional and other inqualities. International Journal of Current Research in Life Sciences, 7(5), 1958-1964. Retrieved from http://www.ijcrls.com

Shoham, A. B., Bar-Meir, M., loscovich, A., Samueloff, A., \& Wiener-Well, Y. (2017). Timing of antibiotic prophylaxis in cesarean section: Retrospective, difference-in-differences estimation of the effect on surgical-site-infection. The Journal of Maternal-Fetal \& Neonatal Medicine,1-5. doi:10.1 080/14767058.2017.1391784

Blum, N., Halperin, D., \& Masharawi, Y. (2014). Ambulatory and Hospital-based Quality Improvement Methods in Israel. Health Services Insights,7. doi:10.4137/hsi.s11027 


\section{GOAL \\ 4}

\section{QUALITY \\ EDUCATION}

पأ
Inclusion and integration

Human Rights

Education for gender

equality in Israel

Education for sustainable development

Partnering for a Better World 


\section{ENSURE INCLUSIVE AND EQUITABLE QUALITY EDUCATION AND PROMOTE LIFELONG LEARNING OPPORTUNITIES FOR ALL}

The main challenge facing the education system in the $21^{\text {st }}$ century is that of educational relevance - the ability to adapt and thrive in a world of change and vicissitude. In order to cope with the relevance challenge, Israel's education system is taking an innovative approach to the implementation of a future-oriented pedagogy. The emphasis is on shaping a desirable pedagogical future so that Israel can address challenges and make use of the opportunities that emerge from social, technological, economic, environmental, political, and educational trends. 
A future-oriented pedagogy proposes implementing six principles of action at all levels of the education system, from the top level of the Ministry of Education to the level of the student/teacher. These principles are: personalism, participation, informality, glocalism, changeability, and integration of personal identity and mission. Implementing these principles will generate changes in the educational field of action regarding curricula and the skills and practices associated with learning, teaching, and evaluation. A future-oriented pedagogy will also create changes that relate to organizational issues in the education system, such as the nature of educational leadership. characteristics of organizational behavior, educational institutions' degree of connectivity, and implementation of innovative study spaces and advanced learning technologies.

Future-oriented pedagogy serves as a systemic compass directing the pedagogical and organizational development in the education system so as to maintain the relevance of education in a changing world. The approach is constantly being broadened, deepened and improved, with an updated version disseminated each year. Significant activity is currently underway to upgrade the effort, and elements of the OECD Education 2030 project are being incorporated into the future-oriented pedagogy approach.

Implementation of future-oriented pedagogy in the education system is underway in several different tracks:

- The Future-Oriented Pedagogy Lab where ten schools are developing different models for the implementation of the approach in the education system;

- The Trial-Run project in which 40 schools are developing learning, teaching, and evaluating initiatives and practices that accord with futureoriented pedagogy as examples for the entire education system;

- The broad-based incorporation of the approach in the State-Religious Education Administration and in the Ministry of Education's Northern District;

- The incorporation of future-oriented pedagogy into new school construction projects and in the creation of innovative learning spaces. Each year 15 innovative schools are built and learning spaces are developed in hundreds of existing schools;

- Cooperation with other entities in the Ministry of Education on the implementation of futureoriented pedagogy, including the Division for Pre-Primary Education on the preschool of the future, the Tel Aviv District on the role of the supervisor, and pedagogical innovation centers.

\section{( Inclusion and integration (SDG 4.5)}

Inclusion and integration are viewed as the best way to advance special needs learners in regular education, while applying psycho-pedagogic principles suited to the needs of each student. The vision is to nurture a society that respects each person and believes in the basic right of each to a sense of belonging while recognizing and accepting the individuality of every person, to create a variety of educational institutions to serve as a mirror of an inclusive, integrative and humanistic society that allows diverse differences to co-exist, and to adapt teaching, learning and assessment methods to enable each student to fully realize his or her potential.

Goals and objectives call for the following:

- Integrating students and advancing them in schools while expanding the ability to embrace them and provide a variety of solutions suited to each learner;

- Enhancing the abilities of teaching staff to cope with a heterogeneous class;

- Reinforcing, expanding and intensifying integration and inclusion in the regular education system;

- Providing optimal solutions for students who are eligible to receive special education services in special education and regular education frameworks;

- Promoting and improving cooperation and dialogue with parents.

In the wake of a 2018 amendment to the Special 
Education Law, a multi-disciplinary committee was established to determine a student's eligibility for special education services: regular class, special education class in a regular school, or special education school. Furthermore, the amendment provided parents with the right to choose the type of setting in which a student who is eligible for special education services will learn: regular class, special education class in a regular school, or a special education school. The basket of services given to a student is an "individual basket," based on his/ her unique needs, level of functioning and type of disability. Types of solutions (teaching, treatment, guidance, assistance) for each student who is mainstreamed in a regular school are determined by the school in conjunction with the educational staff and the parents. At the same time, schools are provided with numerous solutions including training and supervision for teaching staffs, counselors, psychologists, inclusion coordinators, learning function specialists, mainstreaming teachers, inclusion hours, inclusion spaces, and many more.

A student found to be eligible for special education services and whose parents choose to exercise his eligibility in a regular school will receive a solution from the school's basket of resources and the basket of special education resources and will be an integral part of all the school's students.
Guidelines for inclusion in regular schools include:

- Individualized learning plan: The multidisciplinary staff, under the responsibility of the school principal and together with the parents, will choose the types and scope of support suited to the student's needs, as part of the general curriculum of the class in which he/she will be learning;

- Varied and appropriate teaching in the heterogeneous class: Teaching and learning in the school will be accessible and suited to all student populations, based on three principles: variety and range of presentation modes, variety and range of activities and modes of expression, variety and range of learning activities;

\section{- Support for the regular education system:} Special education staff members will work together with regular education staff members, according to the needs of students and staff;

- School-community continuum: The school will be only one component of the broad range of the student's social involvement, so that the student is seen throughout the day in the various frameworks;

- Contact with parents: An informed and positive relationship between educators and parents will be a significant factor in the student's success.

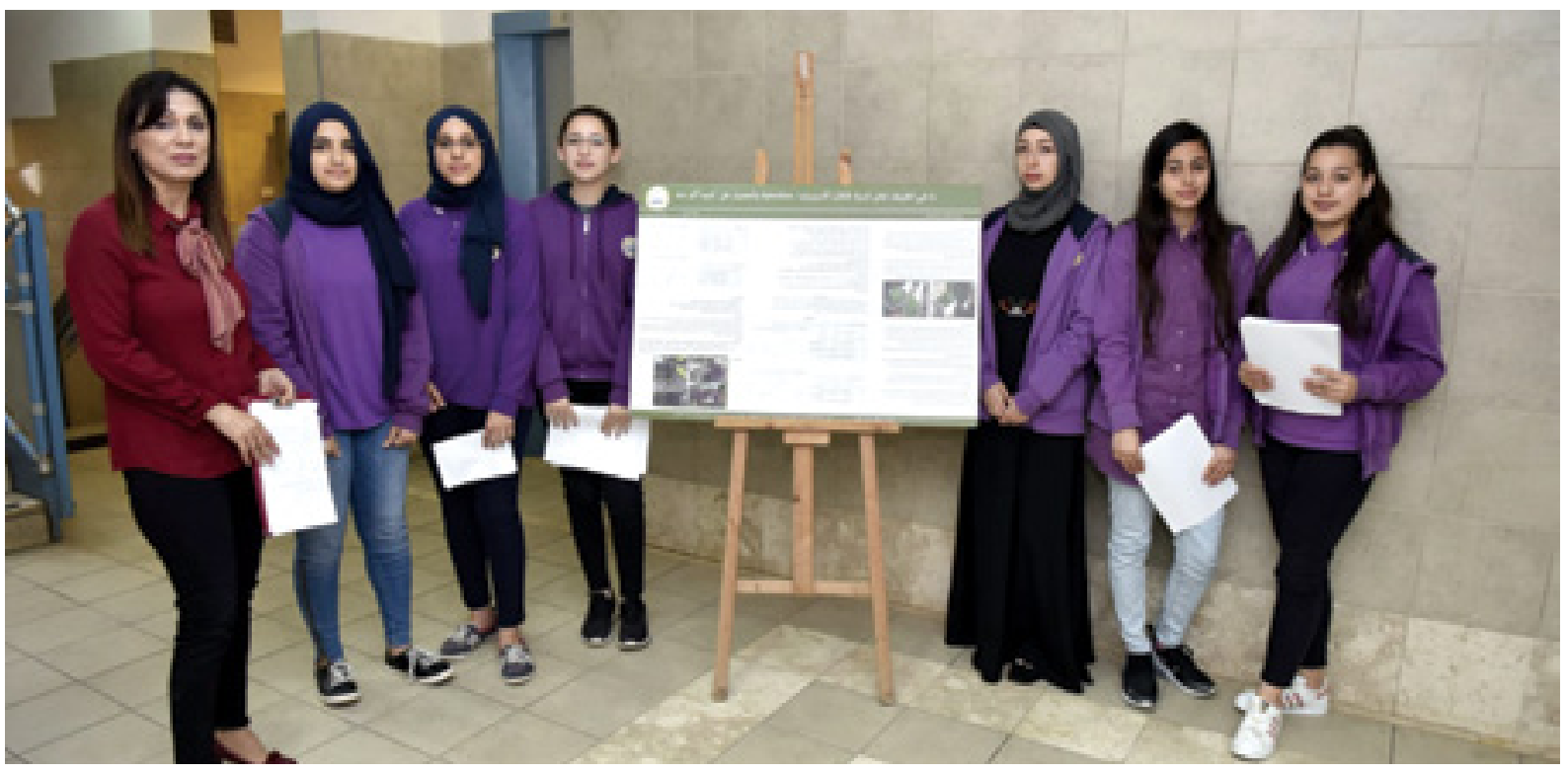

Students presenting their scientific research projects/Photo: Dr. Hussam Diab, Ministry of Education 
Table 1.1

The Arab education system for the $2017 / 2018$ academic year

\begin{tabular}{|l|l|l|l|l|}
\hline Education level & No. Schools & No. Classrooms & No. Teachers & No. Pupils \\
\hline Kindergarten & 2,907 & 2,897 & 3,053 & 74,694 \\
\hline Elementary & 450 & 6,859 & 20,070 & 173,618 \\
\hline Junior High & 180 & 2,203 & 8,137 & 59,856 \\
\hline Secondary & 278 & 3,107 & 4,405 & 79,226 \\
\hline Total & 3,815 & 15,066 & 35,665 & 387,394 \\
\hline
\end{tabular}

\section{Education in the Arab sector}

Education is the most important tool in reducing gaps between students from various populations and in promoting the integration of the Israeli-Arab minority within Israel's society and economy. Top priorities and implementation include:

- Improving learning attainments in core subjects (Arabic, Hebrew, English and mathematics) and improving the matriculation rate and the quality of the matriculation certificate: There has been a continuous improvement in matriculation exam attainment, totaling a $16 \%$ increase between the years 2009-2016. This improvement is due primarily to an increase in resources to the Arab population, including preferential budgeting to the Arab school system;

- Improving the quality of teaching and management in the schools: The training of Arab-Israeli teachers takes place primarily in universities, teacher's colleges and specialized tracks for the Arab population, as well as in certain tracks for joint subjects - physical education, administration, arts, communications, etc. Over the past decade, the Ministry of Education has significantly upgraded the teacher training system for Arab Israelis. As part of this program, new specialization fields have opened, and new opportunities were given for professional development. There has also been an upgrade in the physical and pedagogical infrastructure;

\section{- Decreasing the dropout rate from the schools;}

- Strengthening informal education frameworks in Israeli-Arab communities: Programs in Arab schools are implemented as part of the main program of strengthening the informal education in the Israeli-Arab sector. Programs include free of charge extra-curriculum courses for all Arab students in 4th-6th grade, establishing 47 new community centers by 2020 , and increasing the number of Arab youths involved in various youth movements to 66,000 ;

- Strengthening the Arabic language among Arab students;

- Construction of schools, classrooms and other needed facilities: Over the last five years, the Ministry of Education budgeted approximately $35 \%$ of its total construction budget for the construction of classrooms for the Arab populations (some 11\% more than their proportional representation in the population);

- Encouraging participation in higher education/ academic education: Over the last five years, the percentage of Arab students within Israeli universities and colleges has increased from $9.7 \%$ to $15.2 \%$. The Ministry of Education's encouragement programs included guidance centers at secondary schools, offering 775 unique scholarships for required subjects.

\section{Education of students of Ethiopian descent}

An inter-ministerial program in Israel known as "A New Path" which is specifically targeted at students of Ethiopian descent in primary school, took effect in 2016. The program is based on several cornerstones aimed at advancing the academic achievements of these students, as follows:

- Transfer of responsibility for students of Ethiopian descent born in Israel to relevant departments in the Pedagogic Administration and 


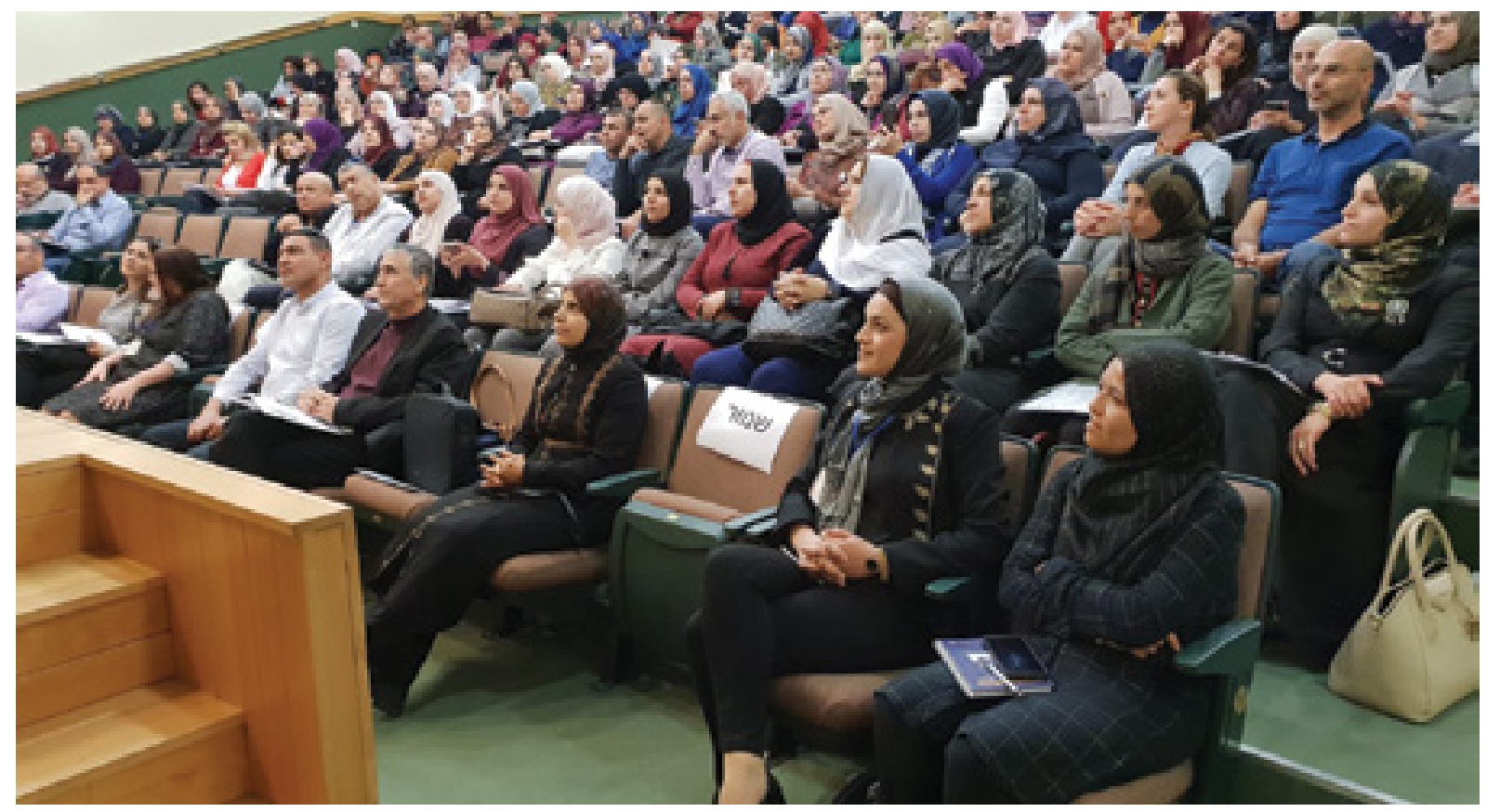

Teachers' seminar/Photo: Dr. Hussam Diab, Ministry of Education

ending implementation of differential programs for students of Ethiopian descent - both of which have already been carried out in full;

- Reduction of gaps in education: Assistance to students with difficulties is provided via the "Class Counselor" program, initiated in the 2018-19 academic year, in schools with more than 20 students of Ethiopian descent (total of around 245 such primary schools) which have no other support programs and where the achievements of these students fall below those of other students. Through this program, a counselor comes to the school, mainly for students in grades 2 and 5 , who specializes in addressing the diversity between the students, particularly in Hebrew language and mathematics teaching. To make after-school activities more accessible, a program known as "Keys" is in operation. With the help of local authorities, vouchers are distributed to students of Ethiopian descent in grades 1 to 5 , and can be used to help the children join high quality after-school classes, together with other students in their neighborhood;

- Addressing strengths: Two measures are being implemented together with the Department for Gifted and Outstanding Students - "Nitzanei Amirim" which is a program for excellence starting from grade 1 that is implemented for all students to promote a culture of achievement and excellence, and enrichment centers based on teacher recommendations for outstanding students of Ethiopian descent;

- Reinforcing the relationship with parents: A program known as "Home Grown" is implemented in schools with large numbers of students of Ethiopian descent in cooperation with the Consulting Psychological Service and JDCAshalim. The program invites all parents and all students to four meetings per year offering hands-on activity working together, along with dialogue, and suggestions they can implement at home based on their experience.

\section{( Human Rights (SDG 4.7)}

The school system continues to focus on reinforcing the values of democracy, tolerance, pluralism and co-existence through activities that target different age groups. As part of these activities, emphasis is placed on developing skills and attitudes to enable students to cope with the problems, complexities and challenges relating to this issue. These include reflective and critical thinking, tolerance, empathy, curiosity, developing dialogue skills, 
understanding the tension between the needs of the individual and the needs of the "society" and more. Furthermore, these values are integrated in relevant spheres of knowledge while engaging with different subjects, such as: Israel as a Jewish and democratic state; principles of democracy; human rights; children's rights; relationships between different groups; identification groups and characteristics; acknowledging the culture, language and historic heritage of the various sides; prejudice, and so on.

Dedicated hours in the lower and upper secondary schools are devoted to human rights. For example, within the framework of civics studies, students learn about human rights, civil rights, cultural rights and minority rights. Time is also dedicated to the divisions in Israeli society and to individual rights and duties - as a person and as a citizen.

Human and civil rights topics are also integrated within History and Geography, Man and Environment studies, with different programs for different age groups, in addition to topics such as accepting the other, tolerance, cooperation, multicultural sensitivity and immigrant absorption. In 20192020, the subject of tolerance will be integrated in other curricula and lesson plans as part of the central theme of "Maintaining Unity, Maintaining Uniqueness." Curricular materials are also prepared and distributed ahead of specific dates on the calendar such as the International Day for the Elimination of Racism (in Hebrew and Arabic), International Day of Tolerance, Human and Civil Rights Day, and more.

An Office for Civic Education and Living Together operates through a variety of channels:

- Supervising schools in promoting values of equality and acceptance of others, by means of in-service classes and seminars. Each year 80 teacher in-service classes are allocated to promote these topics, with some 2,000 teachers participating each year;

- Allocating around 94 hours to schools that wish to advance the subjects of living together or teaching democracy. Hours are allocated in upper secondary schools;
- Allocating extra hours for teaching democracy and equality. The scope of these extra hours is 93 hours each year;

- Marking International Human Rights Day is part of the curriculum requirements and is reflected in the director-general's circular on this issue;

- Promoting joint initiatives with other associations and organizations and supporting encounters between various groups in Israeli society to promote equality, pluralism, reducing prejudice and racism, and promoting living together. For example, one initiative pairs experimental schools to work together in the sphere of education for living together. Another encourages joint learning in a variety of spheres, either face to face learning or online. Encounters with the participation of some 50,000 students per year have been held.

\section{Implementing students' rights}

The International Convention on the Rights of the Child forms the educational foundation of the Education Ministry's policy that stresses the importance of education to create a culture of rights. This educational process inculcates an awareness regarding the rights of children and adults, and impacts the development of worldviews, decision-making processes and civil responsibility among students. As an educational tool, rights can teach about independence and autonomy along with consideration and respect towards others.

In addition to an educational tool, rights are also a legal tool. The Education Ministry believes it is important to learn about the Convention on the Rights of the Child, the Law for Students' Rights and other relevant laws, as well as explanatory materials that translate the Convention's principles into practical actions to promote a culture of respect for rights in educational institutions.

Teacher training is developed and facilitated in addition to in-service training activities for education personnel. Each year different aspects of the International Convention on the Rights of the Child and its implementation are emphasized. Courses are intended for principals, teachers, school counselors, truancy officers, and preschool 


\section{CASE STUDY}

\section{The World's Largest Lesson to Promote the Sustainable Development Goals}

For three years the National Inspectorate for Implementing Students' Rights has carried out the World's Largest Lesson program. The program was developed with the goal of presenting the UN Sustainable Development Goals (SDGs) to children in a friendly and accessible way. The program's underlying approach is that children and youth have a key role to play in achieving the goals. As such, children and youth learn about why the SDGs are important for their lives, communities and countries, and how achieving the goals will help them exercise their rights under the UN Convention on the Rights of the Child.

Different partners worked in developing the content for the program throughout the years, including UNICEF Israel, special units within the Education Ministry (Technology and Information Division of the IT Administration, units and branches of the Pedagogic Administration), and other government ministries including the Ministry of Justice and Ministry of Health.

The program's yearly themes included:

- 2016 - Goal 10 - Reducing inequality and discrimination

- 2017 - Goal 16 - Equal approach to justice, information and civic responsibility

- 2018 - The right to participation - combining all the goals for all students to participate

Learning materials and lessons developed as part of the program were written in Hebrew, and some were translated into English. Additional materials in Arabic, developed by the National Inspectorate for Students' Rights, were also offered to Arabic-language schools. Study materials were made accessible to schools through a special digital portal that was developed for the program and can be found on the Ministry of Education's educational cloud. (For a sample lesson in English: see https://goo.gl/c3MYVB).

Facebook event pages were also opened each year to enable schools to share the results of their learning and promote discussion on the subjects taught. Grades 1 through 12 took part in the program and teaching materials were adapted to the different grade levels, with some 700 classes participating in 2016, and some 1,700 classes and 2,800 classes in 2017 and 2018 respectively.

teachers. They are held in national, district, and locality-based formats and are tailored to all sectors of Israeli society and to education personnel working with all age levels.

In-service training topics include education and equality of opportunity, the right to be heard and to be involved, the right to privacy, the duty to report, the duty of confidentiality, the limits of punishment, education workers' responsibility for damages, minors' rights in criminal proceedings, child victims' rights and the interrogation of children, the rights of LGBT students in educational frameworks, and children's rights as reflected in Israeli and international cinema. 


\section{CASE STUDY}

\section{Dedicated Curricula and Programs for Implementing Students' Rights in Israel}

- Fostering dialogue concerning rights and responsibility - Youth Can Do It: Curriculum in English for secondary school education dealing with the moral, civil and cultural aspects of applying the Charter for the Rights of the Child in different societies and cultures, while addressing the pedagogic needs of teachers and English teachers in adapting the program to English studies.

- On the Path to Rights: Bilingual curriculum for primary school dealing with the complex relationships between students and teaching staff in a society of multiple cultures and identities that respect the different affiliations through familiarity and learning about rights. The activities lead to a formulation of agreements through systematic dialogue and recognizing the rights of the students.

- Civic responsibility - On the Path to Rights: Bilingual curriculum for lower secondary schools based on the idea that, according to the Convention on the Rights of the Child, both young people and adults should learn about their responsibility toward others - sensitivity to the needs of others and helping others. The curriculum offers a status report on young people taking responsibility for themselves and others, via dilemmas as recounted by the young people themselves.

- On the Path to Rights: Discussion of moral and social aspects of the Convention on the Rights of the Child: Bilingual upper secondary school curriculum guiding students and generating student activity on implementation of the Convention on the Rights of the Child. The curriculum emphasizes the importance of education for non-discrimination/equality as a social tool. Studying rights confers independence and autonomy, while also providing tools for proper judgment, social criticism, responsibility, and respect for others.

- Students Designing Their Rights: Collaboration with the design arts track in the IT Administration on the development of educational programs aimed at: raising awareness of children's and youth rights and of situations in which these rights are violated; deepening students' involvement and participation in resolving challenges faced by children and youth in the present; and creative, groundbreaking thinking about how to design students' future environments. All these projects have been submitted for matriculation at 3 to 5 units. In all the programs, the participating students are the researchers, the creators, and the target audience for a meaningful and authentic learning process.

- Program 2035: Program developed as a means of studying and clarifying, from the point of view of students as future parents, the roles of the school of the future and the values that will underlie its activity. The students are required to formulate research questions on creating an innovative and user-friendly learning environment for children and youth and to design an innovative product for the school of 2035. The program was developed in cooperation with Amdocs and the U. S. Embassy in Israel.

- Independent in the Field Program: A joint program of the National Inspectorate for Students' Rights, the Chief Inspector of Design Arts in the Ministry of Education, and the multinational corporation Amdocs. The program aims to foster authentic understanding of children and 
youth, to identify needs and challenges that they face on the road to physical, emotional, and cognitive independence, and to develop products tailored to user needs, desires, and personalities. The projects will be presented to "sharks" so as to determine the feasibility of implementing them.

- From Student to Delinquent: A joint information program with the Public Defense Office (Juvenile Department) in the Ministry of Justice. In the program, public defense attorneys come to post-primary schools to lecture on legal processes pertaining to youth offenses and youth rights in criminal proceedings. Lectures are delivered frontally and online and are tailored to different sectors and to special education frameworks. The program was recognized by the Civil Service Commission as the most significant program in Israeli society for 2016 .

- Youth Labor Rights: A joint information program with the Ministry of Labor and Social Affairs. Expert inspectors come to post-primary schools to lecture on youth labor rights. The talks are tailored to the various sectors, as well as to special education students. This year, posters will be distributed to all post-primary schools with information for young people whose rights have been violated in different kinds of workplaces.

- C.R. Rights Trustees program (C.R. - Civic Responsibility): A program in which 1,000 young people participate in learning processes and civic activities in schools, in their home communities, and in the Knesset (Israel parliament). Most of the activity relates to the following issues: poverty, privacy, equality/non-discrimination, involvement in decision-making.

\section{(Education for gender equality in Israel (SDG 4.5, 4.7)}

Israel considers it highly important to adopt genderoriented thinking at all ages and education stages in the Israeli education system. The Ministry of Education works to promote equality of opportunity between the genders; to raise awareness among education personnel and students about social attitudes and constructions regarding gender roles; to implement gender-oriented thinking; to effect attitudinal change; and to broaden the range of behavioral, social, and learning options for students and education personnel. As early as 2002, a Director General's circular was issued on the topic of education for gender equality in Israeli educational institutions.

Israel strives to enable girls and boys in Israel to fulfill their personal and occupational potential, ensuring that girls and boys are guided toward choosing fields that suit them, based not on social attitudes regarding their skills but, rather, on their inclinations and personal abilities. Actions are taken for this purpose throughout the country in the Jewish and Arab Israeli sectors and at all educational stages. The aim is to ensure adoption of the gender-equality principle by both education personnel and students.

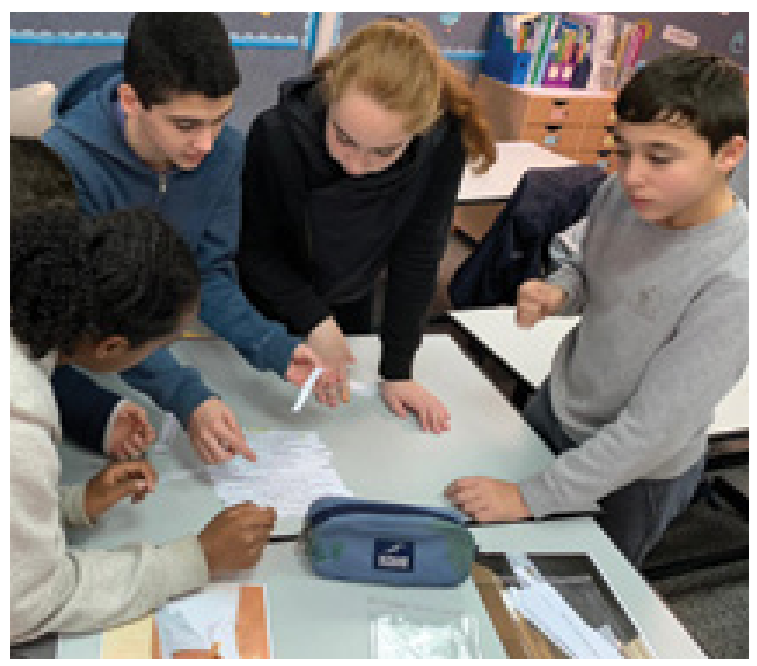

Joint learning and projects/Photo: Unit for Gender Equality, Ministry of Education 


\section{CASE STUDY}

\section{Programs Designed for Students to Promote Gender Equality}

- Shaveh Dibur: Program on gender and gender equality intended for grades 5-6 and grades 8-9 in the Jewish sector;

- Sharsheret: Program intended for six-year schools. The program is based on mentoring in which older, upper secondary school girls work with younger girls in lower secondary schools. The groups handle special assignments on gender-equality issues: marking International Women's Day, teaching classes in the schools, and raising awareness. The program is designed to create a unique space for the participating girls in the school setting. This feminine space is based on the sisterhood model and the "room of one's own" approach, and is intended as a unique provision for girls;

- Masculinity program: A program operated as a pilot in three Jerusalem schools this year. The program features masculinity-focused dialogue: models of masculinity, the personal and social price demanded by "masculinity," gender stereotypes, social constructs, and so on.

A specific unit, the Unit for Gender Equality in the Ministry of Education, is responsible for ensuring the adoption of gender-oriented thinking by all parties in the education system, including principals, teachers, teaching personnel, and students. The unit is also responsible for integrating Ministry of Education actions relating to gender, and it collaborates with other ministry units and guides them on the incorporation of genderoriented thinking into their activities.

An array of in-service training courses, including topics that require gender-oriented in-service training in education, are available for teachers. Courses are designed along a continuum from individual to collective - from the personal to the educational. The individual level is the level of the teacher's personal empowerment. The educational level deals with the social constructions that produce disparities and prevent social mobility and provides teachers with tools for incorporating gender thinking in the classroom and school settings.

Gender thinking is incorporated into the curricula of various subject areas, such as literacy, civics, art, physical education, and more. In addition, an updated website constitutes a readily available and accessible information source on gender education.
Advancing female students in mathematics, technology, and the sciences

The Unit for Gender Equality works in cooperation with the Inspectorates of Mathematics, Science and Technology, Physics, and Computer Science to train female and male teachers for gender-sensitive teaching. There has also been collaboration with a variety of entities, such as science museums, the Weizmann Institute, the Council for the Advancement of Women in Science and Technology, and others. The program aims to achieve the following:

- Increase the percentage of female students choosing to study mathematics, science and technology at the 5-unit (highest) level;

- Raise the percentage of female students who persevere with and complete mathematics, science, and technology study at the 5-unit level (highest);

- Coordinate and pool resources/programs with a variety of entities, such as government ministries and second- and third-sector organizations active in the education system, in order to advance students in general, and female students in particular, in the fields of mathematics, science, and technology; 
- Broaden the circles of influence reaching female students at decision-making junctures, so as to encourage and inspire them to choose careers in mathematics, science, and technology (parents, teachers, school counselors, principals, counselors).

\section{CASE STUDY}

\section{Programs and Measures for Advancing Female Students in Mathematics, Technology, and the Sciences}

- Latet Chamesh Program: Focuses on advancing students in mathematics and aimed at doubling the number of those studying math at the 5 -unit level in general, with an emphasis on female students;

- Na'arot Portzot Derech B'Mada v'Technologia ("Girls Innovating in Science and Technology"): Pools the programs active in the field. The program is intended for female lower secondary students (grades 8-9) studying science and technology;

- Advancing Girls in Science: The program aims at exposing and encouraging girls in science in cooperation with different inspectorates at lower and upper secondary levels;

- Breaking the Glass Ceiling: Joint program in cooperation with relevant municipalities which is operated in six schools (Bat Yam, Netanya, Kiryat Gat, Kiryat Malachi). The goal is to pave the way for girls in the periphery, to strengthen their confidence in their own abilities, to put them in contact with successful women who can serve as role models, and to guide them toward choosing scientific subjects for academic study and future careers;

- Women Scientists of the Future: Program in cooperation with the Ministry of Science, Technology and Space, aiming to encourage girls in peripheral localities to choose study programs in the exact sciences;

- Distributing science posters: Posters are used to inspire/foster identification of female students with science careers;

- Promoting careers in STEM fields: Program to encourage female students to choose careers in STEM fields. The program features a critical approach to common social attitudes about these fields and increases girls' sense of competence;

- Promoting the use of gender-sensitive teaching: Female and male math, science, and technology teachers have access to resources and receive guidance and training at meetings, in-service training, and conferences;

- Eradicating gender stereotypes in the Arab, Bedouin, Circassian and Druze sectors: Program to raise awareness and personal empowerment and increase awareness of equality of opportunity and freedom of choice. It provides female and male students with practical skills for participating and succeeding in the Israeli labor market and in a global, competitive, and technology-oriented marketplace. Gender workshops are run in all parts of the country, and address such issues as gender stereotypes, career and family-life planning, relations between genders, and more. Each year an intensive study day is held for female and male counselors in the Bedouin sector, addressing a variety of study topics, including marriage of minors, marriage at young ages, forced marriage, and polygamy. 


\section{(Education for sustainable development (SDG 4.7)}

The integrated plan for implementing sustainability education in the school system was created to foster the training and professional development of teachers. It aims to provide every graduate of the Israeli educational system with the knowledge and skills necessary to make informed decisions, and therefore leads to civic behavior that is aware, involved, caring and responsible for the environment. For the educational staff, the program provides tools for building pedagogical, social and ethical language in kindergartens and schools, and creates a culture of discussion on environmental issues and sustainability. The program enables a learning space while promoting education for sustainability with an emphasis on action for the environment based on teacher training and professional guidelines and directives.

The components of the integrated plan include:

- Teacher training: At schools the program includes teaching of at least $80 \%$ of the staff for 30 hours - six hours of professional tours and 24 hours of training. New schools are granted professional guidance for 27 hours throughout the year. Continuing schools, which prove their persistence in pursuing, implementing and expanding their environmental activities, are granted professional guidance for 15 hours annually. Kindergartens are granted 30 hours of training $(6+24)$ at teacher instruction centers. New ones are granted 11 hours throughout the year and continuing kindergartens are granted six hours.

- Preparation of a school program on environment and sustainability: The product of the training is a curriculum and an annual work program for kindergartens and schools on environmental issues and sustainability. The product is based on programs prepared by academic institutions that determine the key contents, values and principles upon which the curriculum and work program should be founded. The plans are multidisciplinary and integrate knowledge together with a plan for implementing a sustainable lifestyle and involvement within the community, all revolving around issues that the individual school chooses to advance.

\section{Greening the educational system}

The Ministry of Environmental Protection in collaboration with the Ministry of Education has led the certification process for Green Schools since 2003. The purpose of the process is to implement sustainability principles in schools. Accreditation reflects the recognition of significant environmental education in a school and outlines a possible course of action for schools that want

Figure 4.1

\section{7 schools participated in education for sustainability programs nationwide}

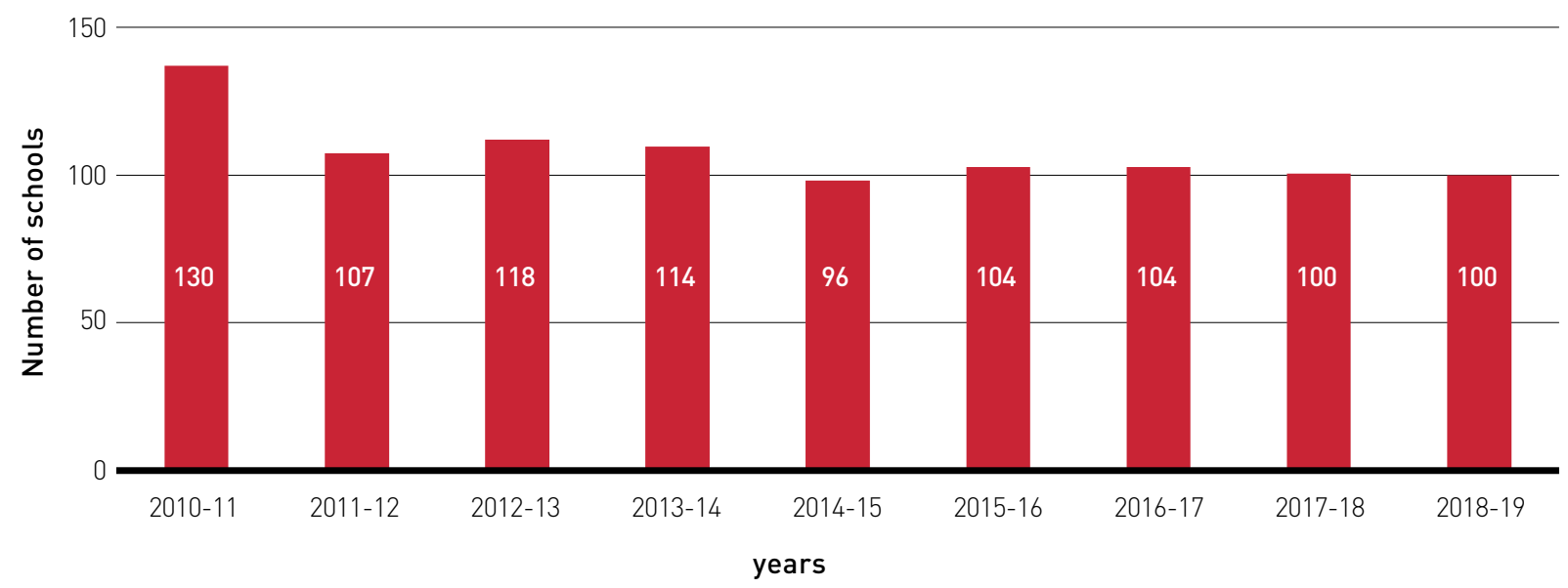




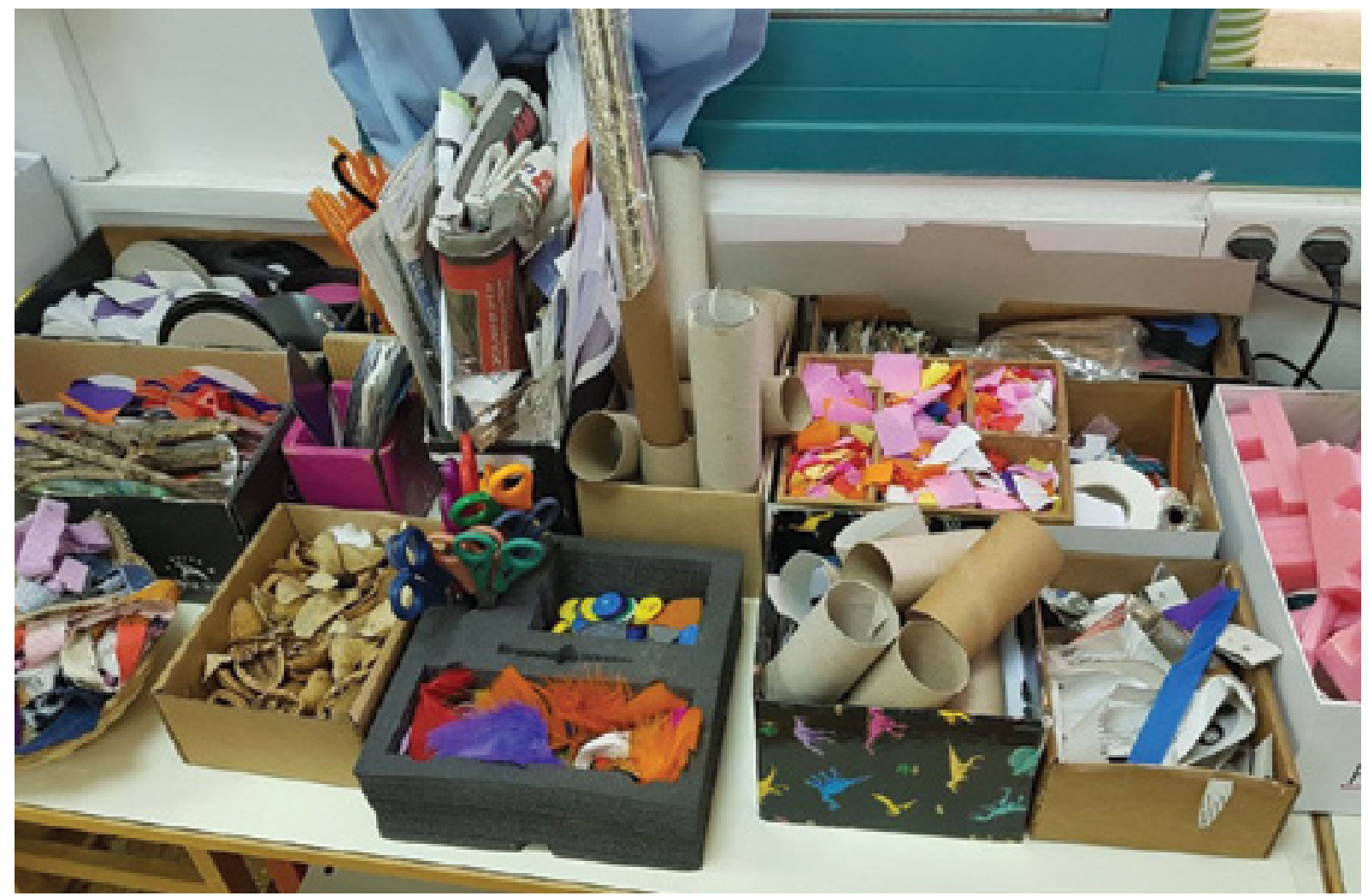

Recycling corner in a kindergarten/Photo: Barbara Andress

to start environmental activities based on existing frameworks. The model is based on the principles of learning about environment, implementation of a sustainable lifestyle at school, and student activities aimed at bringing about a change in consciousness and behavior. A school that meets the criteria for accreditation is granted certification.

\section{Green Kindergartens}

A Green Kindergarten is reflected in several key aspects:

- Promoting values of respect for one another, nature and the environment and a desire to maintain, modify and influence the environment;

- Improving the quality of life and educational climate in the kindergarten;

- Developing open spaces in the kindergarten which enable a sustainable lifestyle and promote play with natural and/or recycled materials;

- Involving parents and the community in order to develop awareness and behavioral change on environmental issues, alongside the children.
The accreditation criteria for a Green Kindergarten include:

- An integrative annual work plan that combines the different themes of environmental education;

- Communal sustainability;

- Visual and behavioral expression of environmental education in the kindergarten's lifestyle.

\section{Green Schools}

In order to help schools along the road toward sustainability, the following guidelines were drafted for Green Schools. Only schools that implement goals in each of the following categories are eligible for Green School accreditation:

- Formal studies: 30 annual hours of formal study of environmental subjects must be integrated into the school curriculum in two grades in elementary school and within the framework of such subjects as geography, science, social studies, etc. Ten annual hours are required for half of the students in a high school; 
Figure 4.2

\section{3,265 preschools certified as Green Kindergartens}

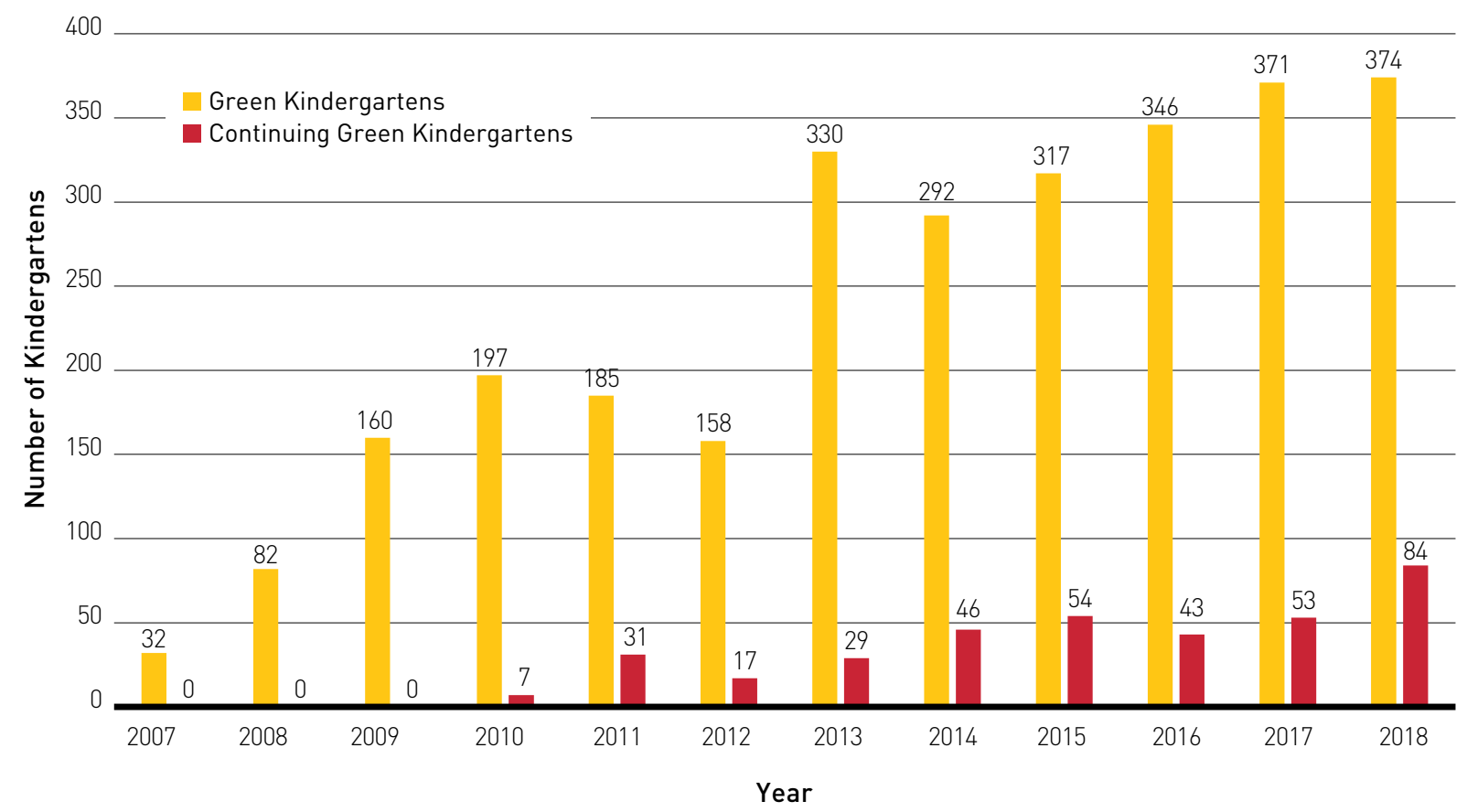

- Rational use of resources: Schools are required to reduce resource use (water, electricity, paper) and/or to collect waste for recycling (bottles, paper, batteries, toners, etc.);

- Contribution to the community: An additional grade beyond those engaged in formal environmental studies is required to implement a community project aimed at increasing awareness of the environment and bringing about behavioral changes. Examples include adopt-a-site projects, recycling, etc.;

- School's green leadership: Each school must establish a green leadership group comprised of students, teachers and others from the community. The group is responsible for promoting the green school accreditation process;

- Training program for teachers: A teacher training program in four modules which addresses environmental and sustainability topics is required.

Some schools are granted a "Continuing Green School" status if they qualify for additional criteria after accreditation as a Green School. The criteria include a dedicated studies program of 30 hours per student on environmental topics (for all six grades in primary school and for three grades in post primary schools), an ongoing environmentalcommunity project and the implementation of additional opportunities for resources efficiency at the school. To date, some 1,224 schools have been certified as Green/Continuing Green Schools.

\section{Green Campuses}

On the university level, most of Israel's major universities and colleges offer graduate or undergraduate programs on the environment. However, alongside growing emphasis on environmental study, efforts are also focusing on introducing environmental behavior into university campuses and a Green Campus project aims to introduce environmental action on the academic, administrative and practical levels.

The criteria for the accreditation of institutes of higher education as Green Campuses are similar to those for Green Schools, namely: 


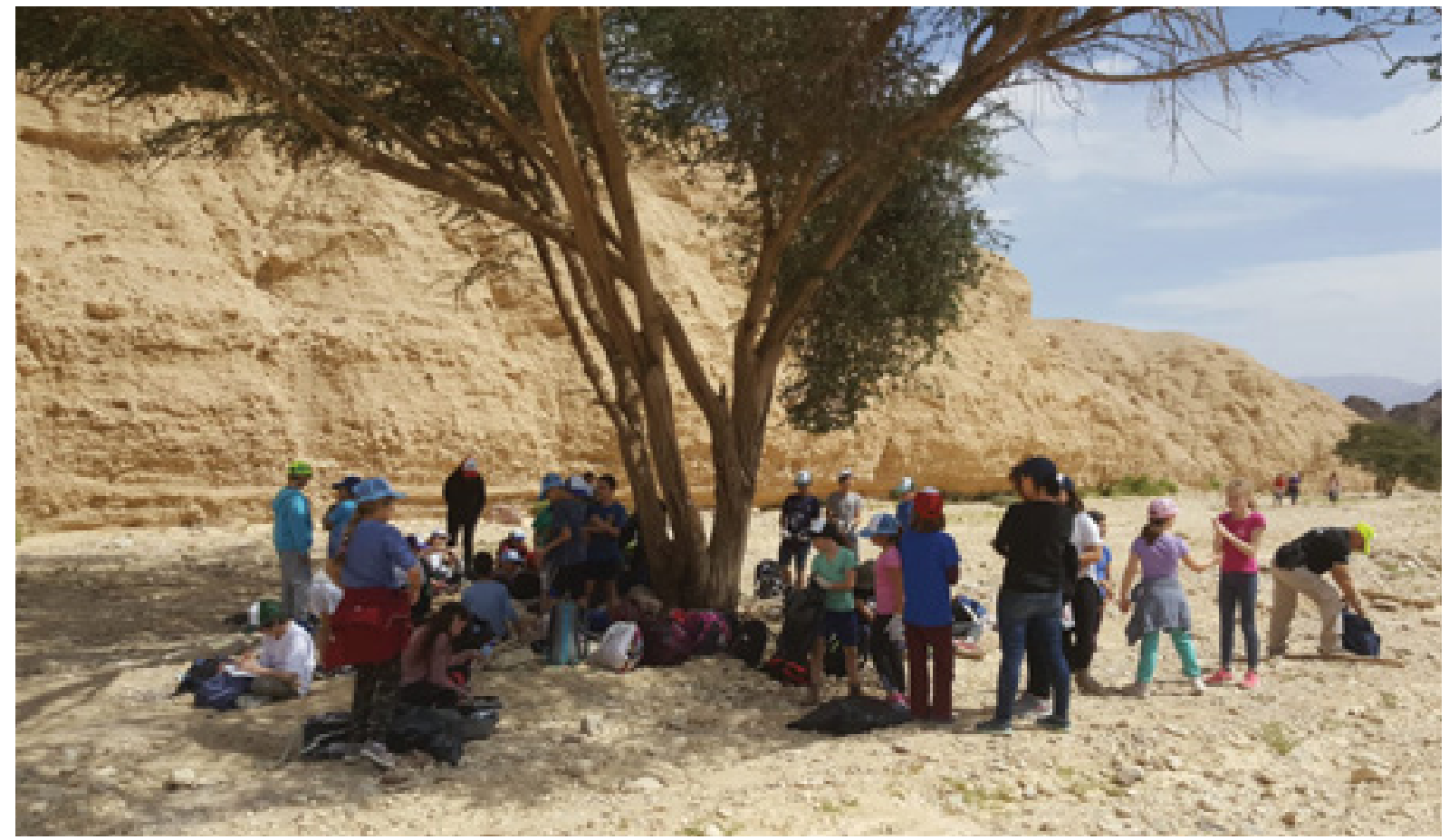

Clean-up campaign by fifth graders from the Maale Shaharut School in Israel's south/Photo: Liat Lerner

\section{Key Figures for Education for Sustainability}

- 3,850 preschool teachers (out of 18,000) attended in-service training in the integrated Education for Sustainability program;

- There are 3,289 Green Kindergartens and 364 existing Continuing Green Kindergartens;

- 1,224 schools were certified as Green/Continuing Green Schools;

- 977 institutional in-service training courses were held for schools in the integrated Education for Sustainability program, with the participation of 24,425 teachers;

- 96 schools participated in a green construction competition in 2016-2018, with 16 winners;

- 120 principals attended a 3-day in-service training course, with attention to administrative, managerial, pedagogical, and communal aspects of education for sustainability;

- 90 groups participated in activities on the topic of environmental leadership in sociallymixed schools;

- Study days on education for sustainability were held for six districts;

- Each year, 700 teaching personnel participate in the environmental education course;

- Second-year students from 15 colleges took a course on education for sustainability;

- 60 students from five northern academic institutions held a sustainability hike on the Israel National Trail;

- Conferences were held in cooperation with academia on issues of education for sustainability. The 18th such conference will be held in 2019;

- There are 10 online lectures on environmental issues. 
- Environmental studies: Each campus will allow each student to take one elective course on the environment;

- Contribution to the community: Each campus will choose and implement a community environmental project, to be conducted by the administration, the students and the community. The project should lead to a change in behavioral and conceptual norms in the community in terms of environmental orientation.
- Rational use of resources: Each campus will choose and implement one environmental aspect - a "reduction" program such as reduction of electricity use, water use, private car use, paper use or an "increasing" program, such as increase in the quantity of bottles, toners, batteries and paper collected for recycling. The assimilation of green building as one of the components of the rational use of resources program is recommended.

\section{PARTNERING FOR A BETTER WORLD}

Education plays a vital role in striving to eradicate poverty, promoting gender equality, advancing human rights and safeguarding democratic values. Israel has faced the challenges of absorbing massive waves of immigration since gaining independence, and its educational policies are shaped by the need to integrate various populations - specifically the most vulnerable and those with special needs - into the educational system. Israel's approach to international development cooperation in education-related disciplines is holistic and inclusive, while recognizing the sensitivities that influence education such as cultural diversity, social norms, and religious belief.

The Convention on the Rights of the Child forms the educational foundation and policy of Israel's Ministry of Education. For the past three years, Israel's National Inspectorate for Implementing Pupils' Rights, in cooperation with, among others, the UN International Children's Emergency Fund (UNICEF) - has carried out the World's Largest Lesson program. The program introduces the Sustainable Development Goals (SDGs) to children, and highlights their relevance to our lives, communities and countries. In 2018, the theme focused on The Right to Participation - Combining all of the Goals for all Pupils to Participate. (SDG 4.7)

Israel's overseas development assistance is dedicated to implementing programs to substantially increase the supply of qualified teachers in developing and least developed countries. Following are some examples: (SDG 4.c)
- Education for Sustainable Development (ESD) aims at preserving environmental integrity, economic viability, and a just society for present and future generations through teaching and learning processes that emphasize an interdisciplinary and holistic approach, promoting critical and creative thinking in education. Within this framework, the Ministry of Education of Kenya, MASHAV and Kenya's Primary Schools Head Teachers Organization - KEPSHA are implementing a project for the inclusion of ESD in the curricula of learning institutions. Close to 800 Kenyan educators from over 90 schools and institutions have taken part in training activities conducted both in Israel and in Kenya from 2010-2016;

- Programs on early childhood education focus on the tools necessary to encompass the cognitive, emotional, physical and social development of the child, are hands-on, and are geared to a trainingof-trainers approach. MASHAV's capacity-building activities support early childhood education for children with special needs, as well as early childhood education: innovative approaches for training-of-trainers;

- Collaboration between MASHAV and Thailand's International Cooperation Agency (TICA) resulted in the implementation of a regional training seminar entitled Overcoming the Challenges of Early Childhood Education (ECE) in the 21st Century. Professionals from seven Southeastern Asian countries took part in the seminar, held at Suan Dusit University in Bangkok; 
- The American Joint Distribution Committee (JDC) began a pilot project on supporting early childhood education in Haiti, together with Israeli (Early Starters International) and local (Prodev) partners to provide support tools and training to early childhood education professionals in accordance with the national Haitian curriculum. In the first phase of the project, a guidebook was jointly developed. The second phase is currently being implemented - a 12-month training program with on-the-ground guidance for early childhood professionals working in three schools. The Prodev team provides support in implementing the new tools and knowledge acquired.

In alignment with SDG 4.c and 4.a, MASHAV is cooperating within the UN system and bilaterally with partner countries to increase the number of quality teachers and educators promoting Information and Communications Technology (ICT) for pedagogical purposes. ICT presents an opportunity for youth and adults to access and equity in education, contributes to the professional development of knowledge providers and learners alike, bolsters income-generating initiatives aimed at eradicating poverty, and serves as a means of inclusion into both the local and global community. Examples include:

- Kenya: A Memorandum of Understanding (MOU) was signed on Teachers Professional Development and ICT within the framework of Kenya's Comprehensive Program for Education on ICT;

- Cameroon: MASHAV assisted in the establishment of the first computer and internet classroom at the CETIC De Ngoa Ekelle School in Yaoundé and held a tailor-made training seminar on Integrating Information and Communication Technology (ICT) in the Classroom. Additionally, UNICEF and Israel's Embassy in Cameroon are working together within the framework of the Connect My School Initiative, dispatching MASHAV experts to the region to connect two schools in vulnerable areas to the internet;

- Philippines: An agreement was signed on a Comprehensive Program for Educators on ICT and implemented through capacity-building and training programs conducted both in Israel and the Philippines.

Juvenile delinquency stems from the lack of alternatives that satisfy the natural needs of young people to grow as individuals and within a group. MASHAV implements an annual institutional capacity-building course in Israel on Preventative Approaches to Juvenile Delinquency for Spanish speaking senior officers and community experts from Latin America. At the request of the governments of Guatemala and El Salvador, followup activities in the two countries were carried out by MASHAV experts. (SDG 4.7)

Within the framework of the UN Educational, Scientific and Cultural Organization (UNESCO) Fellowship Program, MASHAV and UNESCO cosponsored a course in Israel on Educational Methodologies Youth-At-Risk: Preventing Student Dropouts and Facilitating Reintegration. Israel extended fellowships to UNESCO for the benefit of professionals from developing and least developed member states. The course focused on creating an environment of mutual trust, realizing the student potential, reducing alienation from the community, and establishing support systems. (SDG 4.b)

MASHAV conducts a Technical and Vocational Education and Training (TVET) Program for professionals from developing and least developed countries responsible for allocating resources and formulating educational policies. The Program is based on the concept that TVET must be embedded in a socio-economic context, encompass policy areas, and be sufficiently flexible to ensure a successful transition from school to the work force. (SDG 4.4)

MASHAV's Platform for the Inclusion of Children with Special Needs into Normative Frameworks (SDG 4.5) reflects Israel's experience with large waves of immigrants, particularly from the former Soviet Union. The Platform comprises institutional capacity-building programs conducted in Russian on establishing inclusive national and community frameworks for quality education for children with special needs. Courses conducted for educators and teachers include creative thinking and innovation in special education, and integration of children 
with special needs in normative frameworks. In addition, courses in Israel were held on special and inclusive education, early childhood education for children with special needs, and development of children: social-emotional support and wellbeing, focusing on early detection and intervention in the case of vulnerable children.

Ministers of Education and senior policymakers from 15 countries took part in MASHAV's 2018 High-Level Seminar on Transforming Disabilities and Inclusive Education. The seminar organized in cooperation with Israel's Ministry of Education, presented innovative tools for creating an inclusive educational framework for children with special needs. The event concluded with a Conference on Digital and Technological Solutions for Students with Special Needs. (SDG 4.5)

Governments, both federal and local, face the phenomenon of young people excluded from educational frameworks or the labor market. Opportunities to develop suitable alternatives for re-integration are urgently needed. MASHAV's international development cooperation program on Integration of Marginalized Youth presents models for the social and economic integration of marginalized youth at the national and municipal levels, taking into consideration formal and nonformal educational frameworks, as well as judicial and social welfare systems. (SDG 4.3)

Civil insecurity and violence are two issues challenging municipalities in dealing with the lack of public safety in their communities. Municipal preventive action is an effective approach, given their first-hand knowledge of a particular community. Municipal strategies are more effective when based on coordinated efforts and resources. MASHAV's program on Municipal Projects for Civil Security presents adaptable models for cooperation between the educational community and the police force, judicial authorities, volunteer organizations, the private sector and media. (SDG 4.7)

Israel's Ministry of Foreign Affairs grants scholarships for higher education to developing and least developed countries within the framework of Programs for Cultural, Educational, Scientific,
Sports and Youth Agreements. The agreements encourage mutual exchanges, respect for different cultural heritages, and understanding between countries. The ministry also awards scholarships for summer language courses in Israel. (SDG 4.b)

Teachers of Holocaust education face a daunting task of creating relevance for students in the 21st century. As stated in the Stockholm Declaration 2000, “The Holocaust fundamentally challenged the foundations of civilization. The unprecedented character of the Holocaust will always hold universal meaning... \{and $\}$ must be forever seared in our collective memory." In this context, Israel strongly supports the important work by the International Holocaust Remembrance Alliance (IHRA) in uniting governments and experts to strengthen, advance, and promote Holocaust education, remembrance and research worldwide, upholding the commitments of the Stockholm Declaration 2000. Israeli experts participate in IHRA's four Working Groups (Academic, Education, Memorials, Museums) as well as in IHRA's specialized committees (Antisemitism and Holocaust Denial, Holocaust, Genocide, and Crimes against Humanity). In 2016, Bulgaria requested that Israel serve as its mentoring country while in the process of joining the Alliance, gaining full membership at its General Assembly (2018). (SDG 4.7)

In 2018, 350 teachers representing 50 countries attended the opening ceremony of the 10th International Conference on Teaching the Holocaust: Time, Place and Relevance at Yad VaShem, the World Holocaust Remembrance Center in Israel. Participants included teachers, educators, directors of education departments in museums and Holocaust memorial institutions, representatives of ministries of education, as well as graduates of Yad VaShem's international educational seminars. The Chairman of Yad VaShem and the Director of the Auschwitz-Birkenau Museum in Poland held a special discussion on the challenges facing museums that preserve Holocaust remembrance.

\section{(SDG 4.7, 11.4)}

Given the historical and cultural significance of teaching about the Holocaust, Israel's Ministry of Foreign Affairs awards scholarships to educators 
from Eastern Europe attending Yad VaShem's annual International Summer Seminar in English on the Holocaust. The international flavor of the seminar allows educators to experience the diverse understanding and pedagogical imperatives of their colleagues, as well as an international perspective on Holocaust education. (SDG 4.b) 


\section{GOAL}

5

Israel's legislative framework on

GENDER EQUALITY

$\stackrel{\oplus}{\oplus}$ gender equality

National program to prevent domestic violence

Programs to treat women in the cycle of prostitution

Representation of women in parliament, local government and the Civil Service

Property rights to land

Use of enabling technology to promote the empowerment of women

Partnering for a Better World 


\section{ACHIEVE GENDER EQUALITY AND EMPOWER ALL WOMEN AND GIRLS}

The Gender Index, a tool developed by WIPS - The Center for the Advancement of Women in the Public Sphere at the Van Leer Jerusalem Institute examines gender inequality in Israel according to numerous indicators. In 2017 it consisted of 12 domains, assessed by 73 indicators. The domains are: education, labor market, gendering professions, poverty, power, family status, time, violence against women, health, culture and communications, Arab society, and the periphery. Over the years, the Gender Index has improved by some 10\%, although it has remained stable in the past four years. In 2017, despite laws legislated to promote equal rights and gender equality, the Index clearly indicated a persistent trend of male disproportional representation, reflected by men's leading roles in social, economic and political areas. While progress has been achieved on many fronts, the male prevalence in senior positions remains significantly higher than their percentage in the population. These social gender arrangements affect the differential abilities of men and women to earn a living, to impact the social, economic and political agenda, and to achieve status, prestige and financial well-being. 


\section{( Israel's legislative framework on gender equality (SDG 5.1, 5.3, 5.c)}

The laws that directly promote gender equality in Israel are the Women's Equal Rights Law, 1951, and the Authority for the Advancement of the Status of Women Law, 1998. The objective of the Women's Equal Rights Law is to determine principles for ensuring full equality between men and women. Inter alia, it relates to a married woman's property rights, to a woman's right over her body, to equality in social rights and equal guardianship, and to the duty of fair representation of women in public and government bodies. The Law also defines mechanisms for reporting on violations to the Authority for the Advancement of the Status of Women and to the Knesset Committee on the Status of Women and Gender Equality. The Law further stipulates that a provision or act of the type of "affirmative action," designed to rectify prior or existing discrimination against women, will not be deemed as harming (male) equality.

The Authority for the Advancement of the Status of Women Law is designed "to advance equality between men and women in Israel, lead to coordination between the bodies dealing with the status of women in Israel, advance the education, legislation and enforcement in these fields, ensure that the implications of proposed legislation on equality between men and women be examined as part of legislative proceedings, advance activity for the prevention of violence against women, make the tools and information required for achieving the aforesaid objectives available to the government, and establish a central authority that will act to implement these principles." The responsibility of the Authority is to coordinate, advance, advise and review the government's activity in the aforesaid subjects; gather information, initiate studies and advance the establishment of activity and services frameworks in the Authority's fields of activity; act for the implementation of the 1979 Convention on the Elimination of all Forms of Discrimination against Women; and maintain contacts with international bodies and parallel bodies in foreign countries.
The Authority for the Advancement of the Status of Women works in a diversity of areas. It endeavors to make a change in the public narrative with respect to the perception of beauty and general perception of the body by such means as public campaigns that target youth, aiming to serve as an agent of change. Support is also provided for the creation of a nationwide hotline to combat the exclusion of women with the intention of operating it together with relevant NGOs specializing in assisting women in this field. Furthermore, the Authority promotes training such as self-defense workshops designated for women and especially teenagers. The purpose of these workshops is to empower women from the psychological, conscious and physical aspects. In addition, the Authority is aiming to introduce a new databank relating to sexual harassment complaints at the higher education level luniversities and post-high school institutions). This step follows a government plan for reducing the current levels of sexual harassment cases within government. A public campaign will be launched to highlight and bring this topic to the attention of the general public. Currently the Authority is also targeting 217 designated supervisors within higher education institutions and will intensify and promote training programs to increase their effectiveness.

Beyond these two laws, additional equality laws are of relevance, including the Prohibition of Discrimination in Products, Services and Entry into Places of Entertainment and Public Places Law, 2000, and the Employment (Equal Opportunities) Law, 1988. These laws prohibit discrimination on varied grounds including, among others, gender and sexual orientation, and in the Employment (Equal Opportunities) Law, pregnancy and fertility treatments as well. Prohibited discrimination under these two laws can constitute both a civil wrong and a criminal offense.

In all matters pertaining to increasing the effectiveness of enforcement of the Prohibition of Discrimination in Products and Services Law, a broad course of action has been launched, aimed at formulating work procedures, concentrating efforts and re-thinking the enforcement of the law by the Israel Police and the State Attorney's 
Office. Current discussions focus on instituting changes in the police procedure for dealing with complaints under the Law, alleged to be a block to transferring complaints to the prosecution unit and the comprehensive treatment of the criminal phenomenon pursuant to the Law. A decision has also been taken to prepare a new police campaign which would be based on the lessons from the previous campaign, whose success was limited. Cases that had been closed during the years 20152017 are to be re-examined.

In order to expand and streamline the variety of tools in the fight against discrimination under the Law, it was also decided to create an interface between the criminal enforcement parties and the parties at the State Attorney's Office who are in charge of administrative-civil enforcement. Likewise, the possibility of promoting a public campaign, which would emphasize the rights of individuals and their ability to avail themselves of law enforcement authorities as well as present the risks of enforcement facing law breakers, would be examined.

Furthermore, the Legal Counsel and Legislative Affairs Department recently published a call for proposals for empirical academic research that would examine the implementation of the Law on the practical side, inter alia from the viewpoint of victims who do not utilize the Law sufficiently, both on the criminal and the civil track. This is based upon the understanding that the State does not possess sufficient information about the actual conduct of victims and the blocks that might be present in the legal and other proceedings concerning the Law. It is hoped that the information and insights produced by the research will result in recommendations regarding improvements in the implementation of the Law, both in theory and in practice.

In 2018 the Legal Aid (Legal Aid for a Person in a Civil Proceeding pursuant to the Prohibition of Discrimination in Products, Services and Entry into Places of Entertainment and Public Places Law, 2000) (Temporary Order) Law, 2018, was enacted. The amendment to the Law prescribes, as a temporary provision for a period of three years, that legal aid be granted, pursuant to the Legal Aid Law, 1972, to a plaintiff in a civil proceeding without the necessity of complying with the economic eligibility threshold, but subject to an examination of the chances of the claim in court. Furthermore, in October 2018, a draft of the Courts Regulations (Fees) (Temporary Order), 2018, was circulated, which proposes an exemption from payment of a fee for actions pursuant to the Prohibition of Discrimination in Products, Services and Entry into Places of Entertainment and Public Places Law, 2000. The purpose of the proposed exemption is to advance the value of equality that underlies the Law and to prevent discrimination in the entry into public places and the supply of products and services, inter alia, by means of filing claims against who breaches the provisions of the Law.

\section{( National program to prevent domestic violence (SDG 5.2)}

According to 2016-2017 police data on family violence, with an emphasis on violence against women, in 2017, 17,587 domestic-violence files were opened for offenses against a spouse (both men and women). Of these, $69 \%$ were opened following a complaint filed by a woman and $11 \%$, following a complaint filed by a man. In $20 \%$ of the cases filed, complaints were mutual. In each of the years 2016 and 2017, the police opened 11,800 files of violence against women. In 2017, 15,600 offenses were recorded in these files (each file may contain more than one offense): 26 incidents of murder, attempted murder and manslaughter; some 300 sexual offenses, 7,700 acts of violence, and 7,600 threats. In 2016-17, 35 cases of the murder or manslaughter of woman were recorded. About half the victims (17) had complained of violence to the police. In each year of 2016 and 2017, some 9,000 files of domestic violence were closed (not necessarily opened during this period): in $87 \%$ of the cases the reason cited was lack of evidence. In 2016, 2,800 indictments were issued and in $2017-2,600$, for domestic violence against women.

Given the extent of the phenomenon in Israel, the Ministry of Labor, Social Affairs and Social 
Services (MOLSA) led an inter-ministerial national program for the prevention and treatment of domestic violence. Partners to the program include the Ministries of Education, Justice, Health, Immigrant Absorption, and Public Security. Domestic violence is a complex social problem across sectors and socio-economic classes. It takes many forms, including physical, sexual, verbal, psychological and financial abuse, and threatening harassment. To develop and provide optimal integrated responses and improve prevention, identification and enforcement, as well as legal recourse for purposes of protection, treatment and rehabilitation, a number of guiding principles were laid down:

- Responsibility and commitment - diverse responses by all government ministries to address the social problem of domestic violence in all its complexities;

- A common language to reflect an agreed-on professional conception and inter-ministerial coordination;

- Establishment of a treatment continuum to help maintain continuity between prevention and treatment, the space in which the responses take place, and a multifarious, comprehensive approach to family needs;

- Removal of structured obstacles to improve bureaucratic mechanisms and the provision of optimal services:

- Provision of available, accessible and immediate responses to provide effective responses both in emergency situations and as a matter of routine to all affected populations;

- Conception of the family as a system - the provision of suitable service to all family members (from children to senior citizens) and to all types of families (including LGBT families).

Strong emphasis was placed on the provision of new responses for Arab society and the adjustment of existing ones. About a quarter to a third of the existing responses are aimed at Arab society. The added emphasis is due to the high rate of domestic violence in the Arab sector (23\%) relative to their proportion in the population (18\%).
The program is directed by a a staff heading 10 teams in the following areas: women, men, senior citizens, children, danger assessment and special populations, cooperation and data flow, legal issues, means of protection, awareness and information campaigns, and research and measurement.

As highlighted in a report on the implementation of the recommendations of an inter-ministerial committee on the prevention and treatment of domestic violence (2017), MOLSA improved existing programs and developed new ones to prevent and treat domestic violence. These include: allocation of additional staffing positions for social workers experienced in working with abused children for centers addressing domestic violence; units for the prevention of elder abuse; and police stations providing in-depth and comprehensive responses to adults involved in family violence. Furthermore, inter-ministerial training was established for professionals from different disciplines who work with families affected by domestic violence; the emergency hotline was expanded to $24 / 7$ and provides service in different languages; and a family help center was set up for victims of domestic violence with a multi-disciplinary staff experienced in treatment and enforcement. The center operates $24 / 7$, offering a specific, tailored, accessible and all-embracing service to facilitate maximal professional support in the initial period. An integrated treatment service was established for abused women caught up in prostitution and/ or addiction. A treatment center was created for abusive men, emphasizing improved recruitment and the adaptation of responses to their place on the continuum of violence.

A school program was implemented focusing on educating students on healthy relationships, raising awareness of warning signs, and improving communication and interpersonal relations. All the ministries participated in an information campaign to raise awareness of domestic violence and of warning signals. A measurement system was and is being developed to deepen knowledge and evaluation of the extent of domestic violence. Furthermore, a control and supervision center examines the implementation and impact of the 
program. The program is in its formative stages and, as yet, there are no data on its effectiveness in eradicating and treating domestic violence.

\section{( Programs to treat women in the cycle of prostitution (SDG 5.2)}

The national survey of the phenomenon of prostitution in Israel (2016) indicates that figures for prostitution in Israel stand at 121.2 to 128.6 per 1,000. Prostitution takes place in such diverse arenas as the street, brothels, escort services and massage parlors. It is found among addicts, sexually-exploited women, women in financial trouble, and other vulnerable populations. It is mostly a hidden phenomenon regarded with social disgrace, which makes it difficult to estimate its extent. In 2014 the estimated figures ranged from 11,190 to 12,040 , of whom about $95 \%$ were women and the rest, men.

According to an evaluation study of the interministerial committee for the treatment of women and girls in the cycle of prostitution, some $56 \%$ of the streetwalkers and $6 \%$ of those working indoors received treatment via different programs; $17 \%$ of those treated applied to treatment on their own or with the help of a close acquaintance; $9 \%$ were referred by community institutions or various NGOs; and $63 \%$ were identified by the treatment frameworks.

The programs implemented by MOLSA in various parts of Israel include: Saleet in Tel Aviv which operates a national helpline, emergency housing, a hostel, and morning and evening care centers; Ofek Nashi (Female Horizon) in Haifa which operates three city frameworks: emergency housing, a hostel and a daycare center; Nayadut (Mobility) in Tel Aviv and Haifa; Erim Balayla (Awake at Night) which is operated by Elem in Tel Aviv, Haifa and Eilat; and Bishvileikh (For You) which operates in Beersheba.

The programs pursue two main roads: damage control and treatment and rehabilitation. For the former, services provide initial treatment to women in prostitution, on the assumption that it is possible to improve their psychological and physical well-being and the quality of their lives, even if initially full rehabilitation is not achieved. These services are:

- A national helpline - operated by Saleet for 14 hours/day and offering support and an initial response to women and girls: the helpline also provides information on existing help frameworks for women in prostitution. In addition, it initiates conversations with the women in order to maintain contact and enlist them in treatment and to assist them with the exercise of their rights and with access to the social services in their localities.

- A mobile clinic - operated with the cooperation of the Health Ministry: through the clinic, onsite initial contact is made with the women and preliminary medical assistance is proffered, including periodic blood tests to check for illness, provide vaccines, and distribute condoms. The units operate in Haifa and Tel Aviv for a few evenings a week and engage in outreach, building trust and recruiting the women for treatment.

- Emergency housing - operates 14 hours a day, seven days a week In Haifa and Tel Aviv to absorb sexually-exploited women and provide them with the immediate and unconditional assistance of a temporary roof over their heads: generally, the women are addicted to drugs and subject to severe physical and psychological neglect, daily sexual abuse, and street violence. This housing is designed to provide for their basic needs such as a bed, food and clothing; protection, medical tests and treatment; and support from a social worker. Their stay allows them to reorganize and consider change. Weaning the women off drugs and alcohol is also part of this stage.

- Awake at Night - is implemented in partnership with the Elem NGO: it identifies and provides initial treatment for girls and young women exploited for prostitution in Haifa, Tel Aviv and Eilat.

As part of the process to extricate women from prostitution, services are also provided for those engaged in permanent, long-term prostitution. The immediate goal is to help them rehabilitate themselves and leave the prostitution cycle. The services are: 


\section{CASE STUDY}

\section{The Lamed-Bet Program (Education for at-Risk Youth)}

The Lamed-Bet Program (Education for at-Risk Youth) serves as an open space for the treatment of prostitution. It is intended for young girls at the extremity of the risk continuum and exploited for sex. It reaches out to locate these girls, offering damage control, assistance, support and treatment to help them leave the cycle of risk. It operates on an expanded model of 5.5 days and includes emergency beds in seven localities that provide a response to some 300 girls. It also operates on a more limited model of three days a week in six localities, providing a response to some 150 girls. The expected outcomes are: $70 \%$ attendance; $50 \%$ reduction of risk factors. The girls are referred to the program directly, by community education and treatment authorities and by Social Service Departments.

- Hostels - a holistic framework for women, including a roof over their heads: the goal is psychological treatment and rehabilitation for a period of a year. Women who were addicts reach the hostels after the stage of weaning off drugs if they express a desire to change their lifestyle. The hostels provide a roof; intensive, comprehensive emotional and psychological treatment in all areas of life; support throughout rehabilitation including employment rehabilitation, family reconnection, a resolution of the problems of permanent housing, and a return to normative life in the community.

- Day and evening care centers - an intensive daily treatment framework for women in the stage of leaving the prostitution cycle: daycare centers operate from morning to late afternoon and evening centers in the late afternoon and evening. Women receive individual and group psychological treatment, assistance in the acquisition of basic professional skills, a chance to complete their education, work habits, integration into employment, etc. The program is recognized as rehabilitation by the National Insurance Institute for purposes of an income support benefit for participants.

- Ambulatory road - a second road, including individual or group psychotherapy, as well as enrichment activities and vocational courses: the program is intended for women in the initial stage of forming a therapeutic relationship while still active in prostitution, who require acceptance and support. Graduates of hostels and daycare centers requiring support and a therapeutic relationship may also take advantage of the program as it enables them to continue to live independently, removed from prostitution, and to cope with various associated difficulties. The program began in 2011 with the goal of making services accessible and adapting them to the women's needs.

\section{Programs addressing girls in trouble}

Programs addressing girls in trouble include the Correctional Services and Youth at Risk, and Notzetz (Sparkle), the Service for Youth and Young Adults. The latter strives to develop and provide youth on the continuum of trouble, danger and risk with the best possible support, legal defense, assistance and treatment. It maintains flexible, accessible services appropriate to gender, sector and culture. Notzetz services draw on the capabilities and strengths of youth with the goal of helping them to realize their potential (Ronen \& Javetz, 2017). The responses provided to the girls include:

- Warm Homes - treatment centers for at-risk girls: the program provides a therapeutic response and a hot meal in the afternoons for at-risk girls in the community. Countrywide, it 


\section{CASE STUDY}

\section{The Warm Homes Program}

The Warm Home Program is a therapeutic framework providing a response to the needs of girls aged 13-18, particularly those at risk or in trouble or having difficulty coping with everyday life and require an empowering, enriching treatment framework. Those eligible for the service are characterized by problems of family and/or interpersonal communication; a past of sexual, physical or psychological abuse; an immigration and cultural crisis; or disengagement from school. Activities take place in 170 centers providing an afternoon framework for thousands of girls, starting after school and stretching into the evening. It is implemented, supervised and budgeted by MOLSA's Service for Youth and Young Adults (Notzetz).

Activities are held in groups of 15 girls and help is provided by a staff of women, including a social worker, housemother, and one or more counselors. In content, the diverse activities are either therapeutic or scholastic, and include hands-on workshops and activities focusing on empowerment. In addition, every girl receives a structured program for individual progress, tailored to her needs.

The program's implementation follows several formats differentiated by the implementing party, the duration of the girls' stay, and the active weekday. The model of all the homes is determined by the consideration of local needs alongside budget constraints. An evaluation study examined the attitudes, behavior and satisfaction of the girls at 33 homes countrywide. The study also evaluated the different implementation models. It concluded that the homes have a significant, positive impact on the participants. The findings indicated significant positive change regarding the girls' success, behavior and satisfaction. The homes were found to be supportive, empowering frameworks. No correlation was found between the characteristics of the homes and the extent of their success in advancing the girls according to the criteria evaluated. Similarly, the very fact of participation in the homes constituted the main factor in improvement. The most meaningful factor leading to success was the nuclear family.

runs 170 homes in 130 localities, addressing 3,105 girls. The program is operated by local authorities or by NGOs that win tenders published by MOLSA or the local authority. The expected outcomes are that $70 \%$ of the girls will persevere with program activities; $60 \%$ will improve their achievements in the personal and social realms; and $60 \%$ will reduce their risk behavior. The girls are referred to the program by a social worker, school staff, youth workers or by word of mouth of their peers.

- Adi (Hebrew acronym for Power, Concern and Friendship) Center for Young Women at Risk - a daycare center aimed at girls at the extreme end of the risk continuum and long disengaged from the education system: the goal of the center is to integrate the girls into inclusive, supportive treatment centers, reducing their risk factors, improving their scholastic, social and personal standing, helping them out of the cycle of vulnerability, and empowering them.

- Mentoring - Girls for Girls - training girls for the role of mentoring: the countrywide program is part of two other programs providing a response to 110 girls. The expected outcomes are: $80 \%$ will complete the course; $60 \%$ will integrate either into employment as mentors or into National Service. They are referred by Social Service 


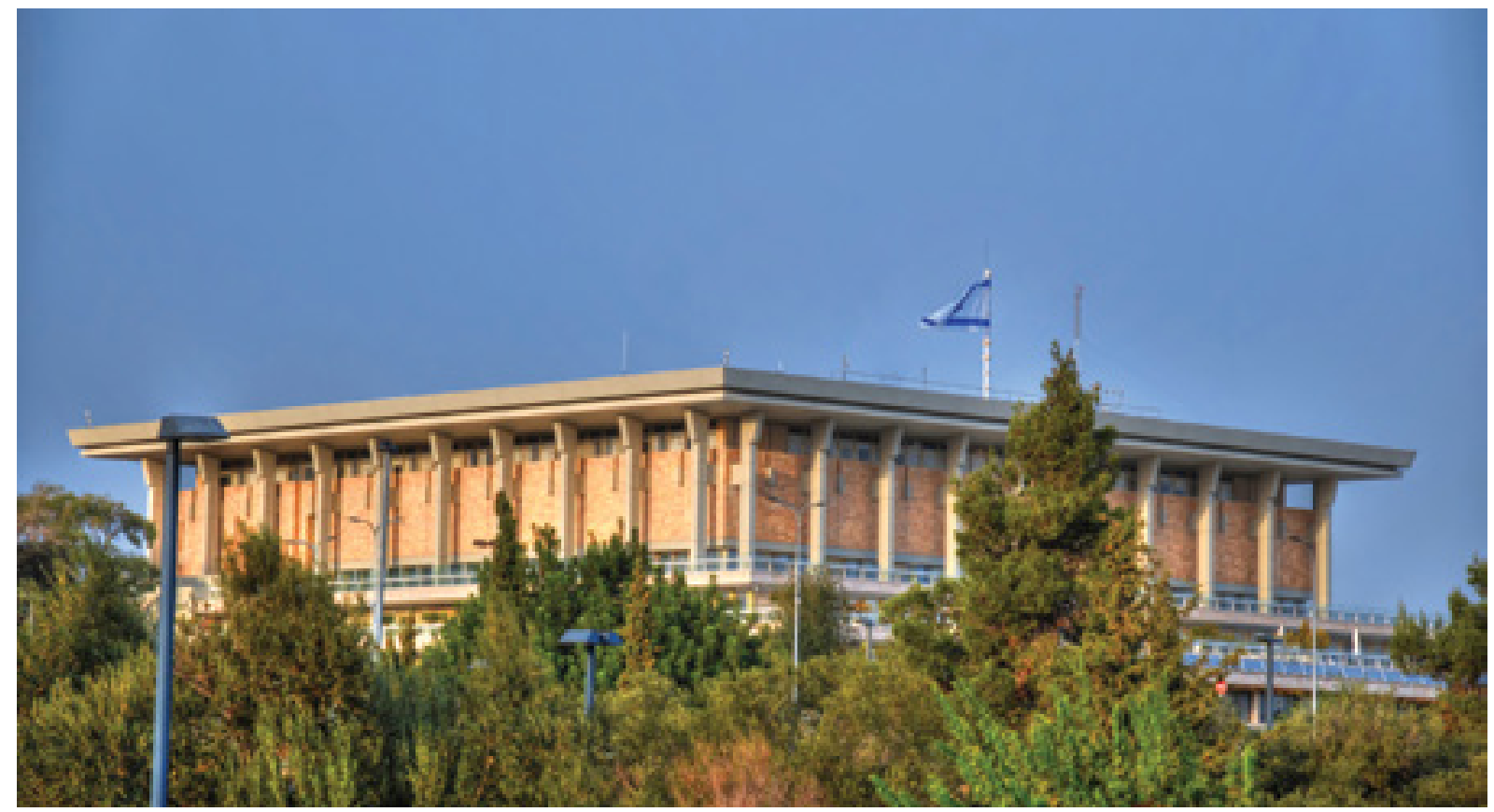

The Knesset (Israel's Parliament)

Departments. The program also has a continuing course to promote integration into education and employment programs, support, and a response in situations of emergency or crisis.

\section{- Tzalash (Hebrew acronym meaning Military} Commendation] - the goal of the program is to prepare at-risk girls for the army (the Israel Defense Forces [IDF]) or National Service. It operates countrywide providing a response to some 240 girls. The expected outcomes are: $95 \%$ will complete the training course; $75 \%$ will go into the army; and 20\% will integrate into National Service. The girls are referred by Social Service Departments, graduates of MOLSA frameworks, and the IDF based on its data on special recruits.

- Girls Circle - the program provides a therapeutic response including meals to at-risk girls in the community, for one afternoon a week (for a duration of 5 hours). Local authorities in Israel operate and provide a response to a total of $71 \mathrm{Girl}$ Circles and 852 girls. The expected outcomes are: $70 \%$ will persevere with program activities; $60 \%$ will improve their achievements in the personal and social realms; and $60 \%$ will reduce their at-risk behavior. The girls are referred by Social Service Departments, school staff, community youth workers or by word of mouth of their peers.

\section{( Representation of women in parliament, local government and the Civil Service (SDG 5.5)}

In 2017, women held $40.7 \%$ of senior positions in the public sector ( 41,800 women), $55.9 \%$ of such positions in education, and $68.3 \%$ in human health and social work activities. Women's representation in Israel's local authorities rose in the 2018 elections, thus continuing the trend of their increased representation in local authorities. In 2013, 386 women served as council members (13\%), In the 2018 elections, 635 women were elected to serve as council members (18\%). The number of women heading local authorities rose from 6 to 14 during this period. Women's representation in the Arab sector, although still low, has improved significantly over the years. In the public sector, the rate of Jewish women (41.6\%) was higher than that of Arab women (27.6\%). The gap between Jewish and Arab women has decreased over the years - from $26.5 \%$ in 2012 to $14.0 \%$ in 2017 . Representation of women in the Knesset (Israel's parliament) and local government is still low compared with the 28 EU countries; the percentage of women who headed local authorities in the EU countries as of 2017 was $14.9 \%$ compared with $5.5 \%$ in Israel after the 2018 elections. Women's 
Figure 5.1

\section{Proportion of seats held by women in the Knesset (Israel parliament)}

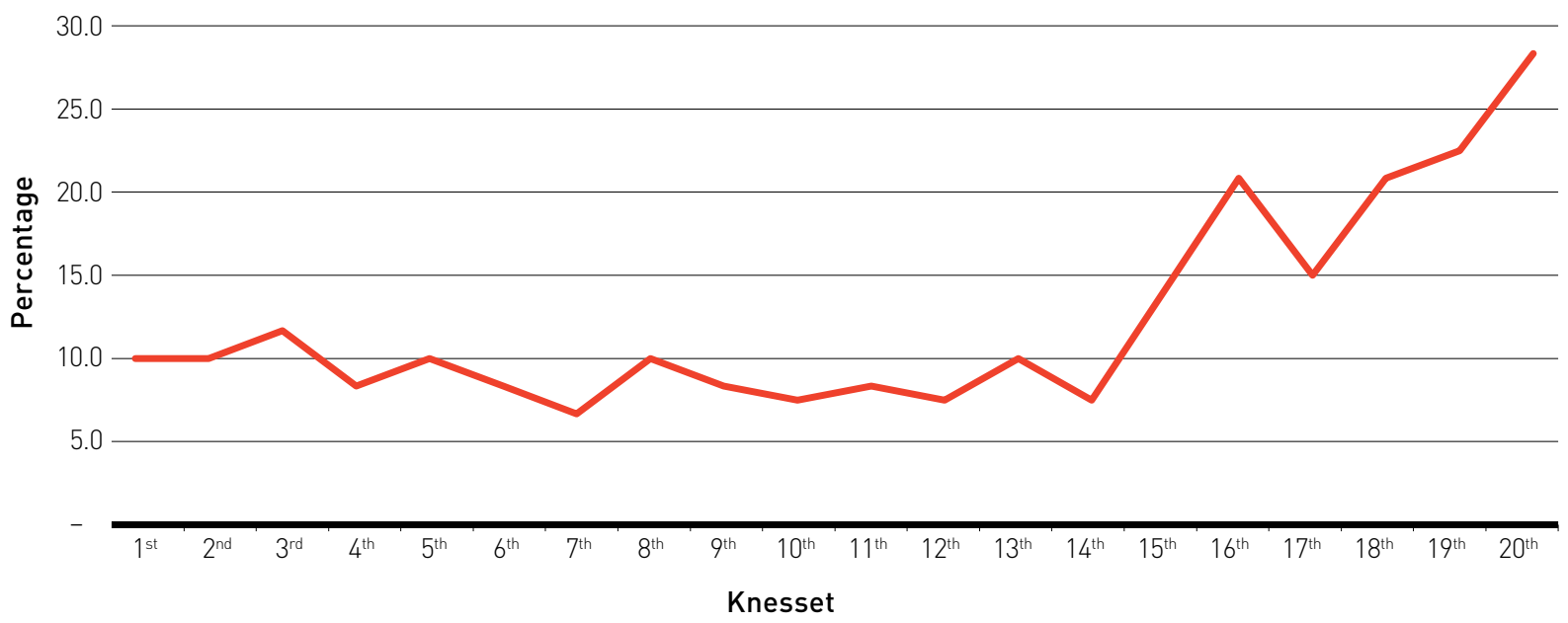

representation in the $28 \mathrm{EU}$ councils was about $32.1 \%$ percent compared with $18 \%$ in Israel. In other words, the average rate of women heads of local authorities in the European Union is almost three times their rate in Israel, and the proportion of women members of the authorities is almost double.

The Ministry of Interior along with the local authorities can play a leading role in getting more women into elected office at local level. This is a top priority in terms of empowering women, because local politics is often the first step to regional and national office. Female leaders in local government can challenge gender stereotypes and set an example to young girls. In 2014, as part of a Ministry of Interior initiative, the Knesset plenum approved the Local Authorities (Election Financing) Law (Amendment No. 12) which grants higher election funding to local authority factions that have high representation of women.

The results of the 2018 local elections indicate that this legislation was partially successful. Although there has been an increase in the number of women who competed for the positions of heads of authority and membership in the council and in the number of women elected to these offices, their proportion is still very low.

The Knesset, as Israel's legislative branch, has 120 members. The data presented in Figure 5.1 show the proportion of women in the Knesset from the first Knesset (1949) to the twentieth Knesset (2015).

\section{( Property rights to land (SDG 5.a)}

The Ministry of Justice's policy acknowledges and supports the basic legal principle of equality, and particularly equality between men and women. With regard to property rights to land, Israel Land Laws do not differentiate and do not discriminate between men and women, with both equally entitled to property rights to land in Israel. Accordingly, there is no difference or reference to the sex of the owner of a property or any other property rights. Similarly, none of the forms for registering land rights include any reference to gender, and a statement of sex or gender is not a requirement. Therefore, the land registry information system in Israel (Rimon) has no registry of, or reference to, sex or gender, and there is no search option for data regarding gender or sex of ownership or other forms of property rights.

\section{Use of enabling technology to promote the empowerment of women (SDG 5.b)}

Specific Digital Literacy goals for women are included within the National Digital Program of the Government of Israel (see SDG 10):

1. Digital literacy for women:

- Focus on financial literacy to empower women in the social and geographical periphery of the country; 


\section{CASE STUDY}

\section{The Israel Police's Gender Equality Unit (SDG 5.5, 16.7)}

The Gender Equality Unit's mission is to create conditions that will ensure equal opportunity for women serving in the Israel Police and to ensure that their capabilities are fully realized. The Unit further seeks to create broad organizational awareness of the issue of sexual harassment so as to reduce the incidence of sexual harassment within the police.

Its roles include the following: to advise the Police Commissioner on integration and promotion of women within the Israel Police; to examine from a gender perspective the Israel Police's regulations, policies, decision and actions; to identify obstacles to the integration and promotion of women within the organization; to take action to promote women to key positions within the Israel Police; to fill knowledge gaps on women's service in the Israel Police and disseminate information on the issue; to address cases of unlawful gender-based discrimination within the Israel Police; to raise awareness with regard to sexual harassment; to deal with cases of sexual harassment within the Israel Police and to support police officers who have been sexually harassed.

- Pilot projects currently in six municipalities;

- 40 hours of learning digital skills.

2. Digital communities' program:

- Closing the digital divide and teaching digital skills through a multi-sector effort at the community level;

- Special focus on Israeli-Arab and ultraOrthodox women.

\section{PARTNERING FOR A BETTER WORLD}

Sustainable development is only possible when men and women enjoy access to equal opportunities to reach their full potential, and no economy can grow to its full potential without the full participation of both women and men. Gender inequalities and discrimination prevent women from lifting themselves, and often their families, out of poverty. Towards this end, Israel's Ministry of Foreign Affairs designated the empowerment of women and the elimination of their sexual exploitation as a key strategy in its Global Agenda 2019.

Israel reaffirms its commitment to the zerotolerance policy on sexual exploitation and abuse and stands united with many UN member states in demonstrating its political will by signing the UN Secretary General's Compact on the Commitment to Eliminate Sexual Exploitation and Abuse. (SDG 5.2)
Violence against women and children is a scourge on societies and remains an obstacle in protecting their fundamental freedoms and human rights. Israel spearheaded a UN Resolution on Preventing and Eliminating Sexual Harassment in the Workplace, adopted within the framework of the Commission on the Status of Women (CSW). The Resolution condemns all forms of sexual harassment, especially against women and girls, affirming the need to take all necessary prevention measures. The Resolution, the first of its kind in the UN, emphasizes the important role of member states in eliminating this phenomenon, and places the primary responsibility upon employers. The Resolution also lays out several methods through which countries can combat and handle sexual harassment, including legislation, policies, education, awareness-raising programs and research. (SDG 5.2, 8.8) 
Within the framework of the CSW (March 2019), Israel's Permanent Mission to the UN in New York organized a side event on Private Sector Wisdom to Crack Gender Equality in the presence of Israel's Minister for Social Equality and the Head of the Gender Partnerships Division of the Organization for Economic Cooperation and Development (OECD). The event, organized in cooperation with the NGO Parliament 51, featured panelists from the private sector (INTUIT, WEBPALS) and civil society (WE POWER), saw discussions on the glass ceiling preventing women from attaining senior management positions and discussed steps needed to bridge employment disparities. (SDG 5.5)

On the global scene, Israel is advocating the promotion of gender-responsive policies, and advancing the principle of equal opportunity and the empowerment of women and girls, especially from rural areas. It is a member of the Executive Board of UN Women, and within this framework, MASHAV signed an agreement with UN Women for the advancement of joint projects promoting gender equality. (SDG 5.5)

In cooperation with the UN Educational, Scientific and Cultural Organization (UNESCO), MASHAV holds an annual International Course on Combating Violence against Women and Children for professionals from developing and least developed countries. (SDG 5.2, 16.3)

Gender equality is enshrined in Israel's 1948 Declaration of Independence and has been further entrenched in Israeli society through legislation and public policies, beginning with the milestone legislation entitled Women's Equal Rights Law (1951). Over the last 17 years, Israel has taken an active part in promoting the six UN Security Council resolutions that followed the principles originally declared in UN Security Council Resolution 1325 , stressing the importance of equal participation and full involvement of women in all efforts for the maintenance and promotion of peace and security. Israel has demonstrated repeatedly its commitment to the Women, Peace and Security Agenda at all levels of society, and was the first country to implement parts of UNSCR 1325 in its national legislation. Recently, Israel joined the UN
Group of Friends of Women Peace and Security (WPS) led by Canada, to increase support for the implementation of UNSCR 1325. (SDG 5.c)

Within the UN system, Israel cosponsored the following General Assembly Resolutions: Intensifying of Efforts to Prevent and Eliminate All Forms of Violence against Women and Girls; The Girl Child; Intensifying Global Efforts for the Elimination of Female Genital Mutilation; Intensification of Efforts to End Obstetric Fistula.

\section{(SDG 5.2, 5.3)}

Israel is advocating for agreed UN language focusing on the eradication of sexual harassment in the UN system through a UN General Assembly resolution. Israel, France, Kenya and the Netherlands established (2018) a Group of Friends to promote this initiative. Israel recently sponsored the third meeting (2019) of the Group of Friends, with the participation of representatives from multilateral organizations including the International Labor Organization (ILO) and UN Women. (SDG 5.2)

Furthermore, Israel is involved in discussions held by the ILO on the proposal for a convention, supplemented by a recommendation, concerning Violence and Harassment in the World of Work. During discussions, Israel suggested amending the text to clarify the explicit inclusion of the issue of sexual harassment. (SDG 5.2)

The Permanent Representative of Israel to the $U N$ in Geneva is a Champion in the International Gender Champions, a leadership network that brings together female and male decision-makers determined to break down gender barriers and make gender equality a working reality. (SDG 5.c)

Additional steps were taken to advance the equal rights of women in the work force when Israel joined the Equal Pay International Coalition (EPIC), a multi-stakeholder coalition for "equal pay for work of equal value," led by the ILO, UN Women and the Organization for Economic Cooperation and Development (OECD). (SDG 5.a, 8.5)

Human trafficking was one of Israel's worst crises in the beginning of the $21^{\text {st }}$ century. As a country of destination, women and children were smuggled into 
Israel from Eastern Europe and the former Soviet Union for purposes of prostitution. Israel's policies to combat this evil phenomenon were based on the principles of prevention, prosecution and protection, in accordance with the Palermo Protocol (ratified). Israel's National Anti-Trafficking Coordinator in the National Anti-Trafficking Unit (NATU), cooperates bilaterally with government ministries, civil society and international organizations to strengthen the fight against human trafficking, engaging in meetings with representatives from the US State Department, European Union and the UN Office on Drugs and Crime (UNODC) to discuss new trends of trafficking. The Coordinator also participated in numerous international fora, including: the Universal Period Review (UPR, Human Rights Council, Geneva); $18^{\text {th }}$ Organization for Security Cooperation in Europe (OSCE) High Alliance Seminar on Trafficking in Persons (Vienna); OSCE National Coordinators Meeting (Vienna); the $10^{\text {th }}$ Anniversary of the Council of Europe's Convention on Trafficking in Persons (Strasbourg); UNODC West European and Other Groups (WEOG) meeting on Trafficking in Humans (Vienna); and the International Social Services (ISS) Working Group on establishing international guidelines to prevent exploitation and trafficking in the context of international surrogacy agreements. In addition, two Israeli experts from NATU took part in the OSCE Live-Simulation Training Course on Combating Human Trafficking along Migration Routes (Viczenza). (SDG 5.2, 8.8)

Acknowledging the fact that human trafficking disproportionally affects women and girls, MASHAV, in cooperation with OSCE, UNODC, ILO, NATU, Israel's Institute of Advanced Judicial Studies and the United States Embassy in Jerusalem conducted the $4^{\text {th }}$ International Seminar for Judges on The Critical Role of the Judiciary in Combating Human Trafficking. Issues discussed included patterns of trafficking, forms of exploitation, psychological challenges facing victims, rights of victims in criminal proceedings including non-criminalization/ non-punishment, and the importance of international cooperation. (SDG 5.2, 8.7)

At the $4^{\text {th }}$ Committee of the $73^{\text {rd }}$ UN General Assembly, Israel declared its support for the UN
Office on Space Affairs (UNOOSA) as the sole organ in the UN system dealing with the peaceful uses of outer space and the implementation of relevant treaties. Moreover, as a member of the current Bureau of the Committee on the Peaceful Uses of Outer Space (COPUOS), Israel reaffirmed its steadfast commitment to the implementation of the Committee's SPACE 2030 Agenda and provided a financial contribution $(\$ 20,000)$ to the Women for Space Project. This contribution allows for the construction of a centralized UN platform 'Dedicated Webpage' for the Space for Women Project, and a framework for coordination and exchange in the STEM (Science, Technology, Engineering, and Math) sectors. In addition, it will promote the idea of the Champions Network to create a "Role Models" platform for girls and young women interested in pursuing a career in STEM. (SDG 5.b, 9.5)

The empowerment of women and girls in science related fields and opportunities for equal access are vital for the achievement of the 2030 Agenda. According to UNESCO Data (2014-16), women make up approximately $30 \%$ in science related fields in higher education. In a panel discussion on Women in Space, conducted within the framework of the 56th Session of the COPUOS Scientific and Technical Subcommittee (2019), Israel introduced the She Space Project, a collaborative initiative between the Remote Sensing and Planetary Thermodynamics Laboratory at Ben-Gurion University of the Negev and Beit Yatziv in Beersheba. The project focuses on encouraging women and girls to study science, technology and space. The She -Space Project, funded and assisted by Israel's Space Agency (ISA) in the Ministry of Science and Technology, is carried out in Israel and is underway globally in collaboration with Brazil, Germany and the United States. Furthermore, COPUOS expressed interest in facilitating future joint initiatives on the She - Space Project between Israel and member states. (SDG 5.5)

Furthering efforts to engage women and girls in STEM, Israel supported the adoption of the UN General Assembly Resolution (70/212) declaring 11 February as International Day of Women and Girls 
in Science. In 2019, the occasion was marked by the participation of an Israeli expert as a panelist in a side event held within the framework of the COPUOS Scientific and Technical Sub-Committee (Vienna). The Space Generation Advisory Council (SGAC) Israel likewise commemorated the occasion.

The empowerment of women and girls and gender mainstreaming are integral components in Israel's overseas development assistance, focusing on inclusive economic growth, strengthening entrepreneurial skills, ICT capabilities, financial literacy, and leadership skills in political, socioeconomic and environmental decision-making processes. A recent study by the World Bank entitled Women, Business and the Law 2018 found that the importance of women's entrepreneurship and employment had increased significantly, as had the understanding of the relationship between legal gender equality and women's economic outcomes. Focusing on the role of smallholders, particularly women, Israel and the UN Industrial Development Organization (UNIDO) are carrying out a program of cooperation relating to agricultural entrepreneurship and the empowerment of women in rural areas. (SDG 5.b, 8.3)

As women in Africa face momentous challenges on the path to leadership positions, MASHAV, UN Women, and Kenyatta University established the Africa Center for Transformative and Inclusive Leadership (ACTIL) in Nairobi. ACTIL seeks to raise transformative leaders and build competencies especially among women and youth. The goal is to provide necessary skills through professional capacity-building and mentorship, while promoting innovation and social transformation to facilitate the emergence of a regional network of Leadership Institutes, creating high standards and, eventually, accreditation in leadership development. Activities include joint workshops organized in Israel in collaboration with the UN Women Regional Office for Eastern and Southern Africa and dispatching Israeli experts to conduct periodic consultancy missions in Kenya to provide technical advice and support in setting up efficient systems of operation, structure and design of training programs to maximize ACTIL's impact and ensure sustainability. (SDG 5.5, 10.2)
Highlighting the importance of equal access of women to leadership roles on the international, national and local levels, MASHAV hosts a biennial International Women's Leadership Conference attended by high- ranking women from developed and developing countries representing the political, socio-economic and environmental segments of their societies. (SDG 5.c)

Gender biases in local governance present challenges to the equal participation of women in all spheres of life. MASHAV's program on Building Sustainable Communities: Leadership, Gender, and the Environment focuses on the interconnection between gender and local governance, stressing the critical role of gender in urban planning, environmental protection, access to land rights, basic urban services, housing, budgeting, economic development, and safe and secure cities. (SDG 5.a) Israel's international development cooperation programming supports collaboration with its neighbors in the Middle East/North Africa region. An example of this collaboration includes a MASHAV workshop conducted in Israel on Women's Leadership and Social Entrepreneurship tailormade for a seven-member delegation from Morocco representing women's NGOs and the private sector. The delegation was acquainted with Israel's social entrepreneurship spirit, emphasizing the economic empowerment of women. (SDG 5.5) 


\section{GOAL}

6

\section{CLEAN}

WATER AND

SANITATION

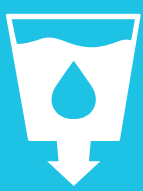

Integrated Water Resources

Management approach

National Water Master Plan 2050

Manufactured water resources

(desalination and water reuse)

Water governance - regulation and management

Research and technologies

Partnering for a Better World 


\section{ENSURE AVAILABILITY AND SUSTAINABLE MANAGEMENT OF WATER AND SANITATION FOR ALL}

Freshwater scarcity is a constant reality in Israel. Yet, the disadvantages of water resource scarcity have incentivized Israel to develop sustainable approaches to water sector management. Israel has transformed crisis into opportunity, recognizing that scarcity goes beyond physical scarcity of water source availability, or even economic scarcity of financial resources, but includes scarcity in management.

Annual water supply in Israel is more than 2 billion cubic meters for a population of about 9 million with a density of about 400 persons per square kilometer, approximately 200 thousand irrigated fields and more than one thousand industrial plants. Agriculture is the main water consumer in Israel, with more than half of the total volume. The domestic and municipal sectors consume about a third and the rest is divided between industry, nature and water supply to Israel's neighbors in accordance with agreements. 
The water balance between demand and available supply of natural water resources shows a constant deficit, growing from year to year. The permanent challenge is to close the gap. Furthermore, and most importantly, natural replenishment is challenged by the impacts of climate change: droughts are more frequent, and when occurring are more intense and for longer periods. Climate change impacts on water planning include reduced annual volume of precipitation and major interannual variability which prevents averagebased planning and increases climate externalities. Climate change externalities therefore exacerbate the gap between supply and demand.

\section{Integrated Water Resources} Management approach (SDG 6.1-6.6)

\section{Universal access to safe and affordable drinking water for all}

The model that Israel adopted and implemented for sustainable management of the water sector is an Integrated Water Resources Management (IWRM) approach, based on two pillars - water resources and water governance. Sustainable management of the water sector calls for looking at the entire picture, considering the needs of all consumers and managing all the "links" of the water chain, from planning, through conveying, quality monitoring, and sewage collection and treatment.

The water resources pillar is based on three foundations: natural water resources, manufactured resources and the integration between the two through planning, operation and development.

Israel's main natural water resources are the Sea of Galilee (Lake Kinneret) and two major aquifers: one along the coast and the other - a mountain aquifer. The scarcity of precipitation and its uneven distribution in different regions of the country (rainy north and dry south) obliged Israel to construct the National Water Carrier in 1964, a conduit that delivers water from the Sea of Galilee to the densely populated regions in the center and the more arid areas in the south. This national bulk water conveyance system optimizes the distribution of water across the country from various sources depending on demand. It conveys

Figure 6.1

\section{Hydrogeological situation of the main natural water sources}

Total volume of water above the red lines for three main water sources ${ }^{1}$

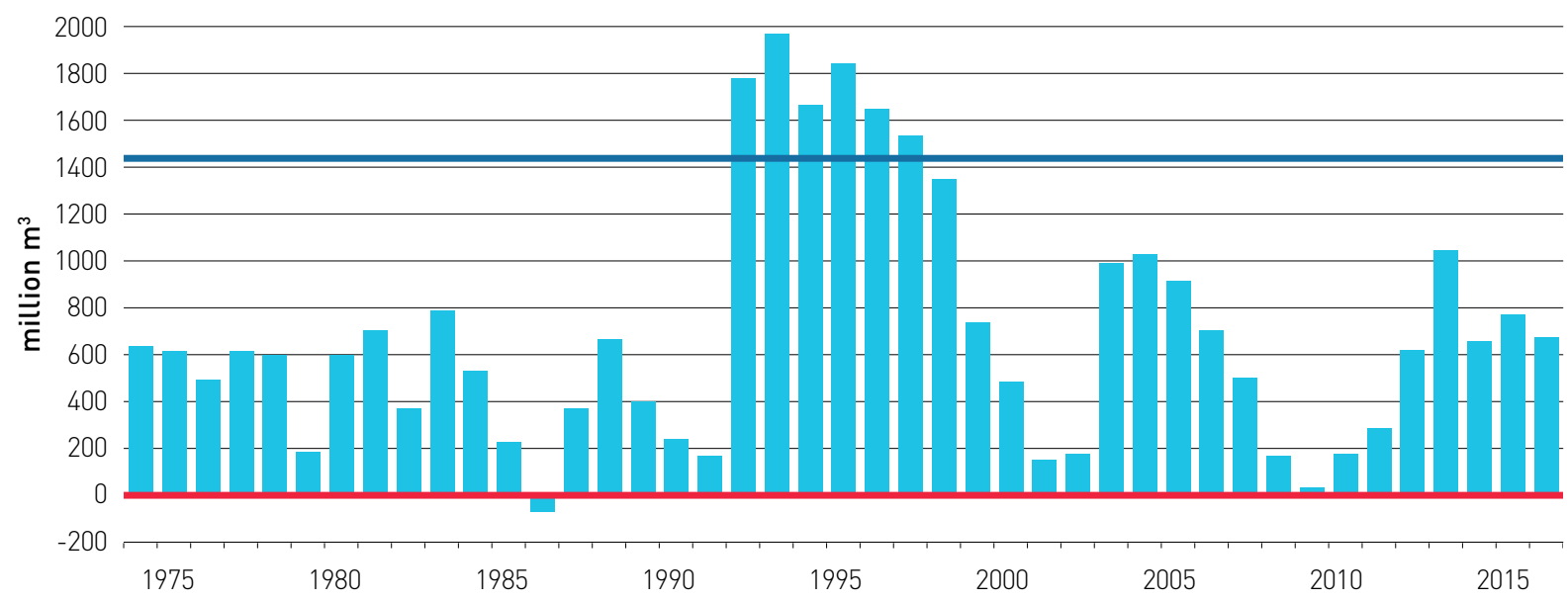

${ }^{1}$ Coastal aquifer, Western Mountain aquifer and the Kinneret. To prevent water salinisation, the hydrological service has defined red lines as the minimal water levels needed for each water source and blue lines as the required water levels to maintain a satisfactory water quality.

Excerpted from OECD Report - The Management of Infrastructure for water supply in Israel, 2018 OECD (2018), OECD Economic Surveys: Israel 2018, OECD Publishing, Paris. http://dx.doi.org/10.1787/eco_surveys-isr-2018-en 
most of Israel's potable water resources (surface water, groundwater, desalinated water) to the regional providers that supply end-users.

\section{Operation and planning}

Israel's natural water resources are not only scarce but, because of their variability, cannot reliably be taken into account in annual water balances. Therefore, reliability can only be increased by means of variability in tailor-made operational plans and in the variety of water resources. In Israel, the tools utilized to do this include use of aquifers as reservoirs and their recharge with treated wastewater during low-demand months, capture of occasional flash floods, and comprehensive monitoring and control of aquifer levels and abstraction.

One of the missions is to guarantee the sustainable exploitation of natural resources and to monitor and react to pollution. To achieve this, the Israeli Hydrological Service monitors water levels and water quality in all natural reservoirs.

The Israel Hydrological Service has developed a system of preparedness for the effects of climate change, and its forecasting system serves as a tool for decision makers. The flood forecasting system can predict major flood events in Israel both in time and space. Given a GIS database and hydrological measuring networks, the system can be operated anywhere in the world, and can serve as an efficient tool for long-term water and drainage planning and water balance simulations (runoff, infiltration, evaporation and recharge to aquifers). The model also operates as an app alert about floods in real time.

\section{Improving water quality and protecting and restoring water-related ecosystems}

Assuring the quality of water resources is a prerequisite for maintaining the water resources pillar. Therefore, pollution prevention measures, monitoring and remediation of polluted water bodies are routinely taken. One example is the Directive on Industrial Wastewater Standards that regulates a long list of components that are prohibited at a certain level. A very high tariff is charged for inadequate quality, making it unprofitable for industrial plants to pollute.

The water balance is managed by the Water Authority's Operations Department which is responsible for the management of Israel's natural water resources, in combination with alternative water sources such as desalinated seawater, brackish water and reclaimed water. The aim is to create optimal conditions for reliable, quantitative and qualitative water supply to all consumers and to conserve natural water sources. The Division is responsible for planning the operational policy of the water sector and implementing it by instructing the various water suppliers, such as Mekorot (Israel National Water Co.) and the desalination companies, and supervising the different divisions in the Water Authority. The policy proposal and operational details are presented and approved from time to time (at least every six months) within the framework of an Operating Committee headed by the Director General of the Water Authority.

The future of river rehabilitation in Israel largely depends on the continued removal of point sources of pollution and allocation of fresh water to sustain biodiversity, provide open space services to residents and tourists and allow for economic development along the river course. Master plans for river rehabilitation have been established for $80 \%$ of the perennial rivers in Israel. Between 1994 and 2018 the number of point sources of pollution decreased from 240 to 62 . The most recent assessment of pollution in Israel's major rivers reveals significant reductions in pollution loads between 1994 and 2017: a 95\% decrease in total organic carbon, a $75 \%$ decrease in total nitrogen and a 97\% decrease in total phosphorus. In 2018 a dedicated government decision was approved on river rehabilitation and the allocation of water for nature.

A National Center for Aquatic Ecology was founded in 2015 through a multi-stakeholder process. The center, established in Tel Aviv University in cooperation with the Ministry of Environmental Protection and the Nature and 


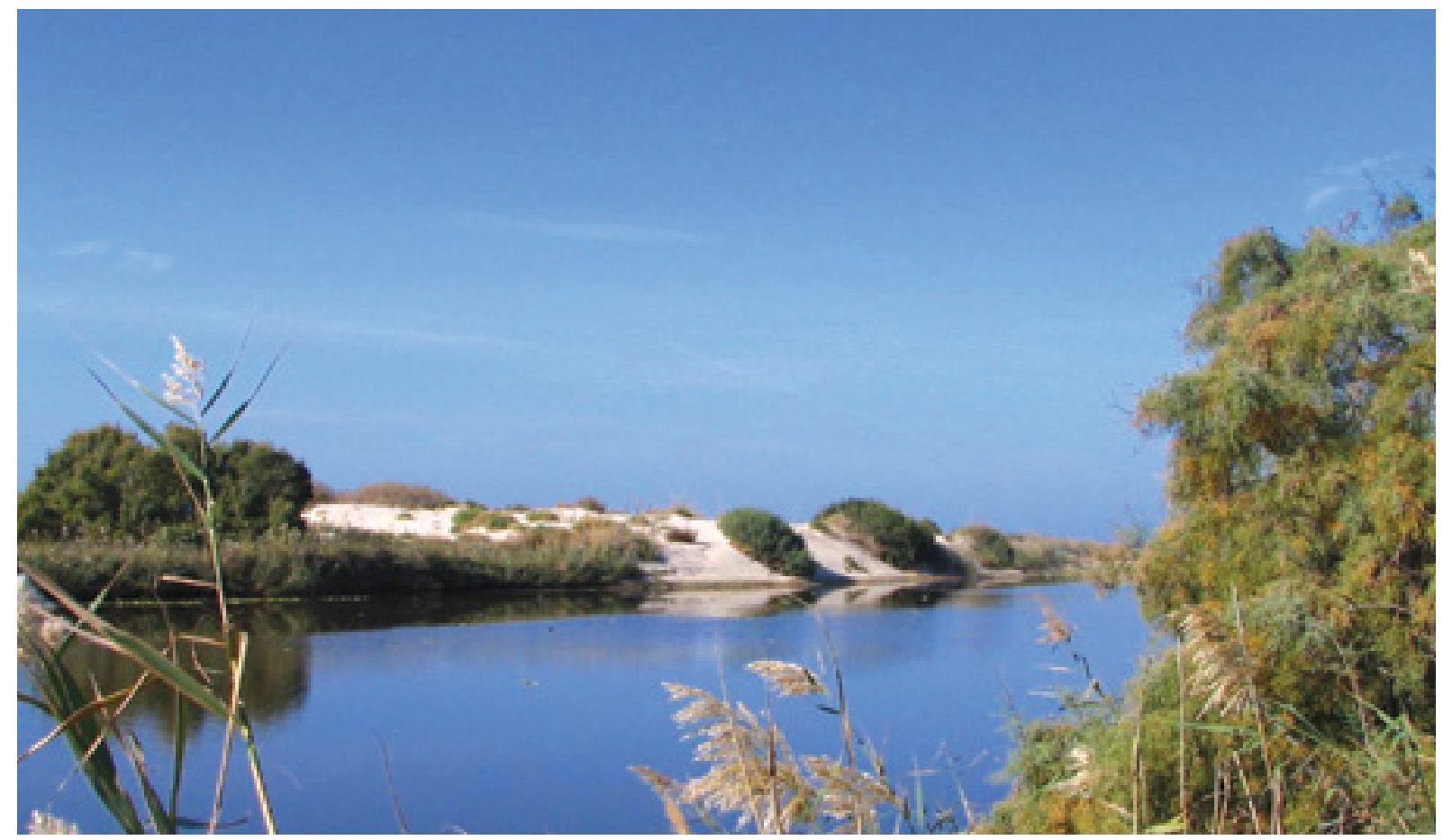

Alexander River/Photo: Ilan Malester

Parks Authority, monitors and provides ecological data on aquatic ecosystems in Israel. It aims to conduct hydrobiological monitoring of all aquatic ecosystems including rivers and wetlands by 2023 in order to assess their ecological functioning and improve the sustainable management of Israel's water bodies. Its approach is based on the Water Framework Directive (WFD) methodology.

In the last two decades, catchment basin management committees have been established and are run on a voluntary basis with a multistakeholder set-up and with the drainage and river authorities as the driving forces in this process. As a result, most of the major rivers have a rehabilitation master plan and actions are taken in accordance with it.

\section{( National Water Master Plan 2050 (SDG 6.1-6.6)}

The Policy Document for the Long-Term Master Plan for the National Water Sector was approved by the Water Authority Council in 2012. This paper defines the vision, goals and objectives of the national water sector and the policy of the sector on the major issues. The paper includes a description of the state of water reservoirs, an analysis of conditions of uncertainty, and initial recommendations for implementation. It also includes an estimate of the required development plan framework, mapping of managerial, budgetary and professional obstacles, and recommendations on how to overcome them. The policy document addresses the primary issues and components of the national water and sewage sector with regard to significant developments that have occurred in recent years.

The primary objective of the water sector in Israel, as approved in the master plan, is to ensure the supply of water, to provide sewage services and designate uses of treated wastewater, and to manage runoff and drainage - of suitable quality, quantity and reliability, and with economic efficiency, for the sustainable well-being of all consumers.

The main policy directives include:

- National goals: The water sector will continue to be a contributing factor for realizing Israel's national goals lagriculture, industry, settlement and development, inter-regional peace agreements, etc.). 
- Social fairness and support for the periphery: The water sector is obliged to exercise full equality in the provision of services to all consumers, aiming to achieve a high level of supply reliability in water and sewage services. The government should accommodate special consumers and water suppliers in accordance with their special conditions, such as helping disadvantaged groups and supporting projects in the periphery that require identification and use of special implementation and management mechanisms.

- Management of the water sector under conditions of uncertainty and water security:

Given Israel's geopolitical environment, planning considerations and management and operating mechanisms will take into account water security threats. The national water sector will prepare for extreme scenarios in all areas of its operation. Future planning will include scenarios for a decline in the supply of natural water and a decline in water quality, as well as possible changes on the consumption side. Preparation for extreme situations should be carried out both by increasing supply and by possible reductions in demand levels.

- Management of natural water sources: Natural sources of water will be rehabilitated and preserved as a strategic asset. As such, rehabilitation zones will be determined, and zones in which this will not be viable will be defined. Operating rules will be set for each of the natural water sources. Management of annual allocations and extraction from the renewable natural water sources will be sustainable. Excessive extraction and a drop below "red lines" will be avoided.

\section{- Management of sewage and treated wastewater:}

The sewage and treated wastewater management system should be structurally organized to ensure that reclamation systems are cost-based and meet national targets las decided by the government) and are efficient in the removal of treated wastewater. Aggressive action will be taken to connect as many sewage producers as possible to wastewater treatment plants, with clear prioritization of central plants. Construction and regulation of wastewater treatment plants will be based on the proven ability of their management and their technical and financial reliability. Treatment of wastewater and regional reclamation systems will be primarily based on criteria of reliability, efficiency and spatial fairness.

- Water quality: The importance of ensuring the quality of water and treated wastewater in all areas of the water sector is recognized. Decision making will take account of maintaining the quality of natural sources of water. Efforts should be made to ensure that natural sources of water used for drinking in the recent past will be restored to potable water quality.

- Consumption management (“Demand Management"): In the water sector, consumption management is of equal importance to supply management. Management of consumption will include setting annual per capita consumption targets and reduced agricultural consumption targets compared with previously accepted levels, as well as implementation of a range of activities and efficiency improvement methods for savings in all consumption sectors: technical, public relations, financial, and water loss reduction.

- Water and agriculture: Agriculture in Israel is socially and environmentally important in addition to its importance for rural communities. Its preservation is a national objective.

- Environment and water for nature: Nature is a recognized consumer under the Water Law since 2003 and is of no less importance than other consumption sectors. Water-dependent ecosystems will be rehabilitated and preserved. Water allocation for nature will be increased, primarily by means of rehabilitating natural water sources.

- Urban water system: Municipal water and sewage corporations should be strengthened professionally, as should their regulation. Water and sewage corporations will be established on the basis of their managerial, technical and financial ability. The number of municipal water and sewage corporations should be significantly reduced, based on engineering, geographic, financial and social considerations. Municipal water supply systems and measurement systems should be upgraded. 
Appropriate levels of service by the corporations to the consumers should be guaranteed.

- Regulation: The Water Authority is the main regulator responsible, among other duties, for the financial and professional supervision of the various water suppliers (Mekorot Ltd., water and sewage corporations, and others). The water sector will be administered through the internalization of costs in water tariffs. To achieve maximum efficiency, costs will be minimized in order to set the most reasonable water tariffs possible and tariffs will be earmarked for developing the water sector. Measures should be undertaken to reduce cross-subsidizing between the various sectors in determining water tariffs (Figure 6.4).

- Water and energy: The water sector is a relatively large consumer of national electricity laround $8 \%$ ) and, as such, coordination on planning the development of infrastructures between the water sector and energy sector will be increased. In addition, extensive steps will be taken for the purpose of improving energy efficiency in the water sector's operations.

\section{( Manufactured water resources (desalination and water reuse) (SDG 6.1-6.6)}

Scarcity of natural water resources was an impetus for Israel to seek sustainable solutions for manufactured water resources. Over the last 30 years, water supply from natural sources was cut by half, with the other half consisting of recycled treated effluents and desalinated seawater.

\section{Desalination}

Within one decade (2005-2015), Israel changed the structure of the entire water sector by establishing a water industry of seawater desalination plants with total production of about $600 \mathrm{MCM} /$ year. Five large-scale desalination plants were built along the Mediterranean coast and another two, with a

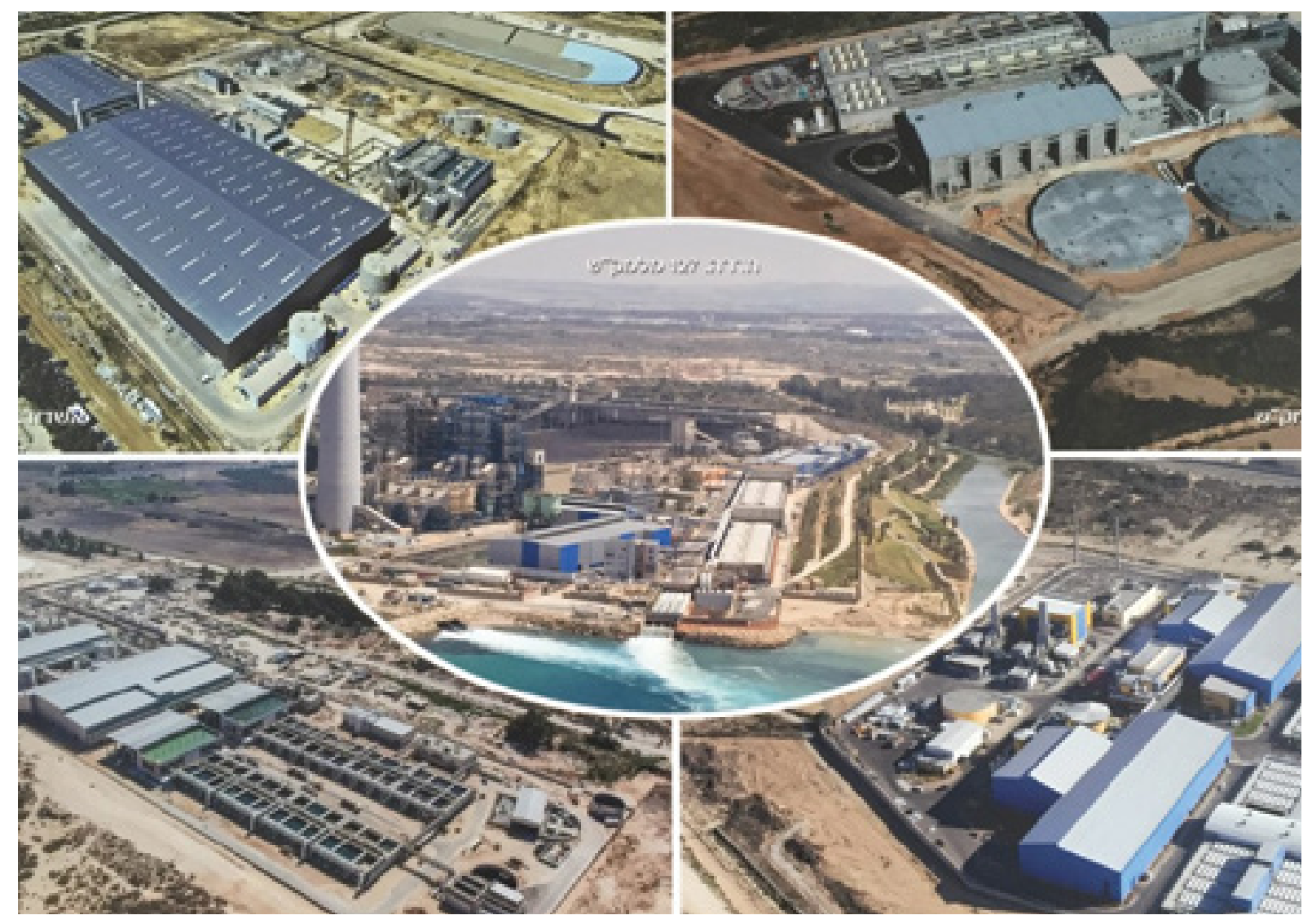

Five large seawater desalination plants in less than 15 years/Photo: Israel Water Authority 
Table 6.1

\section{Build Operate Transfer model for Israel's desalination plants}

\begin{tabular}{|c|c|c|c|c|c|c|}
\hline Project & $\begin{array}{l}\text { Year of } \\
\text { operation }\end{array}$ & $\begin{array}{c}\text { O\&M } \\
\text { duration }\end{array}$ & $\begin{array}{l}\text { Business } \\
\text { model }\end{array}$ & $\begin{array}{l}\text { Price per } \mathrm{m}^{3} \\
\qquad \$ 1\end{array}$ & $\begin{array}{l}\text { Production } \\
\text { capacity } \\
\text { (million } \mathrm{m}^{3} \text { ) }\end{array}$ & $\begin{array}{c}\text { Estimated capital } \\
\text { expenditure } \\
\text { (billion \$) }\end{array}$ \\
\hline Ashkelon & 2005 & 25 years & BOT & 0.78 & 119 & 0.27 \\
\hline Palmachim & 2007 & 25 years & $\mathrm{BOO}$ & 0.86 & 90 & 0.16 \\
\hline Hadera & 2010 & 25 years & BOT & 0.72 & 127 & 0.43 \\
\hline Sorek & 2013 & 25 years & BOT & 0.54 & 150 & $0.41-0.54$ \\
\hline Ashdod & 2016 & 25 years & BOT & 0.65 & 100 & $0.41-0.54$ \\
\hline
\end{tabular}

Source: World Bank report on Water Management in Israel, Aug. 2017

Marin, Philippe; Tal, Shimon; Yeres, Joshua; Ringskog, Klas B.. 2017. Water management in Israel: key innovations and lessons learned for water scarce countries (English). Washington, D.C. : World Bank Group. http://documents.worldbank.org/curated/ en/657531504204943236/Water-management-in-Israel-key-innovations-and-lessons-learned-for-water-scarce-countries

Figure 6.2

\section{Sewage collection, treatment and reuse}
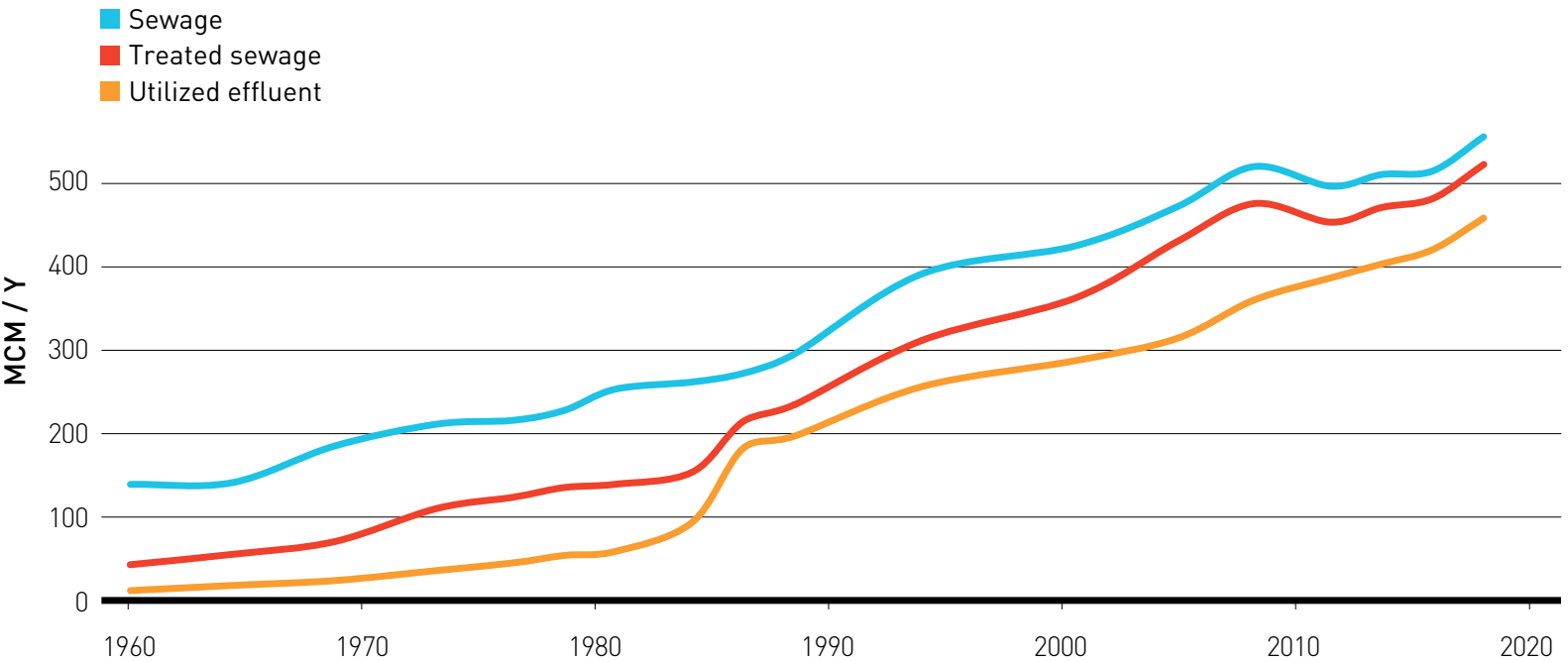

Source: Israel Water Authority

capacity of 300 MCM, are in planning. A multi-annual plan on seawater desalination has been approved to increase desalination in Israel to 1,000 MCM by 2030.

The establishment of such massive facilities required their connection to the national water system, shifting the direction of flow from the previous north to south to west to east. Today, Israel plans to supply water from these plants back to the Sea of Galilee.

Four of the five plants were built using a Build Operate Transfer business model, combining public and private partnership. This model allowed greater technology efficiency, resulting in a fair price per cubic meter purchased by the State.

Israel's desalination facilities are obliged to comply with the highest standards of the Ministry of Health's drinking water regulations. The desalinated water is integrated into the water of the national system, thereby improving the overall quality of the water that reaches consumers.

The benefits of integrating desalinated water in the water supply for various uses include: 


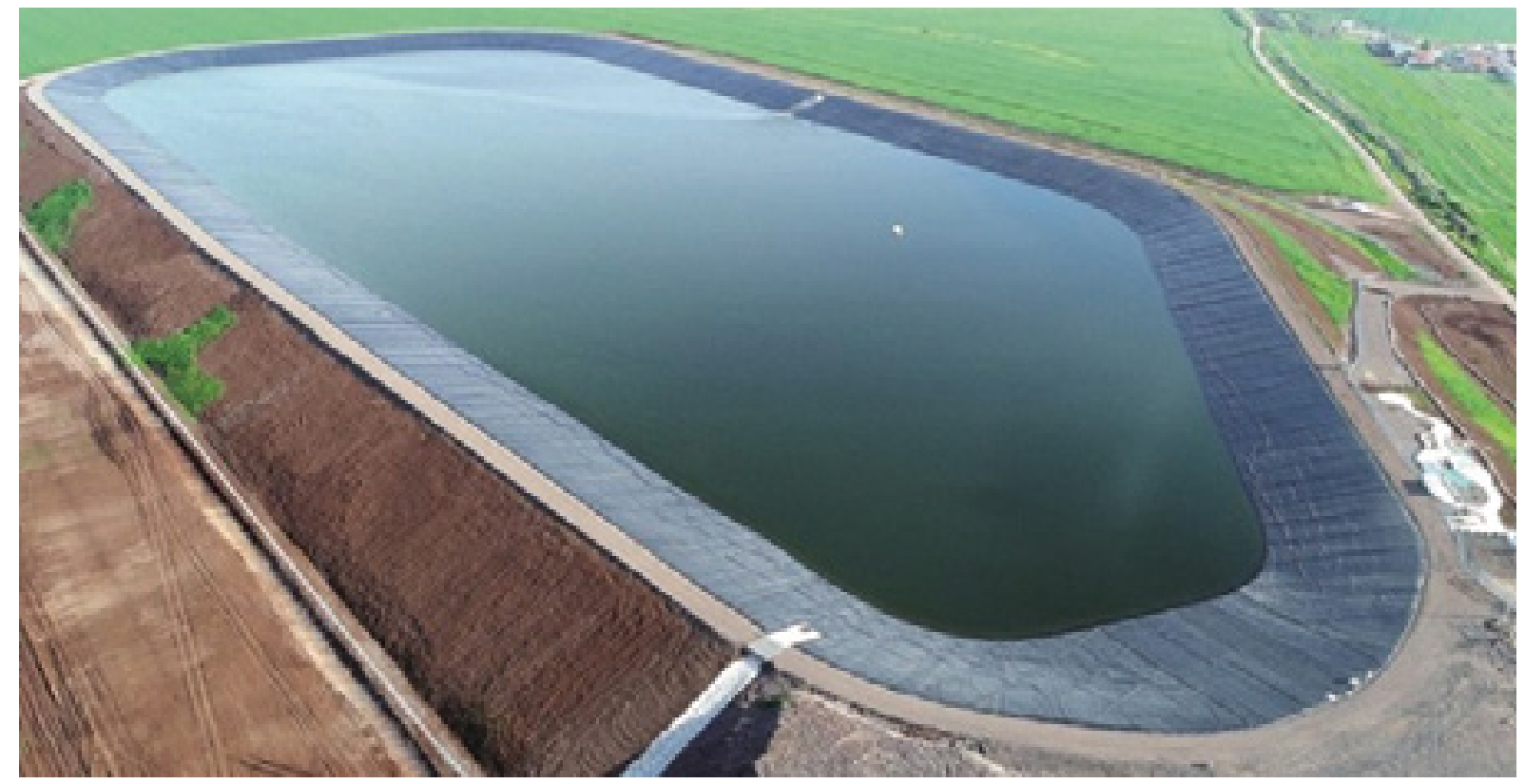

Moledet 2 Reservoir - The goal is to develop treated effluent reservoirs of 100 MCM in the coming decade/Photo: Harod Cooperative Water Association

Figure 6.3

National Water Supply and Consumption: Past to Future Increasing Use of Alternatives. Business as usual.
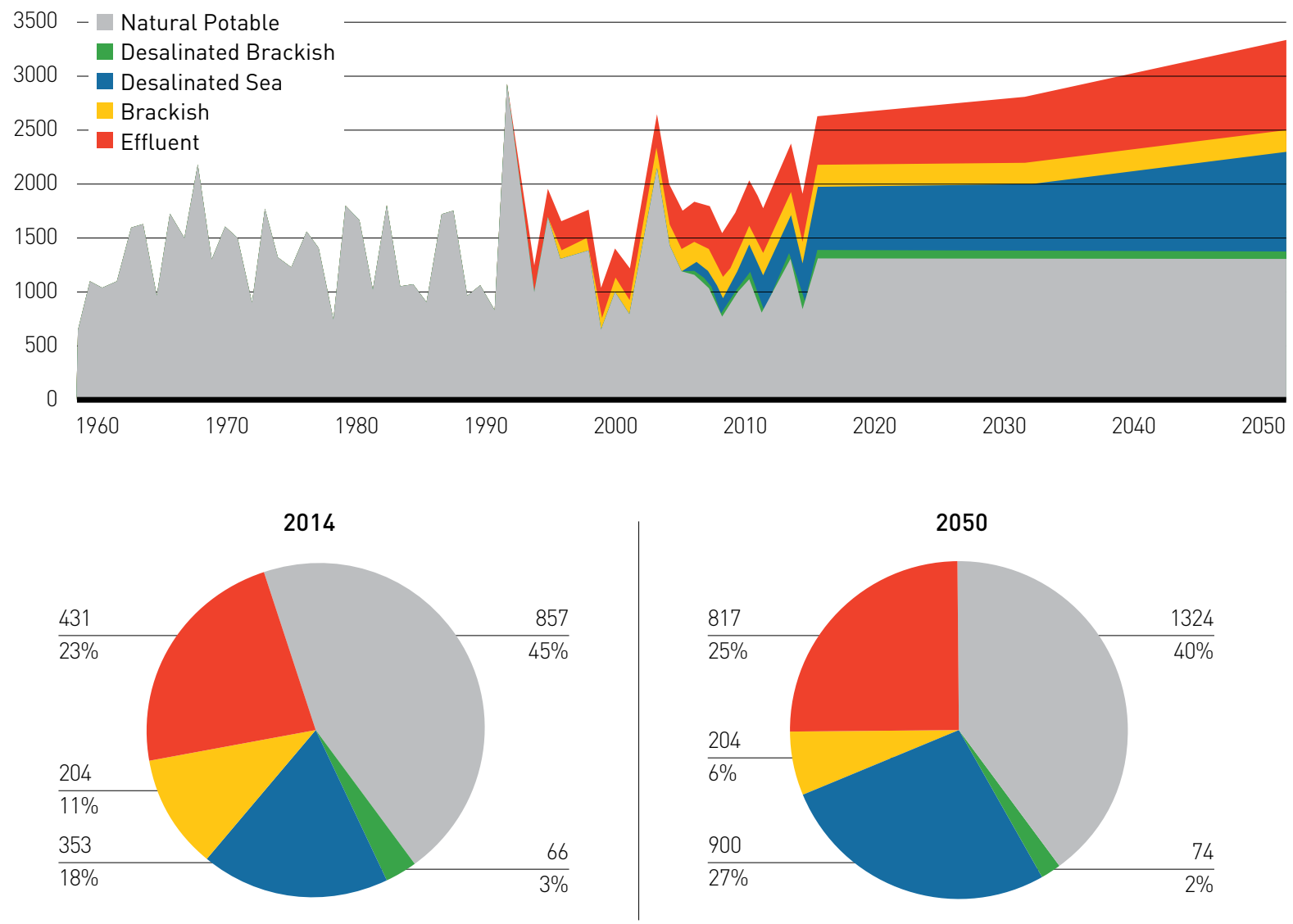

Source: Israel Water Authority 
- Creating a stable and reliable water source: By adding hundreds of millions of cubic meters of water a year that are independent of rainfall quantities and water conditions in the aquifers, the state of the water sector can be improved, thereby meeting the growth in demand and filling and rehabilitating the underground reservoirs by reduced pumping.

- Improving water quality: Continuous monitoring demonstrates a reduction in chloride and sodium concentrations which contributes to the maintenance of water quality. Furthermore, the addition of large quantities of water which is free of pollutants, organic, chemical and other substances results in significant improvements in the quality of water supplied.

- Improvement in the quality of agricultural effluents: The decrease in salt concentrations (mainly chlorides and sodium) leads to an improvement in the quality of irrigation water for agriculture and enables the use of effluents in a greater variety of agricultural crops. In addition, widespread use allows for the "release" of fresh water intended for agricultural use.

\section{- Reducing the use of detergents and energy} saving: Reducing water hardness makes it possible to use detergents for urban consumption (laundry, dishwashers) and for industrial consumption. In addition, the reduction in the hardness of the water leads to energy savings in heating boilers and industrial systems (reduced use of ion exchangers, sedimentation inhibitors, etc.).

\section{Wastewater treatment and reuse}

A main element of Israel's water sector is the collection of all sewage and its treatment to the required quality level so it may be used for irrigation and return to nature. Treatment and reuse exemplify the possibility of transforming wastewater from an environmental problem into a resource. Providing the recycled water to the farmer as a reliable source leaves more natural water in the environment and simultaneously helps cities to remove their sewage. Of the more than 500 MCM of raw sewage which is collected, 93\% is treated and $86 \%$ is reused. About half is treated to tertiary quality enabling unrestricted irrigation.

An assistance program for water reuse plants operated by the Israel Water Authority was initiated in 2000 following a government decision to increase the supply of water for various uses, inter alia by encouraging the establishment of water reclamation facilities for the utilization of treated wastewater and inferior water in private enterprises. Reuse of the treated effluents produced an additional source of water at relatively low cost, with the added advantage of preventing the negative consequences of effluent transfer into the environment. Treated effluents are used in agriculture, industry, public gardening and nature.

The Israel Water Authority accompanies the projects from initiation to construction and operates the financial assistance mechanism that bridges the

\section{The Governmental Authority for Water and Sewage - 2007}

In January 2007, by a legislative act, Israel's Water Commission was restructured as the Governmental Authority for Water and Sewage. The Water Authority is authorized to regulate all aspects relating to the water and sewage sector in Israel, including its management, planning, development, operation and regulation. Its main goal is to enable regular and reliable supply of water to all water consumers in the required quality and quantity, at reasonable prices, while preserving the country's water sources for future generations.

The activities of the Water Authority are anchored in two central laws: The Water Law, which regulates the water aspect in all its aspects, and the Water and Sewage Corporations Law, which regulates the activities of the municipal water and sewage sectors. 
Figure 6.4

Water Tariff Components per $\mathrm{m}^{3}$ - 2018 in USD\$ including VAT (17\%):

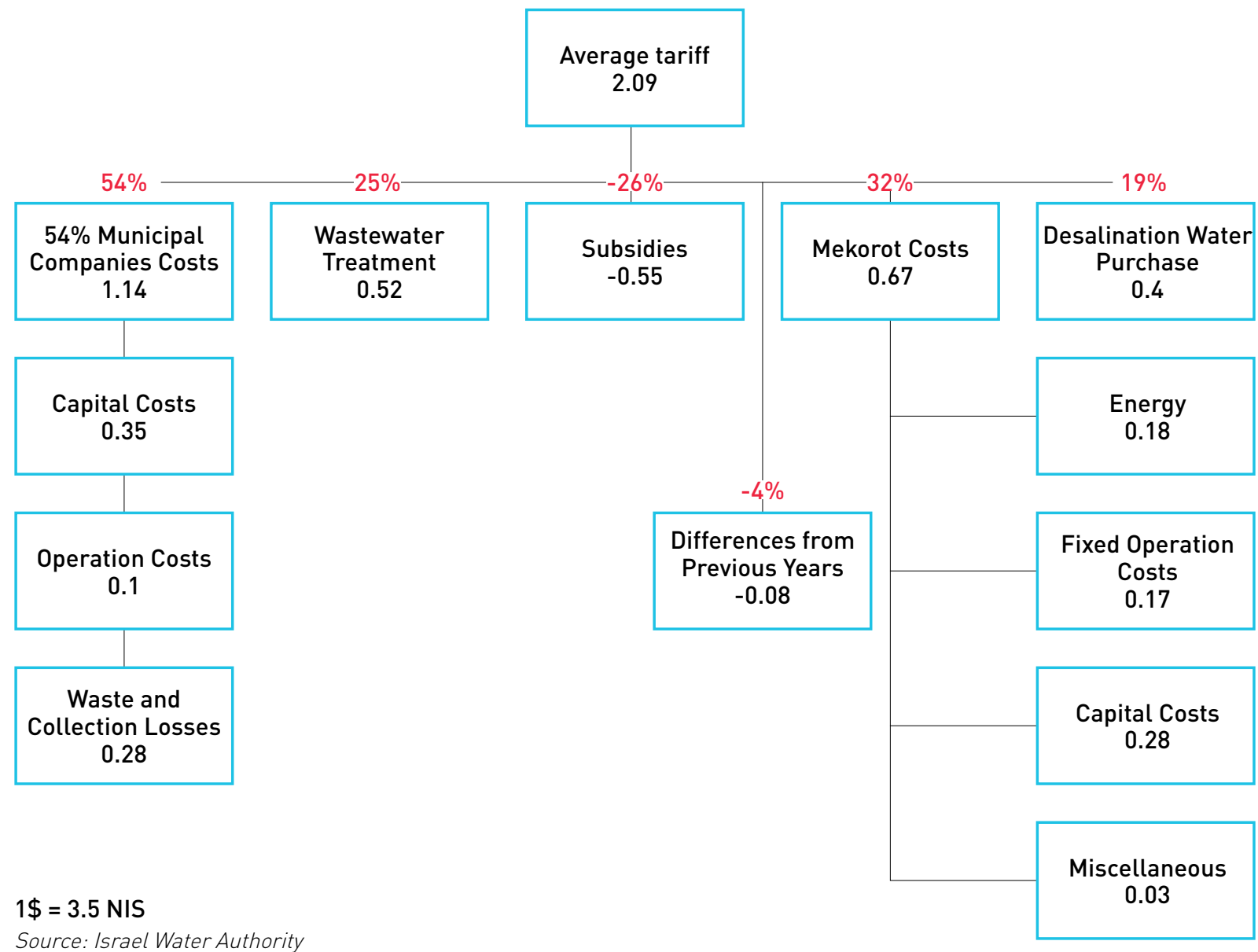

economic benefits of water utilization with the direct benefit of the water entrepreneur. The scope of assistance is determined in accordance with the cost of setting up and operating the plant and aims to reduce the variance in the rate of the different facilities. The average rate of assistance is about $67 \%$ of the total cost of construction.

Concurrent with the implementation of the conclusions of an inter-ministerial committee to examine the effluent sector and Amendment 27 to the Water Law, which came into force at the beginning of 2017, a multi-year budget agreement was signed between the Ministry of Finance and the Water Authority in the amount of NIS 1,400 million by 2022 (seven-year assistance plan).

\section{( Water governance - regulation and management (SDG 6.1, 6.2, 6.4, 6.5, 6b)}

The second pillar of the water sector is governance. It too rests on three main foundations: regulation system, sound financial model, and set of principles for service standards to the consumers.

The legislative basis allowing Israel to manage its water resources is laid in the first paragraph of the Water Law that was approved in 1959: "The water resources in the State are public property; they are subject to the control of the State and are destined for the requirements of its inhabitants and for the development of the country. For the purposes of this Law, 'water resources' means springs, streams, rivers, lakes and other currents and accumulations of water, whether above ground or underground, whether natural, regulated or 
Figure 6.5

\section{Water and sewage tariff, 2017}

Based on a domestic consumption of $15 \mathrm{~m}^{3}$ per month, in NIS adjusted for purchasing power 2015

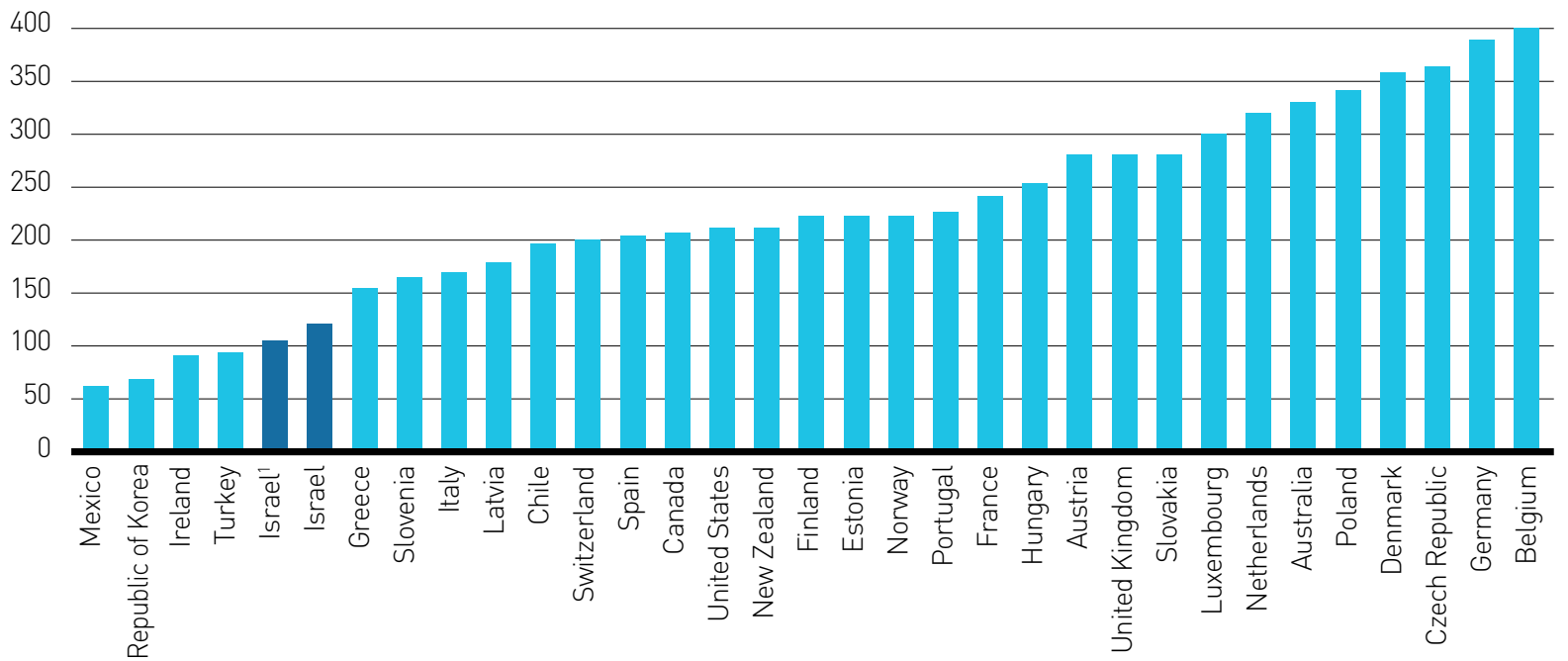

${ }^{1}$ After reduction of water tariffs by $14.5 \%$ for household consumption up to $3.5 \mathrm{~m}^{3}$ per person as of June 2017.

Excerpted from OECD Economic Survey, Israel 2018 OECD (2018), OECD Economic Surveys: Israel 2018, OECD Publishing, Paris. http://dx.doi.org/10.1787/eco_surveys-isr-2018-en

made, and whether water rises, flows or stands therein at all times or intermittently, and includes drainage water and sewage water."

Regulatory responsibility for Israel's water sector is divided among three main bodies: the Water Authority which is responsible for the management of the water and sewage sector, the Ministry of Health which is responsible for the quality of drinking water and for the grant of approvals and permits for effluent irrigation and the Ministry of Environmental Protection which is responsible for the prevention of water source pollution.

The accompanying principle is measurement. Every drop is measured and registered.

\section{Water economy}

A significant change in the tariff arena, instituted in 2010, prevents the water sector from dependence on the government budget. The two main principles of the new tariff system are:

- The tariffs cover all the required costs to develop and maintain the water sector;
- The tariff is identical for any domestic consumer, regardless of geographical location - whether city close to the water source or distant village at the edge of a desert.

The tariff system for the domestic consumer is a two-block tariff - the lower one allows reasonable use at an affordable price, while the higher is unlimited for the quantity used.

The breakdown of an average domestic tariff demonstrates the principle of covering all costs: the relative part of purchasing desalinated water, the services of the municipal companies, the national water company costs and wastewater treatment.

In May 2017, Amendment 27 to the Water Law came into force, and the mechanism of production levies was replaced by a water production tariff mechanism that transfers the revenues to the water sector (instead of to the State Treasury). This major reform advanced the water sector to the next step toward being a closed market and enabled a $14.5 \%$ reduction in the tariff for domestic use. 
Figure 6.6

\section{Domestic water consumption per capita/year, 1996-2014}

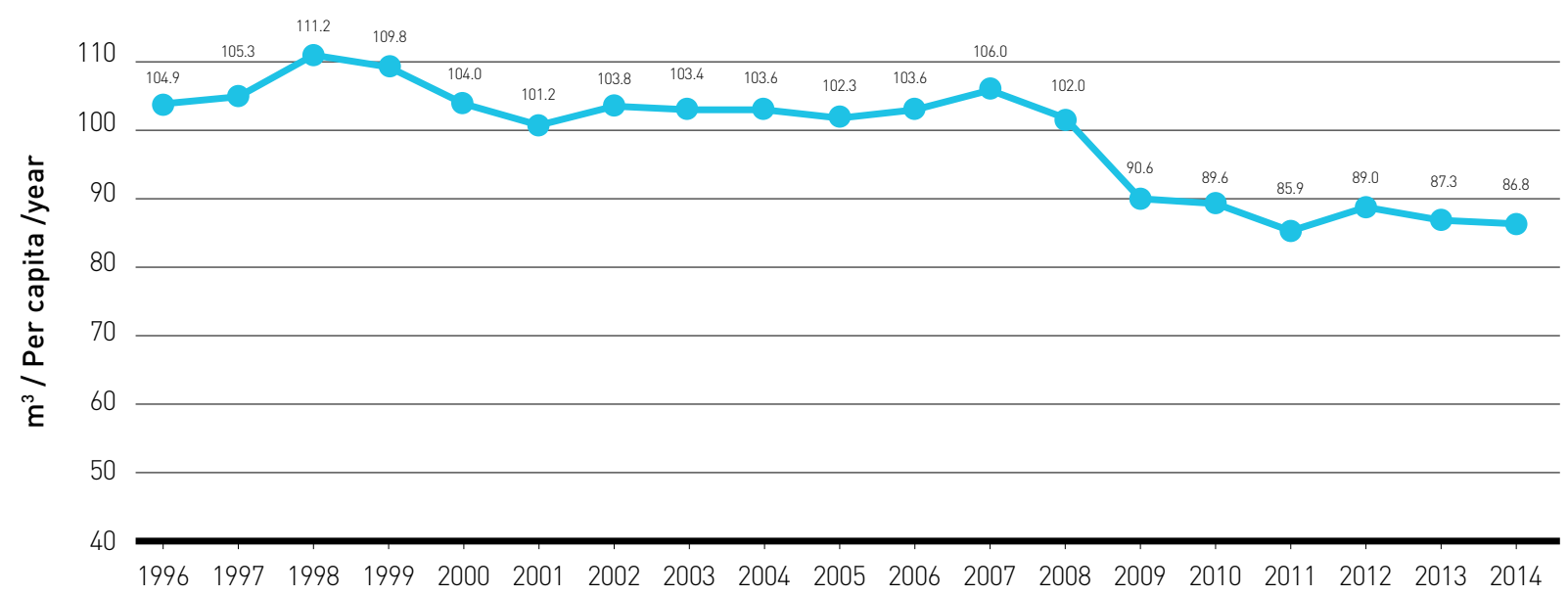

Source: Regulation Division, Israel Water Authority

The reform has also updated the tariff model set for the agricultural sector to a so-called "tariff bath," which sets the same tariff for the agricultural consumer, regardless of the source of water production. In order to facilitate adaptation to this model among farmers who experience a gradual increase in tariffs, discussions have been held between government representatives and farmers representatives regarding the provision of significant budgetary support that will facilitate adaptation.

Another major reform relates to the municipal sector. Previously, municipalities were responsible for managing the urban water sector but revenues from the water tariffs were not dedicated to the water sector of the city. The reform established municipal water companies owned by municipalities but separate in their managerial functions. The reform was instrumental in increasing the level of maintenance and required development of municipal water infrastructures, implementing efficient technologies for water saving and preventing water losses. Economic incentives have promoted efforts to stimulate water-saving innovations, especially by municipal water companies, in relation to combating leaks in the piping system. To this end, the systematic use of smart meters, which provide regular information on water consumption and thereby also warn of unexpected surges that may signal leaks, has been promoted.

\section{Providing support to less-privileged populations}

In April 2012, the Knesset Finance Committee decided to provide support to eligible populations by determining that every eligible person will benefit from double the amount of water at the low tariff Ifrom 3.5 cubic meters to 7 cubic meters per month per person). The Water Authority assisted in assimilating the procedure among the hundreds of water suppliers and in the interfaces with the relevant government bodies, enabling the support to begin in July 2012.

Currently, support is provided to about 570,000 people, with the average annual benefit to eligible individuals amounting to NIS 110 per person per year. To implement the benefit, an Internet system was developed that connects 1,000 water suppliers to the consumers entitled to the benefit based on a monthly update from the reporting entities (National Insurance Institute, Ministry of Defense, Rights for Holocaust Survivors). 


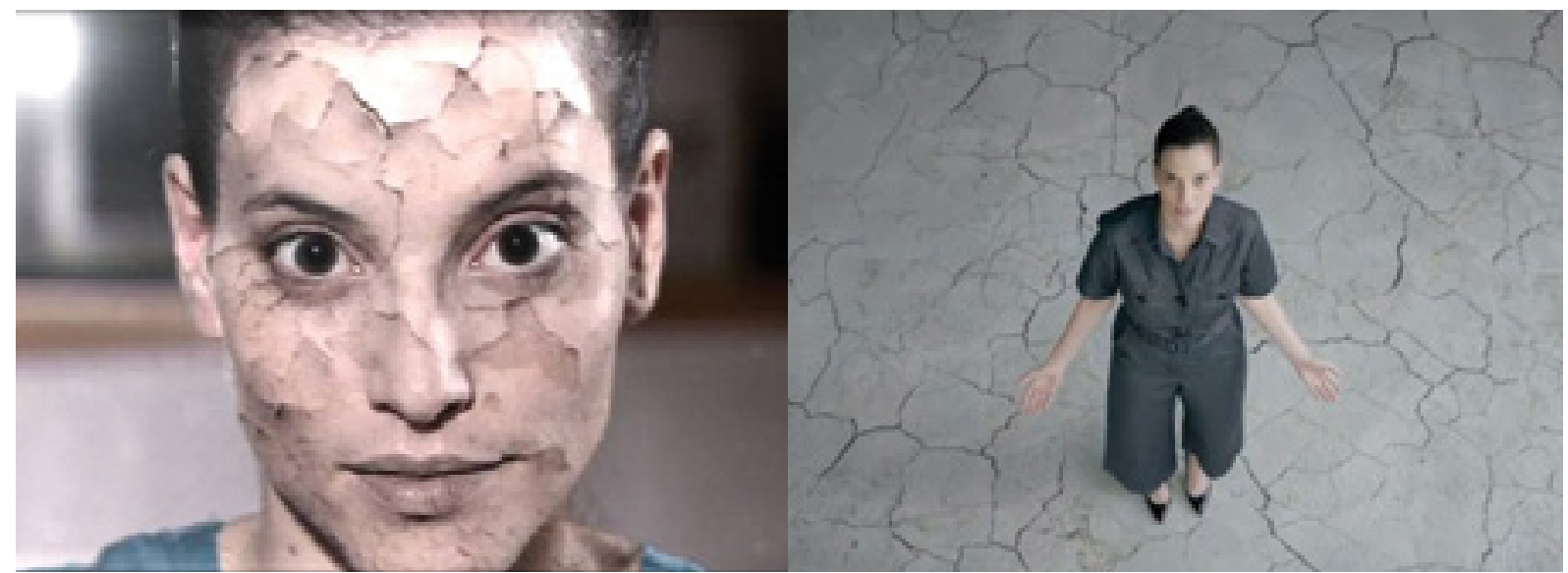

Source: Israel Water Authority media campaign 2008 and 2018

\section{Demand management}

Israel is known for its effective demand management to increase water productivity, reduce per capita potable water consumption, and shift water to higher-value irrigated crops.

The allocation of water for various purposes (agriculture, nature and public gardening) is strictly regulated by annual directives which constantly relate to the hydrological situation, such as continuous drought and severity of drought in previous years. The necessity to limit water use and to ensure procedures for water reservoir rehabilitation is considered. The need to conserve water sources and the needs of the various sectors are balanced constantly based on hydrological considerations.

To promote water conservation, the Water Authority launches media campaigns to encourage people to use water more sparingly. During recent years of drought, a comprehensive and successful nationwide media campaign was launched calling on citizens to save water. Combining regulations with educational and media campaigns, Israel has succeeded in stabilizing domestic consumption below the annual quantity of 90 cubic meters per capita. It "saved" Israel an investment in another large desalination plant.

\section{Water security and emergency preparedness}

The Israel Water Authority is authorized under the Water Law to deal with water security events, and to ensure water supply to consumers in routine and emergency situations. It is also responsible for the preparedness of water suppliers and the Water Authority itself for emergencies, including management of water damage incidents and water crises, while focusing on scenarios of threat to the operation of water supply systems, such as natural disasters learthquakes/tsunamis, extreme weather events and floods); conventional/non-conventional war/terrorism and cyber terrorism; and system malfunctions and human problems. 


\section{(Research and technologies}

(SDG 6.1, 6.2, 6.5)

\section{Development of water technologies}

In view of the water crisis, in 2006, the Israeli government drew up the National Plan for the Promotion of Water and Energy Technologies (Israel NewTech), entitled One Objective Extensive Partnership. The program brings together ministries, regulatory agencies, academia, and industry to encourage and develop Israeli water industries and renewable energy industries. Activity is undertaken on a number of levels to achieve the plan's objectives: development of human capital and recruitment of the next generation, encouragement of $R \& D$ in close cooperation with industry, promotion of designated foreign investment to the sectors, international cooperation, implementation of innovative Israeli technologies in the domestic market and assisting Israeli companies break into the global market, allocation of funds for reciprocal procurements, water security standardization, and more.

\section{Community and education}

The Israel Water Authority attributes major importance to research and development in Israel's water sector. The research activity is carried out by means of "Calls for Research and Surveys," and the studies and surveys are examined and accompanied by professional committees. Results are transferred to research institutions and researchers and are published on the Water Authority's website. Scholarships are also awarded to outstanding graduate students to encourage the training of professional personnel in water-related fields. Furthermore, the Water Authority initiates seminars in which researchers and students present their findings to a wide forum of water professionals.

A government program includes curricula for water subjects in junior high schools and high schools, scholarships for graduate and doctoral students, research projects, commercial projects using innovative technologies, and a biennial international event including an exhibition, professional conference and side events. The Water Authority operates a central component of the program - carrying out commercial-scale projects of technologies that have completed the research and development stage (alpha phase) and are ready for implementation on a large scale and under real-life conditions.

The Ministry of Education brings together government, academia, schools and community by creating educational projects such as Hands Across Water with the participation of 3,300 students from some 100 schools in different communities, establishment and expansion of water technology education programs at several colleges and universities, allocating approximately NIS 17 million for some 50 water studies and NIS 5.2 million for 66 scholarships for advanced degrees in water subjects.

\section{PARTNERING FOR A BETTER WORLD}

Israel is among the leading countries today in building a sustainable water sector, spearheading efforts to deal with water leakage, farming efficiency, wastewater recycling, desalination, pricing policy and awareness through education. The Israeli model is based on a holistic philosophy which creates synergies between different water users - urban, industrial, agricultural and environmental. It also adopts a clear policy: every drop of water is measured and everyone

pays for the use of water. The water sector is self-sufficient economically, and water tariffs fund the municipal water infrastructure run by water corporations to ensure proper levels of service, quality and reliability.

Israel's international development cooperation efforts in water related fields are directed at working with partners on the international, regional, national and community levels to establish water- 
responsible and inclusive policies, best practices in water management, and optimal use of watersaving technologies. In order to help achieve Goal 6, Israel's Ministry of Foreign Affairs appointed a Special Envoy for Water and Food Security to partner in efforts to afford access and availability of water to vulnerable communities, especially in rural areas, integrating a gender-based perspective. Israel recognizes the need to change current approaches to overcome the many challenges and consequences of water poverty - economic, physical, social and environmental - especially those effecting the extreme poor. (SDG 6.b)

The Israel Water Authority is a member of the Sustainable Water Integrated Management (SWIM), a regional technical assistance program launched by the European Commission to contribute to the extensive dissemination and effective implementation of sustainable water management policies and practices in the Southern Mediterranean region. The program addresses such issues as increasing water scarcity, combined pressures on water resources from a wide range of users, desertification processes, and climate change. (SDG 6.4)

Israel is also a member of the Mediterranean Desalination Research Center (MEDRC), an international organization mandated to find solutions to fresh water scarcity. Established in 1996 as part of the Middle East Peace Process, it conducts research, training, development cooperation and transboundary water projects. (SDG 6.5)

The Israel Water Authority is an active member of the Water Experts Group of the Union for the Mediterranean (UfM Water), acting to promote integrated water resources management, implementing the Water Agenda, and advancing cooperation with member countries on mutual challenges in the water sector. (SDG 6.5)

Israel is a member of the Executive Action Team (EXACT) - multilateral working groups, formed (1992) to advance the Middle East Peace Process on water related issues. The Multilateral Working Group on Water Resources endorsed the Water Data Banks Project (1994), consisting of a series of specific actions to be taken by Israel, Jordan, and the Palestinian Authority to foster the adoption of standardized data collection and storage techniques. Furthermore, it aims at improving the quality of water resource data collection and communication among the regional scientific community. (SDG 6.5)

Israel is a Global Partner in the 2030 Water Resources Group (2030 WRG) and supports its vision of achieving universal water security. It recognizes the importance of cross-sectoral efforts to facilitate collective action between government, the private sector, and civil society. Towards this end, the Ministry of Economy and Industry is financing 2030 WRG programs and projects in Africa to improve efficiency in the management and use of water resources. (SDG 6.a)

The Ministry of Economy and Industry's National Energy and Water Program (NewTech) was established (2007) given Israel's advances in the water and renewable energy sectors and the desire to play an important part in the "next generation oasis" for the world's rising water and energy needs. NewTech brings stakeholders Istartups, entrepreneurs, corporations, multinationals, academia, regulators, policymakers and investors) together to deliver smart solutions to global challenges in the cleantech arena. The NewTech team serves as a national hub for the water ecosystem, building relationships with service companies, global clients and investors. Israel NewTech advances the water and renewable energy sectors by supporting academia and research, encouraging implementation in the local market, and helping Israeli companies succeed on the international scene. (SDG 6.a, 7.a)

Furthermore, the Ministry of Economy and Industry is funding the World Bank Group's Water Global Practice to assist developing and least developed countries to overcome complex water security challenges. (SDG 6.a)

In cooperation with the Inter-American Development Bank (IADB), a foundation was established to provide technical assistance to countries in Latin America including financing pilot projects in 
cooperation with municipal water corporations to improve efficiency in the management and use of water resources. (SDG 6.a)

In accordance with a Government Decision (2783) on Strengthening Ties with the Republic of India, two major water agreements were signed between Israel and India during the visit of India's Prime Minister to Israel (2017) on: increasing cooperation in water conservation, including an awareness-raising campaign in India on water saving techniques; and engaging in state water utility reform in India. Israel has contributed approximately $\$ 1.2$ million to these initiatives. (SDG 6.a)

To increase Israel's international cooperation and capacity-building support to developing countries in water related activities and programs, the Standards Institution of Israel (SII) is continuing its steadfast collaboration with the International Organization for Standardization (ISO). One joint activity includes the International Workshop Agreement on Drip Irrigation held in Stockholm (2017). Representatives from developing countries attending the Workshop highlighted the effects of climate change on water sources, particularly in relation to irrigated crops. SII contributed to the IWA 20:2017 standard that reviews drip irrigation in comparison to major irrigation methods available and practiced today by farmers worldwide. It lists the benefits of drip irrigation, such as increased yield, reduced water consumption, reduced energy consumption, lower environmental impact, reduced contamination of groundwater and surface water, reduced greenhouse gas emissions and reduced labor, and suggests a roadmap for future (now in development) standardization on pressurized irrigation systems. (SDG 6.a, 6.b)

The international water related standardization program in the SII is part of Israel's NewTech initiative. Using the platform of international standardization, the NewTech program shares Israeli knowledge and experience to deal with water scarcity. Israel is an active member in the ISO/ Technical Management Board International Task Force on Water (ITFWA), and plays an important role in the writing of many international standards to advance sustainable water management. (SDG 6.1) Examples of contributions made by SII include:

- ISO 24518:2017 is a management standard dealing with an interruption in the normal supply of drinking water and the collection/treatment of wastewater in emergencies. ISO 24520:2017 provides good practice for the technical aspects of crisis management, and ISO 24527/DIS suggests a specific guideline for alternative drinking water service provision during a crisis; (SDG 6.1)

- ISO/TS 24522:2019 provides guidance for water utilities on the detection and classification of water and wastewater events. It deals with all aspects of drinking water and wastewater systems, as well as all causes of abnormal changes in drinking water and/or wastewater service provision capable of detection by monitoring systems, including accidents, unexpected changes, natural hazards and intentional disruption. It does so by providing a guideline on the development of an Event Detection Process; (SDG 6.1).

- ISO/WD 24528 deals with water loss investigation and establishes general principles for water loss management in drinking water distribution networks to improve the sustainability of drinking water utilities and protection of the environment. Caused by increasing urbanization, growing demand, rising costs and ageing distribution networks, water loss is a growing challenge for drinking water utilities. (SDG 6.1)

Within the framework of the ISO, SII led the development of the following international standards dealing with the reuse of treated wastewater and related sludge products for agricultural and industrial purposes, thereby helping to turn waste into a resource and increasing recycling and safe reuse globally: (SDG 6.3, 6.4)

- To increase recycling and safe reuse globally, alternative water resources must be well managed. In regions where water is scarce, treated wastewater can be an ideal resource to replace freshwater use in agriculture, providing a new and vital source of water. ISO 16075 Parts 1-4:2015 can help key players in the field of irrigation maximize the benefits of treated wastewater and reduce 


\section{CASE STUDY}

\section{Environmental and Water Resources Protection of Lake Victoria in Kenya}

Within the framework of trilateral cooperation between Israel, Kenya and Germany, MASHAV, Kenya's Water Resources Management Authority (WRMA), and the Deutsche Gesellschaft für Internationale Zusammenarbeit (GIZ) are carrying out a project aimed at pollution reduction and protection of Lake Victoria and its water resources. The project is carried out in alignment with the 2030 Agenda and aims at reducing the uncontrolled inflow of untreated urban and industrial water into streams and open waters at Lake Victoria, by strengthening the capacities of WRMA staff in water quality monitoring and enforcement.

Implementation of the project, based on the introduction of best Israeli practices and innovative techniques on sustainable water resources management, water and wastewater treatment and water quality conservation, includes:

- Professional consultancy by Israeli experts

- On-the-spot training in Kenya

- Tailor-made study tours in Israel

- Transfer of Israeli experience on the implementation of advanced technologies for water and wastewater treatment

- Strengthening laboratory operations and staff skills

- Capacity building on water quality monitoring, pollution control methodologies, wastewater and water treatment technologies

- Design of monitoring systems for field and laboratory water quality analysis

- Onsite training for water quality sampling using new analytical methods both in the field and in the laboratory

- Building a stakeholder network

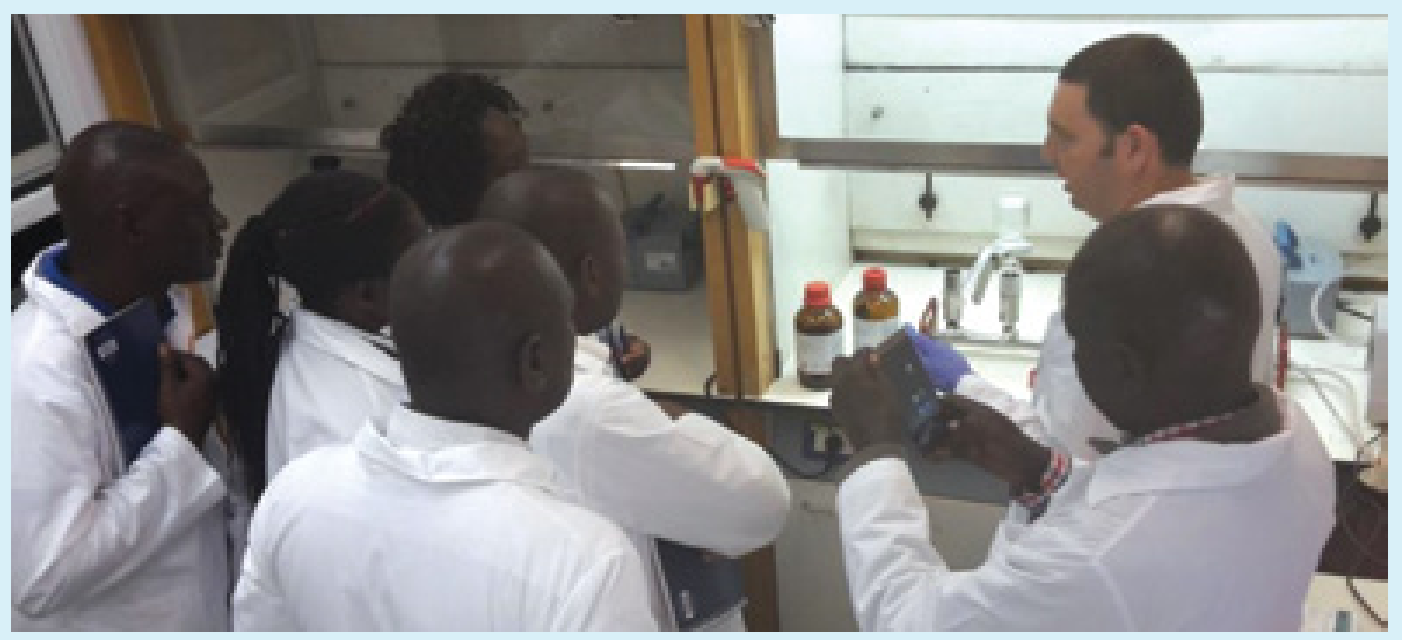

Photo: Mashav 
any related risks to their agricultural irrigation systems. Treated wastewater can be used for land reclamation, improving agricultural growth and reducing fertilization costs. An environmentally productive use of treated wastewater can also prevent ecological damage to water sources. Other than agriculture, treated wastewater can be used for purposes such as urban and industry reuse; (SDG 6.3, 6.4)

- ISO 20419 and ISO 22238 correspond with, and complete, the series of ISO 16075 to increase recycling and safe reuse globally. ISO 20419:2018 deals with the adaptation of irrigation systems and practices to treated wastewater by providing guidelines on how to protect and use irrigation equipment to guarantee water systems functionality at high levels of efficiency, and guides planners and practitioners on how to adjust irrigation equipment. ISO/WD 22238 aims at reducing, or eliminating, major health risks to the operators of wastewater treatment plants, and to anyone coming into contact with treated wastewater or crops irrigated with treated wastewater. This document is a guide to the available methods of disinfection, their effectiveness, impact factors, advantages and disadvantages regarding technical and environmental aspects and effective inactivation or removal of various pathogens in treated wastewater for use in irrigation. (SDG 6.3)

- Industrial activities greatly affect the availability and quality of water discharged into the environment. ISO/CD 22524 is a pilot plan for industrial wastewater treatment facilities. Its purpose is to set a clear guideline to the planning of an industrial wastewater plant pilot for optimal performance procedures to allow the reuse of wastewater within a factory or for exterior uses. (SDG 6.3)

- Biological wastewater treatment is a major process step in many cases of industrial wastewater treatment for reuse. The purpose of ISO/DIS 21939-1 is to create a quantitative measure for universal characterization of the energy consumption for aerobic biological wastewater treatment systems. The need arises especially in consideration of the life cycle cost of a wastewater treatment system. (SDG 6.3)

- As part of the effort to reduce pollution, eliminate dumping, and minimize release of hazardous chemicals and materials, the use of biosolids - organic based materials from industrial or municipal wastewater - as an alternative resource also needs to be considered. ISO/DIS 16968 provides guidance on the beneficial use of biosolids in the production of food and feed crops, as well as energy and forestry crops and for the remediation of disturbed sites. It includes specific guidelines for the use of biosolids for agricultural land application. (SDG 6.3)

- ISO/FDIS 22519 deals with clean and environmentally sound technology system for purified water and water for injection pretreatment and production systems for the biopharma market, while minimizing effluent, spare parts, chemicals, organic media, and regenerations. Systems complying with this standard will have minimum bacteriological buildup and will require drastically less sanitization with significant reduction in usage and release of harsh and hazardous chemicals. (SDG 6.4)

To promote effective public, private and civil society partnerships, Israel hosts the biennial International Conference and Exhibition WATEC, presenting newly developed technologies in the water and environment sectors. Following are three side events which were held within the framework of WATEC 2017:

- Israel-Africa Water Cooperation Event, in the presence of ministers from African countries and the Minister of Agriculture and Environment representing ECOWAS. High-level officials from the World Health Organization (WHO) and the UN Industrial Development Organization (UNIDO) were among the main speakers, with representation from the UN International Children's Fund (UNICEF); (SDG 6.5)

- The Second Meeting of the Roundtable on Financing Water, a global public-private platform established by the Organization for Economic Cooperation and Development (OECD), the World Water Council, and the Netherlands to facilitate increased financing of investment that contribute to water security and sustainable growth. The Meeting focused on innovation 
and provided the opportunity to reiterate the relevance of the work of the Roundtable to the High-Level Panel on Water. The Meeting highlighted how investment in water can contribute to achieving the SDGs, and ways to expedite investments that contribute to water security and sustainable growth; (SDG 6.1, 17.17)

- An International Water Regulators' Workshop for professionals from developed and developing countries. The event brought together 43 water regulators and professionals related to water regulation (resource management and governance) from 15 countries. (SDG 6.1, 17.17)

The MASHAV - Germany (BMZ/GIZ) - Kenya Trilateral Cooperation in Environmental and Water Resources Protection of Lake Victoria Project, launched in the framework of the German-Israeli Africa Initiative, aims at reducing pollution and the inflow of untreated urban and industrial wastewater into the streams and open waters of Lake Victoria by strengthening the capacities of the Kenyan Water Resources Management. Project activities included onsite training courses and the dispatch of Israeli experts to Kenya, as well as study tours and training courses in Israel. (SDG 6.6, 6.a) Though phased out in 2016, MASHAV, Germany (BMZ/GIZ) and Kenya conducted a joint project to improve the farmed tilapia value chain by reducing the overuse and environmental degradation of Lake Victoria and creating alternative livelihoods for the rural communities around the Lake. (SDG 6.b)

A sustainable urban economy also depends on its water quality, reliability and cost of supply. Innovating in urban water management can be technical (ICT and water infrastructure) and non-technical (management and planning approaches). Innovative water management combines technologies and business models for service providers and innovative governance. MASHAV's program on Urban Water Technologies and Management empowers professionals from developing countries to formulate a comprehensive approach to urban water management. (SDG 6.a) In MASHAV's annual work program, almost 500 professionals from developing and least developed countries participate in the many and varied capacity- building and training activities in water related disciplines. Course topics include: Municipal Platforms for Local Strategic Development; Climate Change and Agriculture; Economic Empowerment of Women; Environmental Management of Ecosystems; Combating Desertification and Drought; R\&D in Crop Production Under Saline Stress; and River Rehabilitation. (SDG 6.a) Israel initiated a special event at the UN in New York commemorating UN Water Day 2018, including a panel discussion during which an Israeli scientist from the Agricultural Research Organization/ Volcani Center addressed the issue of irrigation in arid areas.

The Center for Global Engineering and the Engineers Without Borders (EWB) chapter in Israel's Institute of Technology (Technion) were established to actively promote social responsibility among students, equipping them with the skills to meet complex local and global challenges facing developing communities. One of the technological projects initiated is in the village of Meskelle Kristos, Ethiopia, where the building of a water tank near the local school enabled the use of runoff water for drinking purposes and irrigation, as well as an opportunity to work together with the local population on using fresh water for hygiene, sanitation and a healthier environment. EWB developed a system for collecting runoff water and a method for testing its quality for both drinking and agricultural purposes. (SDG 6.a, 6.b) 
AFFORDABLE AND CLEAN ENERGY

先:
Universal access to affordable, reliable and modern energy services Transition to renewable energy Promotion of energy efficiency Partnering for a Better World 


\section{ENSURE ACCESS TO AFFORDABLE, RELIABLE, SUSTAINABLE AND MODERN ENERGY FOR ALL}

Israel's primary energy policy and future targets are articulated in a number of key policy documents: Israel's Intended Nationally Determined Contributions (INDC), the National Plan for Implementation of the Greenhouse Gas Emissions Reduction Targets and for Energy Efficiency, the Ministry of Energy's Objectives Plan for 2030 and the National Master Plan for the Energy Economy. The implementation of these main planning policies in the electricity generation, transportation and industrial sectors aims at integrating renewable energy in the energy supply mix and reducing the use of polluting fossil fuels, specifically by phasing out the use of coal and petroleum distillate fuels, while maintaining the reliability and continuity of energy supply notably by increasing natural gas in the energy mix. Additional measures and policies, such as the National Plan to Reduce Air Pollution, are currently under assessment. 


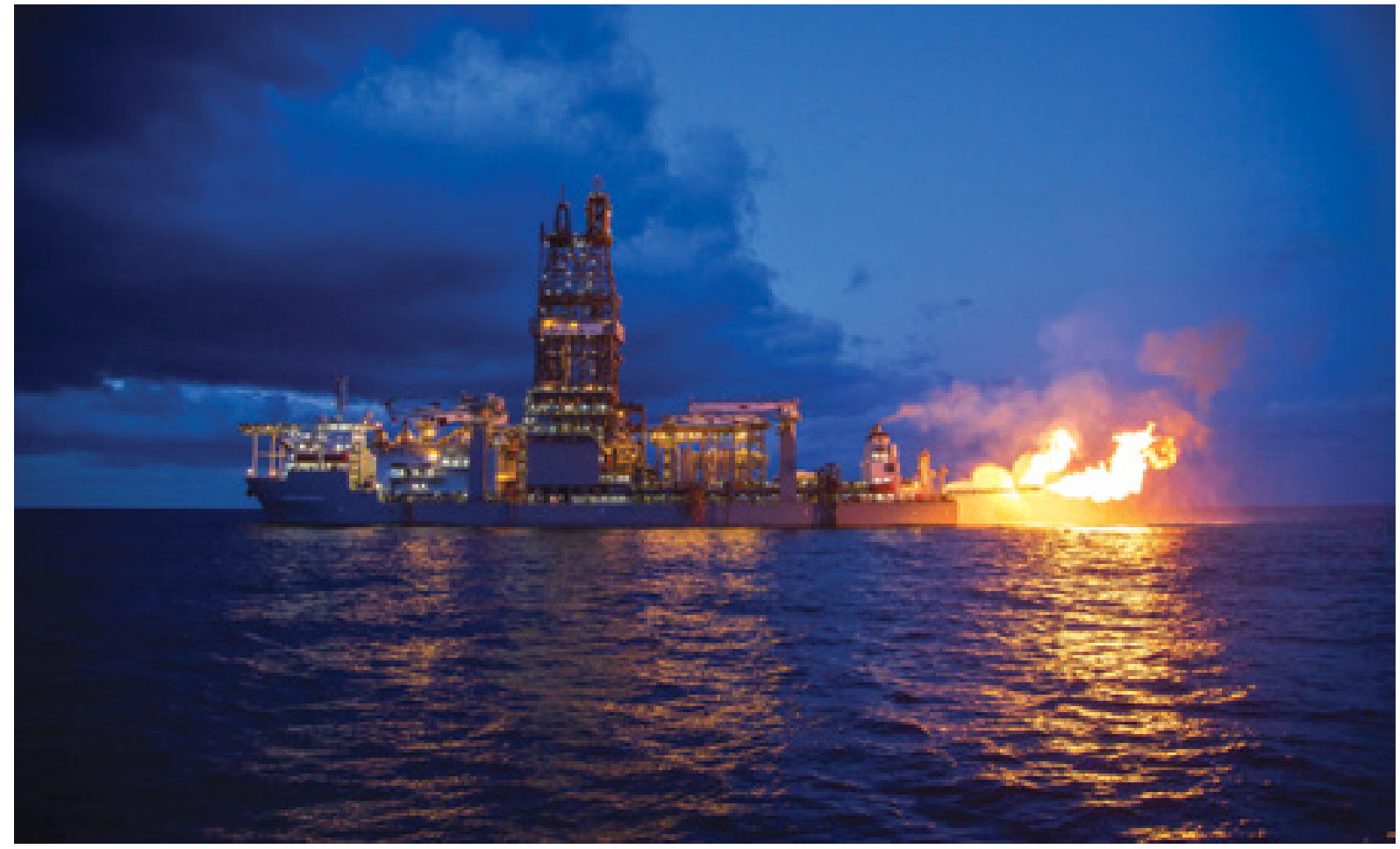

Natural gas barge/Photo: Yevgeny Malkin

\section{( Universal access to affordable, reliable and modern energy services (SDG 7.1)}

\section{Access to electricity}

In Israel, access to electricity is not a primary concern since almost $100 \%$ of the population has access to electricity, and the price of electricity for the country's households is significantly lower than the average for European households, with a price in the first half of 2018 of approximately 0.12 euros/kWh (NIS $0.54 / \mathrm{kWh}$ ) in Israel, compared to 0.20 euros $/ \mathrm{kWh}$ in the European Union (EU-2).

However, the lowest-income population ${ }^{1}$ spends on average $9 \%$ of their total gross money income on electricity, gas and fuel for dwelling, in comparison to an average of $3.4 \%$ spent for the medium-income ${ }^{2}$ population.

\section{Transitioning to cleaner fuels and technologies}

In Israel, electricity accounts for approximately $33 \%$ of final energy consumption, and petroleumbased fuels, other than for electricity, account for approximately $58 \%$. Within petroleum-based fuels consumption, approximately $70 \%$ is consumed by the transport sector, while $20 \%$ is consumed by the industrial sector ${ }^{3}$. Electricity demand is expected to increase by $2.66 \%$ until 2030 .

In the upcoming years, the discovery of offshore natural gas reserves and the completion of the infrastructure of three natural gas fields and their connection to land (slated for 2022) will have major ramifications for the Israeli market, in particular its energy generation mix, allowing the transition towards cleaner fuels and the reduction of the country's dependence on imported fuels.

1 First decile, Monthly income and consumption expenditure in deciles of households, by gross money income per household, ICBS, 2016.

2 Fifth decile, ICBS, 2016.

3 Energy Balance, ICBS data, 2016. 


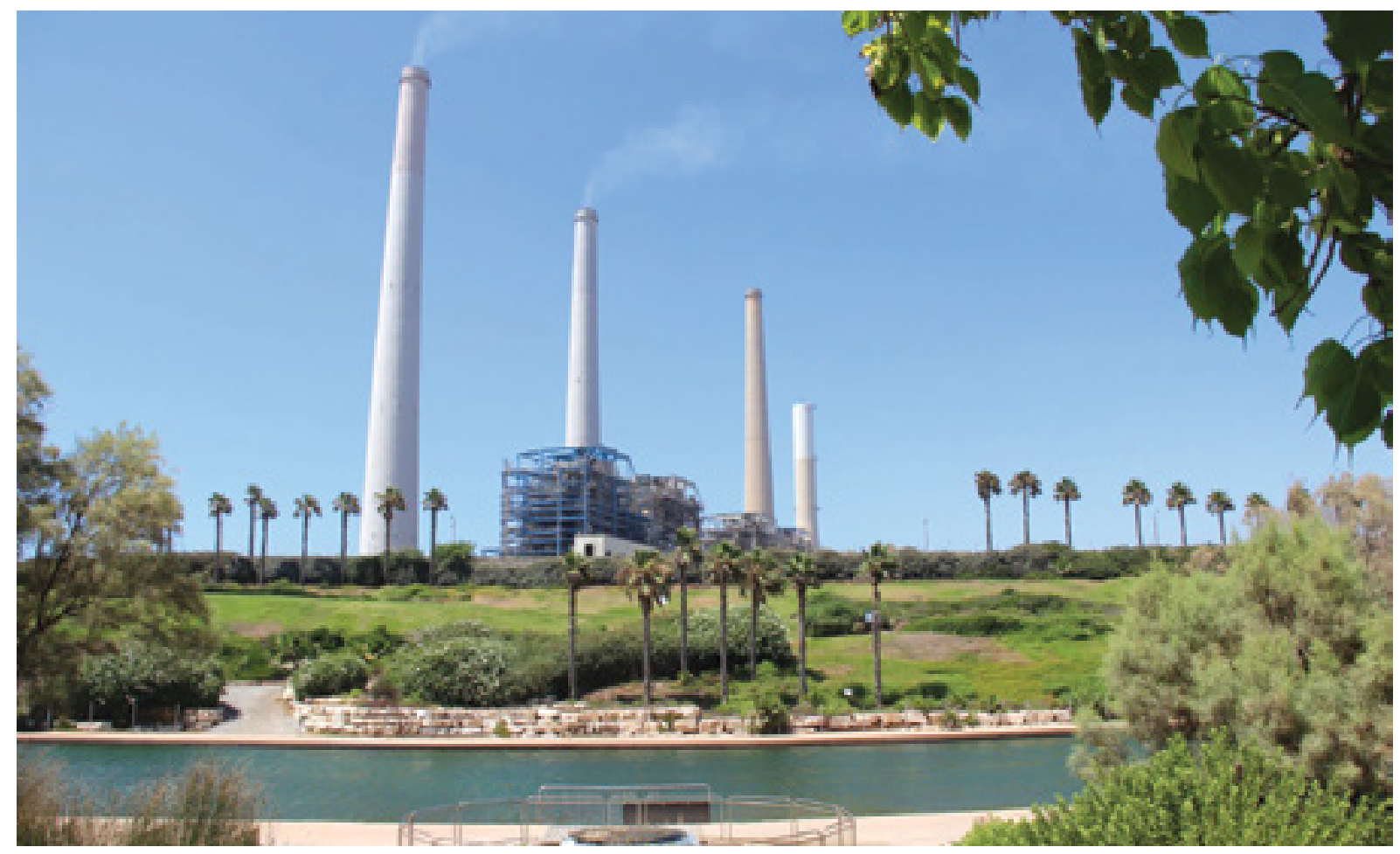

Orot Rabin coal-fired power plant/Photo: Ilan Malester

Therefore, given the current energy situation, Israel is focusing on measures related to the electricity generation, transport and industrial sectors.

\section{Phasing out coal}

Israel announced its intention to be coal-free by 2030 at the 24th Conference of the Parties to the UN Framework Convention on Climate Change (COP 24). It is a member of the Powering Past Coal Alliance and is actively working to reduce the share of coal in its electricity mix while increasing natural gas and renewable resources in the energy mix. The Ministries of Environmental Protection and Energy have presented their joint commitment to deliver this transition and reduce air pollution and greenhouse gas (GHG) emissions from energy production processes in Israel.

Thanks to a switch in the merit order - coal-fired plants continuing to operate at minimum load, but natural gas-fired combined plants operating at full capacity, allowing for flexibility and reliability of supply to the economy - the country has already started the transition, decreasing the share of coal in the total electricity fuel from $58.6 \%$ in 2010 to $32.4 \%$ in 2017 , while increasing the share of natural gas from 39\% in 2010 to $63.6 \%$ in 2017.

Additionally, Israel's commitment to phasing out coal is demonstrated by the government decision to decommission four of the coal-fired production units of the Orot Rabin Power Plant by 2022 lwhich represents $30 \%$ of Israel's coal-fired capacity), reducing the generation capacity from 4,840 to 3,400 MW. Additionally, plans are proceeding for the conversion of one of the units in the second largest coal-fired power plant to natural gas in 2023. In order to achieve zero-electricity production by coal by 2030, the decommissioning or retrofit to natural gas of the remaining units of the country are under technical assessment.

\section{Phasing out gasoline-based vehicles}

In 2018, some $95 \%$ of private vehicles in Israel were powered by gasoline, a rate that has remained constant since 2010. For other vehicles (taxis, buses and trucks), the share of vehicles powered by diesel fuel has increased from 34\% in 2010 to 
$67 \%$ in 2015, replacing gasoline. In addition, the number of hybrid vehicles increased from 31,675 in 2014 to 114,886 in 2018 and plug-in hybrid vehicles increased from 100 to 7,382 during the same period.

The NDC national target calling for a $20 \%$ reduction in kilometers travelled by private vehicles by 2030 relative to the business-as-usual scenario (as projected in 2015) was incorporated into a government decision, and a set of measures to reduce the use of private vehicles is under discussion as part of the National Plan for Air Pollution Reduction. They include, among others: parking cash-out programs, promotion of teleworking, carpools, employment center shuttles and bicycle riding.

Steps to establish Low Emission Zones (LEZ) in polluting cities are currently in planning, with the first such LEZ already operational in Haifa, Additional steps include subsidies for hybrid taxis and electric car sharing projects. Furthermore, a program to reduce pollution from heavy duty diesel vehicles has been launched, which includes subsidies for the installation of particulate filters. As of November 2018, M2, M3, and N3 diesel vehicles are required to install particulate filters (under Euro IV) in order to renew their vehicle license. Instructions have also been issued to major heavy-duty vehicle fleets that require a reduction in PM emissions and 3\% usage of alternative fuels by 2020 .

Other measures under consideration include a ban on the import of new diesel and gasolinefueled vehicles by 2030. Since electric vehicles are particularly adapted to Israel - a small country where distances travelled are relatively short current technological advancements and significant cost reductions in the electric vehicle sector will significantly contribute to the switch from gasoline and diesel to electric vehicles.

\section{Transition to clean energy in the industrial sector}

The energy consumption of Israel's industrial sector in 2016 was $40.6 \%$ from electricity, $57.8 \%$ from petroleum-based fuels and the remaining
1.5\% from renewable energy (waste incineration).

Phasing out polluting fuels in industry and their replacement with more efficient and cleaner energy sources requires connecting light industry and commerce to the natural gas distribution network. Additional solutions are being assessed, such as the use of electricity instead of fuels or the supply of compressed natural gas, as well as the necessary measures for optimal deployment of the distribution network, including supporting investments, infrastructure coordination and economic viability.

\section{Transition to renewable energy (SDG 7.2)}

Solar energy is the primary renewable energy in Israel and as such current technological advancements in the solar power sector are of particular importance and will play a part in the uptake of renewable energy in the country.

Solar energy is a major source of power for residential water heating $-85 \%$ of the population in Israel uses solar water heating, as a result of a regulation from 1980 that mandates installation of solar water heaters on all new residential buildings up to nine floors.

In recent years, the share of renewable energy has increased from approximately $0 \%$ in 2010 to above $4 \%$ in 2019.

Israel has approved a renewable energy target of at least $17 \%$ of total electricity generated in 2030 and interim targets of at least $10 \%$ by 2020 and at least $13 \%$ by 2025 . The $17 \%$ target will be reviewed in 2022 and adjusted if needed. In transitioning to renewable energy, Israel will need to address several challenges regarding:

- Management capacities of a decentralized electricity network with a high level of reliability in both routine and emergency situations and the growing difficulty of finding available land of relevant scale in densely populated Israel.

- Efficiency levels of renewable energy and technological developments in the field of energy 


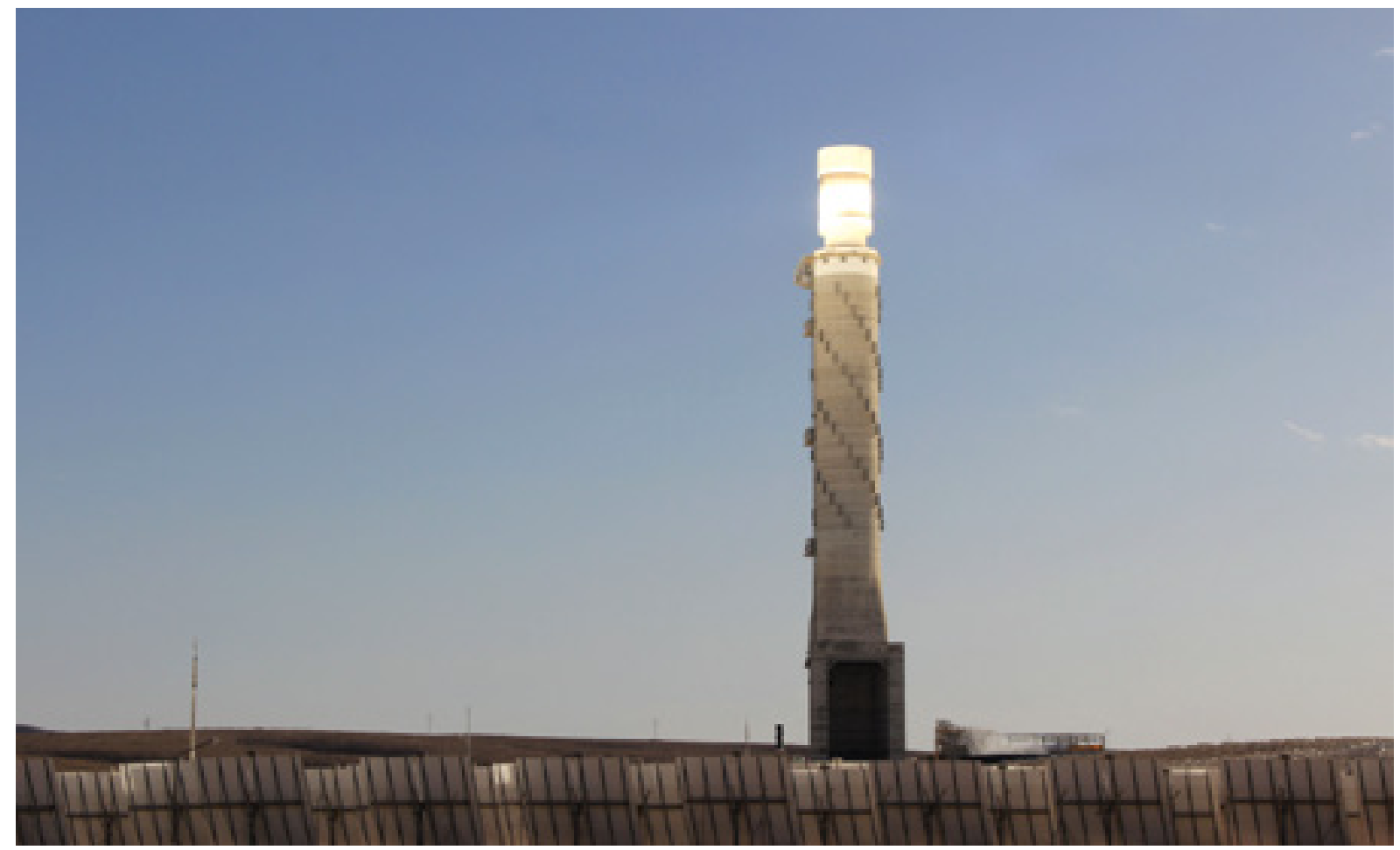

Ashalim solar power station in the Negev/Photo: Ilan Malester

storage. As mentioned, it is expected that most of Israel's renewable energy will be based on solar energy. However, the fact that solar systems on their own cannot provide stable energy 24 hours a day constitutes a clear challenge in the implementation of a renewable energy capacity exceeding the $17 \%$ target that can be overcome by technological advancement in the field of energy storage. This challenge is especially acute for Israel, which is an electricity island without grid connections to neighboring countries.

\section{Main steps to be taken to promote renewable energy:}

- Encouraging planning to implement the renewable energy potential in medium and large-scale plants;

- Complementary investment plan for the Israel Electric Corporation's development program. in particular for renewable energy integration into the network;

- Implementation of renewable energy plants on the roofs of public buildings;

- Development of electricity backup solutions and storage facilities, in particular for emergency situations;

- Promoting the integration of large-scale renewable energy, via the regulation of batterybased storage systems in the electricity production sector.

\section{( Promotion of energy efficiency (SDG 7.3)}

Israel's energy intensity has been below the average of OECD countries since 1990. In 2017. Israel's total primary energy supply per capita was 2.7 TOE compared to the European average of 4.05 TOE per capita. Significant improvements have taken place in the last decade, as reflected in the fact that total primary energy consumption per capita has decreased by approximately $13 \%$ between 2000 and 2016.

Continuing this effort, Israel has committed to meet a national electricity consumption reduction target of at least $17 \%$ by the year 2030 , compared to the expected electricity consumption in a business-as-usual scenario. The National Plan for 


\section{Electricity generation externalities and their connection to other SDGs}

The energy transition in Israel has implications that go well beyond SDG 7; it is expected to significantly reduce the environmental and health damages caused by air pollution and greenhouse gas $(\mathrm{GHG})$ emissions.

The Energy Objectives Plan for 2030 represents important progress towards achievement of SDG 13, integrating climate change measures into national policy and planning. Achievement of the energy targets will significantly contribute to reducing GHG emissions by stabilizing emissions from electricity generation at their current levels, despite the increase in electricity generation.

In addition, Israel's objective of eliminating polluting fuels use, such as coal and distillate fuels in industry, will contribute to achieving air pollution-related SDG targets:

- Reducing the environmental impacts of cities, specifically in terms of air quality (SDG 11.6);

- Reducing illnesses and deaths from hazardous chemicals and pollution, specifically reducing air pollution-related mortality (SDG 3.9).

According to an OECD report published in 2017, 2,240 deaths were caused by air pollution in Israel in 2015, representing an overall increase in the mortality rate due to air pollution between 2010 (238 per million) and 2015 (265 per million). An assessment included in the Israel National Air Pollution Reduction Plan - which includes a strong energy focus - forecasts the prevention of 126 deaths in 2023 and 472 deaths in 2028.

Implementation of the Greenhouse Gas Emissions Reduction Targets and for Energy Efficiency, approved in April 2016, includes key measures to promote energy efficiency and GHG emissions reduction projects in industry and local authorities (see SDG 13). In addition, in the years 2015-2019, a number of government decisions were made to promote energy efficiency projects in the periphery of the country. A total of NIS 27 million labout $\$ 7.5$ million) were granted for this purpose.

In order to achieve the $17 \%$ reduction goal, further energy efficiency measures will be required and are under consideration:

\section{Market-based mechanisms for energy efficiency:}

Several alternatives are being examined to encourage a reduction in electricity consumption among electricity providers, electricity producers, electricity consumers and other license-holders:

- Implementation of a purchase mechanism for non-consumed negawatts;
- Introduction of legal obligations for electricity producers and providers to implement energy saving measures, as part of their license conditions.

2. Building-related measures: As 60\% of the national electricity consumption is due to electricity consumption in buildings, implementation of energy efficiency measures in buildings is crucial. The Standards Institution of Israel has recently developed and published several standards relating to sustainable buildings, building energy rating and thermal insulation. For its part, the Ministry of Environmental Protection is working with key stakeholders in the housing construction industry including the Government Housing Administration and the Israel Defense Forces - on the promotion of green building. The main measures under discussion relate to:

- Mandatory adoption of the Green Building Standard in all new buildings: Full implementation of Israel's Green Building 


\section{CASE STUDY}

\section{The "Good Neighborhood" Project}

The "Good Neighborhood" project is an excellent example of how a single project interlinks several SDGs, promoting renewable energy use and improving energy efficiency, helping to reduce air pollution and contributing to the reduction of inequalities and the creation of more sustainable and inclusive communities.

The aim of this project is to carry out renovations in public housing buildings dedicated to low-income populations, and includes: roof insulation for energy savings, installation of photovoltaic panels and thermo-solar rooftop heating systems, as well as stairwell lighting and creation of green spaces around the buildings.

The project is executed and financed by the Ministries of Housing and Construction and Environmental Protection, and Keren Kaymeth Lelsrael - Jewish National Fund. The revenues from the electricity generated by the photovoltaic system are expected to cover all required operating expenses (including maintenance costs, taxation fees, licensing and insurance).

As of February 2019, 105 buildings were renovated, with their roofs sealed and insulated, and over 1,200 household replaced their thermal-solar rooftop heating systems. The project is scheduled for completion by the end of 2019 and is expected to generate $0.5 \mathrm{GW}$ of power while improving conditions in more than 1,300 households.

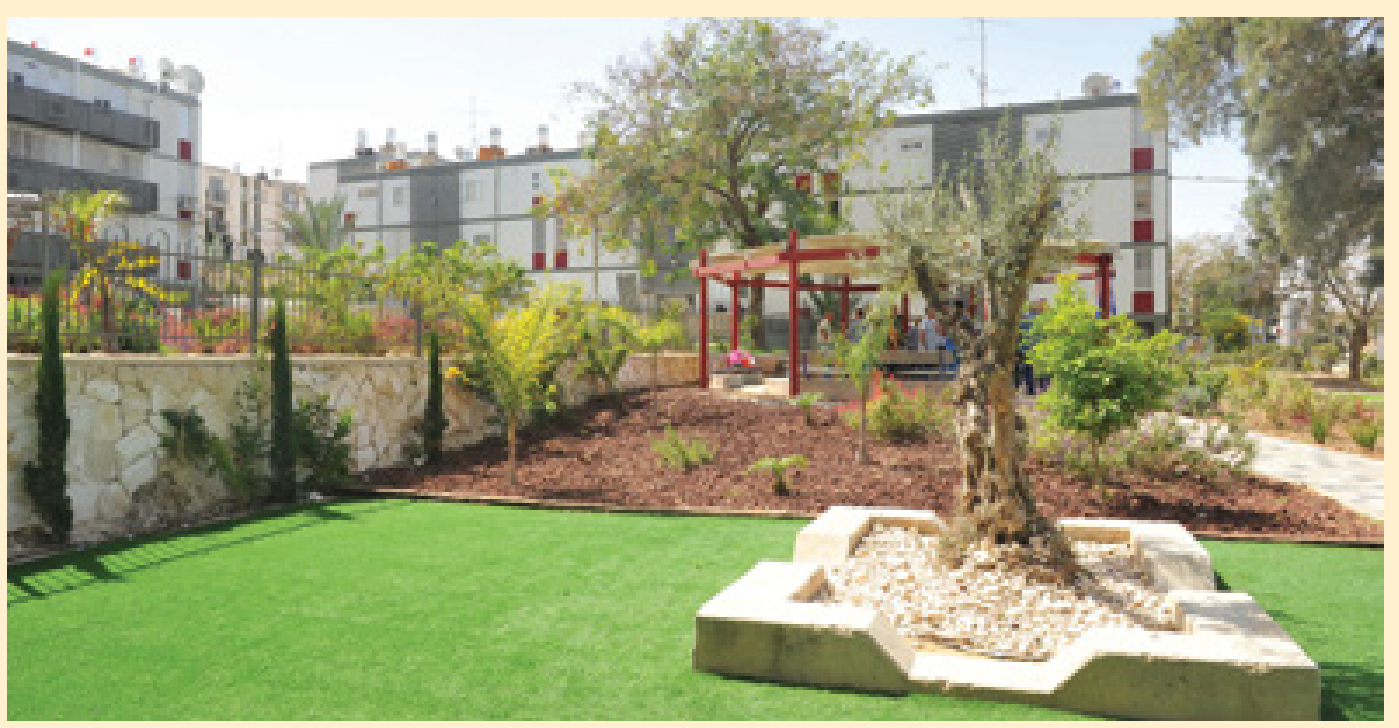

Good Neighborhood project in Ofakim/Photo: Government Press Office

Standard in new buildings would allow a total of 2,173 million MWh per year of electricity savings in 2030. This represents a reduction of more than 560,000 tCO2eq GHG emissions per year in 2028, constituting a total savings of NIS 15,136 million labout $\$ 4,250$ million). Households would directly benefit from this, saving an average of NIS 1,092 (about $\$ 300$ ) per year on electricity. Adoption of the standard for all new construction by Forum 15 - the association representing most of Israel's major cities incorporating over three million people and most of Israel's public institutions - serves as an example of good practice for the country, working with relevant government 
offices in order to remove barriers and provide incentives for green building.

- Zero-energy building: An obligation that 10\% to $20 \%$ of buildings - depending on their type Iresidential, educational, public buildings and offices) - comply with zero-energy building principles is currently under discussion, and would enable a significant reduction of the national electricity consumption.

3. Energy-sustainable city: In order to promote a model city for efficient and smart use of energy, the "Mayor's Challenge for a Clean and Smart City" competition between cities to receive funding for their smart energy projects has been set up. Such projects include: implementation of energy efficiency and energy conservation projects (including conducting energy surveys), implementation of intelligent energy management and energy monitoring systems, energy efficiency awareness campaigns, development of electric vehicles charging stations, and promotion of solar systems.

\section{PARTNERING FOR A BETTER WORLD}

Israel's Ministry of Foreign Affairs appointed a Special Envoy for Sustainable Energy to promote the global effort to reduce energy poverty and ensure universal access to affordable, reliable and modern energy services. At the 73rd UN General Assembly, Israel joined in the consensus on adopting the Resolution entitled Ensuring Access to Affordable, Reliable, Sustainable and Modern Energy for All (73/236). Israel is working with multi-stakeholders to advance modern concepts on how to better manage infrastructure for the long term and adapt decentralization and off-grid applications using sophisticated and optimal technologies. Israel's Special Envoy was also a member of the Bureau of the UN Economic Commission for Europe (UNECE) Committee on Sustainable Energy, and Israel is a member of the EU-MEDSTAT Working Group on Energy. (SDG 7.1)

In 2015, Israel opened an office in Abu Dhabi accredited to the International Renewable Energy Agency (IRENA), the intergovernmental organization supporting countries in their transition to a sustainable energy future. Most recently, Israeli representatives participated in the Eighth (2018) and Ninth (2019) Sessions of the Assembly. MASHAV, in cooperation with IRENA, conducted a training program in Israel for professionals from the developing world on Renewable Energy and its Impact on Sustainable Development, connecting policymakers, experts and innovators to share best practices. (SDG 7.a)
Israel was welcomed (2018) into the Global Methane Initiative (GMI), an international initiative advancing cost-effective, short-term methane abatement and recovery, and use of methane as a clean energy source in the biogas, coal, oil and gas sectors. By joining, Israel identified options for methane reduction and capture, especially in the natural gas sector. (SDG 7.a)

Israel signed onto the Powering Past Coal Alliance (PPCA) at the 24th Conference of the Parties to the UN Framework Convention on Climate Change (2018), joining governments, businesses and organizations around the world working to transition away from coal-powered electricity generation. The Alliance supports the reduction of coal dependency in OECD countries by 2030 (and the world by 2050), coinciding with Israel's current policy. (SDG 7.a)

Israel and the US Agency for International Development (USAID) entered into a Memorandum of Understanding (MOU) wherein Israel is one of the partners in the US-led initiative Power Africa. The initiative aims at advancing the common goals of reducing energy poverty and increasing access to energy in sub-Saharan Africa. Power Africa fosters innovative partnerships between private enterprise, African governments, and aid actors to expand electricity access, on-grid renewable, and off-grid efforts to extend energy across subSaharan Africa. (SDG 7.a, 17.17) 
The West African Power Pool (WAPP) entered into a landmark strategic relationship with an Israeli company - Gigawatt Global - signing an MOU with the 15-nation Economic Community of West African States (ECOWAS) to build $\$ 1$ billion of renewable energy projects in the region. The initiative was boosted by the address given by Israel's Prime Minister during the ECOWAS Heads of State Summit (2017) in Liberia, wherein he vowed to strengthen ties with the African continent. This initiative will help build the WAPP, a unit of ECOWAS, and create a regional electricity system. The initiative builds on an agreement signed by the United States and Israel (2017) to work together to reduce energy poverty in Africa. (SDG 7.b)

Millions of people worldwide are living in regions lacking national grids which provide basic services and infrastructures of water, electricity and sewage. Living off-grid has a direct impact on quality of life and health and is the most prominent indicator of global injustice in the distribution of resources. It is essential to formulate solid strategies and develop tools to deal with the implications of living off-grid, such as irregular water supply, long-distance travel to reach safe water sources, lack of electricity for operating medical clinics, schools and domestic homes, sanitation issues, and food cooling and storage difficulties. Dealing with these ramifications is part of an overall effort to tackle world poverty in developing countries. To address these issues, the Eilat-Eilot Renewable Energy Initiative established an Off-Grid Village in southern Israel which serves as a demonstration site for off-grid infrastructure and technologies and as a center for international trainings/seminars on renewable energy and offgrid eco-tech. (SDG 7.a, 17.17)

The 8th Eilat-Eilot International Summit (2018), with the cooperation of Power Africa, focused on bringing innovation to off-grid communities in Africa and providing energy for basic needs such as lighting, cooking, clean water and irrigation. Potential solutions were presented in the fields of technology, finance and business models, as well as the social approach to effective implementation. (SDG 7.b)

International standardization for biogas, a renewable energy source, can contribute to the alleviation of the world's energy problems. Household waste (kitchen, animal, and human), transformed into clean cooking gas, prevents burning biomass and the resulting indoor air pollution. The efforts of the Standards Institution of Israel (SII) to standardize biogas promote international technological exchanges and will enable an increase in household biogas systems on the global market. (SDG 7.2)

Renewable energy sources offer solutions for problems challenging the development of many regions around the world. MASHAV, in collaboration with the Arava Institute for Environmental Studies, carried out a training course in Israel entitled Renewable Energy as a Catalyst for Regional Development. The course provided an opportunity for mid-level professionals from the private and public sector in the developing world to see the work done by the Center for Renewable Energy and Energy Conservation at the Arava Institute and the Eilat-Eilot Renewable Energy Initiative. (SDG 7.a, 17.17)

An MOU was signed between the Ministry of Energy and Mineral Development of Uganda and Israel's Ministry of Foreign Affairs to cooperate on Biogas Technology for Energy Production and Agriculture Improvement. Both sides recognized their full membership in IRENA and committed to working together given the urgency of finding cost-effective solutions to common biogas technology for energy generation and agriculture improvement issues that support the need for sustainable development using animal waste. (SDG 7.a)

In today's world, young people are encouraged to tap into viable businesses that utilize renewable and local resources, such as solar energy, and turn them into affordable and vital commodities. MASHAV, the Organization of American State (OAS) and the Young Americas Business Trust (YABT) conducted a joint capacity-building program in Guyana on Business Labs Focused on Renewable Energy and Business Innovation. The course was held during the Global Entrepreneurship Week 2018, and additional courses were carried out in Nassau and the Bahamas in cooperation with local partners. The Business Labs Program provides 
training on issues of innovation management, entrepreneurship, and business support, with the purpose of developing local capacities among young entrepreneurs. (SDG 7.a, 8.6)

Israel's efforts to help facilitate access to clean technologies and promote economic growth in developing countries by 2030 include the implementation of two MASHAV initiated international training programs: Clean Technologies - Environmental Technology, Innovation and Management Systems as Means for Regional and Local Economic Development; and Renewable Energy as a Catalyst for Regional Development. (SDG 7.a)

In addition, MASHAV and the UN Economic Commission for Europe (UNECE) conducted a joint workshop on Inter-Regional Energy Efficiency and Renewable Energy: Projects and Policies to promote the accelerated deployment of renewable energy and efficiency in Eurasia. (SDG 7.a)

Israel's Embassy in Cameroon initiated a triangular project in cooperation with the UN International Children's Emergency Fund (UNICEF) and an Israeli NGO - Innovation: Africa. The aim of the Initiative was to provide solar power, clean water and better healthcare to tens of thousands of refugees living in rural villages from the neighboring war-torn Central African Republic. Approximately 50,000 people now enjoy improved healthcare and services due to access to potable water and electricity from solar panels installed in village hospitals. In 2018, MASHAV hosted 24 African professionals employed by Innovation: Africa for a training program on Innovation and Technologies. (SDG 7.a) 


\section{GOAL}

8

DECENT

WORK AND

ECONOMIC

GROWTH

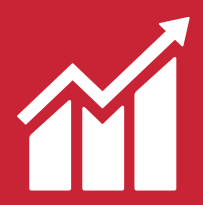

Export promotion

Raising productivity and

competitiveness of Israeli industry

Technology innovation and skilled manpower in high-tech

Encouragement of small and midsized enterprises

Regional economic development

Utilization of human capital, employment diversity, and gender equality

Preparatory programs for the world of work for people with disabilities

Activities of the National

Commissioner for Equal

Employment Opportunities

Activities related to workers' rights

Partnering for a Better World 


\section{PROMOTE INCLUSIVE AND} SUSTAINABLE ECONOMIC GROWTH, EMPLOYMENT AND DECENT WORK FOR ALL

The Israeli economy has grown rapidly in the past ten years and shown impressive stability against external and security shocks. Israel's economy grew by $3 \%$ in 2017 , mainly driven by expanded private and public consumption that supported expanded economic activity, together with recovery in the export of goods and continued rapid growth in the export of services. The Israel Central Bureau of Statistics (ICBS) estimates that GDP totaled NIS 1.26 trillion (about $\$ 350$ billion) in 2017 in constant prices. The Ministry of Economy and Industry's main objective is to boost GDP per capita and climb in the OECD rankings to 15th place by 2025. 
Figure 8.1

\section{Quarterly and annual GDP growth rate}

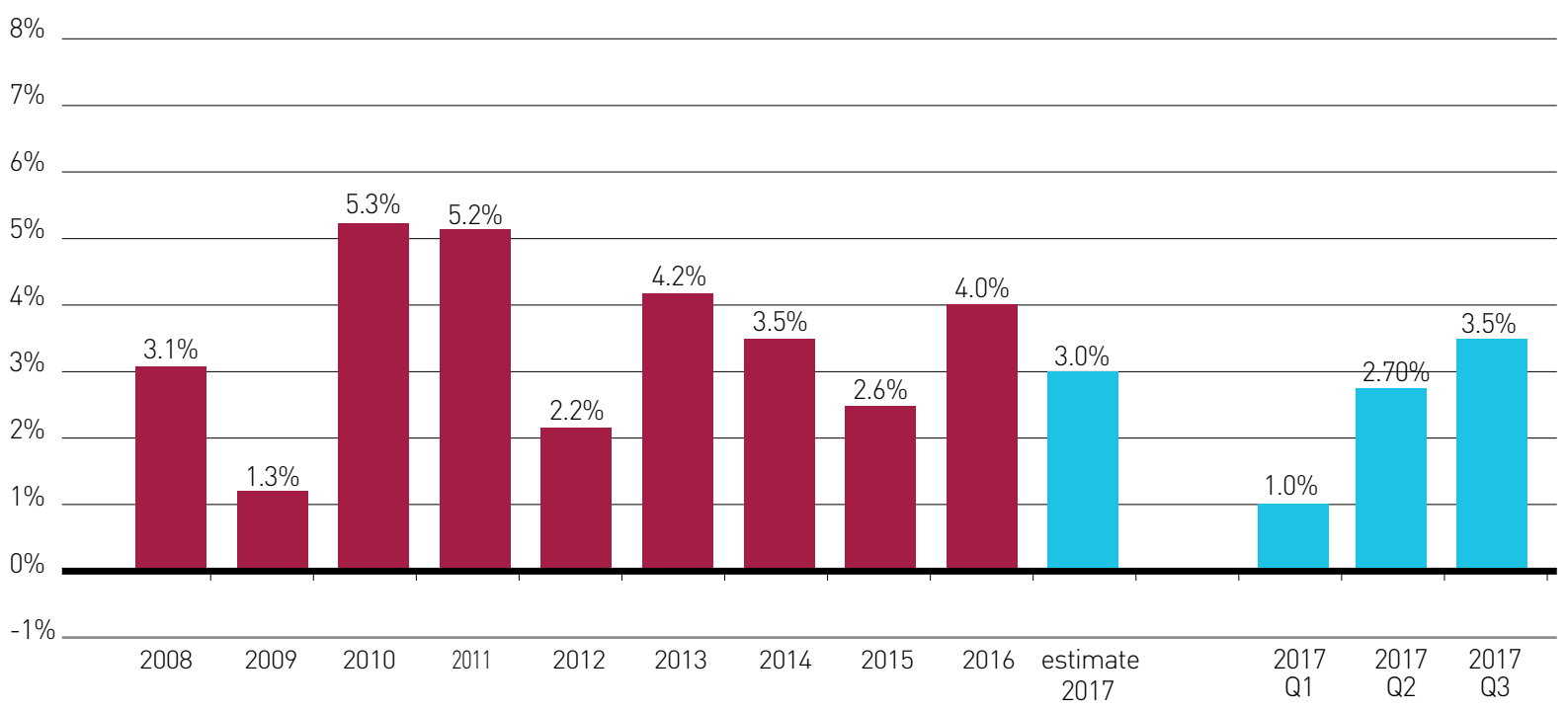

Source: Israel Central Bureau of Statistics, 2019 budget proposal

* The annual rates of change are based on original data, and the quarterly rates of change represent quarterly growth at annualized rates based on seasonally-adjusted figures

\section{Business product}

Business product accounts for about $74 \%$ of GDP. The main business product elements are services and trade (70\%) and industry (20\%), alongside construction and agriculture. According to ICBS estimates, business product grew by $3.2 \%$ in 2018 (in fixed prices), compared with an average annual growth of $3.8 \%$ in 2013-2016.

\section{Industrial output}

The Index of Industrial Output, which shows growth trends in the industrial sector, rose by $3.9 \%$ in 2017 (seasonally adjusted, January-November, compared with the corresponding period in 2016) following several years of more modest growth. High-tech sectors grew more modestly (1.4\%), compared with mixed high-tech industrial sectors $(2.8 \%)$ and mixed low-technology sectors (2.3\%), as shown in Figure 8.2

\section{( Export promotion (SDG 8.1, 17.7, 17.8)}

Israeli exports exceeded the 2017 forecast $\$ \$ 100$ billion), reaching a new record. Exports of Israeli goods and services totaled $\$ 102.3$ billion in 2017 , $6.4 \%$ more than in 2016 . The export of services recorded the most impressive growth, growing by $\$ 3$ billion, attributed to Israel's high-tech industry - computing, software, research and development (R\&D), and more - which has been a key growth engine in recent years. Alongside exports, imports of goods and services grew by $7.9 \%$ over 2016 to $\$ 6.7$ billion. Foreign financial investment jumped by $\$ 18.6$ billion, compared with growth of $\$ 17.9$ billion in 2016 and $\$ 8.6$ billion in 2015 .

Exports of goods and services in U.S. dollar terms rose by about $6 \%$ in the first half of 2017, compared with the first half of 2016 to approximately $\$ 50$ billion. Industrial exports boosted exports of goods by about $4 \%$ in dollar terms. Exports of services rose by about $8 \%$, the fastest growth rate in six years. Exports of services reached about $44 \%$ of total Israeli exports in 2017, compared with about $31 \%$ at the beginning of the decade (Figure 8.3). 
Figure 8.2

\section{Rates of change in the Index of Industrial Output in 2017*, by technology intensity}

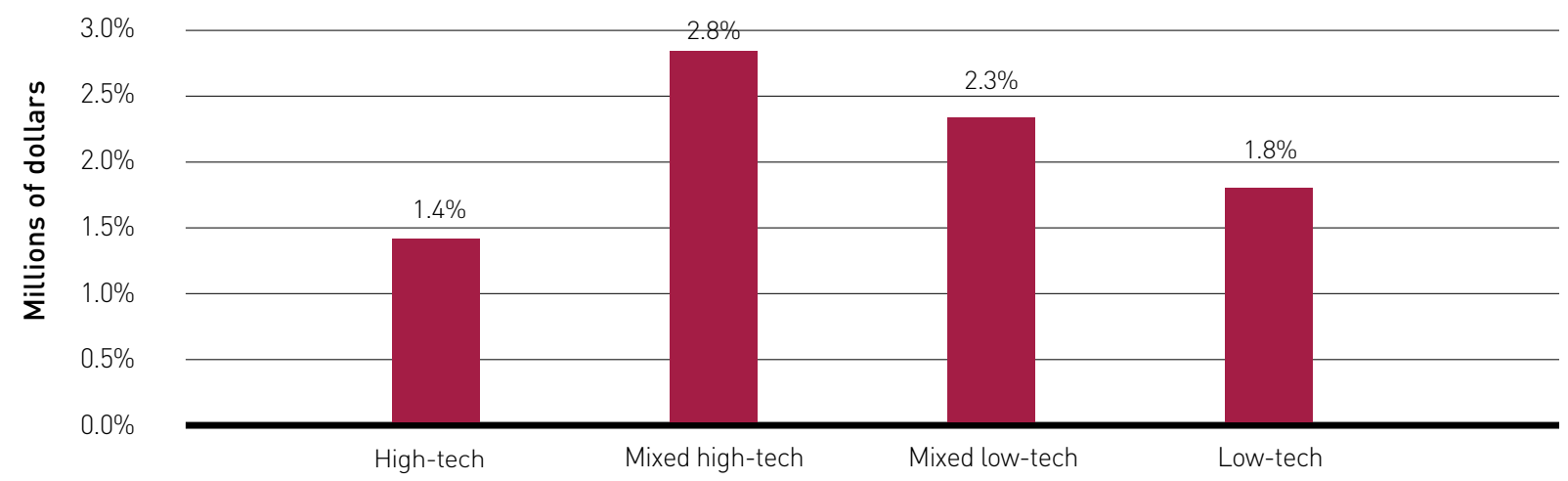

* January-November 2017 figures, compared with the corresponding period in 2016, based on seasonally-adjusted figures Source: Processing of Israel Central Bureau of Statistics data by the Chief Economist's Department at the Ministry of Finance

Figure 8.3

Exports of Israeli goods and services in 2007-2020

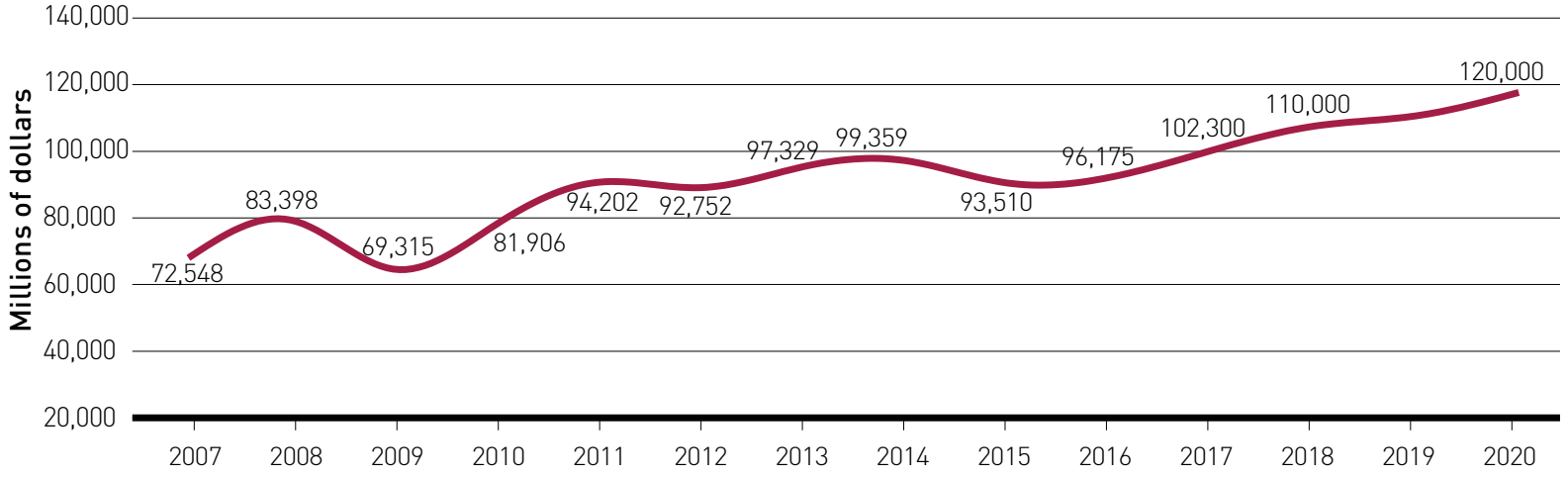

\section{( Raising productivity and competitiveness of Israeli industry (SDG 8.2)}

In recent years, Israeli manufacturing has been coping with competition in the global market, while industrial productivity has been lower than in developing countries. The government is therefore seeking to boost manufacturing productivity and raise the ratio between productivity in low technology and mixed-low technology industry and high-tech and mixed high-tech industry to $72.1 \%$ by 2025 through several policy measures, beginning with the Strengthen Israeli Industry
Plan. The main policy measures are as follows:

1. Productivity raising programs

- In low-technology industries: The program combines assistance with performing physical capital investments with other investments, such as advice and installation of innovative technologies and administrative tools. In 2017. ten companies increased their productivity by $30 \%$, receiving a NIS 14.8 million grant for an investment of NIS 49.6 million.

- In the construction industry: The program includes financing and technology promotion and green and prefabricated construction methods 
to boost productivity in the industry through prefabrication and sustainable construction.

- Small and mid-sized enterprises in the trade and services sectors: The program includes assistance for capital and other investments to promote innovative product and manufacturing processes and the organization of a model for small and mid-sized enterprises.

2. Grants programs: Under the 1959 Law for the Encouragement of Capital Investments, aid is provided to manufacturing exporters for the procurement of manufacturing equipment, buildings, and fixed assets. In 2017, NIS 362 million in grants was allocated for NIS 1.8 billion in investments and the direct hiring of 1,250 new employees and the same number of indirect employees.

\section{National Strategic Plan for Advanced}

Manufacturing: This strategic plan aims to change conventional planning and manufacturing processes to smart processes in tenders and information flow, connectivity, monitoring, and production data collection and analysis in order to create value in the manufacturing processes and supply chain. The plan presents actions to be undertaken to create a national support infrastructure including the establishment of an advanced manufacturing institute to create foundations and information accessibility that support the installation of advanced manufacturing and the establishment of an advanced manufacturing innovations community. Additional actions include aid programs for the procurement and installation of advanced manufacturing technologies, aid programs for R\&D of advanced manufacturing technologies, opening of smart technology industrial zones, and encouragement of academic research on advanced manufacturing.

\section{Trade and Services Sectors Economic Promotion}

Plan: The trade and services sectors have great weight in the Israeli economy, accounting for about $60 \%$ of its gross added value, and employing $73 \%$ of business sector employees. For the first time, a public committee will be established that includes senior government officials, academics, and businesspeople to draw up a policy to boost productivity in Israel's trade and services sectors.

\section{Reducing industry concentration and creating a competitive economy}

- Programs to increase competition in concentrated industries: These programs combine assistance in performing physical capital investments to add new players in concentrated markets or increase the manufacturing capacity of small and midsized enterprises to compete in monopolistic or oligopolistic markets, thereby expanding the supply of products in the relevant industry and lowering prices. To date, there have been interventions in the meat, dairy, whole wheat flour, and gluten sectors. A review of developments in concentrated markets and solutions for increasing competition is conducted each year.

- Setting import quotas under the competitive process: Alongside the creation of domestic competition, efforts are invested in importing less expensive food products and lowering the cost of living by granting importers full or partial customs exemptions.

- Improving the business environment for importers: Efforts focus on opening the imported products testing sector to competition by allowing private laboratories to conduct tests, thereby shortening schedules, lowering prices, improving service, and saving households around NIS 500 million a year.

\section{Economic development in the minorities sector}

In view of the economic and social importance attributed to the economic integration of minorities in Israel, Government Decision 922 was taken on December 30, 2015 to enhance the integration of minorities in economic activity by expanding the supply of jobs and the marketing and development of industrial zones in minority towns as well as by encouraging minority exporters and small and mid-sized enterprises.

\section{Promoting foreign investment in Israel}

There is a positive correlation between foreign investment and economic growth. The Ministry of Economy and Industry therefore strives to encourage foreign investment by bringing together 
potential investors and local businesses. The relationship is mainly undertaken through the industrial reciprocal procurements program, which matches Israeli and foreign companies for the purpose of procurements, including advising foreign companies when investing in Israel and afterwards. (Investors Services Center - https:// investinisrael.gov.il/Pages/default.aspx).

Under the reciprocal procurements program, a foreign company undertakes to make procurement purchases from Israeli companies based on procurement purchases made from it. The scope of the commitment is $50 \%$ for transactions with the Ministry of Defense, 20\% for civilian transactions with government companies under the Government Procurement Agreement (GPA), and 35\% for other transactions. In 2017, 228 foreign companies made reciprocal procurements totaling approximately NIS 8.6 billion, $17 \%$ more than in 2016. During 2017. foreign companies signed 268 commitments totaling $\$ 1.4$ billion. Altogether, Israeli companies are due to benefit from $\$ 12$ billion in reciprocal procurements in the coming years - a 44\% jump compared with 2016.

\section{( Technology innovation and skilled manpower in high-tech (SDG 8.2)}

The Ministry of Economy and Industry and the Israel Innovation Authority are promoting R\&D and leading technology innovation as a lever for sustainable economic growth, as seen in the national expenditure on civilian R\&D of approximately NIS 52 billion in current prices, equal to $4.3 \%$ of GDP. In fixed prices, national expenditure on civilian R\&D rose by $2.4 \%$ in 2016 , following $6.9 \%$ growth in 2015. (Figure 8.4 )

Activities to promote R\&D and lead technology innovation while prioritizing development areas include:

\section{39 programs for encouraging industrial $R \& D$ :}

The programs offer support for companies and new ventures at all stages of technology development, from the basic concept and applied research stage in academia, through pioneer trials and initial commercial demonstrations (pilots). The Innovation Authority operates the plan in five innovation arenas:

Figure 8.4

National expenditure on R\&D as a percentage of GDP in Israel and other OECD members, 2015

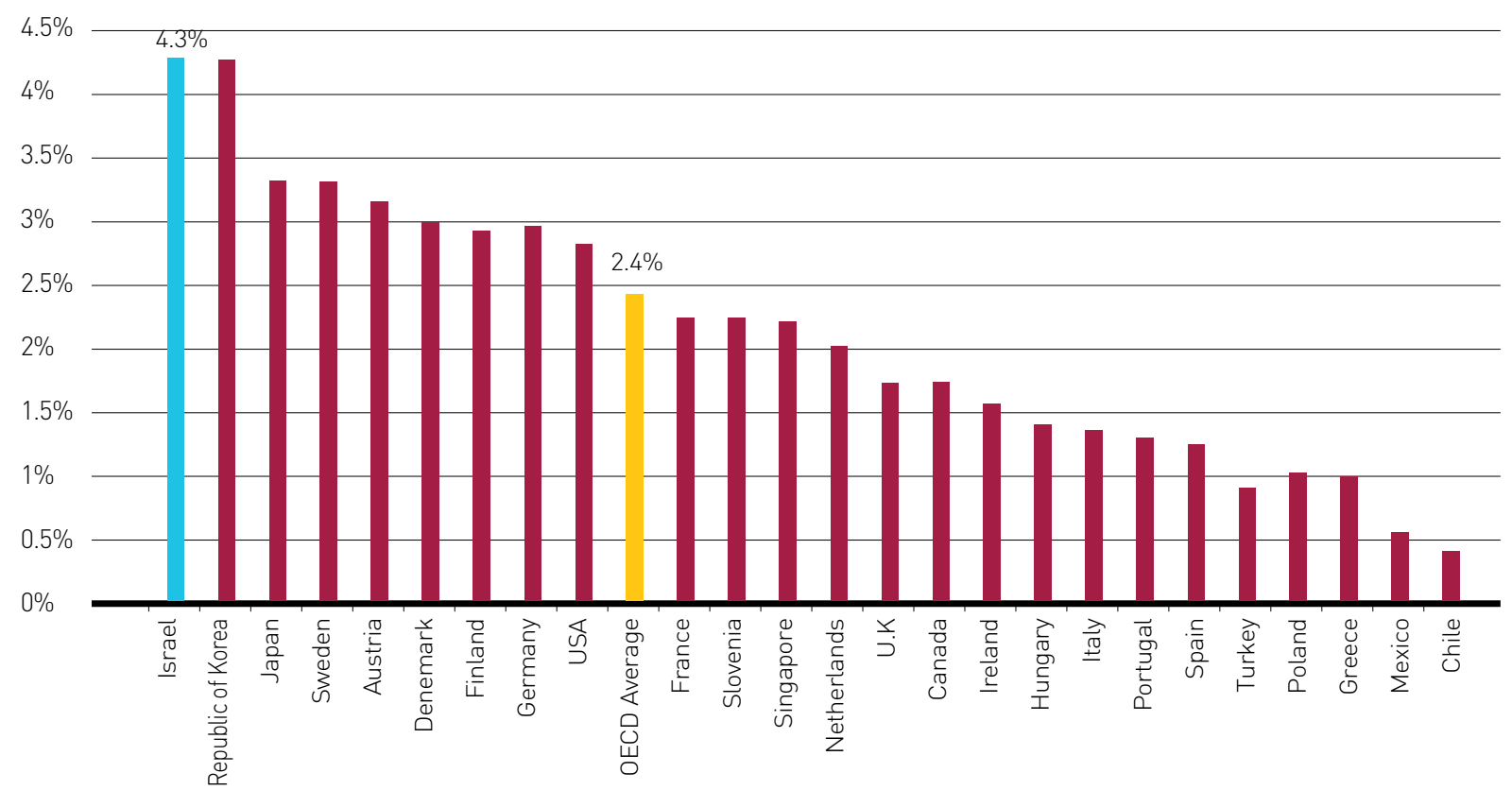

Source: OECD data 
- Technology infrastructures: This arena promotes research and breakthrough technologies and the bridging between academia and Israeli industry to strengthen Israeli industry's longterm technological edge. The arena's programs foster cooperation, information and experiencesharing as well as the development of generic breakthrough knowledge by integrated groups of research in academia and industry.

- Start-ups: This arena offers unique tools to support the early development stages of technology ventures, which help start-ups and new entrepreneurs develop their innovative technology concept, realize it, and reach a major financial milestone. In this way, the arena contributes to strengthening Israel's standing as a thriving center of international entrepreneurship.

- Growth companies: This arena helps companies grow through technological innovation, thereby strengthening the trend of Israeli technology companies that seek to grow and establish themselves as mature companies in Israel. The arena's activity is directed to advancing the growth and technological leadership of mature companies and to supporting rapid growth processes through financing and other means.

- Advanced manufacturing: This arena works to establish a sustainable, advanced, innovative and renewable industry, which is a source of economic growth for the economy and a center of pride for those engaged in it. The arena supports the implementation of R\&D and innovative processes among manufacturers to improve their productivity and global competitiveness. The two flagship programs of the arena are R\&D support for the manufacturing industry (MOFET) and a preparatory course for $R \& D$.

- Social-public: This arena seeks to apply technology innovation to improve public services, social welfare, and standards of living and to expand and diversify the labor supply in high-tech industry. The arena operates several programs to achieve these objectives.

2. Tax breaks: High-tech companies receive large tax breaks to make operations in Israel attractive.

\section{National Plan to Increase Skilled Manpower}

for the High-Tech Industry: In 2017, a national policy was drawn up to offer measures to increase skilled manpower in high-tech, including a $40 \%$ increase in the number of undergraduates in hightech professions and prioritizing the integration of underrepresented sectors of the population in high-tech (with an emphasis on women, Haredim [ultra-Orthodox] and minorities), and personnel from overseas (returning Israelis, new immigrants, and foreign citizens). The arena also offers non-academic training, such as programming 'commandos' and the Best to Industry Plan to match students in high-tech professions with enterprises in national priority areas through financial aid for both students and enterprises, provides students with simultaneous study and job experience, and responds to the shortage of skilled manpower, especially in low technology industries.

\section{Technology Innovation Laboratories Program:} The program finances a laboratory infrastructure (based in some industrial companies) for entrepreneurs to develop and build products.

5. The Digital Israel Program for Innovation in the Public Sector: The program encourages digital ventures to improve the quality of services provided, reduce bureaucracy, and protect information. Sectors supported include digital education, digital welfare, digital health, employment and the development of human capital, digital local government, digital economy, digital legal services, and digital services for people.

6. Binational plans: The plans are designed to create a joint platform between Israeli and foreign companies. Cooperation is critical for the development of new products and technology using knowledge/installations/relations with foreign companies.

\section{(Encouragement of small and mid-sized enterprises (SDG 8.3)}

Small and mid-sized enterprises are an important growth engine for the Israeli economy. Israel currently has more than half a million small and mid-sized enterprises, which comprises $99 \%$ of 
businesses in the country. They are responsible for $53 \%$ of business product and $61 \%$ of all business sector employment. The Ministry of Economy and Industry is therefore seeking to realize the latent economic and social capabilities of the small and mid-sized enterprise sector, to boost their contribution to Israel's GDP and help reduce concentration in the economy through the following means:

\section{Small and Mid-sized Enterprises E-commerce}

Promotion Plan: The Ministry of Economy and Industry and Digital Israel are jointly operating a plan to develop e-commerce by small and mid-sized enterprises. The plan maps the business, provides it with online marketing consulting services and online stores, provides training in different digital subjects, and provides grants to businesses to establish an e-commerce and online marketing platform.

\section{Development of additional financing and credit channels for small and mid-sized enterprises:}

- State-backed loan fund for small and midsized enterprises: The fund's objective is to provide small and mid-sized enterprises with credit that they cannot obtain from banks. The fund operates through several aid channels, including working capital loans to establish new small businesses and expand existing ones. The fund is financed from bank sources against a government guarantee and is jointly managed with the Office of the Accountant General at the Ministry of Finance. The fund approved 5,974 loan guarantees totaling NIS 1.99 billion for small and mid-sized enterprises in 2016.

- Growth capital funds: Two private equity funds were established to support mid-sized enterprises to give them access to growth capital lcapital intended for expanding or rebuilding operations, entering new markets, or financing a major acquisition).

- Microfinancing for the establishment of small businesses by women from the Arab and Bedouin sectors: Microfinancing is provided through the Koret Foundation SAWA, a joint venture of the Ministry of Economy and Industry and the Koret Foundation with the Authority for the Economic Development of the Arab, Druze and Circassian Sectors to develop small businesses through the grant of non-bank loans to women in the Arab and Bedouin sectors.

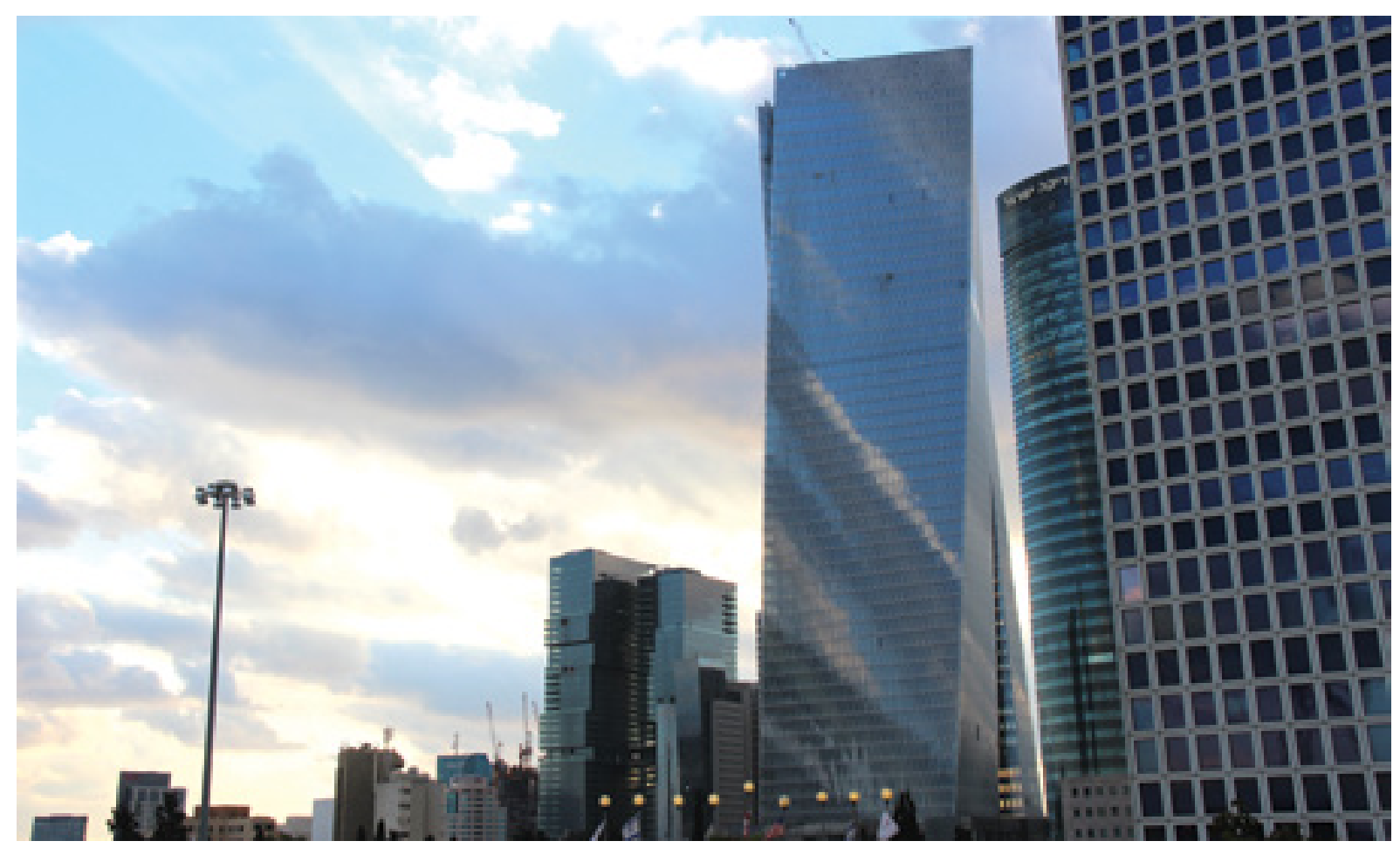

Urban Reflections, Tel Aviv/Photo: Ilan Malester 
3. Social Enterprises Aid Plan: This plan encourages social purpose enterprises to promote the employment of people underrepresented in the labor market, such as former convicts.

4. Field hubs: 30 field branches nationwide, called MAOF which provide businesses and entrepreneurs with a range of tools and services based on the business's needs and advice over time.

\section{Business personal consulting services for} small and mid-sized enterprises: The framework includes industrial design, environmental efficiency, and business licensing services.

\section{Financing and writing business plans} consulting services: This activity includes helping entrepreneurs and existing businesses to apply for financing from foundations and financial institutions - from the filling out of forms through the management of the funds obtained.

7. Business Acceleration Plan: The plan offers comprehensive consulting services for Arab entrepreneurs at all relevant levels from the launch of a venture through the active start-up stage.

8. Establishment of business centers: The business centers provide a physical site for new micro and small businesses, including business consulting, professional training and support, and rent, with no long-term commitment. Seven business centers have been established to date: two in the national periphery and five in minority towns.

\section{Aid for the organization of small and mid-sized} farmers: The Ministry of Economy and Industry and the Ministry of Agriculture and Rural Development are jointly operating a plan to encourage the establishment of agricultural cooperatives to create economies of scale and reduce costs for the individual farmer. There are several programs that provide financial assistance to establish cooperatives and provide them with consultancy services across a range of subjects. The program has helped around 40 agricultural cooperatives to date with a budget of NIS 5 million.

10. Programs to boost productivity in the trade and services sector:

- Aid program for food producers to obtain Good Manufacturing Practices (GMP): The Ministry of Economy and Industry assists small businesses to comply with strict food industry regulations.

- Improving the business environment: The plan

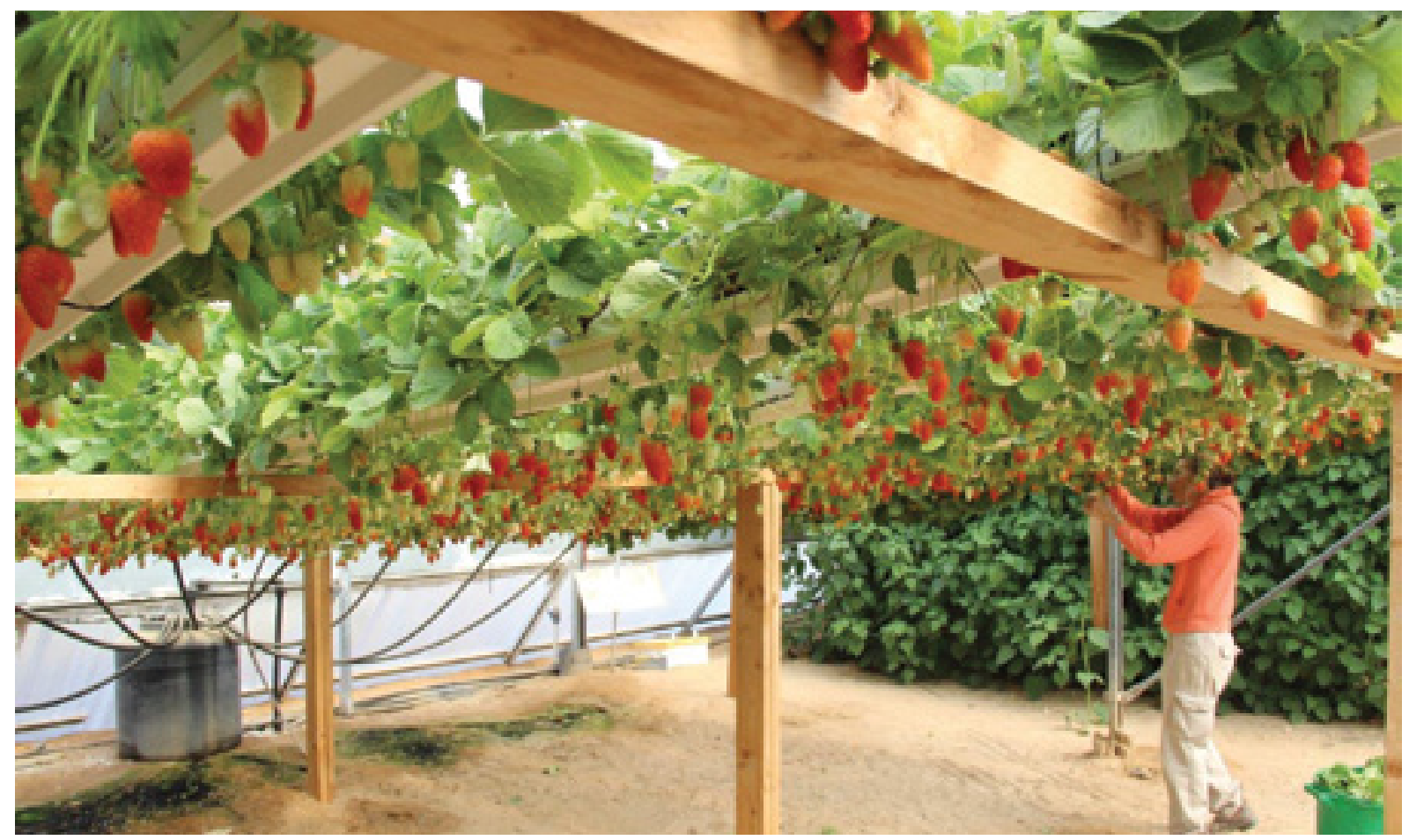

Growing hydroponic strawberries, Hatzeva, Southern Israel/Photo: Ilan Malester 
seeks to identify legislation and regulations that affect small and mid-sized enterprises, including providing opinions on proposed legislation, government decisions, and other policy outputs.

- Providing rental space for food-tech enterprises: The plan aims to build a site with work spaces (production floors) for small food-tech enterprises, as well as providing professional tools and services to advance the enterprise's operations.

- Accelerator programs: Within this framework, 19 accelerator programs operate at local sites in the periphery in cooperation with local authorities. The accelerator is a home for innovative start-ups seeking a professional environment and provides advice on establishing the venture and dealing with the various challenges it faces.

- Assistance to improve local government's ability to promote small and mid-sized enterprises: The program applies the Business-Friendly Index at the local level to examine the degree of local authority support for small and mid-sized enterprises in their jurisdiction. The index is based on local property tax (arnona) data, access to information about the local authority, and a survey of enterprises to examine their perception of the interfaces with the local authority.

\section{( Regional economic development (SDG 8.3)}

The Ministry of Economy and Industry seeks sustainable local development that will provide suitable jobs for the human capital in the region at fair salary terms. It acts to leverage its relative advantages compared with other regions in Israel or globally to generate economic growth. Following are some examples:

\section{- Economic and Industrial Development Plan for} the Dimona, Arad, and Yeruham Municipalities: This plan seeks to strengthen the towns of Arad, Dimona, and Yeruham to significantly improve their socio-economic conditions and turn them into localities that draw investors and employers by attracting industrial enterprises. In 2017, NIS 70 million in grants were allocated for NIS 233 million in investment and the addition of 233 direct employees.

- Economic and Industrial Development Plan for the Northern District: The Israeli government has set the objective to develop the Northern District by developing a transportation infrastructure, promoting tourism, improving access to quality

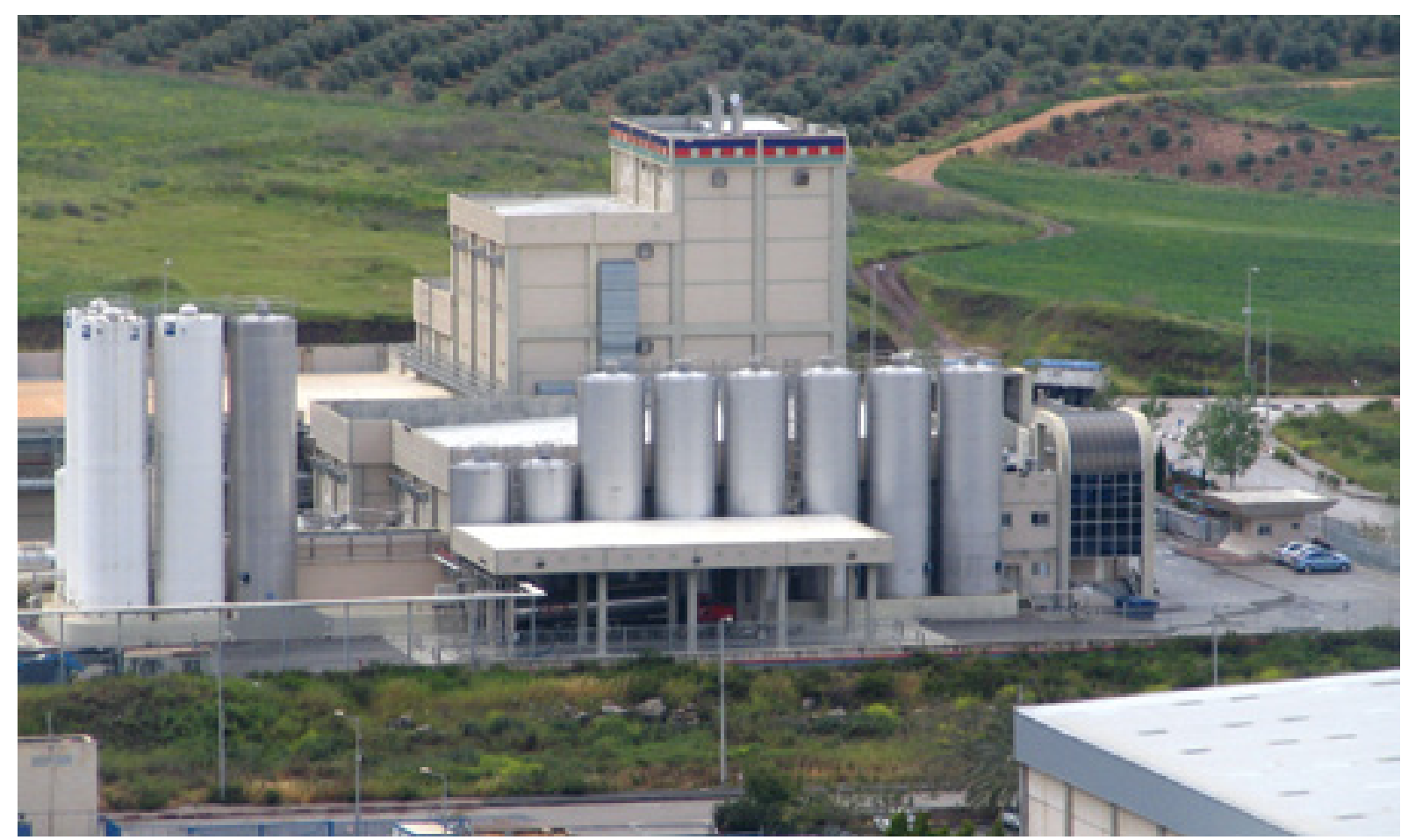

Ba'emek Advanced Technologies in the Jezreel Valley/Photo: Ilan Malester 
public medical care, improving access to higher education, creating local high-quality jobs, boosting productivity, encouraging R\&D activity, establishing research institutes, and turning the Northern District into a food-tech knowledge hub through the establishment of designated technology incubators, leasing space for food-tech ventures by small and mid-sized entrepreneurs, establishment of a food-tech knowledge community, and more.

\section{- Economic and Industrial Development Plan} for the Negev Bedouin 2017-2021: The plan aims to strengthen Bedouin local authorities to significantly improve the socio-economic conditions of the Bedouin population in the Negev and to narrow its lag compared with Israel's general population. The plan encourages and supports Bedouin ventures to establish sustainable businesses, create local jobs, and strengthen local industry in the region through financial support.

\section{( Utilization of human capital, employment diversity, and gender equality (SDG 8.5)}

In 2010, the government set employment targets for 2020 for the general population and for sectors underrepresented in the workforce, realizing that increasing the number of workers is critical for driving Israel's GDP growth (Government Decision 1994 of July 15, 2010). In 2017, it was found that the employment rate in Israel was above the OECD average (72\%), but lower than such countries as Germany and Japan ( $80 \%$ and $80.4 \%$, respectively). Although the general employment rate was above the OECD average, there are still large differences between different groups within Israel, and continued support of some groups, in particular Arab women and Haredi men is needed.

To meet the targets, the government is creating an infrastructure for new and sustainable jobs, and prioritizing groups underrepresented in the labor market in different sectors through the following programs:

- Four aid programs to encourage the hiring of employees from groups with low participation in the workforce. The programs are intended for manufacturing or services enterprises which wish to establish, move, or expand business to national priority areas, Jerusalem, Sderot, communities adjacent to the Gaza Strip, and towns in the south and/or to employ workers from groups with low participation rates in the workforce (Arabs, Druze, Bedouin, Haredim and single parents). The aid is granted through participation in the employees' salaries at a diminishing rate over a prescribed period of employment.

- Aid program to encourage the hiring of workers with disabilities, and programs for manufacturing and services companies in Israel wishing to establish, move, or expand operations in Israel and to hire workers with disabilities. The aid is granted through participation in the employees' salaries at a diminishing rate over a prescribed period of employment.

- A program to encourage the creation of highpaying jobs in national priority areas and Jerusalem: The program is intended for hightech companies in manufacturing or services in Israel wishing to establish, move, or expand operations in national priority areas and to engage employees at high salaries. The aid is granted through participation in the employees' salaries at a diminishing rate over a prescribed period of employment.

- Three aid programs for the integration of underrepresented groups in high-quality jobs, especially in the free professions. The programs are intended for businesses and high-tech companies in manufacturing or services in Israel wishing to hire underrepresented groups at high salaries or to accept them for internships so they can acquire job experience. The aid is granted through participation in the employees' salaries at a diminishing rate over a prescribed period of employment.

- A plan to encourage the integration of youths at risk, released convicts, and people with disabilities in social enterprises. 


\section{( Preparatory programs for the world of work for people with disabilities (SDG 8.5)}

A transition program aims to bridge the gap between the two main stages of life: from students and schooling to adult life and vocational training or working. The outcome-oriented and multi-system personal intervention program enables adolescents/ young adults with disabilities to chart their own meaningful career paths, thereby promoting their participation in community life. The program goal is to create a continuous career-development process ending with the completion of the adolescents' educational frameworks, their independence and the acquisition of tools to manage their own lives, including personal growth, knowledge of the working world, and practical experience. It is aimed at the 16-21 age group, characterized by a physical and/or sensory disability or by a complex learning disability affecting cognition (borderline intellectual functioning). The program is the fruit of cooperation between the Ministry of Labor and Social Affairs (MOLSA) and the Ministry of Education.

During the transition, students are provided with personal and group programs during or after school, built on partnerships with youth, parents and teachers. The content includes the development of personal awareness and self-direction to facilitate making choices and integrating into a meaningful vocation at the end of the program; consolidation of a practical, feasible body of knowledge through theoretical learning to help participants integrate into the working world; and practical experience of the working world while consolidating performance skills.

Each year of participation is built on three main layers:

1. Personal growth combines the concept of a vocational identity lan individual's perception of self and their professional desires and ambitions) and self-direction (skills, knowledge, abilities and beliefs that facilitate the choice of an area to pursue);

2. Knowledge of the working world expands an individual's knowledge of the world of work with the help of workshops on theory, observations, interviews, analysis of activities, participation in feedback, and problem solving. This layer also includes the exposure of participants to the range of possibilities open to them at the end of their studies and their examination. It enables them to contact the future framework of their choice and to be equipped with the knowledge to utilize the relevant community services;

3. Practical experience in order to promote 11 practical skills (work habits) through the experience of working in the community, feedback, and learning about the following areas: safety, acceptance of authority, teamwork, initiative, conduct, appearance, perseverance, attendance and adherence to a schedule, quality of performance, effectiveness of performance, and the work environment.

The transition programs include two types: Firstly, as part of the school, the program is aimed at students in special education or in schools for students with a specific disability. Secondly, a specialized integration program is aimed at meaningful integration into the Israel Defense Forces (IDF) or National Service. With the help of professionals, it prepares participants enlisting in, or volunteering for the IDF, providing support throughout their army service and preparing them for their eventual discharge and independence.

\section{Activities of the National Commissioner for Equal Employment Opportunities (SDG 8.5, 8.8)}

The Equal Employment Opportunities Commission (EEOC) was established in 2008 under the MOLSA. Its vision is to serve as a propeller of equal opportunity in the labor market, and it establishes partnerships to optimally utilize the resources it commands. Its aim is to spearhead the process of integrating equality and eradicating discrimination in the labor market based on the belief that employment security is a key to a life of independence, personal empowerment, and the empowerment of others, all of which are vital to a just and stable society. 
GOAL 8

Figure 8.5

Unemployment rate: proportion of the labor force that is unemployed, persons aged 15-74, 2012-2017

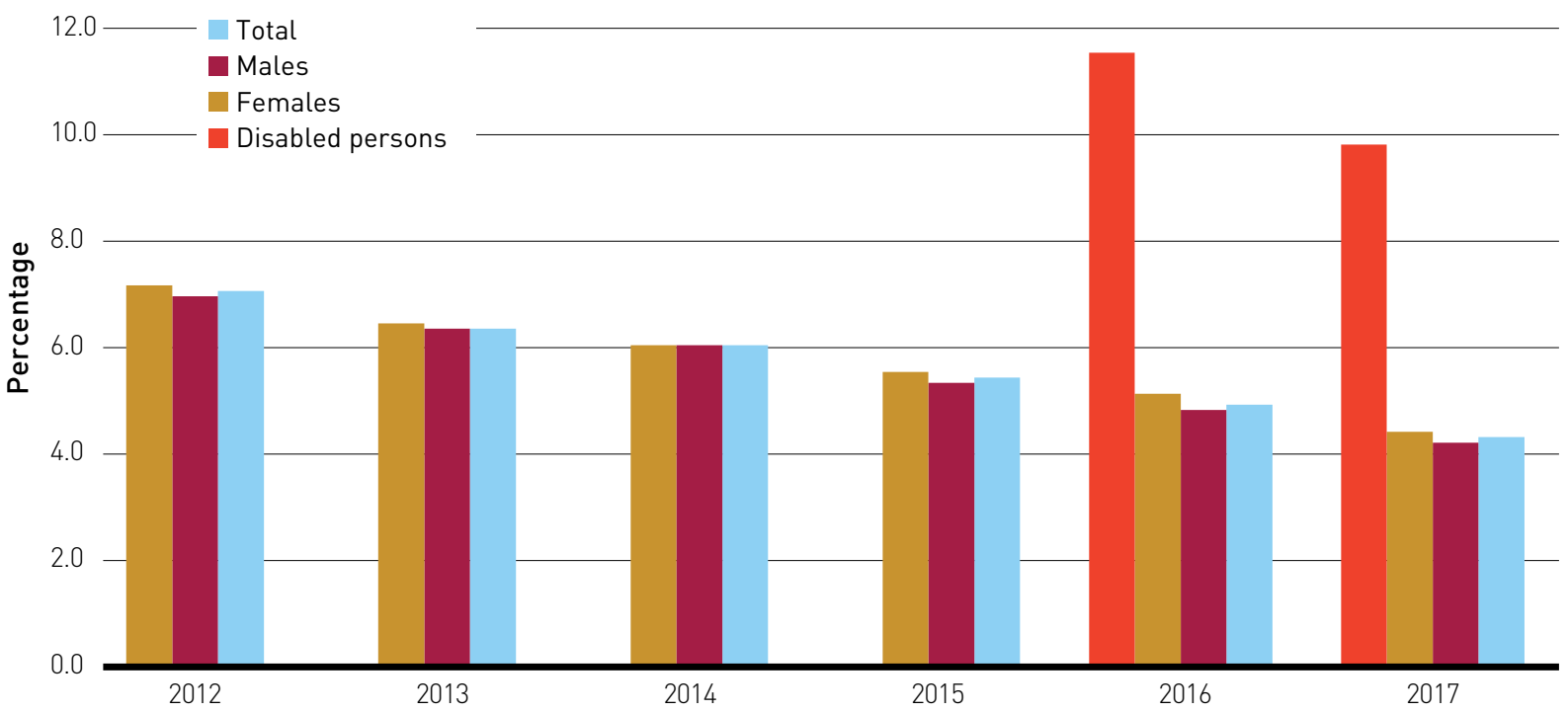

Source: OECD data

Figure 8.6

Persons aged 15-24 not in employment, education or training, 2012-2017

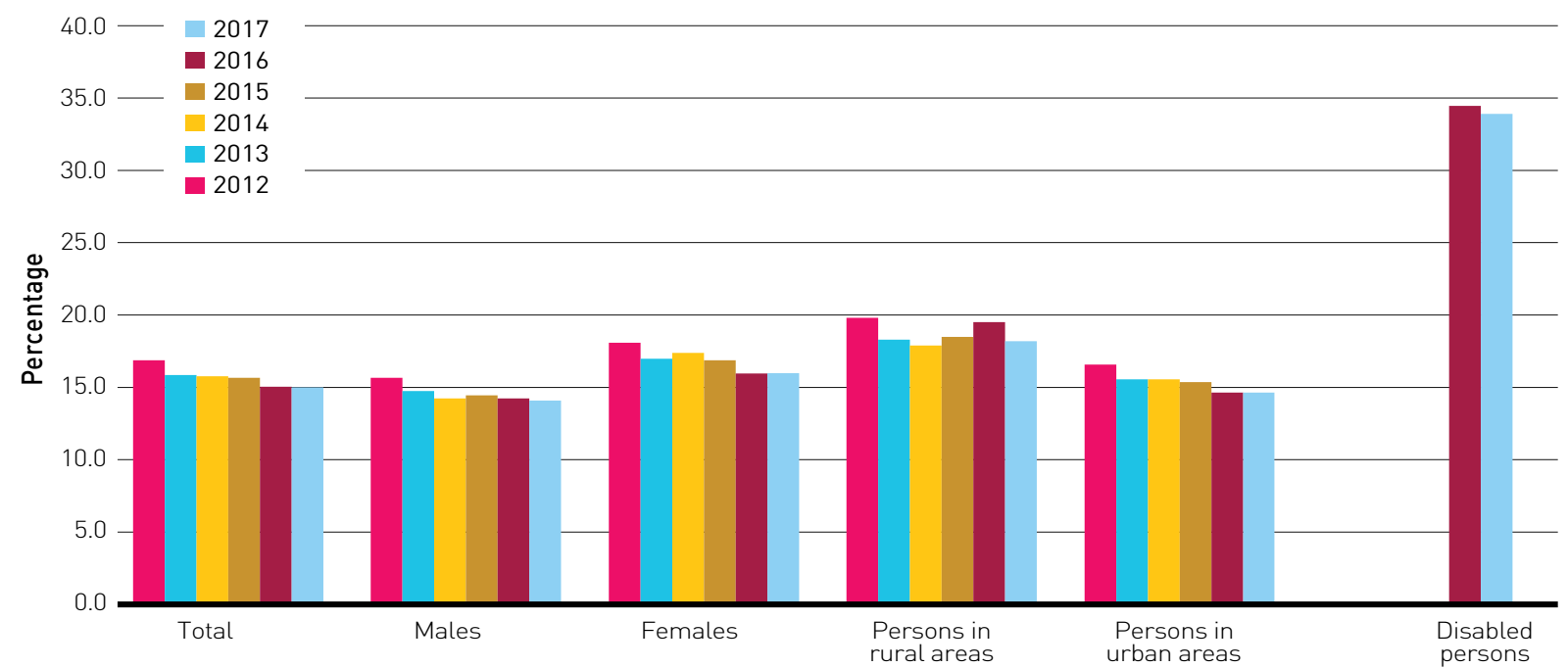

Source: OECD data

The Commission is charged with the enforcement of equality in civilian employment. Its authorities are varied, both legal and informational: to process employee complaints about workplace discrimination, including the clarification of given situations and determination of suitable conduct; to issue injunctions instructing employers to provide data concerning the fulfilment of their obligations under the Employment (Equal Opportunities) Law; to demand that the various bodies subject to oversight provide the information necessary to the Commission in the fulfilment of its duties; to 


\section{CASE STUDY}

\section{Preparatory Program for the World of Work for At-Risk Youth: Meitar-Susan's House}

Programs for preparing at-risk youth for the world of work are essential for imparting life skills and creating better opportunities for integration into the labor market. To be successful, these programs should be holistic, incorporating a range of elements and providing a comprehensive response to different needs. In the long term, these programs are intended to improve the transition from school to work, to give at-risk youth better opportunities to enter the labor market, and to provide them with tools to cope with all aspects of young adulthood (completing their education, beginning full-time employment, starting a family, moving into their own home, etc.) (SDG 8, indicator 8.5.1). Meitar-Susan's House (MSH), which is implemented by MOLSA's Youth Rehabilitation Service, is an example of this type of program.

MSH is a rehabilitation-oriented occupational program for adolescents aged 15-18 on the disengagement spectrum (from high school students characterized as hidden dropouts to adolescents who are not in any organized framework at all). MSH is a short-term program designed to provide an opportunity for paid work as a means of rehabilitation. The program is implemented by the multidisciplinary staff of MSH and the participants learn to manufacture and market a variety of handicrafts and artwork. They gain experience in various units in the workshop, each of which specializes in a different craft, such as glasswork or beads. Here they learn the trade from artists and artisans, as well as life skills, soft skills and business basics. The MSH model provides individually tailored employment programs to prepare the participants to re-integrate into normative frameworks in the community.

MSH was established in Jerusalem in 2002 and has around 50 participants each year. Since its founding, some 500 adolescents have participated in the program. In 2014, a branch was established in Eilat.

submit legal suits to the Labor Court on behalf of petitioners and/or on its own behalf; to apply to the Labor Court for an injunction directing employers to take general measures concerning their employees; and to undertake information and educational activities to raise public awareness of equality and discrimination in the labor market and all that these entail.

\section{Legal activities}

- Public applications: The Commission deals with employee, individual and other requests to obtain or submit information about employment discrimination and inequality. In 2017, it received 766 requests on discrimination, an $8 \%$ increase over 2016. Since its establishment in September 2008 , the Commission has received a total of 6,821 requests on the topic.

- Litigation: The Commission has the authority to carry out civilian enforcement of the Employment (Equal Opportunities) Law. The Commission handles 700-800 applications annually, some of which develop into legal proceedings. In choosing the files or procedures for intervention, the Commission considers the goals and aims defined in its strategic plan, the areas of its responsibility and authority, as well as the precedence-setting value and implications of a legal question. Moreover, with respect to discrimination complaints emanating from employees of government ministries, the Commission is obliged to exercise 
its authority in an intra-governmental procedure vis-à-vis the Attorney-General. In 2017 the Commission was involved in 14 legal files and procedures, five of which are pending. Four of the 14 cases revolved around ethnic discrimination, four around gender, and another three around ageism. Three more cases related to discrimination - one case of employee pregnancy, one of origin and one of place of residence.

- Legal opinions: The Commission's legal opinions serve diverse parties in the political, legal and social arenas seeking precedential decisions on employment equality. In 2017, the Commission formulated and disseminated opinions on various topics. The Commission also presented bills, thereby contributing to the development and expansion of the discipline of jurisprudence on equal opportunity. On the information level, it contributed to the instilment of the value of equality and to changing thought patterns in the private and public labor markets.

- Legislation and parliamentary activity: The Commission actively follows the Knesset's (Israel parliament) legislation and deliberations in ministerial committees to ascertain that employment equality and integration of the different populations into the labor market are taken into consideration. In 2017, it participated in some 36 committees, submitted some 35 comments to bills presented in the committees, and took part in more than 22 discussions. The committees included the Status of Women and Gender Equality; Labor, Welfare and Health; Immigration, Absorption and Diaspora Affairs; Economic Affairs; and the fair representation of Arabs in the Civil Service.

\section{Information and awareness raising}

On the information level, the Commission helps instill the value of equality and contributes to changing thought patterns in the private and public labor markets. It has launched a website and a Facebook page for workers, providing access to information on discrimination, relevant legislation, legal rulings, research and surveys, legal opinions and affirmative action in Civil Service. The website also includes a section for employers with information on training courses for professional diversification and other services offered by the Commission, relevant publications, rulings and legislation, and a campaign on equality. The Commission also organizes panels, conferences and lectures to disseminate knowledge on equal opportunity in employment and provide information on its work.

Encouraging and promoting equality in the labor market: The Commission cooperates with employers and NGOs le.g., in 2017 it took steps to advance the Haredi, Israelis of Ethiopian descent and Arab populations in the labor market and to prevent discrimination); with academia (e.g., the legal clinic of Sapir College); and with ministries and public bodies through private applications by third sector employers and through the provision of opinions and consultation on equal opportunity/ discrimination in employment.

\section{(Activities related to workers' rights (SDG 8.8)}

MOLSA's Employment Relations Unit derives its authority from the Settlement of Labor Disputes Law 1957 and the Collective Agreements Law 1957. The unit is responsible for providing public information on labor laws and endorsing collective agreements and expanded regulations. It also serves as an intermediary in disputes, and deals with such topics as strike data, expanded regulations, collective agreements and labor arbitration.

Its functions include the following:

- Preventing labor disputes and settling them through mediation and arbitration;

- Registering special and general collective agreements;

- Initiating regulations expanding general collective agreements in order to apply the instructions of the agreements to non-organized employees and employers;

- Providing confirmation under the Severance Pay Law 1963 of payments paid into a pension/saving plan in lieu of severance pay and confirmation 
of the inclusion of salary in the compensation for dismissal;

- Initiating and encouraging surveys and applied studies on work relations to identify foci of tension in the system of work relations and understand the processes of the system;

- Providing consultation to citizens and employers on topics related to the labor laws;

- Disseminating public information pamphlets on topics related to the labor laws and providing access to this information on the Internet;

- Handling labor legislation and coordinating between the ministry and the system of labor courts.

Employee rights in the area of work relations and employer obligations to the employee are anchored in labor laws, collective agreements or expanded regulations, as well as in personal employee contracts.

In addition to an employee's right to file a suit for an infringement of rights at the workplace, in certain cases punitive measures may be imposed on an employer and, in some cases, on the service provider as well.

Punitive measures under the labor laws include three types: administrative - imposed by MOLSA's Enforcement Division within the Regulation and Enforcement Administration; civil sanctions the labor court has the authority to rule that an employee is to receive compensation regardless of proof of damages; criminal punishment - alleged offenders must be tried in criminal court and an indictment submitted against them.

\section{PARTNERING FOR A BETTER WORLD}

Israel was among the first countries to ratify the International Labor Organization (ILO) Protocol of 2014 to the Forced Labor Convention 1930, as its commitment to international efforts to combat and eradicate all forms of modern slavery, including child labor, human trafficking, and recruitment and use of child soldiers. Israel believes that preventing forced labor is a core value in protecting human rights and dignity. The Protocol sets forth new tools that complement and strengthen existing practices, while affording new strategies to effectively address these issues. Israel is also finalizing the internal process leading to joining the European Council Convention on Human Trafficking. (SDG 8.7)

Israel maintains extensive contact with the ILO. In 2018, during a visit of the ILO Director General to discuss future avenues of cooperation, he met with high-ranking officials in Israel's General Federation of Labor (Histadrut). The International Department reinforces bilateral relations with confederations of trade unions around the world and their affiliated trade unions, with the umbrella organization International Trade Union Confederation (ITUC) and the regional organization (ITUC-AP), in which the
Histadrut serves as a senior member of the Council. It also encourages member unions to join global federations of workers, establish contacts with parallel unions around the world, and engage with the Trade Union Advisory Committee (TUAC) to the Organization for Economic Cooperation and Development (OECD). (SDG 8.8)

Within the framework of the ILO Strategy and Action Plan for Inclusion of the Roma, Ashkali and Egyptian Communities in Kosovo Society (2016-18), Israel's Ministry of Labor and Social Affairs contributed $\$ 300,000$ towards assisting local public institutions and social partners in implementing a project on Promoting Decent Work Opportunities for Roma, Ashkali and Egyptian Youth in Kosovo. (SDG 8.3)

Furthermore, the Ministry of Labor, the FriedrichEbert-Stiftung Israel, the ILO, and the Israeli Industrial Relations Research Association (IIRRA) held their annual conference in Israel on the topic Working World on the Move - a Blessing? The conference focused on issues including immigration and the global phenomenon of brain-drain. (SDG 8.8)

An annual capacity-building course titled Working Youth, Trade Union Self-Reform and Decent Work 


\section{CASE STUDY}

\section{Entrepreneurial Training for Youth in Latin American and the Caribbean}

Combating poverty among young people through business training and innovative entrepreneurship based on the "Business Labs" concept is the subject of a project spanning over 20 countries across Latin America and the Caribbean. The project is carried out in cooperation with the Young Americas Business Trust (YABT), a nonprofit corporation associated with the General Secretariat of the Organization of American States (OAS). The training model follows a "learning by doing" methodology that introduces fundamental concepts of innovative entrepreneurship and support systems for new entrepreneurs, linked with follow-up training in a variety of forms. The underlying idea is that young people will learn more effectively by gaining skills through experience than through presentation alone.

The project is implemented by means of intensive training workshops held both in Israel and in the beneficiaries' countries, with emphasis on the following:

- Combining classroom knowledge with practical skills in microenterprise training for teachers and students

- Introducing entrepreneurial skills through hands-on experience to enable adaptation to different target populations

- Providing tools and methodologies of business management

- Training to act as "entrepreneurial facilitators" to encourage and prepare young people for economic independence

- Simulating microenterprise creation to function as a small company

- Preparing and presenting a project proposal relevant to the participants' own settings

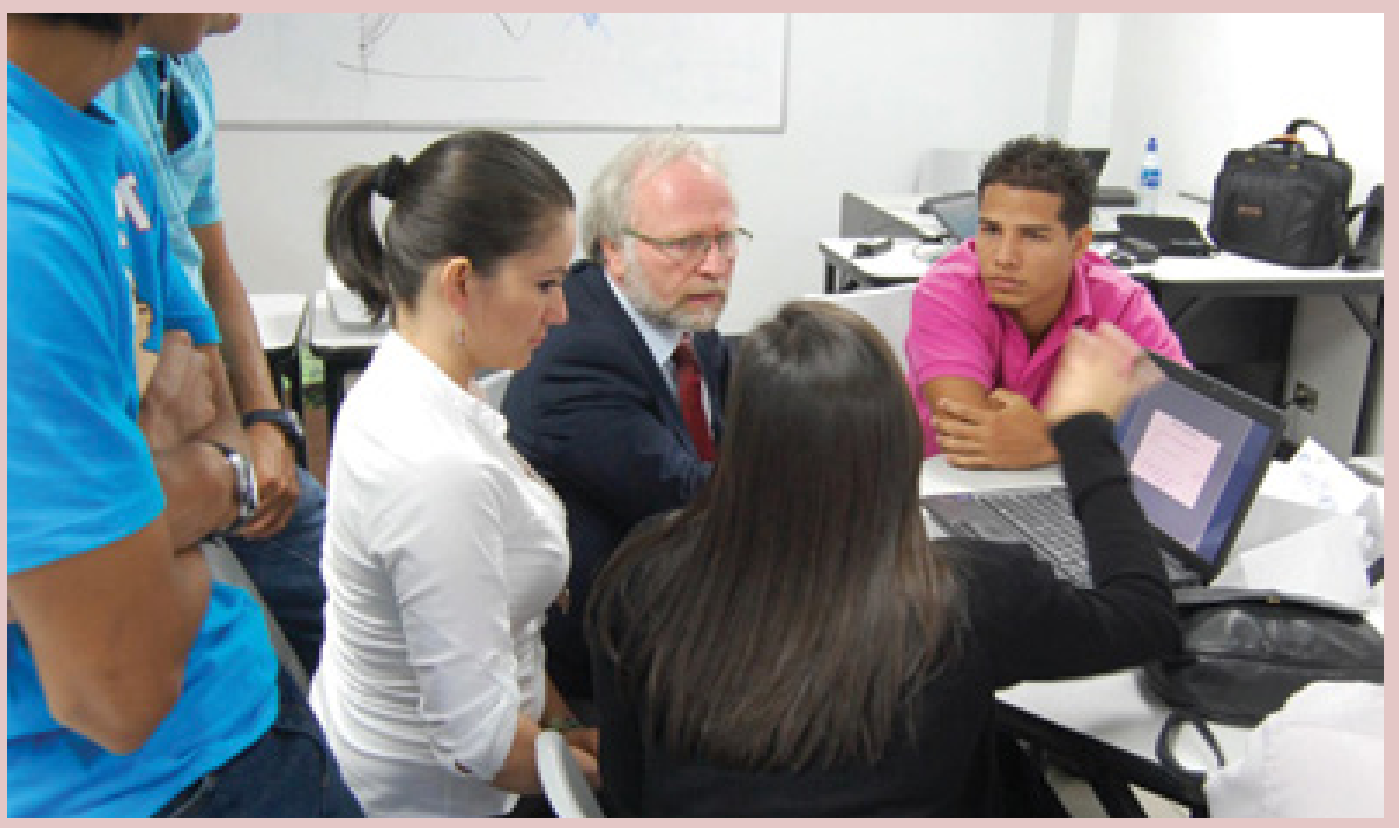

Source: MASHAV 
is conducted by the Histadrut and MASHAV, in cooperation with the Trade Union Confederation of the Americas (TUCA) and the Americas Branch of the International Trade Union Confederation (ITUC). The target population is young leaders from Latin American trade unions. The program focuses on youth and the development of decent work values and reforms, in accordance with international norms and standards. (SDG 8.3)

Israel collaborates with the ILO and the UN Economic Commission for Europe (UNECE) in promoting development cooperation policies aimed at increasing economic growth by creating decent jobs, specifically for women and youth. One program is the Young Americas Business Trust (YABT), based on the long-term relationship between MASHAV and the Organization of American States (OAS). The program fosters innovation and entrepreneurship to promote the social and economic development of young people by supporting youth ventures, especially those working on poverty reduction and the inclusion of young people in the workforce. Training aims at building entrepreneurial capacities through the Business Labs model geared to create opportunities for young future business leaders. MASHAV and the YABT are working together in 29 states in Latin America and the Caribbean, with the participation of approximately 30,000 young people. (SDG 8.6, 8.3)

Representatives from different government ministries in Israel, including the Israel Central Bureau of Statistics, participate in multilateral working groups on labor related issues, as follows:

\section{(SDG 8.8, 17.18)}

- ILO: Israel chairs the Working Group on Measurement of Labor Mobility; Revision of the International Classification of Status in Employment; Measuring Labor Migrants; and Labor Migration Statistics;

- UNECE: Task Force on Measuring Labor Mobility; Meeting of Experts on Measurement of Quality of Employment + Steering Group; Data Confidentiality Work Session; Modernization of Statistical Production Workshop; and Roadmap for Implementing Standards;
- OECD: Advisory Group on Guidelines for Measuring the Quality of Working Environment; Voorburg Group on Service Statistics; Meeting of Providers of the OECD Income Distribution; and Expert Group on Distribution Information on Household Income, Consumption and Savings Within SNA Framework;

- MEDSTAT: Labor Statistics.

The Center for Learning Cities in the Israeli city of Modi'in participates in projects to promote employment opportunities for people aged $40+$ in the framework of the Erasmus+ Program for Education, Training, Youth, and Sport. Together with colleagues from Italy - the city of Udine and Universita della Libereta - a joint project was launched in partnership with the Friuli-Venezia-Gulia Region on Developing Soft Skills and Capacity-Building in Employability and Entrepreneurship. (SDG 8.3) Israel's Special Envoy to Public-Private Partnerships in the Ministry of Foreign Affairs was elected (2018) to the position of Vice-Chair in the UNECE Committee on Innovation, Competitiveness and Public-Private Partnerships. Israel chaired the Committee from 2016-2018 and supported the Ideas4Change Innovation and Entrepreneurship Competition in 2015 and 2017. In 2016, Israel supported the UNECE Conference on Startup Nations: Innovative Entrepreneurship for Sustainable Development and played an active role in the Expert Group on Public-Private Partnerships. Israel is a member of the UNECE Team of Specialists on Public-Private Partnerships and is a co-author of the Standards on PPP in Health and the People-First Initiative on PPP. (SDG 8.3, 17.17)

An Israeli expert is a member of the team compiling a report on Blockchain Technologies and the SDGs and their use in trade facilitation processes. The report will be published in 2019 by the UN Center for Trade Facilitation and Electronic Business (CEFACT), an intergovernmental body serving as a focal point within ECOSOC for trade facilitation, recommendations and electronic standards. (SDG 8.a)

Israel is represented in the International Telecommunication Union (ITU) Focus Group on 
Application of Distributed Ledger Technology (FG DLT). Currently, an Israeli expert on blockchain innovations is the Group Champion for Sustainable Development Goals: Use Cases. Israel is one of the first four countries (USA, Switzerland, UK) to establish academic blockchain research institutes. The Hogeg Blockchain Institute, Tel Aviv University, will host a UN Working Group on Blockchain Application in 2019. (SDG 8.2)

Green growth is the new innovative development paradigm that sustains economic growth while ensuring climatic and environmental sustainability. Given the centrality of natural assets in lowincome countries, green growth policies can reduce vulnerability to environmental risks, increase livelihood security and help economies and societies become more resilient. MASHAV's international training program on Green Growth: Policy and Economic Measures as Tools for Local and Regional Sustainable Development presents new policies and tools on the costs and benefits of environmental and social impacts, as well as strategies for achieving sustainable consumption and production. (SDG 8.4)

Tourism provides economic value, creates new sources of income and fosters cultural pride and awareness of the environment. Currently, Israel is in the process of evaluating the text of the UN World Tourism Organization (UNWTO) Convention on Tourism Ethics, the first Convention of the UNWTO approved (2017) during the 22nd General Assembly in China. (SDG 8.9)

Numerous examples reflect Israel's endeavors on the international/regional scene to promote sustainable tourism that creates jobs and promotes local culture and products. (SDG 8.9)

In 2018, Israel's Minister of Tourism hosted the Chair of the World Committee on Tourism Ethics (WCTE) at the 24th International Mediterranean Tourism Market (IMTM). During the fair, six Israeli private sector tourism associations and enterprises signed the Private Sector Commitment to the UNWTO Global Code of Ethics for Tourism in the presence of the minister and the WCTE Chair.

Between the years 2017-2018, Israel's Ministry of
Tourism conducted four onsite institutional capacitybuilding seminars on Digital Transformation in Managing, Training, and Marketing Tourism in Tanzania, Rwanda, Guatemala, and Honduras, in partnership with relevant government offices in the host countries. Two more seminars (Uganda, Ethiopial are planned for 2019.

MASHAV's program on Innovations in Tourism shares Israel's experience in ecotourism and sustainability, the social impact of rural tourism, integration of women in rural enterprises, and building support systems for small entrepreneurs with professionals from developing and least developed countries.

Four Israeli enterprises - part of the Israel Travel Tech Startups (ITTS) community - made it to the finals of the 1st UNWTO Startup Competition (2018), in collaboration with Globalia, Spain's largest tourism group. Innovations presented included: saving costs on travel accommodations by tracking fluctuations in hotel prices; an interactive videobased travel planner; customer communication platform; and an app that helps tourists with value added tax (VAT) refunds - "Refundit." In the final phase of the Competition held within the framework of the FITUR International Tourism Fair (2019), "Refundit" was declared the winner, topping 3,000 entries from around the world. 


\section{CASE STUDY}

\section{Horticulture Business Development Project in Ukraine}

As part of a trilateral cooperation between Israel, Ukraine and Canada, MASHAV, MEDA (Mennonite Economic Development Associates), Global Affairs Canada and UHBDP (Ukraine Business Development Project) are carrying out a project to increase income generation and rural economic development for small and medium-scale farmers, agribusinesses and small enterprises in four regions in Southern Ukraine. Within this framework, MASHAV provides training and capacity building to enhance horticultural production and business and entrepreneurial know-how. Emphasis is placed on environmental sustainability and gender equality strategies to provide agriculturalists and small-medium business owners with opportunities to advance, pursue and benefit from sustainable horticulture business development.

The project, which has reached over 44,000 women and men, is accompanied by Israeli and Ukrainian specialists on crop protection and fertilizer application, modern agro-technology, mechanization, business management and business psychology. Implementation of the project includes:

- Providing quality training and consultancies to extend and expand capabilities in the horticulture value chain

- Developing networking and business skills

- Linking smallholder farmers to higher value markets through aggregation techniques

- Improving production techniques and technology to facilitate supplier relationships

- Improving post-harvest handling capabilities: produce cleaning, grading, sorting, packaging; traceability; food safety and quality assurance; cold chain storage, transport and logistics

- Aligning university level experience with capabilities demanded in an entrepreneurial market

- Developing new curricula to enhance the competencies of a college-educated entry-level workforce to horticulture industry needs

- Involving university students in internships and short-term work experiences

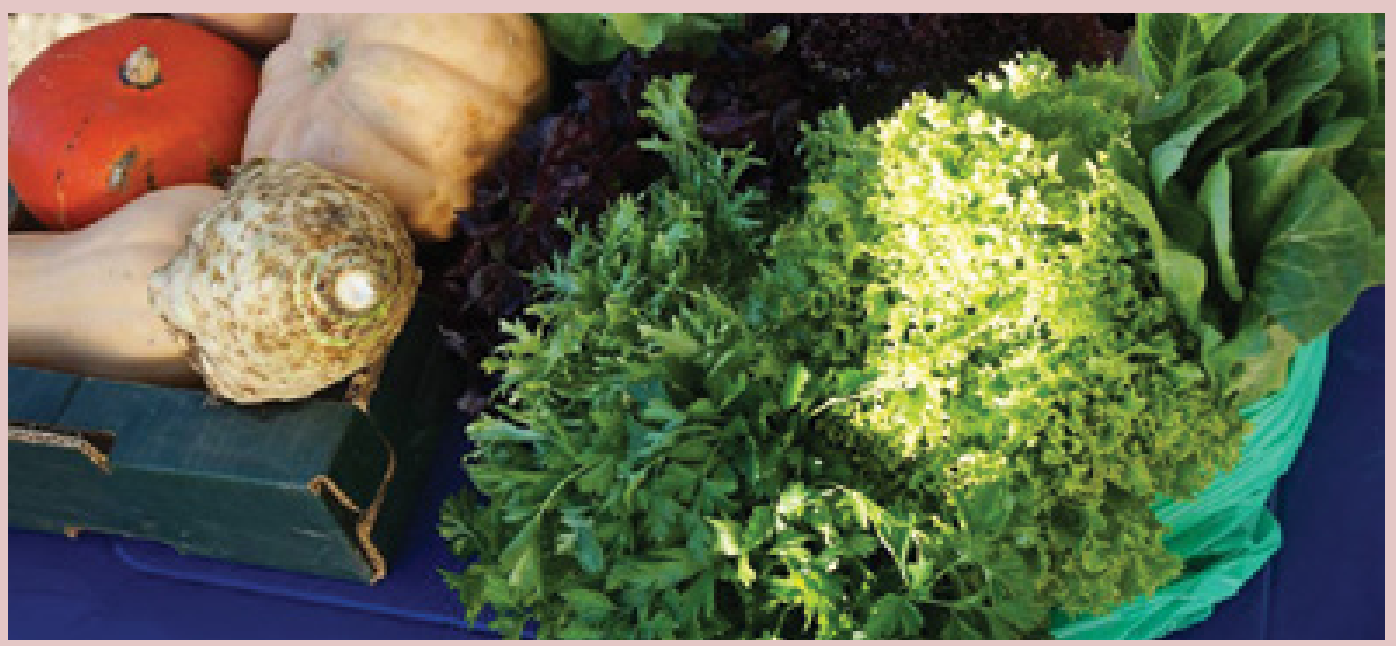

Source: MASHAV 


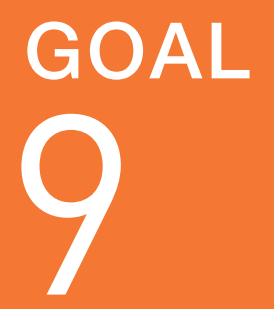

\section{INDUSTRY, \\ INNOVATION AND \\ INFRASTRUCTURE}

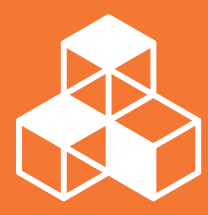

Development of industrial zones

Promoting proper business conduct

Promoting gender inclusivity gender analysis by budget characteristics

Catalyzing innovation across sectors

Paving the way for environmental technologies

Applied support of research institutes

Promoting long-term knowledge infrastructures

Access to information and communications technology

Partnering for a Better World 


\section{BUILD RESILIENT}

INFRASTRUCTURE, PROMOTE INCLUSIVE AND SUSTAINABLE INDUSTRIALIZATION AND FOSTER INNOVATION

Israel has earned the name of "Start-Up Nation" following the success of many Israeli start-up companies which positioned themselves in the international market as making breakthroughs in numerous fields, including communication, internet, medical systems, agriculture, biotechnology, security, water desalination, digital printing and more. The quality of the human capital, entrepreneurial culture and bold innovation spirit, along with government commitment to support breakthrough research and development (R\&D) activities, position Israel at the global front of technological entrepreneurship. According to the World Economic Forum's 2018 Global Competitiveness Report, Israel has one of the ten strongest innovation ecosystems in the world. Israel has the largest number of startups per capita in the world, including more than 2,000 which were founded in the past decade. Israel is also home to more than 350 R\&D centers of some of the world's largest multinational corporations, such as Intel, Microsoft, Apple and Google. 
Israel's emergence as a global leader in technological innovation and entrepreneurship was made possible by the cooperation between government, academia, industry, the business sector, non-profit organizations and civil society. This cooperation makes possible the consolidation of infrastructure and access to knowledge in different disciplines and continues to be a policy priority.

Israel considers the development of industrial zones to be a growth engine for the Israeli economy, and therefore encourages local authorities to establish and develop thriving industrial parks and to motivate new entrepreneurs to establish enterprises that will provide local residents with new jobs.

\section{( Development of industrial zones (SDG 9.1, 9.2)}

Various policy tools are implemented to promote thriving industrial zones in Israel. Some of them focus on Israel's periphery regions and on supporting local authorities in these regions. Key tools include:

\section{- Five-Year Plan for the Planning and Construction} of Industrial Zones in the Periphery: To manage the inventory of developed space and future land reserves for industrial entrepreneurship nationwide, an economic model was drawn up, under which it is possible to see supply and demand, together with the parameters of building rights, to plan over the next five years, and to respond to structural, environmental and technological changes in the industry.

\section{- Specifications for the development of} sustainable industrial zones: In order to assist industrial zones to improve their environmental performance while encouraging development and the use of income-producing advanced technology and operational and financial efficiency, a statement of planning principles for the sustainable development of new industrial zones has been prepared.

- Arab local authorities: In order to transform Arab local authorities into thriving and attractive sites for potential enterprises and employers, Israel is upgrading these local authorities through investment in infrastructure and provision of administrative tools for industrial zones.

- Development of industrial zones in the north: In order to manage the inventory of developed space and future land reserves for industrial entrepreneurship in the north, a policy document was prepared on available land and

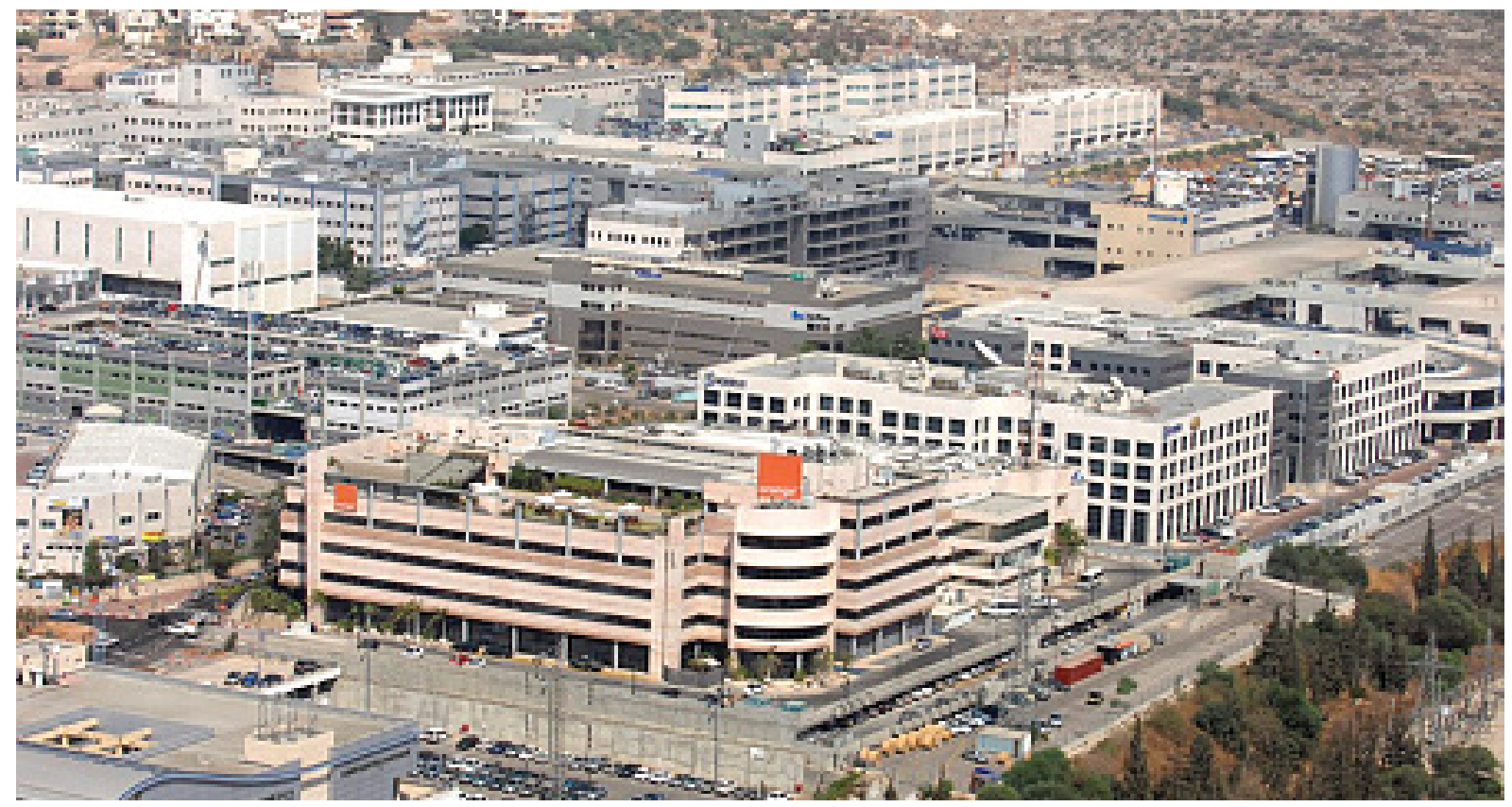

Aerial view of the Afek Industrial Park/Photo: Moshe Milner, Government Press Office 
the requirements for economic progress in the Northern District, on the basis of expected population growth by 2040 , in accordance with the Israeli government's Strategic Housing Plan. The report outlines the principles for the development of industrial zones, including prioritizing the expansion of existing industrial zones over the construction of new ones, taking care of different sectors of the population and their special needs, and setting guidelines for the planning and development of industrial zones with reference to the Fourth Industrial Revolution and the needs of the remote periphery.

- Promotion of regional industrial zones: In order to provide jobs for thousands of local residents and revenues for participating local authorities on the principle of distributive justice, the construction of joint industrial zones for several towns in the same geographical area is encouraged.

- Marketing of land for industry and sharing in the cost of land developments: In order to encourage the development of industrial zones in areas remote from central Israel, and to encourage entrepreneurs to establish enterprises in these regions, the Ministry of Economy and Industry is developing industrial zones and subsidizing the land development costs to lower the cost of the land purchased by the entrepreneurs.

\section{( Promoting proper business conduct (SDG 9.2, 8.7, 12.6)}

Israel is promoting the OECD 2011 Guidelines for Multinational Enterprises and has established a special unit, the Proper Business Conduct Unit at the Ministry of Economy and Industry, which serves as Israel's national contact center for the guidelines. The OECD corporate responsibility guidelines for multinational enterprises offer nonbinding guidelines for proper business conduct in a global context. As part of its work on promoting the 2011 OECD Guidelines, the Proper Business Conduct Unit's work has assumed responsibility for promoting the export of social innovation and is currently building a work plan and mapping the sector and its potential. The unit's flagship project is the creation of a foundation to encourage Israeli companies to file corporate responsibility reports and prepare a manual for these companies. The unit is also mapping all government activity on corporate responsibility. The Proper Business Conduct Unit is in continuous contact with corporate responsibility stakeholders, the Manufacturers Association of Israel, top companies in the field, consulting firms and civil society organizations.

\section{Promoting gender inclusivity - gender analysis by budget characteristics (SDG 9.2)}

To provide insights on the budgetary allocations of the Ministry of Economy and Industry in terms of gender, two types of analysis were conducted individual support to individuals and support to firms. In the first type of analysis, the results demonstrate that the rate of expenditure on women varies between the different employment programs and tracks, as shown in Figure 9.1, ranging from a few percent to full budget allocation to women. The total rate of individual budget expenditure on women is less than on men, at a 40:60 ratio, respectively.

In the second type of analysis, support budgets to firms were analyzed indirectly in terms of gender. In most instances, the proportion of women in industrial sectors or high-tech, was used rather than data belonging directly to the supported companies because these data are not all available to the government. The results of this indirect calculation indicate that expenditures designated for the support of women are less than for men, at a ratio of 42:58 respectively.

Since there is currently no information from the Investment Promotion Center and the Industrial Zones Administration programs about the rate of men and women employees at supported companies, the gender breakdown in these cases was made on the basis of the general distribution of employees in the industry.

Moreover, in the National Authority for Technological 
Figure 9.1

Distribution of individual employment program budgets, by gender

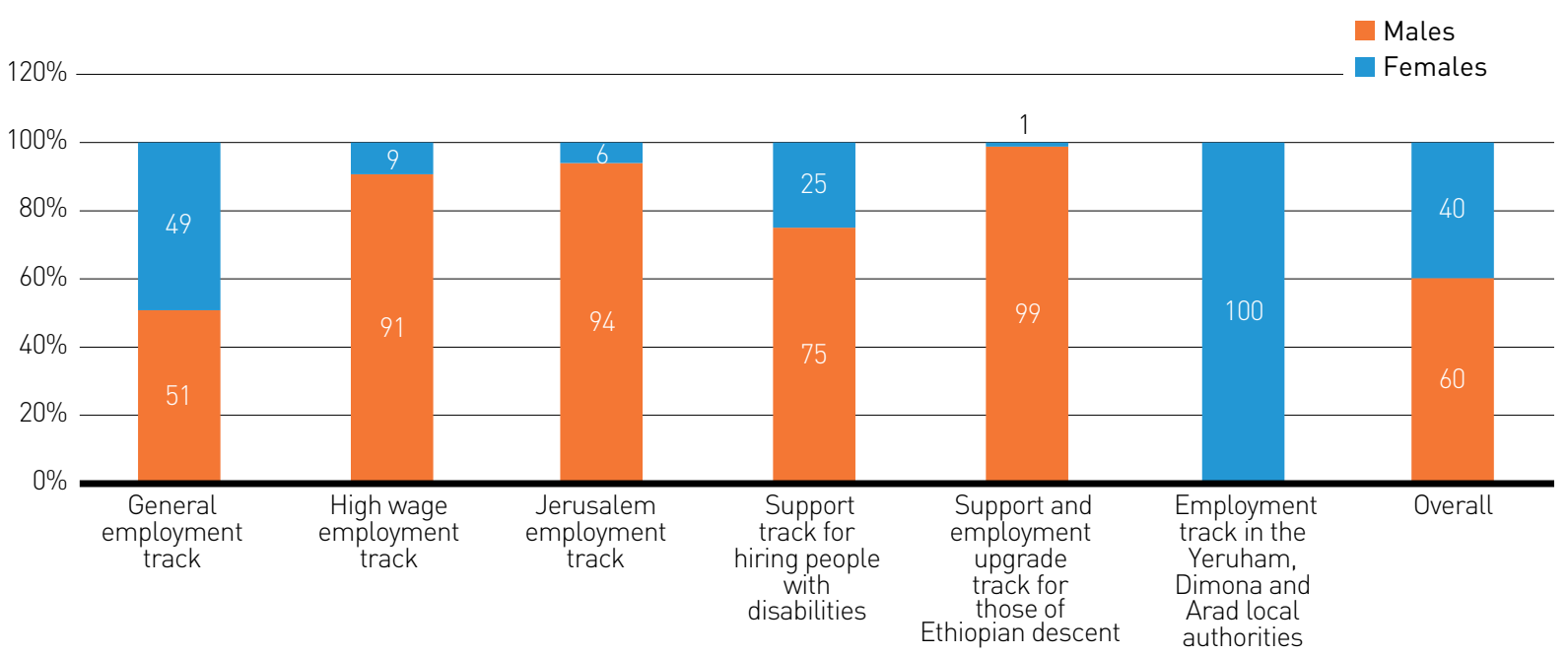

Source: State Budget Proposal, Ministry of Finance

Figure 9.2

\section{Distribution of support budgets to companies, by gender}

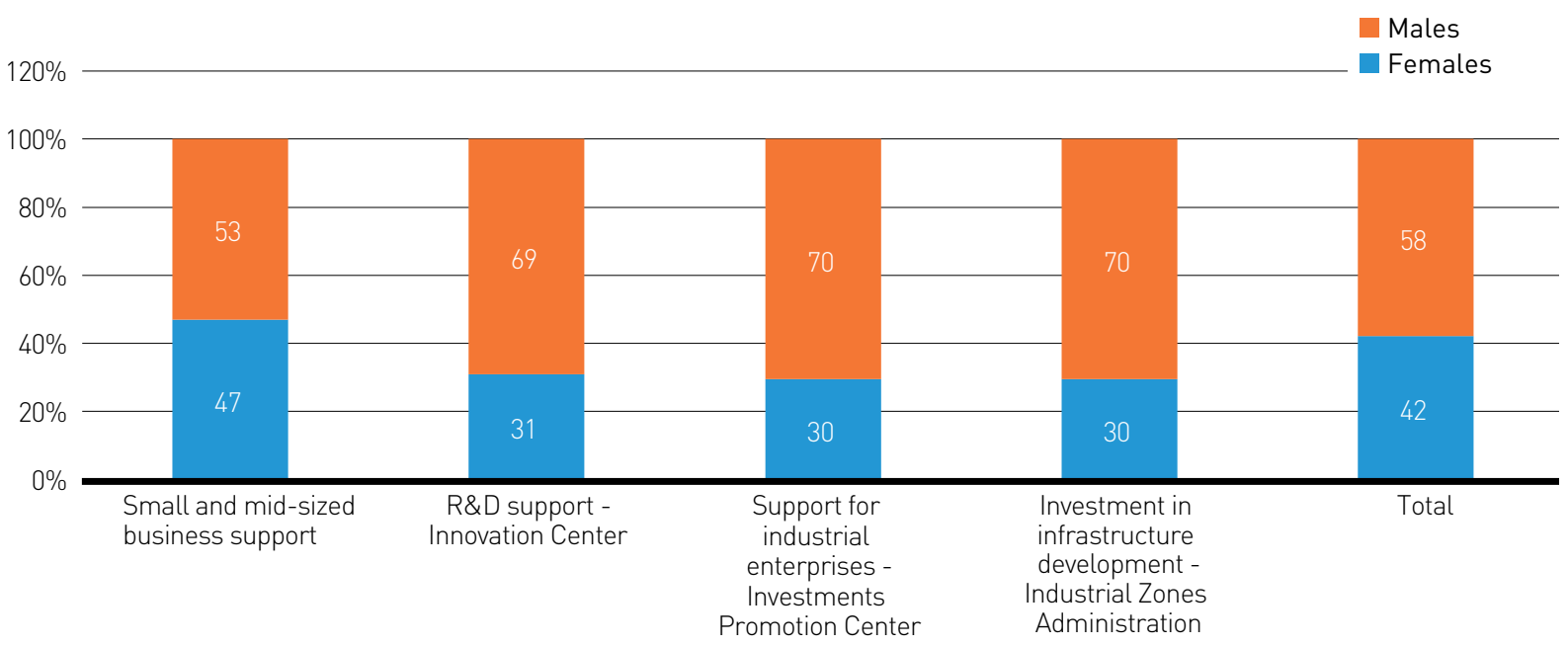

Source: State Budget Proposal, Ministry of Finance

Innovation, which operates a wide range of support programs for industrial R\&D operations in Israel, most of the support is channeled to high-tech companies. Because there is no data about the proportion of women employees in the companies supported by these programs, or about the gender of the companies' owners, the gender breakdown of most of the budget is based on the general distribution of high-tech employees labout 36\% are women). The gender breakdown in the Tnufa Incentive
Program budget and the Technology Incubators Program on the basis of the gender distribution of their participants is $5 \%$ and $8 \%$ women, respectively.

\section{( Catalyzing innovation across sectors (SDG 9.2, 9.5)}

The government supports industry and innovation in industry through multiple tracks and incentive programs. One key track is the collaboration 


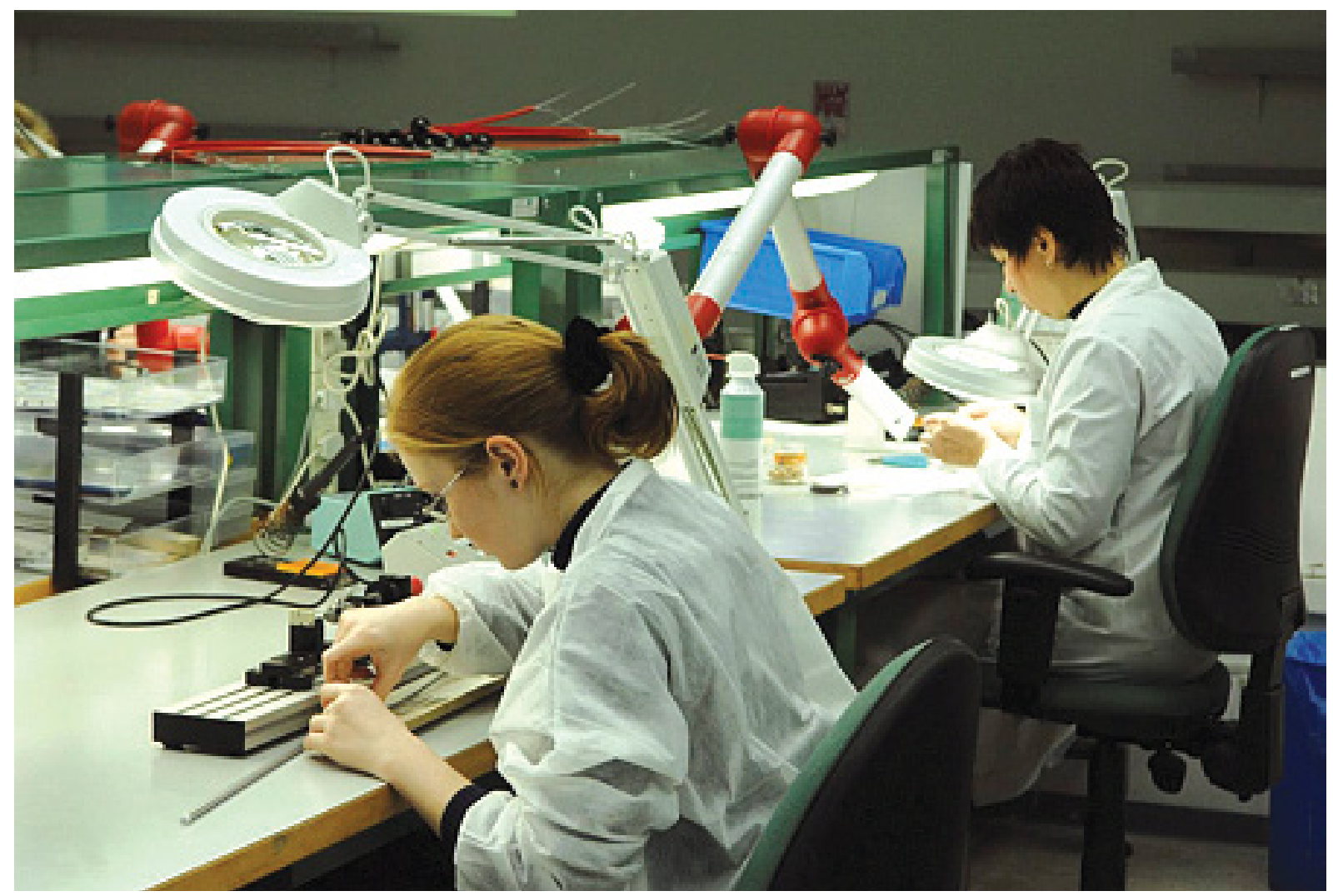

A medical factory in Yokneam in northern Israel/Photo: Amos Ben Gershom, Government Press Office

between the Innovation Authority and various government ministries that enables government efforts to focus on selected fields, including: funding support for high-risk initiatives, supplementary government support tools including dedicated regulatory requirements for pilot tests, and access to government-owned trial sites and facilities. The incentive programs enable Israeli technology companies to receive support for their $R \& D$ programs or trial programs (pilots for assessing feasibility/proving the value of an existing technology in a work surrounding that simulates the target market). The programs encompass selected fields including transportation, environmental protection, digital health, space technologies, cyber, agriculture, energy efficiency and reduction of greenhouse gas emissions and diverse areas under the responsibility of the Government Companies Authority.

\section{Trial programs in the field of transportation}

The program supports trial (pilot) programs in the field of transportation and is operated jointly by the Innovation Authority, the Ministry of Transport, and the Fuel Choices and Smart Mobility Initiative in the Prime Minister's Office. The objective of the new program is the development and application of transportation related innovative technologies and operative perceptions that will advance the field and develop the Israeli high-tech industry with the aim of streamlining and improving transportation, both in Israel and abroad. The incentive program is intended for Israeli technology companies in the field of transportation. The participating companies will receive financial support of between 20\%-50\% of the approved pilot's expenditures. An exceptional support rate of $75 \%$ of approved R\&D expenditures will be awarded to programs with the potential for exceptional impact on streamlining and improving transportation in Israel. Regulatory assistance may also be given by the Ministry of Transport for the implementation of a trial program and receiving relevant designated regulatory delineation. 


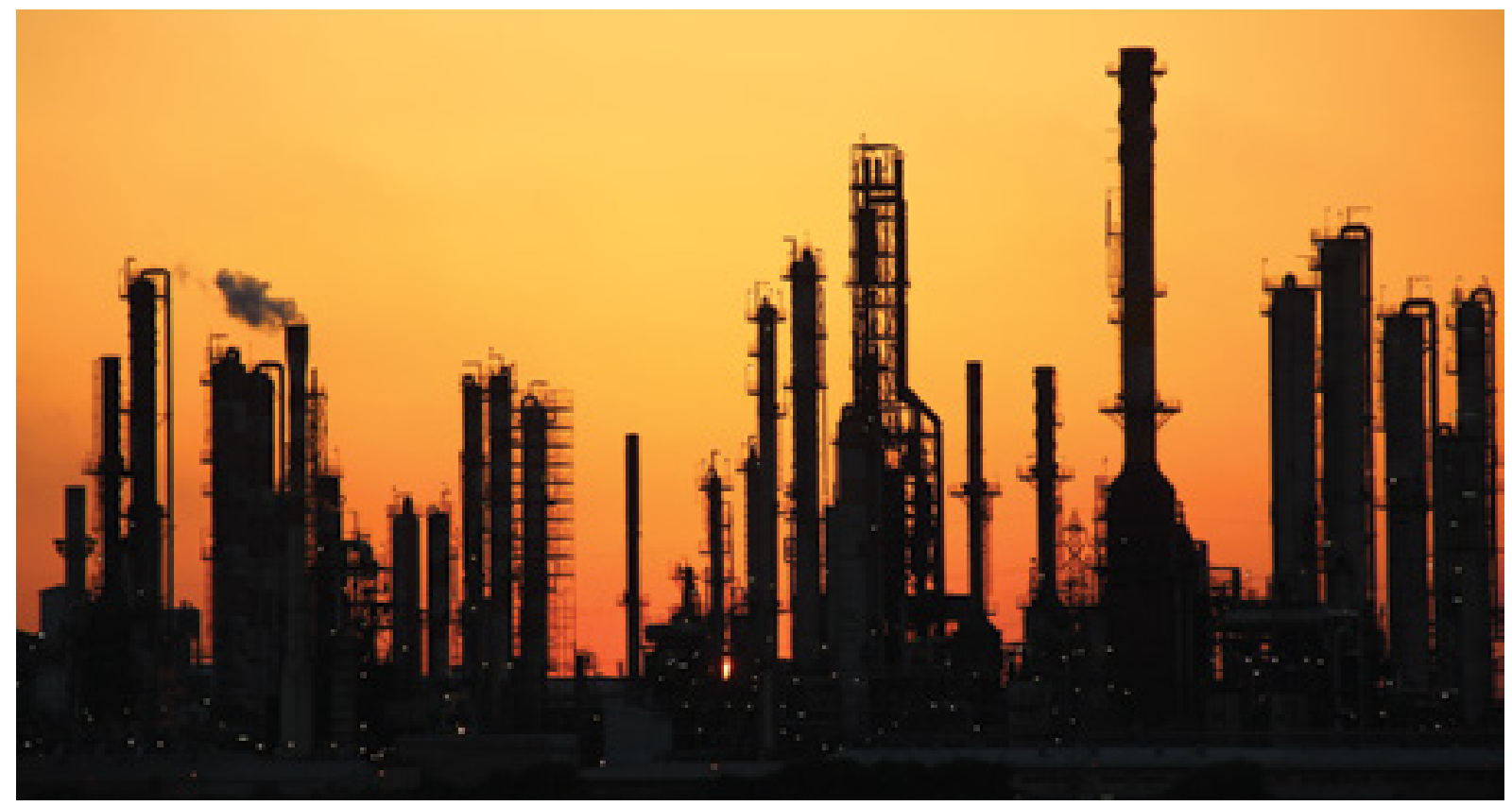

Oil Refineries, Haifa/Photo: Ilan Malester

\section{Trial programs in the field of environmental protection}

The program supports R\&D and trial programs in the field of environmental protection and is operated jointly by the Innovation Authority and the Ministry of Environmental Protection. The program's objective is to develop and implement innovative technologies in the field of environmental protection (cleantech) and evaluate their feasibility on an industrial scale and among relevant clients. Other objectives include: development and implementation of technologies that lead to a reduction in the use of natural resources and/or to a reduction in pollutant emissions and the cultivation of complete and sustainable environmental protection companies in Israel by promoting innovative technological solutions. The incentive program is intended for Israeli technology companies in the field of environmental protection. The participating companies will receive financial support of between $20 \%-50 \%$ of the approved pilot's expenditures. An exceptional support rate of $75 \%$ of approved R\&D expenditures will be awarded to programs with potential for exceptional impact on the environment. Regulatory assistance may also be given by the Ministry of Environmental
Protection in the implementation of a trial program including temporary regulatory flexibility during the trial period.

\section{Trial and R\&D programs in the field of digital health}

The program supports R\&D programs and trial programs in the field of digital health and is operated jointly by the Innovation Authority, the Ministry of Health and the Headquarters for the National Digital Israel Initiative in the Ministry of Social Equality. The objective of the program is to promote the health system and public health, both in Israel and abroad, through the development and application of innovative technologies in the field of digital health and evaluation of their feasibility among health organizations in Israel. Furthermore, this incentive program also encourages the creation and cultivation of complete and sustainable companies in the field of digital medicine in Israel via the promotion of innovative technological solutions. The incentive program is aimed at Israeli technology companies (that are not one of the existing health organizations in Israel) for projects in the field of digital health, to be implemented by the Israeli health organizations, or that are based on their capabilities, figures or data. The 
participating companies will receive financial support of between $20 \%-50 \%$ of the approved pilot expenditures. An exceptional support rate of $75 \%$ of approved R\&D expenditures will be awarded to programs with potential for exceptional impact on the health system and public health, both in Israel and abroad, or that constitute a technological breakthrough in its field.

\section{Trial programs in conjunction with the Government Companies Authority}

The program supports R\&D and trial programs at government companies' sites and is operated jointly by the Innovation Authority and the Government Companies Authority in the Ministry of Finance. The program's objective is to develop and examine technologies which enable the streamlining of the government companies' financial activity and commercial performance via trial sites of relevant government companies. The program is intended for Israeli technology companies (that are not government companies) which will implement their initiative at government companies' sites or via their capabilities, data or information. Participating companies will receive financial support of between $20 \%-50 \%$ of the approved pilot's expenditures. An exceptional support rate of $60 \%$ of approved pilots' expenditures will be awarded to programs with potential for exceptional impact on government companies' financial activity.

\section{Encouraging R\&D in the field of space technologies}

This incentive program encourages R\&D to find technological solutions in the various fields of space and is operated jointly by the Innovation Authority and the Israeli Space Agency in the Ministry of Science and Technology. The incentive program aims to encourage $R \& D$ that may strengthen Israeli industry's knowledge and technological developmental capability in the field of space; to reduce gaps in knowledge vis-à-vis the global market and to enhance Israeli industry's competitiveness in these fields. These objectives are undertaken despite many challenges which include: significant technological risk, high development costs, limited scope of manufacturing, and the systems' high cost. The program is intended for companies that are developing products in the fields of space and which are intended for installation in satellites or in ground stations for satellite control and navigation, as well as for companies that are developing devices and equipment for calibration and testing of these products or that are involved in satellite activity, including the installation of different versions of sellable satellites for export. As part of this program, a "large" company (with an annual turnover equal to or exceeding $\$ 100$ million in the year prior to submitting its request) will receive a grant of $50 \%$ of the approved $R \& D$ expenditures. A "small" company (with an annual turnover less than $\$ 100$ million in the year prior to submitting its request) will receive a grant of $60 \%$ of R\&D in ground-designated products and parts, or a grant of $85 \%$ of $R \& D$ in products or parts intended for operation in space.

\section{Groundbreaking R\&D programs in the field of cyber}

The program is operated jointly by the Innovation Authority and the National Cyber Directorate at the Prime Minister's Office. It supports breakthrough research and development programs in cyber defense. The program's goal is to promote significant growth of core technological capabilities that will enable the finding of innovative technological solutions and the development of groundbreaking products in the field of cyber defense. Furthermore, this incentive program also encourages the creation and cultivation of complete and sustainable companies in the field of cyber defense in Israel via the promotion of innovative technological solutions. This program is intended for Israeli cyber technology companies. Participating companies will receive financial support of 20\%-50\% of approved R\&D expenditures. An exceptional support rate of $66 \%$ of approved $R \& D$ expenditures will be given to programs with significant potential to influence the global cyber market, or that constitute an outstanding technological ground breaking in its field. 


\section{Support for pilot programs in the field of cyber}

The program is operated jointly by the Innovation Authority and the National Cyber Directorate at the Prime Minister's Office. It supports R\&D programs in the field of cyber defense technology with the objective of supporting and promoting productization and piloting of innovative cyber technologies as well as conducting feasibility tests among significant customers. This program also promotes growth of complete and sustainable cyber companies in Israel by bolstering innovative technological solutions. The program is intended for Israeli cyber technology companies that have developed a product/service that has yet to be launched in Israel and which is still undergoing development. In terms of technology readiness level (TRL), the program aims to promote cyber companies with a TRL of $4 / 5$ (validation in laboratoryl to a level of TRL 7/8 lactual system qualified through test and demonstration). This program offers companies a grant of 20\%-50\% of the approved R\&D budget.

\section{Support for agricultural R\&D and pilot programs}

The program is operated jointly by the Innovation Authority and the Ministry of Agriculture. It aims to encourage the development of agricultural products intended for sale and export. The incentive program is intended for Israeli companies in the various agricultural fields such as species cultivation, seeds, animal breeding and others. Participating companies will receive financial support of up to $60 \%$ of the approved R\&D and pilot's expenditures for development of agricultural equipment or innovative commercial agricultural technology. Companies active in designated development regions will be eligible for an additional grant of $10 \%$.

\section{Assistance for investment in projects for energy efficiency and reduction of greenhouse gas emissions}

The program aims at providing extensive support for projects that undertake initial commercial installations of new Israeli technologies. The program is operated by the Innovation Authority in conjunction with Israel Investment Center and the Ministry of Environmental Protection. The program's objective is to encourage demand for innovative Israeli cleantech technologies and to develop Israeli high-tech industry in the field of energy efficiency and reduction of greenhouse gas emissions. The incentive program is intended for entities engaged in initial application of new Israeli technologies in the fields of energy efficiency and reduction of greenhouse gas emissions. Participating companies will receive financial support of $40 \%$ of the cost of installation.

\section{( Paving the way for environmental technologies (SDG 8.2, 9.2, 12.1, 17.7)}

The terms "environmental technologies" and "cleantech" refer to products, processes and services that reduce the negative environmental impacts of various industries or improve the efficiency of the use of natural resources. In Israel, several hundred companies operate in various environmental technologies: renewable energy production, energy efficiency, water resource management, recycling and waste management, streamlining the use of materials in industry and sustainable transportation. Dialogue between government and industry shows that environmental technology companies are facing special challenges, particularly with regard to transition from the laboratory stage to the demonstration stage of commercial-scale technology.

The transition between the R\&D stage and the laboratory to the commercial demonstration stage of the product is known as "the Valley of Death." Two barriers are known to obstruct the assimilation of innovative technologies in manufacturing industries:

- Financial barrier: The demonstration stage of environmental technology on an industrial scale is characterized by high cost on the one hand, and by a lack of funding sources on the other hand, by banks, institutional investors or venture capital funds;

- Regulatory impediment: By its very nature, innovative technology cannot guarantee compliance with the emission standards that the plant is required to comply with. Significant time and 


\section{CASE STUDY}

\section{The Environmental Technologies Program}

The environmental technologies program consists of a number of projects each aiming to assist Israeli environmental ventures along the value chain:

- The Beta Sites Project: The Beta Sites Project, launched in June 2018, will enable the acquisition of experience in innovative technologies that will be assimilated into industry and improve the environment in Israel. In addition, the ability to demonstrate innovative technology will help technology companies mature and grow, and subsequently reach global markets including, developing countries. The project supports the construction of pilot facilities (the first demonstration of technology on an industrial scale), selected in a competitive process and granted both financial and regulatory support. In response to the first call for proposals, more than 50 applications were submitted, of which 15 projects were chosen.

- The Environmental Technology Innovation Lab Project: This program will be implemented through the development of a novel "open innovation" model where an industrial corporation (which could be a joint venture of two or more corporations), chosen by tender, is granted a license to operate an environmental innovation lab. The operator of the lab will receive financial support for establishing the physical infrastructure of the lab and for operating it for three years by employing a full-time lab manager. In return, the operator undertakes to recruit start-ups to the lab and assist them in their development endeavors. There is also government funding for the start-ups themselves. The Innovation Lab will serve as a platform for cooperation between entrepreneurs and manufacturing companies in order to increase the number of projects in the field of environmental technologies that meet the environmental challenges of traditional industry. The operating companies benefit from early familiarity with $R \& D$ outside their own $R \& D$ departments and the ability to influence the technological development to suit their own needs, and can invest in the equity of the start-up companies entering the lab. The start-ups benefit from the possibility of developing their technologies using the host corporation's infrastructure. Government involvement in a project of this type is unusual.

- The Industrial R\&D Project: This project aims at enhancing technology transfer from academia to industry and encouraging commercialization of academic research with economic potential. This program will consist of research grants for researchers and industrial companies to promote research from the basic research stage to the feasibility study of product development. The project will be implemented through three tracks which differ from each other mainly in the parameters regarding the maturity of the research, the involvement of an industrial company in research and the resulting level of financing that the company is obliged to provide which ranges from $0 \%$ to $34 \%$ 
cost resources are required to produce studies, expert opinions, and more in order to persuade the regulator to authorize the use of a new technology.

Israel seeks to address these challenges by implementing programs to promote and empower the Israeli cleantech industry. The goal of the programs is to expand the arena of players and business activity in all areas of environmental technologies, and in relation to a wide range of technological development stages. The benefits offered by the programs are twofold - environmental and economic. A thriving business community of environmental innovation will help improve the environmental performance of the industry and offer innovative solutions to improve the environment in Israel. In addition, alongside the environmental benefits, as technological developments mature and succeed in conducting business on a commercial scale, these companies will be able to continue to develop into international markets.

\section{Government expenditure on} environmental R\&D in Israel

Data on government expenditure on environmental $R \& D$ relative to total government expenditure on R\&D were taken from the OECD Green Growth Indicators 2017. The Green Growth Indicators report includes data on countries other than OECD countries. The report shows the following for government expenditure on R\&D in 2013-2015:

- Government R\&D budgets are increasing globally, but the proportion dedicated to the environment is stagnant;

- Out of the R\&D dedicated to energy, there is a clear transition to renewable energy;

- In the index of general R\&D out of GDP, Israel ranks second in the world;

- In the index of R\&D on the environment, out of all R\&D, Israel ranks 32 out of 36 countries that were ranked.

\section{( Applied support of research institutes (SDG 9.5)}

When it comes to academia, Israel is ranked fourth in the world for research personnel, with the highest number of PhDs per capita globally. This ecosystem provides the most fertile of soils for research and innovation alike - from cutting-edge biomedical engineering, to technology breakthroughs, fintech and the Smart Revolution.

The focus of collaboration between industry and academia is at the core of Israel's technological advantage. Whether a research group interested in transforming its discoveries into products, a research institute looking for research directions for the industry, or an industrial corporation

Figure 9.3

Government expenditure on R\&D in the environment out of total government expenditure on R\&D, 2015

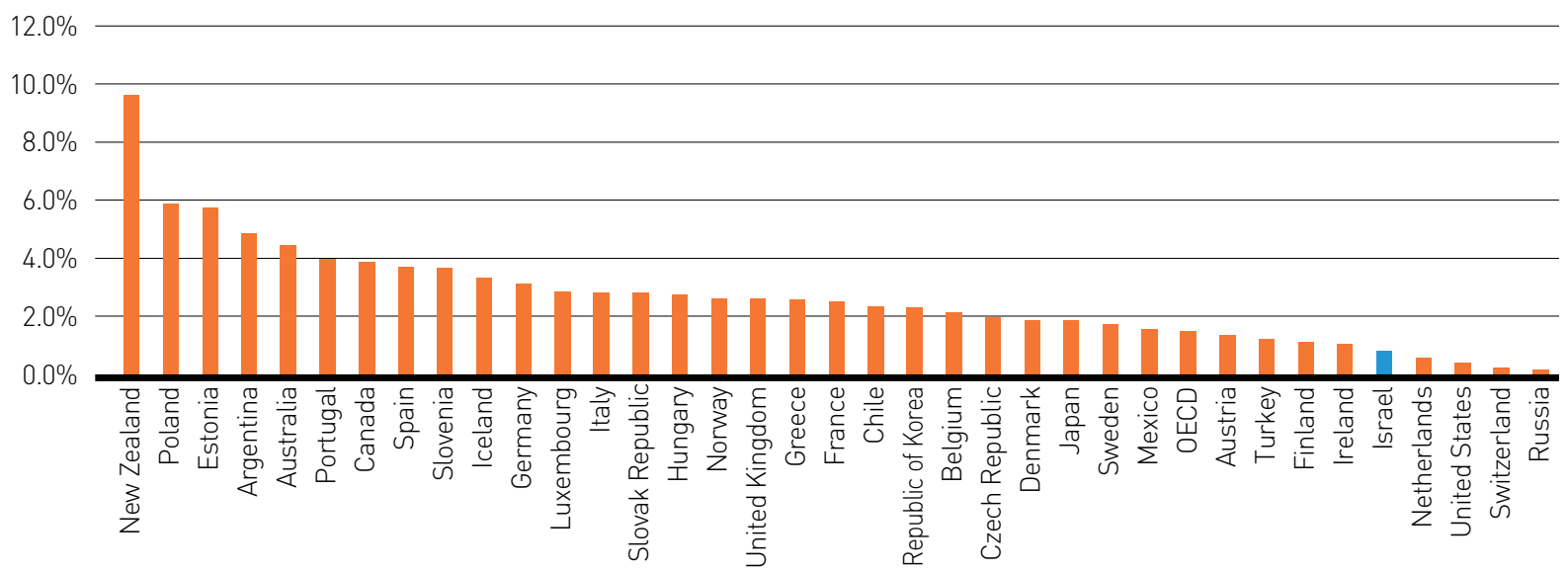

Source: OECD data 


\section{CASE STUDY}

\section{Israel NewTech - Government Support for Israel's Growing Industries}

Israel NewTech helps to advance the water and renewable energy sectors by supporting academia and research, encouraging implementation in the local market, and helping Israeli companies succeed in the international arena. The pioneering program has spearheaded Israel's water and energy eco-system since its founding in 2007. Israel NewTech brings all stakeholders - start-ups, entrepreneurs, companies, multinationals, academia, regulators, policy makers and investors - together to cooperate in order to deliver smart solutions to global challenges in the cleantech arena. Israel NewTech is recognized as a successful case study of an innovative and effective way government can be involved in supporting the cleantech sector of a country and increasing Israeli exports in the water and alternative energy sectors. The program has been studied and emulated by a number of governments and countries throughout Europe and the world.

Israel NewTech was founded on the belief that the Israeli water and renewable energy sectors have the talent and capability to be strong growth industries for the country, and to play an important part in establishing the "next generation oasis" for the world's rising needs. This pioneering national program is led by the Ministry of Economy and Industry, and is supported by a number of additional Israeli government agencies serving as a best practice example of multi-stakeholder cooperation.

The program's goals include:

- Attracting international investment: Leading international players in the water market including Veolia, GE, Siemens, Coca-Cola have already identified Israel as a strategic target for investment and cooperation.

- Promoting relevant R\&D programs: Millions of dollars in government financing are designated to transform Israel to an international beta-site by encouraging technology innovation.

- Strengthening human capital: Heavy investment in academic programs, research scholarships and professional training aims to encourage highly educated and qualified professionals in the water and energy sectors.

- Increasing international awareness: International recognition of Israel's innovative water and renewable energy sectors is promoted by means of delegations, conferences and international events.

The program is currently being restructured.

interested in developing innovative products, Israel's programs promote the exchange of knowhow and experience and the development of groundbreaking knowledge by an integrated group of researchers from academia and industry.

One key incentive program assists and supports research institutes conducting applied research to promote industry in Israel. The main goal of the incentive program is to provide assistance and support in the creation of R\&D capabilities in research institutes that conduct applied research whose goal is the further development of Israeli advanced manufacturing. The program is designed for research institutes operating as independent 
legal entities engaged in applied R\&D and providing $R \& D$ services for Israeli industrial corporations.

The support is provided via three subprograms:

- R\&D generic technology programs;

- R\&D programs in cooperation with an industrial company;

- Purchasing of R\&D scientific equipment.

\section{Promoting long-term knowledge infrastructures (SDG 9.5)}

Several institutes and centers in Israel have been set up to provide the necessary knowledge infrastructure to promote innovative and sustainable technological and manufacturing processes. They include:

- Food Research and Innovation Institute: The institute will operate in the Galilee and serve as a hub for the development of new food technologies and products, the distribution of knowledge, and encouragement of entrepreneurship in the food industry.

\section{- National Institute for Advanced Manufacturing}

The institute will operate to develop and implement manufacturing techniques that can streamline enterprises, improve labor productivity, and help Israeli companies deal with foreign competition.

- Resource Efficiency Center: The center will operate to implement advanced manufacturing processes with a low environmental footprint in industry. The center will make it possible for enterprises to reduce pollution - from the initial selection of raw materials and the planning of production processes - in order to comply with strict environmental standards and enable industry to increase profits by cutting production costs (see SDG 12.2).

\section{Access to information and communications technology (9.C)}

According to Israel's most recent Well-being, Sustainability, and National Resilience Indicators (2017 data, published February 2019), 81.6\% of persons aged 20 and over used the Internet and $37.6 \%$ used online government services (E-gov). Additional information can be found in the Wellbeing Indicators Annex, under the indicators for Information and Communications Technology.

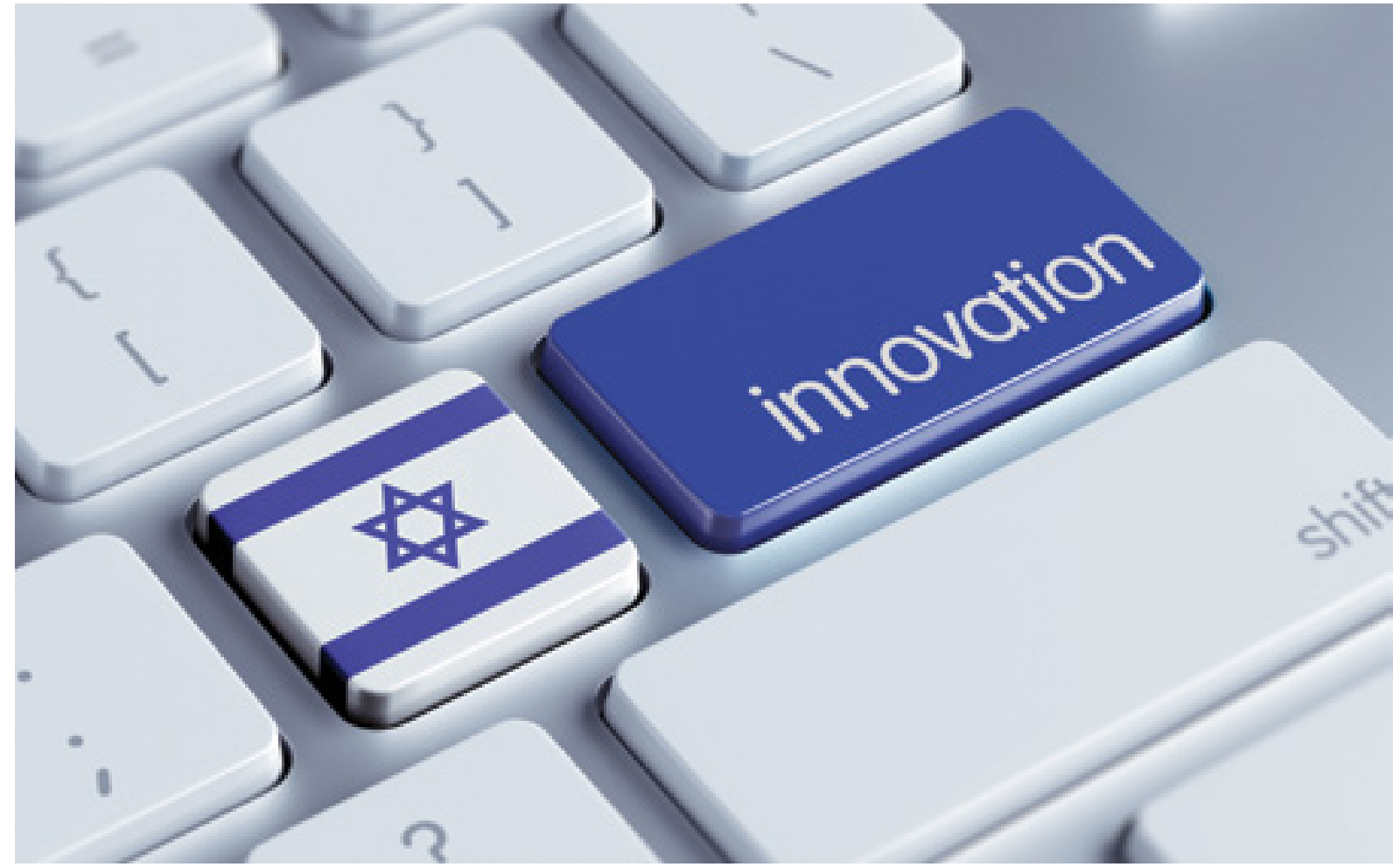




\section{PARTNERING FOR A BETTER WORLD}

Israel's overseas development assistance programming directs its efforts at promoting sustainable industrialization and fostering inclusive, multisectoral, gender-responsible policies and best practices. In light of the rapid pace of global changes, Israel strongly supports establishing thriving innovation ecosystems throughout the developing world by sharing tools to encourage and advance industrial research and development. At the 72nd UN General Assembly, Israel, as part of the Core Group, joined the consensus on the Resolution titled Impact of Rapid Technological Change on the Achievement of the Sustainable Development Goals.

Israel's Ministry of Foreign Affairs designated innovation and development a priority platform in the implementation of its Global Agenda 2019. Furthermore, in line with the Government Decision on Advancing Israel's Activities in International Development Cooperation (4021), the ministry established an Innovation Department to promote Israel's innovation ecosystem as a model for development.

Israel is one of the leading countries in the field of innovation and entrepreneurship, incorporating knowledge and technologies to help eradicate poverty and support the empowerment of women and youth, vulnerable communities and people living in rural and remote areas. As inclusive and sustainable economic growth are priority policies, during the $73^{\text {rd }}$ UN General Assembly, Israel spearheaded a Resolution on Entrepreneurship for Sustainable Development, reaffirming the 2030 Agenda. The Resolution calls upon relevant organizations and UN bodies to further recognize and integrate entrepreneurship into their policies, programs and reports, and acknowledges that entrepreneurship and innovation are essential for harnessing a country's economic potential. The resolution also calls to continue to provide support to member states, at their request, to identify, formulate, implement and assess coherent policy measures on entrepreneurship and the promotion of micro, small and mediumsized enterprises, building upon previous Israelisponsored Resolutions on Global Entrepreneurship (67/202 and 69/210). (SDG 9.5)

Israel hosted (2018) the visit of a five-member mission representing the UN Secretary-General's High-Level Panel on strengthening cooperation in digital space among governments, the private sector, civil society, academia and relevant stakeholders. As early as 2013, Israel adopted a decision establishing the Digital Israel National Initiative to formulate and implement a national digital policy for using information and communications technology (ICT). The visit, therefore, provided an opportunity for Israel to contribute to the broader global digital dialogue on how interdisciplinary and cooperative approaches can help ensure a safe and inclusive digital future. (SDG 9.c, 17.17)

An expert from the Ministry of Environmental Protection is working with the Organization for Economic Cooperation and Development (OECD) on a project aimed at promoting the implementation of best available techniques (BATs) for preventing and controlling industrial chemical pollution. One of the objectives is to share experience and knowledge on BAT among OECD member and partner countries. As part of the project, an Israeli expert is advising Kazakh officials on their transition to BAT, based on the Israeli experience. (SDG 9.4)

The UN Industrial Development Organization (UNIDO), mandated to promote inclusive and sustainable industrial development (ISID), and Heineken International B.V., committed to a Corporate Social Responsibility Strategy - Brewing a Better World, when signing a Memorandum of Understanding (MOU) to cooperate on the development of water stewardship programs in selected catchment areas in the developing world. Israel contributed towards the development of project proposals and their upscaling through bilateral and multilateral cooperation by funding (€50,000) the international environmental management expert working with UNIDO and 
partners. The Strategy includes the creation of sustainable and inclusive supplier development programs and the promotion of renewable energy solutions, aiming toward inclusive growth, protecting water resources, reducing $\mathrm{CO}_{2}$ emissions, sourcing sustainably, advocating responsible consumption, and promoting health and safety. The goal is to develop public-private partnerships for water stewardship programs to overcome cumulative stress on finite water resources shared by multiple stakeholders through collaborative action. (SDG 9.a)

MASHAV and UNIDO signed an MOU to cooperate in providing technical assistance, onsite capacitybuilding, consultancies and demonstration projects to government entities and civil society organizations to promote industrial sustainability, agribusiness, women's entrepreneurship, water and energy management, marketing, and innovative tourism. Within this framework, joint programs were held in Israel for professionals from Ethiopia, Kenya, South Sudan, Tanzania, and Uganda and Armenia. (SDG 9.5)

In accordance with the Agreement for Development Cooperation between MASHAV and the UN Development Program (UNDP), a seminar for UNDP country offices and headquarter directors was held in Israel on Accelerating Innovation for Social Impact. The seminar aimed at creating partnerships between participating UN agencies and impact investors to support socially responsible businesses, especially small and medium-sized enterprises, focusing on key social aspects such as the empowerment of women, girls and youth to generate social and environmental impact. In addition, MASHAV and the Singapore Cooperation Program (SCP) conducted two capacity-building activities in Israel on Startup Accelerators in the Entrepreneurial System, and Developing Entrepreneurial Ecosystems for Startups: Accelerators, Communities and Support Systems. (SDG 9.5)

MASHAV conducted the following training and capacity-building activities in cooperation with multilateral organizations: (SDG 9.a)
- Innovative Entrepreneurship from an Idea to Starting a Business, and Support Systems for Entrepreneurship (Young American Business Trust - YABT);

- Supporting Women Entrepreneurship through the Use of Information and Communication Technologies (UN Economic Commission for Europe - UNECE);

- Social Entrepreneurship as a Tool for Community Development (UN Educational, Scientific and Cultural Organization - UNESCO).

Israel's Innovation Authority, an independent publicly funded agency, provides practical tools and funding platforms aimed at effectively addressing the dynamic and changing needs of international innovation ecosystems. Its International Collaboration Division is responsible for coordinating international initiatives on innovative R\&D knowledge and technology between Israeli companies and counterpart organizations abroad, creating synergies and leveraging complementary strengths and needs in both Israel and partner countries. International cooperation initiatives, collaboration in R\&D and incentive programs are carried out through multinational frameworks. These include, among others, the EU Framework Programs for Research and Innovation that finance joint projects between Israeli and foreign companies, five bilateral funds including a $\$ 40$ million fund with India for innovative technological collaboration, and more than 30 parallel support programs in which each party offers financing. Following are some examples. (SDG 9.a)

The India-Israel Innovation Bridge is a bilateral initiative calling on Israeli and Indian companies to combine forces and develop solutions to some of the world's most critical challenges in agriculture, water, and digital health. The mutual desire to cooperate, which lies at the core of the initiative, is backed by a substantial investment aimed at building a bridge between the two countries to develop a pipeline of commercially viable technological solutions. The initiative leverages Israel's strengths in technology with India's knowledge of emerging market structures. Selected companies from both countries are provided the 
opportunity to interact with end-users, investors, corporations and other potential partners in India and Israel. The bridge offers qualifying companies knowledge exchange, comprehensive guidance, mentoring, a practical toolkit and funding to help start-ups explore piloting and opportunities in the developing world.

Grand Challenges Israel is a collaborative effort by the Israel Innovation Authority and MASHAV in the context of the Global Grand Challenges, comprising a group of initiatives fostering innovation to solve key global health and development problems in developing countries. The aim of the incentive program is to encourage research and development for innovative technological solutions to global health challenges and support the entry of Israeli companies into new markets. Grants are up to NIS 500,000 (up to $90 \%$ of the approved budget) for the duration of the approved program, and applications for R\&D cover a range of fields including global health (SDG 3), water and sanitation (SDG 6), agrotech and food security (SDG 2).

The Innovation Visas Pilot Program enables foreign entrepreneurs to stay in Israel for up to 24 months, hosted in one of 12 Landing Pads for Foreign Entrepreneurs, thereby introducing them to Israel's innovation ecosystem while providing a workspace and technological infrastructure, as well as business and logistic support.

Bilateral Programs for Parallel Support of Industrial $R \& D$ constitute a platform for the promotion of technological innovation among industrial and research entities. The Authority operates these programs in collaboration with R\&D and innovation funding bodies in leading countries, provinces and cities in Asia, North and South America, Europe, and Australia. In the framework of the Agreement on Industrial and Technological Research and Development Cooperation between Russia and Israel, the two countries announced procedures for the selection of $R \& D$ projects eligible for financial support on developing innovative products and applications in all technological and application areas toward the development of a new product, industrial process or service.
The visit of India's Prime Minister to Israel, and the reciprocated visit of Israel's Prime Minister to India, contributed toward an ever-growing bilateral cooperation between the countries. In 2017, a series of agreements were signed between Israel and India to strengthen innovation and technological ties, including the India-Israel Industrial R\&D and Technological Innovation Fund, a five-year, \$40 million fund to encourage partnerships between Israeli and Indian innovators.

Research and Development Cooperation with multinational corporations is an incentive program for international collaboration between foreign multinational corporations and Israeli companies. The program catalyzes co-development, testing and piloting of impactful technologies, products, services and devices of Israeli companies within partner country facilities, which are then translated to other sites and settings across the country and beyond. It essentially creates a living laboratory for external partners to improve an existing process, or to apply a new process for the first time. Among the program goals is enabling small and mediumsized Israeli companies to establish relationships with multinational corporations.

Israel's Embassy in New Delhi hosted the Start India Competition, in conjunction with the Indian Government's Department of Science and Technology (DST), seeking out India's most promising start-up to participate in Israel's largest international start-up competition - Start JLM. The Indian start-up Mimyk, focusing on virtual reality methods for training doctors in endoscopy procedures, competed in the competition. (SDG 9.a)

On the grassroots level, Indian and Israeli innovators continue to work together to solve a range of global issues including the Israel-India Hackathon for creating and enhancing ties with entrepreneurs to further expand both Israeli and Indian start-up ecosystems. The Hackathon, hosted (2017) by the Pears Program for Global Innovation at Tel Aviv University, brought more than 600 innovators, developers, entrepreneurs, and health care professionals from Mumbai, Hyderabad and Bengaluru to create affordable tech solutions for millions of Indians without access to medical care. (SDG 9.b) 
Recognizing the importance of enhancing scientific research (SDG 9.5), Israel's Ministry of Science and Technology (MOST) is implementing some 30 scientific agreements with governments in Asia, Europe and Latin America to promote cooperation on research projects. Examples include:

- Vietnam: An MOU on Scientific and Technological Cooperation was signed (2017) with the Ministry of Science and Technology of Vietnam. Areas of cooperation include: agriculture and food technology; aquaculture and fishery processing; quality of life and environment; material technology; IT and communication; biotechnology; health and medical science; and new and renewable energy. In 2018, a delegation of Israeli scientists visited Vietnam and attended a joint conference on Clinical Aspects, Diagnostic and Prevention of Vector-Borne Diseases Focusing on Malaria, Zika and Dengue Diseases. In addition, Israeli experts visited five research institutions, universities and hospitals involved in the initiative, and the first call for proposals is expected to be published in the near future;

- Belarus: Israel and the State Committee on Science and Technology of Belarus signed (2018) an MOU on Scientific Cooperation to promote a joint program on scientific research. A joint call for proposals was issued on digital agriculture as well as social sciences;

- Ukraine: A Program for Scientific and Technological Cooperation was signed (2015) with Ukraine on Applied Mathematics and Theoretical Physics. The call for proposals was recently published;

- Argentina: An MOU on a Scientific Cooperation Program was signed (2017) with the Ministry of Science, Technology and Productive Innovation of Argentina, followed by a Work Program for Scientific and Technological Cooperation, aimed at funding joint research projects. Areas of cooperation are under discussion;

- Brazil: A Protocol of Intent with the Ministry of Science, Technology, Innovation and Communication of Brazil was signed (2018) on Cooperation on Science, Technology and Innovation to fund joint research proposals. The first call for proposals is expected to focus on renewable energy and atmosphere-ocean interaction;

- Philippines: An MOU on a Scientific Cooperation Program was signed (2018) between Israel and the Department of Science and Technology of the Philippines, supporting research projects and the exchange of best practices. A delegation of scientists visited Israel to discuss further implementation of the MOU in possible areas including aquaculture and air pollution;

- Middle East: Regional cooperation with Israel's neighbors in the Middle East is an important component in Israel's overseas assistance. Israel is one of the founding member countries of the Synchrotron-Light for Experimental Science and Applications in the Middle East (SESAME), a "third-generation" synchrotron light source that opened officially (2017) in Allan, Jordan. It is the first major international research center in the Middle East, with member countries including Jordan, Egypt, Turkey, Cyprus, the Palestinian Authority, Pakistan and Iran. Research focuses on disciplines including biology, archaeology, medicine, physics, chemistry, material engineering and life sciences.

A new initiative in Israeli academia complements government efforts to promote innovation and sustainability in developing economies. The Nitsan Innovation Villages Program, based at Tel Aviv University, and supported by the Boris Mints Institute, enables the adaptation of Israeli innovations to the needs of low-income populations in developing countries while achieving widespread impact. Nitsan harnesses the resources of the entrepreneurial student community to form a bridge between the Israeli innovation ecosystem - in both the private and public sectors - and the realities of developing countries. Nitsan graduate students are based in communities throughout the developing world, working together with their peers from local partner universities. University experts lead scientifically rigorous, data-driven and evidence-based processes. The flagship program is currently carried out in India, in collaboration with Tata Trusts, and aims at increasing the income of smallholder farmers. (SDG 9.b) 
The Multi-Disciplinary Center for Learning Cities in Modi' in is an active member of the European Cooperation in Science and Technology (COST) initiative. Israel is represented in two COST programs: The Promotion of Scientific Literacy in Society: Building on Scientific Literacy in Evolution Towards Scientifically Responsible Europeans; and The Pan-European Family Support Research Network; A Bottom-up, Evidence-Based and MultiDisciplinary Approach. (SDG 9.5)

Providing the visibility and digital tools to NGOs in developing countries helps to bridge the innovation and technological gaps within societies. GivingWay is a social impact company that developed an online platform providing grassroots level NGOs the visibility and digital tools needed to engage with volunteers, donors and supporters on a local and international level. Through its free service, NGOs use GivingWay's online tools to manage their workflow and network of supporters on all social media outlets. (SDG 9.c) 


\section{GOAL \\ 10}

\section{REDUCED}

INEQUALITIES

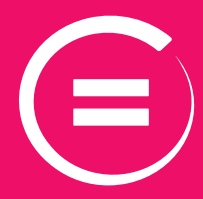

Inequality in Israel

Bridging the digital divide - the

Digital Israel National Initiative

Children and youth at risk

Additional measures taken on a municipal level

Financial regulation in Israel

Partnering for a Better World 


\section{REDUCE INEQUALITY WITHIN AND AMONG COUNTRIES}

A person's well-being depends on many factors such as health, social relationships, trust, work, income, and sense of security. The more one's needs are met in various life domains, the better one's well-being. The most recent report on Israel's Well-being, Sustainability, and National Resilience Indicators (2017 data, published February 2019) indicates that financial wealth, disposable income, individual consumption, and satisfaction with the economic situation have increased overall. Concomitantly, the Gini coefficient of inequality has slightly decreased, but it is still higher than the average in OECD countries. The debt of households as a percentage of the GDP has increased. In 2017, 62\% of persons aged 20 and over were satisfied with their economic situation. Israeli Jews were more satisfied with their economic situation than Israeli Arabs (64\% vs. 53\%, respectively). Among the Jews, $72 \%$ of the religious population was satisfied with their economic situation, compared to $69 \%$ of those who were Haredi (ultraOrthodox), $64 \%$ of those who were secular, and $63 \%$ of those who were traditional. Satisfaction was found to increase with age: $61 \%$ for persons aged $20-64$ and $70 \%$ for those aged 65 and over. 


\section{( Inequality in Israel (SDG 10.1, 10.2)}

Inequality in Israel is estimated according to the Gini measure of inequality in the available financial income of families. Figure 10.1 presents the standard per capita income from 1997 to 2017. It shows that inequality has decreased in recent years peaking in the early 2000s. Since 2003, Israel has seen a gradual, ongoing decrease in financial inequality due to an increase in the employment rate and, in the past three years, also in wages, including minimum wage. Since 2006, in the wake of this increase and as a result of government policy (chiefly in the area of payment transfers to the elderly), the inequality in available income has also decreased, and the gap between the Gini measures has narrowed.

From 2016 to 2017 (the year of the most up-to-date data), the Gini measure decreased to an estimated inequality rate of $1.6 \%$ in available income and $1.3 \%$ in the measurement of financial income.

Figure 10.2 presents another measurement of income inequality in Israel. It shows several measures of inequality, the Gini measure, and the relative measures of various income percentiles vis-à-vis the onset of poverty in the population. For purposes of calculation, the percentiles were sorted by the standard per capita available income. For every percentile, the upper boundary is given.
For 20 of the measures of income gaps between the different percentiles, the measure of p50/p90 - which reflects income gaps among half of the high-income population - decreased in 2017, to reach a lower point than in 1999. The p90/p10 and p50/p10 measures, which respectively reflect the income gaps between the highest and the lowest percentiles, and between the median and the lowest, indicate a continuing trend of the decrease in the past year following an increase in recent years. When compared to 2016, the decreases were at a rate of $6.1 \%$ for available income, and $3.1 \%$ for financial income.

The decrease in the rate of inequality is impressive because, among other things, it occurred against a welcome rise in standard of living, as expressed by the $6.4 \%$ increase in standard per capita available income (i.e., adjusted for family size). However, alongside these improvements, 2017 also registered a deterioration in the socio-economic situation of low-income sectors of the population. The fact that National Insurance Institute (NII) benefits do not keep pace with the rise in standard of living, but are increased according to consumer price ceilings, generates a gap between the rise in the general standard of living and that of people in need of support and benefits. Ultimately, this gap is expressed in the growing intensity and depth of poverty.

Figure 10.1

\section{Inequality over time in Israel - the Gini measure, by available financial income, 1997-2017}
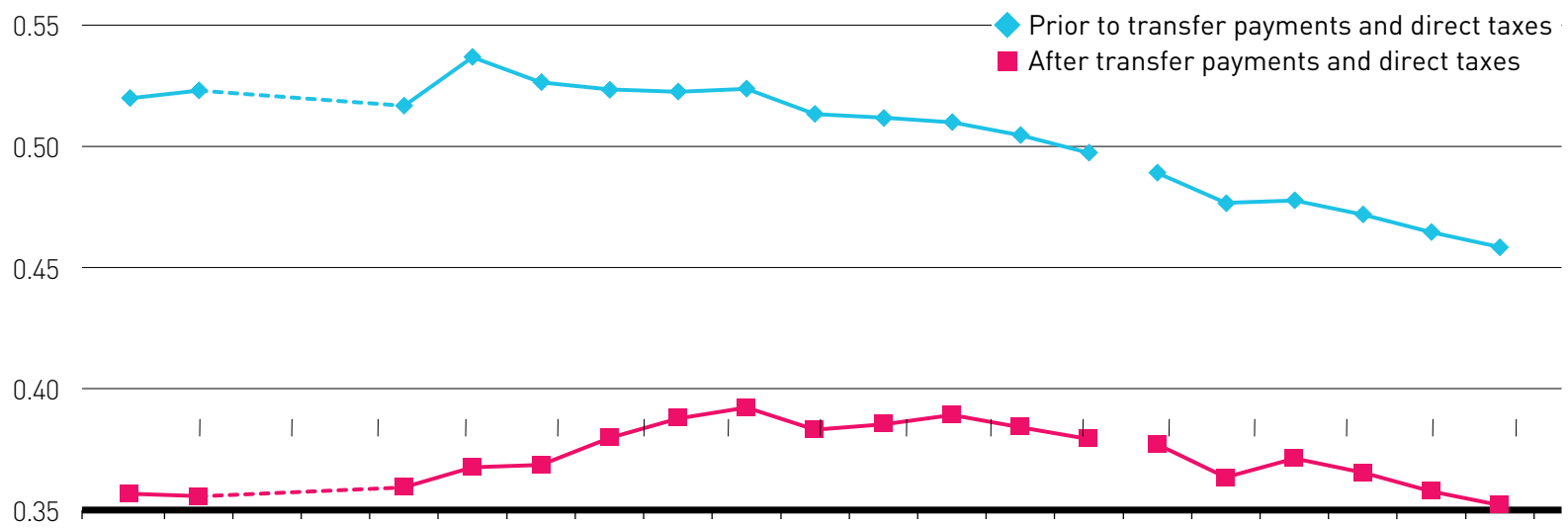

199719981999200020012002200320042005200620072008200920102011201220132014201520162017

Source: Calculated by the research administration of the National Insurance Institute for the Household Expenditure Survey and Income Survey of the Israel Central Bureau of Statistics (ICBS) 
Figure 10.2

\section{Selected measures of income inequality gaps, selected data, 1999-2017}

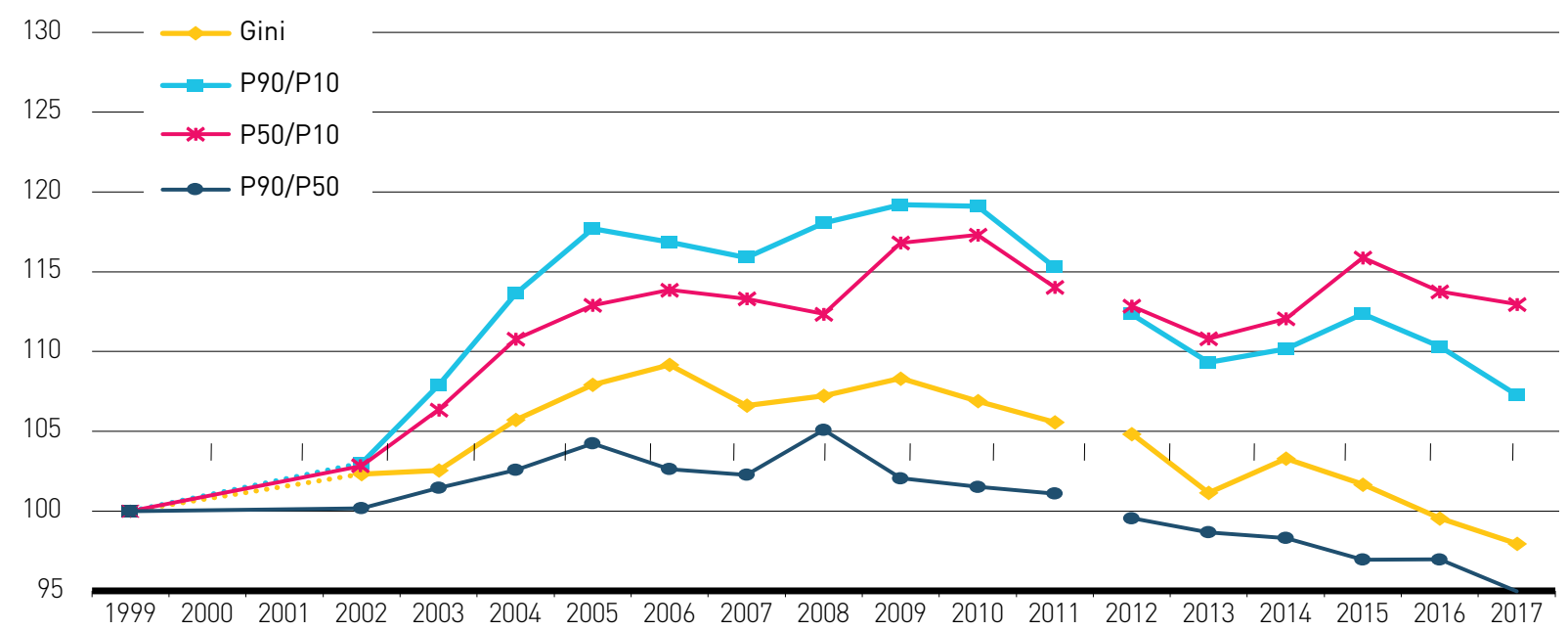

Source: Calculated by the research administration of the National Insurance Institute for the Household Expenditure Survey and Income Survey of the Israel Central Bureau of Statistics (ICBS)

Inequality in the quality of employment

The Well-being, Sustainability, and National Resilience Indicators illustrate an overall increase in employment rate, median gross income of households from work and satisfaction with work, as well as a decrease in the prolonged unemployment rate. Yet, an increase is noted in gaps between the Jewish and Arab populations in both the employment rate and involuntary parttime employment. In addition, $9.7 \%$ of employees felt discrimination at work.

The employment rate in Israel is on the rise increasing from $49.7 \%$ in 2000 to $54.2 \%$ in 2011 and from $59.2 \%$ in 2012 to $61.3 \%$ in 2017. In 2017 . the employment rate was $66.1 \%$ for men and $56.7 \%$ for women, while the employment rate for Jews was $65.1 \%$ compared to $43.4 \%$ for Arabs. This gap increased from 17.4 percentage points in 2002 to 18.7 percentage points in 2011. In 2012, the gap was 22.0 percentage points and in 2017 it was 21.7 percentage points. The employment rate among Arab women has been substantially lower than that of Jewish women over the years. In 2017, the employment rate of Arab women was $26.4 \%$ compared to $63.2 \%$ for Jewish women. (Figure 10.3)
Furthermore, in 2002-2017, the gross median income from work per household increased by about $36 \%$, from NIS 12,780 to NIS 17,394 (in constant prices). During that period, the income of Jewish households increased by about 37\% (from NIS 13,969 to NIS 19,140), and the income of Arab households increased by about $56 \%$ (from NIS 7,344 to NIS 11,435). (Figure 10.4)

\section{Expenditure on housing out of disposable income}

The Well-being, Sustainability, and National Resilience Indicators demonstrate large gaps between deciles in the percentage of expenditure on housing out of disposable income in addition to a general increase in the percentage of that expenditure (Figure 10.5). At the same time, it shows an increase in the percentage of respondents satisfied with their dwellings. In 2017, 30.9\% of the households in Israel spent $30 \%$ or more of their net money income on housing labout $50 \%$ of the households in the bottom decile, and about 15\% of the households in the top decile). 
( Bridging the digital divide - the Digital Israel National Initiative (SDG 10.2)

Israeli innovation and advanced technologies are key drivers to growth. However, the advantages and potential offered by the digital age have yet to permeate all parts of Israeli society and the economy. Moreover, what has percolated occurred at different levels of intensity among different population groups. There are significant disparities in Israeli society and a growing digital divide between those parts of society that take advantage of digitization and disadvantaged populations that frequently do not benefit from its fruits. One of the goals set by the government is to address these disparities and to act to narrow gaps between population groups, eliminate the "distance" between periphery and center and advance equal opportunity, using digital tools.

Developing digital industries in the country can serve as a significant growth engine for Israel,

Figure 10.3

Employment rate of persons aged 15 and over, by sex and population group, 2000-2017

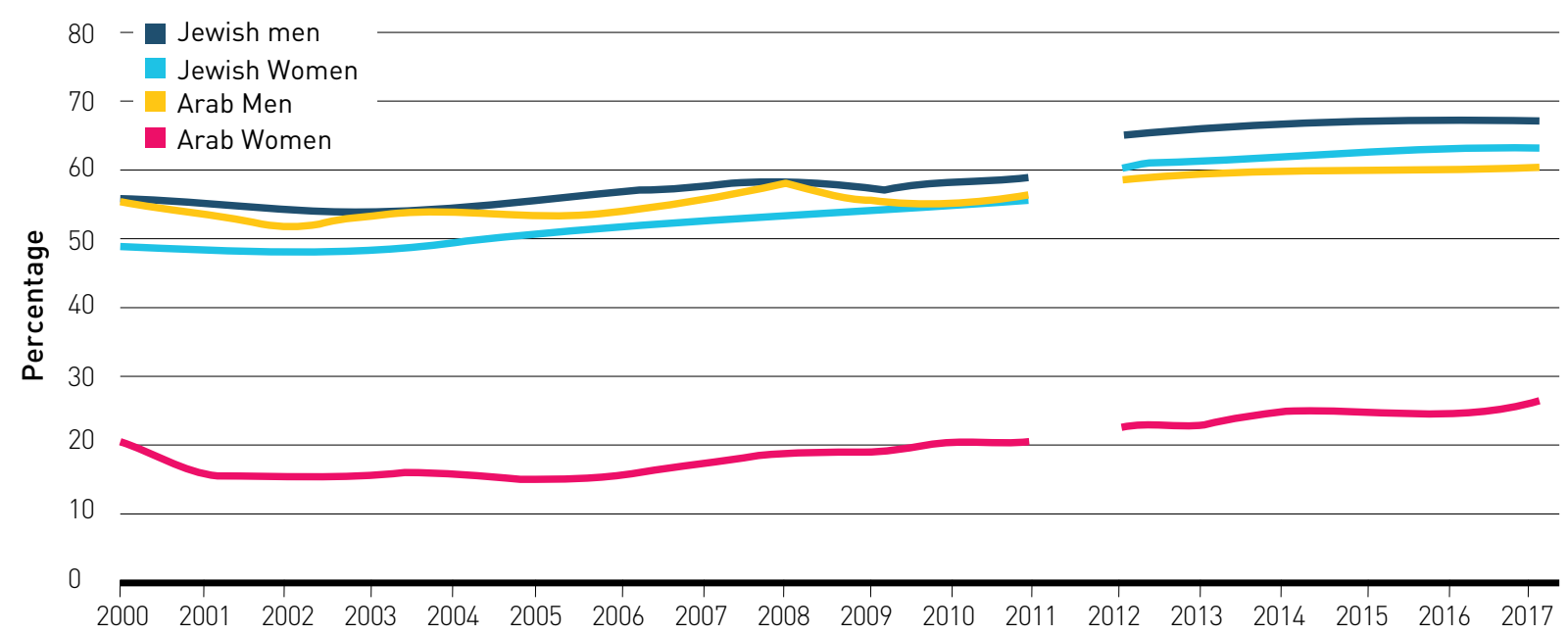

Source: Israel Well-being, Sustainability, and National Resilience Indicators, 2017

Figure 10.4

Gross median income of all households from work, by population group at constant prices (2017), 2002-2017
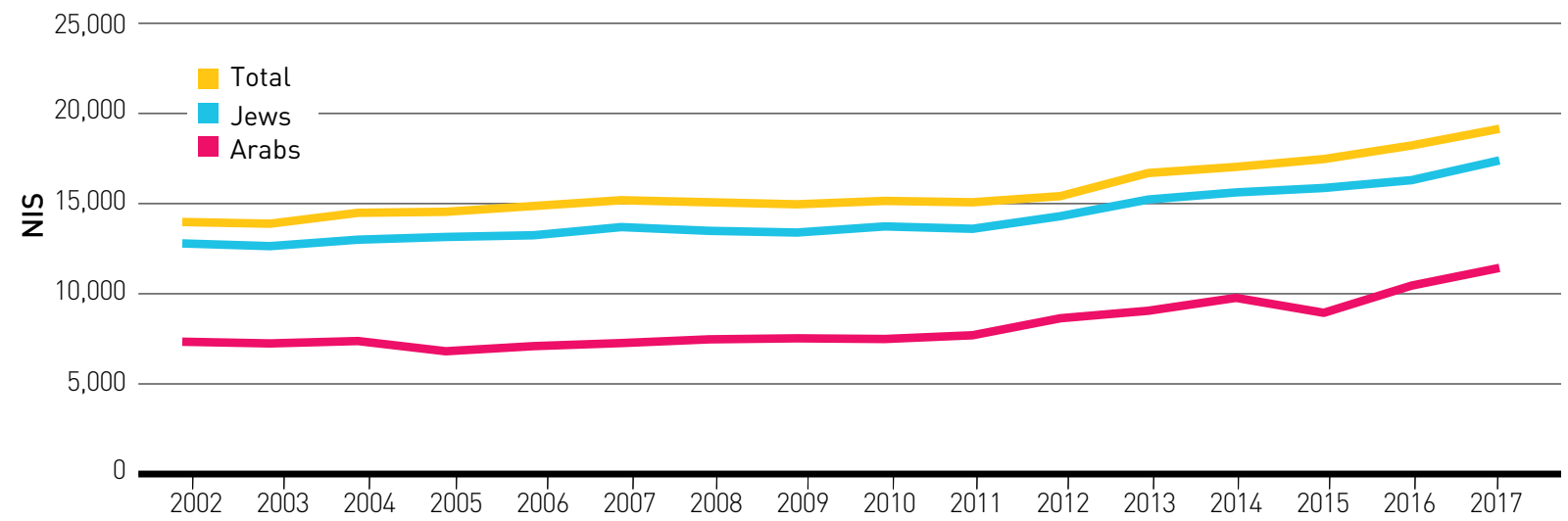

Source: Israel Well-being, Sustainability, and National Resilience Indicators, 2017 
resulting in economic growth, job growth and improved services to the public, while fostering an innovative and advanced ecosystem. There is also significant potential to foster data-driven innovation in the public sector and in industry, and to improve digital infrastructures and accessibility of state and local government services to the population at large.

The government of Israel has identified the acute need to formulate a comprehensive national digital policy, establishing the "Digital Israel National Initiative" in 2013 with the aim of formulating and implementing a national digital policy for using ICT. "Digital Israel" was defined as one of the six strategic issues the State should address by the National Economic Council in the strategic socio-economic assessment that it prepared and presented to the government (see the introduction to this report).

To coordinate and integrate government work to advance the National Initiative and its goals, the government also decided to establish the Digital Israel Bureau that operates within the Ministry for Social Equality. Among other tasks, the government charged the Bureau with the responsibility for formulating a National Digital Program, while accompanying the development of intra-ministry and inter-ministerial digital programs, as well as programs to promote cross-government steps necessary for realizing the Initiative. The aim of the program is to use digitization to bridge key socio-economic gaps among disadvantaged populations, while increasing both national and local government efficiency.

In order to help narrow the gaps at the local level, the Ministry of Interior (MOIN) has also promoted digital programs, held meetings with the digital innovation community in several local authorities, and provided financial support to fifteen local authorities for developing digital projects and smart cities. The MOIN further supported these programs by encouraging local authorities to hold public meetings and to gather knowledge in the fields of digitization and smart cities.

\section{( Children and youth at risk (SDG 10.3)}

Given the growing concern over deterioration of the plight of children and youth at risk in Israel due to the increase in the extent of poverty and changes in pension policy, a review of the necessary policy changes affecting children was necessitated. This concern was the basis of the government decision to establish a public committee to review the plight of at-risk or troubled children and youth. The

Figure 10.5

\section{Percentage of households that spend $30 \%$ or more of their total net money income on housing, in deciles of households by net income per standard person, 2000-2017*}

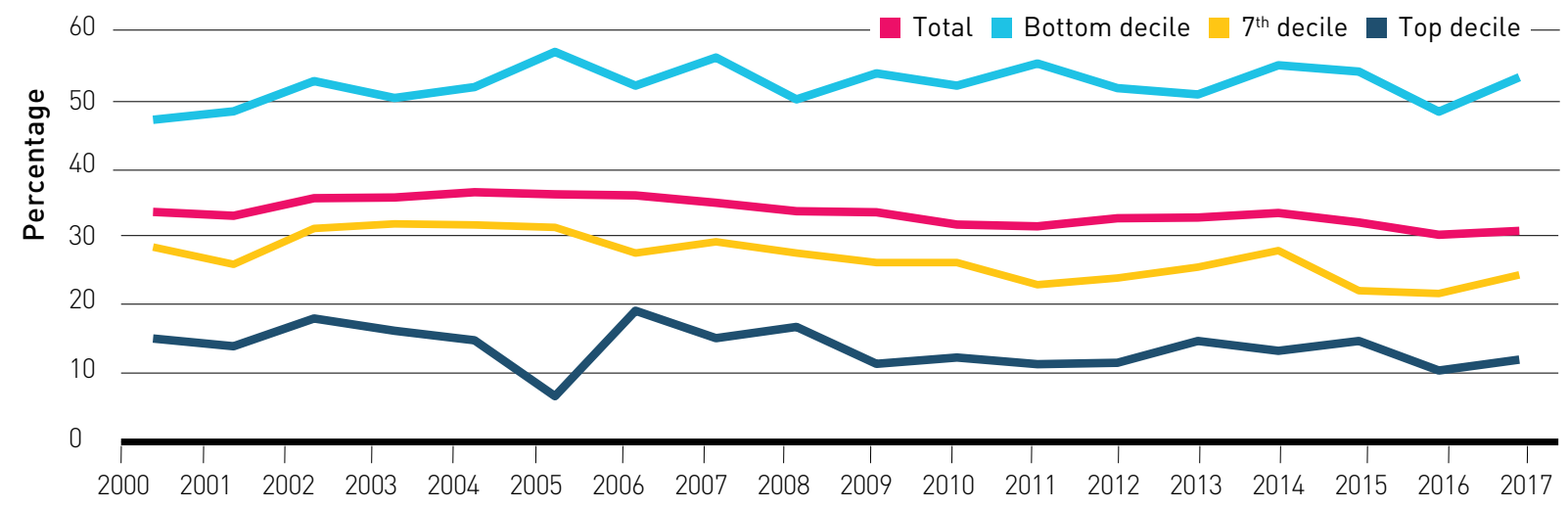

Source: Israel Well-being, Sustainability, and National Resilience Indicators, 2017

*The net money income of a household is divided by the number of standard persons in the household. A scale was designed which determines a two-person household as the base unit. The larger the number of household members, the lower the marginal weight of each additional person in the household ("size advantage"). 
Figure 10.6

The Digital Israel National Initiative

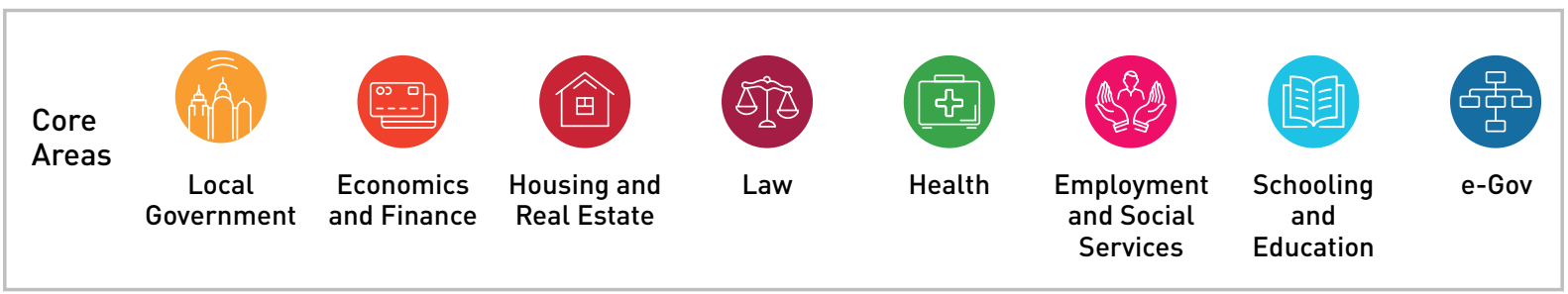

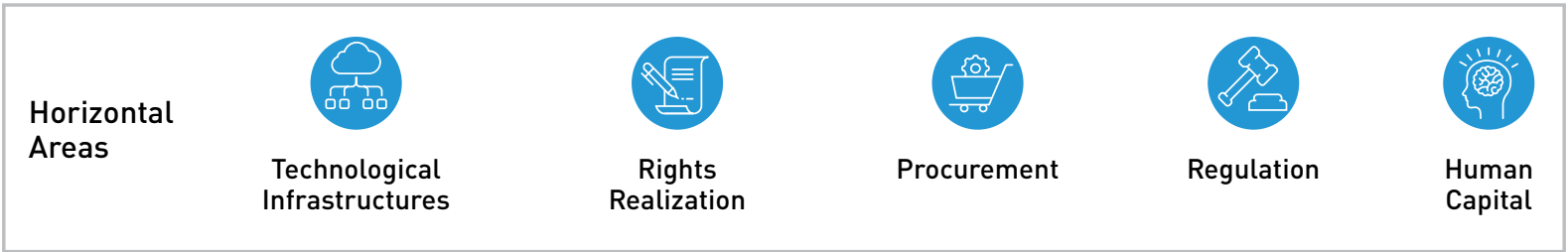

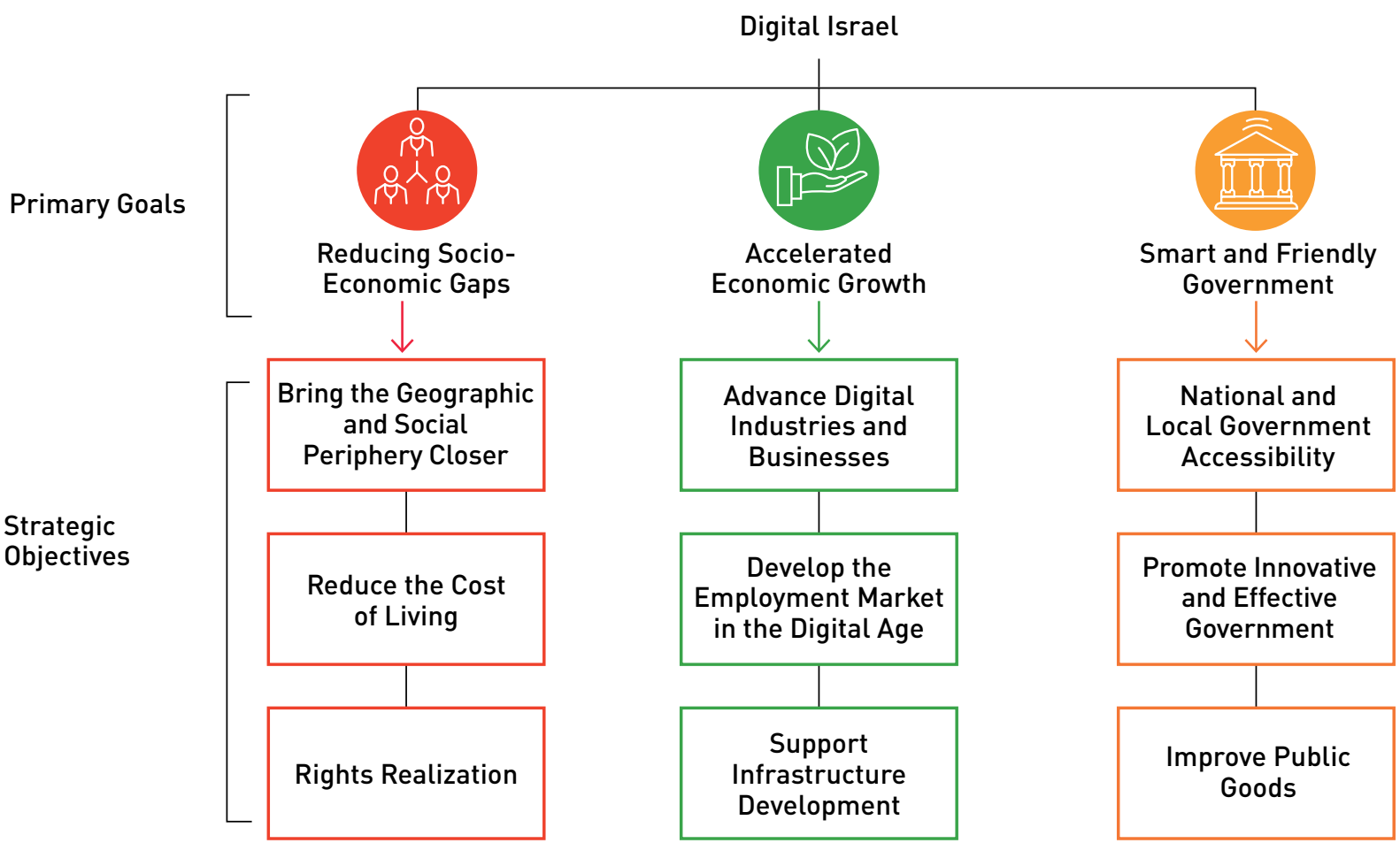

committee submitted its recommendations in 2006. Among other things, it condemned the absence of a uniform definition and common inter-ministerial priorities for at-risk children and youth; the absence of uniform systematic data, implementation, and outcomes regarding their care; the allocation of hard budgets that fail to encourage resource pooling; and numerous models that never reach the stage of widespread assimilation on the ground. The committee proposed a joint inter-ministerial definition for children and youth at risk, based on the Covenant of the Rights of the Child and accepted definitions in the professional literature and among Israel's policymakers. According to this definition, these are children and youth who live in situations endangering them, their families and their surroundings and are harmed in terms of the exercise of their rights under the Convention in the following areas: physical existence, wellbeing and emotional health; social belonging and 
integration; protection from others; and protection from their own dangerous conduct. The definition adopted is broad and posits a continuum of risk: at its extreme end are children who suffer from abuse and/or neglect and danger.

\section{( Additional measures taken on a municipal level (SDG 10.3, 1.4, 1.b)}

In order to address socio-economic gaps, various measures are also taken on a municipal and regional levels by the MOIN, as illustrated by the following examples:

\section{Instituting changes in municipal boundaries} and revenue distribution

Six Permanent Geographical Boundaries Committees deal with municipal boundary changes and with the distribution of revenues from income-producing areas (such as army bases, joint industrial zones and quarries). In the coming year, several committees are expected to distribute revenues from these areas to authorities characterized by low socio-economic status.

\section{Allocating a balancing grant to poor local authorities}

The balancing grant is a non-earmarked budget transferred by the MOIN to poor local authorities in order to assist them in providing their residents with a reasonable level of municipal services. The grant is intended to bridge the gap between the expenditure per capita of the local authority and the potential income per capita, thus compensating weak local authorities for this gap. The grant per resident is higher as the socio-economic cluster is lower. The 2018 reform led to a significant increase in the balancing grant and enabled a more equitable distribution of income between local authorities. Most of the increase reaches the low socio-economic clusters of 1-2, for which the grant was raised by NIS 61 million.

\section{Support for communities in the periphery}

A special grant was awarded to strengthen the civilian resilience of poor communities in the periphery.
Sderot la western Negev cityl and other communities surrounding the Gaza Strip were awarded this grant for the years 2015-2016 in order to respond to immediate needs. Support was also awarded to other peripheral communities including the Bedouin communities in the north and south. This is part of the government's plan for empowerment and socioeconomic reinforcement of these communities in the years 2016-2020. In 2016, a multi-year development budget of NIS 35.55 million along with a development grant of NIS 30 million were allocated to the Bedouin communities in the north.

\section{Inclusion of poor local authorities in joint} industrial zones

In order to reduce socio-economic gaps, five local authorities of low socio-economic rating (1-5) were included in already existing joint industrial zones. These industrial zones are shared by several authorities in order to create a pool of independent sources of income and employment for the region. Within the new boundaries thus created, revenues from existing and future joint industrial areas will be distributed during the coming years, with the expectation that this will reduce inequality among local authorities.

\section{Raising the income of local authorities from non-residential property taxes}

Non-residential property tax is an important source of income for local authorities. These revenues have great economic value. They attest to positive urban-economic activity and generate a profit for the local authority. This is in sharp contrast to residential property taxes, which, given the regular provision of services by the local authority to its residents, constitutes an economic loss in relation to expenses. The rate of increase in the ratio of non-residential property tax revenues is expected to reach $15 \%$ by 2020 .

\section{Establishing the fund for reducing gaps}

In effort to assist weaker local authorities, the MOIN established a NIS 500 million fund designed to reduce the gaps between various authorities and 
to promote distributive justice among them. The ministry's goal is to provide budgetary solutions to local authorities in Israel's geographic and social periphery and to improve local authority services. The fund is distributed according to criteria that, among others, prioritize local authorities in the periphery that are characterized by a low socioeconomic status.

\section{( Financial regulation in Israel (SDG 10.5)}

Financial regulation in Israel is broad and meets the highest standards. One indication is the resilience of Israel's banking system during the 2008 financial crisis. The government takes part in all global forums on financial regulation and operates according to international standards. Israel has recently joined the inter-governmental Financial Action Task Force (FATF).

\section{CASE STUDY}

\section{$360^{\circ}$ - National Program for Children and Youth at Risk}

The $360^{\circ}$ National Program for Children and Youth at Risk, established in 2008, aims to reduce the risk situations to which children are exposed between the ages of birth and 18, change the way that Israeli society deals with children and youth at risk, and improve the community services aimed specifically at them. It is designed as a multi-stakeholder effort including five ministries: the Ministries of Labor, Social Affairs and Social Services - MOLSA (the coordinator), Education, Health, Aliyah and Immigrant Absorption, and Public Security, as well as additional bodies including the Israel Police, JDC-Israel, and the Union of Local Authorities in Israel. It currently operates in 185 local authorities with socio-economic characteristics comparable to clusters 1-5 set by the ICBS (see Statistical Annex), localities with a concentration of disadvantaged population groups, such as Israeli Arabs, Haredim, and new immigrants.

The program is based on several basic principles:

- Commitment to changing priorities: Emphasis is placed on community care, prevention, early childhood care (Hatchala Tova/Good Start), allocation of resources to strengthen parents and prioritization of Israeli Arabs, Haredim and new immigrant sectors.

- Inter-ministerial work: This is expressed in the joint budget of the five ministries partner to the program, under the management of a common directors-general committee. The program is implemented by MOLSA and the Ministry of Education with a commitment to inter-ministerial, multidisciplinary decision-making on resource allocation and program policy. The program's organizational structure - comprising inter-ministerial committees on the national and district levels and inter-organizational committees at the local level - reflects the commitment to work together and make joint decisions. To facilitate interministerial resource pooling, a special budgeting system was devised enabling follow-up of activities performed by each ministry along with a flexible transfer of funds between them.

- Distribution of roles between the government and local authorities: As part of the program, local authorities were granted the possibility of planning and choosing the measures they most required for their at-risk children and youth, based on local needs and utilization patterns. The government and the districts are responsible for setting policy and mode of operations, and controlling the quality of planning, decision-making, and implementation processes, as well as the outcomes for the children. 
- Commitment to transparency, quality, and data-based decision-making: The program emphasizes upgrading the quality of services for children and youth at risk, accumulating data and knowledge, and service quality. Planning processes are based on a comprehensive mapping of all the children and youth at risk, according to a uniform definition.

The program has a reservoir of sub-programs containing some 300 measures by the partner ministries. From these, the participating local authorities choose and implement the ones most suitable to their populations with funding from $360^{\circ}$. The locality's data infrastructure contains information on the children receiving a service or a response chosen by the local authority from its reservoir of programs throughout the years of the system's operation (mainly since 2011).

During its operation, 260,888 at-risk children and youth were identified, comprising $16 \%$ of the total children in these localities. Out of all at-risk children and youth, 31\% were in the early childhood category, $37 \%$ were of elementary-school age, and $32 \%$ were youth. Of the at-risk children and youth, 47\% were Israeli Arabs, 49\% - Israeli Jews, and 4\% - others. The data collected on children participating in $360^{\circ}$-funded measures and subjected to two measurements (at the start and end of the program) show evident improvement in their situation. Thus, for instance, in the area of well-being and emotional health, there was a 19\% decrease in the number of children with problems; in the area of learning and the acquisition of skills, there was an 18\% decrease; in the area of physical existence, health and development, and social belonging and integration, there was a $16 \%$ decrease.

Data for recent years reveal a clear, stable trend of improvement in the situation of children participating in the program: in $2015,11 \%$ of the children emerged from situations of risk, rising to $13 \%$ in 2016 and $17 \%$ in 2017.

\section{PARTNERING FOR A BETTER WORLD}

The Israel Commission for Equal Rights of Persons with Disabilities was actively involved in formulating the UN Convention on the Rights of Persons with Disabilities (CRPD). It spearheaded work with the country's government ministries to ratify the UN Convention (2012) and was appointed Israel's coordinating entity to advance the implementation and monitoring of the Convention. Israel is deeply engaged in promoting the human and civil rights of people with disabilities and strongly supports global and national efforts to safeguard these rights. Its commitment is underscored by the decision of the Ministry of Foreign Affairs to designate the rights of persons with disabilities as one of the main goals to pursue in its Global Agenda 2019. Israel is also party to the Group of Friends of Persons with Disabilities in Geneva. (SDG 10.2)
Israel ratified the Marrakesh Treaty to Facilitate Access to Published Works for Persons Who Are Blind, Visually Impaired, or Otherwise Print Disabled, within the framework of the World Intellectual Property Organization (WIPO). The Treaty establishes an exception to intellectual property laws to enable the creation of a copy of a published work in a format suitable for use and enjoyment by persons who are visually impaired without requiring the consent of the right holder. (SDG 10.3)

Prejudice, misconception and negative stereotypes regarding persons with disabilities are the source of discriminatory policies and practices. Sport is a powerful tool to deconstruct these prejudices and barriers while promoting the right of equal access and inclusion. Under the theme Challenging the 
Rules of the Game - Sport as a Tool for Inclusion, Israel's Permanent Mission in Geneva initiated a side event in cooperation with Japan and Greece to commemorate the 2018 International Day of Persons with Disabilities (IDPD). (SDG 10.2)

Israel's Ministry of Labor, Social Affairs and Social Services hosted (2018) a delegation of local governance officials of Wuxi, China, working in cooperation with Beit Issie Shapiro on structured modules to assist in the development of communitybased facilities and services for children with disabilities, their families and communities. Beit Issie Shapiro is an Israeli NGO with consultative status to the UN Economic and Social Council (ECOSOC) since 2012, and an active participant in the annual Conference of States Parties to the UN Convention on the Rights of Persons with Disabilities (CRPD). (SDG 10.2)

Between five to seven million disabled children lack wheelchairs globally, and only $30 \%$ will be able to attend school. The Wheelchairs of Hope initiative provides a solution with an innovatively designed child-oriented and affordable wheelchair to empower education through mobility. As one of the first recipients of Grand Challenge Israel, Wheelchairs of Hope affords children access to education, and fosters new generations of children with better skills, confidence and hope. In cooperation with Wheelchairs of Hope, MASHAV donated hundreds of wheelchairs to children in Vietnam (2017), and in 2018, to children in Ethiopia, Guatemala and Cameroon. (SDG 10.2)

To empower and promote social, economic and political inclusion, irrespective of their disabilities, MASHAV dispatches teams of ophthalmologists and medical personnel to partner countries to conduct blindness prevention and eye-care missions. Hundreds of operations and surgical procedures are performed on the premises of local hospitals/ clinics, thereby increasing the number of trained local medical personnel. (SDG 10.2)

The call to improve the status of older people at the international level is increasing. Israel supports the Declaration of Concerted Action on Healthy Ageing to implement the WHO Global Strategy and
Action Plan on Ageing and Health 2016-2030. As a member state in the UN Economic Commission for Europe (UNECE), MASHAV promotes health and well-being across the life course as a fundamental part of a policy response to population ageing. For example, MASHAV and UNECE conducted a series of International Workshops on Community Services for the Elderly. In addition, Israel serves on the Bureau of the Working Group on Ageing for the Committee on Population. (SDG 10.2)

Access Israel, a non-profit organization working closely with government ministries and authorities, was granted consultative status to the UN Economic and Social Council (ECOSOC) since 2016. It shares its experience and knowledge with partner countries worldwide on accessibility and inclusion of persons with disabilities. Examples of Access Israel's international actions include: (SDG 10.2)

- Latvia: At the invitation of Israel's Embassy in Riga, Access Israel shared its educational inclusion approaches with high-ranking government officials. During the mission, the delegation organized several experiential activities, including the Accessibility Trail and a Feast of the Senses;

- Brazil: In cooperation with the Consulate General in Rio de Janeiro, Access Israel served as representatives at the Paralympic Games. It also organized a Feast of the Senses dinner event for local policymakers to raise awareness and an Accessibility Trail for children;

- Austria: A partner Austrian non-profit organization duplicated the Experiential Trail, offering it to schools throughout the country to promote inclusion. Access Israel also works closely with the Zero Project to promote the rights of people with disabilities globally in support of the UN Convention on the Rights of Persons with Disabilities. In 2017, the Zero Project awarded Access Israel with the best innovative practice for its project Pay it Forward in Sign Language to advance the learning of basic sign language, and in 2018, with the best innovative practice for its Accessible Service Training Project;

- Kenya: Access Israel works in cooperation with the organization InABLE, which empowers blind 
and visually impaired students in Africa through assistive computer technology, and helped to organize the first conference in the country on this subject;

- Ecuador: Access Israel's urban accessibility training project Help Me Help You was recognized by the UN Department of Economic and Social Affairs (UN DESA) at the UN Habitat III Conference (2016) in Quito. It also held a sign language workshop for Conference participants;

- Germany: At the invitation of the German Ministry of Labor and Social Affairs, Access Israel participated in the Inklusiv Conference in Berlin;

- In coordination with Israel's Permanent Mission in New York, Access Israel participated in several panel discussions throughout the years at the UN Convention on the Rights of People with Disabilities, and serves as the representative of the International Association of Accessibility Professionals (IAAP) in Israel;

- The $7^{\text {th }}$ Annual Access Israel's International Conference on The Future of Accessibility 2019 will focus on current topics, including transitioning from implementing accessibility to maintenance and excellence, and making future technologies accessible by design. 


\section{GOAL \\ 11}

SUSTAINABLE

CITIES AND COMMUNITIES

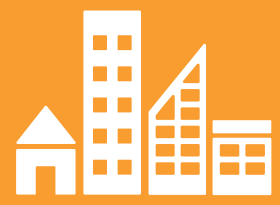

Affordable housing and basic services

Urban renewal in Israel

Accessible and sustainable transport systems

Inclusive and sustainable urbanization

Protection of cultural and natural heritage

Disaster impact reduction, climate change adaptation and resilience to disasters

Waste management in cities

Air pollution reduction in cities

Green and public spaces

National and regional development planning

Partnering for a Better World 


\section{MAKE CITIES AND HUMAN SETTLEMENTS INCLUSIVE, SAFE, RESILIENT AND SUSTAINABLE}

Urbanism is one of the most urgent challenges in Israel, a country with one of the highest population growth rates in the world, reaching approximately $2 \%$ per year. In the past two decades, Israel's population increased from 6 million people in 1998 to 9 million in 2018. 
According to demographic predictions, Israel's population will reach 11 million by 2030 and 15 million by 2048 (Israel's centennial). Israel is also one of the world's most densely populated and urbanized countries, with approximately $93 \%$ of its population living in cities. These facts illustrate the importance of promoting better urbanism in Israel and strengthening the sustainability, resilience and inclusiveness of cities and regions throughout the country.

These challenges are addressed by the Ministry of Construction and Housing ( $\mathrm{MOCH}$ ), by the Israel Planning Administration (IPA) and by the Ministry of the Interior. The MOCH leads the processes of policy planning and implementation in the field of urbanism, urban planning and urban development as well as in the areas of public and social housing, affordable housing, development of new construction methods and the assimilation of advanced technology and innovation in the construction industry. The IPA formulates national planning policy on various issues and initiates national, regional and local master plans, strategic plans and policy and procedural guidelines, with the aim to increase the efficient use of land and to improve the compactness and livability of all environments. The Ministry of the Interior leads the national policy and strategy for local government, with the aim to increase economic growth, to narrow gaps between communities and to strengthen national and regional planning.

Urban renewal, in all its facets, is a cornerstone objective of Israel's government and one of the main challenges in the country. In light of the rate of population increase and the shortage in land resources, the government recognized the need for urgent and creative solutions to intensify and concentrate population densities in the cities and is placing a great emphasis in this area. In August 2016, the Law for a Government Authority for Urban Renewal was approved, establishing a designated body responsible for urban renewal in Israel. In light of the seminal role played by urban renewal in sustainable cities both in Israel and globally, the $\mathrm{MOCH}$, as the leading ministry in Israel on promoting SDG 11, is considering the proposal of a new local indicator for urban renewal which could provide the basis for a global indicator within SDG 11.

\section{( Affordable housing and basic services (SDG 11.1)}

One of the main challenges facing the State of Israel is to ensure affordable housing for its citizens. Approximately $70 \%$ of apartments in Israel are privately-owned and homeownership is still an aspired goal for many citizens.

Figure 11.1

\section{Apartment purchase and rental prices to net income ratios}

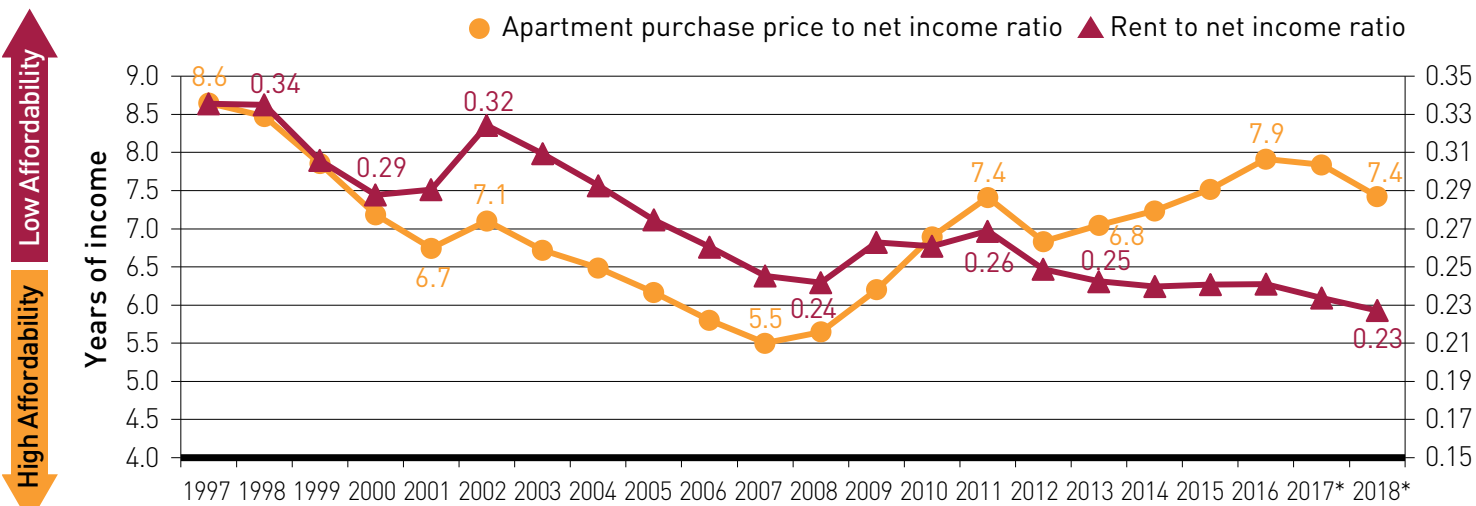

Average income for 2017-2018: estimated by the Economic Analysis Division, MOCH

*Rent prices are adjusted to the apartment prices index, base year 2015. 
During the last decade, real housing prices have almost doubled due, inter alia, to rapid population growth, low unemployment rates, growth in family income and low interest rates - all of which have caused housing demand to increase while supply has remained relatively low. At the same time, rental prices have increased at a much lower rate. In fact, affordable housing, in terms of rental housing, remained stable throughout the years (purchase and rental prices relative to income, Figure 11.1).

As part of the effort to increase the housing supply, the Israeli government approved a new holistic
National Strategic Housing Plan in February 2017. The plan determines the future housing needs of the population: 1.5 million new residential units by the year 2040. In order to reach this target and due to the gap between the number of approved units and number of actualized units, a target of 2.6 million planned residential units was set.

To assess progress toward meeting the intermediate objectives of the strategic housing plan and the planning objectives, a locally suggested indicator is proposed (Figure 11.2)

Currently, subsidized apartment-purchase tracks are offered to the public, as in the example of 'Mehir

Figure 11.2

\section{Approved housing units}

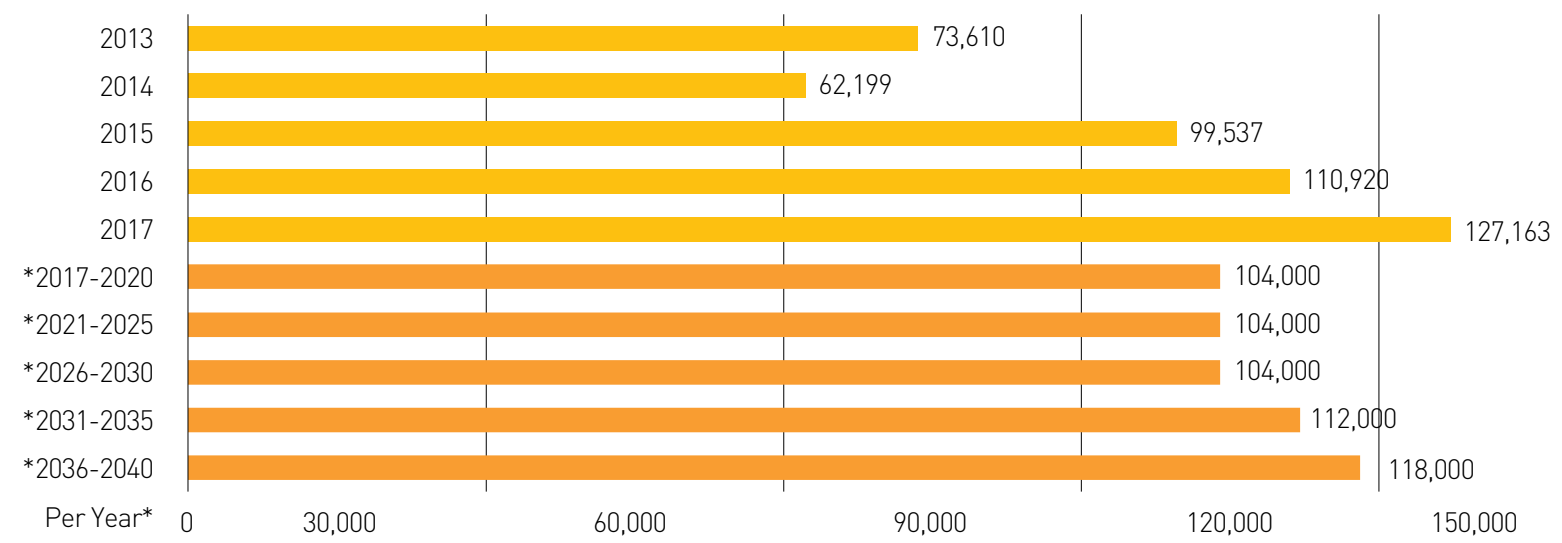

\section{CASE STUDY}

\section{Neighborhood $360^{\circ}$}

Neighborhood $360^{\circ}$ is an innovative measuring tool that aims to promote the planning, development and building of quality, healthy, prosperous and sustainable neighborhoods in Israel. The tool integrates multi-dimensional development indexes by setting estimation criteria and standards for the quality of the planning process, by incorporating environmental considerations in the planning and execution stages, and by promoting vibrant urban neighborhoods. The tool is adapted to the development patterns and the planning reality in Israel and will serve professionals from the early planning stages, through the development and building stages, and ending with the populating stage. The assessment of a project using the tool includes an examination and analysis of approximately 50 sustainability criteria in infrastructure and building, natural and public spaces, and efficient resource use. For each of these parameters, a unique index was developed, specifically adapted to the reality in Israel, and a weighted scoring scale was defined. 
Figure 11.3

\section{Housing assistance over the last decade}

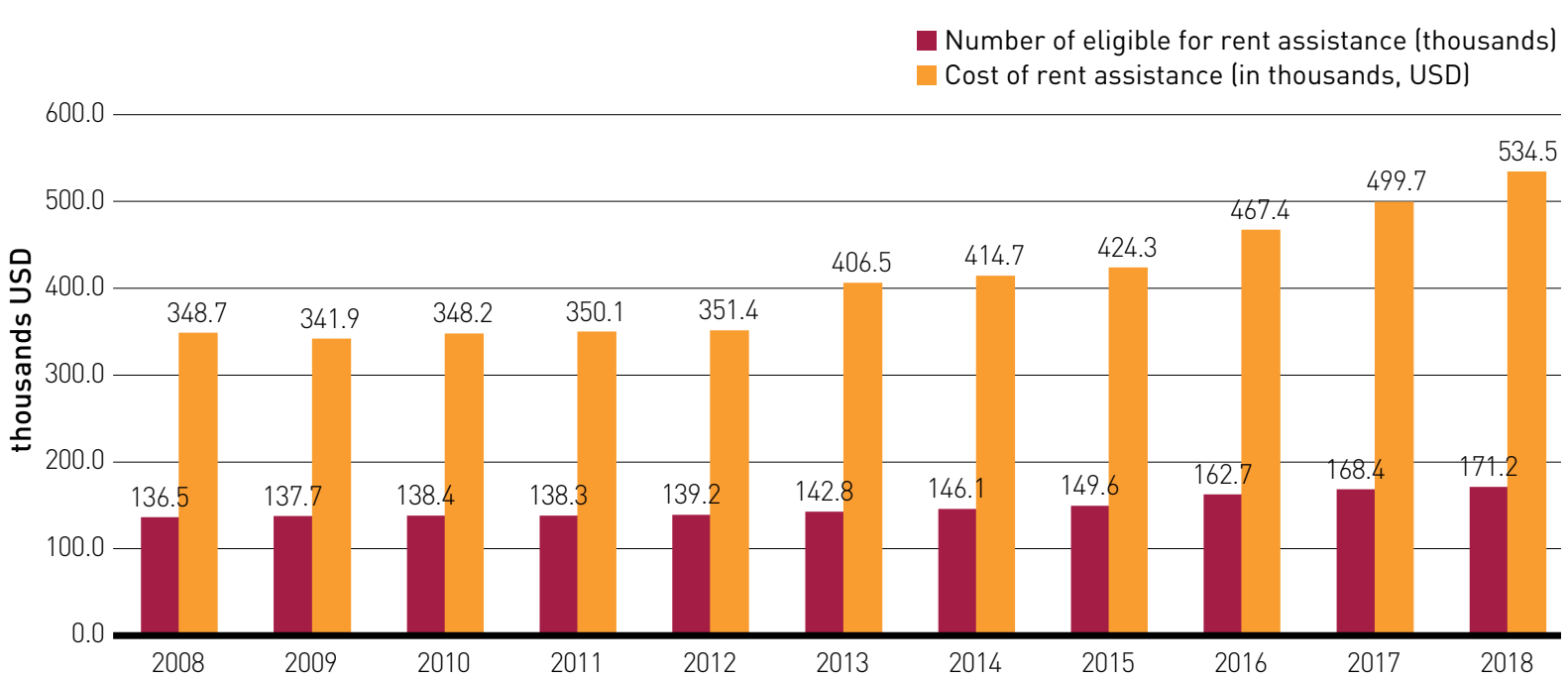

Lamishtaken' (Buyer's Price), in which first-time apartment buyers are given a significant discount off the market price. In addition, in order to improve compatibility between planning and housing needs, the government established the Housing Cabinet, which serves as a coordinator between the relevant bodies. For populations unable to afford housing, several housing assistance programs are offered, designated for a variety of populations of different socioeconomic backgrounds, such as public housing allocations and rent assistance to eligible people (Figure 11.3).

When it comes to neighborhood planning, a diverse array of actions exists, such as: land siting surveys, feasibility studies, theoretical plans and urban building schemes. In this field, the government is promoting planning excellence, knowledge dissemination and the publication of guides on different subjects, such as the "Neighborhood $360^{\circ}$ "guide (see box).

To ensure the quality of apartment and building construction, building and sustainable building codes are being prepared. Building codes will include all building guidelines and regulations, enabling Israel to meet international standards in various areas: acoustics, fire safety, damp and moisture protection, building energy scoring, waste collection, venting and acclimation, and more. Sustainable building codes will relate to areas such as shading, energy efficiency, green construction and parking allocations Iwhich were reduced in correlation with the building's proximity to mass transit).

\section{( Urban renewal in Israel}

The Israeli housing market is characterized by a high proportion of homeownership, which poses a difficulty for urban renewal projects. Israel's governments have created a number of tracks to encourage and implement urban renewal, with the emphasis on increasing building rights in return for upgrading the resilience of buildings to earthquakes by strengthening or reconstructing existing buildings. In most tracks, the renewal of public spaces is also addressed.

Among its many benefits, urban renewal leads to quality densification and thus to better, rejuvenated cities. Furthermore, Israel is located in a seismicsensitive area, with a probable risk of earthquakes. Since past building standards did not comply with the national standard for structural durability in an earthquake, Israel's Urban Renewal National Master Plan requires the strengthening of existing buildings as a prerequisite for the addition of building rights.

Israel's Strategic Housing Plan for 2040 sets targets for the number of housing units to be built 


\section{CASE STUDY}

\section{The "Kiryat Moshe" Project in the City of Rehovot}

This project is a detailed plan for urban renewal, as part of the government decision to promote urban renewal and regeneration in neighborhoods with a majority of Jews of Ethiopian descent. According to this plan, old buildings will be destroyed, and new residential buildings will be constructed in their stead. The plan makes use of available land near the neighborhood (called "complementary land").

Today there are 1,629 apartments in the neighborhood. The plan allows for the building of 8,000 additional housing units, thereby transforming the neighborhood into a large, central block of the city. Today, approximately 7,000 residents live in the neighborhood, with 30,000 residents expected to reside in the neighborhood in the future. The plan includes an addition of public buildings and open spaces on approximately $0.4 \mathrm{~km}^{2}$. As part of the renewal program, a set of assistance tools was provided to the residents, such as the establishment of a community administrative authority, funding for fully executing resident's rights, and social care and assistance.

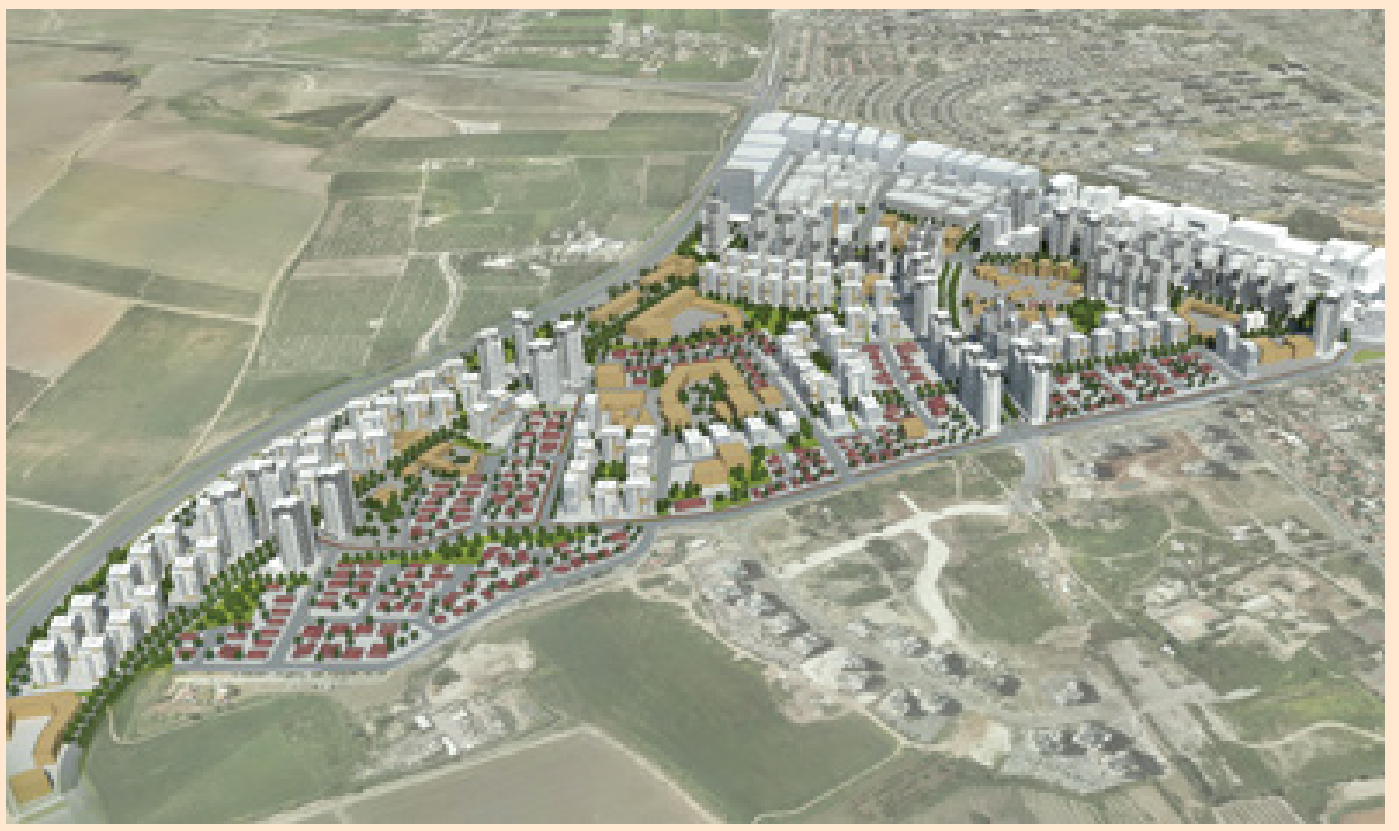

Simulation: Barre Levie Dayan Architects

in urban renewal projects until target year 2030. According to the plan, $20 \%$ of the additional, planapproved housing units are to be built by 2020 and up to $35 \%$ by target year 2030 . This target includes unbuilt plots in urban areas that are not under the Authority's jurisdiction. In light of the multiplicity of parameters for measurement, efforts are concentrating on coordinating the data in order to ascertain whether the target is met or not.
In the local context, due to the expected increase in plan approvals in 2018-2020, the Urban Renewal Authority expects to achieve the objective for 2017 2020: building 21,000 housing units in the urban renewal track in 2020 (20\% of total plans approved) as part of meeting the final target for 2030 - building 870,000 housing units, representing a third of the total approved housing units. In order to measure progress toward meeting the target of the Strategic 
Figure 11.4

\section{Urban renewal housing units, by years}

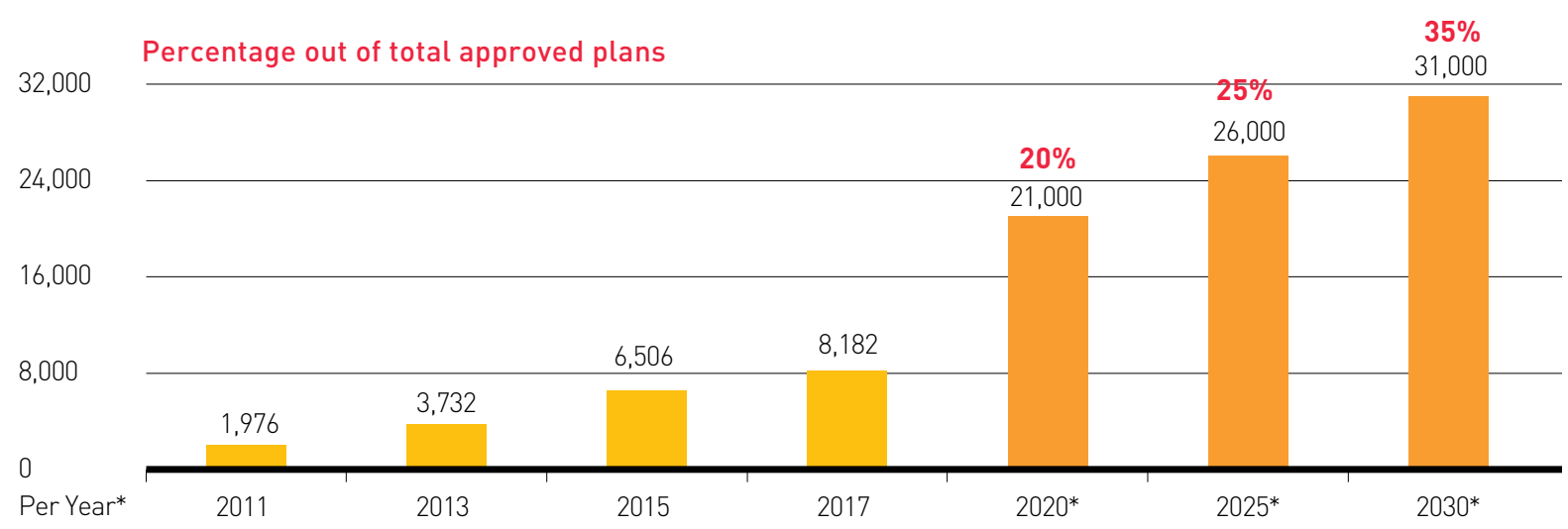

Housing Plan for 2040, a locally relevant indicator is proposed: percentage of housing units in urban renewal projects out of the total of approved plans (Figure 11.4)

Urban renewal is one of the most important tools currently used in Israel to provide solutions for the shortage in housing units and for increasing building resilience against earthquakes. In order to meet the above objectives, government ministries, entrepreneurs and local authorities need to continue to jointly provide supportive tools, policy and legislative measures, and additional financial solutions, to increase the rate of sustainable urban renewal in demand areas and in the periphery, while fully cooperating, coordinating and creating certainty for property owners.

\section{( Accessible and sustainable transport systems (SDG 11.2)}

During the last decade, the Israeli government initiated major changes in its transport policy and began planning, developing and implementing mass transport systems in the four metropolitan areas (Jerusalem, Tel Aviv, Haifa and Beersheba). In Jerusalem, Israel's first light rail line was opened in 2011, and two more lines will be added in the next few years. In Tel Aviv, four light rail lines and three underground lines are currently in different stages of planning and implementation.
In 2014, the National Public Transport Authority (NPTA) was established, as a support unit in the Ministry of Transport and Road Safety (MOT). Through the NPTA, the government established new train lines, significantly increased budgets for public transport, and took important policy measures. These include promoting competition in the public transport sector, establishing a new rate policy, initiating taxation changes in the private vehicle sector, re-organizing the public transport system in the most crowded area in Israel (the Tel Aviv Metropolitan Area), introducing innovative ticketing and information technologies and improving service levels.

However, Israel still falls significantly behind OECD countries, both in the infrastructure sector and the complementary policy measures (Table 11.1). In light of this, in 2012, the MOT and the Ministry of Finance prepared a "Strategic National Plan for the Development of Public Transport." The 25year plan revealed that Israel lags behind in such areas as cumulative investment in public transport infrastructures, service level, extent of use of public transport and split-mode travels, average speed of public transportation, insufficient exclusive rightof-way, and lack of diversity in travel modes and level of multi-modal integration.

Despite new efforts to promote public transport, data show that while private vehicle trips are dropping in OECD countries, the opposite trend is 
Table 11.1

\section{Summary of indicative parameters in Israel's metropolitan areas}

\begin{tabular}{l|c|c|c|c|c} 
Parameter/Metropolis & Jerusalem & Tel-Aviv & Haifa & Beersheba & $\begin{array}{c}\text { Parameter average in } \\
\text { developed metropolises as } \\
\text { of 2010 }\end{array}$ \\
\hline $\begin{array}{l}\text { PT kms/resident/year } \\
\text { (December 2017) }\end{array}$ & 68.2 & 43.7 & 75.1 & 87 & 100 Vkm/inhab \\
\hline $\begin{array}{l}\text { Daily average speed of PT } \\
\text { network (December 2017) }\end{array}$ & 26 & 18 & 19 & 42 & $25 \mathrm{~km} / \mathrm{h}$ \\
\hline $\begin{array}{l}\text { Number of public transport } \\
\text { boardings/resident/year }\end{array}$ & 106 & 36 & 57 & 42 & 250 Br/inhab \\
$\begin{array}{l}\text { (December 2017) } \\
\begin{array}{l}\text { Modal-split: percentage } \\
\text { of work travels by public } \\
\text { transport (2017) }\end{array}\end{array}$ & $22 \%$ & $17 \%$ & $15 \%$ & $14 \%$ & $40 \%$ \\
$\begin{array}{l}\text { Length (meters) of exclusive } \\
\text { public transport infrastructure } \\
\text { (December 2018) }\end{array}$ & 26 & 35 & 60 & 5 & $150 \mathrm{~m} / 1000$ inhab \\
\hline
\end{tabular}

Figure 11.5

Changes in length of roads, km travelled and motor vehicles, 1970-2015.

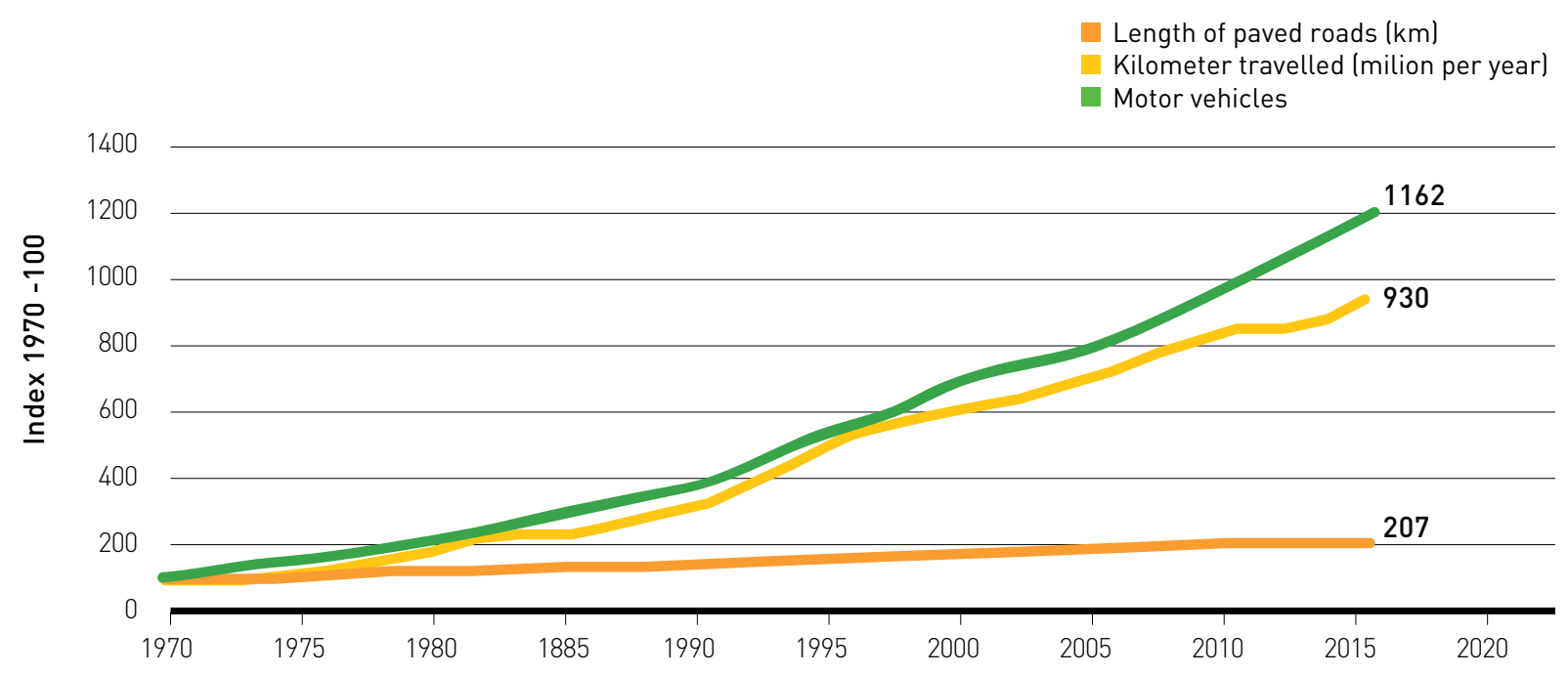

noted in Israel. Examination of relevant data from 2014 shows that the number of vehicles per $1 \mathrm{~km}$ of road was 2,700 (vehicles $/ \mathrm{km}$ of road). Due to high vehicle density, the transport infrastructure is overloaded. The rate of increase of distances travelled is particularly high in Israel, so that increased road construction cannot provide the only solution (Figure 11.5).

In recent years, a significant increase in the number of electric bikes in major Israeli cities has been noted. Electric bikes are a convenient, quiet and environmentally friendly form of transportation which contribute to reducing congestion on the roads. However, the increased use of this mode of transport has been accompanied by an increase in the number of injured in electric bike accidents, although a moderation was noted in 2017.

The MOT has prepared five strategic action plans in recent years which form the basis for its implementation policy. These plans address 
investments in public and urban non-motorized transport, integration of policy measures to encourage the use of these transport modes and land-use, and transport policies that will enable sustainable accessibility. The main component in the strategic plans is the promotion of public transport services.

To measure Israel's progress toward accessible and sustainable public transport, the following local indicators are recommended for Israel:

\section{- Modal-Split: percentage of travels by public} transport: This suggested indicator, which largely relates to the percentage of travelers by public transport, is an important tool to evaluate sustainable transport development for a city or a defined area. The average modal-split for developed metropolises in the world is $40 \%$, while in Israel it is only $17 \%$. As can be seen in Table 11.1, as of 2017, the modal split-level in Jerusalem was 22\% and in Tel Aviv, Beersheba and Haifa between $15 \%$ and $17 \%$

\section{- Length (km) of exclusive public transport} infrastructure (December 2018): This suggested indicator evaluates the service level as well as the advancement in public transport development in the city. The average distance traveled by public transportation in exclusive public transport infrastructure in developed metropolises in the world is $100 \mathrm{~km} /$ resident, while in Israel it is $68.5 \mathrm{~km} /$ resident.

\section{( Inclusive and sustainable urbanization (SDG 11.3)}

Inclusive urbanization and participatory human settlement planning are important elements in Israel's Planning and Building Law (1965). The planning system is comprised of three organizational levels - national, regional and local - with representatives of government ministries and agencies and local authorities as well as representatives of professional organizations and the public at some of the levels.

In general, three different levels of citizen engagement can be identified in planning: citizen information whereby information is conveyed from the government to the public, citizen consultation whereby the government initiates processes allowing citizens to voice their opinions, and citizen participation and empowerment based on a twoway dialogue whereby planning solutions take both parties' views into consideration.

The Planning and Building Law requires the following:

\section{- Plan deposition (partial citizen consultation):}

The law stipulates that the plan approval process be conditional on a two-month plan "deposition" to allow for stakeholders' objections. The law defines stakeholders (mostly owners, part owners, neighbors and relevant professional organizations) and sets procedural limitations with respect to the time and manner of objecting to plans, but it clearly empowers the most affected parties and assures that their voice is heard, and their objections are part of the public record.

\section{- Transparency in plan-related information Icitizen} information): Israel is strengthening its policies on the sharing of planning information. The trend is reflected in recent planning law amendments, most notably in Amendment 101 (2014). This amendment significantly reinforced transparency requirements with regard to both plan content (documents must be on public display from the day of plan submittall and decision-making processes (planning committees' decisions must be published within three days of meeting dates). Publication of information on the web is a legal requirement.

Israel has introduced system-wide digitalization of planning and permitting processes that sets new benchmarks for the accessibility of planning data to the general public. Significantly, it includes an online plan submittal system and a recently released online system for submitting building permit applications. Both systems enable the orderly viewing of data, thus improving the ease and quality of public involvement. Today, many planning agencies extend public participation practices well beyond the legal requirements as shown in the following examples:

- Local comprehensive plans include four public meetings throughout the planning process to obtain public input with respect to present conditions, strategic vision, draft plan alternatives 
and selected, individual issues of local importance;

- The preparation of subject-oriented national master plans is accompanied by an extensive stakeholder and/or citizen participation process. Typically, it involves meetings with stakeholders, an "Editors Committee" including stakeholders' representatives, and publicized meetings with the general public;

- A structured plan for citizen participation is required as a pre-condition to formal plan submittals in some types of plans processed by the National Planning and Building Committee for Prioritized Housing Areas (Vatmal). The Vatmal is a legislative fast track for large housing plans, mostly on government-owned land, which was established in 2014 for five years with an option of extension;

- Extensive public participation is typical of urban regeneration projects in which the assent of property owners is required by law. The Urban Renewal Authority is promoting several citizen participation processes in urban renewal plans such as detailed plans in the local authorities' track, detailed plans in the taxation track (entrepreneurial), guidelines for citizen participation processes in the 18 urban renewal administrations and a guide on the preparation of a social report on urban renewal projects.

In October 2018, the Urban Renewal Authority published a guide which aims to involve and include property owners and the entire community in the planning processes of urban renewal projects. The guide elaborates on the tools and stages at which public participation should be integrated, and on means of drafting recommendations that can later be implemented in the planning process and used throughout the different stages of the project.

In recent years, due to the increase in urban renewal projects, the degree of citizen participation in planning processes has risen as well. This rise has been supported both by the government, through its Urban Renewal Authority arm, and by property owners who are given the opportunity to participate in project meetings at initial planning stages.

A locally-relevant indicator has been suggested for Israel which relates to the number of status updates to property holders and tenants during a project's planning process: a semi-annual update for an outline plan and a quarterly update for a detailed plan. Status reports may include site conferences, resident meetings, letters, and other official updates.

\section{( Protection of cultural and natural heritage (SDG 11.4)}

Natural and cultural heritage sites in Israel's cities are an integral part of the urban landscape. The intensive urbanization and development processes that have taken place in Israel in recent decades present challenges to the planning and conservation of open spaces, natural values and local cultural and heritage sites. In the face of these challenges, national master plans (NMPs) have been promoted and approved for national parks and nature reserves (NMP 8), forests (NMP 22), seashores (NMP 13), and more, as well as a strategic plan for open spaces.

As part of an ongoing global trend, cities in Israel have also begun to implement actions directed towards the conservation of natural and cultural sites, and changes of approach are taking place among decision makers as well as the general public.

While it was previously believed that local authorities have no part in nature conservation, in the last decade meaningful progress has been made towards understanding the importance of urban nature conservation as part of the national effort to protect Israel's biodiversity. For example, as a first step towards protecting urban natural areas and assimilating this field in municipal policy and management, cities have begun to map their urban natural areas. Between 2008 and 2018, 26 urban nature surveys were completed, and 24 more are in different execution stages.

Findings from the last decade's urban nature surveys suggest that valuable open spaces, rare habitats, endangered species and unique natural assets of national and regional importance exist in many municipal areas. Today, a few models for the management of the different natural areas in the urban environment can be found in Israel. Examples of nature site preservation in cities include the Coral Beach Reserve in Eilat, Apollonia 


\section{CASE STUDY}

\section{Urban Nature in Jerusalem}

Israel's first urban nature survey was conducted in Jerusalem. Among several unique sites identified is the Gazelle Valley in the southern part of the city, confined between several residential neighborhoods. The site has a rich and significant biodiversity, including a herd of mountain gazelles. The site's development caters to the needs of the general public, and especially the surrounding neighborhoods whose residents are active partners in the maintenance and development of the site. The Gazelle Valley Park is very popular and is an excellent example of a successful urban nature park.

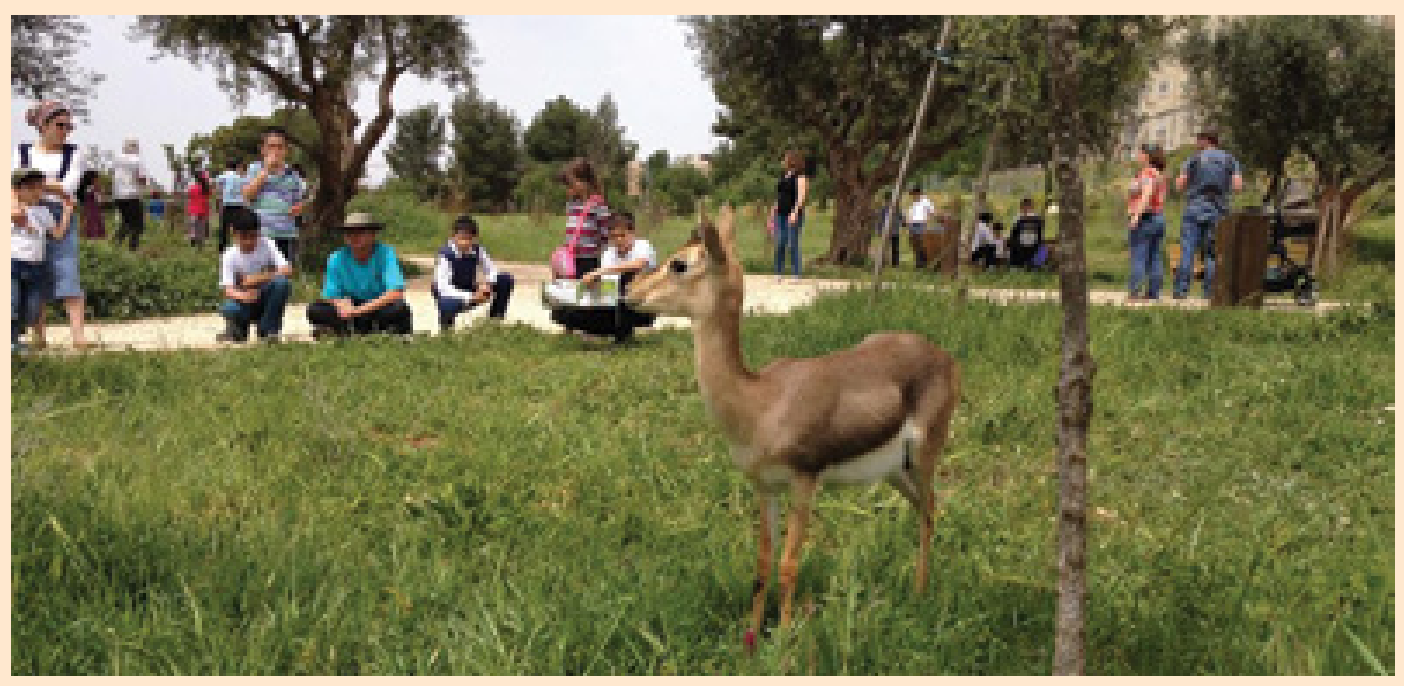

The Gazelle Valley, Jerusalem/Photo: Gazelle Park Team

National Park in Herzliya, the Community Forest in Rosh Ha'ayin, and the Haifa wadis.

The conservation of urban cultural heritage sites is also of major importance. The many cultural heritage sites in Israel, from different historical periods, are witness to the variety of cultures and events that took place in the country. Urban heritage conservation may range from preserving a single monument to preserving the historic old city at the heart of a municipality. In Israel, such sites include archeological or historic assets and buildings as well as areas designated for preservation such as the remains of old cities, ports, streets, markets or specific architectural styles. Throughout the years, laws and other measures have influenced the protection of cultural heritage sites in the urban environment. Today, several bodies are involved in the preservation of urban cultural heritage sites, but each usually focuses on a specific historical period or interest, with no single authority responsible for managing and leading this field.

Significant government support for the preservation of national heritage sites in Israel has been provided by means of the "Landmarks" program initiated by the Ministry of Jerusalem and Heritage. The ministry's Heritage Department operates at two main levels: supporting projects to preserve national heritage infrastructures and promoting national policy and strategy.

The conservation of local cultural heritage and natural values has a key role in urban development. Of high importance is the empowerment of local authorities to promote natural and cultural 


\section{CASE STUDY}

\section{The "White City" of Tel Aviv}

The "White City" of Tel Aviv was declared a World Cultural Heritage Site by UNESCO in 2003. According to the inscription, Tel Aviv is a prominent example of the "various trends of the Modern Movement in architecture and city planning at the beginning of the 20th century." As part of the process, the Tel Aviv municipality committed to prepare a statutory protection plan for the historical buildings and fabric of the "White City."

In 2008, the Tel Aviv conservation plan was approved, identifying approximately 1,000 historical buildings designated for statutory protection, the majority of which are located in the White City's officially designated limits

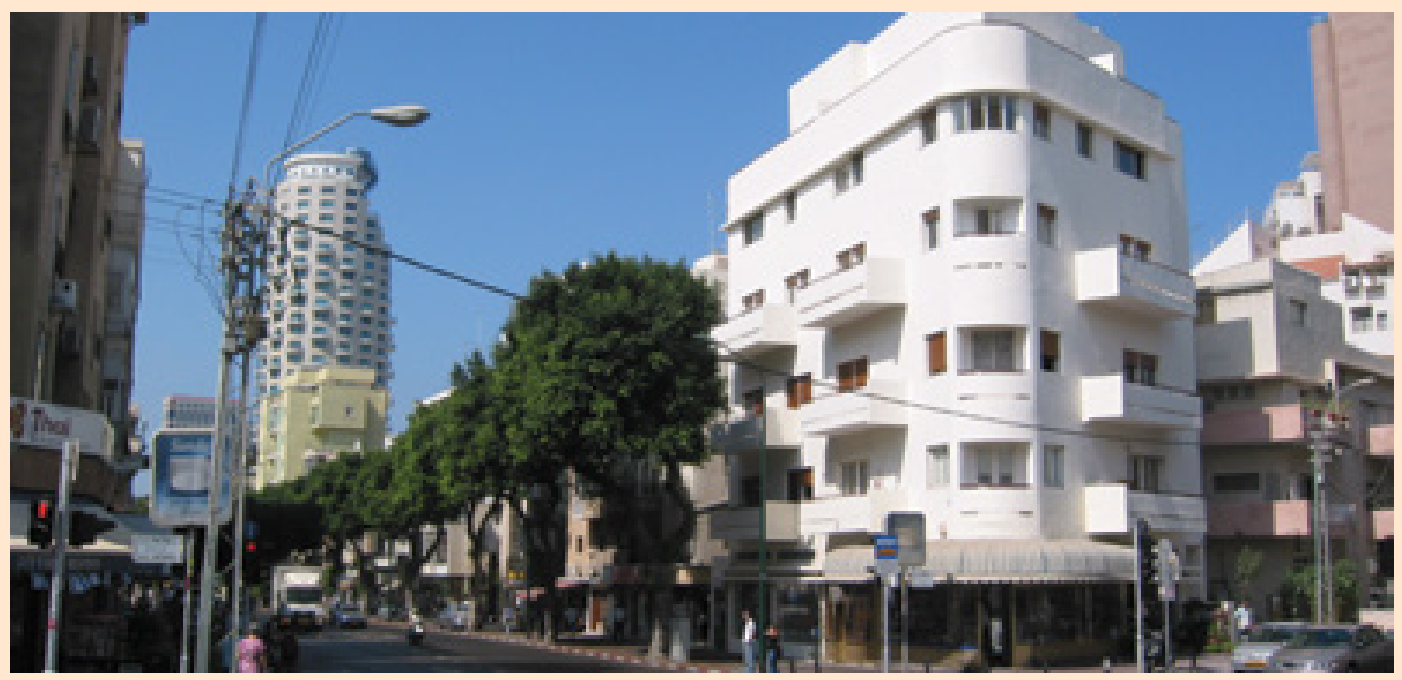

Ben Yehuda Street, Tel Aviv/Photo: Wikipedia

heritage sites under their jurisdiction by completing their heritage database and implementing appropriate policies and plans in their municipal systems. The multiplicity of bodies involved in the conservation efforts sharpens the need for a more holistic, unifying management approach under governmental responsibility, which will include the accompaniment, regulation and support of local urban initiatives and overall supervision of this field.

\section{Disaster impact reduction, climate change adaptation and resilience to disasters (SDG 11.5, 11. b)}

Israel has a comprehensive emergency and preparedness mechanism, headed by the National Emergency Management Authority (NEMA), under the Ministry of Defense. NEMA is responsible for compiling national hazard assessments and directing and coordinating preparedness and response of government offices, infrastructures and local authorities in case of emergency.

NEMA defines the range and probability of risks Israel faces, including the following:

- Natural disasters: Earthquakes and tsunamis, extreme heat and droughts.

- Man-made disasters: Fires, terrorism and war, cyber-attacks and hazardous materials events. A dedicated plan is in place for each type of hazard, with regular exercises to ensure readiness. On the local level, cities are obliged by law to establish a local emergency deployment committee and to prepare a municipal emergency portfolio. However, the natural disaster 
framework, outlined by the state, only includes fires and earthquakes, with no reference to storms or floods.

In December 1999, the Israeli government established a Steering Committee for Earthquake Preparedness to lead and coordinate actions. The current assessment defines a 16\%-26\% chance of a major earthquake in the next 20-30 years, with approximately 7,000 fatalities. Preparedness for earthquakes is widespread, substantiated by building codes, a national master plan and numerous financial tools. The Earthquake Building Standard was instated in 1975, followed by a dedicated master plan intended to create economic incentives for structural strengthening of buildings built before 1980, but actual implementation remains limited. In 2014, a tsunami assessment was compiled. Consequently, the assessment was translated into preparedness plans and drills in local authorities along the Mediterranean coast.

As most of Israel's local authorities are small and lacking in financial resources, a sliding index for resilience support has been developed to ensure that the budgetary standing of the municipality does not reflect on its disaster preparedness. The sliding index is a compilation of the municipality's vulnerability with its financial and administrative robustness.
Israel recognizes that those living in poverty, who comprise $21 \%$ of the Israeli population, are the most vulnerable in any extreme event and require dedicated municipal and government responsiveness in order to ensure their safety and resilience. Therefore, schemes for disadvantaged communities and populations include special housing solutions and dedicated plans in times of emergency.

While most local authorities in Israel have effective emergency systems in place, they do not have contingency plans for climate changes, in particular for the impacts of increased heat and infrastructure degradation due to extreme weather. In Israel, climate trends already indicate temperature rise and more extreme weather events, resulting in local flooding and prevalence of heat islands in cities, with the likelihood that the intensity of precipitation events will increase along with sea level rises (Israel Meteorological Service, 2016 data).

Numerous impacts of extreme weather events are already evident in Israel:

- Flooding is on the increase in winter in many urban areas. While most local authorities have contingency plans for rare flood events, plans and building codes need to be adapted in light of the significant increase

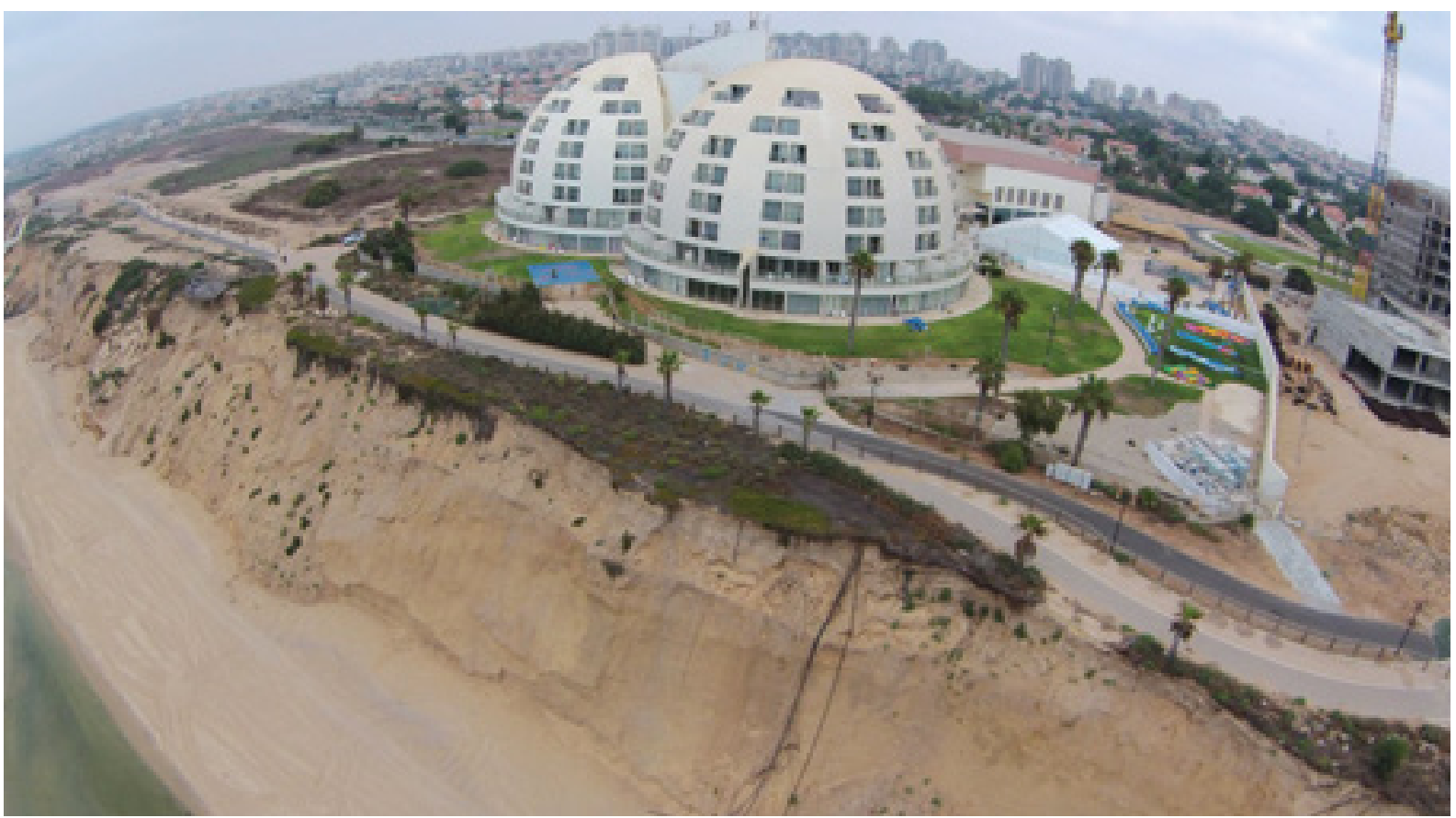

Mediterranean coastal cliff/Photo: Photo Yossi Uzi Ltd. 
in the number of such events. A policy paper on runoff management in urban areas is in preparation.

- Shade is becoming a critical asset for the urban environment. As heat increases and water resources decrease, the livability of cities depends more and more on the availability of shaded areas.

- Coastal cliff erosion is risking 10-50 meters high lime cliffs, along $45 \mathrm{~km}$ of Israel's Mediterranean shoreline. Cliffs are retreating by dozens of centimeters each year on average. Some have begun to collapse, endangering people and structures and creating a budgetary constraint on local authorities. In light of this concern, a National Master Plan for the Protection of Coastal Cliffs along the Mediterranean was approved, enabling the implementation of coastal and maritime protections while preserving, to the extent possible, the cliff's natural configuration.

The second decade of the 21 st century began with a number of extreme weather events which led to disastrous consequences. For example, in December 2010, a major fire tore through the Carmel Mountain. The fire was extinguished after four days, during which forty-four people lost their lives and approximately 17,000 residents were evacuated, alongside extensive property damage. In December 2013, Israel was hit by an unusually extreme snowstorm which primarily affected the northern and central areas of the country. Trees fell on top of power lines, and many residents were besieged in their own homes with no access to basic products and no means of heating.

In 2014, the Ministry of Environmental Protection (MOEP) published a National Assessment of Climate Change Impacts, which relates, inter alia, to recommendations on climate change adaptation at the local level. In addition, the State Comptroller published a special report about the preparedness of local authorities for extreme weather conditions. This triggered a reevaluation of the existing mechanisms and existing emergency scenarios, including a July 2018 government decision to implement the National Program for Adaptation to Climate Change and the establishment of an Inter-ministerial Administration for Climate Change Adaptation and a Sub-committee for Local Adaptation. The administration is responsible for the implementation of the national program, aimed at preparing for and coping with climate changes and extreme weather events (see SDG 13.1).

Currently, there is no structured data compilation of impacts and economic losses due to natural disasters, neither at the governmental level nor at the local level. Towards the end of 2018, the State Comptroller issued a report on the 2016 fires, identifying them as the worst disaster in terms of damage to property and the environment: more than $40 \mathrm{~km}^{2}$ of land were burnt, and total damages surpassed \$176 million.

Figure 11.6

\section{Natural disaster events in Israel 2007-2018}

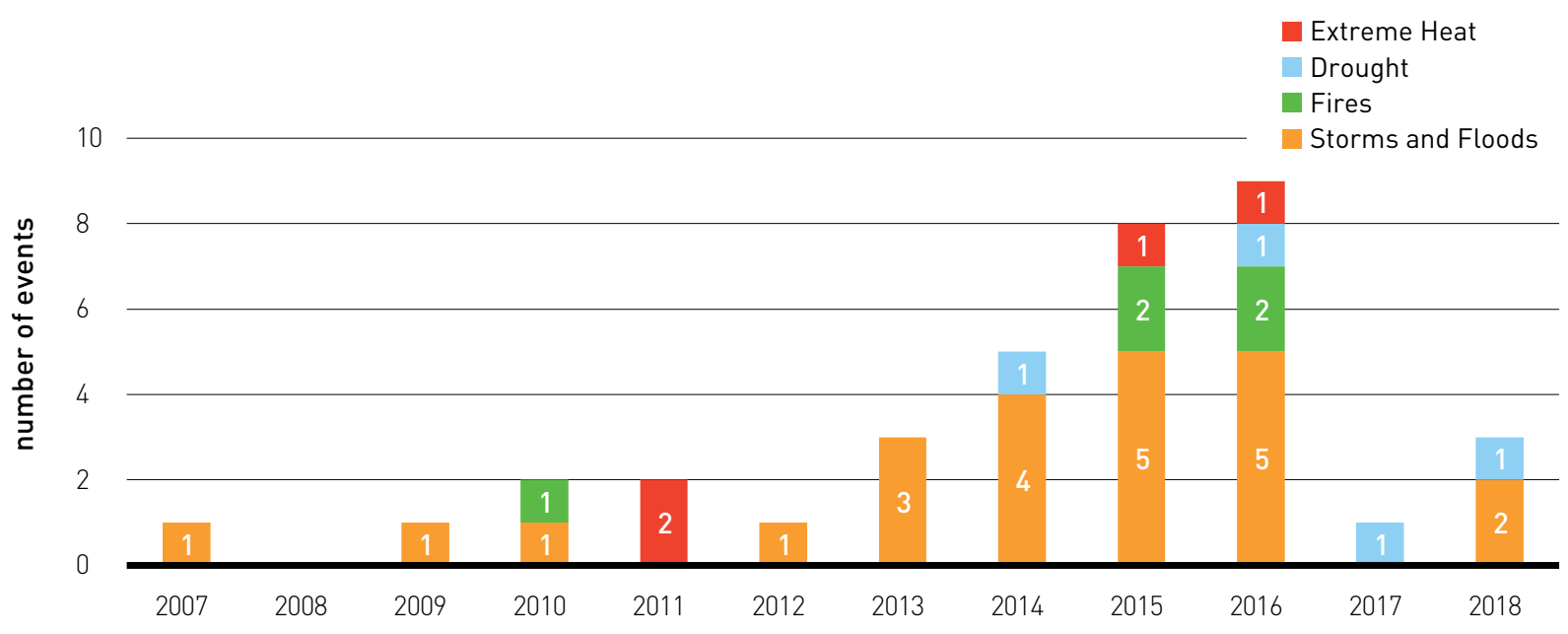




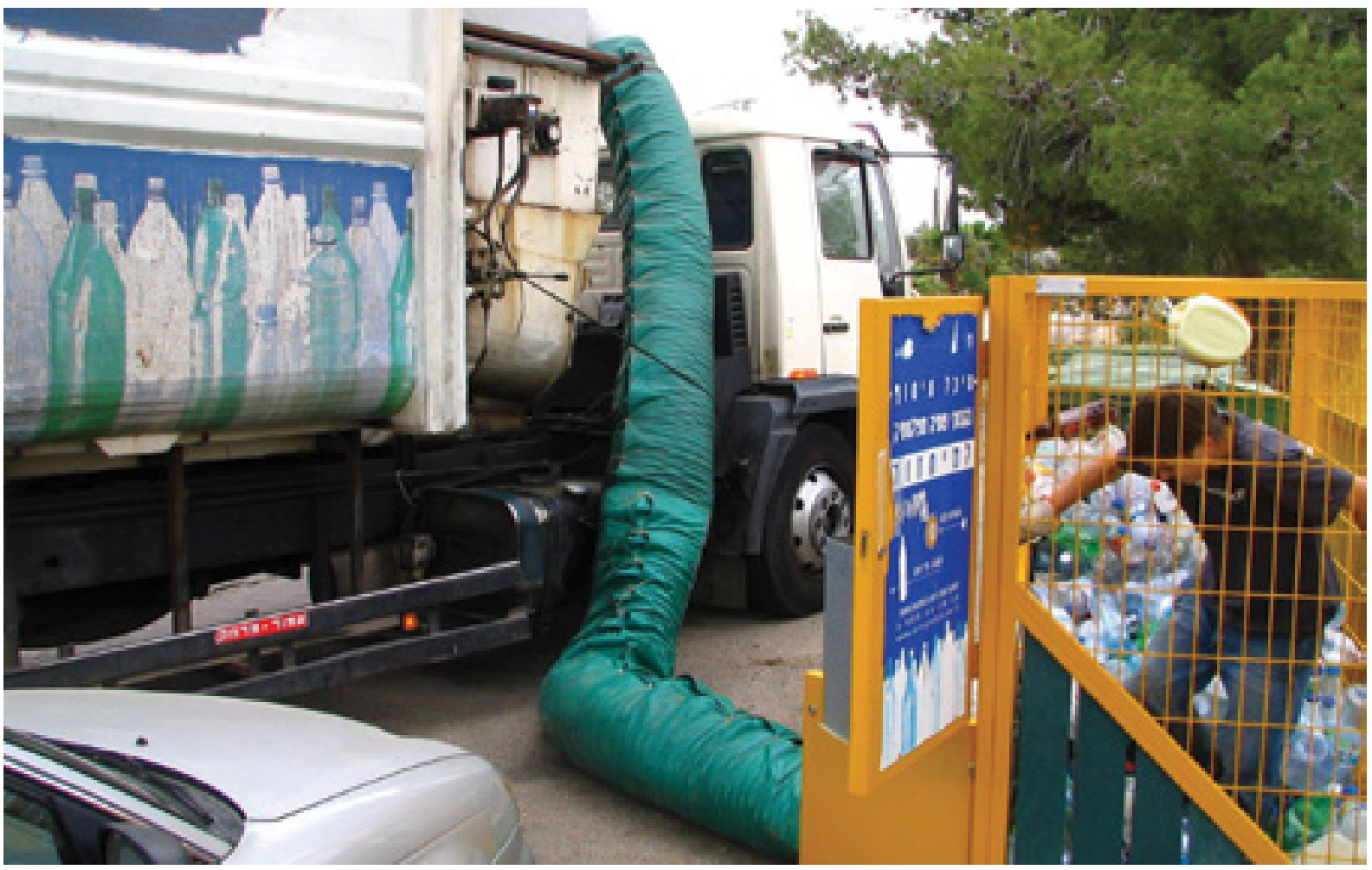

Plastic bottle collection/Photo: Ilan Malester

\section{( Waste management in cities (SDG 11.6)}

The Ministry of Environmental Protection (MOEP) is responsible for waste management regulation in Israel. Local authorities are responsible for the collection of urban waste and waste disposal to designated waste disposal sites.

Israel's residents produce approximately $1.7 \mathrm{~kg}$ of waste per day. In 2017 about 5.4 million tons of municipal waste were produced, of which $22.4 \%$ were recycled, a $2 \%$ improvement over the previous year. Over the last two decades, separation at source of various waste streams, with government support, has been introduced into local authorities and businesses. Municipal waste is collected in bins in residents' homes and in commercial centers or separated at source of collection into different waste streams. These streams include paper and cardboard, plastic bottles, organic waste, garden waste and electronic waste. Designated receptacles and centers have been set up for specific waste components in local authorities throughout the country (see SDG 12.4, 12.5).

\section{( Air Pollution Reduction in Cities (SDG 11.6)}

Transportation is recognized as a major contributor to air pollution in urban areas and exceedances of air quality standards measured in monitoring stations in city centers confirm that air pollution in Israel's cities is primarily caused by motor vehicles.

\section{( Green and public spaces (SDG 11.7)}

While public space is universally accepted to be one of the most important components of vibrant, equitable cities, it defies attempts at a clear definition. The role of the public space is strongly affected by local culture, climate, resources, topography and other local factors. As a result, creating effective policy-making and management related to the public space is quite challenging, further complicated by the fact that best practices often do not transfer well between cities. 


\section{CASE STUDY}

\section{Reducing Air Pollution from Transportation in Haifa Bay and Jerusalem}

In the wake of government decisions taken in 2015 and 2017, Israel's first Low Emission Zone (LEZ) was established in Haifa Bay. According to the decisions, which were backed up by a municipal bylaw, polluting diesel vehicles are prohibited from entering and parking in the LEZs, unless equipped with a particulate filter. To facilitate implementation of the decision, subsidies are provided to owners of polluting diesel vehicles to enable the installation of particulate filters in vehicles in the Haifa Bay region. The first stage of the program was launched in February 2018 and related to polluting vehicles weighing over 3.5 tons with the second stage following in January 2019 and relating to polluting vehicles less than 3.5 tons (including taxis and commercial cars). The program is accompanied by a multifaceted awareness-raising campaign. Implementation of these measures throughout all the residential areas of Haifa is expected to bring about a $23 \%$ reduction in vehicular pollution and a significant improvement in air quality.

In a related development, a breakthrough in efforts to reduce air pollution from transportation in Jerusalem was achieved in May 2017 within the framework of a government decision, backed up by a municipal bylaw. As Israel's most populated city, Jerusalem has long been plagued by air pollution from transportation sources, with exceedances of particulate and nitrogen oxide standards recorded near major transportation arteries. While improvements in air quality in the city center were achieved following the launch of Jerusalem's light rail in 2011, the government decision to implement a LEZ in Jerusalem, to assist owners of old diesel vehicles to install particulate filters and to allocate funds for the purchase of electric buses should further reduce air pollution in the city.

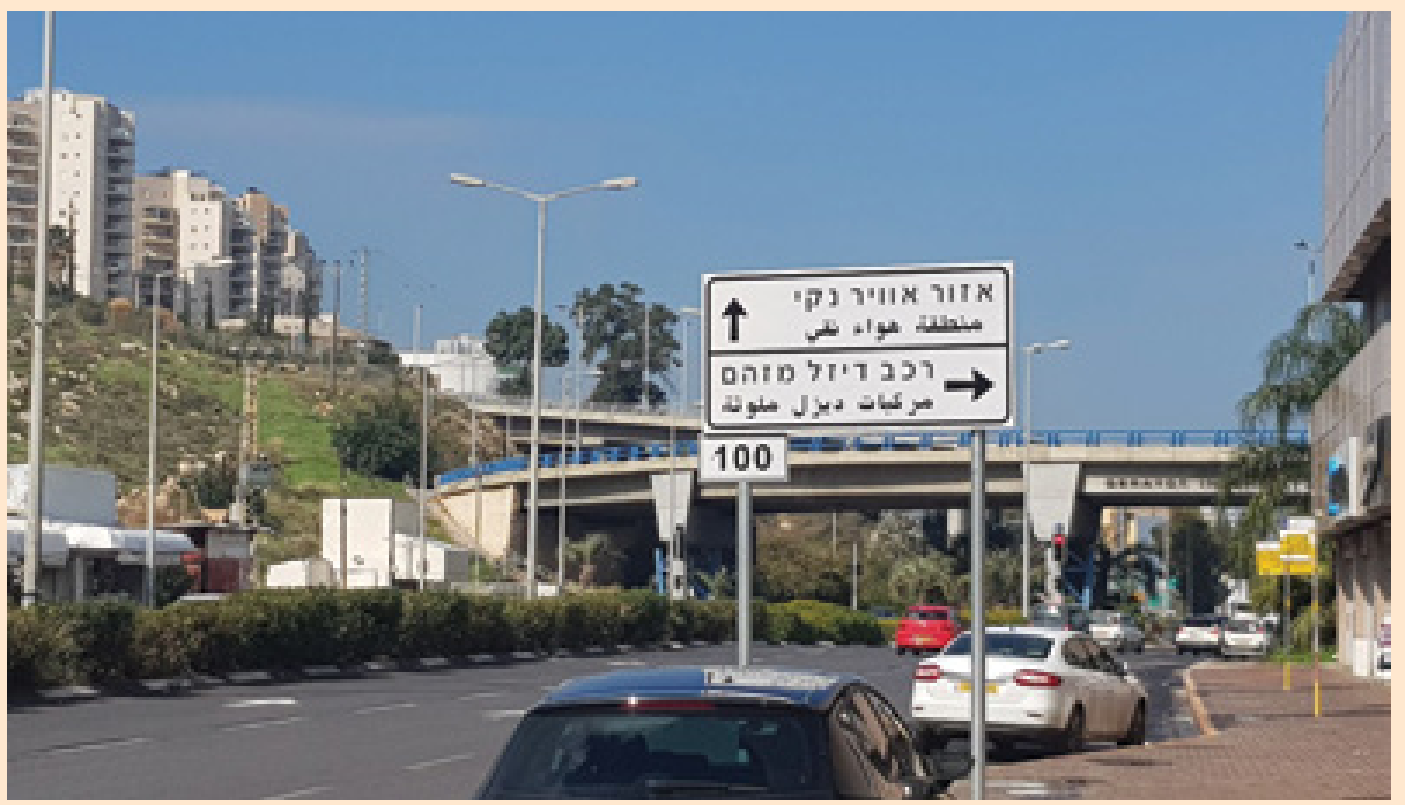

Haifa Low Emission Zone/Photo: Ronen Lotan 
Figure 11.7

\section{Number of cities with sufficient public space}

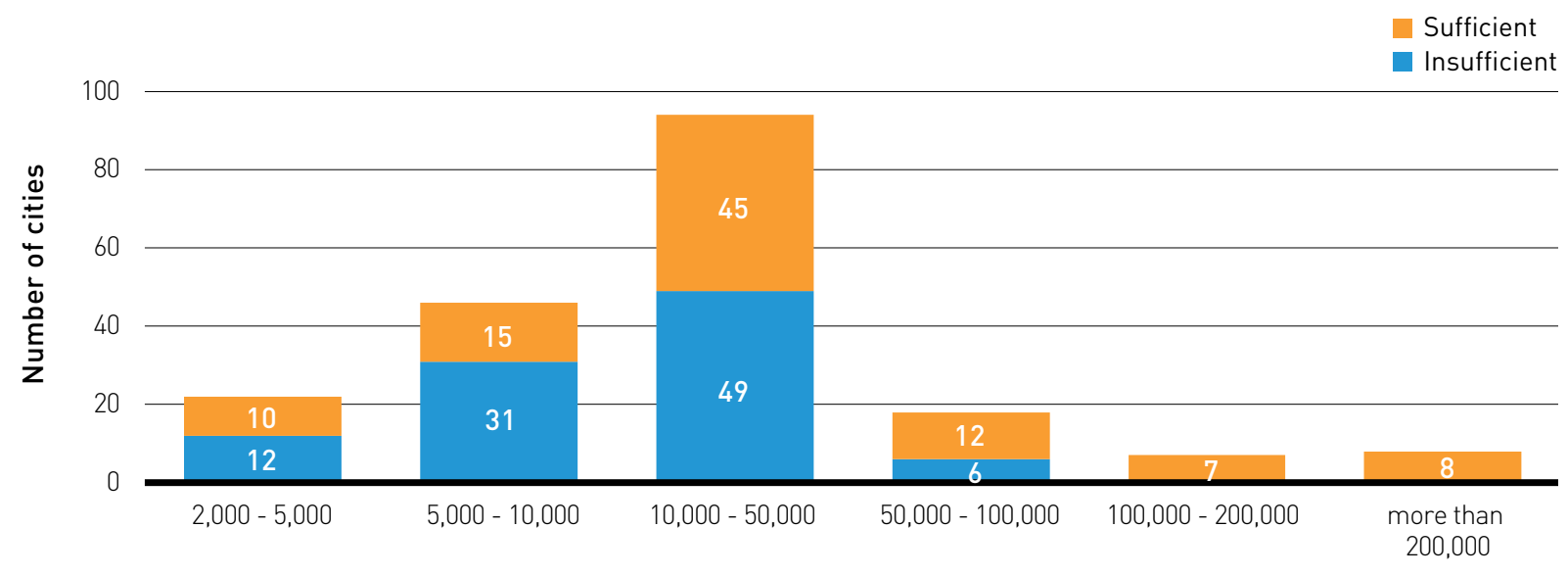

Number of residents

The main national policy for public spaces in Israel is the "Directory for Allocation of Land for Public Use," which was updated in 2018. The directory recommends allocating a minimum of $10 \mathrm{~m}^{2}$ of public space per resident, and it sets minimum allocations of public spaces at defined, maximum distances, as measured by travel time by foot, bicycle or public transportation, to ensure accessibility. In addition, at least $80 \%$ of the public spaces in each municipality are to be green open spaces, such as parks, gardens and natural sites. Numerous practical guides for planning various aspects of the public space have been published by several ministries, including the $\mathrm{MOCH}$, the MOEP and the IPA, as well as by municipalities and NGOs. In 2017, a voluntary standard for sustainable neighborhoods (Neighborhood 360') was developed, which places emphasis on public spaces and their quality.

As minimum allocations of public space have been mandatory in Israel since the 1970s, including defined maximum distances from residential areas. UN Habitat's recommendation for a minimum of $15 \%$ open space is met in half of Israel's cities (defined as having more than 2,000 residents), including all large cities with more than 100,000 residents. Of the cities which are deficient in public space, especially small cities lunder 100,000 residents), approximately $70 \%$ are cities in which the land is mostly privately owned. In these cities, ongoing legal and planning efforts are taken to alleviate the lack of public space.

Currently, Israel's large cities have sufficient municipal public space. As a result, efforts are concentrated on improving the quality of existing municipal public spaces as well as those planned for new developments, and on creating additional public space in cities where land is for the most part privately owned.

The MOCH has prepared a publication entitled "Reviving the Public Space" and distributed it to heads of local authorities in Israel. The booklet is a compilation of best practices designed by local authorities and residents, in the form of a catalog from which local authorities can pick projects to implement in their jurisdictions.

Two of the most pressing issues affecting the quality of public space in Israel are lack of defined management responsibility and lack of quantified, monitored quality indicators. These issues have been identified by government bodies, by NGOs and by academia, and work is currently underway in all three sectors to create and to implement solutions. As part of the ongoing efforts to address these two issues, two locally-relevant indicators are suggested: 
- Number of large cities evaluating the creation of a neighborhood level of management, including strategies to manage the public space.

- Evaluation of internationally accepted plans to monitor and evaluate public space, including their adaptation for the Israeli context, and the creation of implementation policies at the municipal and national levels.

\section{National and regional development planning (SDG 11.a)}

In view of the need to balance development with the protection of natural areas and open spaces, in 2005 the government adopted National Master Plan) NMP( 35, which incorporates sustainable growth principles. NMP 35 is a state-wide spatial plan. This strategic, map-based plan divides the country into five planning designations (termed "textures"), some development-oriented and some preservation-oriented:

- Urban: Compact urban development, high density, and urban quality of life;
- Rural: Rural settlements, agricultural land, tourism and employment, and contiguous open space;

- National preserved: Large continuous spaces of natural importance, agricultural and scenic landscapes, etc.;

- Mixed preserved: "Green" corridor encompassing natural areas, agriculture and heritage, running the length of the country, bounded by urban settlements;

- Coastal: Coastal land conservation and public access to the sea and the beaches.

The provisions of NMP 35 differentiate between the textures - supporting development processes in development-oriented areas, while restricting the extent of development in preservation-oriented areas. Several variables, such as maximum size of the municipality or its minimal density, are determined for each texture lurban texture excluded). The plan structures the urban texture in coordination with the road and mass transit networks and emphasizes public transport and environmental sustainability.

\section{CASE STUDY}

\section{Community Gardens}

Hundreds of community gardens have been established throughout Israel with the support and/or cooperation of local authorities, NGOs, and government ministries. Over the past couple of decades, neglected plots in densely populated urban areas have been turned into community gardens with the direct involvement of nearby residents.

While most of the gardens were originally established in Israel's central cities, recent years have seen a proliferation of community gardens in peripheral areas as well. In addition to helping to preserve urban nature and open space, these gardens have helped develop a sense of environmental responsibility and empowerment among residents. They offer rest and relaxation as well as social interaction with neighbors. Residents who take part in the cultivation of community gardens report a sense of pride and belonging, a feeling of camaraderie, and a realization that they can make a difference in the quality of life and environment of their neighborhood.

An inter-ministerial National Committee for Community Gardening was established in 2009, with representatives hailing from government ministries, municipalities and NGOs. The committee aims to coordinate the resources and promote collaboration among stakeholders. 
The interfaces between rural and urban settlements in Israel are a major challenge in rural development, and the rural-urban interrelationships are a critical component for regional growth. In 2015, the Ministry of Agriculture and Rural Development published a policy paper that addresses this urban-rural interface. The methodology that lays the foundation for the analysis of the urban-rural interfaces reflects a spatial and inclusive regionally-linked planning perception that emphasizes several basic assumptions. Firstly, the urban-rural affinity is a main component in growth patterns, both in the relative qualities and merits of each sphere and of both as a whole. Secondly, the ruralurban interface (physical and functional) needs to change from "a space of conflict" to "a space of opportunities," regarding growth patterns, collaborations and regeneration. Thirdly, sensitive, well-adapted planning regarding the urban-rural affinity features will enable the development of better models of ruralism and urbanism. The methodology is based on the following principles, adapted to the reality in Israel and to the national planning policy:

- Urban-rural affinities as the basis for defining the rural area's role in the spatial unit: The urban-rural affinity's characteristics change in accordance with the specific spatial area. Hence, planning must take account of the role of the rural sub-area in the spatial unit and reflect it while considering the affinity characteristics between it and the relevant cities. The analysis and incorporation of such affinities in planning considerations are the foundations for defining planning guidelines which enhance the rural area's uniqueness and its added value to the entire region's growth.

- Multidisciplinary analysis encouraging an inclusive regional perspective: The affinity analysis must be based not only on population size and the relative population sizes of the rural vs. the urban areas, but must also take into account social, economic, demographic and environmental aspects.

- Flexibility - no need for division to fixed subareas: Since Israel is a small country, it is unnecessary to divide the land into sub-divisions or define "closed" and "open" sub-areas.

In addition to NMP 35, fifty additional national outline plans exist along with hundreds of partial plans, most of which deal with detailed or specific areas. Every plan highlights a certain aspect in the national planning system. Due to the multiplicity of plans and the many complexities, it is necessary to update, renew, and convert all plans into one unified "language." Therefore, in 2013, the National Building and Planning Council decided to establish one, unified NMP (NMP 1) that will unify all detailed outline plans.

\section{CASE STUDY}

\section{The Regional Clusters Program}

The regional clusters program is intended as part of MOINs policy and strategy to promote economic growth at the periphery, to narrow gaps between communities, to develop economic ties between a city and its rural environment, and to strengthen national and regional planning. The program was initiated and led by the MOIN in cooperation and with the Ministry of Finance and JDC Israel.

The clustering concept is based on the understanding that systemic and sustainable solutions are needed to cope with the challenges faced by peripheral local authorities. A cluster of authorities creates an opportunity and a platform that helps overcome regional failures by leveraging local and regional resources and improving the authorities' ability to promote the 
welfare of the residents. Essentially, such a cluster enjoys economies of scale, enabling the authorities involved to reduce costs and maximize benefits. As a result, the local authorities associated with the cluster can improve a large array of municipal services such as garbage disposal or regional veterinary systems.

Regional clusters enable the establishment of a regional strategy that deepens internal connections within the region while expanding external connections with actors operating beyond municipal boundaries: government ministries, the third sector, academia and research bodies, entrepreneurs, and the business sector. This local and national integration facilitates the development of a comprehensive plan and better realization of regional assets and resources, thus strengthening regional cohesion and reducing socio-economic gaps.

Joining the cluster also enables local authorities to create a strong regional voice and to influence policy makers in central government. The joint voice increases the cluster's ability to enlist partners from government ministries and philanthropy, thereby significantly increasing the mobilization of new resources. A clear expression of this was found in the successful joint activity of several clusters that called upon the government to produce a comprehensive plan for the development of the Northern District.

Furthermore, the cluster's activity is aimed at reducing economic and social gaps between neighboring authorities and between center and periphery. This is done through the following:

- strengthening the ability of all the authorities associated with the cluster, with special emphasis on the weak ones, to provide optimal services to their residents;

- creating a social mix of authorities in the cluster, with strong ones supporting weaker ones through partnership and joint regional activity, thus improving the welfare of the residents of the entire region and reducing gaps within the cluster;

- pooling of resources to achieve regional growth insofar that the whole is greater than the sum of its parts;

- creating mutual working interfaces and common living spaces that promote social cohesion between residents, communities and populations that make up the cluster.

The first cluster, the Western Galilee Cluster, was established as a regional corporation with over 150,000 residents by several local authorities in 2009. This initiative, which was adopted by the Ministry of Interior, served as the first pilot and inspired the development of other clusters in the periphery.

The number of regional clusters increased from 5 to 10 between 2016 and 2018. During these years, the number of local authorities associated with the clusters increased from 59 to 109 , and the scope of budgetary activity increased from NIS 15 million (about \$4.16 million) to NIS 220 million (about $\$ 61$ million). Numerous initiatives born of the partnerships formed within the clusters flourished in recent years including a unique science education system, regional centers for people with disabilities, joint transportation systems, garbage treatment projects, and more. Among the subjects proposed for future cooperation are environmental enforcement and waste treatment, sports and cultural activities, joint planning of public transportation, tourism, industrial parks, emergency preparedness, and regional frameworks for populations with special needs. 
To address the problem of inequalities resulting from past zoning divisions between cities and to promote a regional and systematic point of view, the Ministry of Interior (MOIN) has established seven geographical committees charged with continually examining the municipal area under their responsibility. Each committee is responsible for an area that comprises between 40 and 60 local authorities, linked regionally and spatially. The committees examine various issues such as changing municipal boundaries, better income divisions from profitable areas such as commercial, industrial and services areas, army bases, quarries and more. In addition, they are responsible for deciding on changes of municipal entities, from local councils to municipalities (cities), and sometimes even on the unification of local authorities. In addition, a regional clusters initiative is being promoted with the aim of promoting regional cooperation among neighboring local authorities in the periphery by leveraging size advantages to achieve economic efficiency, improve services to residents and narrow socio-economic gaps.

(Please see the stakeholders' annex at the end of this report for some input from NGOs in Israel to SDG 11)

\section{PARTNERING FOR A BETTER WORLD}

Israel's international development cooperation program collaborates with UN specialized agencies to promote a multi-disciplinary approach to integrated regional development. Acting as the focal point for UN Habitat in Israel, the Ministry of Construction and Housing (MOCH) is leading the processes of policy planning and implementation in the fields of urbanism, urban planning and development, public and social housing, affordable housing, new construction methods, and the assimilation of advanced technology and innovation in the construction industry. The ministry is currently taking part in an international Working Group on Affordable Housing, headed by the Transport and Urban Development Department of the Union for the Mediterranean (UfM), to promote housing solutions across the region. (SDG 11.1)

In 2016, MOCH led Israel's delegation at the UN Conference on Housing and Sustainable Urban Development (Habitat III) in Quito, Ecuador. Habitat III offered member states the opportunity to discuss the New Urban Agenda, focusing on policies and strategies that can result in effectively harnessing the power and forces behind urbanization. Israel strongly supported the Quito Declaration on Sustainable Cities and Human Settlements for All, and UN General Assembly Resolution (71/256) on the New Urban Agenda. (SDG 11.3)
In light of Israel's support of the New Urban Agenda, MASHAV hosted a delegation of UN Habitat officials on a study mission to explore the opportunity of joint programming in Africa. The parties agreed to conduct three pilot programs in Kenya, Uganda and Liberia. Israel's Embassy in Nairobi is working together with UN Habitat on capacity-building activities designed to respond to the needs of youth in agriculture, service delivery, employment and income-generation. In the past (2015), MASHAV and UN Habitat conducted a special training course on Bringing Women and Girls into the Cityscape, focusing on gender equality and the empowerment of women in urban planning. (SDG 11.3, 5.5)

Within the framework of Habitat III, MASHAV, the Young American Business Trust (YABT) and UN Habitat organized a side event on Israel's UrbanInnovational Platforms. Among the organizational platforms presented included: Municipal Innovation - Case Study: Vietnam; Municipal Platforms for Local Economic Development and Revenue Enhancement - Case Study: Kenya; The Gender and Local Government UN Habitat/MASHAV Joint Program - 10 Years and Beyond; and Business Labs for Young Entrepreneurs - Methodology and Showcases. (SDG 11.a)

At the Israeli Pavilion at Habitat III, the NGO Access Israel introduced the Accessibility Path, focusing 
on adapting the urban environment to the needs of every resident, especially people with disabilities and the elderly. The event stressed the challenges people with disabilities and the aged confront in an urban setting. (SDG 11.7)

$\mathrm{MOCH}$ signed a bilateral cooperation agreement with the Russian Ministry of Construction, Housing and Utilities as part of the Joint Israeli-Russian Commission on Trade and Cooperation. Two meetings of the Joint Working Group were held on issues including legal and technical regulations, purchaser's rights, increasing energy efficiency, building exploitation, urban renewal, housing provision for targeted populations, the sale of state-owned lands to entrepreneurs, capacitybuilding courses on new methods for specialists, and working with the International Organization for Standardization (ISO) on national standards regarding building structures and materials. (SDG 11.1)

Concrete is accepted as the main material in construction, as well as a material that may increase environmental damage due to its high consumption in the mining of minerals, energy, and polluting effects. The Standards Institution of Israel (SII) is leading the initiative on the development of Self Compacting Concrete and High Performance and Ultra High Performance Concrete within the framework of the ISO. This initiative affects the construction life cycle (durability) in a positive manner by decreasing material and energy consumption to improve the sustainability spectrum. (SDG 11.c) In addition, a new initiative supported by the SII and the Ministry of Economy and Industry encourages the active involvement of Israel's industrialized sector in the ISO's international standardization activity.

Israel is a member of the Global Sustainable Development Criteria (GSDC) Initiative, committed to promoting sustainable communities worldwide. In 2018, MASHAV, in cooperation with the Gender Units of UN Habitat and the UN Environment Program (UNEP), organized a capacity-building course in Israel on Building Sustainable Communities: Leadership, Gender, and the Environment. Furthermore, a capacity-building course on Gender and Local Governance was conducted promoting basic urban services, land rights and housing, security, budgeting, environment, and confronting gender biases. (SDG 11.b, 5.5)

Resilient communities engender good governance, transparency of government institutions and policies, and stronger community services. Israel works together with communities and civil society on developing skills to promote economic growth, resilience in the face of mass casualty events, reinforcing social support systems for the protection of women and children, ensuring safety by engaging in community policing, and developing drug control strategies. While cities command an increasingly dominant role in the global economy, rapid urban growth throughout the developing world is seriously outstripping the capacity of most cities to provide adequate services. MASHAV's programs reflect Israel's commitment to strengthening community resilience by enhancing the capacities of women and men to contribute to the development process. The programs empower people to develop an integrated and comprehensive approach to address the challenges of urbanization, in accordance with the three pillars of sustainability - economic, social and environmental - while confronting the massive increase in the numbers of urban poor.

Following is a description of some of the programs: (SDG 11.3, 11.a)

- In alignment with the findings of the Organization for Economic Cooperation in Europe (OECD. Studies on Water and Cities, 2015), " cities can only develop sustainably when they provide a reliable water supply and sanitation services..." MASHAV conducts two annual capacity-building courses: Urban Water Technologies and Management, focusing on the critical importance of access to water as a defining factor in the location, prosperity and resilience of cities, and providing tools to develop a comprehensive approach to urban water management; and a capacity-building course on Addressing the Urbanization Challenge.

- Individual and social activism contribute to the development of an inclusive and safe society and foster a spirit of volunteerism. Citizen 
mobilization encourages action for the betterment of societies, an alertness to the needs of the community, spearheading of new legislation by means of feedback to decision-makers, and pressuring for reform. The direct relationship between the strength of a community and solving the problems of its individual members provides the context for MASHAV's Leadership for Civil Social Activism training course aimed at equipping community leaders with the skills to build and strengthen their civil society through the mobilization of human and material resources.

- Municipal Projects for Civil Security is a capacitybuilding course conducted in Spanish for mayors, police officers and relevant municipal and national officials from Latin America. Civil security is one of the largest problems facing municipalities, and decentralization processes have led local governments to assume responsibility for areas usually covered by the central government. The course relates to the municipal government system in Israel as a regulator and coordinator of civil security programs such as the City without Violence integrative model. Follow-up seminars were carried out in Peru and Guatemala.

- The Police and Community Training Course focuses on approaches to connect the police force with the community on matters relating to crime prevention, particularly among youth. Courses are conducted in English and Spanish. Follow-up seminars were subsequently carried out in El Salvador and Honduras.

In this context, a move to promote awareness and implementation of the SDGs in cities and municipalities throughout Israel was taken (2018) by the Interdisciplinary Center (IDC) Herzliya, which introduced the first Smart and Sustainable City Index (SSCI) Survey. The Survey measured the performance of Israeli cities in line with the SDGs and rated them accordingly. The Index offers goals and key directions for instituting strategies for sustainability in cities to help prepare for the challenges involved in the growing complexity of urban life as a result of global processes - climate change, migration, and intense economic changes while taking advantage of the ubiquity of technology.
Israel is in the process of sharing the SSCI as a tool with relevant specialized UN agencies. (SDG 11.3)

Israel is implementing the Sendai Framework for Disaster Risk Reduction 2015-2030, adopted at the World Conference on Disaster Risk Reduction, and is active in the UN Office for Disaster Reduction (UNISDR). As it approaches 2030, Israel will increase its international development programming to enhance partner country preparedness as well as crisis management in emergency and mass casualty situations. At the 72nd UN General Assembly, Israel cosponsored a Draft Resolution on International Cooperation on Humanitarian Assistance in the Field of Natural Disasters, from Relief to Development. (SDG 11.b)

Israel currently supports four of its experts on the roster of the UN Disaster Assessment and Coordination (UNDAC), and a representative of Israel's National Emergency Authority (NEMA) serves on its Board. (SDG 11.b)

The establishment of Israel's Meteorological Service (IMS) was due to a large extent to successful collaboration with the World Meteorological Organization (WMO). The Ministry of Foreign Affairs and Ministry of Transport commemorated (2018) the 80th anniversary of the IMS in the presence of the Secretary General of the WMO and Head of Cabinet. The event also launched Israel's membership in the Consortium for Small-Scale Modeling (COSMO) and the opening of the COSMO General Meeting in Jerusalem. Furthermore, COSMO developed a Regional Numerical Weather Prediction Model, and the software is shared with colleagues from the developing world. (SDG 11.b)

Each year severe hydro-meteorological events around the world give rise to multiple casualties and significant damage to property and infrastructure, with adverse economic consequences for communities, and especially vulnerable populations. Many of these tragic events can be forecasted and accurate warning information disseminated in a timely fashion by the responsible national meteorological and hydrological services (NMHS). Often, there is a lack of communication between the NMHS and emergency authorities. MASHAV, 
IMS, NEMA, the Finnish Meteorological Institute (FMI) and the Meteorological Office of the United Kingdom conduct an annual institutional capacitybuilding course for professionals from developing and least developing countries on Meteorological Warnings and Emergency Response to Hazardous Weather Events. (SDG 11.5)

In line with the Sendai Framework for Disaster Risk Reduction 2015-2030 (SDG 11.b), additional activities conducted include:

- Emergency Situation Preparedness is a capacity-building course conducted in Israel by MASHAV, the International Institute of Leadership (Histadrut) and NEMA, in both English and Spanish for professionals from Africa, Asia and Latin America. The main goal of the course is to assist authorities in formulating a National and Regional Response Plan to Crises while maintaining political, economic, and social stability. Israeli experts conducted follow-up courses in South East Asia (Myanmar), Africa (Nigeria, Kenya,) and Latin America (Colombia, Argentinal in cooperation with local partners;

- In cooperation with Rambam Hospital, MASHAV holds two international capacity-building courses in Israel on Decision Making in Emergencies and Mass Casualty Events involving multistakeholders and Developing and Organizing a Trauma System in Mass Casualty Situations.

Israel recognizes the critical importance of regional cooperation in tackling adverse environmental impacts on cities and human settlements to promote resilience to disasters and develop the necessary tools to implement early disaster warning systems. Israel is a member of the WMO initiative, supported by USAID, on the Black Sea and Middle East Flash Flood Guidance System (BSMEFFGS), a regional initiative aimed at enhancing capabilities to issue timely predictions and warnings of flash flood events. Within the scope of the initiative, a series of training activities took place in Israel, including an operational workshop, under the auspices of the IMS, with the participation of professionals from the Palestinian Meteorological Department in Ramallah. Additional examples of regional cooperation include: (SDG 11.b)

- The IMS participates in the regional WMO and USAID initiative entitled South East Europe Multi-Hazard
Early Warning Advisory System (SEE-MHEWS) to construct severe weather warning mechanisms to support Disaster Risk Reduction (DRR) in many developing countries. Israel is a member of the Project's Policy Advisory Group (PPAG), and its experts participate in different working groups. Israel is set to host the next SEE-MHEWS Project Steering Committee meeting in 2019;

- NEMA, Jordan and the Palestinian Authority are working together with the UN Office for Coordination of Humanitarian Affairs (OCHA) in Geneva on improving regional preparedness in the field of emergency management. This cooperation includes setting up a regional early warning system in case of earthquakes and creating a regional coordination center in case of natural disasters. Two disaster prevention exercises were conducted in the region: a European-Mediterranean exercise in Israel and Jordan on preventing forest fires and an exercise in Israel, Jordan and the Palestinian Authority on earthquakes and extending international assistance in emergency situations;

- OCHA/Geneva certified Israel's National Search and Rescue Unit (NRU) as an International Search and Rescue Group (INSARAG). This allows Israel to enhance its collaboration with other search and rescue teams in serving the international community in response to emergency situations. In 2019, an Israel Defense Forces aid delegation arrived in the area of the collapsed dam in Brazil, making it the first National Search and Rescue Unit dispatched after INSARAG qualification.

Within the framework of the OECD, Israel is a member of the International Transport Forum, supporting the promotion of sustainable transport that is safe, clean and competitive. In addition, Israel assumes an active role in the UN Economic Commission for Europe (UNECE) Committee on Rail Transport, and its representative was re-elected to the position of Vice Chair of the Working Party on Rail Transport that provides a pan-European forum for exchange of technical, legal and policy information. Israel is also active in the OECD International Traffic Safety Data and Analysis Group, and the ITF - International Transport Forum. (SDG 11.2, 3.6) 


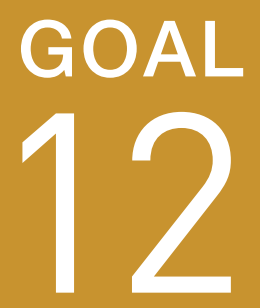

RESPONSIBLE CONSUMPTION AND

PRODUCTION

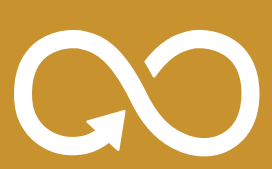

10 Year Framework of Programs on Sustainable Consumption and Production Patterns

Towards green growth

Resource efficiency

Reducing food waste in Israel

Environmentally sound management of chemicals and wastes

Waste and recycling policy in Israel

Resource efficiency and circular economy

Companies adopting sustainable practices and integrating sustainability information into their reporting cycle

Sustainable public procurement

Information and awareness for sustainable development

Partnering for a Better World 


\section{ENSURE SUSTAINABLE CONSUMPTION AND PRODUCTION PATTERNS}

The challenge of achieving economic growth and development in the face of the limited carrying capacity of the environment has become increasingly apparent in recent years. To meet this challenge, Sustainable Consumption and Production (SCP) strategies must be developed.

As part of the SWITCH-MED Programme, launched to promote a "switch" of the Mediterranean economies toward SCP patterns and green economies, Israel formulated its own national SCP roadmap, which was based on a year-long scoping review process that included over 300 participants from all sectors.

Israel is an active member of the Barcelona Convention and its affiliated bodies under the UN Environment Program (UNEP), including the Mediterranean Commission for Sustainable Development and the Regional Activity Center for Sustainable Consumption and Production (SCP/RAC). 


\section{Year Framework of Programs on Sustainable Consumption and Production Patterns (SDG 12.1)}

Israel's Sustainable Consumption and Production Action Plan for 2015 to 2020 mainstreams SCP into national policies relating, among others, to green public procurement; implementation of sustainable development strategies within government companies; support in the assimilation of best practices at small and medium enterprises (SMEs); launch of a resource efficiency knowledge center and promotion of environmental standards and labeling. As stated, the Action Plan is an outcome of work carried out within the SWITCHMED project, under the auspices of UNEP, UNIDO and UNEP MAP.

Several initiatives have been launched within the framework of the 10YFP, and work between the Ministry of Economy and Industry and the Ministry of Environmental Protection (MoEP) is underway to prepare an updated SCP Action Plan with a focus on industry. In recent years, emphasis was placed on green public procurement, green technologies and innovation, and the implementation of sustainable best practices. In addition, a green label for products and services has been developed as a collaboration between the MoEP and the Standards Institution of Israel and, in the case of the restaurant sector, for example, in collaboration with the Tel Aviv-Yafo Municipality.

\section{( Towards green growth (SDG 12.2)}

In 2011, the Israeli government approved a proposal to prepare a National Green Growth Strategy for the years 2012-2020. The decision defines green growth as "socio-economic growth and development that does not harm the environment, makes efficient, economical and sustainable use of natural resources, and creates green jobs while maximizing opportunities for the use of clean growth engines and emphasizing the decoupling of economic growth from environmental deterioration."

An inter-ministerial committee was established to formulate a national plan. To facilitate the process, a process was launched, with the participation of some 500 stakeholders representing government, business and civil society in round table discussions and hundreds more on a dedicated website set up especially for the purpose of ideas exchange. Consultations focused on overcoming obstacles, changing behavioral patterns and promoting investments. The insights that emerged formed the basis for recommendations on major levers for change.

Israel's National Green Growth Strategy identifies three areas of action: production, consumption and innovation. Various government ministries are implementing some of the recommendations, most notably the introduction of integrated pollution prevention and control, integrated environmental licensing, green consumption, green innovation and green procurement, both within and in addition

\section{CASE STUDY}

\section{Resource Efficiency Center (SDG 8.4, 12.2)}

The Ministry of Economy and Industry, the Ministry of Environmental Protection (MoEP), and the Ministry of Finance are helping industry improve its global competitiveness, while protecting environmental values, through the establishment of the Resource Efficiency Center for industry with a NIS 51 million (about \$14.2 million) budget over five years. One of the main challenges that industry faces is the need to be economically efficient and competitive while complying with environmental regulations and safeguarding the environment. According to the Israel Central Bureau of Statistics, most investment to comply with environmental 
regulations (over $85 \%$ ) is at the "end of the pipe", providing solutions for treating pollution after its production. A reverse trend is now evident in the world, especially in OECD member states, where most of the environmental investment by industry is channeled to solutions "at source" to prevent pollution at the outset. These solutions create added value for the enterprise due to greater efficiency in production processes together with the institution of optimal environmental solutions that can save on raw materials, energy and water, resulting in clean production.

To help industry move toward solutions at source, it was decided, similarly to other developed countries, to establish a Resource Efficiency Center in Israel, as a body with the necessary professional knowledge to serve industry. The Center will operate professional consultancy networks to move industry toward cleaner production and direct Israeli industry to leverage opportunities to reduce resources at source as a means of efficiency and boosting productivity, while meeting environmental targets, based on familiarity with the relevant regulation in each area of business.

The Center's activity is expected to put Israeli industry at the global forefront in terms of clean production, technological knowledge and tools, while simultaneously encouraging and advancing innovative Israeli technologies in the sector. The gain will be two-fold: reduced pollution generated by industry in Israel and diversion of up to NIS 1 billion labout \$278 million) in investment to growth and productivity. The Resource Efficiency Center is a key part in the implementation of the advanced manufacturing in industry plan and is part of the National Plan for a Circular Economy.

Supplementary plans were recently launched to move industry forward, including:

- An Institute for Advanced Manufacturing to help industry adopt advanced production technologies and methods in order to boost productivity;

- A national pilot to promote industrial symbiosis aimed at advancing trade in the waste and byproducts of industrial enterprises, resulting in financial savings for industry by reducing waste and cutting waste treatment costs, saving on raw materials, preventing pollution, and reducing the consumption of resources;

- Support for environmental pilot installations based on experience which indicates that the development of technological innovations tends to fail at the technology commercialization stage - in the transition from R\&D and laboratory to the commercial demonstration stage at an industrial installation (a running installation). To overcome this barrier, the MoEP and the Innovation Authority have launched a plan to support running installations in 2018-2019. The MoEP will also lift regulatory barriers for the operation of these running installations;

- Innovation laboratory - an inter-ministerial effort is launching a platform for cooperation between entrepreneurs and manufacturers to increase the number of entrepreneurs in environmental technology disciplines who meet the environmental challenges faced by low technology industries;

- Applied R\&D support - the MoEP and the Innovation Authority will jointly award research grants to researchers and industrial companies to advance research in environmental technology disciplines, combining basic research with feasibility studies for product development. 
to the 10YFP's areas of focus. However, while environmental and green growth considerations are increasingly integrated into the work plans of government ministries, they are not yet fully integrated into the government's development strategies.

\section{( Resource efficiency (SDG 12.2, 8.4)}

Based on accumulated experience, markets often fail to realize the reduction of pollution at source and the efficient use of production resources. The profitability of such steps and the rapid return on investment are not reflected in the willingness of industry and businesses to implement them. This is usually due to low awareness, lack of data and indicators for measurable progress, and lack of practical tools for a professional diagnosis of the potential, for drafting an implementation plan and for raising the necessary funds.

To track Israel's resource efficiency and material use, the MoEP and the Israel Central Bureau of Statistics (ICBS) have jointly developed a framework for data collection, analysis and reporting on material flows and resource productivity, on future environmental accounts (other than water) and on environmental expenditure. Israel has decided to align its material flows and resource productivity national framework with the EU material flows methodology for biomass, non-metallic minerals and metal ores. The following indicators will be reported on a biannual basis: domestic extraction (used), imports, exports, direct material input, domestic material consumption, physical trade balance, domestic processed output and net additions to stock. Israel will also expand its satellite accounts, with additional satellite accounts to be completed by the end of 2020 . The necessary Institutional capacity and budget were provided in 2017 and work began in 2019. The budget includes the development of a digitized platform for data collection and analysis, a full-time position, biannual report preparation and publication, and training.

Israel's Green Growth Strategy identified the need for a center for streamlining resources that will help industry redirect environmental investments.
This includes the development and implementation of tools for prevention at source and for increasing efficiency in industrial and business resource use, thereby reducing both emissions to the environment and landfilling of waste. In addition, consultants and know-how will be provided to increase investment in solutions that reduce pollution and waste at source and minimize environmental risks while improving environmental and economic performance.

\section{( Reducing food waste in Israel (SDG 12.3)}

Most fruits and vegetables in Israel are sold in retail chains and in open markets in bulk, on open shelves, and usually without refrigeration or packaging. Wasted food has significant environmental, social and economic implications. Three main negative environmental impacts occur: wasted resources in the food production process /such as land, water, energy); unnecessary environmental damage due to production, transportation and storage processes resulting in emissions to the environment; and unnecessary creation of waste.

Food Waste Day was marked for the first time in Israel this year, bringing public attention to the fact that 2.5 million tons of food were thrown out in 2018, at the cost of NIS 19.7 billion labout $\$ 5.5$ billion), while others in the population suffer from food insecurity. One of the aims of Food Waste Day is to bring consumers into contact with the source of their food - in agricultural farms, packing houses, orchards and fields - so as to increase their appreciation of food provision and the need to reduce waste.

The State Comptroller published a report in 2015 highlighting the significant social, environmental and economic implications of food waste in Israel. The report highlighted the need for a coordinated government approach, in-depth analysis and evaluation of the problem, and formulation of policy on the reduction of surpluses in the production and marketing of fruits and vegetables. In an effort to reduce food waste along the production and consumption chain, the Ministries of Environmental Protection, Economy and Industry and Agriculture 


\section{CASE STUDY}

\section{Sustainable Best-Practices in the Restaurant Sector}

Sustainable practices have been incorporated into selected businesses in the restaurant sector, with a focus on SMEs and retail activities with an ongoing relationship with end-consumers. A Green Restaurants Guidebook was developed for restaurant owners and dining establishments which allows customers to take sustainability into account when making their choices. In addition, an online interactive platform which guides SMEs in acquiring a Green Label in the restaurant sector was set up by the Municipality of Tel Aviv-Yafo and the Standards Institution of Israel. This online platform guides business owners on sustainable practices about various products and services including energy and water use, cleaning products, paper products, inventory management and waste reduction which is easily replicable at a relatively low cost.

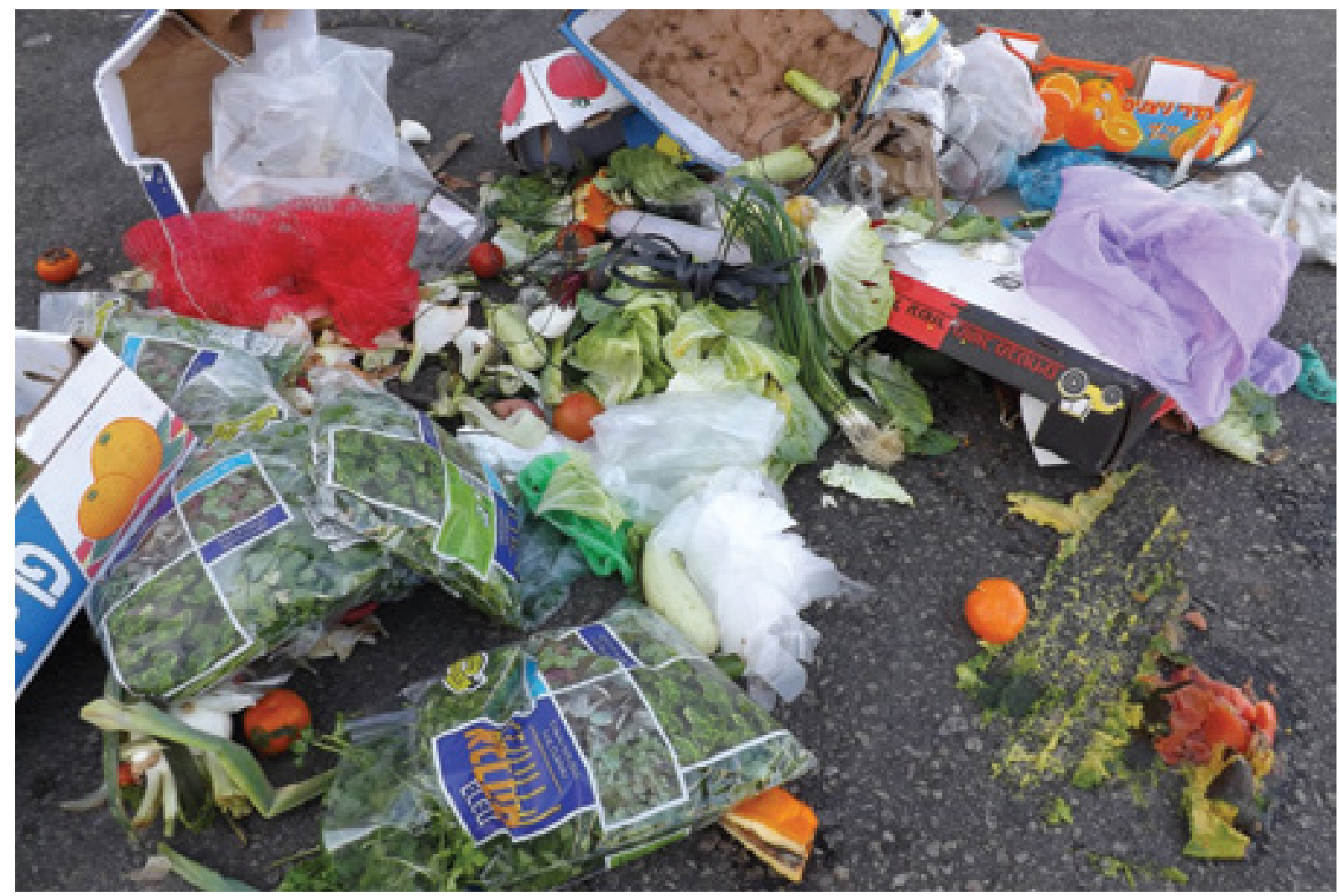

Food waste at wholesale market/Photo: Ron Porat, Agricultural Research Center

are currently examining a national target which will incorporate assessment and systematic measurements of the amount of wasted food along the value chain; public information and awareness-raising; and economic incentives and regulatory tools designed to reduce food waste in Israel. The following steps have been taken toward implementation of this target:
- As of 2016, food waste reduction is included in school curricula, with the participation of 100,000 students each year;

- Two research projects have been carried out to examine the potential for reducing food waste in marketing chains and reducing food waste resulting from expiration dates. As a result, the 


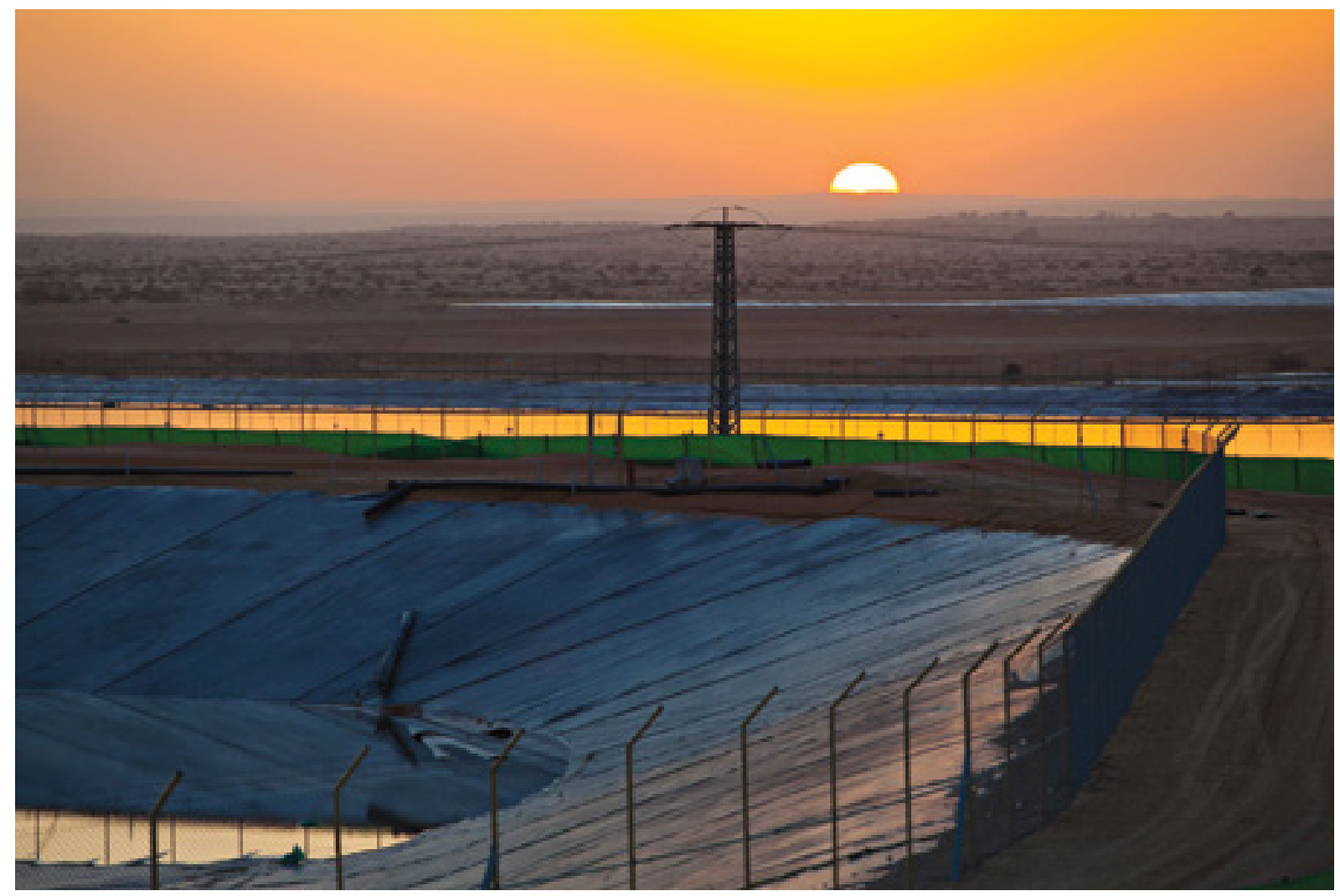

Evaporation ponds at Neot Hovav Eco Industrial Park/Photo: Pini Hamou

issue of food labeling has been closely examined and options for improving and clarifying markings are being considered;

- The Green Label for cafes and restaurants in Tel Aviv was launched in 2016 and includes a component on food waste reduction;

- A voluntary covenant has been conceived and drafted by The Natural Step Israel and promoted by the private sector, on food waste reduction in organizations, businesses and local authorities. The Ministries of Environmental Protection, Agriculture and Economy and Industry have supported this initiative;

- As a result of a joint government, private and academic initiative, a hackathon, a creative programming race, was held on food waste reduction with the participation of more than 150 entrepreneurs and mentors. Two tracks were available: food waste reduction in the agricultural sector and consumer behavior and food waste reduction possibilities at the point of purchase, at home or in institutional catering. In addition, a multi-sectorial awareness raising workshop was held canvassing technological and other solutions:

- Other initiatives include subsidies to local authorities to encourage farmers' markets and ways to encourage the marketing of "misshapen" or "ugly" produce.

Food waste is also included as one of the streams to be addressed in the current strategic waste reduction plan for Israel.

In developing a strategy for food loss at the production stage, two pilot programs were launched for two main crops that constitute staple foods of the Israeli diet. Growers who participate in the pilot are paid the picking costs for the surplus they want to donate. The government is also financing a three-year multi-disciplinary research aimed to test the applicability of technological solutions that are in the final development stage. Solutions considered include smart packaging for fresh agricultural produce le.g., packages that help regulate humidity levels) and various post-harvest treatments le.g., covering potatoes with menthol oil 
to prevent sprouting). Finally, a series of preliminary studies is underway whose aim is to come up with creative solutions, together with key players in the food production and distribution industry, for raising the public's awareness of the problem of food waste and offering strategies for reducing waste at both the retailer and the consumer levels.

\section{( Environmentally sound management of chemicals and wastes (SDG 12.4)}

Chemicals and pharmaceuticals account for over $30 \%$ of total industrial exports (by value). Israel has an advanced framework of laws and regulations to ensure that production, use and distribution of hazardous chemicals used domestically as well as those involved in international trade are carried out in an environmentally responsible manner. Israel is a party to the Rotterdam Convention on Prior Informed Consent (PIC) and is working towards ratifying the Stockholm Convention on Persistent Organic Pollutants (POPs) and the Minamata Mercury Convention. Israel fulfills its obligations under the Convention to which it is a party including its reporting obligations.

Facilities that use hazardous substances, including hazardous waste treatment plants, are required to hold a "Poisons Permit" that sets out the environmental conditions for the possession of hazardous materials. In addition, major facilities are required to obtain an emissions permit under the Clean Air Law that incorporates the principles of implementing best available technology (BAT), in accordance with the IED European Union Directive. The intention is to update these in accordance with international standards thereby ensuring that internationally accepted standards are regularly incorporated.

A Hazmat Incidents Investigations Department was established in the MoEP in 2016 pursuant to a government decision (5217) in order to reduce and prevent recurrence of hazmat incidents. It established a process of investigating specific highrisk incidents involving hazardous materials and implementing the necessary regulatory updates arising from the investigation as well as evaluating the efficacy of these regulatory changes.
In addition, the work that has been carried out in recent years on setting separation distances between hazardous material industries and surrounding populated areas, which is of special importance in a small densely populated country such as Israel, has minimized potential risks to humans and the environment.

Israel will be undertaking a new chemicals management program in accordance with its commitments to the OECD and has carried out an analysis to examine options. The regulation of pesticides already accords with many chemical management principles. Pesticides for agricultural, sanitation and human use are regulated and in recent years a number of active ingredients have been phased out. In addition, a 2016 law on the regulation of integrated urban pest control practice sets environmental priorities on the choice of pest control methods, beginning with preventative treatment and opting for chemical pest control substances only in case of need.

Data published in 2016 by the ICBS indicate a $14 \%$ decrease in sales of agricultural pesticides in 2011-2013 relative to 2008-2010. Despite this decrease, Israel continues to hold the record for the use of pesticides (tons per 1,000 $\mathrm{m}^{2}$ of agricultural land) among selected OECD countries. Sales of sanitation pesticides dropped by $20 \%$ between 2010 and 2013. However, there was an increase in the sales of pesticides for use by the general public compared to pesticides for professional use $148 \%$ in 2013, up from 34\% in 2008).

Legislation is currently planned to reduce exposure to pesticides used in agricultural areas that are situated near populated areas.

\section{Asbestos}

Israeli law requires the removal of all friable asbestos from structures by the year 2021 and imposes strict restrictions on the continued use of any structure still containing friable asbestos until such removal. The first stage of a major asbestos removal project in the Western Galilee was successfully concluded in April 2017. The asbestos waste had been spread in hundreds of 


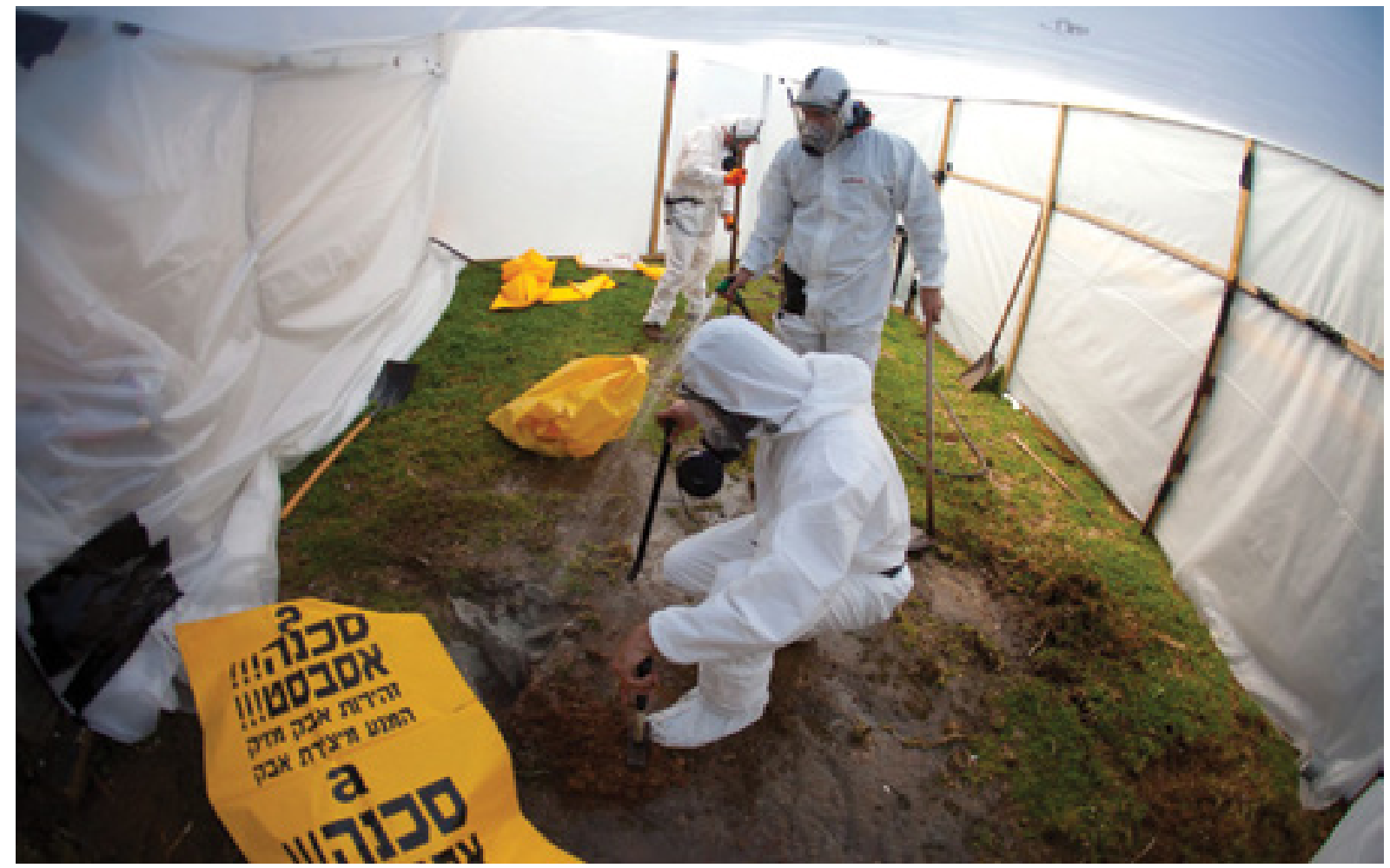

Asbestos removal/Photo: Pini Hamou

locations in the area by an asbestos cement plant in the north of Israel, which was closed in 1997. The six-year project saw the cleanup of all known and visible contaminated sites. The second stage of the project will see the completion of beach cleanup near the plant in addition to contaminated sites owned by local authorities and sites under buildings and roads in the area. According to current plans, within the coming decade, all risk of exposure to friable asbestos will be removed.

\section{( Waste and recycling policy in Israel (SDG 12.4, 12.5)}

Until the establishment of the MoEP in 1988, hundreds of mostly illegal dumpsites were scattered across the country, causing environmental hazards. From the early 1990s, these dumpsites were closed, and standards for sanitary landfills were introduced. Today, there are 13 regulated landfills in Israel, supervised and operated under business licenses. Waste is collected from local authorities and transferred to transfer stations before being sent for landfill.

Some 8.9 million Israeli citizens reside in over
250 local authorities which are responsible for municipal solid waste (MSW) collection and disposal to a regulated waste treatment site. Israelis generate approximately $1.7 \mathrm{~kg}$ of waste per day per capita, and in 2017 generated a total of 5.4 million tons of municipal waste, of which $22.4 \%$ was recycled - an improvement of about $2 \%$ from 2016. Based on a business-as-usual scenario and an annual population growth rate of $1.8 \%$, MSW produced in 2030 will be 6.7 million tons.

Although landfills in Israel still accept all MSW streams, some of the waste is transferred to sorting facilities or materials recovery facilities (MRF) and sorted for recycling. Over the past two decades, the government has supported local authorities and businesses in voluntary actions for the purpose of separation at source of various waste streams such as paper and cardboard, large plastic bottles, textiles, garden waste and organic waste. In addition, laws were enacted on the collection and recycling of packaging waste, electronic wastes (WEEE), beverage bottles (plastic, glass and aluminum) and tires. These materials are also separated for recycling at the sorting facilities. However, most of 


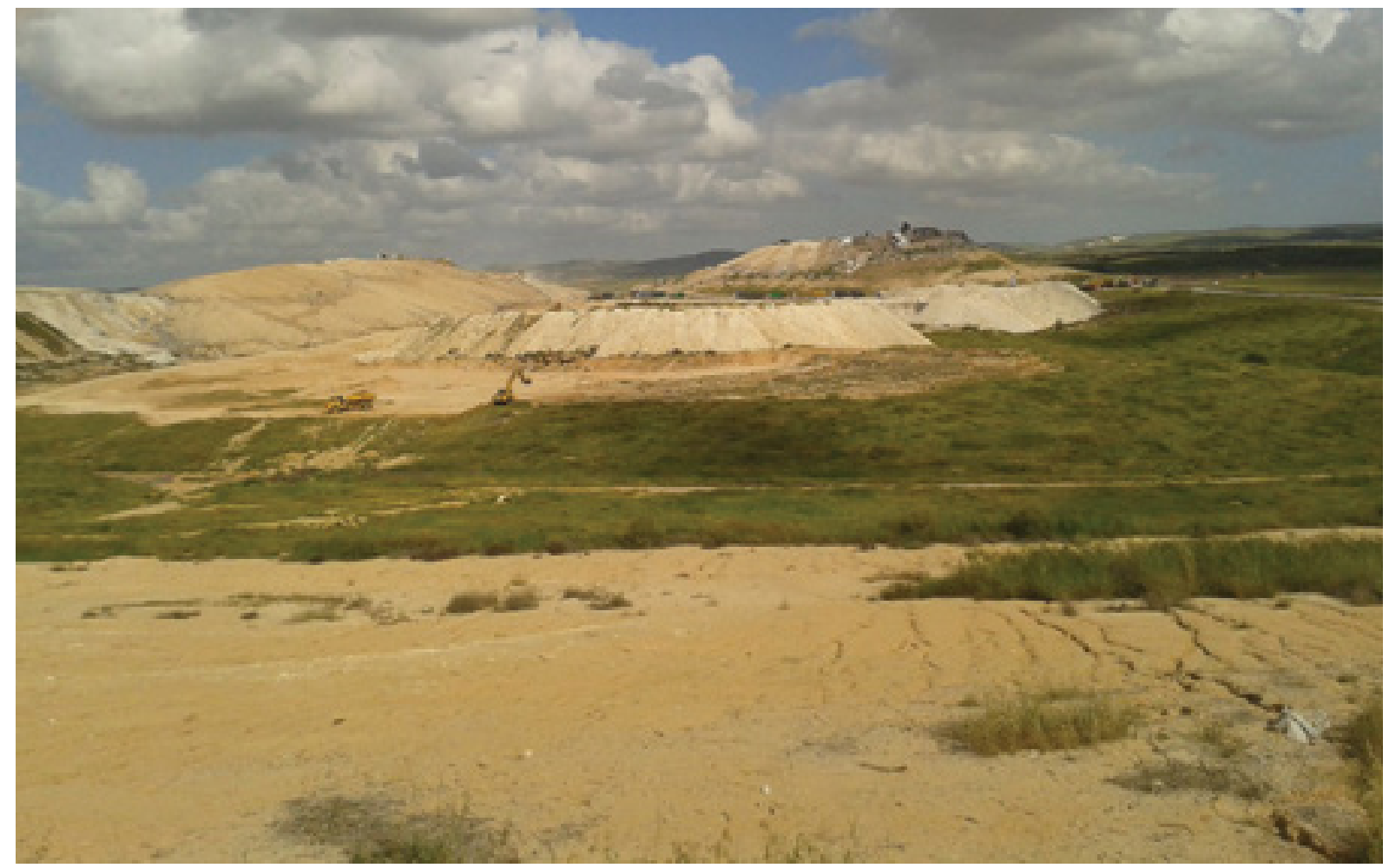

Dudaim landfill/Photo: Tamar Raviv

the MSW in Israel continues to be sent for landfill, with about a quarter of the waste going to recycling.

\section{Israel's Municipal Solid Waste Strategy for 2030}

Israel's Municipal Solid Waste Strategy for 2030 was approved in May 2018. Its goals are to reduce landfilling, increase recycling, and decrease pollution and environmental risks from waste. The targets include a reduction to less than 30\% for landfilling; improved efficiency of the waste market and waste management; improved service; and establishment of infrastructures for waste treatment and waste recycling.

The new strategy is based on several key principles:

- Separation at source into two streams: Waste collection at the household level is separated into two streams: mixed waste which goes into green bins and packaging waste into orange bins. In addition, bins for the collection of glass, paper, cardboard and metal are provided within the community but not at household level. This policy component is reinforced by extended producer responsibility principles. As part of the new strategy, the possibility of expanding the use of the orange bin to all recyclable waste is being examined. Local authorities may choose additional streams for separation at source.

- No landfill without prior sorting: At present there is no law in Israel that prohibits the landfilling of untreated waste, but a prohibition on the landfilling of packaging waste will come into effect in 2020, and a prohibition on the landfilling of electronic waste will come into effect in 2021.

- Establishment of sorting facilities nationwide: Currently five sorting facilities or MRFs exist, and at least six more are planned by 2030.

- Establishment of waste-to-energy facilities: Residual waste, which cannot be recycled, will be transferred after the sorting process to wasteto-energy facilities which will serve as the main alternative to landfill. Three sites are planned to be set up by 2030 .

- Separation of organic waste in the commercial sector: Work is currently being carried out on this component.

- Compostation of organic waste: Organic waste 


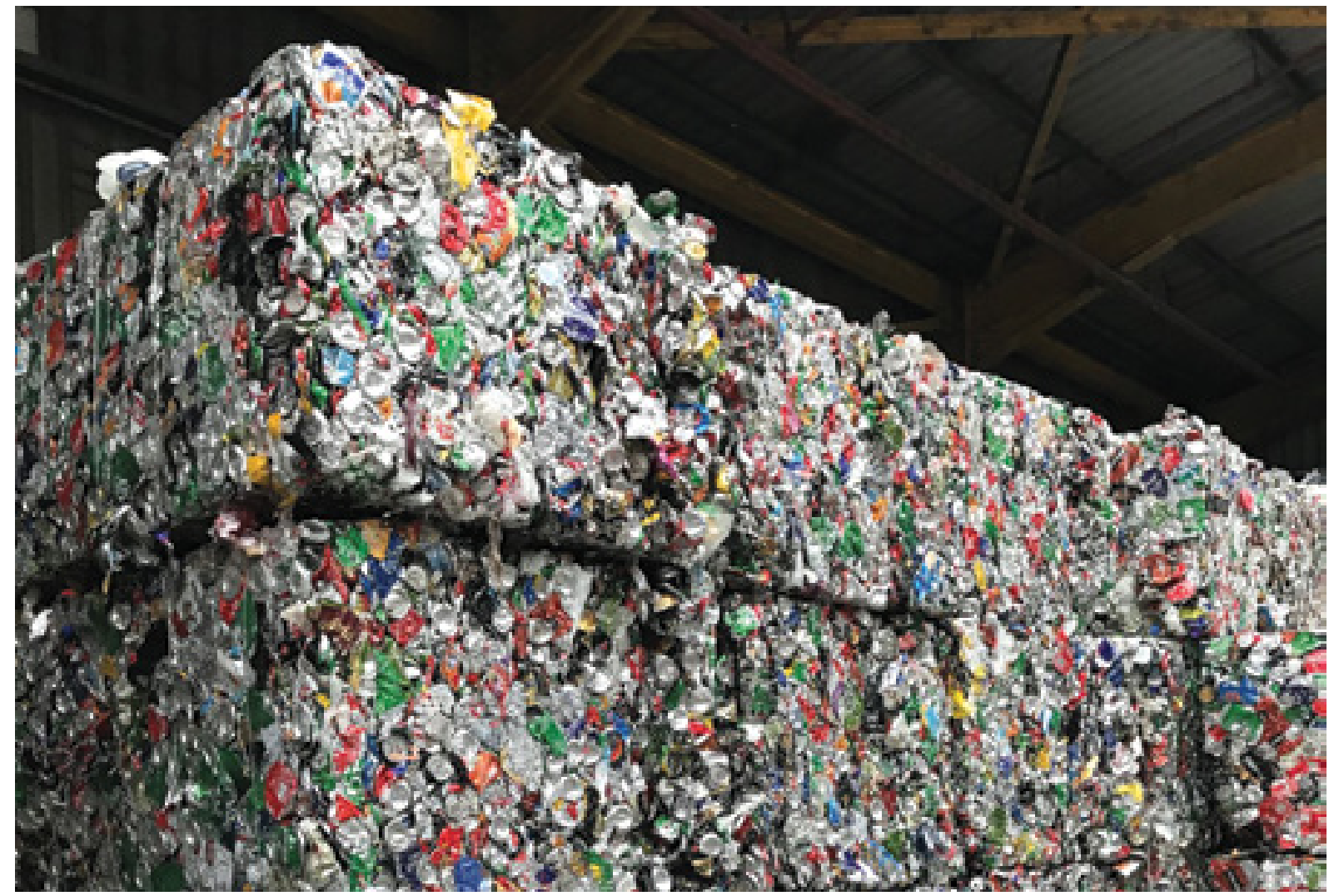

Bales for recycling/Photo: Oded Nezer

treatment facilities for production of biogas and compost are of major importance. Organic waste is a significant part (about 35\%) of municipal waste and is the main source of methane gas emitted in landfills. Currently one large anaerobic digestion facility is underway and three additional facilities are planned for 2030.

With the completion of the program in 2030, only $26 \%$ of the waste in Israel will go to landfill, $51 \%$ will be recycled, and $23 \%$ will be used for energy production. The aim is to improve services and reduce the price of waste treatment. The success of the program is expected to completely change the way waste is managed and treated in Israel and to significantly reduce its environmental impact. The proposed treatment mix and the goals of the plan will significantly reduce the gap between EU member states and Israel with regard to the treatment of waste. A major component of this new waste strategy is an examination of policies to manage plastic waste which has become increasingly urgent given the growing evidence of the negative effects of this waste stream on the marine environment.
A new Plastic Waste Strategy will be presented in May 2019 and several policy responses are being examined such as:

- Source prevention/reduction;

- Examination of means to reduce single-use plastic items such as taxation, inclusion in the Packaging Law or prohibition on the sale of specific products;

- Prohibition on the use of micro-plastics as additives in products (relevant to cosmetics and soaps);

- Cooperation with the Ministry of Education to stop the use of single-use take-away trays in school meals, thereby raising awareness of the need to reduce;

- Creation of a recycling market;

- Raising the collection and recycling targets for plastic waste in Israel's existing deposit and packaging laws in order to promote separation at source;

- Support for the recycling industry; 
- Requirement for public procurement of recycled products;

- Identification of specific sectors with a potential for wider separation at source.

\section{Regional municipal clusters}

The lack of certainty concerning the delivery of a sufficient daily waste supply has proved a major impediment to the viability of materials recovery and recycling plants. The economic viability of such plants could not be guaranteed based on the quantity that would be received from individual local authorities, especially small or peripheral local authorities. Examples exist of outlying and economically weaker municipalities paying twice as much as wealthier communities for waste collection. The municipal clusters initiative promotes regional cooperation between neighboring local authorities in the periphery by leveraging size advantages to achieve economic efficiency, improve services for residents, and narrow socio-economic gaps as well as to bring economic and social advancement to the region. On average, each cluster is made up of about a dozen local authorities, including municipalities, local councils and regional councils.

\section{Environmental justice programs}

Since minority and low-income populations tend to be disproportionately exposed to environmental hazards, the aim of Israel's environmental justice programs is to improve environmental quality in low-ranking socio-economic communities. A number of government decisions were passed focusing on improving waste treatment in minority sectors, with funding for these projects totaling about $\$ 125$ million. All projects focus on enhancing waste management of municipalities by upgrading MSW collection and infrastructure, improving recycling schemes, rehabilitating areas used for illegal dumping of construction and demolition waste particularly in open spaces and their conversion to parks and recreation areas, and promoting environmental education. Implementation of the projects has strengthened local authorities and their relationship with their residents who have become major players in the preservation of waste-free open spaces. The projects include:

\section{- 'Sviva Shava' - Equal Environment 2014-ongoing:} This flagship program includes 75 localities throughout the country and has led to substantial improvements in quality of life and public awareness;

\section{- Project for the Bedouin population 2014-2019:}

The project focuses on legal Bedouin settlements that had, up until 2014, no infrastructure for waste collection. As a result, the population lapproximately 100,000 people in two regional councils and seven local authorities) was forced to burn their waste, creating air pollution that contaminated the entire Negev region. In the first year of the project alone (2014), some 2,500 tons of waste were collected and transported to legal waste sites and were not burned for the first time. Plans for a similar project for the Bedouin population in the North of Israel have been approved;

\section{- Environmental infrastructure project in East} Jerusalem 2018-ongoing: The aim of this project, initiated in 2018 and scheduled for completion by 2021 , is to improve waste management in the Arab neighborhoods of East Jerusalem.

\section{Hazardous waste}

As part of its policy for the treatment of hazardous waste, Israel has set the following recovery and recycling targets:

- $2020-45 \%$

- $2025-50 \%$

- $2030-60 \%$

In order to achieve these goals, the following policies have been adopted regarding large waste producers:

- Adding requirements in permits for conducting a waste survey and gap analyses with BAT technologies and examining options to change production processes that could lead to the reduction of waste quantities produced; 
- Examining the options for changing treatment of the waste or using advanced recycling and recovery technologies instead of waste disposal;

- Providing financial assistance for the promotion of innovative technologies;

- Assisting waste recovery plants in identifying additional waste streams currently landfilled that can be treated in recovery facilities;

- Setting a levy for the disposal of hazardous waste at a rate that will encourage the transfer of waste for recycling and recovery;

- Encouraging the establishment and addition of industrial facilities and plants for the production of energy from waste.

Israel is a party to the Basel Convention on the Control of Transboundary Movements of Hazardous Waste and Their Disposal, but it has not yet ratified its Ban amendment although it complies with its provisions as a member of the OECD. Israel fulfills its obligations under the Convention including its reporting obligations.

\section{Agricultural waste}

The management of agricultural waste in Israel is carried out according to the UN waste treatment hierarchy. Master plans are aimed at providing sustainable economic and environmental solutions for agricultural waste through circular economy tools, based on the perception of agriculture as a national interest and the need to maintain food security. Emphasis is placed on the inorganic waste stream so that agricultural plastic is collected from plots and treated in a waste facility. Business licenses for agricultural facilities set terms and conditions to ensure minimizing damage to the environment.

\section{( Resource efficiency and circular economy (SDG 12.6)}

As part of its efforts to move toward more sustainable use of resources, the Ministry of Economy and Industry is leading together with the MoEP a national plan for circular economy in industry. The plan is to use new technologies to motivate industry to use resources more efficiently and tackle environmental problems in the planning, design, and production stages, thereby turning environmental requirements into opportunities instead of a burden. According to the plan, the government will examine and assess the most

Figure 12.1

Number of sustainability reports by type

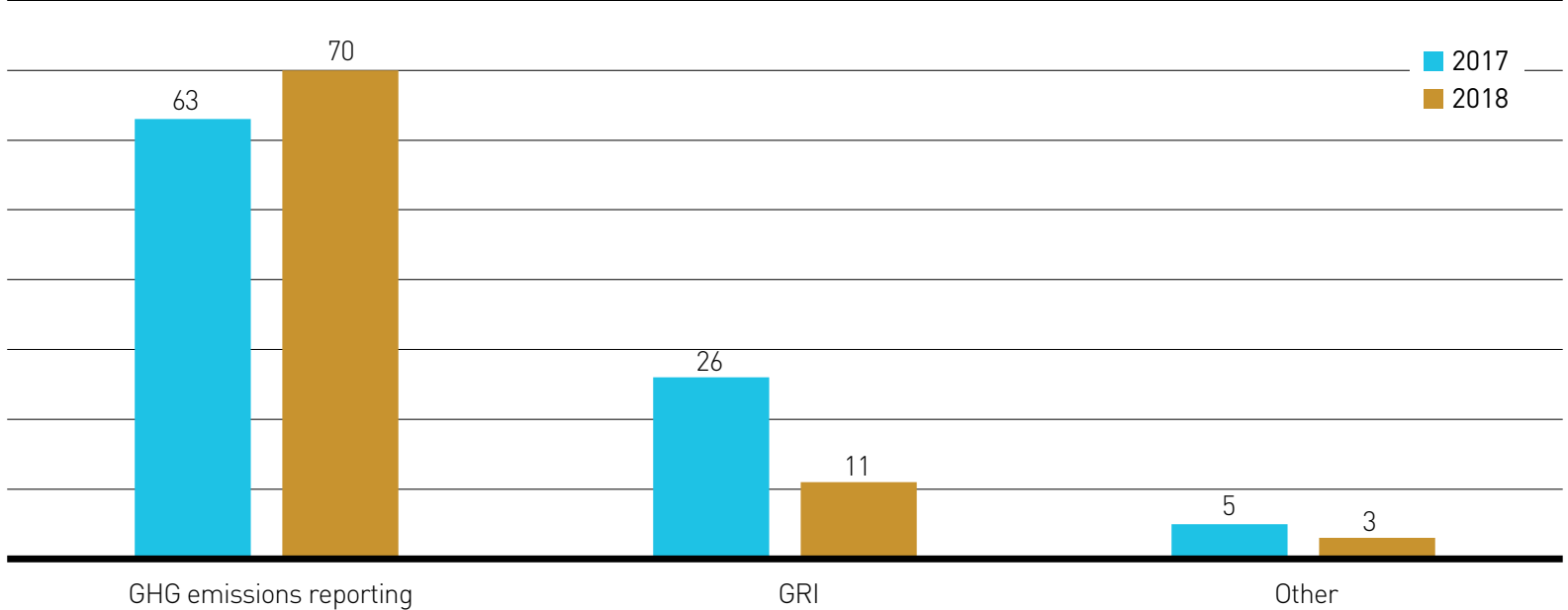




\section{CASE STUDY}

\section{Setting and Developing Sustainability and Responsible Management Standards}

Maala, a non-profit corporate membership organization, promotes Corporate Social Responsibility (CSR), setting and developing sustainability and responsible management standards among Israeli businesses. Founded in 1998, Maala's membership comprises about 115 of Israel's leading companies. Annually, Maala SRI (Socially Responsible Investing) indices are published on the Tel Aviv Stock Exchange (TASE).

The process of developing these indices generated the Maala ESG (Environmental, Social and Governance) Rating system, annually rating approximately 150 companies while serving as an internal management tool for these companies. The rated public companies comprise about half of the aggregated turnover of all TASE traded public companies, thus providing a good indication of the Israeli market. The rating process sets a benchmark for Israeli business performance on ESG standards, based on local conditions and priorities.

The Maala Rating questionnaire is updated biennially through an inclusive process, raising pertinent issues from the field. Issues are discussed by designated subcommittees; comments are submitted by civil society and social organizations, businesses, academia and governmental bodies; a public hearing is held; and following deliberations by a public committee, comprised of multi-sectoral representation, new rating criteria are introduced. This process results in highly localized rating criteria and standards. Once these domestic issues and challenges are addressed, linkages become apparent to the SGDs, exploring global standards and goals.

The Maala Rating is comprised of some 130 standards (questions), structured in nine sections focusing on various CSR and sustainability issues. The sections align with the SDGs as follows: Chapter 2 - Environment (according to environmental impact) corresponds with SDGs 5, 6 , 7, 9, 11, 12, 13 and 15; Chapter 3 - Work Relations, Health and Balance relates to SDGs 1 , 3, 4, 8 and 10; Chapter 4-Responsible Procurement addresses SDGs 8, 9 and 10; Chapter 5 - Diversity and Inclusion attends to SDGs 5 and 8; Chapter 6 - Community Contribution connects with SDGs 1, 2, 3 and 4; and Chapter 8 - Corporate Governance associates with SDG 5.

effective tools, best practices and experiences from other countries, and on this basis, create a detailed plan comprising targets, budgets, schedules, and relevant partners. The process is expected to ultimately become a government decision for a national resources efficiency plan in industry.

Additional activities already taking place include the establishment of a national Resource Efficiency Center (see box) and the Industrial Symbiosis Encouragement Plan, a plan to motivate the market to move towards a circular economy and the production of added value for industry through cooperation between industries to use byproducts, waste, and emissions. The Ministry of Economy and Industry aims to identify as many entities with as diverse a profile as possible lin terms of the nature of their byproducts and waste, scope of operations, sector, etc.) and to identify and foster the potential for transactions between producers of the byproducts and waste and potential customers. The project started function in April 2019. Finally, the government is establishing various tracks aimed at supporting facilities in implementing 


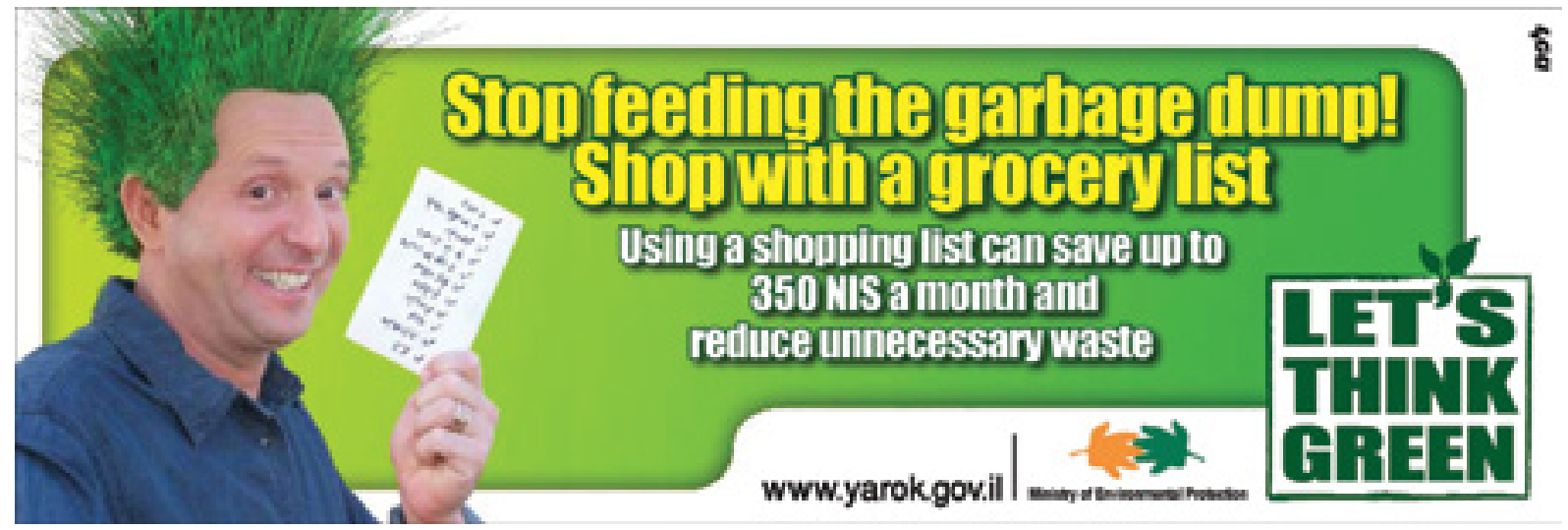

"Let's Think Green" campaign/Government Advertising Bureau

resource efficiency measures as well as developing a plan with support tools for the recycling industry to increase the demand and supply of recycled products in the market.

\section{Companies adopting sustainable practices and integrating sustainability information into their reporting cycle (SDG 12.6)}

The number of sustainability reports submitted by companies in Israel is fairly low. Banks are a leading sector in this field with a mandatory requirement to publish a sustainability report. Several different sustainability reporting schemes are used in Israel.

The most commonly known global standard for sustainability reporting is the Global Reporting Initiative (GRI). By 2017, 26 companies were reporting in accordance with the GRI. However, there was an unexplained drop to 11 companies in 2018. In addition, the MoEP manages a voluntary greenhouse gas emissions reporting scheme, with an increasing number of reporting companies, reaching 70 companies in 2018. Finally, in 2018, three companies published sustainability reports not as part of any of these reporting schemes.

The rate of reporting of Corporate Social Responsibility (CSR) by companies in Israel has grown in recent years. As reporting is still mostly voluntary in Israel, there are a number of different CSR reporting schemes in use. The most common CSR reporting mechanism is produced by and known as "Maala", a corporate membership organization comprised of some 115 of Israel's large and mid-size companies. The network includes local Israeli companies as well as international companies operating in Israel. 150 companies reported to Maala in 2018 as opposed to 69 companies in 2017.

\section{Sustainable public procurement (SDG 12.7)}

In 2012, the Israeli government recognized the potential of green public procurement as a means to encourage the Israeli market towards the consumption of products with a less negative impact on the environment, to serve as an example for households, and to inform manufacturers and importers of the direction the government was taking. Accordingly, the government decided that by $2020,20 \%$ of the expenditure of government ministries on operational procurement will be carried out through "green" tenders in which environmental criteria have been incorporated in order to reduce the negative effects on the environment.

As a first step, the MoEP focused its efforts on developing knowledge and preparing product pages for government and local authority tenders. Product pages with threshold conditions and quality criteria were prepared for products such as computers, printers, computer monitors, tires, pest control, cleaning services, energy saving detectors, energy efficient street lighting and lead-free paints.

In recent years, the MoEP has worked in cooperation 
with the Government Procurement Administration of the Ministry of Finance, responsible for the major procurement tenders of government ministries in Israel, to incorporate environmental criteria into government tenders for products, either as a prerequisite or through preferential assessment. In addition, the Government Facilities Administration has set green standards for building renovation, leasing and purchasing activities conducted by the Administration.

Furthermore, in order to assess and assist the relevant authorities in the procurement process in local authorities and to examine the best way to implement environmental criteria in local authority tenders, a local Green Procurement Forum was established. The Forum has met several times and training sessions have been held designed to harness the purchasing power of local authorities for green procurement.

\section{Information and awareness for sustainable development (SDG 12.8)}

\section{Pollutant Release and Transfer Register}

The Pollutant Release and Transfer Register (PRTR) created under the Environmental Protection Law of 2012 includes comprehensive information on the emission of pollutants into the air, sea, land and water, as well as on waste transfers from the 570 largest plants in the country which are required to transmit an annual report on their emissions and transfers of pollutants to the environment. A key principle of the PRTR is to ensure the transparency and publication of information to the public in a convenient and accessible manner. The MoEP's PRTR site uses a geographic information system (GIS) in which each plant has an "index card" that displays detailed information, such as its address, type of activity, name of the plant owner, and the reported data (i.e., type and quantity of pollutants emitted or transferred from the plant). It is also possible to evaluate the data using an advanced data analysis tool, which enables the execution of a wide range of cross-sections and queries. The MoEP publishes an annual public report of PRTR data and submits the report to the Knesset (Israel parliament).

\section{Let's Think Green Campaign}

The MoEP's "Let's Think Green" public awareness campaign, first launched in 2011, has led to a change in perception and behavior among the Israeli public. Encouraging a "green life" strategy, it focused on the economic and environmental benefits of sustainable environmental behavior as well as emphasizing how this could benefit household budgets. The campaign used the mass media and positive psychology to catalyze changes in long-term consumer behavior and initially focused on five main topics - paper saving, ecodriving, responsible food purchasing, cleanliness in public areas and electricity savings - with additional subjects, such as separation of waste at source, following later. Notably, the campaign was also the subject of a side event at the 19th Session of the Commission on Sustainable Development.

\section{PARTNERING FOR A BETTER WORLD}

\begin{abstract}
Sustainable consumption and production aims to promote resource and energy efficiency, sustainable infrastructure, and access to basic services, green and decent jobs and a better quality of life for all. Its implementation helps achieve overall development plans, reduce future economic, environmental and social costs, strengthen economic competitiveness, and reduce poverty.
\end{abstract}

Following the Rio+20 Summit in 2012, Israel significantly increased its annual contribution to the UN Environment Program (UNEP) in 2014 and has since contributed regularly according to the Voluntary Indicative Scale of Contributions (VISC). In addition to the constant increase in non-earmarked funding towards the Environment Fund, Israel's Ministry of Environmental Protection (MoEP) cooperates with UNEP on the development 
and implementation of projects that involve the transfer of Israeli know-how to developing countries and economies in transition. These projects focus on improving efficiency in the use of natural resources consistent with, and supportive of, UN Environment's mandate and Israel's policies and priorities for international development cooperation.

\section{(SDG 12.2)}

Israel's MoEP and UNEP are in the process of signing a Framework Agreement on Strategic Cooperation aimed at strengthening cooperation on improving efficiency in the use of natural resources. Activities supported by this Agreement are aligned with UNEP's Medium-Term Strategy 2018-2021 and respective programs of work and are based on Israel's know-how and innovation. Proposed programming includes general support regarding the implementation of the approved program of work, support of the 10 Year Framework of Programs (10YFP) on Sustainable Consumption and Production Patterns, capacity-building on green and new technologies for enhanced water security I projects in Africa on eco-innovation for healthy and productive ecosystems and development of clean-tech water projects in Asia). (SDG 12.1)

Israel sits on the Board of the 10YFP as a representative of the Western Europe and Others Group (WEOG) since September 2017 and at least until September 2019. The 10YFP, adopted at the World Summit on Sustainable Development (2012) and affirmed in SDG 12.1, is a global commitment to accelerate the shift towards sustainable consumption and production in both developed and developing countries. It generates collective impact through six multi-stakeholder programs: Public Procurement, Buildings and Construction, Tourism, Food Systems, Consumer Information, Lifestyles, and Education. Israel was also part of the team that performed the Mid-Term Review of the Framework in 2017, which took into account the results and lessons learned from Phase 1 of the Trust Fund and the 10YFP. Key recommendations were made enabling the One Planet Network (implementation of the 10YFP) to develop the new Five-Year Strategy 2018-2022.

Despite progress made to increase global food production, approximately half of the population in developing countries does not have access to adequate food supplies. One of the reasons is food loss occurring throughout the supply chain, from production, post-harvest, processing to marketing. Recognizing the importance of ensuring food security by reducing food losses along the production and supply chains (SDG 12.3), MASHAV implements a capacity-building program on Postharvest Physiology, Pathology and Handling of Fresh Commodities, in cooperation with the Institute of Postharvest and Food Sciences.

It is widely accepted that synthetic pesticides pose a real threat to the global environment, including to human health. Moreover, a reduction in their efficacy as well as an increase in the cost of their development and use serve as strong stimuli for the development of environmentally compatible and sustainable pest control measures. MASHAV tackles this global problem through its Integrated Pest Management Platform, an annual institutional capacity-building course conducted in cooperation with the Institute of Plant Protection. (SDG 12.4)

The production of agricultural goods relies on natural resources, including land, water, biodiversity, and finite fertilizers. Population growth and the fast-paced increase in global food demand puts heavy pressures on the sustainability of natural resources and the environment, leading to water and soil degradation, pollution, damage to biodiversity, deforestation, and overgrazing. MASHAV's international course on Agro-Ecological Approaches to Sustainable Intensive Agriculture focuses on the challenges of maintaining safe, sustainable and intensive food production systems by adopting new practices and precision technologies. (SDG 12.a)

In support of SDG 12, the Weitz Center for Sustainable Development is a member of the UN Industrial Development Organization (UNIDO) global network of centers within the framework of the Network for Resource Efficient and Clean Production (RECPnet). In joining RECPnet, Israel committed to cooperating in a spirit of international partnership, knowledge transfer and professional excellence, and serving as a catalyst in driving 
innovation forward. As part of Israel's contribution to this initiative, MASHAV conducts capacity-building courses in Israel on Green Growth and Clean Technologies, and scholarships are awarded to eligible RECPnet members. The courses include a workshop on the UNIDO TEST Methodology on Sustainable Production and Resource Efficiency. (SDG 12.a)

\section{CASE STUDY}

\section{The Agricultural Cooperation Project in India}

As part of a bilateral cooperation between Israel and India, MASHAV and India's Ministry of Agriculture have jointly set up nearly thirty Centers of Excellence in India. The goal of the Indo-Israel Agricultural Cooperation Project is to demonstrate best practices and new technologies to increase crop diversity, productivity, irrigation and water use efficiency. The Centers of Excellence in Agriculture adhere to the requirements and needs of the Indian government, providing a sustainable platform for the transfer of Israeli agro-technologies adapted to the local conditions and requirements of the farmers.

The Centers of Excellence are arranged in clusters - vegetables, mangoes, pomegranates, citrus, dates, flowers and beekeeping - and are headed by an Indian expert. A long-term MASHAV expert accompanies the project onsite.

The project introduces best Israeli practices and innovative techniques through capacity building programs focusing on nursery production, cultivation methods, and management of irrigation and fertigation. Implementation includes:

- Professional capacity building in Israel and India

- Application of the "training-of-trainers" approach

- Demonstration of advanced Israeli agricultural technologies

- Adaptation to existing local needs

- Introduction of new varieties

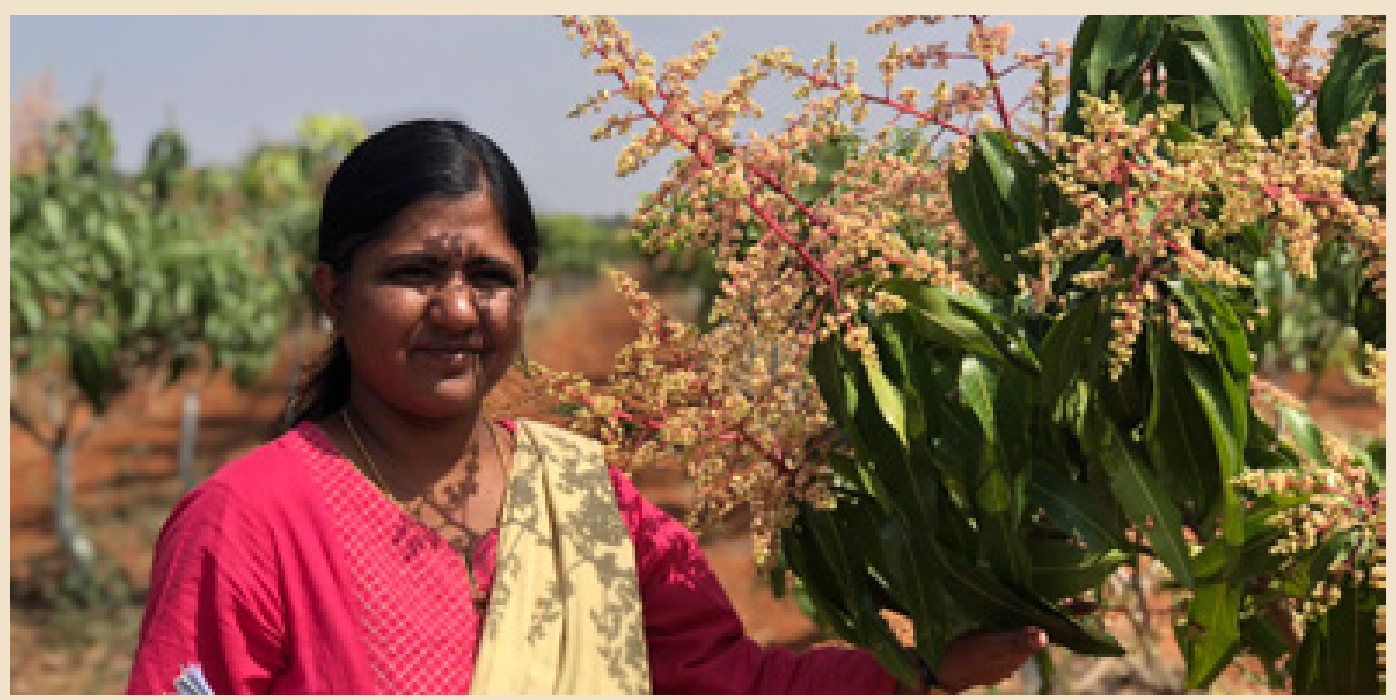




\section{GOAL \\ 13}

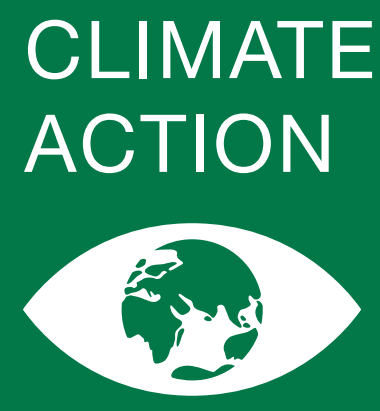

Adaptation to climate-related

hazards and natural disasters

Integration of climate change measures into national policies and plans

Partnering for a Better World 


\section{TAKE URGENT ACTION TO COMBAT CLIMATE CHANGE AND ITS IMPACTS}

Israel is a small, densely populated country characterized by expanding economic growth against a backdrop of land and water scarcity. Arid zones comprise over $45 \%$ of the area of the country. Under a "business as usual" scenario, forecasts indicate that mounting energy consumption coupled with escalating traffic congestion would bring about significant increases in greenhouse gas (GHG) emissions by 2020 and beyond. Israel is determined to change this trend.

Israel's energy strategy pushes forward on several fronts: minimizing the use of coal; leveraging on the economic potential of energy efficiency; advancing the widespread adoption of renewable energy; and supporting initiatives to find alternatives to fossil fuels, especially for transportation. Despite Israel's high population and economic growth rates, GHG emissions have remained relatively stable and emissions intensity has steadily decreased. 


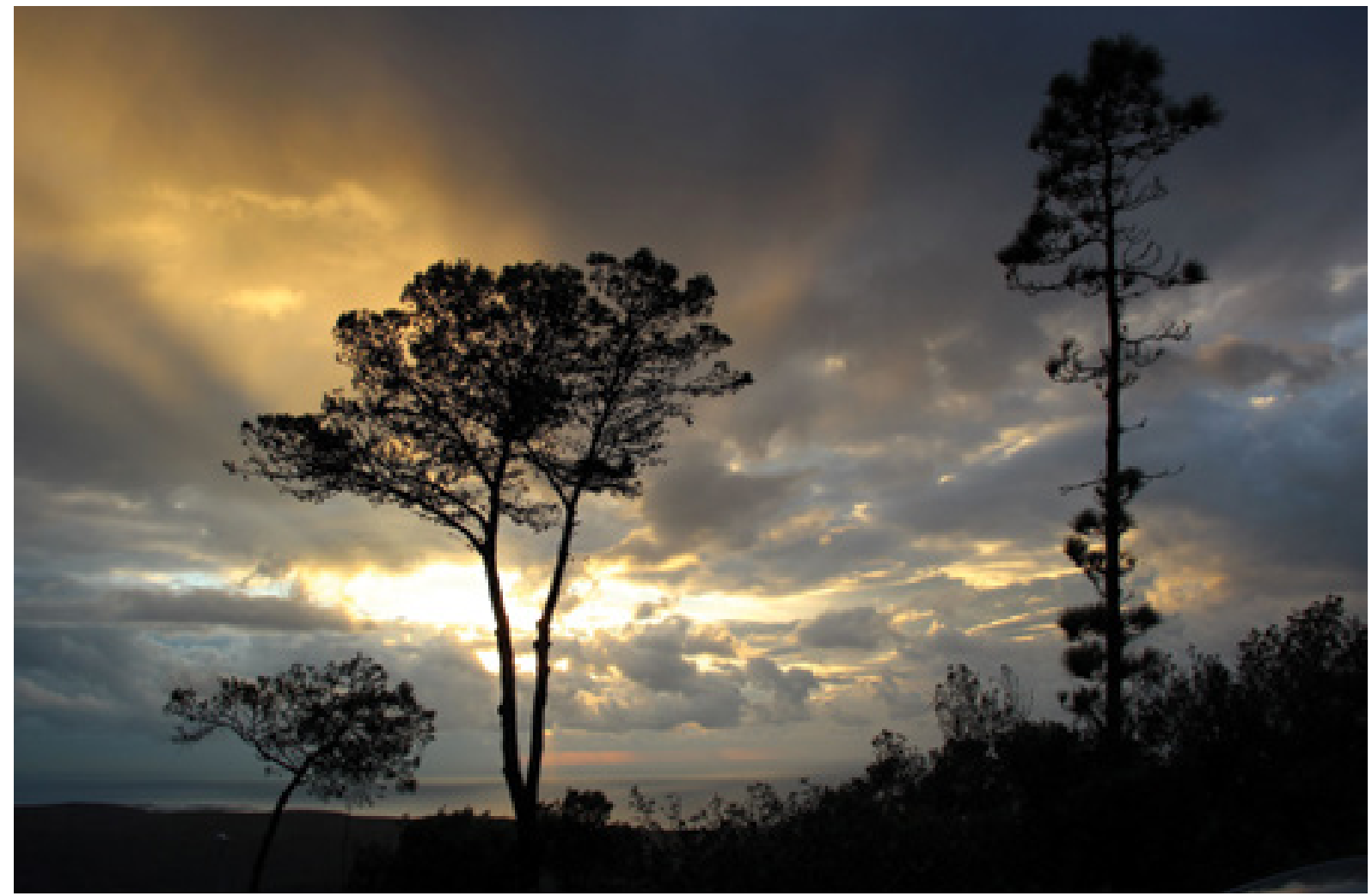

Trees on Mount Carmel in northern Israel/Photo: Ilan Malester

\section{( Adaptation to climate-related hazards and natural disasters (SDG 13.1)}

Israel's intended nationally determined contribution (INDC), was submitted to the UN Framework Convention on Climate Change on September 29, 2015 and serves as Israel's NDC under the Paris Agreement. Subsequently Israel finalized its National Adaptation Plan. Based on an extensive inter-ministerial and non-governmental sectorial consultative process, a detailed recommendation document, which reviewed the information and capabilities in each of the plan's components, was prepared and submitted to the government. It then served as the basis for Government Decision 4079 (July 2018) on “Israel's Adaptation to Climate Change: Implementation of the Recommendations to the Government for a National Strategy and Action Plan."

The decision determines that the State of Israel recognizes the occurrence of global climate change and the need to adapt to it. Israel's vision is to be highly prepared for the impacts of climate change and extreme events by implementing action plans and policy measures that reduce risks to health, environment and the economy, while maximizing potential benefits and opportunities.

The objectives of the strategic plan are:

- Reducing human and property damage and building economic resilience;

- Taking measures to increase the resilience of ecosystems;

- Developing and updating the scientific knowledge for decision making:

- Promoting education, raising awareness and making knowledge accessible to decision-makers and the public;

- Promoting regional and global climate cooperation.

The national strategy is based on the understanding that adaptation to climate change is a long-term process which entails uncertainty regarding the nature, effect, intensity, scope and date of occurrence of the changes. Estimating the scope of the risks and their economic ramifications 
poses a significant challenge. For this reason, it was decided that preparedness and adaptation measures are to be integrated into the policies of all relevant government ministries. The process requires commitment, coordination and flexibility in order to respond to the emerging reality and to new information obtained from ongoing research. Israel's strategic plan calls for the mainstreaming of climate change adaptation measures into decision making by various authorities. Each governmental body will draft and implement an adaptation action plan in areas under its responsibility. To date, several government ministries and agencies have already begun to implement measures directed at better responding to the adverse impacts of climate change. For example, given the reality of water scarcity, Israel has established desalination plants and plans to further increase water production in order to avoid dependence on natural water sources.

\section{Israel's National Climate Change Adaptation Plan}

The National Climate Change Adaptation Plan contains some 30 action plans that cover all aspects of life and economic activity, some of which require coordination between several relevant entities while others are under the responsibility of a specific ministry. Thus, for example, the action plan to formulate a program to prepare for climate change and risk assessment in agriculture requires the active participation of the Ministry of Agriculture, the Meteorological Service, the Water Authority, the Israeli Central Bureau of Statistics, the Ministry of Environmental Protection and other government entities. On the other hand, the action plan to review the impact of climate change on organizational matters, including necessary actions to increase resilience and address extreme weather events, is under the responsibility of the Ministry of Public Security alone. To this end, the

\section{EXISTING ACTIONS ON CLIMATE ADAPTATION}

Following is a brief description of climate adaptation actions already taken by government entities in several realms:

\section{Water}

- Analysis of existing and projected water balances

- Planning for a $15 \%$ increase in water supply through the development of desalination facilities and the reclamation of treated wastewater, among others

- Incorporation in development plans of drought preparedness and preservation of water reserves for continuous drought years

\section{Construction and Housing}

- Development of assessment tools for green neighborhoods

- Training on sustainable planning of urban construction and shading

- Implementation of energy efficiency research

- Pilot projects for upgrading buildings

- Tunneling and underground construction

- Integration of sustainable development principles into construction programs 


\section{Health}

- Preparation for short- and long-term responses to climate events

\section{Agriculture}

- Research and development on the improvement of fruit and vegetable strains and implementation of the results

- Research and development partnerships with the European Union

- Establishment of an agricultural research center at the edge of the desert

- Preparation of plans for agricultural adaptation to climate change

\section{Education}

- Planning for the resilience of educational institutions to the impacts of extreme weather events

- Establishment of a sub-committee to develop an educational sequence on extreme weather events that will determine subjects to be integrated into existing sustainability programs

- Examination of the cold and heat stress scale used when approving school trips

\section{Tourism}

- Incorporating a condition to meet the minimum requirements of the Green Building Standard (5281) for hotels in the conditions for receiving grants under the Encouragement of Capital Investments Law

\section{Keren Kayemeth Lelsrael - Jewish National Fund}

- Development of an innovative water-resilient cities project

- Construction of three bio-filter sites that collect and purify urban runoff

- Development of runoff collection methods to prevent flooding of urban areas

- Investment in research and development to promote varieties of drought-resistant trees

- Establishing 80 pest monitoring sites

- Construction of 170 buffer lines to prevent the spread of fires

\section{Meteorology}

- Ongoing preparations for storms in cooperation with such bodies as the Israel Ports Authority, Israel Railways and Israel Airports Authority

\section{Public Security}

- Establishing a joint database on fire prevention with relevant bodies

- Upgrading existing systems to predict the advance and spread of fires

- Preparations by the police for extreme conditions

- Preparations by the firefighting services for protecting populations in the vicinity of forests and woods 


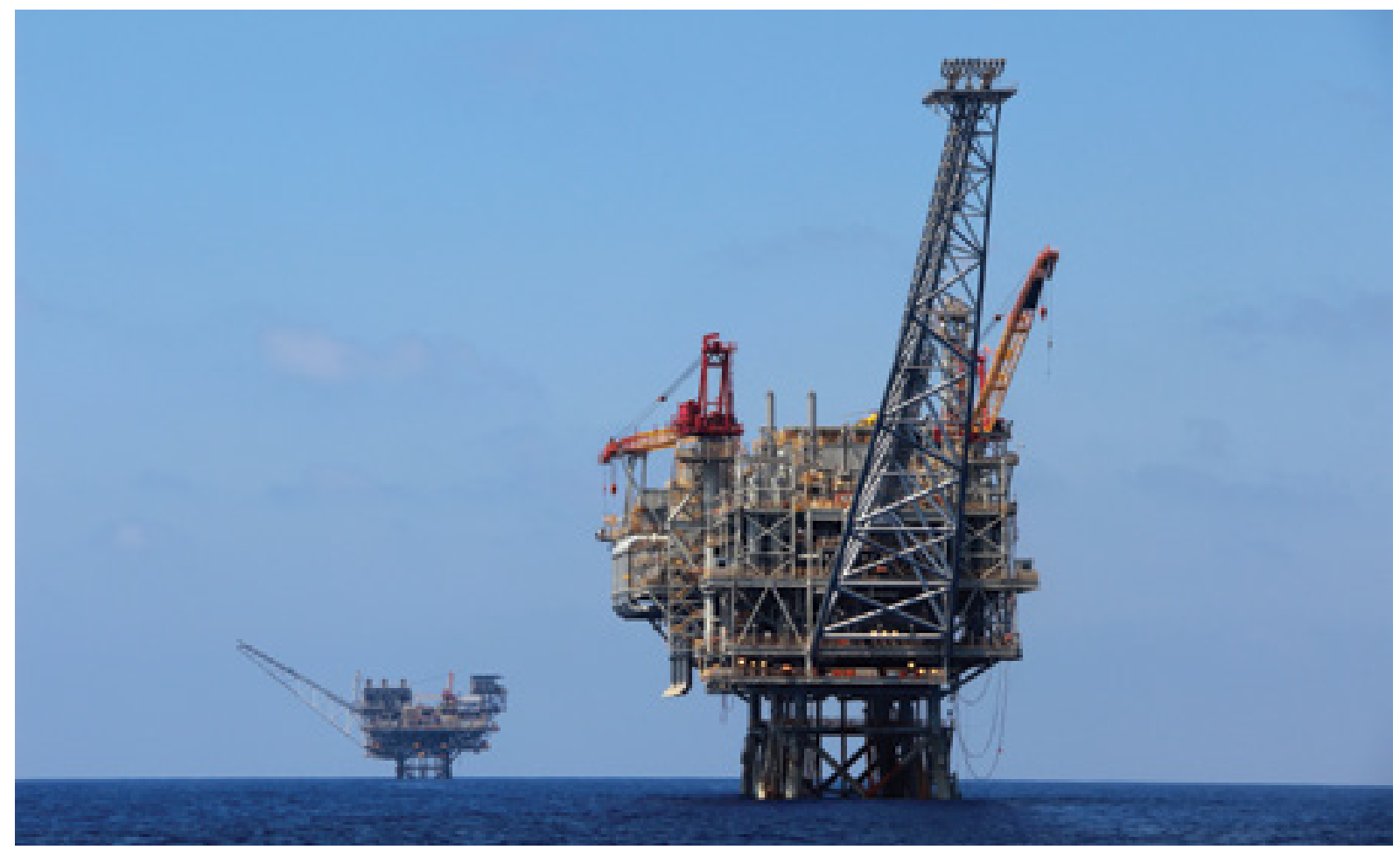

Tamar Platform/Photo: Ilan Malester

Research Department of the Ministry of Public Security is coordinating an inter-ministerial team on measures for climate change preparedness, while examining the ramifications of these changes on the activity of the ministry's operational bodies. In addition, three intra-organizational teams have already been designated - within the Israel Police, the Israel Prison Service and the Israel Fire and Rescue Authority.

The government decision on adaptation establishes an administration incorporating all relevant stakeholders to oversee the implementation of the national strategy and action plans and to promote inter-ministerial and multi-stakeholder coordination. The Climate Change Adaptation Administration was first convened in December 2018, with 33 members representing government ministries, public bodies and non-governmental environmental organizations.

This administration is made up of seven subcommittees: Strategy; Health and Emergency; Natural Resources, Agriculture and Environment; Energy, Infrastructure and Technologies; Research and Development; Local Authorities; and
Communication and Education. The administration will prepare a national climate change adaptation plan for government approval by the end of 2019. The new decision will include the allocation of long-term resources for implementation by 2022.

\section{Integration of climate change measures into national policies and plans (SDG 13.2)}

GHG emissions in Israel reached 78.36 million metric tons of $\mathrm{CO}_{2} \mathrm{e}$, or 9.7 tons $\mathrm{CO}_{2}$ e per capita in 2013. Under a business as usual (BAU) scenario, GHG emissions are expected to reach 105.5 million tons of $\mathrm{CO}_{2} \mathrm{e}$, or 10 tons of $\mathrm{CO}_{2}$ e per capita in 2030. Israel's main emission sources are the energy production and transportation sectors, which are responsible for $53 \%$ and $21 \%$ respectively of total emissions anticipated in 2030. Under the BAU scenario, GHG emissions from electricity generation are expected to reach 56 million $\mathrm{tCO}_{2} \mathrm{e}$ in 2030.

Israel ratified the Paris Agreement in November 2016 and committed to a target to reduce GHG emissions to 7.7 tons $\mathrm{CO}_{2}$ e per capita by 2030 . 


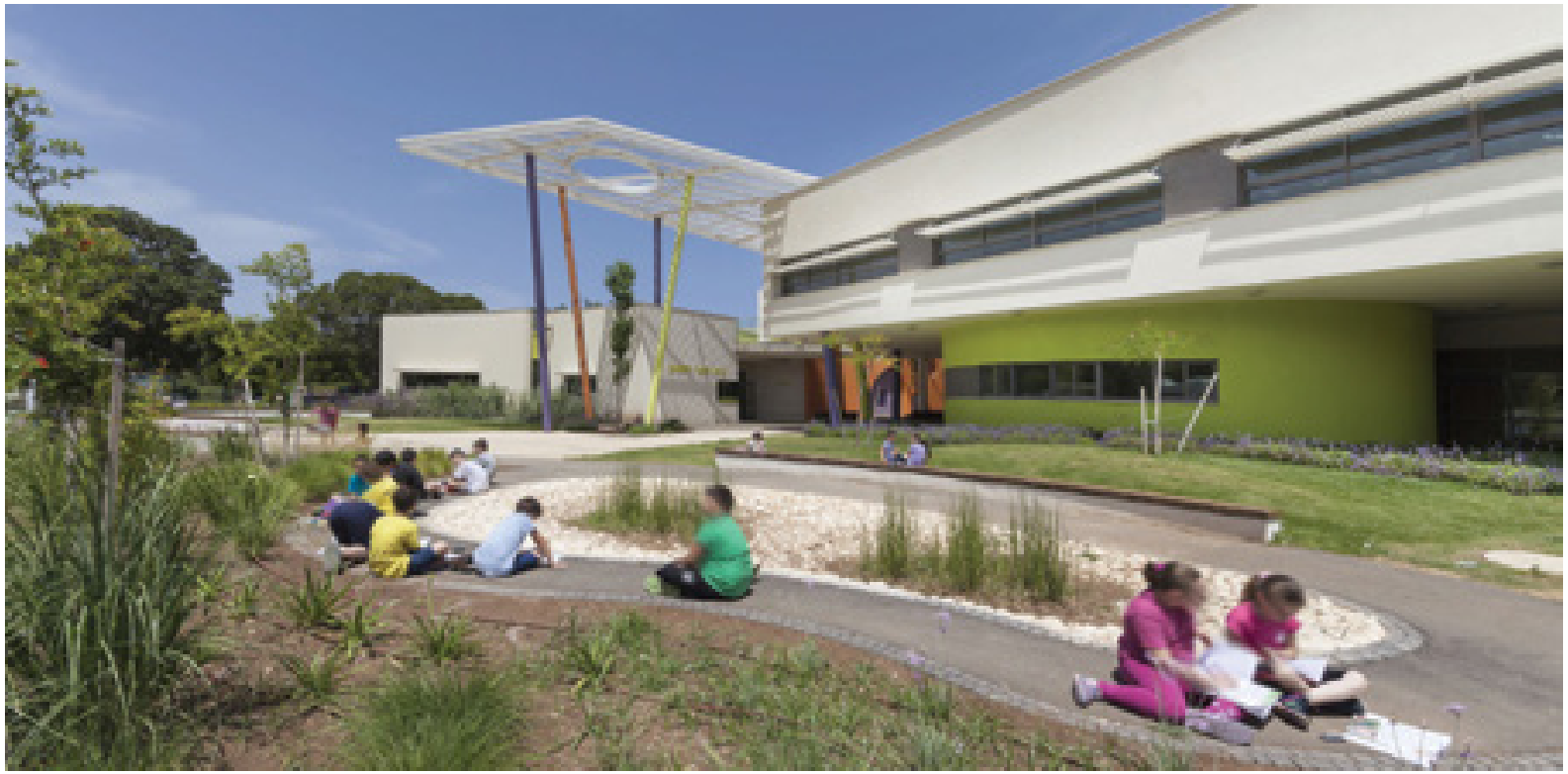

Rakafot Green Building School/Photo: Elad Gonen

constituting a $26 \%$ reduction relative to 2005 per capita emissions of 10.4 tons $\mathrm{CO}_{2}$ e. Additionally, an interim target of 8.8 tons $\mathrm{CO}_{2}$ e per capita by 2025 was set. The government further approved sector-specific targets for 2030:

- $17 \%$ reduction in electricity consumption relative to anticipated electricity consumption in 2030;

- $13 \%$ of electricity consumption in 2025 from renewable energy, increasing to $17 \%$ in 2030;

- $20 \%$ reduction in kilometers travelled by private vehicles relative to anticipated kilometers travelled in 2030

Compliance with these targets requires the reduction of 24.5 million tons $\mathrm{CO}_{2} \mathrm{e}$ in 2030 relative to anticipated emissions under a BAU scenario (105.5 million tons of $\mathrm{CO}_{2} \mathrm{e}$, or 10 tons $\mathrm{CO}_{2}$ e per capita in 2030). Since the submission of Israel's NDC, GHG emissions in 2016 were 79.95 million tons $\mathrm{CO}_{2}$ e or $9.36 \mathrm{CO}_{2}$ e per capita.

National Plan for Implementation of the GHG Emissions Reduction Targets and for Energy Efficiency

In order to meet Israel's 2030 goals, a National Plan for Implementation of the GHG Emissions Reduction Targets and for Energy Efficiency (Government Decision 1403) was approved in April
2016 that includes mitigation measures in key areas and sets timetables for review and formulation of additional measures.

The main elements in Israel's national plan include:

- Removal of barriers to renewable energy uptake, including planning, economic, regulatory and bureaucratic barriers;

- Reduction of coal use in the power sector;

- Allocation of NIS 500 million (about \$138 million) in government guarantees for a ten-year period to leverage investment loans in the fields of energy efficiency and GHG emissions reduction, in accordance with criteria that relate to eligibility, duration and prioritization of innovative Israeli technologies;

- Allocation of NIS 300 million (about \$83 million) in government grants to energy efficiency, with an emphasis on local authorities with low socioeconomic rankings and small and medium-sized businesses. The grants are awarded based on competition over the reduction cost of a ton of GHG gases and KW/h saved.

The emissions reduction plan also calls for a wide variety of additional measures in such areas as green building, energy ratings for new buildings, public transportation, energy efficient alternative propulsion vehicles and more. 


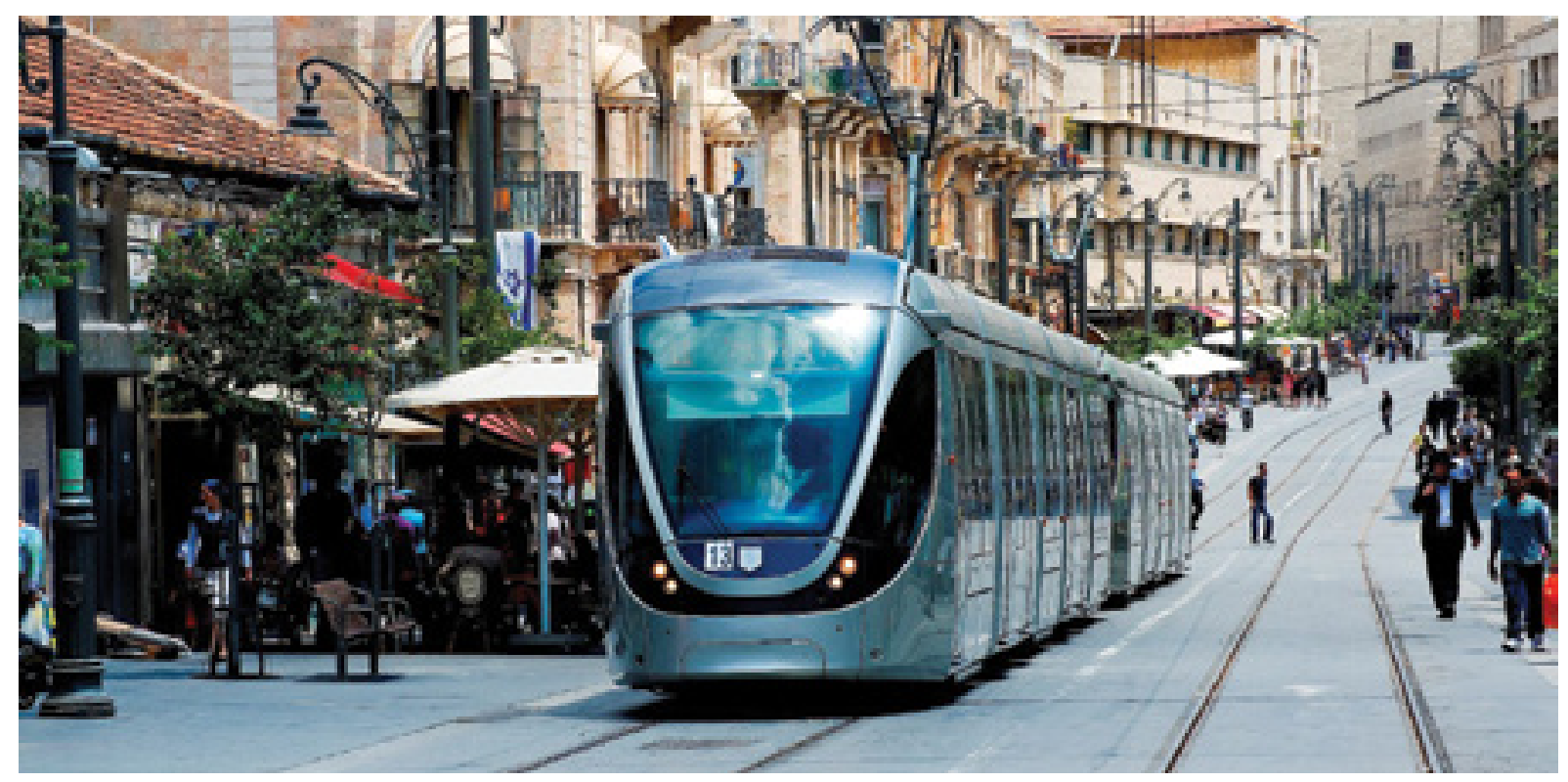

Light rail in downtown Jerusalem/Photo: CityPass

The implementation of the national plan is currently underway, and Israel is already witnessing successful programs to encourage energy efficiency and $\mathrm{GHG}$ emissions reduction. Major potential exists in the building sector where recommendations are being implemented for reducing GHG emissions and decreasing electricity consumption. Furthermore, plans are proceeding for the closure of four of the oldest and most polluting coal-fired units in Israel's largest power plant in 2022 and for the conversion of one of the units in the second largest coal-fired power plant to natural gas in 2023. Coal will be reserved for back-up in emergency situations of natural gas shortage.

The Ministry of Energy recently prepared an energy policy document for 2030 which supports the national plan and requires the shutting down of all coal-fired power plants by 2030 and the replacement of coal with natural gas and renewable energy sources. The transition will be based on a change in the load order of power plants that prioritizes the most efficient units (combined cycle units based on natural gas) over less efficient units. A further increase in the $17 \%$ target for electricity generation from renewable energy will be reviewed in 2022, depending on the availability of technological developments, mainly in the area of energy storage.
To monitor progress in achieving Israel's reduction goals, a national system for measurement, reporting and verification (MRV) has been established. Within this framework, data is collected and analyzed in order to measure the effectiveness of government policy in implementing the measures defined in the national plan and to update it accordingly.

Israel submitted its first National Communication in 2000, the second in 2010 and third in 2018. Israel's first BUR was submitted to UNFCCC in May 2016. An annual national GHG inventory is prepared by the ICBS and submitted to UNFCCC.

\section{Israel's 2050 vision}

The Ministry of Environmental Protection, in cooperation with the Israel Democracy Institute, the Ministries of Energy, Transport, Economy and Finance, the Planning Administration and additional central bodies together with the OECD have started a multi-sectoral process to formulate Israel's 2050 vision and associated roadmap and develop a Long-term Low Emissions Development Strategy (LT-LEDS). Specific targets and measures are currently under assessment in four focus areas - electricity generation, transportation, industry and waste, buildings and cities - including, inter alia:

- Major upscaling of renewable energy; 
- Massive uptake of electrical and other zeroemissions vehicles;

- Modal shift to sustainable mobility lwalking, biking and public transport);

- Green buildings and zero-energy buildings;

- Transport-oriented development in urban planning;

- Circular economy and zero-waste.

Once properly evaluated, the measures will be integrated into a comprehensive long-term economic vision and roadmap taking into account not only emission impacts, but also air pollution morbidity and mortality, affordability and cost of living, macro-economic effects, land use and open areas.

This process is scheduled to be completed and submitted to the UNFCCC in 2020, in accordance with the Paris Agreement.

\section{PARTNERING FOR A BETTER WORLD}

Israel has made it a national priority to partner with UN specialized agencies, regional organizations and bilateral aid agencies, thereby allowing all stakeholders to better identify the impacts and options for mitigation and adaptation and find solutions to the adverse effects of climate change on water resources, health, biodiversity and quality of life. In September 2015, Israel submitted its official greenhouse gas reduction target to the UN Framework Convention on Climate Change (UNFCCC), signed the Paris Climate Agreement in April 2006, and saw its ratification in November 2016.

In a government decision on Israel's adaptation to climate change adopted in July 2018, one of the five major goals referenced is Israel's contribution to global efforts in combating climate change and its impacts. In light of this decision and the fact that climate change is one of the most important components in the environmental pillar of the 2030 Agenda, the Ministry of Foreign Affairs appointed a Special Envoy for Sustainability and Climate Change to lead Israel in its continued support and implementation of the UNFCCC.

Israel's Ministries of Environmental Protection (MoEP), Finance and Economy and Industry recently established a fund in cooperation with the European Bank for Reconstruction and Development (EBRD). The $€ 1$ million Israel-EBRD Cooperation Fund supports projects in countries where the EBRD is active and in areas such as water, energy and climate change, including the integration of gender equality in climate change interventions and climate finance. The Fund provides a wide range of technical assistance tools lincluding feasibility studies, surveys, engineering and legal consultancies). These tools enable fund raising (commercial, regional development banks, special funds) to provide supplementary financing for environmental projects including from the Green Climate Fund. (SDG 13.a)

The Natural Resources and Environmental Research Center (NRERC) at Haifa University became a member (2015) of the UN Climate Technology Center and Network (CTCN). The CTCN, serving as part of the UNFCCC, aims to strengthen the abilities of developing countries to prepare and organize technological climate change adaptation and mitigation projects. (SDG 13. b)

Israel was a member of the Climate Impact Research and Response Coordination for a Larger Europe (CIRCLE-2), an international network of institutions focused on interfacing climate change, science and policy. Members of CIRCLE-2 were committed to maximizing the degree to which research outcomes address both national and international climate policy needs, especially on adaptation. (SDG 13.2)

The Global Covenant of Mayors for Climate \& Energy is an international coalition of mayors and city officials committed to reducing greenhouse gas emissions, enhancing resilience to climate change and tracking their progress publicly. Israel's MoEP encourages local authorities to join 
this effort, providing background information and guidance to local authorities interested in signing the treaty. Several authorities have already joined the Covenant. (SDG 13.1)

Israel has signed dozens of bilateral agreements with countries throughout the world in a myriad of environmental fields, including climate change mitigation and adaptation. One example is the launch (2014) of a three-year project on the use of climate-neutral substances that do not deplete the ozone layer in the cooling sector, in partnership with the Bavarian State Ministry of the Environment and Consumer Protection, the Bavarian Environment Agency and the German Society for International Cooperation (GIZ). A Joint Declaration of Intent was signed (2015) to cooperate on GHG emission reduction towards the transition to climate friendly refrigerants, policy measures, and requirements regarding reduction of F-gases. (SDG 13.2)

Israel's overseas development assistance on combating the impacts of climate change in least developed and developing countries focuses on enhancing preparedness and adaptation to yield environmental and socio-economic benefits, in line with the national policies and strategies of cooperating partners, to ensure effective climate change planning and management. (SDG 13.b)

Israel is a Partner State in the Climate and Clean Air Coalition to Reduce Short-Lived Climate Pollutants (CCAC) since 2012. CCAC is a voluntary partnership of governments, intergovernmental organizations, businesses, scientific institutions and civil society organizations committed to protecting the climate and improving air quality through actions to reduce short-lived climate pollutants (i.e., black carbon, methane, HFCs). (SDG 13.3)

Israel was a Partner Country in the ClimaSouth project (2013-2017) to support climate change mitigation and adaptation in nine South Mediterranean countries including Algeria, Egypt, Jordan, Lebanon, Libya Morocco, Tunisia and the Palestinian Authority. This EU-funded project supported their transition towards low carbon development and climate resilience, while enhancing regional cooperation. (SDG 13.3)
Building on the achievements of, and ensuring continuity with the completed EU funded ClimaSouth project, the European Union launched a new four-year project in the Southern Mediterranean Neighborhood (Algeria, Egypt, Israel, Jordan, Lebanon, Morocco, Tunisia and the Palestinian Authorityl entitled Clima-Med, Acting for Climate in the South Mediterranean. The project focuses on supporting sustainable energy policies and strategies at national and local levels and providing technical assistance to support the Sustainable Energy Access and Climate Action Plan (SEACAPs). The overall objective is to enhance energy security and adaptive capacity of partner countries while increasing energy sovereignty and reducing $\mathrm{CO}_{2}$ emissions. Climate finance is also a key component. Partners are supported in implementing and updating Nationally Determined Contributions (NDCs) to develop adaptation and mitigation plans lintegrated to long-term low emission development strategies) and enhance MRV capacities. (SDG 13.2, 9.4)

The African Continent is one of the most vulnerable to the adverse impacts of climate change, as already visible in the increase in temperatures, decrease in precipitation, reliance on rain-fed agriculture, and increase in extreme weather events. Israel's Ministry of Foreign Affairs, under the auspices of the Prime Minister, convened (2018) a High-Level Conference on Africa on Climate Change and Israel's Contribution. The Conference focused on challenges facing Africa and developed an action program for cooperation. The Executive Secretary of the UNFCCC was the keynote speaker, and the Special Advisor to UN Secretary General on Sustainable Development concluded the event. The Ministry of Foreign Affairs intends to convene a larger scale follow-up conference. (SDG 13.b)

Two Israeli professors were selected as lead authors on the Intergovernmental Panel on Climate Change (IPCC), under the auspices of the World Meteorological Organization (WMO), and UNEP, to provide regular assessment for policymakers on the scientific, technical and socio-economic aspects of climate change. The IPCC is in its Sixth Assessment cycle and Assessment Report 6 (AR6) will be finalized in 2022. (SDG 13.2) 
The lead author (Hebrew University) in IPCC Working Group I Ithe scientific basis of climate change) participated in the First Lead Authors Meeting (LAM) in Addis Ababa. The lead author (Israel Oceanographic \& Limnological Research) in Working Group II helped write the IPCC Special Report on the Ocean and Cryosphere in a Changing Climate, assessing relevant literature on the ocean and cryosphere. He also attended the First Lead Authors Meeting (LAM) Group II in Guangzhou, China. In support of IPCC goals, Israel contributed $\$ 10,000$ to fund the participation of professionals from least developed countries in IPCC fora. (SDG 13.b)

MASHAV and the Food and Agriculture Organization (FAO) strengthened collaboration on climate change related fields to benefit African countries. A delegation of representatives from Africa arrived in Israel to participate in the first study visit entitled Agricultural Development and Adaptation to Climate Change. Furthermore, Israel and the FAO will be conducting a Joint Seminar on Climate Change, Biodiversity, Land and Water to explore further opportunities for collaboration. (SDG 13.3)

MASHAV conducts the following capacity-building courses relating to climate change: Green Growth: Policy and Economic Measures as Tools for Local and Regional Sustainable Development; Agriculture and Environment in a Changing Climate; and Climate Change and Agriculture, conducted in cooperation with the WMO. (SDG 13.3) 
GOAL

14

LIFE BELOW WATER

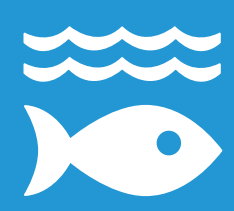

Marine pollution

Marine litter

Marine monitoring

Oil spill incidents and response

Sustainable management and protection of ecosystems

Regulation of marine harvesting

Conservation of coastal and marine areas

Partnering for a Better World 


\section{CONSERVE AND SUSTAINABLY USE THE OCEANS, SEAS AND MARINE RESOURCES FOR SUSTAINABLE DEVELOPMENT}

Israel's Mediterranean and Red Sea coastlines, stretching about 190 kilometers and 14 kilometers respectively, are among the country's most valuable natural assets. They are characterized by a rich diversity of natural, environmental, cultural and economic values. Nearly $70 \%$ of the population and much of the country's economic and commercial activity are concentrated along the Mediterranean coastal strip and along the upper Eilat-Aqaba Gulf in the Red Sea. 


\section{( Marine pollution (SDG 14.1)}

The protection of Israel's coastlines from the adverse impacts of pollution and development is a national priority. The primary coastal issues on Israel's agenda include: balancing development and conservation, protecting the sand balance and preventing damage to the shoreline and coastal cliff, conserving the diversity of species and their ecosystems, preserving archaeological, historic and cultural heritage, and protecting the coastline as an open space for the enjoyment of present and future generations. The prevention of land-based pollution is one of the major items on Israel's agenda.

Israel prevents and controls all aspects of marine pollution: accidental and operational oil and chemical spills from ships or terminals; effluents from industrial or municipal land-based sources; dumping of waste at sea; implications of various activities including underwater soil gas exploration and exploitation, mariculture and construction; and marine littering.

Marine pollution prevention activities are based on comprehensive national regulatory systems, grounded in legislation and international conventions and protocols, rigorously enforced.
Prevention of land-based pollution of the

\section{marine environment}

Israel is an active member of the Convention for the Protection of the Marine Environment and the Coastal Region of the Mediterranean (Barcelona Convention), a participant in the activities of its regional activity centers, and a signatory to most of its protocols including the Land-Based Sources Protocol. In 2015 Israel updated its National Action Plan (NAP) pursuant to its obligations under the Barcelona Convention and its Land-Based Sources Protocol which analyzed, inter alia, those areas in which additional actions must take place to further reduce the pollution of the Mediterranean Sea and coastal areas. Israel's current NAP, which is to be implemented by 2025 incorporates new developments consolidated by the Barcelona Convention and its bodies such as implementation of the Ecosystem Approach to achieving Good Environmental Status in areas such as eutrophication, contaminants and marine litter. As a result of the NAP, detailed action plans have been prepared on the mitigation of marine litter, mitigation of pollution in coastal streams, mitigation of polluted runoff and an Action Plan for Hot Spots and Sensitive Areas.

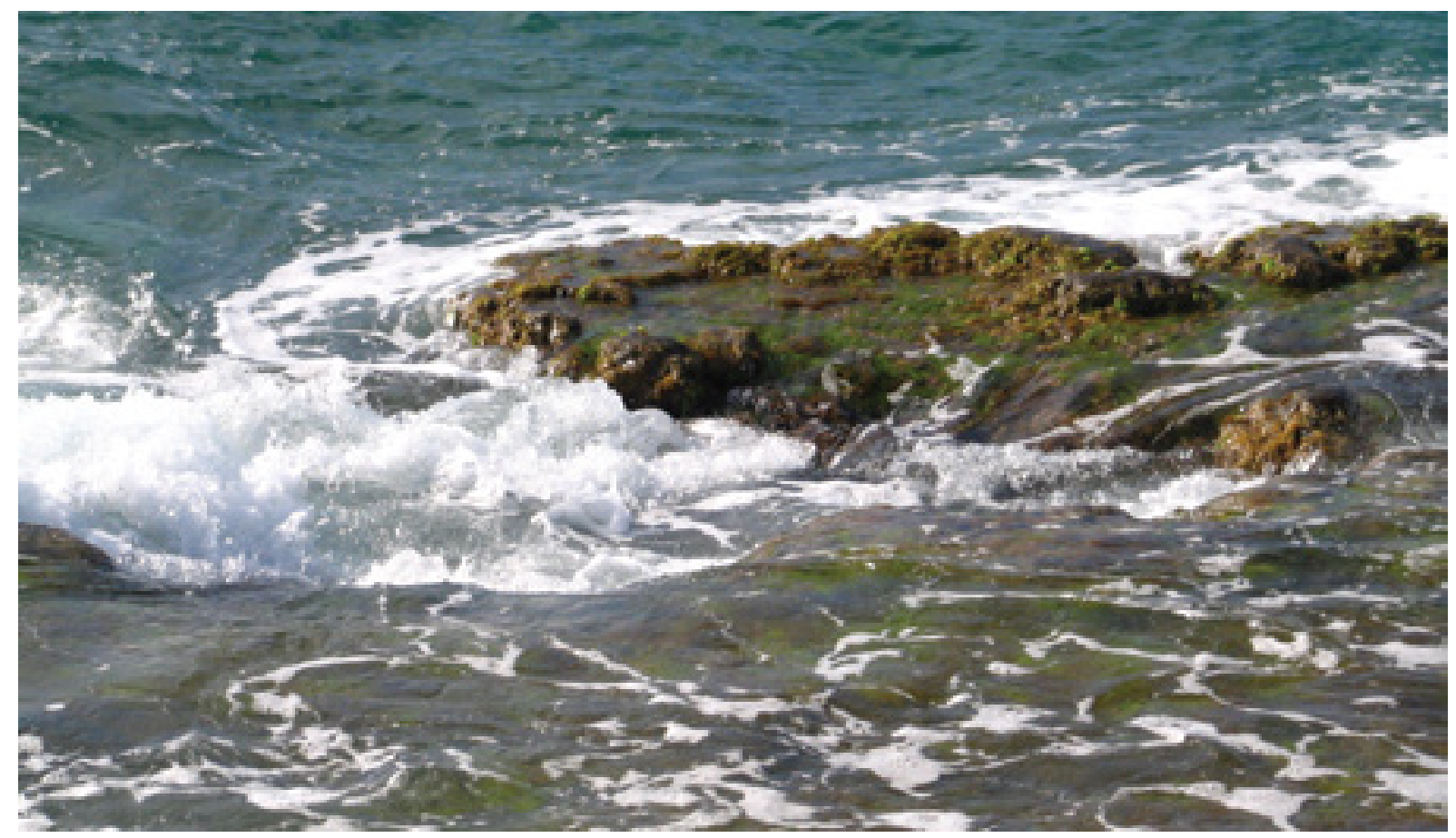

Israel's HaBonim coast/Photo: Ilan Malester 
In the past, pollution from land-based sources in Israel was, by far, the main contributor to marine pollution, and therefore the greatest improvements have occurred in this area. A comparison between the findings of the Multiannual Marine Land-Based Pollution Report of 1998-2004 and the annual reports of 2014, 2015, 2016 and 2017 shows a significant reduction in most contaminant loads discharged into the sea despite the increase in the number of discharge-to-sea permit holders. The findings are based on many thousands of samples taken from discharges to the sea by permit holders.

In the last twenty years, the load and concentration of pollutants discharged into the Mediterranean Sea has been reduced by $95 \%$ to $100 \%$. This was achieved through a combination of enhanced regulation, increased supervision and enforcement and use of economic tools, including the imposition of substantial financial sanctions on polluting facilities.

The main reason for the dramatic reduction in pollution loads from 2017 onwards is the cessation of discharges of sludge from the Dan Region Wastewater Treatment Plant (Shafdan). Following the completion of anaerobic digestion processes for $100 \%$ of primary and secondary sludge at the Shafdan and its use for agricultural purposes, discharge of sludge to the sea ceased in February 2017. As a result, 80\%-99\% of the contaminant loads discharged into the sea were reduced.
To date, seven land-based pollution "hotspots" have been reduced to two. Approximately $87 \%$ of Israel's sewage is recycled for irrigation and almost no treated or untreated sewage is discharged to sea. However, new pollution challenges such as desalination plants and gas exploration and production will have to be effectively managed.

At the 2017 United Nations Oceans Conference aimed to further the implementation of SDG 14 , Israel made two voluntary contributions:

- By the end of 2017, to reduce direct discharge of wastewater from industrial and municipal sources (which are sources of BOD, nutrients, heavy metals, oils and suspended solids) by $80 \%$ compared to a 2012 baseline budget of pollutants;

- By 2022, to reduce indirect atmospheric emissions of NOx and SOx to the marine environment by an additional $27 \%$, due to the installation of scrubbers in Israel's main coastal power stations and the replacement of coal fuel with natural gas. This will result in further improvement of the marine environment.

The following graphs demonstrate that the first voluntary contribution has been fully achieved. Discharges of heavy metals, BOD and nitrogen, for example, as well as other parameters, have all been reduced dramatically, particularly by the end of 2017 (Figures 14.1, 14.2, 14.3).

Figure 14.1

\section{Metals loads into the Mediterranean Sea 1998-2017}

$$
250,000
$$

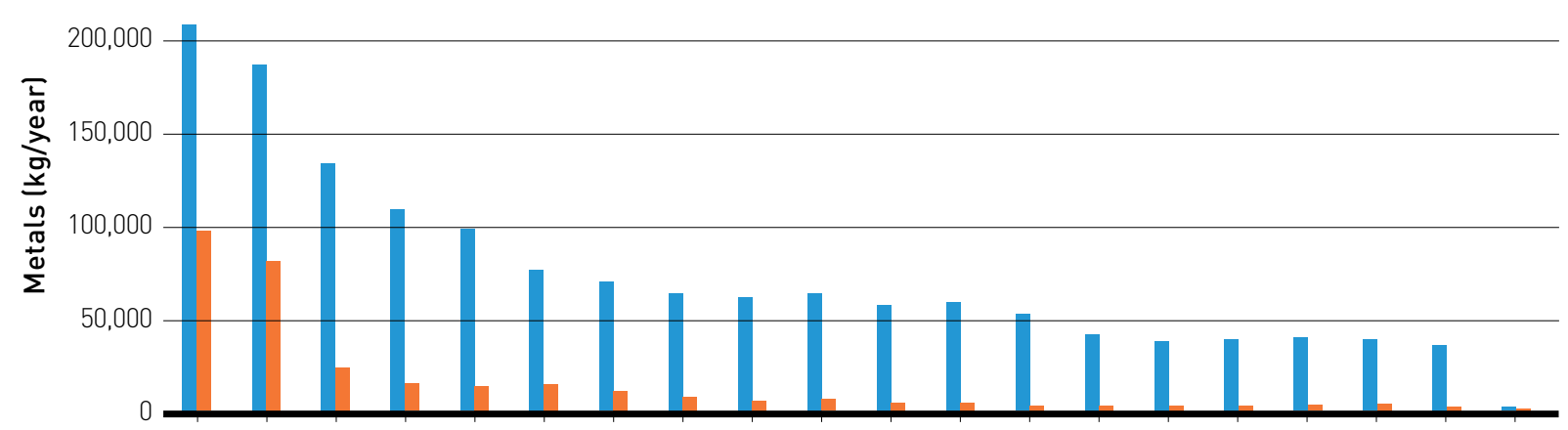

19981999200020012002200320042005200620072008200920102011201220132014201520162017 
Figure 14.2

\section{BOD load into the Mediterranean Sea 1998-2017}

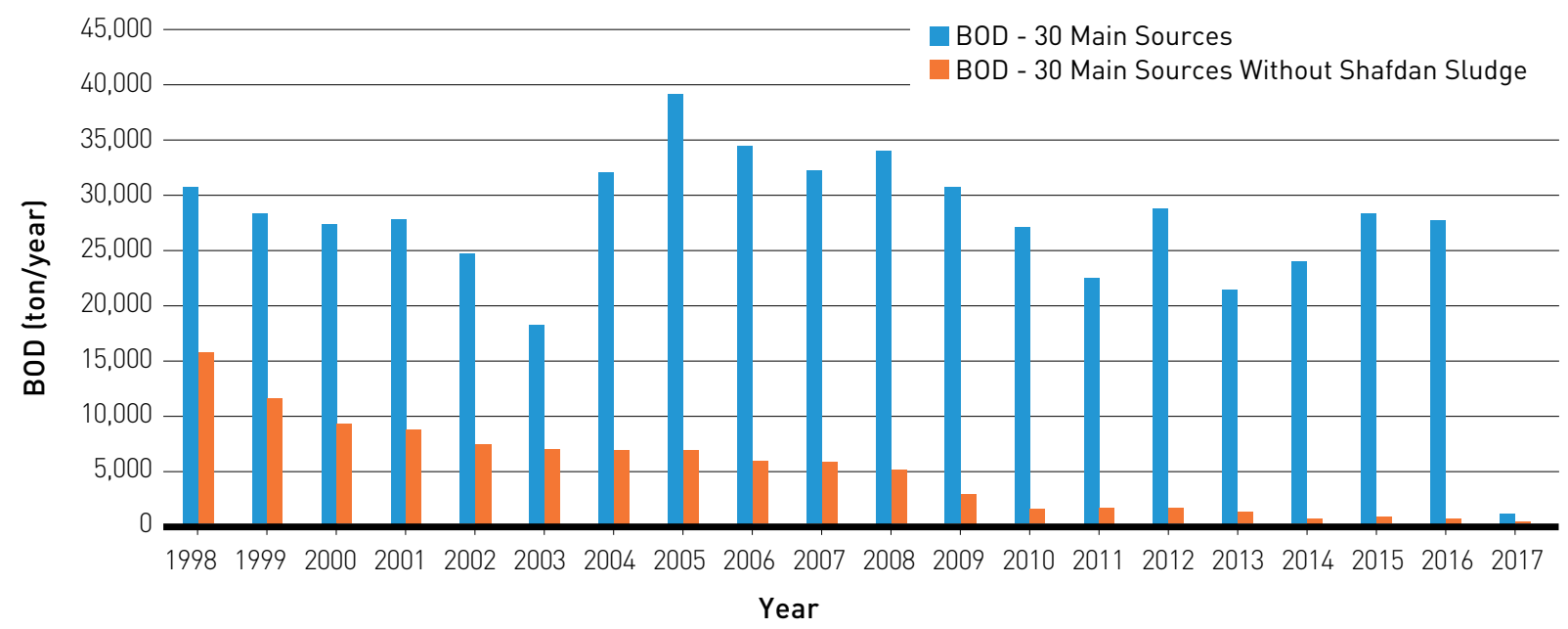

Figure 14.3

Nitrogen load into the Mediterranean Sea 1998-2017

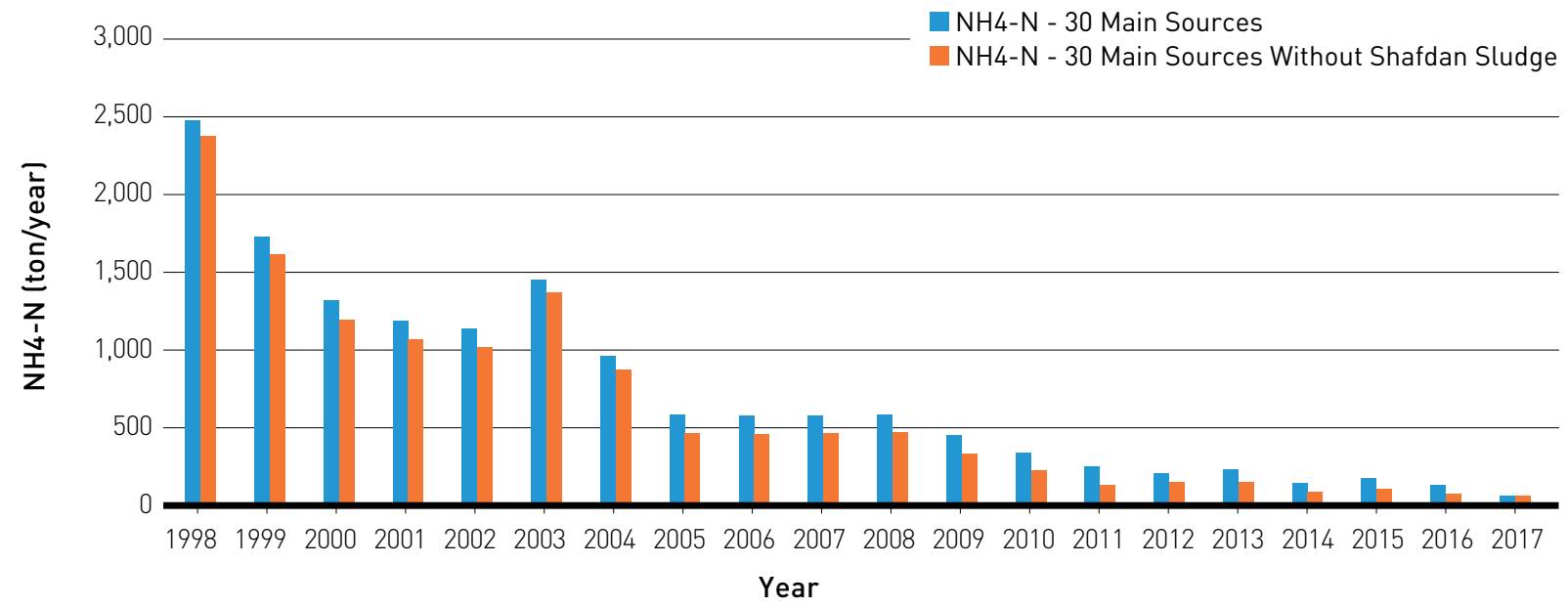

Israel implements the same pollution reduction policies on discharges to the Dead Sea and the Red Sea (Gulf of Eilat/Aqaba) as it does in the Mediterranean Sea. Monitoring of Eilat's coral reefs for more than ten years has led to the recognition of the upper Eilat-Aqaba Gulf in the Red Sea as a refuge for coral reefs. While worldwide degradation of coral reefs is occurring due to mass bleaching, the coral cover in the Eilat reefs remain steady, with a slow positive trend despite the rise in seawater temperature.

Nutrients (nitrogen and phosphorus) were identified as the main pollutants affecting the Eilat reefs. As of 2016, a maximum total load for nitrogen and phosphorus that can be discharged to sea without endangering the reefs has been established. Discharge permits are determined so that the total nutrient load from all permit holders does not exceed this maximum. Monitoring results from recent years show a decrease in nutrient loads from land-based sources to the Gulf of Eilat/Aqaba.

In the last ten years there has been a marked reduction in oil and grease loads from land-based 


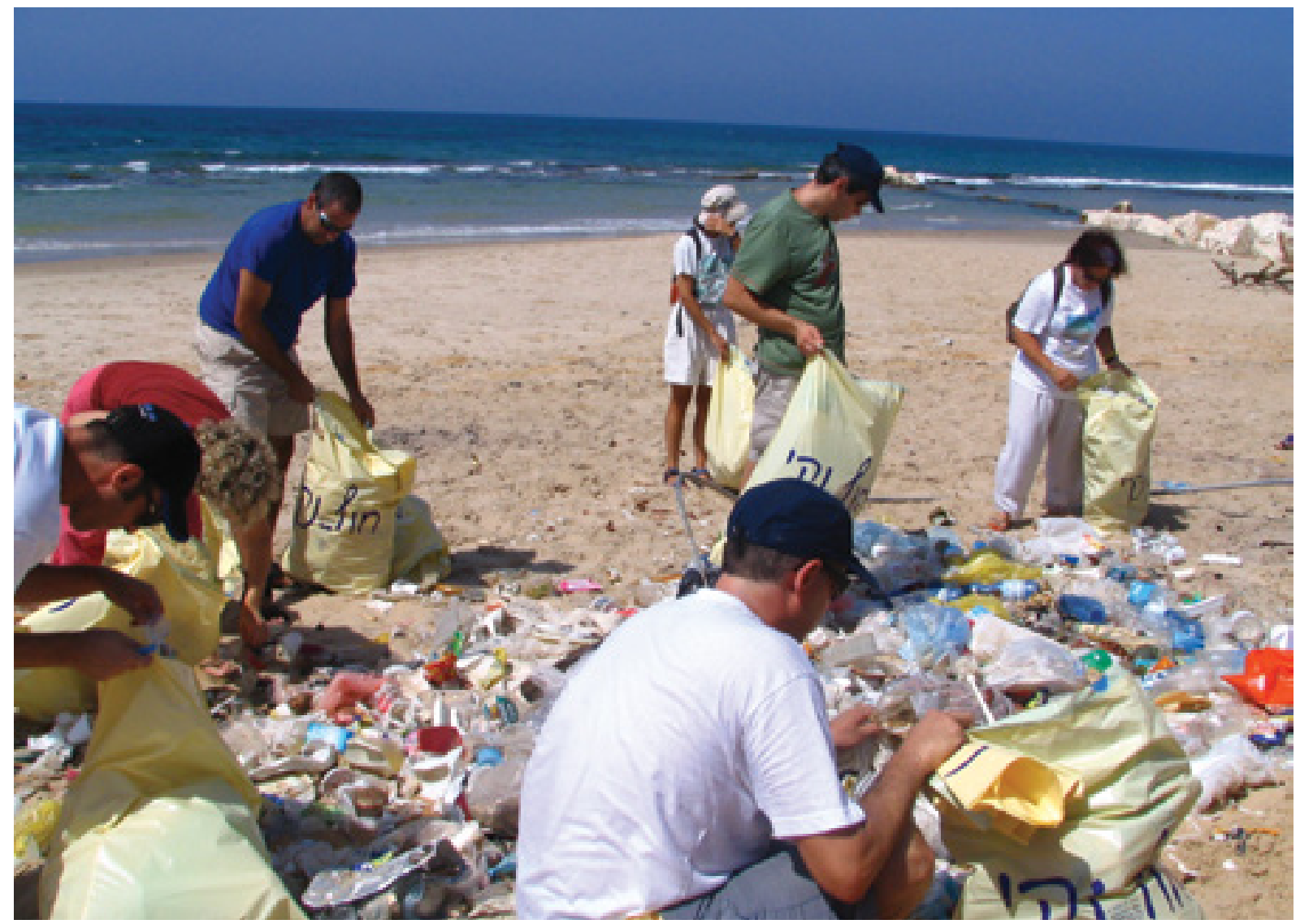

Coastal clean-up campaign/Photo: Ilan Malester

sources discharged to the Gulf of Eilat/Aqaba. In addition, nutrient loads have remained low and stable since the removal of the commercial fish cages from the north beach of Eilat in 2010.

\section{( Marine litter (SDG 14.1)}

\section{Reduction at source}

Israel puts great emphasis on combatting marine litter and has developed policies and programs to implement the Barcelona Convention's Regional Plan on Marine Litter Management under the Land-Based Sources Protocol.

The enactment of the Plastic Bags Law to reduce the use of single plastic bags has proved a major domestic development. The law came into effect on January 1st, 2017 and addresses the aboveaverage consumption of plastic bags in Israel. The law, which is aimed at motivating Israelis to use environmentally friendly reusable bags, has banned the distribution of "very thin" plastic bags (with a width of less than 20 microns) at major supermarket chains. In addition, these supermarkets must now charge a tax of at least NIS 0.10 lapproximately 2.5 cents) for bags with a width between 20-50 microns. Supermarkets are required to submit quarterly reports detailing the number of bags sold. The money paid for plastic bag purchase is transferred to the Maintenance of Cleanliness Fund of the Ministry of Environmental Protection (MoEP) to be used for such environmental projects as advancing waste treatment and recycling, preventing air pollution, and raising public awareness. Results of the first years of enforcement of the law indicate a very effective outcome. There has been a reduction of approximately $80 \%$ in the number of plastic bags purchased in major supermarket chains and a reduction of approximately $50 \%$ in the economy-wide use of these plastic bags. In the early stages of implementation of the law, a 
Figure 14.4

\section{Percentage of beaches measured "clean" $70 \%$ of the time}

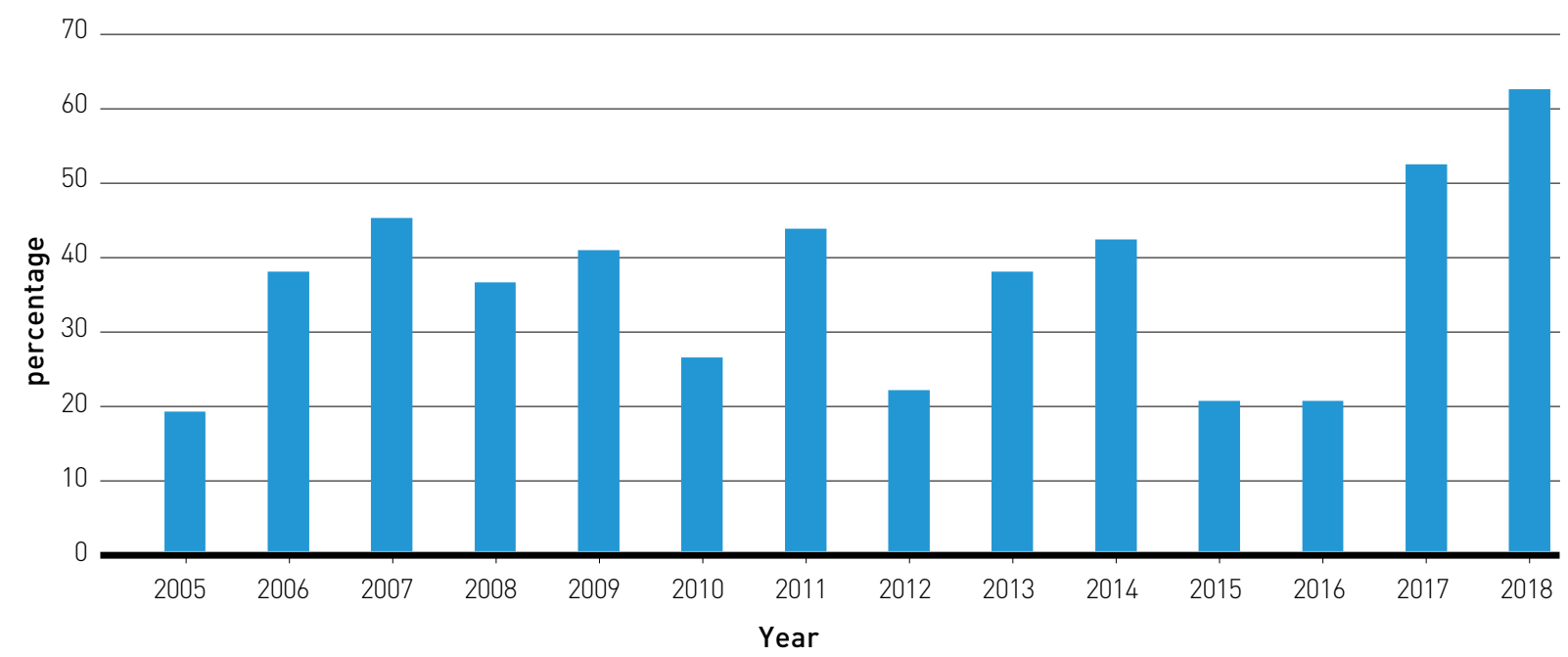

national public awareness campaign was launched, and free reusable bags were distributed to every household. The current goal is to reduce the use of plastic bags from 325 per person per year to 65 bags by 2031 .

UN Environment's \#CleanSeas campaign aims to eliminate single-use plastic by 2023. Israel has made a pledge to the \#CleanSeas campaign to have $70 \%$ of its beaches clean $70 \%$ of the time, and to continue examining ways to further reduce plastic bag use.

\section{Clean Coast Program}

One of the most successful programs currently in force to reduce marine litter in Israel is the Clean Coast Program, which includes such components as financial aid to local authorities, inspection based on a Clean Coast Index which ranks coastal cleanliness on a scale ranging from "extremely dirty" to "very clean," educational activities in schools, and information and publicity campaigns.

\section{( Marine monitoring (SDG 14.1, 14.2 and 14.3)}

A national marine monitoring program has been in effect in Israeli Mediterranean waters since 1978, focused mainly on marine pollution parameters. Marine and coastal monitoring is required under the Barcelona Convention as part of its Ecosystem Approach (ECAP). For the past ten years Israel has contributed to the formulation and establishment of the Integrated Monitoring and Assessment Program (IMAP) of the UN Environment Program - Mediterranean Action Plan (UNEP/MAP). By adopting IMAP, Mediterranean countries committed to monitor and report on 23 common indicators, covering topics related to pollution, marine litter, biodiversity, non-indigenous species, coast and hydrography. Mediterranean monitoring is carried out by the Israel Oceanographic \& Limnological Research (IOLR).

Since the long-term viability of effective monitoring must be guaranteed, in December 2018, a Government Decision (4399) on a National Monitoring Program for Israeli Mediterranean Waters was adopted in line with IMAP. Israel has been commended by the Barcelona Convention Secretariat as the first contracting party to submit a monitoring program to the Barcelona Convention which incorporates the principles of joint monitoring under IMAP and which serves as a best practice to be followed in the Mediterranean region.

The Israeli program for marine litter monitoring, which is a part of the overall IMAP-based national 


\section{CASE STUDY}

\section{The Clean Coast Program}

Marine litter constitutes an aesthetic, health, and safety hazard to the public and to marine and coastal animals. Some eight million tons of plastic pollution is dumped in the seas every year, choking coral reefs and threatening marine wildlife. About $90 \%$ of the marine litter in the seas off Israel is plastic; most of it is not biodegradable.

In response, the Israeli Ministry of Environmental Protection (MoEP) developed a Clean Coast Program and Index in 2005, aimed to evaluate cleanliness at unauthorized beaches (where swimming is forbidden), spanning $155 \mathrm{~km}$ of Israel's $190 \mathrm{~km}$ shoreline. Financial incentives are provided to local authorities in whose municipal boundaries these beaches are located. NIS 8.6 million (over $\$ 2$ million) has been allocated to local authorities this year, triple the previous budget. The Clean Coast Program is carried out together with the Israel Nature and Parks Authority (INPA) and with the involvement of other stakeholders and has cooperated, among others, with the Blue Flag Eco-Label and local NGOs such as EcoOcean and the Israel Society for the Protection of Nature, which provides assistance in monitoring marine plastic. In addition, twelve local authorities are taking part in a pilot project, which strengthens enforcement even further through the involvement of police officers and coastal authority officials.

The most recent data shows that beaches across Israel were cleaner in 2018 than they have been since monitoring began. $65 \%$ of beaches were ranked as "clean" or "very clean" at least $70 \%$ of the time, surpassing the success of previous years. In 2017 , almost $55 \%$ of beaches were defined as "clean" or "very clean" as opposed to around $20 \%$ prior to the initiation of the Clean Coast Program in 2005. The current program target calls for $70 \%$ of the beaches to be ranked as "clean" or "very clean" at least $70 \%$ of the time.

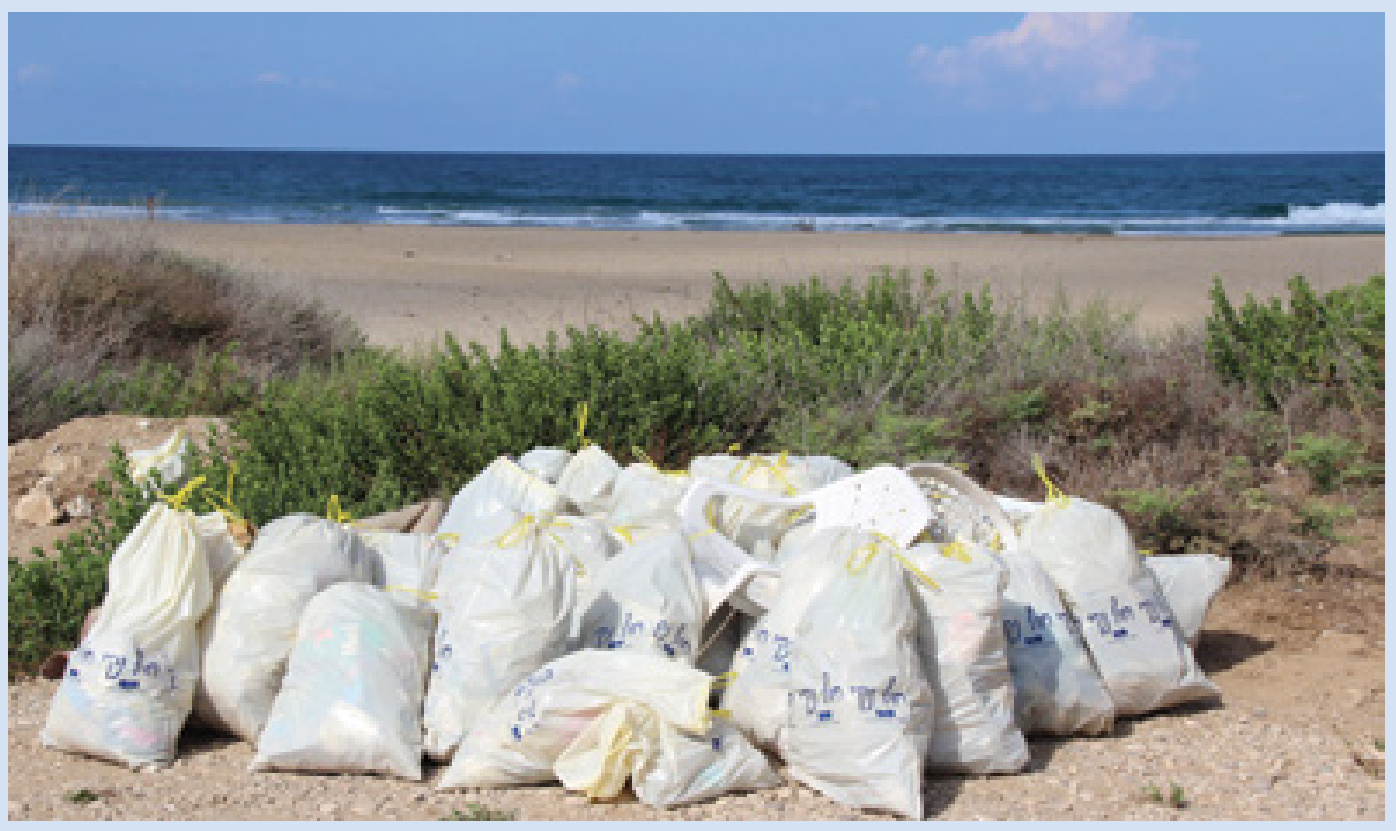

Cleaning Israel's coastline/Photo: Ilan Malester 
program, includes beach litter monitoring, floating stream litter monitoring, seafloor litter monitoring. and micro-litter monitoring of surface water and of sediments.

Previous marine litter studies have indicated that a citizen-science component is highly effective in raising public awareness of marine litter and marine monitoring in general. Therefore, Israel's marine litter monitoring work is carried out by both scientists and volunteers - divers from "Mishmar Hayam" ("Sea Guard" - a group of volunteer divers) and students from the School of Marine Sciences at Ruppin Academic Center. The citizen-science component was developed as part of a UNEPfunded pilot project titled "Adopt a Beach."

An additional national monitoring program, funded by the MoEP, is implemented in the Gulf of Eilat/Aqaba and is carried out by the Interuniversity Institute in Eilat. It monitors environmental and biological parameters in open waters, the coral reserve and the sandy habitats in the Gulf of Eilat/Aqaba.

\section{( Oil spill incidents and response (14.1)}

Israel is a signatory to the International Convention for the Prevention of Pollution from Ships (MARPOL) and the International Convention on Oil Pollution Preparedness, Response and Co-operation (OPRC) on which Israel's national legislation is based, including its National Contingency Plan (NCP) for Preparedness and Response to Incidents of Marine Oil Pollution.

The NCP is based on a three-tier response system for local, regional and national actions depending on the severity of the spill, and was approved by the government in 2008. It covers prevention, reduction of environmental and economic damage in the event of an oil spill, and necessary rehabilitation. Cleanup costs are imposed on polluters in accordance with the "polluter pays" principle. The plan provides an organizational structure, authority and framework of command for the various entities involved in oil spill response and allows for the efficient use of measures in emergency situations involving up to 4,000 tons of spill.
Under the auspices of one of the bodies of the Barcelona Convention and in light of increased hydrocarbon exploration activities in the Eastern Mediterranean, a Sub-regional Contingency Plan for preparedness and response to major hydrocarbon marine pollution incidents was formulated within the framework of trilateral relations with Greece and Cyprus. An Implementation Agreement on the Sub-regional Contingency Plan was signed in May 2018, providing for periodical joint training and joint exercises and enhancing mutual assistance if needed.

Increased awareness coupled with strict enforcement has led to a notable decrease in the number of oil spills over the past decade. Israeli ports have reception facilities for oily bilge and ballast waters. The MoEP and the Port State Control of the Ministry of Transport carry out regular inspections of ships calling at Israeli ports, according to the MARPOL Convention's provisions.

Deterrence is highly effective as all pollution cases are investigated and, where appropriate, prosecuted and substantial fines imposed (Figures 14.5, 14.6).

\section{Sustainable management and protection of ecosystems (SDG 14.2)}

The competition for resources and the burden on the sea are constantly on the increase. In light of technological developments and the need to explore and utilize new resources, extensive economic activity takes place in Israel's territorial and economic waters, including deep-sea gas drilling, laying of communication and energy lines between Israel and southern Europe, mariculture, large desalination plants along the coast, construction of larger and deeper seaports, and increasing leisure and tourism activities. In addition, there are proposals for marine renewable energy infrastructure and artificial islands. These activities put intense pressure on marine ecosystems. In particular, the exploration and exploitation of deep sea gas has given further impetus to the need to assess the vulnerability and sustainable utilization of the Mediterranean Sea and its coastal areas. 
Figure 14.5

MARPOL inspections (annex I, IV, V) in Israel, 2011-2018

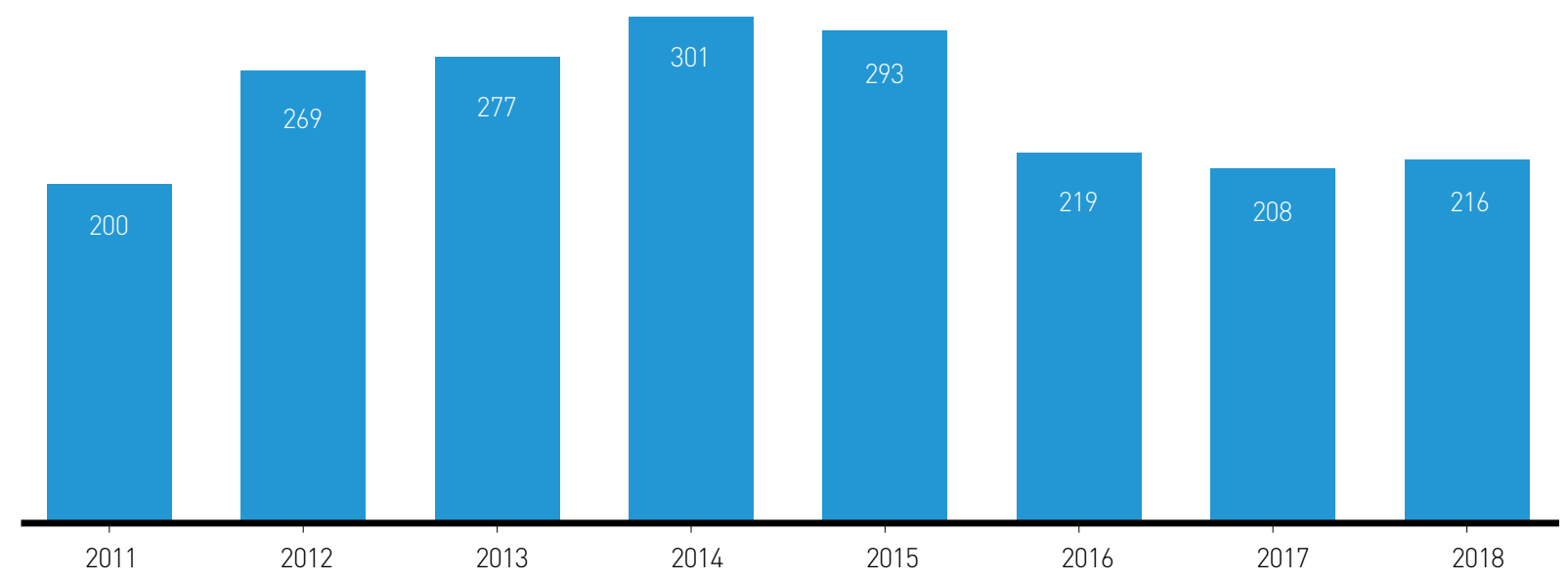

Figure 14.6

Criminal investigations of MARPOL (annex I, IV, V) violations in Israel, 2011-2018

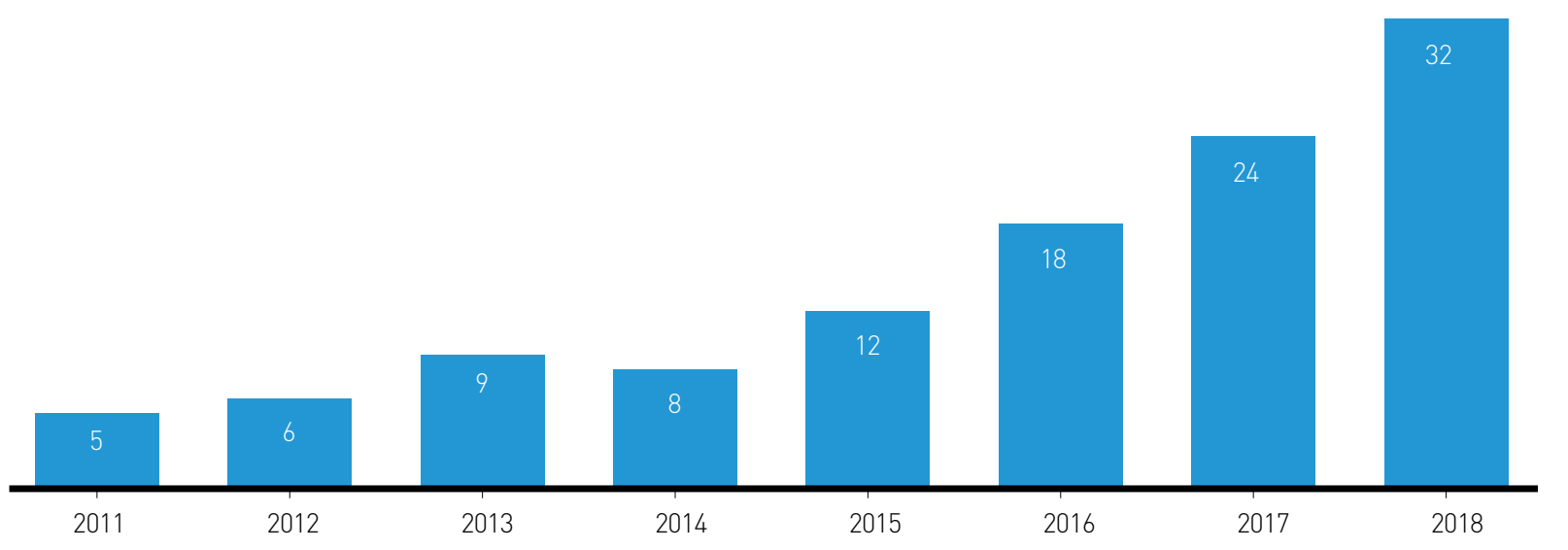

A Strategic Environmental Survey for the Exploration and Production of Oil and Natural Gas at Sea was prepared by the Ministry of Energy as part of its policy assessment on granting permits for natural gas and oil exploration. The survey was intended to inform decision-making processes on the development of resources. The final report contains maps of habitats and environmentally sensitive areas and presents a variety of alternatives for developing offshore oil and gas resources. It includes an in-depth discussion of the potential environmental impacts of implementing each of the development alternatives. The report also provides recommendations for implementing its conclusions, identifying information gaps and ways to improve the knowledge base, and establishing indices for monitoring the recommendations and their Implementation.

The Gulf of Eilat/Aqaba is a unique body of water, hosting some of the most productive and diverse coral reefs in the world. This $160-\mathrm{km}$ long section of the Red Sea (of which Israel has $14 \mathrm{~km}$ of coast) is a biodiversity hotspot and, as mentioned, is considered a coral reef refuge from global warming as its corals have a unique high tolerance to temperature fluctuations. The INPA has prepared a Policy Paper for Conservation and Sustainable Development in the Gulf of Eilat, aimed at putting the high ecological value and uniqueness of the 


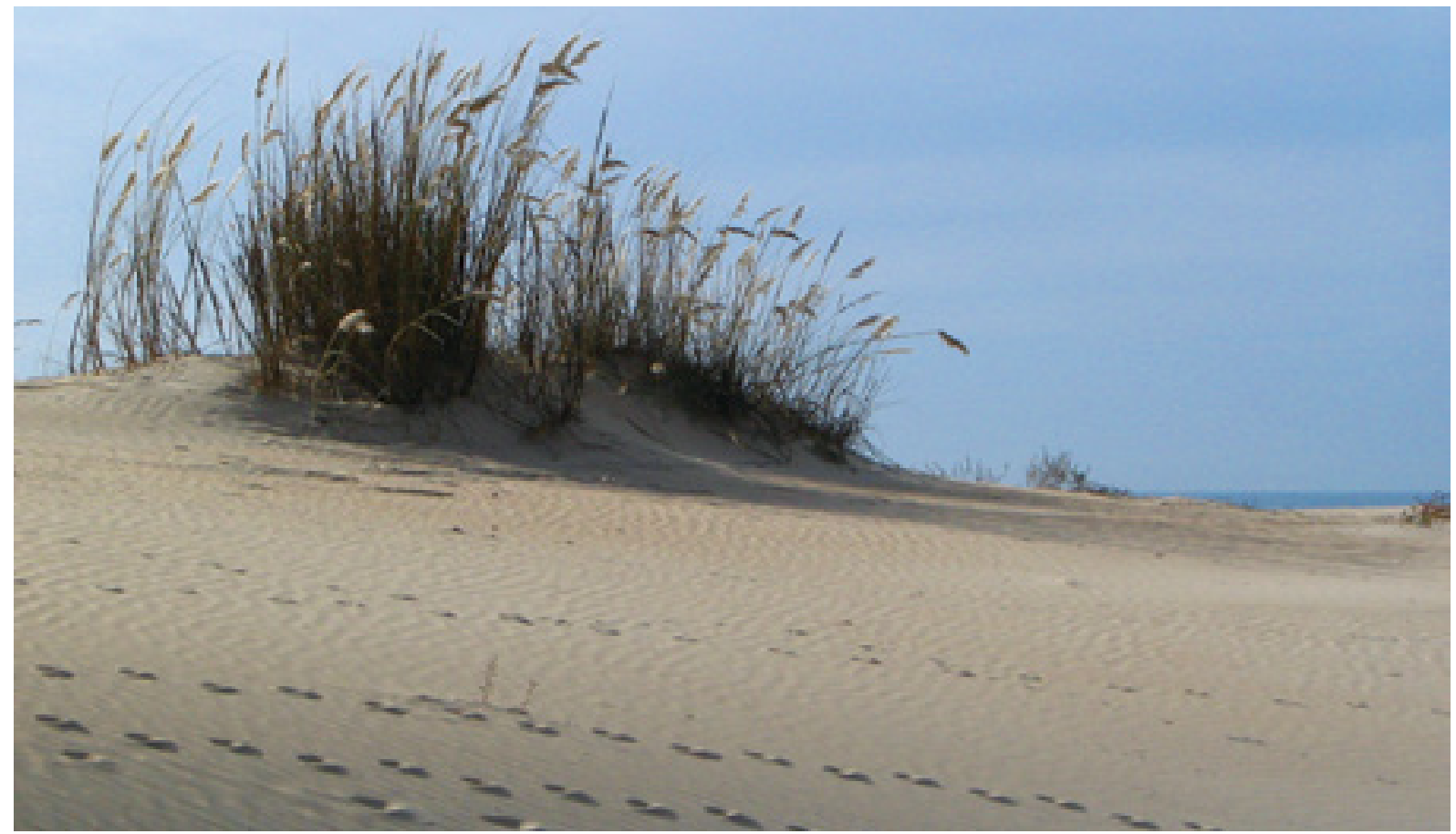

Michmoret beach/Photo: Ilan malester

Gulf at the basis of any planning activity in the area. The document includes guidelines on conservation as well as recommendations on the expansion of nature reserves and protected areas in the Gulf.

\section{Marine spatial planning}

An integrated marine spatial planning policy for the Mediterranean Sea is under preparation in a multi-stakeholder, interdisciplinary process. The principles of the policy document relate to the planning and management of the marine space, while taking into account the dynamism of the marine environment. It is aimed to maximize the economic potential in the area while preserving its natural, landscape, ecological richness and heritage values. The document will serve the following purposes:

- Formulation of planning policy for the maritime area of the Mediterranean Sea;

- Establishment of a national database on environmental, technological, engineering and legal issues relating to the maritime waters;

- Creation of tools for integrated management, coordination between stakeholders and encouragement of cooperation between them.
The document defines environmental principles for development, such as large-scale marine protected areas and limitation of development and activities (e.g., fishing) in these areas; principles for managing sand resources; principles for prevention and treatment of marine pollution; and environmental principles guiding the exploration and exploitation of hydrocarbons.

\section{Planning and building}

At the specific building and planning level, National Master Plan 1 is designed to consolidate many disparate physical plans. While the master plan has not yet received statutory approval, it addresses several issues, including preservation of the coast (the "waterfront"), and it incorporates the existing prohibition on building within 100 meters of the coastline lbuilding is permitted only for specific purposes related to certain marine recreational purposes and marine education, while giving due regard to local natural assets). Further limitations apply to coastal areas in rural areas.

\section{Marine Areas Bill}

The Marine Areas Bill, currently in debate in the 
Knesset (Israel parliament), will apply both to Israel's territorial waters adjacent to the shores of Israel as well as to Israel's Exclusive Economic Zone (EEZ). Israel's domestic Planning and Building Law will not apply in the EEZ, but the bill requires the preparation of a long-term policy document that will include, among others, the principles, objectives and priorities regarding activities and uses in the EEZ.

Environmental considerations are an integral part of the approval process for oil and gas operations in the EEZ. Furthermore, an initial environmental impact assessment must be carried out in accordance with environmental guidelines set by the MoEP. Environmental terms and conditions, determined by the preliminary documents, are then incorporated in the licenses for oil and gas exploration and exploitation activities issued by the Ministry of Energy. Any derogation from the guidelines or the conditions requires special justification and must be publicized.

\section{Marine invasive alien species}

Marine invasive alien species are a major challenge as the Mediterranean Sea is the most invaded marine basin in the world. More than 800 multicellular non-indigenous species have been recorded in the Mediterranean Sea, far more than in other European seas, because of the everincreasing number of Red Sea species introduced through the Suez Canal. 30 non-indigenous species new to the Israeli coast were recorded in the past three years alone. This highlights the role of the southern Levant as a "hotspot" and a beachhead and dispersal hub for their secondary spread. Some of the introduced species cause serious environmental and economic damages, whereas others, poisonous and venomous, can harm human health. It will be necessary to identify appropriate science-based management solutions to deal with this challenge in the coming years.

\section{"Red-Dead Canal"}

Due to growing use of water in the Jordan basin and due to climate change, water entries to the Dead
Sea have declined. In addition, there has been an increase in the utilization of Dead Sea water by the Israeli and Jordanian Dead Sea Works. The level of the Dead Sea decreases at a rate of about 1.2 meters per year.

Israel and Jordan initiated the Red Sea-Dead Sea project ("Red-Dead Canal”) as a joint regional project to halt the decline of the Dead Sea level and to desalinate water. The project includes the annual flow of 2 billion $\mathrm{m}^{3}$ of water from the Red Sea to the Dead Sea through pipes, the desalination of 850 million $\mathrm{m}^{3}$ /year (mainly for Jordanian use) and the construction of hydroelectric power stations to exploit the altitude differences between the Red Sea and the Dead Sea. Realization of the project will lead to the release of some 1.15 billion $\mathrm{m}^{3} /$ year of brine concentrate into the Dead Sea.

A World Bank Feasibility Study examined the impact of the proposed project on the Red Sea, the Arava and the Dead Sea. The study concluded that there is environmental, technical and economic feasibility for the project.

In light of the environmental sensitivity of the project - due to the ecological value of the Red Sea and its coral reefs, the transfer of seawater and concentrate over water aquifers and the release of foreign water into the Dead Sea - work has been and is being carried out to examine the impact on the environment.

For the sake of caution, it was agreed to implement the project in stages, with a limited scope first phase which will be used to examine the environmental, technical and social aspects of the complete project. The first phase includes the discharge of up to 235 million $\mathrm{m}^{3} /$ year of brine concentrate and Red Sea water to the Dead Sea, which constitutes a reduction of about one-third of the annual decline in the water level. Additional solutions for a complete halt to the decreasing water level and future rehabilitation of the Dead Sea will be examined later.

Throughout the project period, the Israel Geological Survey will conduct ongoing monitoring of the Dead Sea waters, including the Gulf of Lisan, where the main mixing of brine with the Dead Sea salt will 


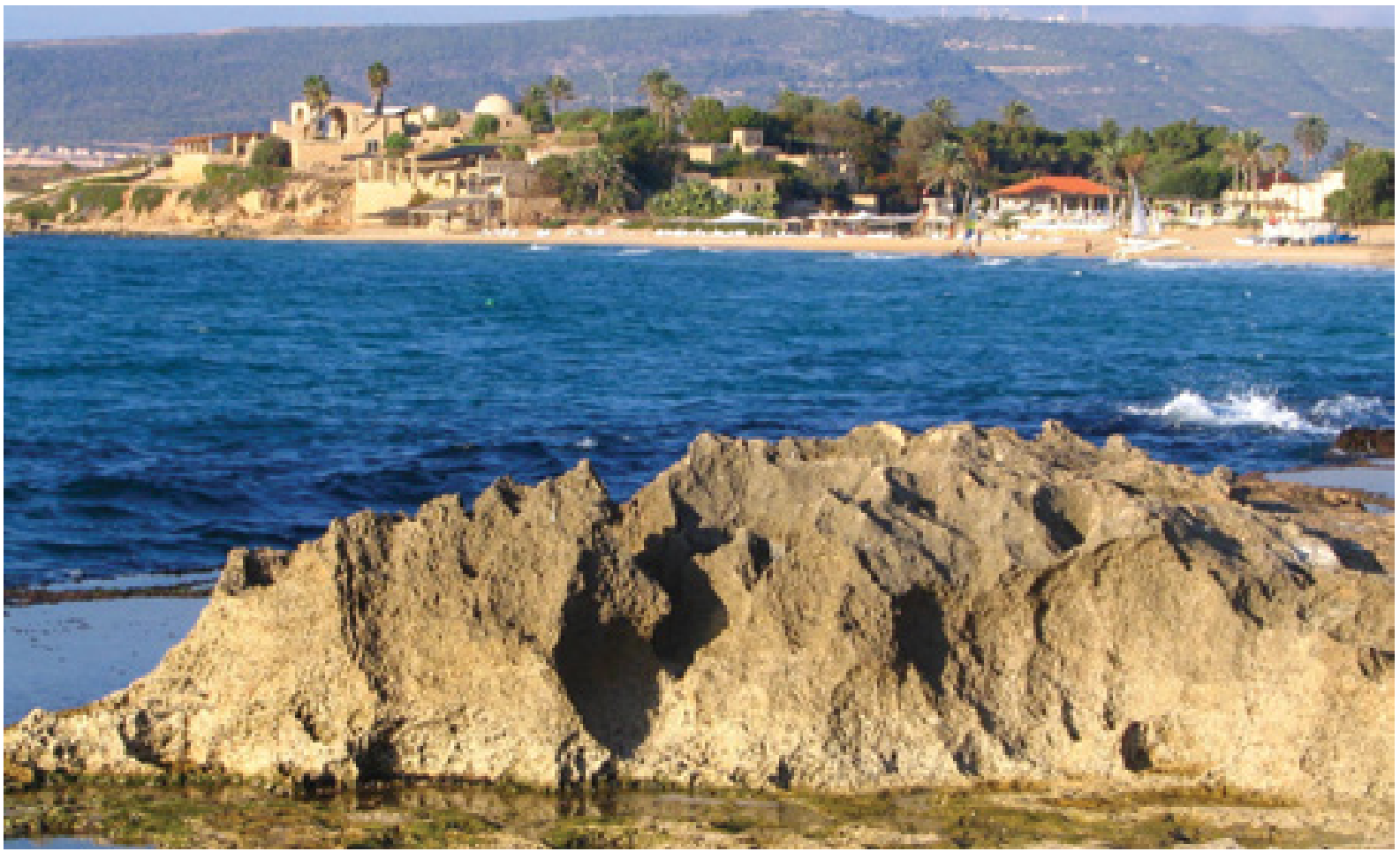

Achziv coastline/Photo: Ilan Malester

take place. The Interuniversity Institute in Eilat will supervise the ecosystem and quality of the Red Sea and the Hydrological Service of the Water Authority will monitor the quality of the groundwater along the route.

It should be noted that the tender documents will include the possibility of canceling the project and compensating the concessionaire and the financing if it is found that the project causes heavy environmental damage.

\section{( Regulation of marine harvesting (SDG 14.4)}

Israel's Fishing Regulations significantly changed in late 2016 and now restrict fishing activities with regard to permitted areas, methods, periods and species. In addition, in May 2018, responsibility for controlling fishing activities moved from the Ministry of Agriculture to the INPA. In addition, the rehabilitation of marine ecology by creating artificial reefs whose habitats favor endangered species is underway.

The Marine Spatial Planning Policy, currently being formulated, adopts the Ecosystem Based Fishery
Management principle and takes a precautionary approach. The policy aims at ensuring the future existence of the fishing industry while reducing risks to the marine ecosystem resulting from fishing activities.

\section{( Conservation of coastal and marine areas (SDG 14.5)}

Marine and coastal protected areas are declared and benefit from statutory management as part of a lengthy multi-tiered planning process prior to approval. Currently $0.3 \%$ of territorial waters are fully protected and managed, and an additional $5.6 \%$ are at various stages of approval and relate to three locations: Rosh Hanikra in the North; Rosh Carmel near Haifa; and Yam Evtach further south. It is anticipated that this substantial increase in fully protected areas will occur in the next few years.

Within the context of the Marine Spatial Planning Policy (under formulation), various uses of Israel's relatively limited marine and coastal areas have been assessed. The policy includes $8.5 \%$ of Israel's territorial waters as protected areas with the 
highest level of statutory declaration of protected areas and an additional 13\% as special areas with guidelines for protection. In the EEZ, a special area of $590 \mathrm{~km}^{2}$ has been designated, of which a few specific areas are protected areas, and the rest are areas with basic levels of protection and in which further investigation is needed.

\section{PARTNERING FOR A BETTER WORLD}

Israel is committed to the conservation and sustainable use of the oceans, seas, and marine resources, operating on the international, regional and national levels. Israel shares the view that there is a need for greater international cooperation, transfer of maritime technology, and capacitybuilding. Recently (2017), Israel, Greece, and Cyprus signed a Memorandum of Understanding (MOU) for mutual assistance in the event of an oil spill, and a contingency plan is being prepared with the help of the UN Environment Program (UNEP) MAP). (SDG 14.1)

While Israel is not party to the UN Convention of the Law of the Sea (UNCLOS), it considers the Convention as a primary source of guidance regarding rights and responsibilities of States on pertinent maritime issues. (SDG 14.c)

Israel's Ministry of Environmental Protection and Israel Oceanographic \& Limnological Research (IOLR) are cooperating on enhancing the National Monitoring Program of the Mediterranean Marine Environment to enable science-based decision and policymaking for all of Israel's marine anthropogenic activities, including land-based pollution, marine traffic, desalination, power plants, fishing, recreation and the growing oil and gas industry. In 2018, a Government Decision (4399) on a National Monitoring Program for Israeli Mediterranean Waters was adopted, in accordance with UNEP's Integrated Monitoring and Assessment Plan (IMAP), under the Barcelona Convention and climate change perspectives. According to UNEP/ MAP, Israel's national practice is at the forefront of IMAP implementation, and serves as an example of best practices for the Contracting Parties to the Barcelona Convention. (SDG 14.2)
Israel is a member state in the Intergovernmental Oceanographic Commission of the UN Educational, Scientific and Cultural Organization IIOC UNESCO), promoting international cooperation in research, services and capacity-building to improve management, sustainable development, and the protection of the marine environment. An Israeli representative of IOLR participated in the 25th Session of the International Oceanographic Data and Information Exchange (IODE) of the Commission (Tokyo, 2019). (SDG 14.2)

Professionals from developing countries participated (2018) in MASHAV's annual capacitybuilding course on Intensive Aquaculture Production, focusing on the implementation of science-based sustainable management plans to restore fish stocks, at least to levels that can produce maximum yields, ocean health, and the sustainable use of marine resources. (SDG 14.7)

In order to promote the protection of areas beyond Israel's territorial waters and its Exclusive Economic Zone, Israel is taking part in current UN negotiations on the protection of Biodiversity in Areas beyond National Jurisdiction (BBNJ). (SDG 14.C) 


\section{GOAL}

15

LIFE ON

LAND

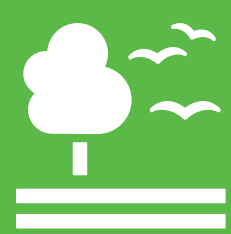

Conservation, restoration and sustainable use of terrestrial and inland freshwater ecosystems

Progress towards sustainable forest management

Degraded land

Red List Index

Illegal poaching and trade of wildlife

Prevention and control of invasive alien species

Strategic plan for biodiversity

Financial resources for the conservation and sustainable use of biodiversity and ecosystems

Partnering for a Better World 
PROTECT, RESTORE AND PROMOTE SUSTAINABLE USE OF TERRESTRIAL ECOSYSTEMS, SUSTAINABLY MANAGE FORESTS, COMBAT DESERTIFICATION, AND HALT AND REVERSE LAND DEGRADATION AND HALT BIODIVERSITY LOSS

The conservation of biodiversity in a country such as Israel is challenging. On the one hand, Israel's geographic location at the junction of three continents and at the crossroads of climatic and botanic regions has yielded a rich biodiversity at all levels - genetic, species, ecosystem and landscape. On the other hand, accelerated development and population growth, together with the effects of climate change, threaten this unique biodiversity through habitat loss, fragmentation and degradation, increase in invasive species, pollution, and overexploitation of resources. 
The most recent findings concerning the situation of Israel's biodiversity are reported in the Israel State of Nature 2018 report. The report is primarily based on findings of the National Terrestrial Biodiversity Monitoring Program run by HaMaarag (Israel's National Nature Assessment Program) and other sources such as the Ministry of Environmental Protection (MoEP), Israel Nature and Parks Authority (INPA), Keren KayemethLelsrael - Jewish National Fund (KKL-JNF) and academic researchers. The monitoring program is a continuous, spatial and temporal effort to evaluate the state of nature and to identify trends in key processes affecting Israel's natural environment. The 2018 report along with monitoring reports highlights the continuing trend of the conversion of open landscapes into other land uses and lists various threats to the country's diverse ecosystems.

\section{Among the report's findings:}

- There has been an ongoing trend over the past nine years to convert open landscapes into other land uses. Most of this is occurring in the more populated areas in the center of the country, in the Mediterranean ecoregion;

- The level of habitat fragmentation is extremely high in the center and north of Israel, whereas the south still contains relatively large and continuous open landscapes;

- Woody vegetation is the backbone of most of Israel's ecosystems. Climate changes and anthropogenic developments that could potentially cause a decrease in woody plants may result in severe consequences for many ecosystems;

- Proximity to housing, agriculture, infrastructure, and military bases has profound effects on bird and mammal communities. Over the past 15 years, the status of the resident bird species has declined and $30 \%$ are currently threatened;

- A serious lack in ecological and biological knowledge is apparent in freshwater habitats in Israel. This necessitates the establishment of additional research and the development of a monitoring program that will determine their status.
Positive findings on biodiversity status were described in the Fifth National Report (2016) on the implementation of the Convention on Biological Diversity (CBD) in Israel. Among others, the report indicates that the state of the coral reefs in the Gulf of Eilat has improved in terms of cover and density, but recruitment is still low. Other environmental pressures, such as sea pollution, have remained steady or have decreased over the last decade.

\section{Conservation, restoration and sustainable use of terrestrial and inland freshwater ecosystems (SDG 15.1)}

The proportion of Israel's protected areas has increased significantly in recent years. Approximately $60 \mathrm{~km}^{2}$ of land were declared nature reserves and national parks in 2017-2018, compared to $8.1 \mathrm{~km}^{2}$ in 2016 and only $0.21 \mathrm{~km}^{2}$ in 2015. The protected areas include not only terrestrial ecosystems but also streams and other threatened aquatic habitats. Declared protected areas are one of the most effective regulatory tools for protecting Israel's ecosystems and biodiversity. Following an official declaration of a protected site, the INPA has the legal power to control human activities, act against violators and take additional actions to safeguard biodiversity. At present, there are approximately $5,060 \mathrm{~km}^{2}$ of declared protected areas in Israel, representing $20 \%$ of Israel's land under INPA jurisdiction. The goal is to reach $30 \%$ protected areas by 2030 . Out of Israel's 23 ecosystems, $30 \%$ are still underrepresented in protected areas, and the goal is to reach at least $17 \%$ representation by 2030 .

In addition, 9\% of Israel's land area is designated forest (natural and planted), managed by the KKLJNF, which provides forest services pursuant to a covenant signed with Government of Israel in 1961.

\section{Progress towards sustainable forest management (SDG 15.2)}

In 2014, KKL-JNF introduced a new Forest Management Policy (FMP) for the forests it 
manages in Israel. The FMP states that the main purpose of forests in Israel is to supply ecosystem services for the well-being of all citizens and the environment. It furnishes an obligatory professional basis for managing Israel's forests in a goaloriented and sustainable fashion and serves as the foundation for particular and ecosystem-specific forest management master plans that are tailored for each forest and reflect a holistic and sustainable approach. These master plans, combined with a national scale survey, will serve as a foundation for long-term strategic forest management plans.

\section{( Degraded land (SDG 15.3)}

Israel's 2018 State of Nature report highlights the continuing degradation of the country's natural landscapes due to the growing proportion of conversion of natural areas into other land uses. Currently, the average rate of conversion of open landscapes to artificial land cover for housing and infrastructure has reached an all-time high rate of over $18 \mathrm{~km}^{2}$ per annum, compared to the
2016 report which shows a rate of about 10-13 $\mathrm{km}^{2}$ per annum. Most of this is occurring in the more populated areas in the center of the country. Furthermore, during these years, approximately $17 \mathrm{~km}^{2}$ were converted from forested land to agricultural land.

Land conversion is mainly due to the rapid increase in housing development projects due to the high population growth in Israel, which is currently about $2 \%$ a year. Based on a government decision on planning future housing infrastructure, the Government of Israel published a new Housing Strategy in 2017 aimed at developing 1.5 million housing units by 2040 and planning an additional 1.1 million housing units. Taking this strategy into account, natural landscapes will continue to be degraded in the coming years. Nevertheless, the government has recognized the need to minimize the degradation of open landscapes and advised the MoEP to work with the Planning Authority towards finding the right balance between the new housing strategies and providing the necessary tools for

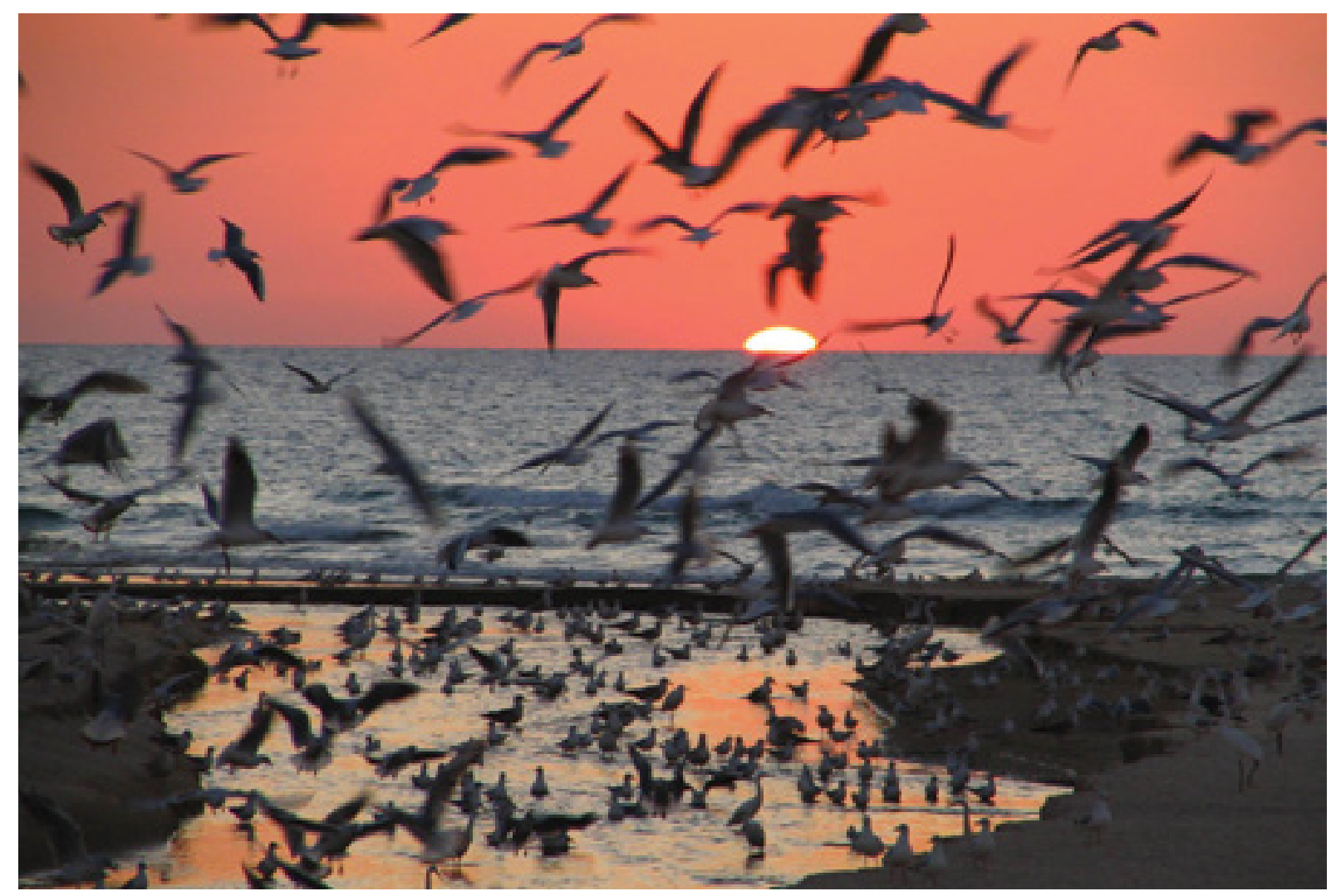

Sunset at Ma'agan Michael/ Photo: Ilan Malester 
protecting open landscapes and biodiversity. To this end, an Action Plan for the Protection of Biodiversity and Open Spaces is being developed. The "Healthy Natural Environment" plan will accord with the CBD Aichi targets.

Restoration and rehabilitation plans are also being implemented in degraded ecosystems, such as polluted rivers and quarries.

\section{( Red List Index (SDG 15.5)}

Israel's nationally produced periodic Red Books are prepared by the INPA with input from local experts using IUCN Red List standards. These national red lists show that many species have an unfavorable conservation status demonstrating ongoing declines. The Red Number for plants was set according to six criteria of vulnerability and importance for conservation and the Red Book was updated in 2011. The Red Book for vertebrates was last published in 2003 and is currently being updated.

- Plants: Out of 2,391 plant species that have been identified in Israel, 414 (17\%) are listed in the Red Book (2011) as endangered, and out of this number 28 species are in all probability extinct by now. Only 67 of the endangered species are protected by law, meaning that most of the endangered species do not have sufficient official protection.

- Vertebrates: Until 2017, 452 species of vertebrates were identified in Israel, of which 28 species are locally extinct (four of the species have been reintroduced back into the wild), and six species have become globally extinct. Of the remaining species, 153 species are endangered, representing $37 \%$ of terrestrial vertebrates, $23 \%$ of bird species, $23 \%$ of freshwater fish species, $35 \%$ of reptiles, $60 \%$ of mammals and $82 \%$ of amphibians. Specifically:

- Birds: Almost all bird species in Israel are protected (except for a few agricultural pests). As of 2017, the list of endangered birds nesting in Israel comprises 213 species. 21 species are critically endangered, 13 species are endangered, and 31 species are vulnerable, making a total of 65 species compared to 39 species in 2002. The 2017 update shows that the situation of 21 species has improved compared to a decline in the situation of 37 species.

- Amphibians and reptiles: All species of amphibians and reptiles are fully protected under law. Recently, an endemic amphibian species thought to be extinct has been rediscovered in the Hula Valley.

- Mammals: Almost all mammal species in Israel are protected lexcept for a few agricultural pests). There are 33 species of bats in Israel, most of which are endangered. Israel Joined the EuroBATS Agreement in 2014.

The current main threat for extinction of vertebrate species is loss of habitat. The increase in protected areas is considered the best way to prevent extinction generally. In addition, the INPA has in-situ and ex-situ conservation programs for many endangered species of animals and plants. The goal is to greatly expand the list of protected species, especially plants and fish, by 2020 and to include all endemic plants. Work is currently being carried out on a new list that will include over 650 protected species.

Reintroduction and conservation programs for endangered species have met with some success and include successful reintroduction of the Persian fallow deer, Asiatic wild ass, White oryx, Griffon vulture, Egyptian vulture and the Ferruginous duck. A new strategic plan for in-situ conservation of Red List plant species in sanctuaries is being implemented. A seed collection of most of Israel's wild plants is maintained in the Israel Gene Bank.

\section{I lllegal poaching and trade of wildlife (SDG 15.7)}

The INPA is the government body in charge of enforcing poaching and illegal wildlife trade regulations. Hunting is not a popular sport in Israel. There are only a few hundred licensed hunters and only a few migratory bird species that are legal game. The aim is to totally outlaw sport hunting by 2030. Illegal shooting of partridges and ungulates Igazelles and ibex) occurs occasionally but is not 


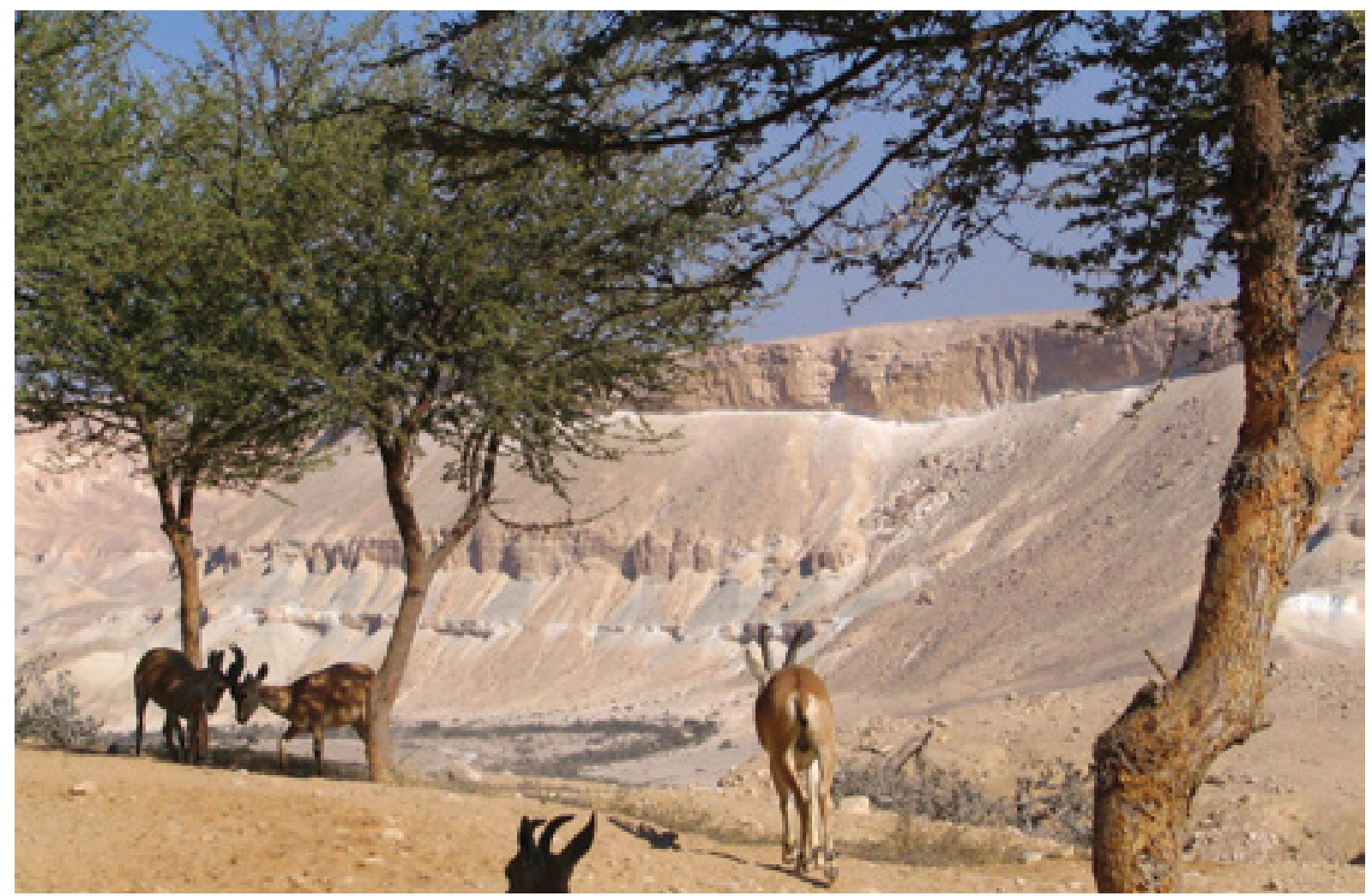

Ibex at Sde Boker/Photo: Ilan Malester

thought to have a major impact on wild populations. However, snare traps, set by migrant farm workers, cause indiscriminate and widespread harm to many species, and occasional cases of illegal poisoning by disgruntled farmers are known. No domestic species of wildlife are traded legally but there are some isolated cases of illegal trafficking of protected species such as raptors, song birds (goldfinches) and sharks.

\section{( Prevention and control of invasive alien species (15.8)}

Invasive alien species are a major issue in Israel and the main challenges continue to be treating infected areas and preventing the entrance of new invasive species into the country. Currently, there are 167 identified invasive plant (terrestrial) species, 18 bird, 12 freshwater fish, 218 insect, 52 mollusk, one reptile and one mammal species in Israel.

In 2009, a law was passed by the Ministry of Agriculture preventing the import of certain invasive plant species to protect local agriculture. The law, however, does not prohibit the sale of plants already in the country.

While development of new legislation for the purpose of biodiversity protection is still required in Israel, implementation of various voluntary actions and joint collaborations is underway and demonstrates success in treating alien invasive species. Most of these actions are joint projects between the public and private sectors and NGOs.

The following are examples of measures taken to combat the invasion of alien species:

- Israel's Least Wanted Alien Ornamental Plant Species (2013) serves as a tool for landscape architects, gardeners, environmentalists, ecologists, foresters and the general public which provides information on invasive species and discourages their uses on a voluntary basis;

- An ongoing risk assessment program by the INPA prevents the import and sale to the public of any birds and reptiles that are assessed as carrying a risk of invasion.

- The government has prioritized the Ambrosia 
sp. and aims to treat infected aquatic habitats and prevent new invasions. The main focus of the initiative is on eradicating burr ragweed (Ambrosia confertiflora);

- An inter-ministerial roundtable committee was set up to deal with the problem of invasive species and to coordinate operations;

- Efforts to combat the invasion of alien species are being mainstreamed into the spatial planning sector via environmental impact assessments and into other government bodies and the private sector through the development of voluntary guidelines.

There is broad agreement on the need for new legislation on invasive species and a draft law has been prepared and is under review. The draft legislation is designed to close gaps in legislation and to provide a more robust platform for prevention and control of invasive species. The current projection is that this legislation will be enacted by 2025 .

\section{( Strategic plan for biodiversity (SDG 15.9)}

A central priority of the MoEP is to prepare a National Action Plan for the Protection of Biodiversity and Open Spaces. The plan will be prepared by 2020 and will focus on developing policy and economic tools for the purpose of minimizing the negative impact of the new National Housing Plan for 2040 and balancing the needs of future land development with the need to protect biodiversity, preserve endangered ecosystems, rehabilitate landscapes, enhance public awareness and implement ecological considerations in various sectors.

In recent years, the National Planning Administration, which is responsible for land use development, has placed greater focus on ecological issues and the incorporation of more sustainable planning measures. These include:

- Carrying out new country-wide mapping of essential ecological corridors and its mainstreaming into regional planning. Recognition of the negative impacts of fragmentation on open landscapes and natural resources makes it imperative to consider these effects when planning for future infrastructure development and for the expansion of communities;

- The Israel Planning Administration has started to develop strategic plans for various sectors such as industry and infrastructure, open spaces, and more. These plans will provide the Planning Administration with the additional information needed for improved planning and will facilitate the formation of strategies benefiting the management of open landscapes and natural resources in Israel;

- A tool currently being developed relates to the protection of agricultural land.

\section{( Financial resources for the conservation and sustainable use of biodiversity and ecosystems (SDG 15.a)}

Major progress in funding open space protection was achieved in 2012 with the establishment of the Open Spaces Protection Fund under the Israel Land Authority Law. Over $90 \%$ of the land in Israel is owned by the State and managed by the Israel Land Authority, and the law determines that $1 \%$ of the income from the sale of the land for housing will be allocated for projects aimed at protecting open spaces and nature, with an emphasis on spaces of importance to biodiversity protection and the protection of ecosystems in Israel.

Given Israel's projected population increase, land continues to be a highly valuable resource so that future income for the Fund is stable and likely to continue. Since 2013, over $\$ 150$ million have been distributed to more than 300 projects nationwide.

Israel is a highly urbanized society and over the past decade, urban nature sites have become increasingly important to safeguard the wellbeing of residents and protect nature. Local authorities are becoming more concerned about the importance of urban nature sites within their jurisdiction and are investing in the development 


\section{CASE STUDY}

\section{Israel's Open Spaces Protection Fund}

The establishment of the Open Spaces Protection Fund in 2012 marks a landmark in the implementation of economic tools for the conservation of biodiversity and sustainable development. The Fund and its activities are an integral part of Israel's progress towards the 2020 Aichi Biodiversity Targets. The Fund serves as an offset for land development projects and therefore balances incentives and subsidies harmful to biodiversity.

The main aims of the Fund are: rehabilitation, restoration and conservation of open spaces including development of recreational parks for public use; treatment and removal of environmental hazards and invasive species; restoration and rehabilitation of rivers and aquatic habitats and their surrounding areas; collection and processing of data; monitoring, research and spatial planning of open spaces to ensure sustainable development and land use.

Over 300 projects nationwide have been funded so far. Examples include: the Jordan River slopes restoration project; eradicating plant invasive species from the Judean Hills; and restoration of the Sorek and Kishon River estuaries. Israel believes that this economic tool, along with the relevant legislation, has been and will continue to be highly effective in restoring and rehabilitating open spaces and natural landscapes and in protecting biodiversity.

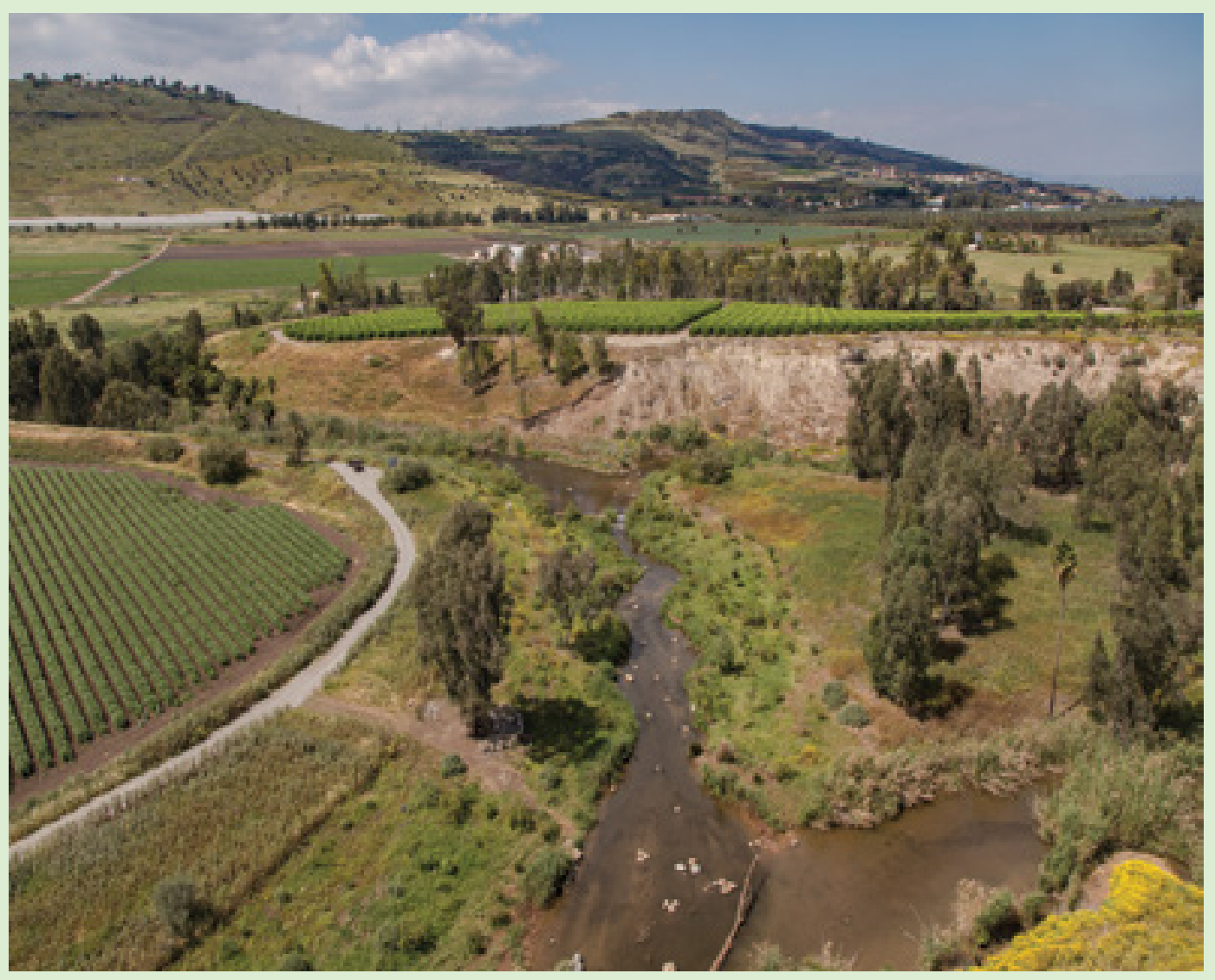

Jordan River Slopes Restoration Project/Photo: Albatross 
of urban nature sites. An estimated \$1.2 million were invested in conducting urban nature surveys in 50 municipalities nationwide. These surveys help identify key sites for conservation and rehabilitation. One of the goals of these actions is to increase the accessibility of the population to nature and its social and health benefits, especially for marginalized groups.

Israel views the business sector as a valuable partner in the effort to preserve and protect nature. Since 2014 , over $\$ 350,000$ have been allocated for the "Business and Biodiversity" project. The project is designated to create accessible and easy to use tools to encourage private companies to integrate nature protection values into their core business activity. It is believed that the new dialogue with the private sector can enhance conservation efforts and can mainstream ecological considerations into their long-term business management plans.

\section{PARTNERING FOR A BETTER WORLD}

Forests are known worldwide for their significant contribution to achieving the goals and targets set in the 2030 Agenda for Sustainable Development. Recently, the Food and Agriculture Organization (FAO) presented substantial evidence that this contribution goes way beyond SDG 15 , encompassing all 17 Goals, and acknowledging that forests and trees are critical to people's livelihood and well-being, adaptation and mitigation to climate change, water quality, sustainable cities, combating desertification and many other aspects of sustainability. (FAO, The State of the World's Forests 2018 - Forest Pathways to Sustainable Development).

Keren Kayemeth Lelsrael - Jewish National Fund (KKL-JNF) channels a specific annual budget of approximately \$650,000 towards international development cooperation in support of enhanced environmental quality, sustainable forest management and climate change resiliency. Capacity-building and rural development projects are carried out together with local stakeholders to increase the livelihoods of marginalized communities. (SDG 15.a)

MASHAV, in cooperation with relevant UN agencies, conducts capacity-building programs for professionals from the developing world on Combating Desertification and Drought, as well as Combating Desertification: Maintaining Soil Productivity, Preventing Land Degradation and Reclaiming Damaged Soils. (SDG 15.3)
The Drivers, Pressures, State, Impact and Response (DPSIR) Model, a causal framework for describing the interactions between society and the environment due to the interdependence of the components, is now more widely adopted by the European Environmental Agency. MASHAV's capacity-building course conducted (2018) on Environmental Management of Nature Parks: Focus on Ecosystem Services (2018) analyzes different management styles, the structure and importance of biodiversity in maintaining healthy ecosystems, and the functions of ecosystem services. (SDG 15.9)

The 6th International Drylands, Deserts, and Desertification Conference (2017) at Ben-Gurion University of the Negev, focusing on Combating Desertification and Dryland Management-Theory and Practice, was attended by stakeholders from more than 60 countries. Within the framework of the Conference, MASHAV held a special session on Enhancing Food Security, Adaptation to Climate Change and Combating Desertification, with the participation of approximately 100 professionals from least developed and developing countries attending four MASHAV courses in Israel. The Conference provided an opportunity for course participants to consider a wide range of theoretical and practical approaches and methods to combat desertification and live sustainably in the drylands.

\section{(SDG 15.3)}

Israel's Embassy in Angola organized a special event to celebrate World Day to Combat Desertification and the $60^{\text {th }}$ Anniversary of the establishment of 


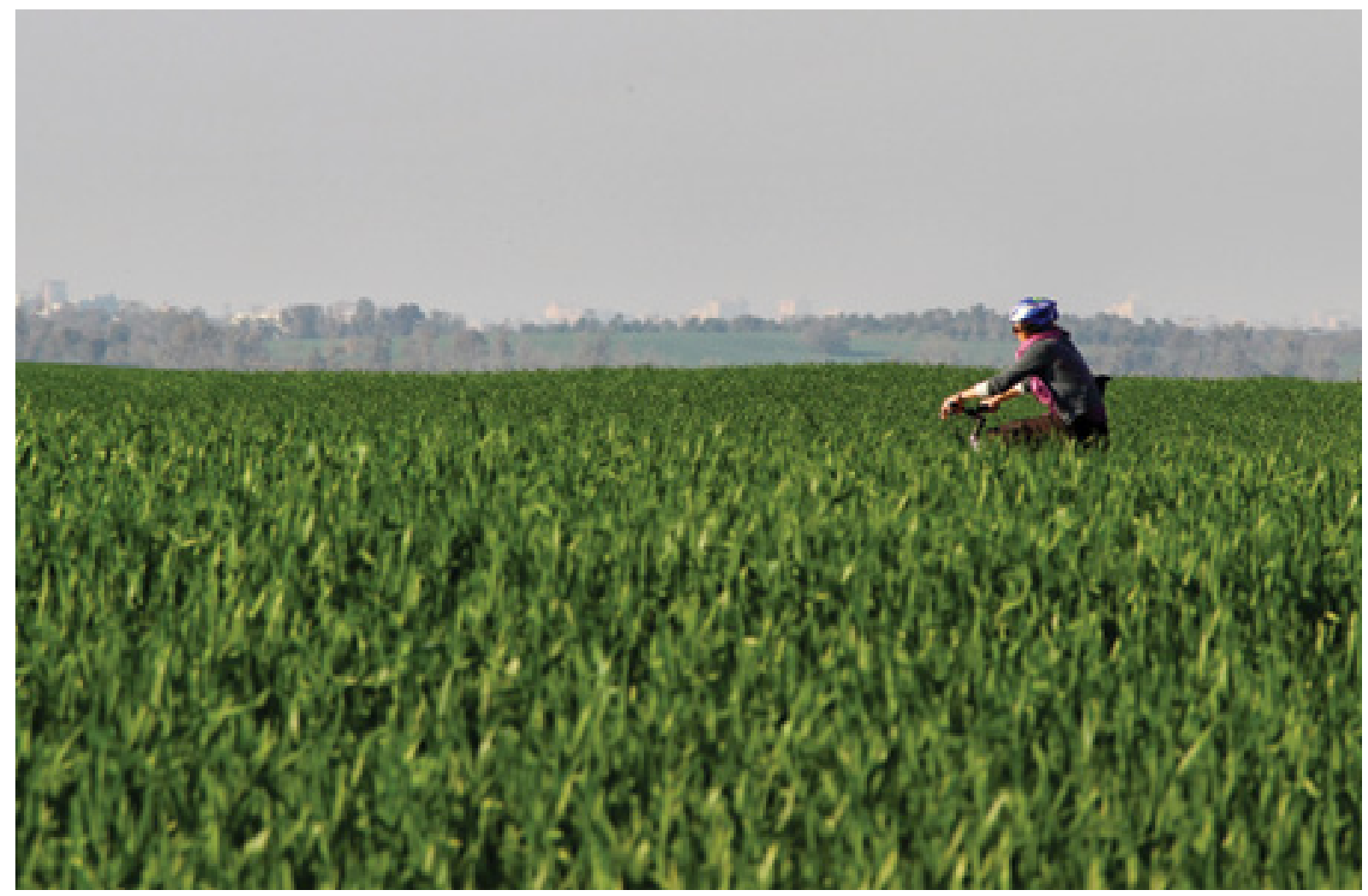

Cycling in southern Israel/Photo: Ilan Malester

MASHAV. Over 500 children from Tombwa City in Angola's Namibe Desert planted 1,000 trees to create a green wall between the desert and the city. (SDG 15.3)

Since 1997, Israel has been a Contracting Party to the Ramsar Convention on Wetlands of International Importance especially as Waterfowl Habitat. At the $12^{\text {th }}$ Meeting of the Conference of the Contracting Parties to the Ramsar Convention on Wetlands (COP12) in Uruguay, Israel's representative was awarded the Ramsar Wetland Conservation Award for her continuing work to bring to life the principles of wise use, and for demonstrating the importance of wetlands to local communities. Using Ramsar principles, she proved it possible to reverse the damage done to a key wetland area even when not managed effectively. (SDG 15.1)

Israel is a party and an active participant in meetings and negotiations and reports in accordance with its obligations to a number of biodiversity and ecological conventions and organizations, including: the Convention on Biological Diversity; Ramsar Convention; Convention on International
Trade in Endangered Species of Wild Fauna and Flora (CITES); Convention on the Conservation of Migratory Species of Wild Animals (CMS) and its various agreements; UN Convention to Combat Desertification (UNCCD); International Union for the Protection of New Varieties of Plants (UPOV); and the Intergovernmental Science-Policy Platform on Biodiversity and Ecosystem Services (IPBES). Israeli experts have been proposed and accepted to the various scientific bodies of these organizations. (SDG 15.5) 


\section{GOAL}

16

PEACE,

JUSTICE AND STRONG

INSTITUTIONS

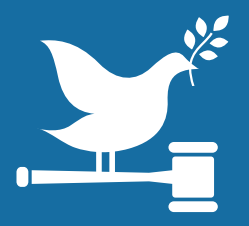

Crime prevention

Combating the exploitation of minors

Fighting trafficking

Promoting the law and ensuring equal access to justice for all

Educating youth to civic responsibility: The high school policing studies program

Combatting organized crime, serious crime and public corruption

Transparency

Multiculturalism in Israeli society

Improving service to the citizen

Capacity building, participation and decision-making at the local level

Freedom of Information Unit

Making information accessible to residents for the exercise of their rights

Partnering for a Better World 


\section{PROMOTE PEACEFUL AND} INCLUSIVE SOCIETIES FOR SUSTAINABLE DEVELOPMENT, PROVIDE ACCESS TO JUSTICE FOR ALL AND BUILD EFFECTIVE, ACCOUNTABLE AND INCLUSIVE INSTITUTIONS AT ALL LEVELS

The foundations of a democratic society are embedded in its ability to impart personal security to its citizens and to promote values of mutual partnership, the rule of law and public order. Israeli society is a multicultural society consisting of diverse religions and groups - some $75 \%$ are Israeli Jews of all backgrounds, $21 \%$ are Israeli Arabs of any religion other than Jewish, while the remaining nearly $5 \%$ are defined as others.

Israeli law is mainly based on common law, but it incorporates facets of civil law as well due to its rich history, influenced by varied systems of law. The Basic Laws of Israel are believed to function as the country's constitutional laws; however, Israel does not have a formal constitution.

The Israeli judicial system is comprised of three levels of courts - Supreme Court, District Courts and Magistrates' Courts. Independence of the judiciary is guaranteed from intervention by the two other branches of the State - the legislative authority (Knesset - Israel parliament) and the executive authority. 


\section{( Crime prevention (SDG 16.1)}

The most recent report on Well-being, Sustainability, and National Resilience Indicators, published in 2019, indicates an increase in the rate of victims of violence or threats of violence; decrease in the rate of sexual harassment victims; decrease in the rate of persons killed and increase in the rate of persons seriously injured in road accidents; decrease in the frequency of road rage; and decrease in economic damage caused by property crimes out of disposable income.

- In 2017, 3.7\% of the persons aged 20 and over had been victims of violence (use of physical force) or threats of violence. This rate was higher among men than women ( $4.4 \%$ vs. $3.1 \%$, respectively). In addition, among both sexes, the rate of victims among young adults aged 20-34 was higher than the rate among persons aged 35 and over.

- In 2017, 85.6\% of all persons aged 20 and over felt safe walking alone after dark in their area of residence. The rate of men who felt safe was higher than the rate of women $191.7 \%$ vs. $79.3 \%$, respectively).

- The rate of sexual harassment victimization in 2007-2017 was stable: about 2\% for the total population and about $3.5 \%$ for women. In 2017 , about $7.5 \%$ of women aged $20-34$ were victims of sexual harassment versus $1.5 \%$ of women aged 35 and over.

- The rate of persons killed in road accidents declined from 8.0 persons per 100,000 residents in 2002 to 3.6 persons per 100,000 in 2017. The rate of persons seriously injured in road accidents declined from 36.8 persons per 100,000 residents in 2002 to 23.5 persons per 100,000 in 2017.

- The number of persons killed in terror attacks in 2016 was 0.2 per 100,000 persons, compared to 0.3 in 2015.

- In 2017, 52\% of persons aged 20 and over were victims of road rage (a decrease from 2016):

Selected Indicators of Personal Security 2017 (percentages unless otherwise specified)

\begin{tabular}{|c|c|c|c|c|c|}
\hline & $\begin{array}{l}\text { Desired } \\
\text { direction }\end{array}$ & $\begin{array}{l}2017 \\
\text { Indicator }\end{array}$ & $\begin{array}{c}\text { Direction } \\
\text { of change } \\
\text { compared } \\
\text { with previous } \\
\text { year }\end{array}$ & $\begin{array}{c}2002 \\
\text { Indicator } \\
\text { (base year) }\end{array}$ & $\begin{array}{c}\text { Direction } \\
\text { of change } \\
\text { compared } \\
\text { with base } \\
\text { year }\end{array}$ \\
\hline $\begin{array}{l}\text { Rate of victims of violence or threats of } \\
\text { violence }\end{array}$ & $\downarrow$ & 3.7 & $\downarrow$ & & \\
\hline $\begin{array}{l}\text { Feeling safe walking alone after dark in } \\
\text { the area of residence }\end{array}$ & $\uparrow$ & 85.6 & $\sim$ & 73.6 & $\uparrow$ \\
\hline Rate of victims of sexual harassment & $\downarrow$ & 2.2 & $\downarrow$ & & \\
\hline $\begin{array}{l}\text { Rate of persons killed in road accidents, } \\
\text { per } 100,000 \text { residents }\end{array}$ & $\downarrow$ & 3.6 & $\downarrow$ & 8.0 & $\downarrow$ \\
\hline $\begin{array}{l}\text { Rate of persons seriously injured in road } \\
\text { accidents, per } 100,000 \text { residents }\end{array}$ & $\downarrow$ & 23.5 & $\uparrow$ & 36.8 & $\downarrow$ \\
\hline Rate of murders, per 100,000 residents* & $\downarrow$ & 1.2 & $\downarrow$ & 3.2 & $\downarrow$ \\
\hline $\begin{array}{l}\text { Rate of persons killed in terror attacks, } \\
\text { per } 100,000 \text { residents }\end{array}$ & $\downarrow$ & 0.2 & $\downarrow$ & 4.7 & $\downarrow$ \\
\hline $\begin{array}{l}\text { Rate of persons wounded in terror } \\
\text { attacks, per } 100,000 \text { residents }\end{array}$ & $\downarrow$ & 1.7 & $\downarrow$ & 21.4 & $\downarrow$ \\
\hline Victims of road rage & $\downarrow$ & 51.6 & $\downarrow$ & & \\
\hline $\begin{array}{l}\text { Economic damage caused by property } \\
\text { crimes }\end{array}$ & $\downarrow$ & 0.37 & $\downarrow$ & $\begin{array}{l}\text { New } \\
\text { indicator }\end{array}$ & \\
\hline
\end{tabular}

* Updated to 2016 
Figure 16.1

\section{Persons killed in road accidents}

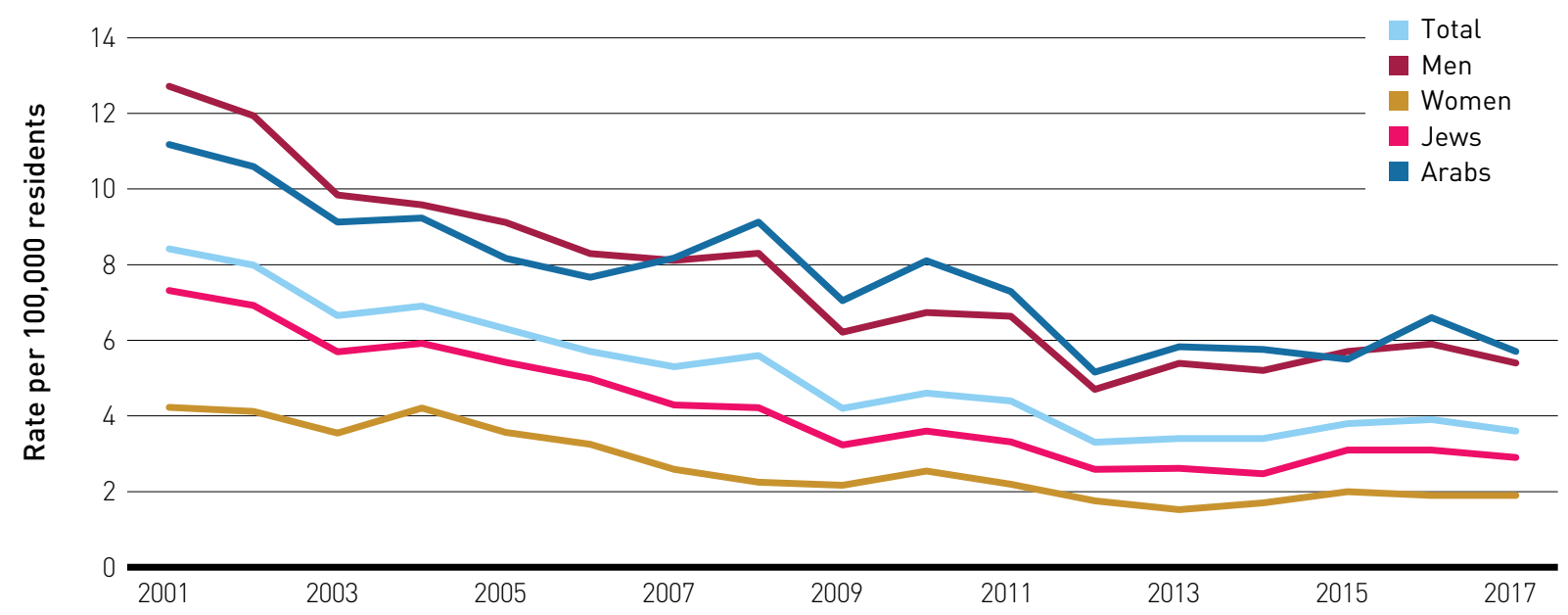

Source: Well-being, Sustainability, and National Resilience Indicators (2017), published 2019, Israel Central Bureau of Statistics

Figure 16.2

Homicide rate per 100,000 people, $2000-2016$

Females

Males

12

All Population

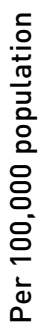

요

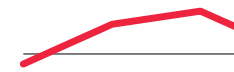

0

2000

2001

2002

Source: Israel Central Bureau of Statistics

$17.8 \%$ were victims every day or almost every day, $16.1 \%$ once or twice a month, $17.7 \%$ less than once a month, and $48.4 \%$ never.

- In 2017, 6.1\% of households were victims of damage to their dwellings and $3.9 \%$ were victims of damage to their cars.

- The homicide rate in Israel has declined from the peak year 2011 to the year 2016 by $28 \%$. Over the course of 2017 the homicide rate decreased by $19 \%$.
Israel Authority for Prevention of Violence, Alcohol and Drug Abuse

In 2017, Israel enacted a law to establish the Authority for Prevention of Violence, Alcohol and Drug Abuse in the wake of a government-led reform to unify the different agencies working in these realms. The Authority, which began its operations in February 2018, aims to prevent violence, crime and antisocial behavior and to combat drug and 
alcohol abuse by means of the following:

- Recommending overall policy and promoting legislative amendments:

- Developing and implementing professional programs on prevention and enforcement;

- Gathering information and engaging in research, evaluation and knowledge development, in accordance with the law;

- Supporting government ministries, the Israel Police and additional agencies working towards prevention, treatment, rehabilitation and enforcement, in establishing and developing suitable services, frameworks and action plans;

- Promoting cooperation between government ministries, local authorities, the Israel Police and additional agencies working towards prevention, treatment, rehabilitation and enforcement;

- Encouraging volunteering in the fields of prevention and enforcement, and instructing and guiding the volunteers and supervising their activity;

- Training and qualifying professional personnel on prevention and enforcement;

- Working to improve public education and public awareness;

- Promoting and maintaining ties with international agencies:

- Developing technological tools.

\section{Domestic violence}

The phenomenon of domestic violence, which includes murder and brutal violence between a couple and towards their children, is a severe phenomenon which needs to be dealt with in a determined manner. In accordance with the policy formulated by the Minister of Public Security, the Israel Police will place particular emphasis on providing a readily-available and swift response for every complaint related to domestic violence and will complete the establishment of Family Services Units, while cooperating with the welfare agencies and expanding the implementation of the Police Social Worker Project. The Israel Police is reviewing the possibility of implementing electronic monitoring to protect women from violence and increasing supervision over aggressive men who pose a high level of risk. A pilot is planned to examine the efficiency and effectiveness of electronic monitoring for confronting domestic violence. In addition, the Israel Authority for Prevention of Violence, Alcohol and Drug Abuse conducts public education and prevention programs on the issue of domestic violence.

\section{Municipal Enforcement program}

The Municipal Enforcement program was established to improve the supervision and enforcement capability of local authorities with regard to offenses under their purview, and to enable them to assist the Israel Police in violence prevention. The goals of the program include:

- Providing a rapid response to events and improving residents' quality of life;

- Providing assistance to the police in preventing crimes and enforcing laws involving disorder, bullying, violence and vandalism;

- Responding to crimes involving illegal building;

- Preventing and responding to crimes involving alcohol consumption;

- Preventing and responding to crimes involving aggressive driving:

- Providing assistance in securing large-scale events.

The Municipal Enforcement program combines police officers and municipal inspectors, enabling the police to improve quality of life within the municipalities in which the program operates. The program was launched in 2011 and is now implemented in 73 municipalities across Israel, which represent over $25 \%$ of Israel's local authorities.

\section{Parent patrol}

One of the main civilian programs, led by the Ministry of Public Security and local authorities, is the parent patrol program. Parent patrols are groups of parents who volunteer to patrol youth hangouts on weekends and during school breaks. The presence 


\section{SENSE OF SECURITY}

\section{Personal Security Survey}

The level of violence, nationally or locally, directly influences the public's sense of security. The Personal Security Survey conducted by the Israel Central Bureau of Statistics, for the years 2015-2017, examined the population's sense of security from a number of aspects. The survey asked about the fear of walking alone in one's residential area after dark, the fear of being harmed by violence in one's residential area and the general sense of security there. The following figure shows the distribution of those who responded: "To a very large degree" or "To a large degree."

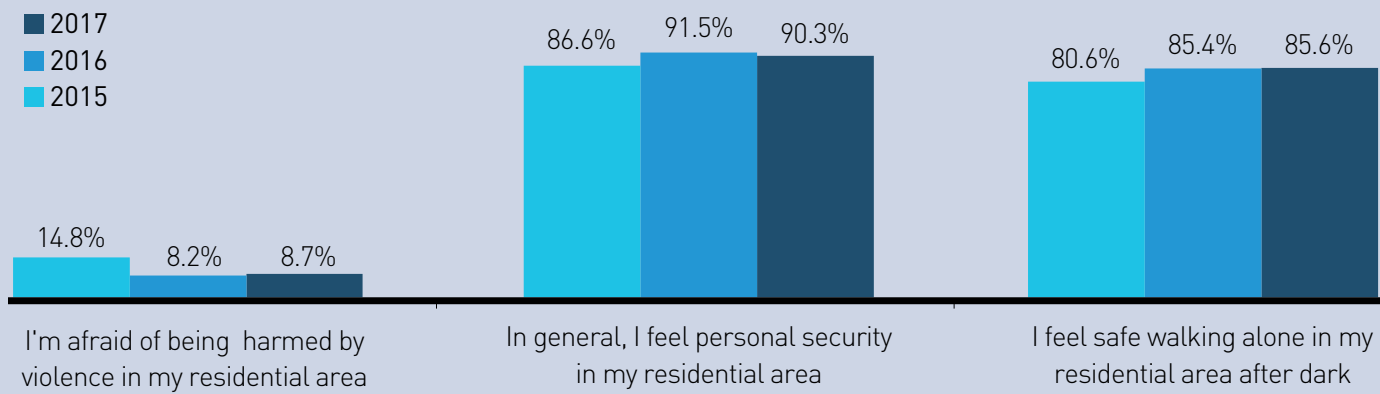

- The sense of fear of walking alone in one's residential area after dark in 2017 remained similar relative to 2016, and a 5\% increase was recorded relative to 2015;

- An approximate $1 \%$ decrease was recorded in the sense of personal security in one's residential area relative to 2016 , and an approximate $4 \%$ increase relative to 2015 ;

- The fear of being harmed by violence in one's residential area remained similar relative to 2016, and a 6\% decrease was recorded relative to 2015.

Figure 16.3

Percentage of respondents who stated that they felt safe to walk alone at night in their residential area, 2016

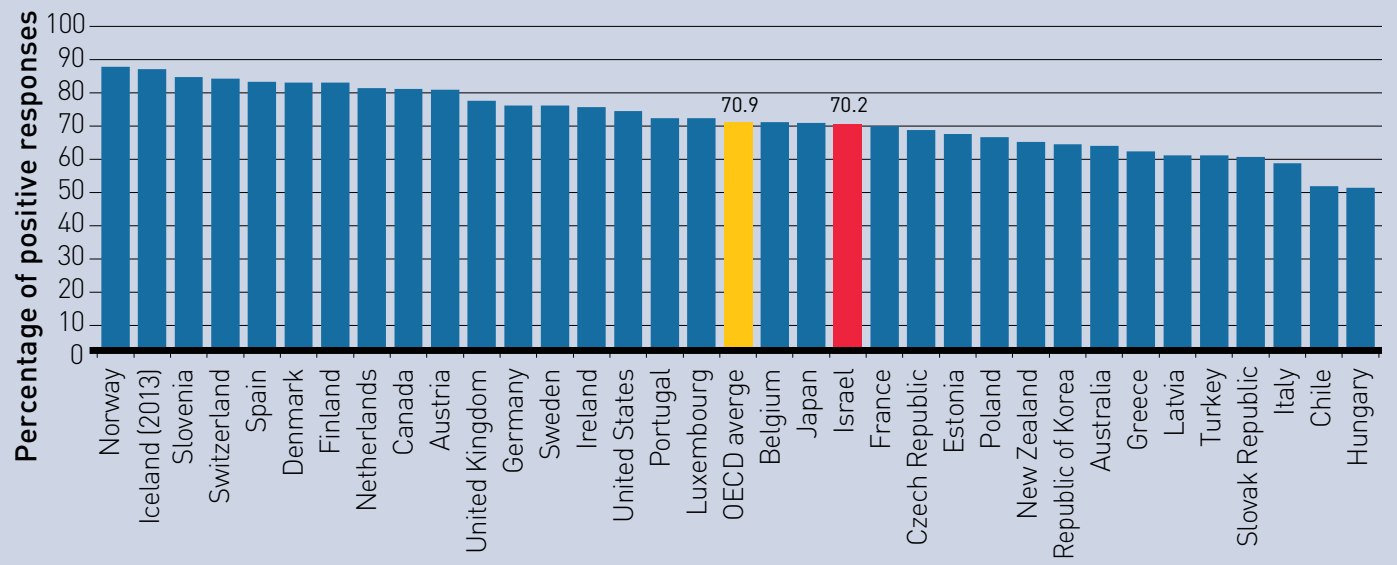

Source: OECD, Better Life Index 2017 
of the patrols reduces violence and alcohol abuse, as well as overall dangerous activity among the youth. The patrol members can offer assistance, discuss problems, and educate the youth about the dangers of drug and alcohol abuse. The parent patrols are overseen by the Israel Authority for Prevention of Violence, Alcohol and Drug Abuse in coordination with the municipality, and coordinators receive training in youth outreach. There are currently over 200 active parent patrols across the country, serving a diverse range of communities.

\section{Foot patrol}

The foot patrol program acts to increase police presence, visibility and service to citizens with the aim of improving personal security, and establishing situational crime prevention. The foot patrol unit patrols crowded, popular areas at peak times, ensuring police presence and approachability, so as to raise public confidence in the police and increase deterrence of crime and delinquent behavior. The officers patrol in areas such as commercial zones, tourist sites, leisure spots, beaches and markets. The program operates in 77 municipalities and 127 activity sectors around the country and has handled 93,074 incidents in 2017-2018.

\section{Investigation of work accidents}

The number of those injured or killed as a result of work accidents has increased over the past few years. The Israel Police thoroughly investigates such accidents with emphasis on accidents in which there is suspicion of negligence. The Israel Police aims to increase its activities in this field, with the objective of creating deterrence and reducing the scope of accidents, especially at construction sites. At the end of December 2018, a joint unit for investigating work accidents was launched by the Israel Police and the Ministry of Labor, Social Affairs and Social Services (MOLSA). The unit will formulate a comprehensive approach to the issue of work accidents, and will engage in investigating work accidents and irregular incidents and exposing related criminal offenses

\section{CCTV centers for violence prevention}

As part of the effort to create safe and resilient cities, security cameras are placed in public spaces in many municipalities to prevent violence and vandalism. The cameras are connected to a control center. The decision on the location of the cameras is made based on needs identified by the police station and local authority - cameras are placed in public locations that are at risk for violence and vandalism offenses. An operational program is created to fit the needs of each locality, determining the number of cameras, the type of technology and the locations monitored. The cameras serve to prevent violence, record events, collect evidence, deter crime and enable responses to violent events.

\section{( Combating the exploitation of minors (SDG 16.2)}

\section{National Child Online Protection Bureau}

The National Child Online Protection Bureau is a unique civilian/police unit which was established pursuant to Government Decision 1006 (2016) to form a Child Protection Bureau to enforce and prevent online violence and crime against children and youth. The Bureau operates in partnership with the Israel Police and the following government ministries: Ministry of Public Security, Ministry of Education, MOLSA, Ministry of Health and the State Attorney's Office. The Bureau is comprised of a national headquarters, an emergency reporting center/hotline (105) and an operational police unit. It deals with a variety of issues, including: child prostitution, pedophilia, drug dealing, blackmail, harassment, threats of suicide, violations of privacy, bullying and shaming. The Bureau provides a holistic government response to online threats against children and youth.

\section{Efforts to combat exploitation of minors for prostitution}

According to a 2014 survey conducted by the MOLSA together with the Ministry of Public Security, it is estimated that about 12,000 men and women work 


\section{CASE STUDY}

\section{Law on Authorities for the Prevention of Committing Crimes Through Use of a Website (2017)}

The Israel Police, in cooperation with the Cyber Department of the Ministry of Justice, takes daily action to prevent crimes committed by use of a website. An amendment to the law improves the ability of the police and law enforcement agencies to shut down locations that are used for prostitution and human trafficking. The law gives the court the authority to order restricted access to a website or to order a website to be taken down from the internet and/or the illegal content be removed in cases in which the order will prevent committing any of the offenses listed in the law. Offenses listed in the law include: prohibition of advertising adult prostitution services and prohibition of advertising minors' prostitution services, as well as online incitement, pedophilia, encouragement and support of terrorism, and certain types of online shaming and more. The law is designed to prevent criminal activity on the internet, by restricting access to websites through which these activities take place.

An amendment to the Penal Law, 1977 provides for the following:

- Increasing the punishment of one who receives service from an underage prostitute - five years instead of three years;

- Prohibiting publication of an offer to engage in prostitution. This amendment highlights the prohibition of advertising recruitment for prostitution, and helps enforcement in cases where the defendant is a link in the advertising chain but is not the advertiser himself, as in the example of a printing company.

The law provides additional tools to prevent prostitution, including:

- Disconnection of telephone numbers to prevent offenses (2018): The presumption is that disconnecting the telephone number on the advertisement will cause the ad to be ineffective or at least will make it difficult to implement.

- Amendment of the instruction of a State Attorney concerning enforcement policy for offenses accompanied by prostitution: Activity in strip clubs, which includes sexual contact, will be considered prostitution, and enforcement of the offenses which accompany prostitution will be allowed.

- Prohibition of consumption of prostitution (2018): This new legislation constitutes important progress in efforts on behalf of women in prostitution, with few countries worldwide having similar legislation. An administrative fine of NIS 2,000 will be imposed on those who break the law, with double the sum in case of a repeated offense.

- Promotion of an amendment to the Patient's Rights Law (Providing Information to Prevent Harm) (2018): This proposed amendment is designed to allow caregivers in the health industry to provide information to the appropriate agencies in order to reduce offenses against helpless minors and cases in which minors are exploited for prostitution.

- Addressing prostitution among minors online: Establishment of The National Child Online Protection Bureau (see above). 
in prostitution. Among adults, this includes about 10,000 women and 550 men. Among minors, it is estimated that between 970-1,260 young women and 30-40 young men are exploited for prostitution. In addition, about 500 transgender people were found to be working in prostitution. It is estimated that close to $20 \%$ of women and about one third of men sex workers became part of the sex industry as minors.

In recent years, law enforcement, prevention and treatment agencies have set a goal of addressing the issue of exploitation of minors for prostitution and caring for minors exploited. To this end, the Ministry of Public Security and the Israel Police are working to improve the measures taken to confront the exploitation of minors for prostitution.

These efforts include leading, promoting and formulating a consensual plan for the police and relevant prevention and treatment agencies and personnel to operate a pilot of round tables to combat the phenomenon of exploitation of minors for prostitution. The goal of the plan and round tables forum is to conduct a multi-professional, systemic consultation for the purpose of assembling a full situational picture both with regard to the overall phenomenon and individual cases.

Prior to the start of the pilot, the Ministry of Public Security conducted - in cooperation with the Israel Police and the Ministry of Justice's National AntiTrafficking Unit, and with the participation of all relevant agencies including representatives of relevant NGOs - an orientation day for the purpose of presenting the pilot, emphasizing important points, providing clarifications, highlighting focus areas and answering questions. In addition, training activities were held for police youth investigators, and explanatory materials were distributed to police officers at police stations. Additionally, exploitation of minors for prostitution was added to the list of offenses that the police must investigate even without a complaint. In addition, the National Child Online Protection Bureau was launched in 2018, one of whose tasks is to address issues such as online child prostitution and pedophilia.

These measures followed the work of an interministerial team that formulated a plan to confront the problem of exploitation of minors for prostitution, including prevention, locating victims, enforcement and treatment.

\section{( Fighting trafficking (SDG 16.2)}

For the past seven years, the State of Israel has been ranked in Tier 1 of the US State Department's Trafficking in Persons Report, confirming that Israel operates on the three levels set out in the report - prosecution, prevention and protection. Israel makes major efforts to combat human trafficking, including action taken against exploitation of minors for prostitution, advertising prostitution services and dealing with prostitution-related offenses.

Israel is committed to fighting trafficking in children. Receiving acts of prostitution from a minor is a criminal offense, with the penalty recently raised from three to five years - changing its status to a felony. The Inter-ministerial Committee on Reducing Use of Prostitution Services included a set of recommendations on combatting commercial sexual exploitation of minors. The Police and the MOLSA have developed a joint procedure for information-sharing and shared decision making on cases of sexual exploitation of minors - which is slated to begin operations this year.

The MOLSA runs the following programs for assistance for children and young adults:

\section{The Heart 24/7}

The Heart 24/7 program has been offering 24/7 support for minors in prostitution in the Tel AvivJaffa area since 2014. In 2017, about 250 youth or young adults were treated in the program, which offers two main treatment tracks: damage reduction and treatment and rehabilitation:

- Damage reduction: The $24 / 7$ center is operated by staff and volunteers. Services include meals, hygiene needs, showers, laundry, medical assistance, consultations, and a place to stay and rest. In addition, the center offers numerous workshops;

- National helpline: The helpline operates on a 24/7 basis and assists youths and young adults along 
the prostitution spectrum through listening and crisis management. It also provides referrals to professionals when necessary;

- Assistance with education and employment: The program provides assistance and support with education and employment in order to help in building a future;

- Personal support: The youths and young adults are each assigned a mentor who accompanies them personally through the process, providing them with support and a safe person with whom to discuss safe sex, sexual identity, drug use, relationships, personal difficulties and more.

\section{The Heart - Open Centers for Girls and Young Women in Commercial Sexual Exploitation in Municipalities}

The open centers serve as a safe and protective alternative to the street or to an abusive home, and give basic aid in the form of meals, a place to shower, and preliminary support. The girls and young women also receive personal mentoring and mediation with services available in the community, allowing them access to other means of support and aid. In 2017, a budget increase allowed for the establishment of 13 programs for youth in commercial sexual exploitation - up from five in 2016. In 2017, about 160 youth and young adults participated in the outreach process, and over 350 were in a process of treatment. Another budget increase came into effect in 2017-18, and further programs are scheduled to be opened in the coming year.

\section{( Promoting the law and ensuring equal access to justice for all (SDG 16.3)}

The Ministry of Justice renders justice and provides service to the public in the legal sphere in accordance with government policy while protecting the rule of law, human rights and the basic values of the State of Israel as a Jewish and democratic state where everyone is equal before the law. Key target areas derived from its mission statement include:
- Improving efficiency and effectiveness of the law enforcement system and the legal infrastructure of the State of Israel;

- Promoting and protecting human rights and narrowing social gaps;

- Increasing the public's trust in the rule of law;

- Improving service to the public and improving the response given to government ministries and units in the ministry;

- Striving for excellence and encouraging innovation.

\section{Educating youth to civic responsibility: The high school policing studies program (SDG 16.3)}

The police high school program is a socialcommunal program conducted in several youth villages whose goal is to provide a unique high school dormitory program that combines a typical high school curriculum with additional subjects related to the rule of law, security and civic responsibility. The curriculum includes criminology and sociology studies, and aims to instill values and skills that will motivate the students to work hard and strive for excellence while preventing violence and crime. In addition to formal studies, the program incorporates informal learning in the afternoon and evening, led by police officers. The extracurricular activities help expand the students' horizons and encourage them to become responsible, law-abiding citizens who contribute to society. Over $70 \%$ of the graduates attain full matriculation. The program that began with just 17 students now boasts over 300 .

\section{( Combatting organized crime, serious crime and public corruption (SDG 16.4, 16.5)}

Serious and organized crime adversely affects the personal security of the country's citizens. The Israel Police is taking steps to strike a significant blow against criminal organizations and to uproot the phenomena of extortion and protection rackets. 
This will be done, inter alia, by cooperation with the Israel Prison Service in reducing crime orchestrated from within prisons and with the Witness Protection Authority, which will continue to expand its capacity to place witnesses under protection, along with the continued operation of combined task forces, focusing on economic enforcement against crime bosses and crime organizations and increasing the use of signals intelligence and other technologies as tools to eradicate the phenomenon.

The Israel Police is continuing its focused efforts to expose crime and public corruption in the various government institutions. It works and will continue to work to increase economic enforcement in the areas of serious and organized crime, including conducting investigations into money laundering offenses and guiding field units to maximize use of economic enforcement tools, including seizure and forfeiture of all assets that can be linked to the perpetrators of the offense for the purpose of confiscating the offenders' property and preventing them from returning to criminal activity.

The Israel Police is also working to advance legislation on economic enforcement for the purpose of reducing serious and organized crime, such as two recently enacted laws on the reduction of the use of cash and on supervision of financial services. It will continue to take action to expand money laundering orders to apply to additional sectors, as was recently done with credit service providers.

Furthermore, efforts will be made to increase cooperation with enforcement agencies within Israel and worldwide for the purpose of promoting effective enforcement and reducing serious and organized crime.

\section{( Transparency (SDG 16.6)}

Public awareness and trust in law enforcement organizations are a necessary condition for the implementation of the Ministry of Public Security's work plan, objectives and goals. Therefore, the ministry will formulate and implement a systemic program for raising the trust of the citizenry in the law enforcement system. In order to reduce the potential of friction inherent in the police officercitizen encounter and to increase transparency, the implementation of the body cameras project will be advanced. The first stage of the project was launched in the Tel Aviv District in January 2019, with 420 cameras distributed to police officers. The project is slated to include 8,000 cameras in total, to be distributed in all police districts.

The objective is to raise the level of public confidence in the operation of the ministry and its operational bodies by means of a formal public relations program, while increasing transparency, implementing a concept of procedural and multicultural justice in the activity of the ministry's operational bodies and implementing the body camera project.

\section{( Multiculturalism in Israeli society (SDG 16.7)}

Israeli society is a multicultural society. The operational bodies of the Ministry of Public Security implement this multiculturalism in their encounters with the citizen and in the pre-service and in-service training tracks of those serving in it, so that the service provided to the citizen will be nonpartisan and unbiased. Furthermore, efforts will continue to be invested to provide service adapted to unique communities, such as Arab Israelis, Israelis of Ethiopian descent, Druze and the Haredi lultraOrthodox) sector, with their unique characteristics.

Ensuring equal services to Arab society, both from the aspect of improving policing services as well as the aspect of law enforcement, is at the center of the Ministry of Public Security's endeavors. It is planned to allocate additional resources and to increase the efforts on the part of the Israel Police and the Israel Authority for Prevention of Violence, Alcohol and Drug Abuse, with the objective of improving personal security amongst Arab society and reducing crime and antisocial conduct amongst this population.

The Israel Police's Directorate for the Development of Policing Services for the Arab Israeli Population was established in 2016 with the aim of increasing 


\section{CASE STUDY}

\section{Alternative Dispute Resolution in the Community (The ADR Project)}

The complexity of the social space of conflict impelled the development of alternative tools to manage disputes, which were adopted and developed in Israel by the Ministry of Justice (1998-2010), in line with the literature and practice around the world. These refer to a range of cooperative, voluntary processes to build agreement and settle disputes through negotiation, mediation and dialogue according to the needs and desires of the parties.

As part of these efforts, the Ministry of Justice (until the end of 2009) served as the professional headquarters for the development of theory and practice and the establishment of 25 ADR centers in the community level at different localities. The infrastructure laid down at the Ministry of Justice and the subsequent inputs of the Gishurim (Mediation) Program have resulted in ramified activity and dozens of centers at different localities.

The MOLSA participates in the cost of employing social workers at the centers. The establishment of the centers in the community reflects a social conception and values, with an eye on social change through the implementation of the centers in the community.

The theoretical/substantive model focuses on establishing centers responsible for handling a range of disputes at the level of the individual, the community, and between the local authority and the individual, submitted by the public or referred by internal parties (local authorities, the police, education and welfare, the courts, etc.). The centers are managed by volunteer mediators representing the community, with an emphasis on responses and service availability and accessibility adapted to community needs and values; on early intervention in disputes; on activities related to instilling the approach; and on education and prevention. The centers take a systemic approach, cooperating with government agencies, professionals, the community and the public.

the trust of Arab society in the police and increasing the presence of Arab Muslim officers in the ranks of the police. Since its establishment, seven new police stations have been built to serve the Arab population. In the coming years, the recruitment of 600 police officers from Arab society will be completed.

The Ministry of Public Security has taken action to implement Government Decision 1300 on promoting the optimal integration of citizens of Ethiopian origin into Israeli society, by means of programs operated by the Israel Police and the Israel Authority for Prevention of Violence, Alcohol and Drug Abuse. The steps taken to strengthen the trust of the Ethiopian community in the police include placing
Israelis of Ethiopian descent non-commissioned officers (NCOs) and community police officers at police stations across the country, convening a joint steering committee with community representatives and religious leaders, recruiting and promoting Israeli police officers of Ethiopian descent, and conducting cultural competency training for about 16,200 police officers. In 2018, the Authority for Prevention of Violence, Alcohol and Drug Abuse conducted education and leisure activities for the Ethiopian community geared to prevent violence and alcohol and drug abuse, which were attended by over 25,000 youth and adults. 


\section{( Improving service to the citizen (SDG 16.3, 16.6)}

From a broad perspective, obeying the law in a democratic society cannot be based solely on deterrence. The legitimacy of the police and the Ministry of Public Security's operational bodies, based on their performance in practice as well as on the fairness of the processes in which they are involved is a key factor. Insofar as the citizen, who comes into contact with the ministry's operational bodies, will feel that the processes by which the policy is applied are fair - so will trust in the ministry and in its associated organizations increase. Within this ambit, a great deal of importance is afforded to the extent to which citizens are able to express their position prior to a decision on their matters. A neutral, non-discriminatory and transparent attitude in the decision-making process, along with concern for their welfare and quality of life is of utmost importance.

Therefore, improving service to the citizen, with emphasis on the Israel Police, the Israel Fire and Rescue Authority and the Firearms Division, is a central objective of the Ministry of Public Security, in providing good, quality and more readily available service to the citizenry.

The ministry is investing efforts to make its services available, both physical services and online services, and to increase the transparency and the visibility of its operations and those of its operational bodies.

\section{Capacity building, participation and decision-making at the local level (SDG 16.6, 16.7)}

Several measures are being taken by the Ministry of Interior to support the development of local authorities in Israel. They include measures to promote processes of decentralization and participation in decision-making, to encourage tolerance and transparency, to cultivate a proper and honest administration at the local level, and to strengthen the effectiveness of local institutions, as exemplified by the following:
- Empowerment and participation in decisionmaking: In order to empower local authorities and increase their participation in decisionmaking processes, the Ministry of Interior initiated a pilot project that transfers budgetary authority from the central office to the district headquarters of the ministry. In addition, an outline for decentralization of authority from the central office to the district headquarters in the fields of organizational development in the local authorities was formulated.

- Cultivation of proper administration: Efforts to strengthen local authorities were accompanied by the development and monitoring of proper administration. In this context, the Ministry of Interior examined the reciprocal relations between the local authority and its corporations. As part of the examination, several issues were studied: agreements between the corporation and the local authority, the work of the board of directors/executive committee, regulation of bylaws, and internal auditing of the corporation by the local authority, In addition, the ministry examined compliance with business licenses in 30 local authorities.

- Strengthening transparency: To increase transparency in local authorities, the Ministry of Interior established a transparency forum with civil organizations and formulated recommendations to increase transparency in local authorities. The ministry's databases have been made accessible to the public and provide a broad picture of the state of the local authority and its position with regard to other local authorities.

- Encouraging tolerance: In an attempt to increase interreligious and multiculturalism sensitivity, the Ministry of Interior published and distributed a publication entitled "Acceptance of the Other," based on the four religions. A mediation course was held for clerics of all religions to disseminate the tolerance message. Additionally, support was provided to schools in which tolerance activities were conducted. 


\section{Investing in human capital}

Training and enrichment of human capital at the level of local authorities in Israel is of prime importance given the large number of employees in local authorities - approximately 137,000. Investment in human capital will enable a higher level of governance (decision-making and implementation), better performance, economic development, and provision of quality services to residents. The Ministry of Interior aims to strengthen the effectiveness of local authorities and to promote organizational development programs with special emphasis on organizational processes such as annual and multi-annual planning and training processes.

Training processes are based on a wide range of activities. Mandatory training courses, for example, are conducted for new CEOs in municipal corporations and efforts are taken to improve the management skills of CEOs in municipal corporations.

One of the most prominent programs in the field of training is the development of administrative reserve programs in accordance with a government decision on the subject of "Reserves for Israel" - a cadet plan for local government. The cadet program is led by the Ministry of Interior in cooperation with the Ministry of Finance, Atidim Itranslated as Futures, Atidim is a national program which aims to develop human resources and close the socio-economic gaps that cripple Israel's periphery by creating equal educational opportunities) and the Union of Local Authorities. It aims to train the administrative human capital reserve for local government by combining undergraduate or second degree studies with group and personal development processes. The graduates of the cadet program form a network of colleagues that can create partnerships and lead local initiatives. Graduates are placed in local authorities, especially in weak and peripheral ones. They often serve in senior positions such as assistant directorsgeneral or aides to mayors, and assist in leading strategic and economic development processes.

The enhancement of human capital has been advanced by other measures as well. Sixty-two local authorities participated in an organizational development program that included Bedouin communities, Druze communities, the Circassian communities and disadvantaged Jewish communities.

The enrichment of human capital in local authorities is carried out by a network of training centers known as Mifa'mim. This network was established by the Ministry of Interior and local authorities to improve the skills and capabilities of local leaders and employees. In so doing, the network, which includes eight training centers spread throughout the country, assists local authorities in strategic planning, organizational change and courses to selected employees, managers and staff.

\section{( Freedom of Information Unit (SDG 16.10)}

The Freedom of Information Unit was established within the Ministry of Justice in 2011. The establishment of a central unit aimed at promoting transparency is an expression of the value of open governance. The principle of transparency and the right of citizens to obtain information from government authorities has been enshrined in case law since the mid-1960s. The unit constitutes a center of professional knowledge in the field of freedom of information and gathers relevant information, conducts public awareness campaigns and trains civil servants and other public employees. Its primary role is to manage the work of freedom of information officers in the various government ministries, handle public complaints about freedom of information in the ministries and rectify errors discovered in the process. The unit submits annual reports to the government on the compliance of Israeli ministries and other authorities with the provisions of the law. The first report was submitted in May 2013. According to Government Decision 2950 of March 2011, the unit is to serve as a center of professional knowledge on freedom of information and to gather relevant information to assess compliance by the authorities with the provisions of the law and the consideration of future legislative amendments. 


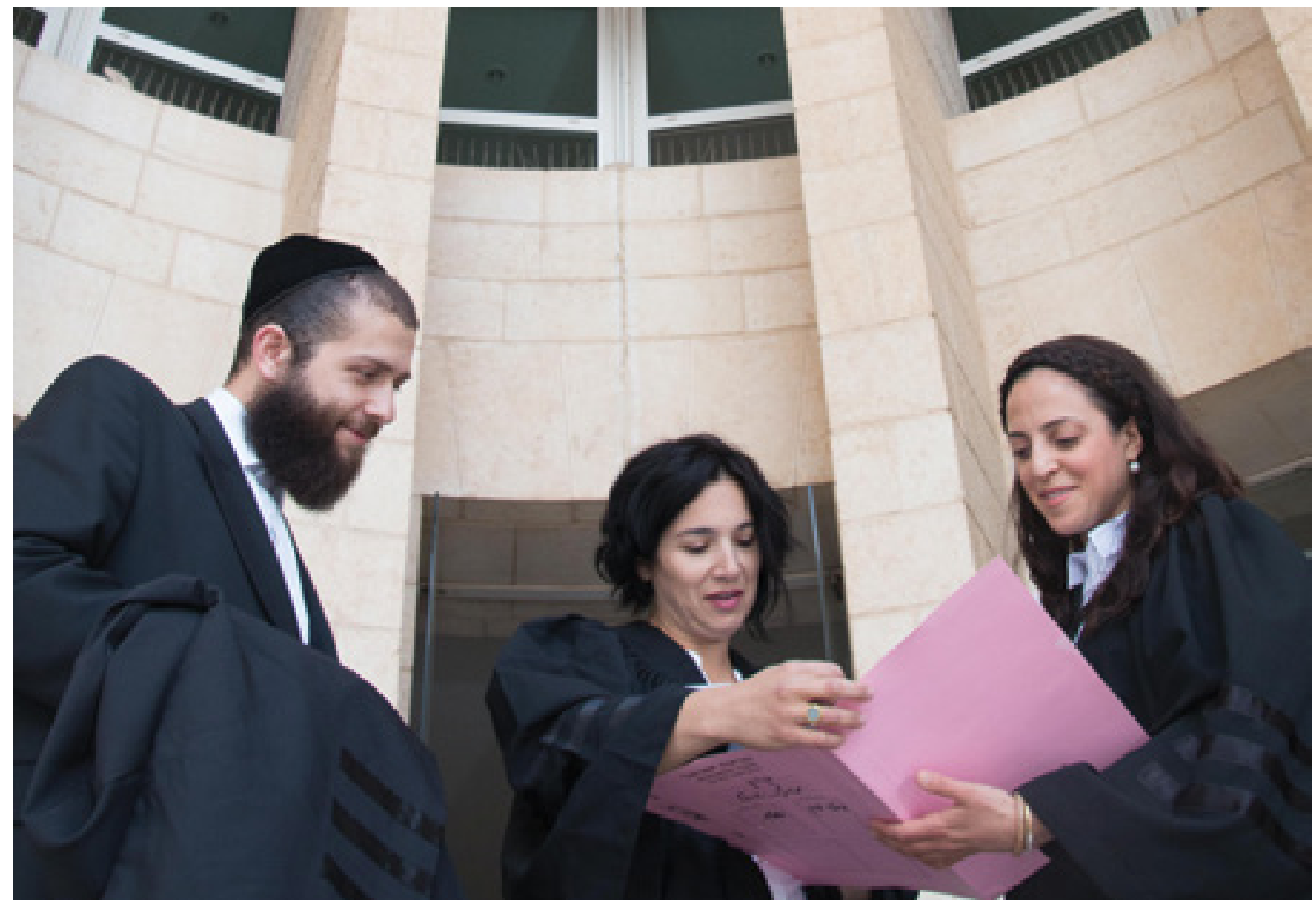

Inside the Supreme Court/Photo: Ministry of Justice

To attain its objectives and goals, the unit is responsible for the following:

- Handling public complaints concerning the conduct of the freedom of information supervisors at government ministries and auxiliary units;

- Providing guidance to government ministries and auxiliary units on the manner of implementation of the law, in accordance with the provisions of the law, case law rulings and relevant Attorney General Guidelines;

- Providing instructions to correct defects discovered in the work of a government ministry or auxiliary unit, after affording that authority an opportunity to exercise its right to a hearing;

- Providing training and guidance to all freedom of information supervisors at public authorities;

- Distributing and updating a list of public authorities as required under the Freedom of Information Law;

- Monitoring the manner of implementation of the law and publishing the findings in an annual report to the government;
- Setting up a central freedom of information website for all government ministries and auxiliary units, making it easier for the public to submit freedom of information requests;

- Initiating and promoting projects for the distribution of information held by government ministries and auxiliary units on a proactive basis.

Future main activities include:

- Defining a professional and objective "transparency scale" by the Freedom of Information Unit, according to Israel's commitment to the Open Government Partnership (OGP);

- Expanding freedom of information activities to local authorities;

- Organizing training courses for freedom of information officers lobligatory in government offices);

- Implementing a central freedom of information management system in government offices. 


\section{CASE STUDY}

\section{Ongoing Power of Attorney Arrangement}

In recent years, the Israeli government recognized that the legal process of guardianship is highly-priced and inaccessible for many civilians. As part of the belief of the Israeli system in equality for all before the law, and therefore equality in accessibility to the institutes of justice, the Ongoing Power of Attorney Law in the Legal Capacity and Guardianship Law, 1962 was amended in 2016.

The aim of this amendment was to make it possible for every adult to appoint another person to be authorized to act in their name in the legal matters prescribed in the law. An ongoing power of attorney "may be in the appointer's personal affairs, wholly or partly, or in matters of his property, wholly or partly" (Section 32B (b)).

To examine the influences of the enactment of this amendment, an evaluation study was conducted for the Administrator General a year after the entry of the ongoing power of attorney into force. The main procedures existing in this arrangement were surveyed along with the method of performing them, their effectiveness and their implementation in practice were examined. Using the data, it was possible to reach conclusions in connection with the arrangement and the changes required.

The ongoing power of attorney arrangement is anticipated to influence the lives of many men and women. The study assisted in obtaining a thorough and complete picture of the arrangement, with its advantages and disadvantages, and in providing a better understanding of what is required to improve it.

\section{( Making information accessible to residents for the exercise of their rights (SDG 16.3, 16.10)}

On the understanding that residents should be aware of all possible tools to help them know the law, their rights, and the services benefiting their welfare, Israel undertook to make that information available by pursuing several avenues of communication. These include: Shil the Hebrew acronym for Civilian Advice Service), which is manned by volunteer professionals at 70 stations countrywide, and offers information on topics related to work and welfare; a hotline for public information and assistance on social rights and welfare services appropriate to the needs of clients; cooperation with other parties to enhance the accessibility of information, mainly, by creating an internet database accessible to residents - "My
Right to Welfare" - with information on laws, rights and services suited to the needs encountered by all residents in the course of their lives. The database will be created with a view to the future - to interface with the inter-ministry database now under construction as part of the Digital Israel endeavor on the exercise of rights.

The database will pool topics related to children and youth at risk, single-parent families, violence in the family, marital crises due to unemployment, divorce and other events, assistance to people with special needs, assistance to Holocaust survivors and senior citizens, and so forth. 


\section{PARTNERING FOR A BETTER WORLD}

The early stages of the criminal justice process are crucial for those arrested or detained in respect of criminal offense. Israel's Public Defender was part of a group of experts that drafted the UN Principles and Guidelines on Access to Legal Aid in Criminal Justice Systems, which states that legal aid is an essential element of a fair, humane and efficient system based on the rule of law. Israel also funded the printing of the Handbook for Policy-Makers and Practitioners on the Early Access of Legal Aid, which was distributed to all UN Member States. (SDG 16.3)

Israel is committed to protecting and promoting the human rights and civil liberties of the LGBT community, and has expressed its robust support in international fora, as well as bilaterally with partner countries. Israel's strong commitment is underscored by the decision of the Ministry of Foreign Affairs to designate this issue as a central component in its Global Agenda for 2019. (SDG 16.b)

In 2014, parallel to the $7^{\text {th }}$ Session of the Conference of the Parties to the UN Convention against Transnational Organized Crime (COP UNTOC), Israel's Permanent Mission in Vienna, in cooperation with the Office of Israel's Public Defender, initiated a side event on International Cooperation between Legal Aid Providers in Criminal Matters. Continuing in this effort, at the $25^{\text {th }}$ Session of the Commission on Crime Prevention and Criminal Justice (CCPCJ), Israel strongly supported a Resolution adopted on Promoting Legal Aid, Including through a Network of Legal Aid Providers. The Resolution called for the establishment of the first global network of legal aid providers. In subsequent years, important steps were taken toward establishing the International Legal Aid Network (ILAN). At the Second International Conference on Access to Legal Aid in Criminal Justice Systems, held in Buenos Aires (2016), Israel supported the Buenos Aires Declaration calling for the establishment of a working group on launching ILAN, and took part in the Expert Group Meeting on the Quality of Legal Aid Services (Vienna, 2018). At the Third
International Conference on Access to Legal Aid in Criminal Justice Systems, held in Tbilisi (2018), Israel strongly supported the Tbilisi Declaration, calling upon donors to ensure the resources and support necessary to establish, administrate, and organize a global legal aid network. (SDG 16.3)

Since the signing of the Comprehensive Peace Agreement in 2003 following a prolonged civil war, Liberia has been rebuilding its public institutions including its criminal justice system. The UN Office on Drugs and Crime (UNODC) initiated a project, in cooperation with the Liberian Judicial Institute, Public Defender's Office, Israel's Public Defense Unit and others, providing workshops and training to Liberian public defenders. Israel's Ministry of Foreign Affairs funded the dispatch of legal experts to participate in two capacity-building missions to Liberia. (SDG 16.3)

Further collaboration with UNODC led to engaging with Vietnam's Legal Aid Office. Israel's Ministry of Foreign Affairs and Public Defense Unit, together with legal experts from Vietnam, discussed the legislative process regarding legal aid in the criminal justice system. Israeli experts led a further mission to Vietnam focusing on representing the accused in domestic violence cases and general legal aid issues in criminal cases. A delegation of Vietnamese experts visited Israel on a follow-up study mission. (SDG 16.a)

An Israeli legal expert participated in a UNODC led Cross-Regional Seminar on Access to Legal Aid in Criminal Justice Systems - Quality Assurance, Holistic Services and Equity to Access, conducted in Guăngzhōu City, China (2018). The Seminar, held in cooperation with China's Ministry of Justice, saw the participation of experts representing countries in South East Asia geared to promote the implementation of the UN Principles and Guidelines on Access to Legal Aid in Criminal Justice Systems, development challenges and practical solutions. (SDG 16.3)

The work of UNODC on crime prevention and criminal 
justice reform is guided by UN standards and norms. Within the framework of the CCPCJ, Israel supported the following Resolutions (SDG 16.a): Enhancing the Role of the Commission on Crime Prevention and Criminal Justice in Contributing to the Implementation of the 2030 Agenda for Sustainable Development; Follow-up to the Thirteenth United Nations Congress on Crime Prevention and Criminal Justice and Preparations for the Fourteenth United Nations Congress on Crime Prevention and Criminal Justice; and Strengthening Measures against Trafficking in Persons. (SDG 16.2)

In addition, Israel was part of the consensus on the adoption of the UN General Assembly Resolution on Strengthening the United Nations Crime Prevention and Criminal Justice Program, in Particular its Technical Cooperation Capacity, as well as the Resolution on Human Rights in the Administration of Justice. (SDG 16.a)

Israel's National Anti-Trafficking Coordinator serves as a member of the Working Group to the Conference of the Parties to the UN Convention against Transnational Organized Crime and participated in the 2018 Conference of the Parties in Vienna. (SDG 16.a, 8.7)

In light of global concern regarding surrogacy and the inherent power imbalance that might lead to exploitation of both the mother and the child born from these arrangements, a human rightsbased approach to safeguard both mother and child from abuse is required. Due to the role that international regulation plays in guiding national responses to surrogacy, Israel's Ministry of Labor, Social Affairs, and Social Services hosted (2018) a three-day international work meeting in Jerusalem with experts from the International Social Service (ISS) to focus on establishing global norms on surrogacy. (SDG 16.3, 5.2)

Israel is actively engaged in the UN Convention against Corruption (UNCAC), serving as the only legally binding universal anti-corruption instrument and a unique tool for developing a comprehensive response to a global problem. Israel plays a dominant role in two of UNCAC's Working Groups - Asset Recovery and Prevention. (SDG 16.6)

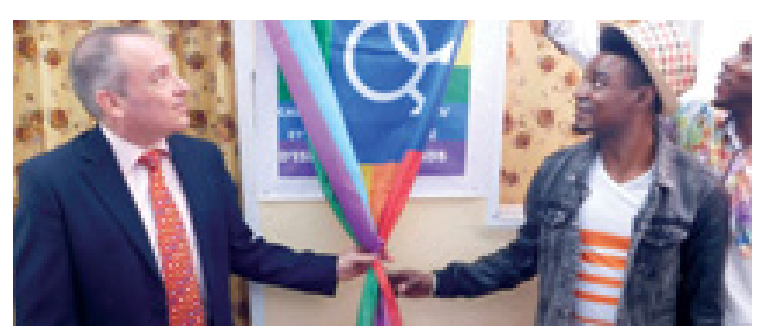

Opening a shelter for LGBT youth with local NGO - CAMFAID/ MASHAV

As an active UNCAC Member State, at the 2017 Conference of the States Parties (CoSP), Israel held a special event on The Central Role of Financial Intelligence Units (FIUs) in Combating Global Corruption. The Director General of Israel's Ministry of Justice participated in the event, and Israel cosponsored the following Resolutions: (SDG 16.5)

- Resolution 7/2 - Preventing and Combating Corruption in All its Forms More Effectively, Including When it Involves Vast Quantities of Assets, Based on a Comprehensive and Multidisciplinary Approach, in Accordance with the United Nations Convention against Corruption;

- Resolution 7/3 - Promoting Technical Assistance to Support the Effective Implementation of the United Nations Convention against Corruption;

- Resolution 7/4 - Enhancing Synergies between Relevant Multilateral Organizations Responsible for Review Mechanisms in the Field of Anti-Corruption;

- Resolution 7/5 - Promoting Preventive Measures against Corruption;

- Resolution 7/6 - Follow-up to the Marrakech Declaration on the Prevention of Corruption;

- Resolution 7/8 - Corruption in Sport.

In support of the important work being done by UNCAC, Israel and Turkey have been working together on the Review Process on the implementation by the United Kingdom of Chapters 2 and 5 of the United Nations Convention against Corruption. Moreover, Israel has been an active member in the Implementation Review Group and has presented an initiative to enhance the effectiveness of the discussions through restructuring the Multi-Year Work Plan and Agenda. (SDG 16.5)

During the UNCAC CoSP in 2015, an Israeli initiated Resolution was adopted on Promoting the Use of 
Information and Communications Technologies for the Implementation of the United Nations Convention against Corruption (Resolution 6/7). Following the CoSP, Israel's Money Laundering and Terror Financing Prohibition Authority (IMPA) gave a presentation on topics included in the Resolution at a meeting in Buenos Aires of the Egmont Group of Financial Intelligence Units. (SDG 16.5)

To assist member states in developing a balanced intellectual property legal framework to meet society's evolving needs, Israel's Patent Office (ILPO) works in close collaboration with the World Intellectual Property Organization (WIPO) on joint programs, including: (SDG 16.8)

- An annual capacity-building training program for interns from the WIPO Worldwide Academy on topics including Patent Search and Examination in the Field of Pharmaceuticals (2016); Patent Search and Examination in the Computer and Telecommunications Fields (2017); and Patent Search and Examination of Patent Applications in the Fields of Pharmaceuticals, Chemistry and Biology (2018);

- ILPO and Georgia's National Intellectual Property Center signed (2014) an Agreement Concerning Action of the ILPO as an International Searching Authority and Preliminary Examining Authority Under the Patent Cooperation Treaty for Certain International Applications Received by the National Intellectual Property Center of Georgia;

- ILPO and the National Patent Office of Ukraine are in the final stages of signing a Memorandum of Understanding (MOU);

- ILPO and the National Patent Office of Tajikistan maintain ongoing professional contact. A delegation from Tajikistan conducts an annual mission to Israel to exchange information and best practices.

Israel is actively promoting the human rights of the LGBT community. It is a long-standing member of the LGBT Core Group that fought to protect the mandate given to the Independent Expert on the Protection against Violence and Discrimination Based on Sexual Orientation and Gender (SOGI) in the Third and Fifth Committees at the UN
General Assembly. Israel also cosponsored many awareness-raising side events held by the Core Group on the rights of the LGBT. (SDG 16.b)

Israel is a member of the Core Group for the Defense of LGBT, within the UN framework, and a founding member of the Equal Rights Coalition, consisting of a group of countries outside the UN system committed to promoting the rights of the LGBT community. It is also a member of the Thematic Working Group dealing with national legislation, programs and practices working together with the Organization for Economic Cooperation and Development (OECD) and the World Bank. (SDG 16.b)

Israel's overseas development assistance programming has shown steadfast support of LGBT communities through MASHAV's work, for example, in Cameroon where a shelter was established for homeless LGBT youth, in cooperation with a local NGO - CAMFAID. Moreover, the first HIV diagnostic laboratory for LGBT youth was established. (SDG 16.b, 3.3)

Israel's contribution to advancing human rights within multilateral frameworks is reflected in the election of an Israeli expert to an unprecedented third term in the UN Committee on the Elimination of Discrimination against Women (CEDAW, 2006-2018), and her election as Vice Chair of the Committee (2009-2010, 2017-2018). (SDG 16.3)

For the first time, an Israeli expert was chosen to Chair the Geneva-based Human Rights Committee, under the auspices of the Office of the UN High Commission for Human Rights. The Committee is responsible for monitoring the International Covenant on Civil and Political Rights (ICCPR). (SDG 16.3)

In its commitment to combat the scourge of antisemitism, and promote peaceful, nondiscriminatory and inclusive societies irrespective of ethnicity or religion, and holding that remembrance is a human right, Israel actively supports the International Holocaust Remembrance Alliance (IHRA), uniting governments and experts to promote Holocaust education, remembrance, and research, in accordance with the 2000 Stockholm Declaration. Israeli experts participate in IHRA's Working Groups 
(Academic, Education, Memorials, Museums), as well as in IHRA's specialized committees (Antisemitism and Holocaust Denial, Holocaust, Genocide, and Crimes against Humanity). In 2016, Israel was requested to serve as a Mentoring Country to accompany Bulgaria in its process of joining the Alliance. In 2018, Bulgaria was accepted as a full member. (SDG 16.b, 10.2)

Recognizing the human right to remembrance, the UN and countries around the world mark International Holocaust Remembrance Day on 27 January, commemorating the six million Jews who perished during the Holocaust. In 2018, Israel's Ministry of Foreign Affairs produced an exhibit entitled Beyond Duty, honoring 36 righteous diplomats who rescued hundreds of Jews, risking their lives and the safety of their families. The exhibit was displayed in Israel's Ministry of Foreign Affairs and in 70 missions abroad. In 2019, Israel's Permanent Mission to the UN in New York organized the display of this exhibit in the presence of the UN Secretary General. (SDG 16.b)

A political environment which fosters antisemitism is fundamentally incapable of protecting the human rights of the individual, and therefore stands in direct opposition to democratic values. The Sixth Global Forum for Combating Antisemitism (2018), organized by Israel's Ministry of Foreign Affairs, with the assistance of the Ministry of Diaspora Affairs, was the largest gathering of its kind in the world. Plenary sessions and expert panels focused on Web Antisemitism and Cyber Hate, Antisemitism and the Rise of Far Right Politics in Europe, and Faith as a Resource for Combating Antisemitism and Hate Crimes. (SDG 16.a)

Israel passed the Counter-Terrorism Law (2016) and is working with both international and regional organizations on such issues as the use of the internet for radicalization and extreme violence, recruitment, fundraising, and establishing local cells. The International Institute for CounterTerrorism (ICT) at the Interdisciplinary Center (IDC) Herzliya has for the last 19 years conducted an Annual World Summit, providing an opportunity for high-profile experts and policymakers to establish a platform for cooperation. High-ranking officials from the UN participate, including the Executive Director of the Counter-Terrorism Committee Executive Directorate (CTED), UN Office on Counter-Terrorism, Head of the al Qaeda \& Taliban Monitoring Group, and the Special Envoy of the UN Secretary General for the Israeli-Palestinian Peace Process. In addition, experts from the ICT hold briefings for UN and world leaders. (SDG 16.a)

Israel is actively engaged in the global effort to combat terrorism and violent extremism, and in the future will play a more vital role in the strategic development and implementation of multi-stakeholder activities to enhance international and regional stability. The Director of the UN Office on Counter-Terrorism, and the Assistant Secretary General and Executive Director of the Counter-Terrorism Committee Directorate (CTED) met in Israel with high-ranking government officials to explore opportunities for further cooperation. (SDG 16.a)

In 2017-2018, Israel carried out six practical capacitybuilding counter-terrorism trainings for experts from Africa, Central Asia and Latin America. (SDG 16.a) Israel, a Partner for Cooperation in the Organization for Security Cooperation in Europe (OSCE). collaborates with the Organization on counterterrorism issues. An Israeli expert was chosen by the OSCE to plan a teaching and research framework on the growth of online incitement, radicalization and recruitment for terrorism. Furthermore, Israel hosted an International Conference on Criminal Justice Responses to Terrorism, and an International Conference on the Lone Wolf Phenomenon, both organized in partnership with the UNODC/Terrorism Prevention Branch (TPB), OSCE, and CTED. (SDG 16.a)

Israel participated in the Chairmanship Conference on Cyber Security, organized by the Italian OSCE Chairmanship. The Conference provided a platform to exchange views and perspectives on the latest cyber/ ICT security developments amongst relevant OSCE Participating States and Partners for Cooperation. Discussions included OSCE cyber/ICT confidencebuilding measures (CBMs). Israel also participated in the OSCE Mediterranean Contact Group Meeting on Cyber Security and is actively contributing to the 
Organization's work on advancing CBMs. In addition Israel is active on the Ad Hoc Committee on CounteringTerrorism, established by the OSCE Parliamentary Assembly's Standing Committee. (SDG 16.a)

In the wake of increasing threats to cities worldwide, urban security was the central theme of the MUNI WORLD 2019 City Innovation Conference hosted in Israel by the Federation of Local Authorities, in cooperation with CTED. Aligned with UN Security Council Resolution 2396 (2017) calling on Member States to establish and strengthen national, regional, and international partnerships with public and private stakeholders to prevent, protect, respond and recover from terrorist attacks, the Conference focused on potential solutions to urban security hazards, cyber security, promoting best practices, and encouraging stakeholders to cooperate more effectively. (SDG 16.a)

Cyber threats against government systems are growing in number, and developing countries are increasingly targeted but are often ill-equipped to deal with them effectively. Israel's National Cyber Directorate (INCD) is responsible for advancing and implementing cyber policy, reporting directly to the Prime Minister. CERT-IL is a Division within the INCD operating with a global approach. Cooperation with partners in the international cyber community includes performing joint exercises on threat discovery, sharing actionable information and best practices, assisting in the mitigation of malicious activities, and joint analyses. The INCD also hosts multiple international cyber delegations, including heads of state and CEOs of global organizations. (SDG 16.a)

To encourage knowledge sharing with client governments in cyber security, the World Bank, in cooperation with Israel's Ministry of Economy and National Cyber Bureau, organized a capacitybuilding workshop for delegations from Argentina, Mexico, Colombia, India, Zambia, Kenya, Côte d'Ivoire and Montenegro. The workshop focused on cyber security policies, strategies, technologies and the implementation of a national cyber agency. (SDG 16.a) Israel's Ministry of Justice organized (2017) the International Governance Forum on Leveraging the Multi-Stakeholder Model for Cyber Security
Regulation. Israel is a founding member of the Global Forum for Cyber Expertise and is leading its Working Group on Skills and Strategies. Israel also cosponsored a side event within the framework of the UNODC Commission on Cryptocurrencies, Cybercrime and the Impacts on Women. (SDG 16.a, 5.b) 


\section{GOAL \\ 17}

\section{PARTNERSHIPS}

FOR THE

GOALS

8
Trade

Enhance policy coherence for sustainable development

Encourage and promote effective public, public-private and civil society partnerships

Well-being, sustainability, and national resilience Indicators

Partnering for a Better World 


\section{STRENGTHEN THE MEANS OF}

\section{IMPLEMENTATION AND REVITALIZE \\ THE GLOBAL PARTNERSHIP FOR SUSTAINABLE DEVELOPMENT}

On July 23rd, 2018 the Government of Israel took a major step by approving Government Decision 4021 on Advancing Israeli Activity in the Field of International Development. Based on this decision, the government has appointed its first inter-ministerial committee for international development. The committee has been tasked with formulating Israel's reformed international development strategy. It is headed by the Director General of the Prime Minister's Office and brings together delegates from various government offices and directorates who are involved in the field of international development, first and foremost the Ministry of Foreign Affairs, the Ministry of Finance and the Ministry of Economy and Industry.

The rationale for reforming Israel's international development strategy is the recognition that Israel's global leadership in fields such as agriculture, water, and health can support and accelerate efforts to overcome global development challenges at a higher pace and greater scale than previously done. When formulating the strategy, the committee will examine: tools to encourage Israeli innovation for the unique needs of the developing world; tools to encourage Israel's private sector to take part in international development; financing tools to increase Israel's international development activity, as well as the possibility of establishing an Israeli development finance institution; tools to bolster Israeli aid and to connect it with complementing efforts by civil society and the private sector. 


\section{( Trade (SDG 17.7, 17.8, 17.10)}

Israel operates a network of 103 diplomatic delegations worldwide. A considerable part of their activities is to promote Israel's trade and economic relations. 43 of these delegations, based in target countries for Israeli exports as well as at international economic organizations, have special commercial attaches. Israel continues to draw up trade agreements to improve reciprocal access to the goods and services market, provide legal protection for investments and intellectual property, and other aspects of international trade by lowering customs duties on goods, lifting nontariff barriers, reducing technical barriers to trade, expanding access to government tenders, and more.

The establishment of relations with international financial institutions is a key priority. In order to strengthen relations with foreign banks and promote entrepreneurship in development financing, the Emerging Markets and Financial Institutions Department of the Ministry of Economy and Industry was established in 2016. Projects promoted in the past two years include, among others, accession to the TOV Foundation, which grants micro-loans to farmers in Ethiopia so they can gain access to agriculture technology and joining the USAID-led Power Africa initiative, which aims to connect 60 million residents on the continent to the power grid by 2030 .

\section{( Enhance policy coherence for sustainable development (SDG 17.14)}

\section{Regulatory Impact Assessment (RIA)}

In accordance with Government Decisions 2118 and 4398, all new regulations, whether introduced as a result of legislation or administrative decisions, must be formulated and evaluated using Regulatory Impact Assessment (RIA) methodology (Figure 17.1). In addition to RIA's improving policy coherence as a whole, a key element in the RIA process is assessing the regulations' impact on public welfare, a component which includes among others, environmental impact, thereby promoting environmental and other sustainable development goals. An obligation was set for each ministry to formulate a five-year plan to reduce regulatory burdens in its area of competence. The OECD

Figure 17.1

OECD Indicators of Regulatory Policy and Governance (iREG): Israel, 2018

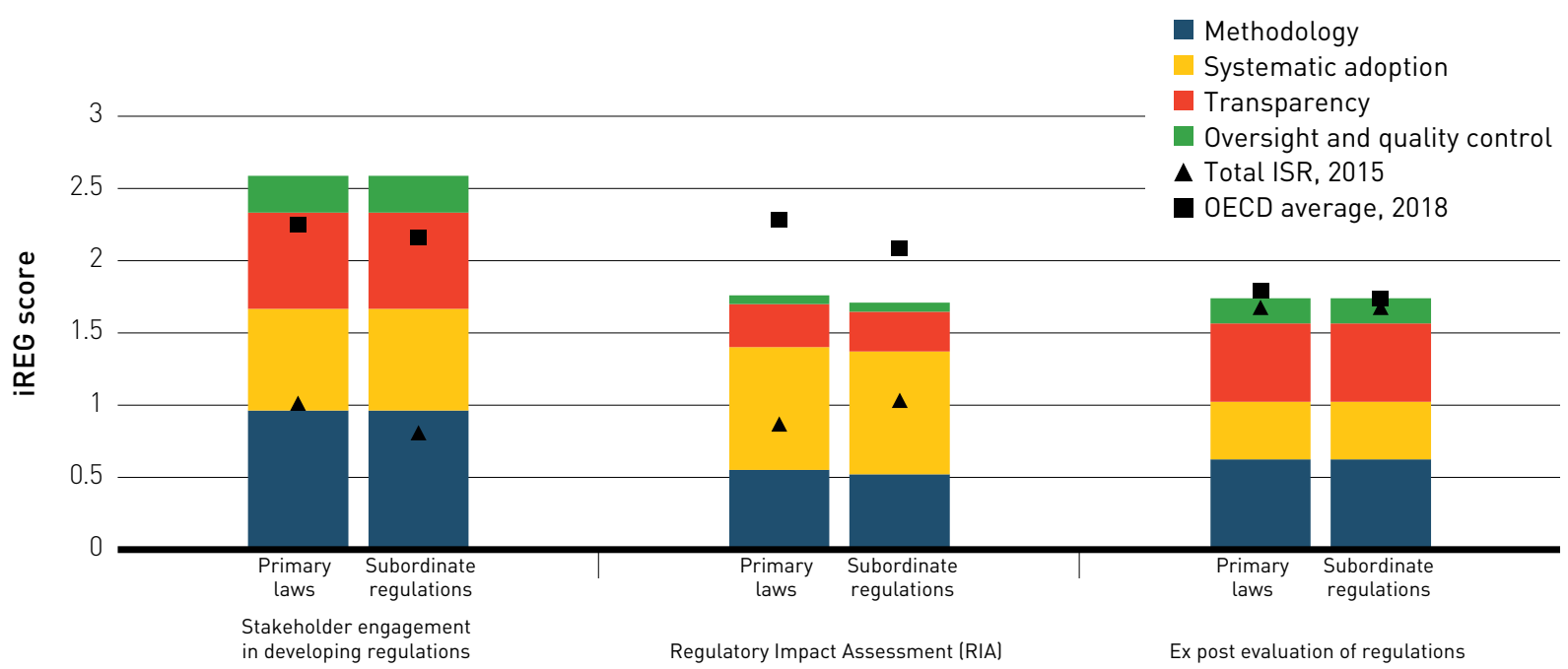

Notes: The more regulatory practices as advocated in the OECD Recommendation on Regulatory Policy and Governance a country has implemented, the higher its iREG score. The indicators on stakeholder engagement and RIA for primary laws only cover those initiated by the executive (53\% of all primary laws in Israel).

Source: Indicators of Regulatory Policy and Governance Surveys 2014 and 2017, http://oe.cd/ireg. Source: StatLink 2 https://doi.org/10.1787/888933815794 


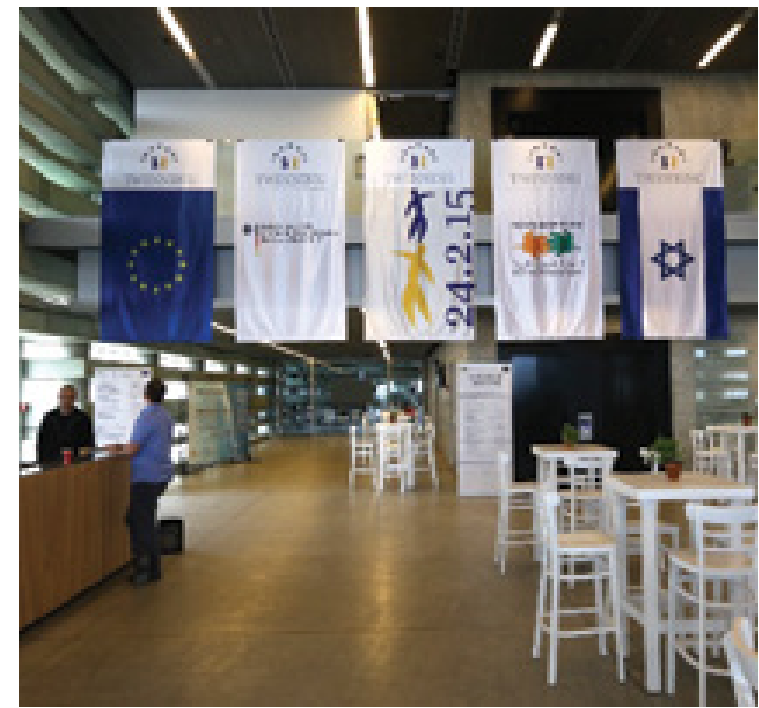

Closing ceremony of IPCC Twinning/Photo: Shauli Lendner

Regulatory Policy Outlook 2018 report notes that the program also examines burdens far in excess of compliance costs - it considers other factors such as organizational and process aspects, quality of service, time and market saving. The report indicates that the program in Israel has helped reduce regulatory burdens by NIS 2.67 billion (approximately $\$ 740$ million) in annual direct costs and saved over 40 million 'waiting' days.

\section{Israel - EU cooperation}

Israel - EU cooperation is largely carried out within the framework of the European Neighborhood Policy (ENP). Within this framework, a National Action Plan for Israel was first agreed upon in 2004 setting out priority areas for cooperation, and bilateral subcommittees were created to plan and oversee collaboration in multiple areas.

Israel actively participates in EU regional programs in the field of environment and climate change, such as the Horizon2020 initiative for a cleaner Mediterranean, SWIM (Sustainable Water Integrated Management), SwitchMed for sustainable consumption and production, ClimaSouth that provides support for climate change mitigation and adaptation and SEIS which promotes Shared Environment Information System for all ENP countries. Most programs have a national component which enables tailor-made activities alongside a regional component which enables cooperation with neighboring countries on environmental issues.

As a partner country of the ENP, Israel benefits from instruments which provide European expertise, such as the Technical Assistance and Information Exchange (TAIEX) and Twinning projects for long term institutional cooperation between EU and non-EU member states.

\section{( Encourage and promote effective public, public-private and civil society partnerships (SDG 17.17)}

Israel is developing its national mechanism for the implementation of Agenda 2030. The Prime Minister appointed an inter-ministerial committee which carried out the mapping and scoping as a prerequisite to preparation of Israel's first sustainable development report, with the aim that the report and committee will form the future regulatory function. Stakeholders are already involved in the process of SDG implementation. Representatives from civil society, the private sector and academia were invited to take part in the inter-ministerial committee's meetings, providing inputs to the meetings and supporting, among others, the discussions on Leave No One Behind. Stakeholders were also invited to submit executive summaries of their SDG related activities as annexes to the 2019 Voluntary National Review.

\section{( Well-being, sustainability, and national resilience Indicators (SDG 17.8)}

The Israel Central Bureau of Statistics (ICBS) is developing, reporting and updating indicators of well-being, sustainability, and national resilience in the following 11 domains: quality of employment; personal security; health; housing and infrastructure; education; higher education and skills; personal and social well-being; environment; civic engagement and governance; material standard of living; leisure, culture and community; and information technology. Eight indicators were selected for each domain, and the desired direction 
of change was defined for each indicator. Data on the indicators were taken from the ICBS databases and are based on administrative information and regular surveys conducted by the ICBS. The indicators present the trends from the beginning of the $21^{\text {st }}$ century and provide a basis for examining changes in well-being in Israel, comparing different population groups in Israel and undertaking international comparisons (see Well-being, Sustainability, and National Resilience Annex)

\section{PARTNERING FOR A BETTER WORLD}

Israel's international development cooperation program has consistently engaged in multilateral partnerships to maximize on-the-ground impact, mobilize and share knowledge and expertise as well as technological innovations, reduce redundancy of human, financial and environmental resources, and prevent providing partner countries with development assistance that cannot be effectively implemented, and is therefore, unsustainable. Israel will continue in its support of the Global Partnership for Sustainable Development to enhance policy coherence on the global, regional, national, and local levels.

As the world moves towards a future increasingly dependent on innovation and technology as strategic priorities, the Knesset (Israel's parliament), in cooperation with the Inter-Parliamentary Union (IPU), hosted a seminar entitled Achieving the SDGs for the IPU's Twelve Plus Geopolitical Group and East Asia Parliaments: Parliamentary Cooperation in Achieving SDGs. The seminar focused on strengthening innovation ecosystems within countries and between countries and developing an effective parliamentary mechanism to ensure coordination and the strong engagement of the institution on matters relating to Agenda 2030. An Outcome Document was circulated, urging parliamentarians to support effective and resultsoriented action to promote the SDGs. The outcome document was later brought to the attention of the $140^{\text {th }}$ IPU Assembly in Doha. (SDG 17.14, 17.16)

Moreover, as requested by the Inter-Parliamentary Union (IPU), in preparation for the above- mentioned conference, the Knesset Research and Information Center prepared a policy document on the ways in which innovation or innovative tools can help parliaments implement the principles of Goal
16 and in particular, the targets of developing effective, accountable and transparent institutions at all levels and ensure responsive, inclusive, participatory and representative decision-making at all levels.

The document focuses mostly on the Knesset. It includes examples both of the ways in which the Knesset has adopted innovative tools and also of the challenges to achieving the aforementioned targets, while providing several examples from other parliaments around the world. The document was later presented at the IPU conference regarding E-parliaments in Geneva. (SDG 17.4, 17.16, 16.6, 16.7)

To widen the scope and effectiveness of Israel's involvement in the global development agenda, during the past two years Israel's Ministry of Economy and Industry has engaged in the following initiatives with international financing institutions:

(SDG 17.7, 17.8]

- World Bank: Three agreements were signed setting up funds (\$2.5 million) on water (technical assistance to African countries on improving the management and use of water resources), agriculture, and cyber. Collaboration also includes extending TechEmerge - a joint program with Israel, Finland, and the $\mathrm{CpC}$ Group on improving access to health services in India, and a market access program in Brazil to improve healthcare delivery through structured pilots and new partnerships;

- International Development Bank (IDB): establishment of a cyber fund;

- Inter-American Development Bank (IADB): providing technical assistance to Latin American countries and implementing pilot programs in cooperation with municipal water 


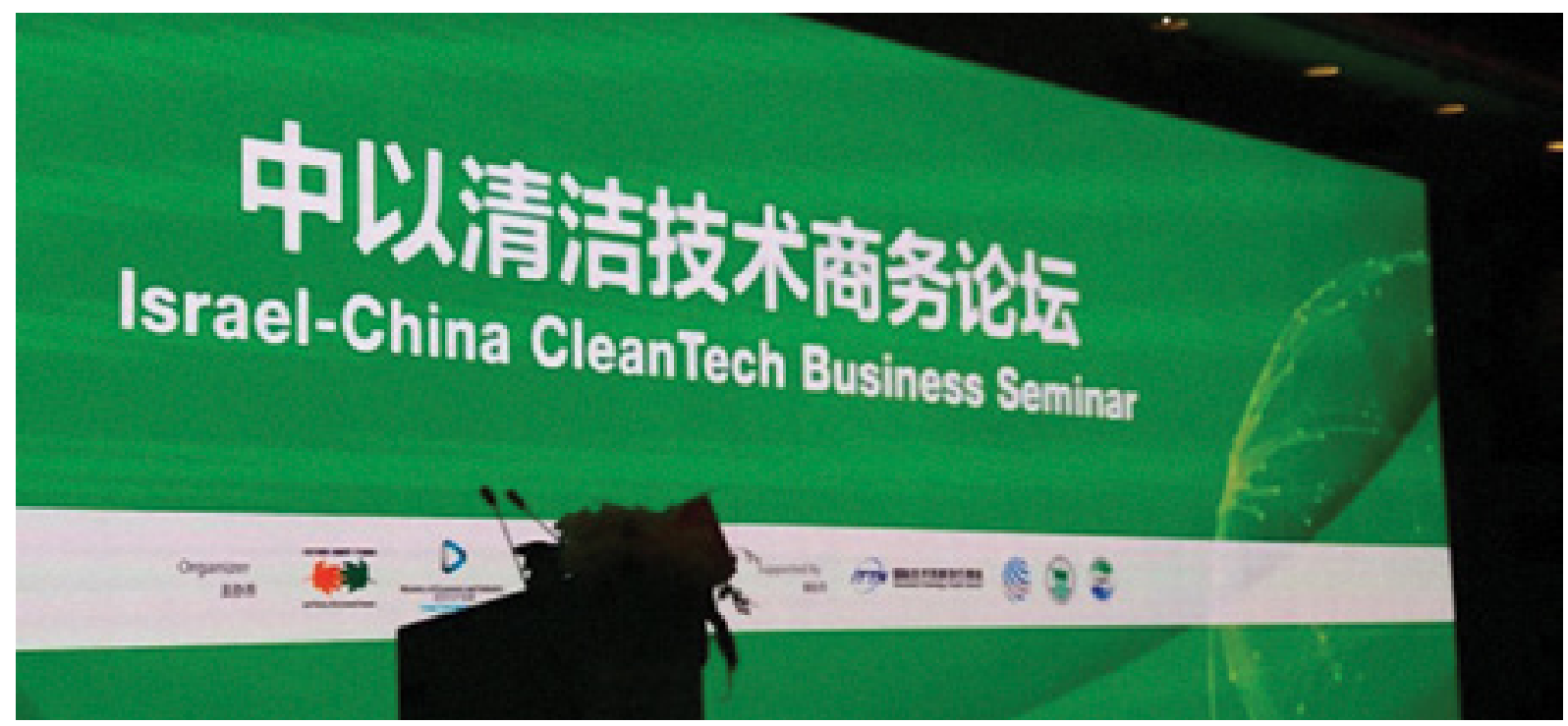

Beijing, September 2017

corporations to improve the management and use of water resources:

\section{- European Bank for Reconstruction and} Development (EBRD): The Ministries of Environmental Protection, Finance and Economy and Industry recently established a fund in cooperation with the European Bank for Reconstruction and Development (EBRD). The €1 million Israel-ERBD Cooperation Fund supports projects in countries where the ERBD is active and in areas such as water, energy and climate change, including the integration of gender equality in climate change interventions and climate finance.

The OECD extends policy guidance to both member and non-member countries. As a member country, Israel plays an active role in the Organization's Environment Policy Committee (EPOC) and is a member of the Bureau responsible for implementing the Environment Program. Israeli experts take part in a number of EPOC subsidiary bodies and are Bureau members in several of them. (SDG 17.14)

In addition, Israel contributes to different OECD projects including dispatching experts as part of the Environmental Performance Review Missions and participating in various fora including the Green Growth and Sustainable Development (GGSD) Forum. Currently, an Israeli expert is serving as a reviewer for the Climate Change Chapter of the OECD Environmental Performance Review of Greece. The Review will conclude in the beginning of 2020. Israel also contributes its best practices to OECD member and partner countries in the framework of the Best Available Techniques project (BAT). (SDG 17.14)

A Memorandum of Understanding (MOU) on Environmental Cooperation was signed by the Ministers of Environmental Protection of Israel and China, followed by the signing of MOUs between Israel's Ministry of Environmental Protection (MoEP) and two Chinese provinces on advancing the exchange of knowledge and promoting the integration of Israel's innovation into projects in China. Discussions focused on high-priority issues including water resource management and climate change adaptation. A three-year Action Plan on Israel-China Environmental Cooperation was signed (2017) by both governments on the management of water issues, air quality, soil remediation, waste management, and research and development in environmental science and technology. For the past few years, the Senior Deputy Director General of Israel's MoEP held the position of Special Consultant to the China Council for International Cooperation on Environment and Development. (SDG 17.16)

Israel's MoEP joined (2018) the International Coalition for Green Development on the Belt 
and Road, a joint initiative of China and various international partners. The vision is to incorporate environmental sustainability considerations across different areas of focus and strengthen environmental governance to ensure that investments made under the Belt and Road are green and contribute to the 2030 Agenda. Israel will share its expertise and information regarding advanced environmental policies and technologies for better implementation. (SDG 17.6)

Israel and the UN Office for Outer Space Affairs (UNOOSA) have developed a robust agenda for cooperation, as follows: (SDG 17.16, 17.17)

- As a spacefaring nation, Israel attaches great importance to cooperation with UNOOSA to maximize the use of space technology. Towards this end, Israel joined (2015) the UN Committee on the Peaceful Uses of Outer Space (COPUOS). Furthermore, a Framework Agreement for Cooperation was signed (2016) between the Office, on behalf of the UN, and the Government of Israel.

- Within the framework of the Framework Agreement for Cooperation, an MOU on Joint Collaboration was signed (2016) at a ceremony during Israel's Space Week between UNOOSA and Israel's Space Agency (ISA). The MOU affords Israel opportunities for membership in committees and enables Israeli scientists to participate in projects having global impact. One of Israel's fields of focus is the use of satellites to contend with catastrophes in real time, while helping search and rescue teams (SPIDER Initiative)

- In 2018, UNISPACE+50 celebrated the 50th anniversary of the first UN Conference on the Exploration and Peaceful Uses of Outer Space, held in Vienna in 1968. The event provided an opportunity for the international community to consider the future course of global space cooperation. During the conference, Israel and France organized a joint side event on the Venus Satellite, presenting the Director of UNOOSA with a mock-up of the satellite to be placed in the Office's permanent exhibit;

- Israeli-French collaboration in space was first celebrated (2017) at UN headquarters in New
York in a joint event on Satellite Technology for Sustainable Development. The two countries highlighted the contributions of the Venus Satellite to climate and environmental research in an effort to advance the Israel-initiated UN Resolution on Agricultural Technology for Development;

- ISA hosted the $66^{\text {th }}$ International Astronautical Congress (IAC) in Jerusalem in cooperation with the International Astronautical Federation (IAF). The theme of the event, Space - The Gateway for Mankind's Future, supported IAF's platform to advance knowledge about space and foster the development of space assets by facilitating global collaboration. The Congress saw the participation of many of Israel's high-tech companies, academia, and research institutions;

- The Space Generation Advisory Council (SGAC) Israel is active in the global space community and hosted the 2016 International Space University's (ISU) Space Studies Program (SSP).

Recognizing the importance of achieving policy and institutional coherence in the global effort to eradicate poverty, Israel supports The Partnership in Statistics for Development in the $21^{\text {st }}$ Century (PARIS21) to promote the better use and production of statistics throughout the developing world committed to evidence-based decision-making. Israel is also a member of the UN Economic Commission for Europe (UNECE) Working Group on Gender Statistics. (SDG 17.18)

Paragraph 89 of the 2030 Agenda for Sustainable Development calls on major groups and other stakeholders to report on their contribution to the implementation of the SDGs, with specific reference to civil society organizations (CSOs). Recognizing the importance of civil society to the development process, Israel's Permanent Mission to the UN in New York hosted a special forum celebrating 70 years of Israeli civil society. Four NGOs gave presentations including Innovation: Africa, a CSO granted special consultative status to the UN Economic and Social Council (ECOSOC). At the UN Global South-South Development Expo in Nairobi, Innovation: Africa won the Innovation Award for bringing off-grid Israeli remote monitoring technology to power schools, medical clinics, 
orphanages and water pumping systems in rural Africa, transforming lives in 10 African countries. Farmers were able to grow more crops with less water and use solar energy systems to pump water from aquifers saving villagers - mostly women and children - from countless hours previously spent finding water. (SDG 17.6)

To heighten public awareness of the 2030 Agenda for Sustainable Development, the School of Sustainability at the Interdisciplinary Center (IDC) in Herzliya conducts a series of annual conferences in cooperation with UN specialized agencies, government ministries, civil society, country aid agencies, and international organizations. With the participation of representatives from all sectors and facets of society, the conferences focus on issues including outreach to aid actors to enhance effective and targeted implementation of the SDGs in alignment with the Global Partnership for Sustainable Development. (SDG 17.16)

In alignment with SDG 17, MASHAV emphasizes the importance of creating effective partnerships for development and of implementing cooperative projects both in Israel and abroad with a wide range of partners with each side contributing its strengths and unique experience and expertise. This is achieved through active dialogues for development with donor countries and sister agencies and signing bi- and trilateral development cooperation agreements with partner countries. (SDG 17.6.1)

With the goal of enhancing triangular regional and international cooperation through improved coordination among existing mechanisms, in particular at the UN level (SDG 17.6), MASHAV's multilateral development activities in 2018 included, among others, cooperation with UNIDO, UNWomen, FAO, UNOSSC, UNECE, UN-Environment, UN-Habitat, UNESCO, UNDP, and the WMO. Among others, regional seminars were conducted with the following in 2018:

- MASHAV-Visegrad Countries: A first of its kind Regional Seminar on Innovative Entrepreneurship for leading entrepreneurs in cooperation with the International Visegrad Fund and Israel Ministry of Foreign Affairs' (MFA) Economic and the European Divisions.
- Israel-Cyprus-Greece: A first joint Regional Seminar on Innovation Road Map: Scaling-Up Start-Ups was held in Israel in cooperation with Israel MFA's Economic and European Divisions.

MASHAV implements regional, bilateral, and multilateral development cooperation programs with Israel's neighbor countries in partnerships with government ministries, civil society, academia and the private sector in order to address development issues (SDG 17.9). Working together to meet common challenges contributes significantly to fostering personal and professional ties, as well as promoting better understanding between neighboring countries. 


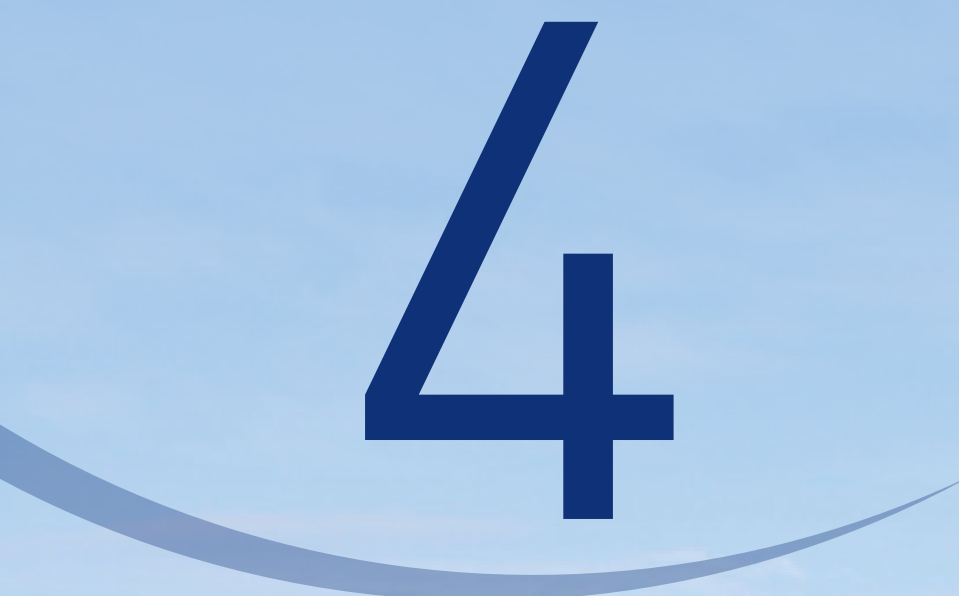

\section{STATISTICAL ANNEX INDICATORS FOR SUSTAINABLE DEVELOPMENT GOALS IN ISRAEL}
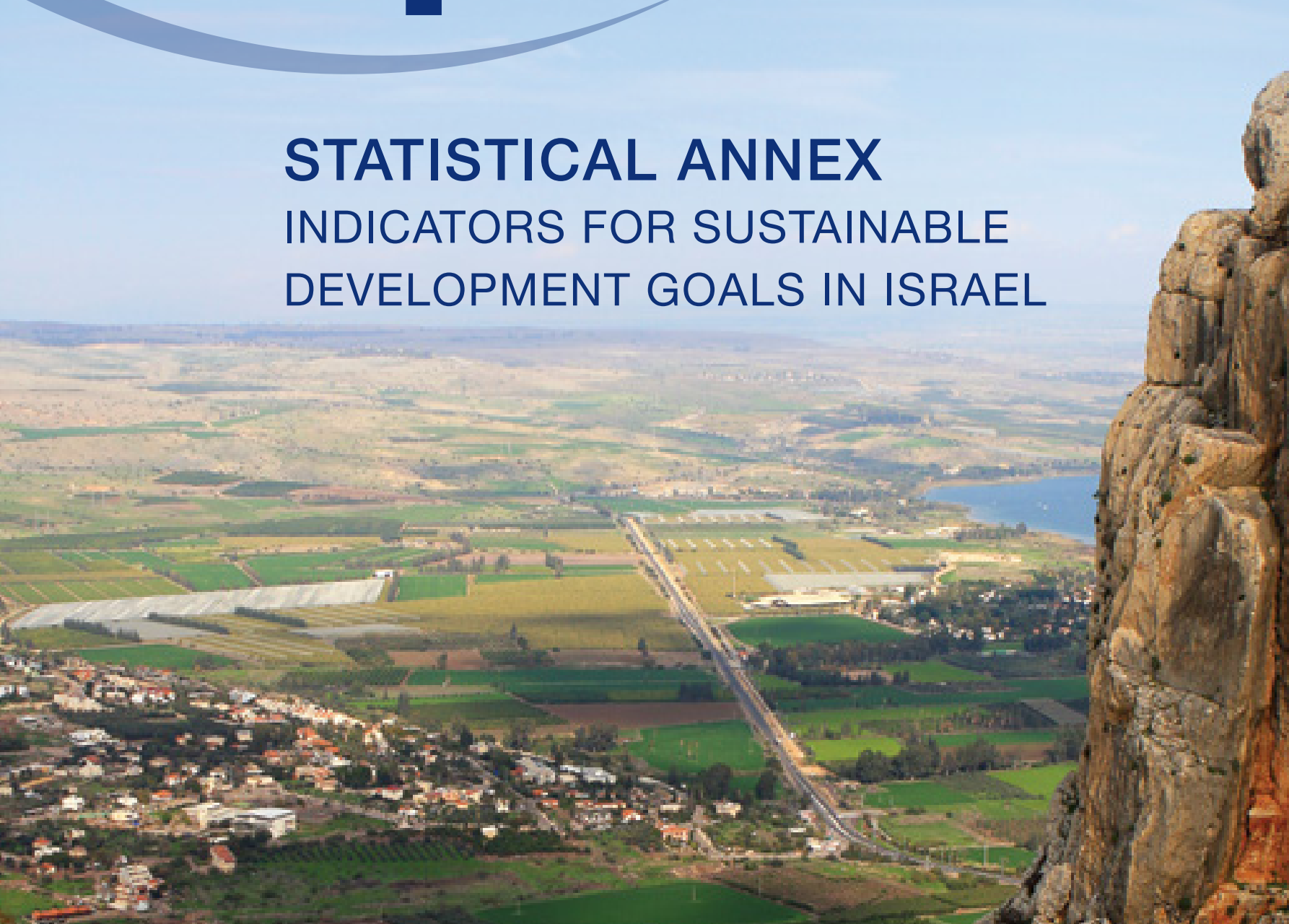


\section{PREFACE}

This statistical report presents data on selected indicators showing the current state and trends with respect to measuring Israel's progress towards meeting the SDGs. The indicators in the report are based on available data in the Israeli national statistical office, the Central Bureau of Statistics (ICBS), and on available data existing in additional government agencies that are responsible for producing official statistics under the framework of the National Statistical System (NSS).

The ICBS serves as the national focal point for all processes connected to collecting and reporting data on SDG indicators. This also includes indicators that are not part of the official statistics produced by the ICBS and the NSS. Such indicators mainly refer to policy measures and legislation related to the SDGs.

The process of collecting SDG indicators

Structure and content
Prior to the preparation of the Israel Voluntary National Review, ICBS started a process of mapping available SDG indicators data within the national statistical office (NSO) and in other governmental agencies for the purpose of providing maximum international reporting of all relevant data. This mapping process was very complex at times since relations between international organizations and national data producers and suppliers often did not involve the NSO. One of the main contributions of the governmental VNR process in this respect is that it created an intergovernmental task force. Through the mechanism of the intergovernmental VNR task force it was possible to improve the quality of information concerning availability, relevance and development needs for the future. The initial ICBS mapping was submitted to all members for correction and completion. Members were also asked to send all past SDG reports to the ICBS and to coordinate all future reports with the ICBS so that after submitting Israel's first VNR no new data gaps will remain.

ICBS data for this report are based on two main types of data sources:

- Surveys conducted on an ongoing basis by the ICBS. These include surveys such as the labor force survey, household expenditure survey, social survey, victimization survey and more.

- Administrative sources such as the population register, social security and tax authority files, administrative files of the health and education systems and others. 
Data for the selected indicators in this report include presentation of the current state at the national level and wherever possible the trend of at least the past ten years as well as international comparisons and any relevant disaggregation of the indicators (e.g., by sex, population group, age and more). The data presented in the report also include statistics and indicators that do not match precisely the international UN SDG indicator definition, but contain relevant data that can serve as proxies of information that help understand the state of the target and goal.

As of December 2018, data for 123 SDG indicators are available, of which 58 have already been reported through the coordination role of ICBS to different international custodian agencies. All data collection and publication is conducted in accordance with the Israeli Statistical Ordinance ${ }^{1}$. The Statistical Ordinance defines the role of the national statistician as responsible for all official statistics in Israel and, as such, responsible for all SDG indicators data reported by the ICBS or other members of the NSS.

The way forward

The data presented in this report are intended to serve as a basis for further development of SDG related statistics in the ICBS as well as a starting point for strengthening and improving relevant work of the NSS for the purpose of building a complete national knowledge platform for monitoring progress towards achieving the SDGs.

$1 \mathrm{http} / /$ :www.cbs.gov.il/www/statistics_ordinance_e.pdf The Statistics Ordinance [New Version], 5732 - 1972 


\section{STATISTICS}

\& INDICATORS 


\section{GENERAL BACKGROUND DATA}

\section{AREA AND LAND USE}

Total area, land area, lakes and districts (sq. km.)

Land use 2013

\begin{tabular}{|l|l|}
\hline Israel - Grand Total & 22,072 \\
\hline Land Area - Total & 21,643 \\
\hline Area of Lakes - Total & 429 \\
\hline Jerusalem District & 653 \\
\hline Northern District & 4,473 \\
\hline Haifa District & 866 \\
\hline Central District & 1,294 \\
\hline Tel Aviv District & 172 \\
\hline Southern District & 14,185 \\
\hline
\end{tabular}

Land use 2013

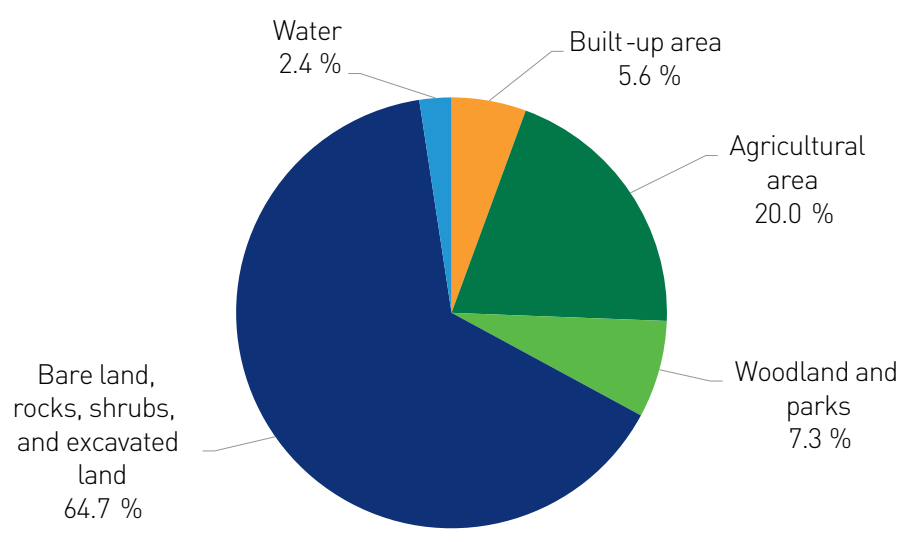




\section{POPULATION 2017}

Population

Immigration to Israel

Migration of Israelis

abroad (2016)

Households

and families

Live births

and fertility

Population density per sq. $\mathrm{km}$. by district
Total Population - 8,798,000

Jews and Others - $79.1 \%$

Arabs - $20.9 \%$

Others $-4.5 \%$

Annual population growth rate $-2.0 \%$

Immigrants since the establishment of the state (1948) - 3.2 million Immigrants to Israel in $2017-26,400$

Departed from Israel - 15,200

Returned to Israel - 8,900

Private households - 2,510,300

Average number of persons per household - 3.32

Nuclear families - 2,057,200

Live newborns - 183,648

Total fertility rate, average number of children per woman - 3.11

\begin{tabular}{|l|l|l|l|l|}
\hline District & 1990 & 1995 & 2008 & 2017 \\
\hline Grand Total & 220.4 & 247.4 & 323.1 & 387.4 \\
\hline Jerusalem District & 922.4 & $1,035.6$ & $1,384.6$ & $1,698.1$ \\
\hline Northern District & 178.9 & 211.4 & 277.7 & 318.7 \\
\hline Haifa District & 768.4 & 860.9 & $1,025.7$ & $1,170.8$ \\
\hline Central District & 830.7 & 953.2 & $1,368.2$ & $1,667.3$ \\
\hline Tel Aviv District & $6,439.4$ & $6,678.6$ & $7,318.5$ & $8,176.8$ \\
\hline Southern District & 40.7 & 53.0 & 75.2 & 89.7 \\
\hline
\end{tabular}


Projection of population

Local authorities and characterization and classification of geographical units by the socio-economic level of the population
According to the projection for the years 2015-2065, the population in Israel is expected to reach 10 million residents by the year 2024 178.8\% Jews and others and 21.2\% Arabs). By the year 2065, the population is expected to reach 20 million (80.7\% Jews and others and 19.3\% Arabs).

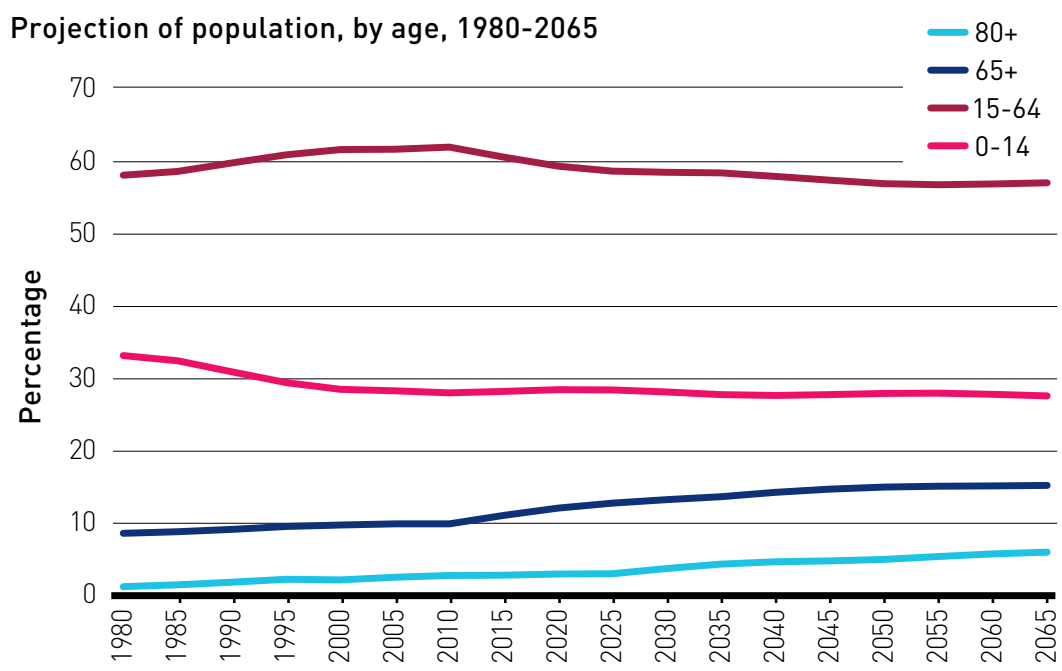

Local authorities in Israel administer the local affairs of a locality or group of localities. They are divided according to their municipal status into municipalities (cities), local councils, and regional councils la regional council includes several forms of localities, such as moshavim, kibbutzim, and rural localities).

As of 2017, there are 255 local authorities in Israel. $74.2 \%$ of the population resided in municipalities (cities), $14.7 \%$ of the population in local councils, $10.3 \%$ of the population in regional councils and $0.8 \%$ of the population in localities with no municipal status.

The concept of socio-economic level of the population of a geographical unit reflects a combination of basic characteristics of a specific geographical unit investigated (for example, the population of a local authority). 
The central aspects that comprise the socio-economic level of residents of a geographical unit are:
Distribution of local authorities by socio-economic cluster
- Financial resources of the residents (from work, benefits, etc.)

- Housing - density, quality, and other components of this aspect

- Home appliances, e.g., air conditioner, dishwasher, personal computer

- Motorization level - quantitative and qualitative

- Education

- Employment and unemployment characteristics

- Various types of socio-economic distress

- Demographic characteristics

The ICBS produces socio-economic indices based on these characteristics. The socio-economic index for local authorities characterizes each local authority by the socio-economic level of the population consisting of its residents relative to the socio-economic level of the populations in all other local authorities.

Based on the socio-economic index, all local authorities were grouped into 10 clusters, whereby cluster 1 represents localities of lowest socioeconomic level and cluster 10 represents localities of highest socioeconomic level.

\section{Distribution of local authorities by socio-economic cluster}

50

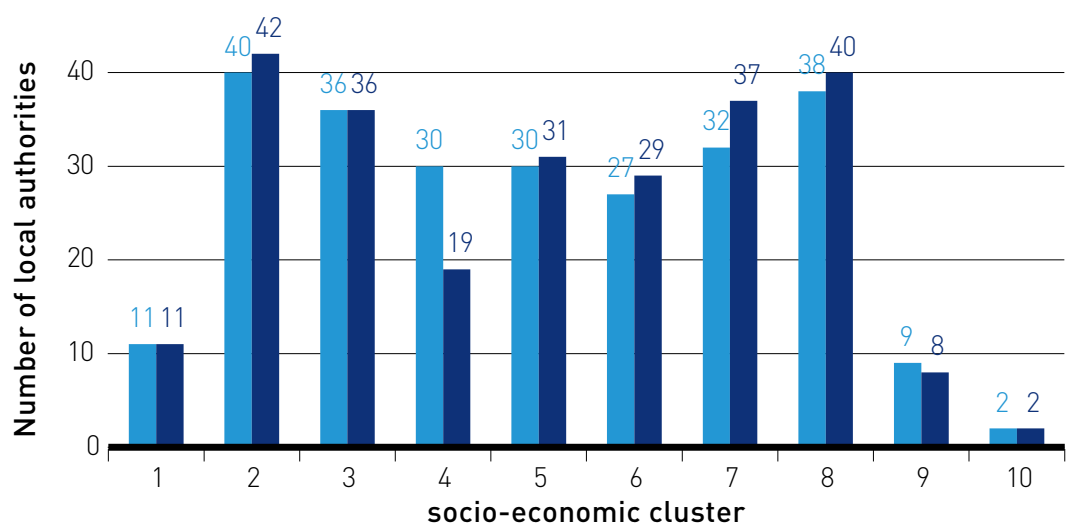


Distribution of population in local authorities by socio-economic cluster 2017
Distribution of population in local authorities by socio-economic cluster, 2017

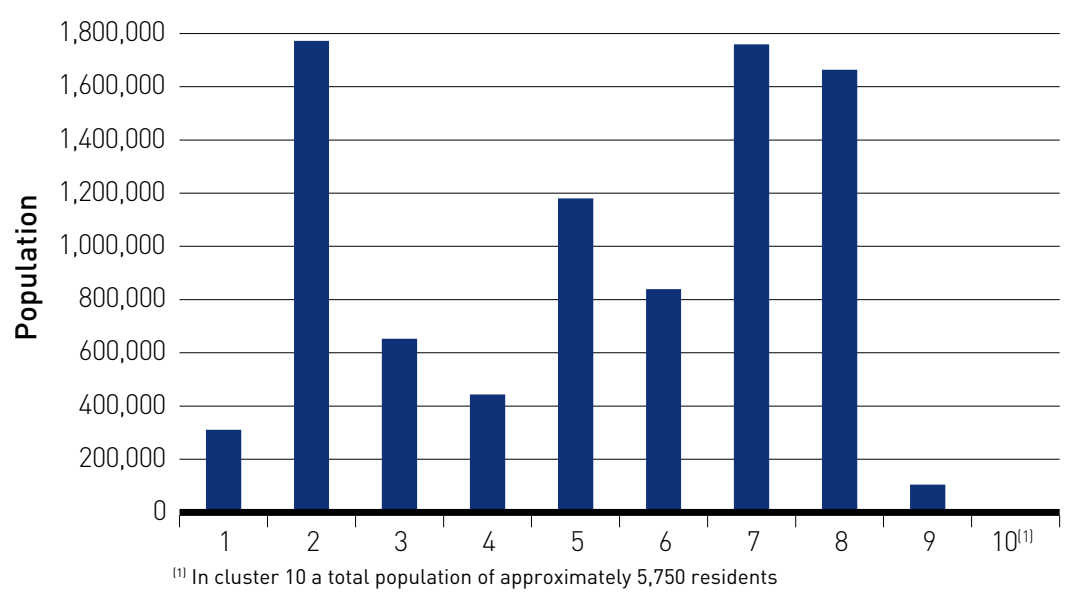




\section{SELECTED SDG}

INDICATORS \& DATA

\section{GOAL 1}

\subsection{1}

Proportion of population living below the national poverty line, by sex and age.

\subsection{2}

Proportion of men, women and children of all ages living in poverty in all its dimensions according to national definitions

\section{END POVERTY IN ALL ITS FORMS EVERYWHERE}

The national poverty line is defined as 50 percent of median income.

Persons below the national poverty line, 2005-2017

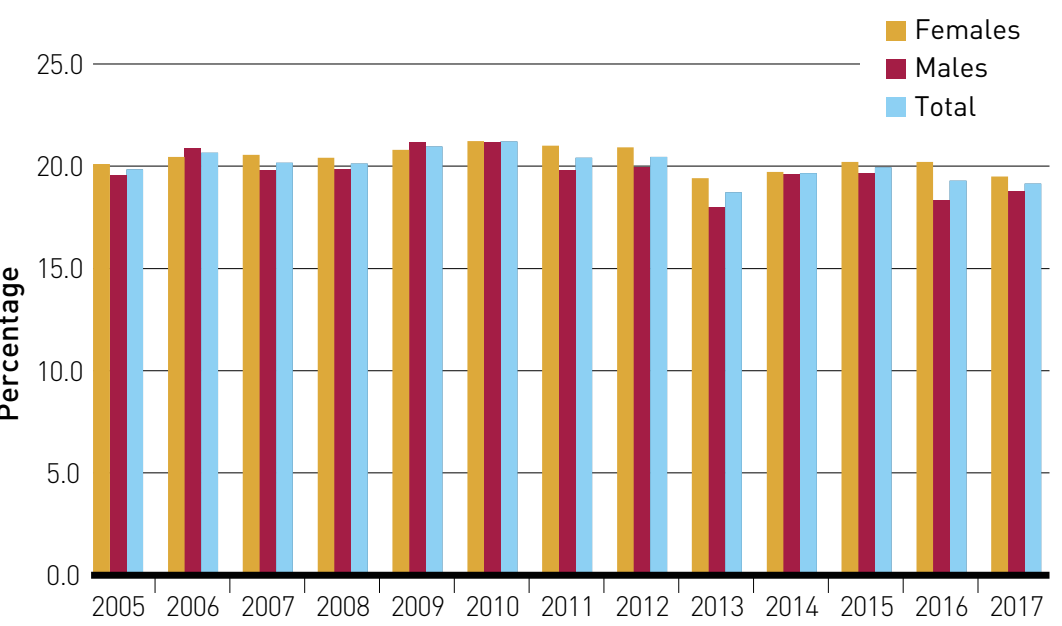

Based on EU definitions of poverty and social exclusion, $27.0 \%$ of the population was at risk of poverty in 2017, higher than the EU average (17.0\%). The net money income per standard person in the upper quintile was 6.8 times higher than the income in the lower quintile. This gap is higher than the average gap in the EU (5.0).

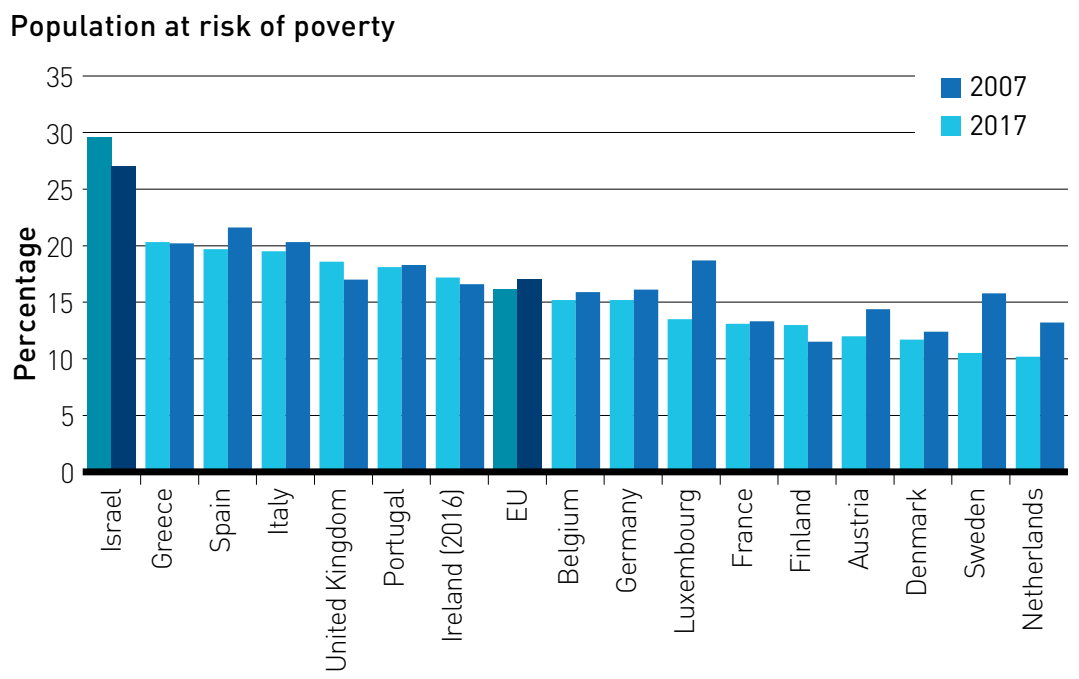




\section{2 .2}

\subsection{1}

Proportion of population covered by social protection floors/systems, by sex, distinguishing children, unemployed persons, older persons, persons with disabilities, pregnant women, newborns, work-injury victims and the poor and the vulnerable
Population at risk of poverty ages 0-17 and 65+, 2017

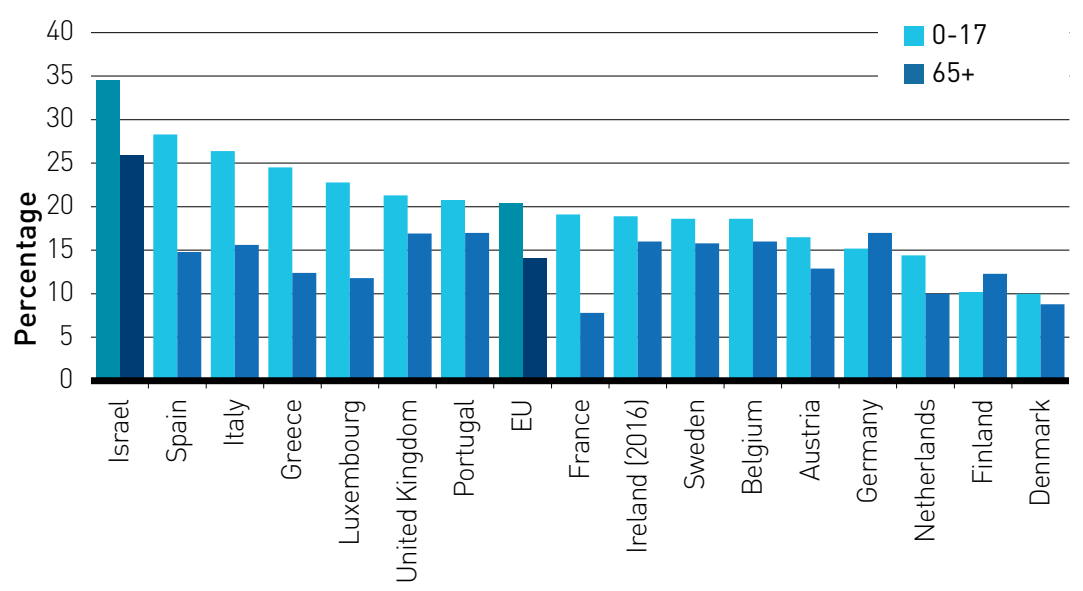

The social protection systems included are: family (children's) allowances, old age pension, survivor pension, work damages allowance, general disability, unemployment benefits, income support, other allowance, nursing allowance and birth allowance.

Percentage of persons age 15 and over covered by social protection systems

\begin{tabular}{|l|l|l|l|}
\hline & $\mathbf{2 0 1 0}$ & $\mathbf{2 0 1 5}$ & $\mathbf{2 0 1 7}$ \\
\hline Total & 37.1 & 38.6 & 38.4 \\
\hline Men & 19.5 & 18.6 & 18.7 \\
\hline Women & 53.7 & 57.4 & 57.0 \\
\hline
\end{tabular}




\section{1.a.2}

\section{Proportion of total} government spending on essential services leducation, health and social protection)
General Government expenditure on health, education and social protection as a percentage of the total general government expenditure, according to COFOG (Classification of Functions of Government). The government sector includes government ministries, the National Insurance Institute, national institutions, local authorities and public non-profit institutions whose expenditures were mostly financed by the government.

Expenditure of the general government sector on education, health and social protection as a percentage of total expenditure, 2005-2017

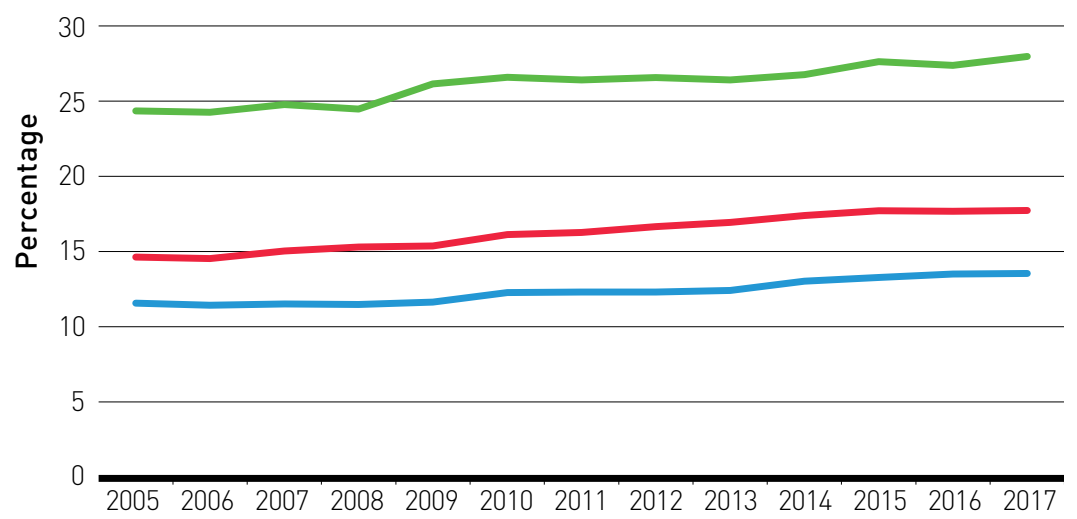


GOAL 2

\subsection{2}

Prevalence of moderate or severe food insecurity in the population, based on the Food Insecurity Experience Scale (FIES).

\section{2.a.1}

The agriculture orientation index for government expenditures

\section{END HUNGER, ACHIEVE FOOD SECURITY AND IMPROVED NUTRITION AND PROMOTE SUSTAINABLE AGRICULTURE}

The 2016 Food Security Survey ${ }^{2}$ is the third survey carried out nationwide by the Research and Planning Administration of the National Insurance Institute of Israel (NII).

The findings show that in $2016,82.2 \%$ of families were food secure, and $17.8 \%$ experienced food insecurity. The 2016 survey reveals an improvement in food security in the population compared to the $2011 / 2012$ survey. The trend of improvement among the population as a whole is supported by a drop between the two periods in the proportion of families and individuals living in poverty. This decline is well reflected among families with children, where the level of food insecurity dropped from rates of around $30 \%$ to around $20 \%$.

The agriculture orientation index for central government expenditures (AOI = Agriculture Share of Government Expenditures / Agriculture Share of GDP), 2005-2017

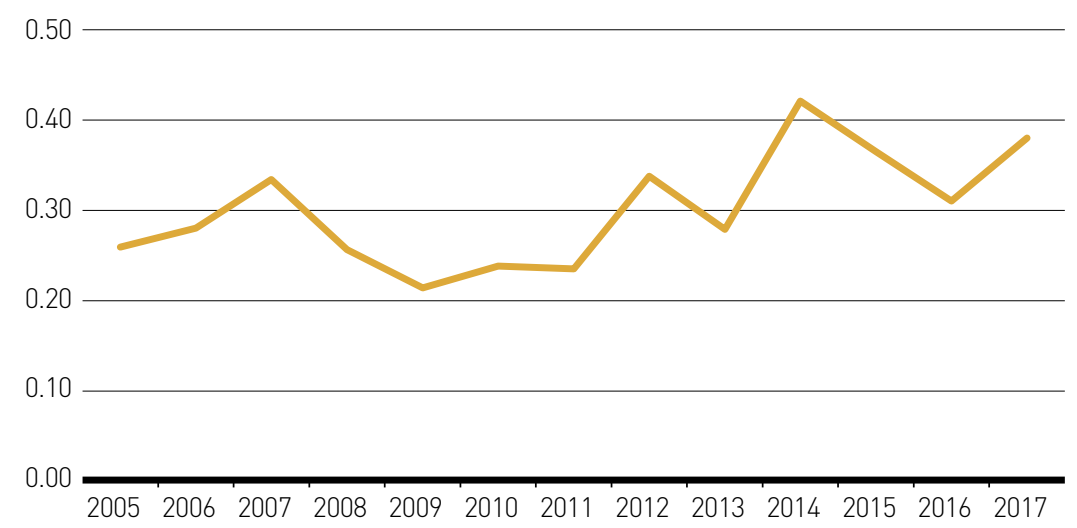

(2) "Food Security Survey 2016 Main Socio-economic Findings," Miri Endeweld, Oren Heler, Netanela Barkali, Daniel Gottlieb. 2018. 
2.a.1

2.a.1
Ratio of agriculture, forestry and fisheries value added to GDP, 2005-2017

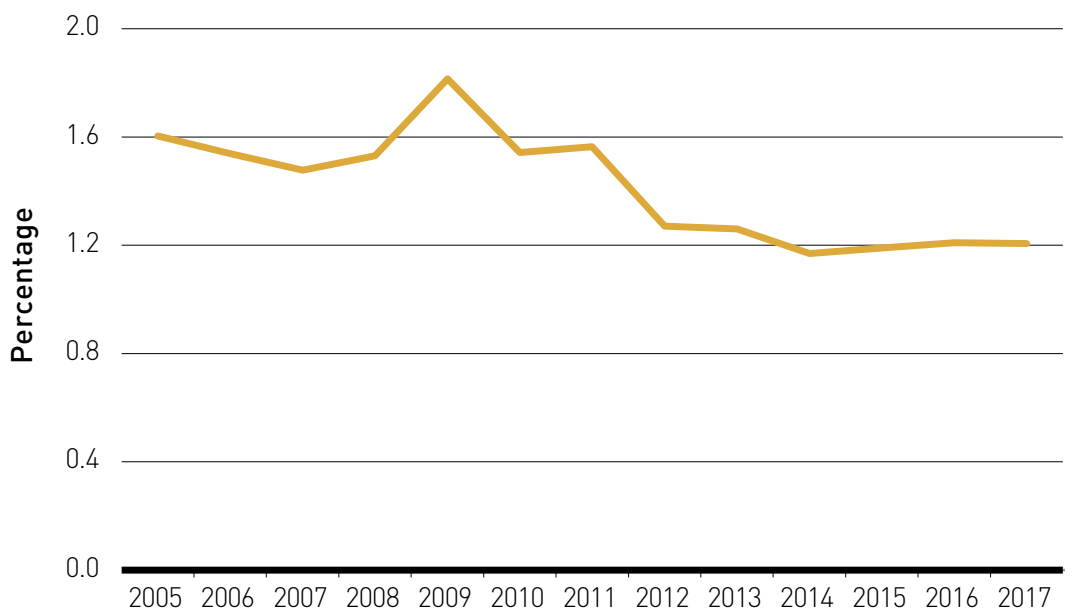

Central government expenditure on agriculture, forestry and fisheries as a percentage of central government expenditure, 2005-2017

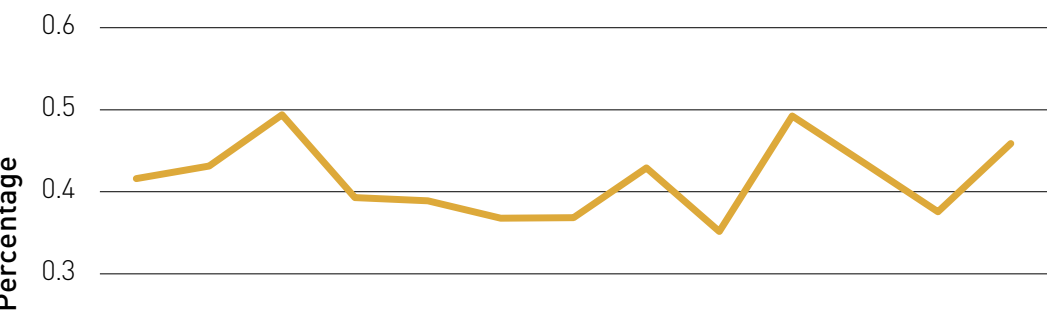

0.2

0.1

0.0

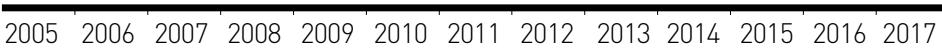


GOAL 3

\subsection{1}

\section{Maternal mortality ratio}

\section{1 .2}

Proportion of births attended by skilled health personnel

\subsection{1}

Under-five mortality rate

\section{ENSURE HEALTHY LIVES AND PROMOTE WELL-BEING FOR ALL AT ALL AGES}

Maternal mortality ratio (deaths per 100,000 live births), 2005-2016

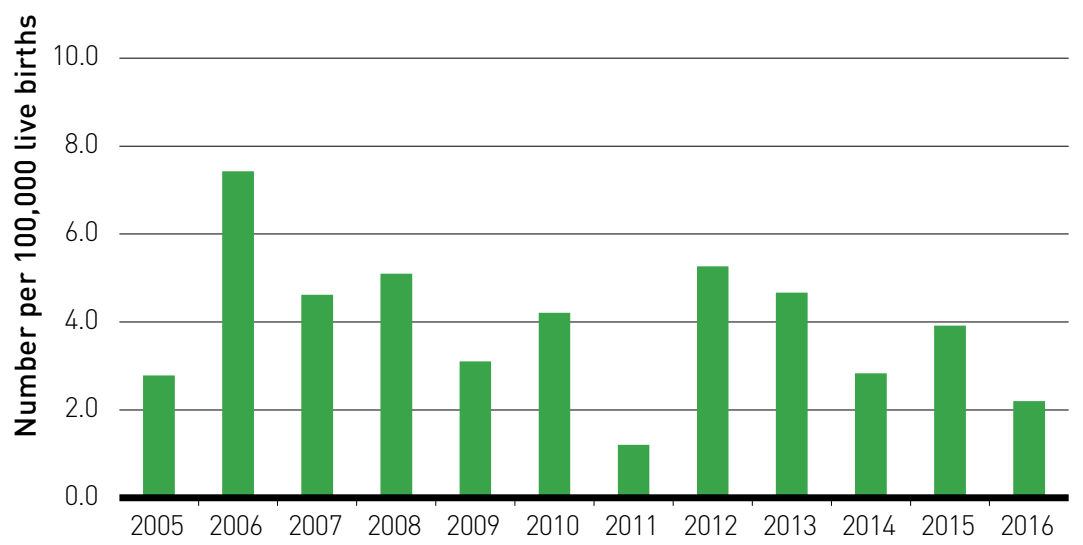

The proportion of births attended by skilled health personnel in Israel is estimated at $100 \%$

Infant mortality rate in Israel in 2017 was 3.0 per 1,000 live births, lower than the OECD average (3.9 per 1,000 live births).

Under five mortality rate (relative to the number of live births), 2005-2017

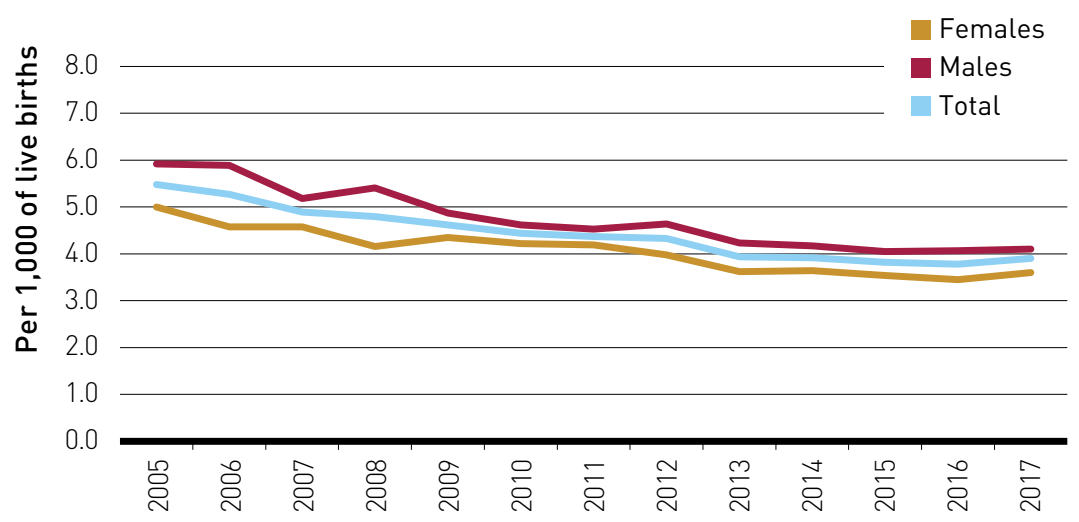




\section{2 .2}

\section{Neonatal mortality rate}

3.3.1

Number of new HIV infections per 1,000 uninfected population, by sex, age and key populations

\section{3 .2}

Tuberculosis incidence per 1,000 population
Neonatal mortality rate (number of deaths of children dying in the first 28 days of life relative to the number of live births), 2005-2017

Females

Males

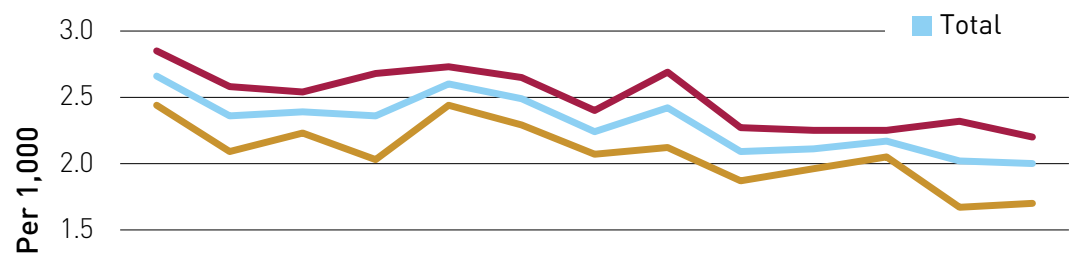

1.0

0.5

0.0

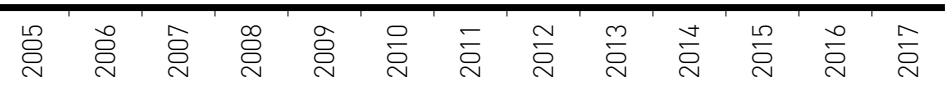

The number of new HIV infections per 1,000 uninfected population in Israel is very low, less than 0.1 per 1,000

Tuberculosis incidence, 2005-2017

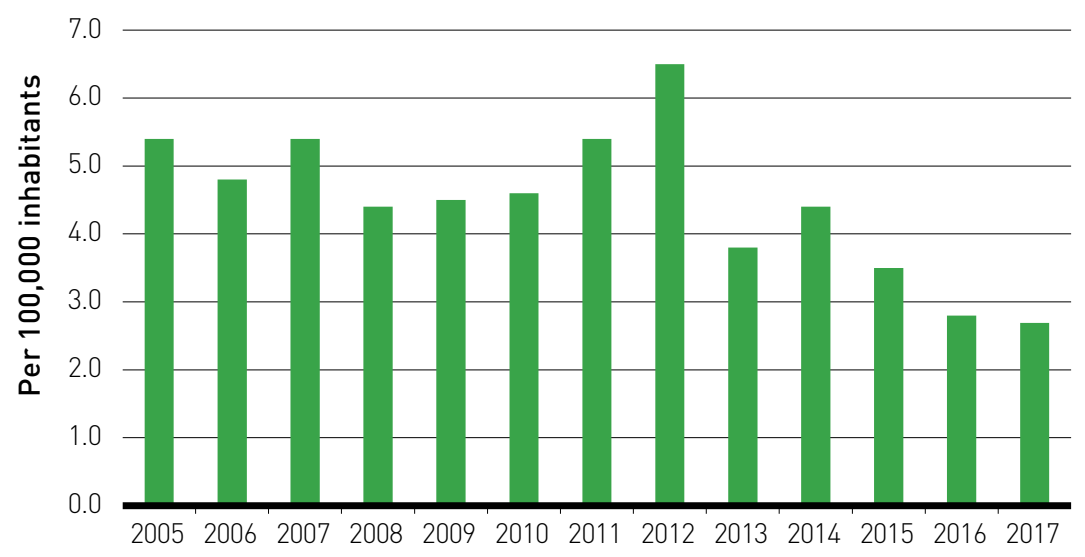




\subsection{3}

Malaria incidence per 1,000 population

\subsection{4}

\section{Hepatitis B incidence per} 100,000 population

\subsection{5}

Number of people requiring interventions against neglected tropical diseases
All cases of malaria in Israel are imported and therefore are not included in the indicator.

Hepatitis B incidence, 2005-2017

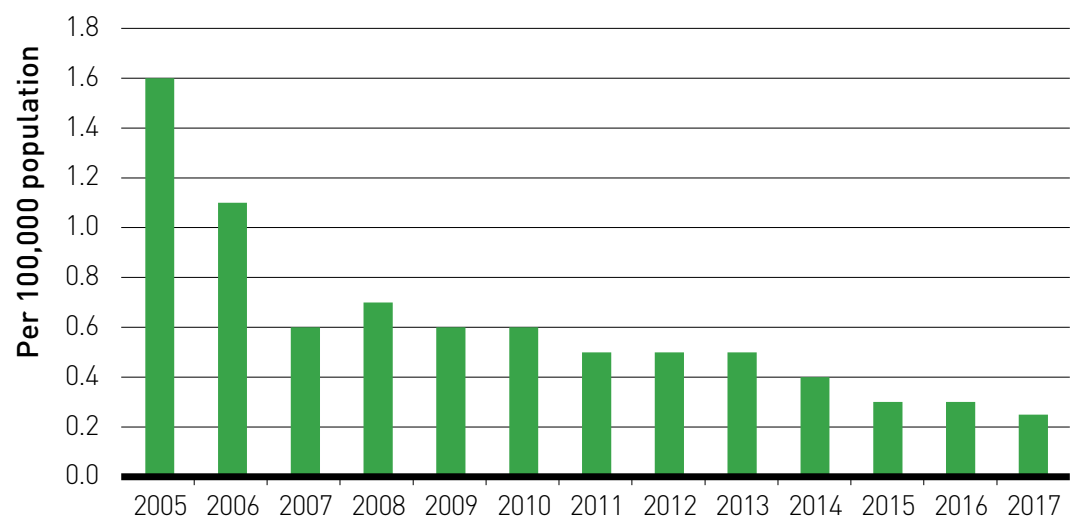

In 2006, the majority of NTDs reported consisted of cutaneous leishmaniasis and scabies. During the years 2007-2017, the vast majority of NTDs reported consisted of cutaneous leishmaniasis.

\section{People requiring interventions against} neglected tropical diseases, 2006-2017

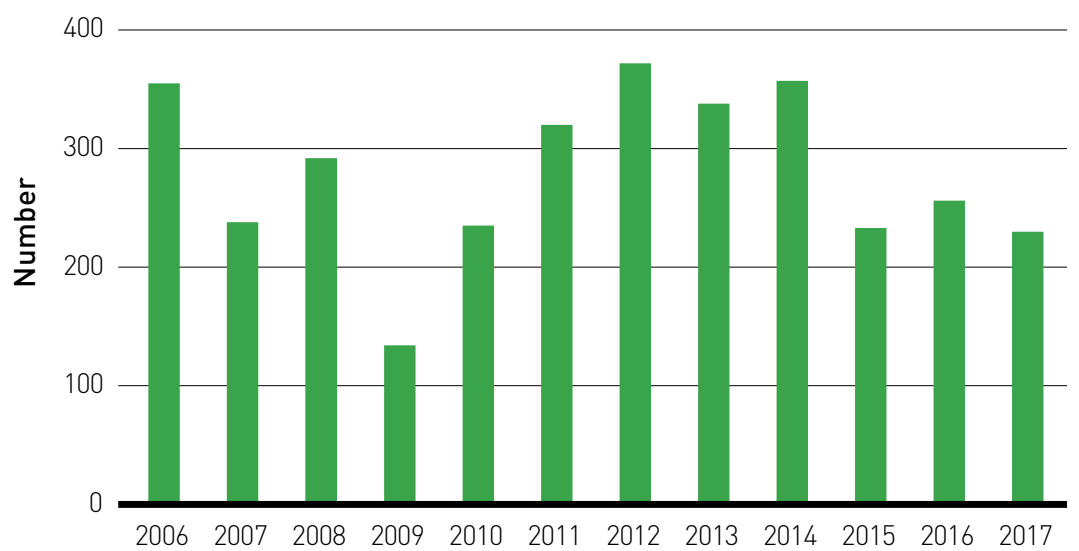




\subsection{1}

\section{Mortality rate attributed}

to cardiovascular disease, cancer, diabetes or chronic respiratory disease

\section{4 .2}

Suicide mortality rate
In 2016, 25.2\% of the deaths in Israel were caused by malignant neoplasms (cancer) and $14.6 \%$ were caused by heart disease.

Persons aged 30 who would die before age 70 from chronic diseases, 2005-2016

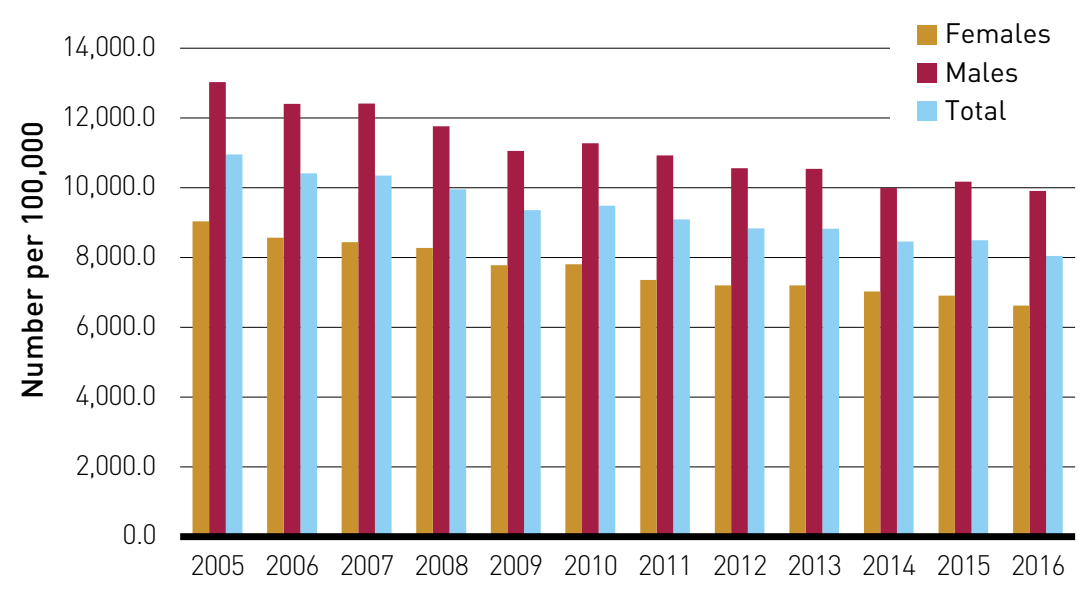

Suicide mortality rate, $2005-2016$

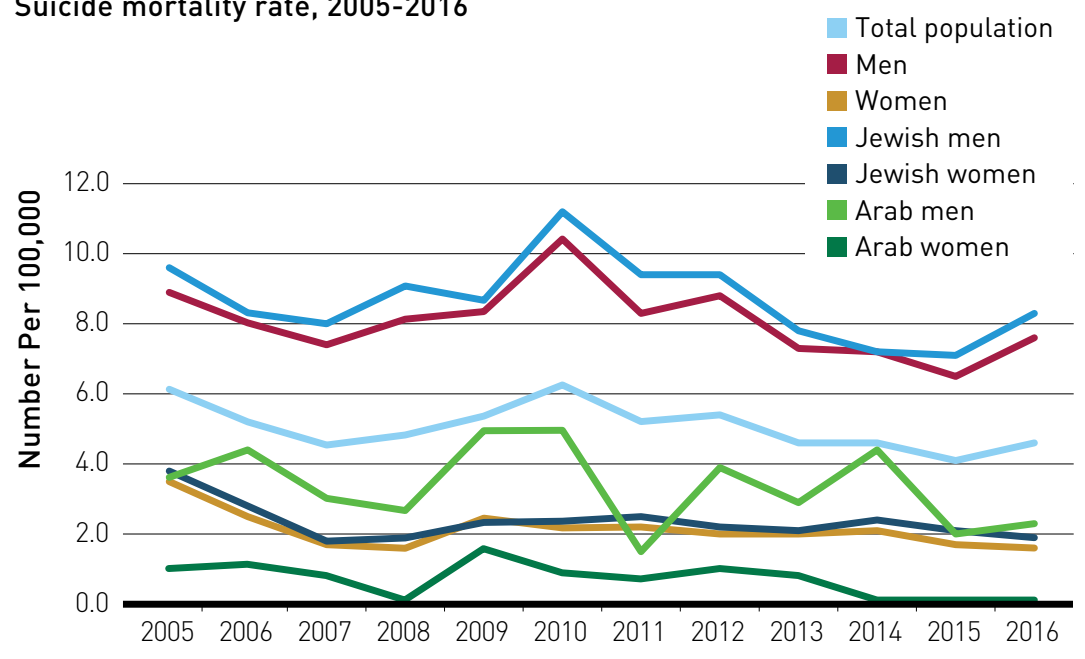




\section{5 .2}

Harmful use of alcohol, defined according to the national context as alcohol per capita consumption laged 15 years and older) within a calendar year in litres of pure alcohol

\subsection{1}

Death rate due to road traffic injuries
Numbers from WHO Global Information System on Alcohol and Health (GISAH).

Annual consumption of pure alcohol (in liters) per person (persons aged 15+), 2005-2016

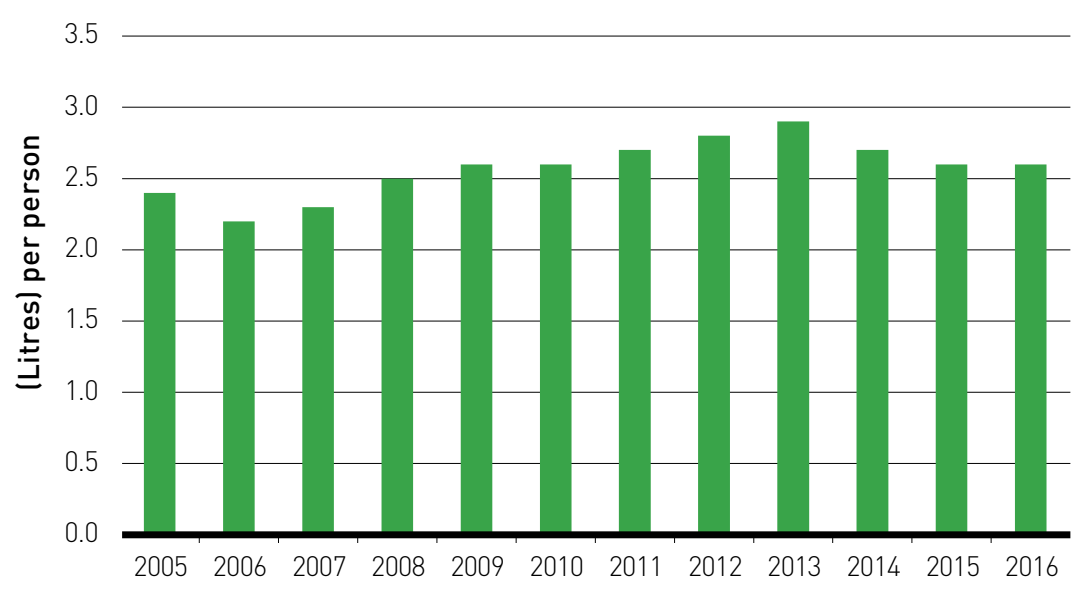

321 people were killed in road accidents in Israel in 2017, 33\% of whom were pedestrians.

Persons killed in road accidents by sex and population group, 2001-2017

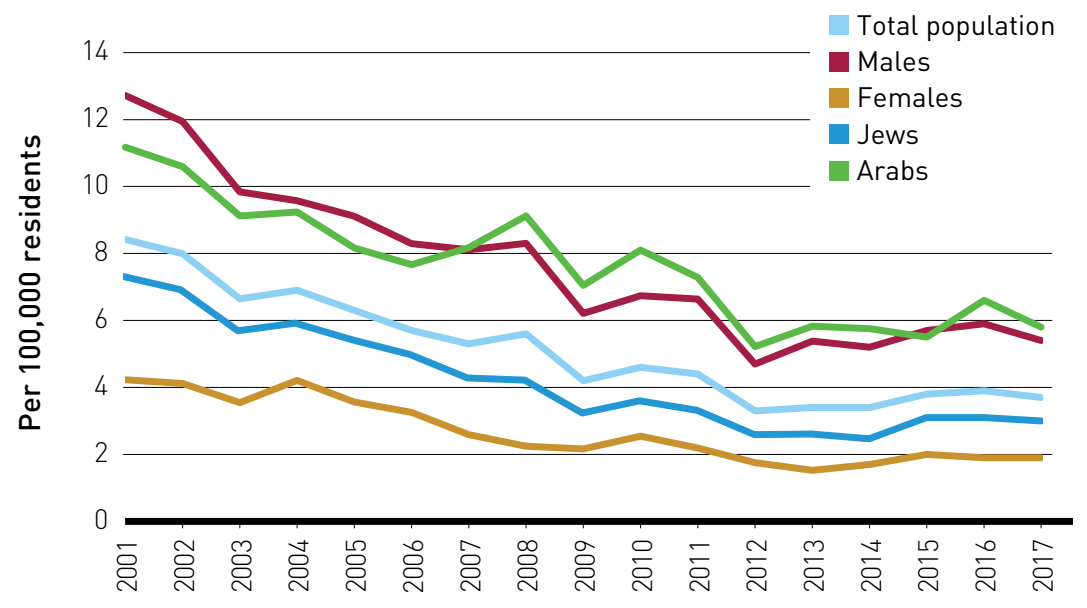


3.6.1

\section{7 .2}

Adolescent birth rate laged 15-19 years) per 1,000 women in that age group
Persons killed in road accidents in selected OECD Countries, 2016

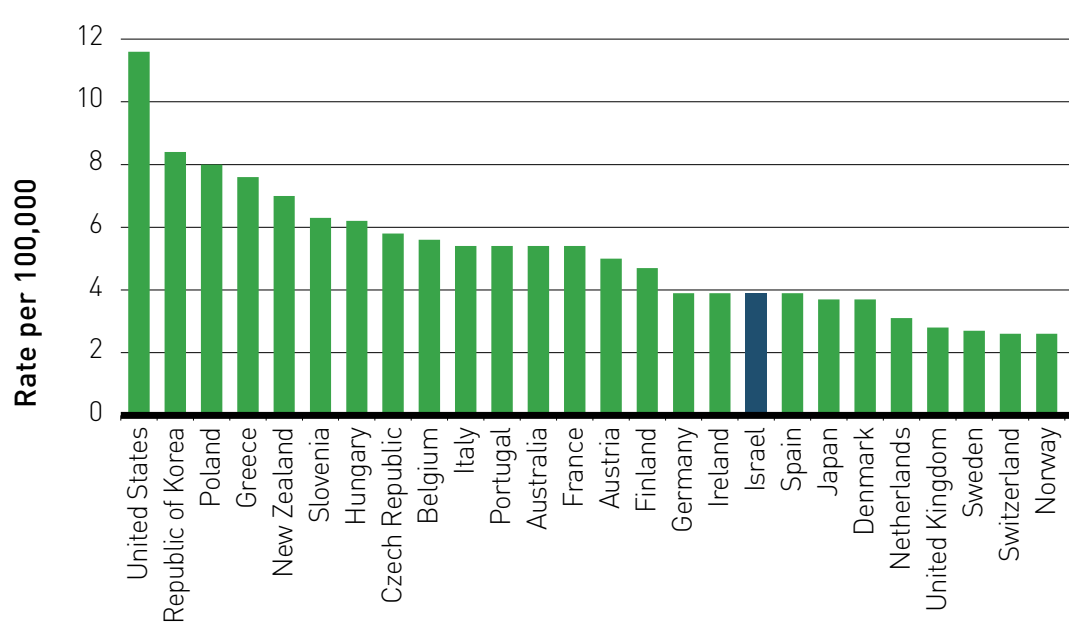

Adolescent birth rate, women aged 15-19, 2005-2017

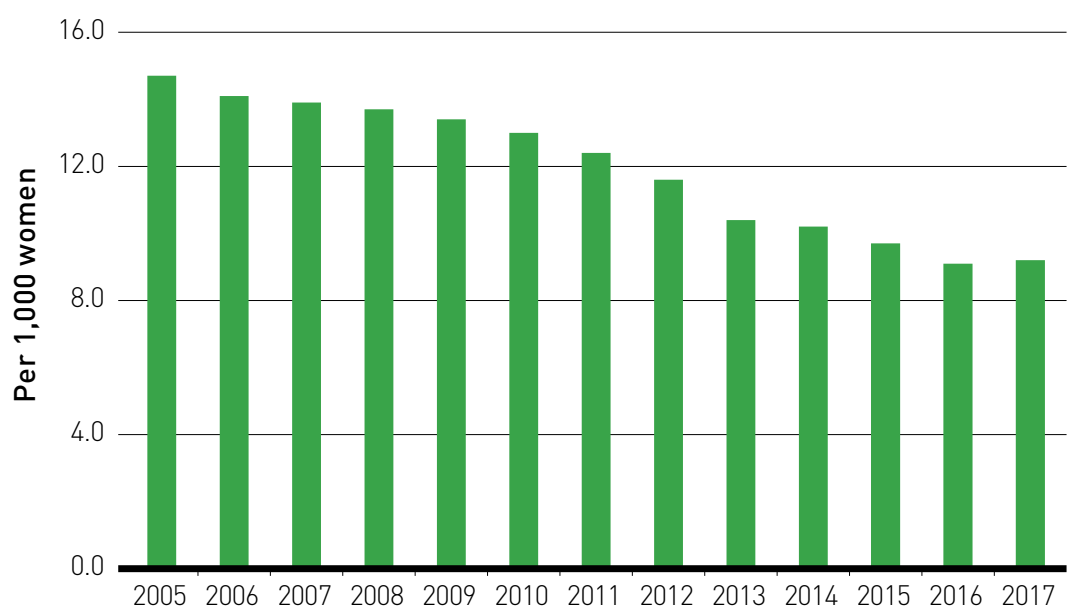

Total fertility rate, $2005-2017$

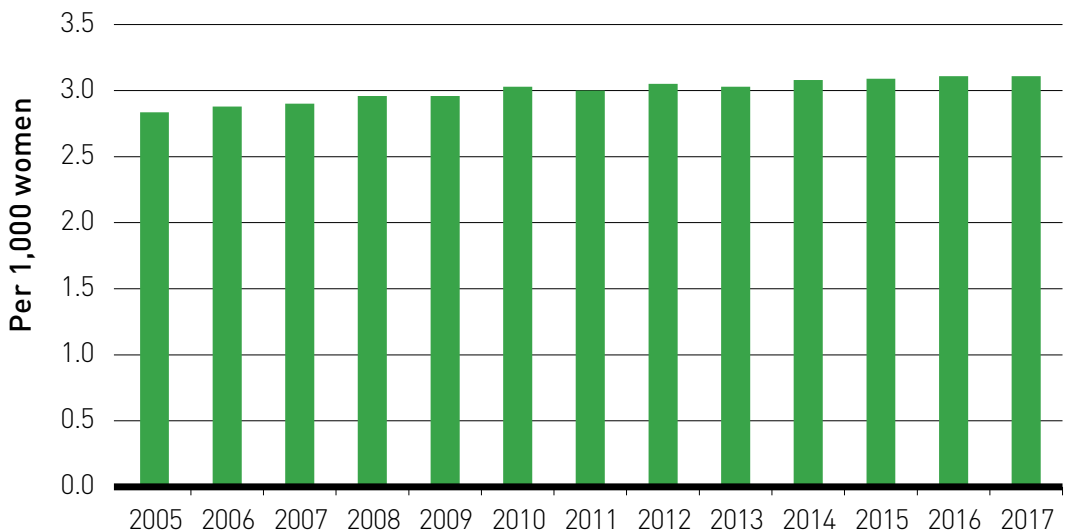




\subsection{1}

Coverage of essential health services

\section{8 .2}

Proportion of population with large household expenditures on health as a share of total household expenditure or income

\section{3.a.1}

Age-standardized prevalence of current tobacco use among persons aged 15 years and older
Based on Israeli legislation, $100 \%$ of the population have coverage for essential health services.

2017

\begin{tabular}{|l|l|l|}
\hline & $\begin{array}{l}\text { Health expenditurel } \\
\text { Total household income }\end{array}$ & $\begin{array}{l}\text { Health expenditurel } \\
\text { Total household expenditure }\end{array}$ \\
\hline Up to $\mathbf{1 0 \%}$ & 18.1 & 25.4 \\
\hline Up to $\mathbf{2 5 \%}$ & 4.9 & 5.1 \\
\hline
\end{tabular}

The data refer to people who reported having smoked at least one cigarette a day (daily smokers).

Smokers aged 21 and over, by sex, 2002-2017 ${ }^{(1)}$
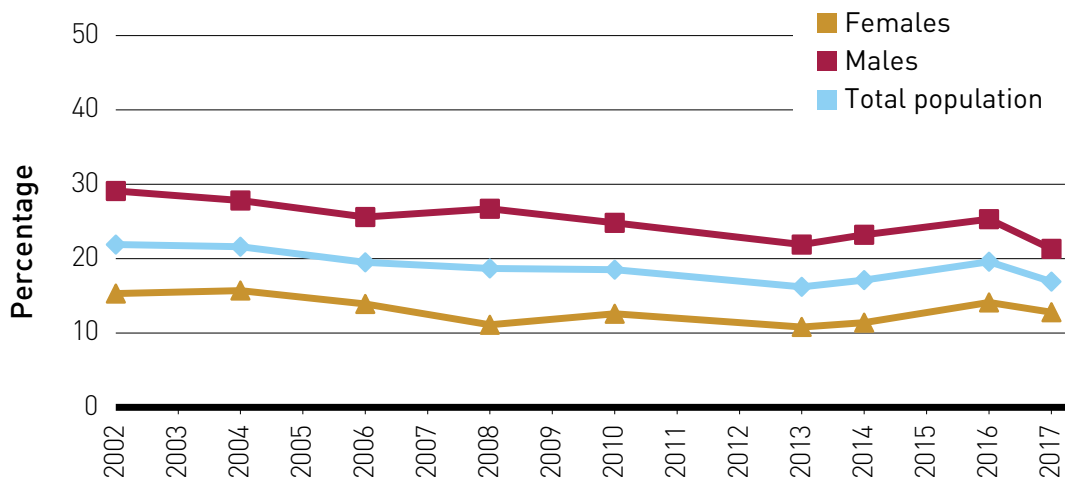

(1) Data for 2002-2013 and 2017 data are based on KAP surveys, 2014 data are based on the 2014 INHIS survey, 2016 data are based on the 2016 Physical Activity Survey, Ministry of Health. 
3.a.1

3.a.1
Smokers aged 21 and over, by population group, age and sex, 2017

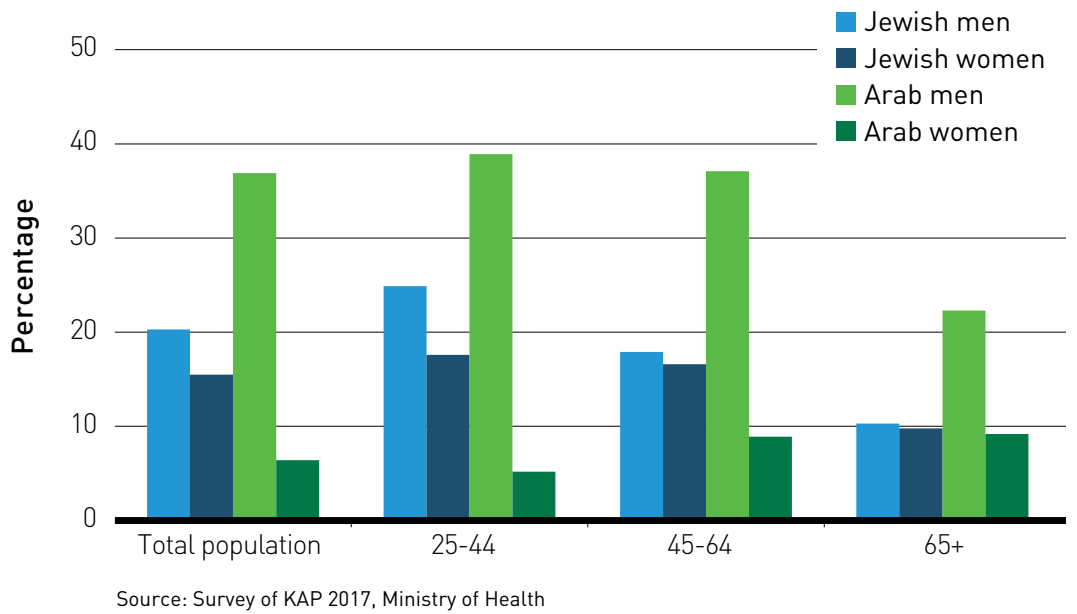

Source: Survey of KAP 2017, Ministry of Health

Smokers aged 15 and over ${ }^{(1)}$ in OECD countries, 2012-2017

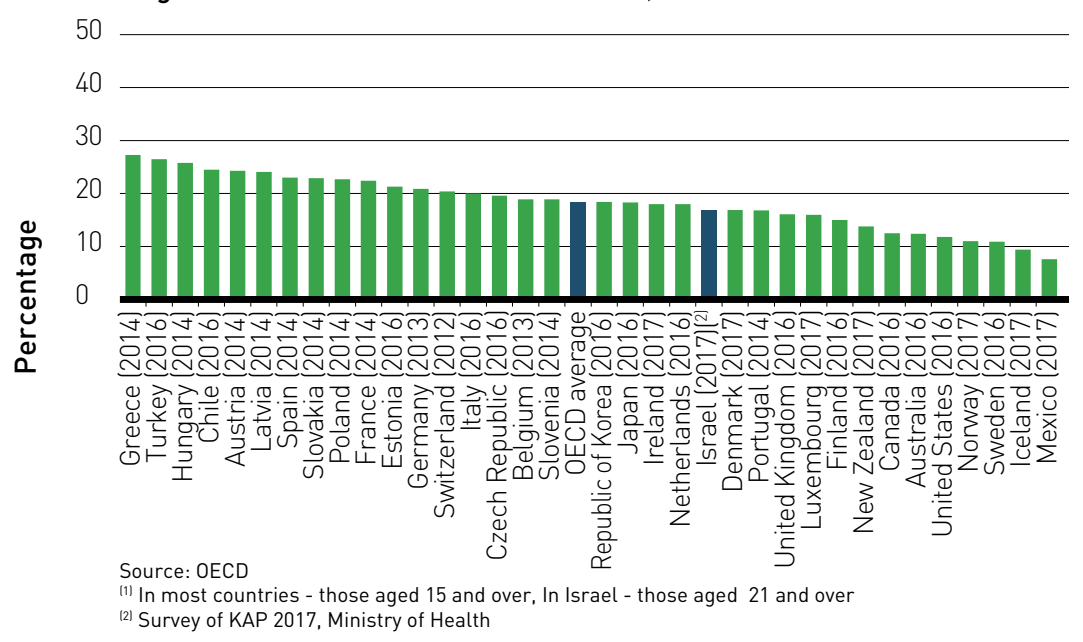


3.b.1

Proportion of the target

population covered by all

vaccines included in their

national program

Percentage of children immunized, of all those for whom information was reported, by type of immunization

\begin{tabular}{|c|c|c|c|c|c|c|c|c|c|c|}
\hline $\begin{array}{l}\text { Year } \\
\text { reported }\end{array}$ & & $\begin{array}{c}\text { Pneumo- } \\
\text { coccal } \\
\text { infection }\end{array}$ & Varicella & $\begin{array}{c}\text { Hepatitis } \\
\text { A }\end{array}$ & $\begin{array}{c}\text { Measles, } \\
\text { Mumps, } \\
\text { Rubella }\end{array}$ & Poliomyelitis & & $\begin{array}{c}\text { Diphtheria, } \\
\text { Tetanus, } \\
\text { Pertussis }\end{array}$ & $\begin{array}{l}\text { Haemophilus } \\
\text { influenzae b }\end{array}$ & $\begin{array}{c}\text { Hepatitis } \\
\text { B }\end{array}$ \\
\hline & Rota 3 & PCV 3 & VAR & HAV 1 & MMR 1 & IPV $4^{(2)}$ & OPV 3 & DTP 4 & $\mathrm{Hib} 4$ & HBV 3 \\
\hline 1991 & & & & & 94 & & 95 & 94 & & \\
\hline 1992 & & & & & 94 & 92 & 93 & 90 & & \\
\hline 1993 & & & & & 95 & 93 & 93 & 92 & & \\
\hline 1994 & & & & & 94 & 92 & 92 & 91 & & 92 \\
\hline 1995 & & & & & 95 & 95 & 95 & 94 & & 93 \\
\hline 1996 & & & & & 94 & 93 & 92 & 93 & 92 & 96 \\
\hline 1997 & & & & & 95 & 92 & 92 & 92 & 92 & 97 \\
\hline 1998 & & & & & 94 & 92 & 92 & 93 & 94 & 97 \\
\hline 1999 & & & & & 94 & 92 & 92 & 92 & 93 & 96 \\
\hline 2000 & & & & 89 & 95 & 93 & 93 & 94 & 93 & 98 \\
\hline 2001 & & & & 91 & 95 & 93 & 93 & 92 & 94 & 96 \\
\hline 2002 & & & & 89 & 95 & 91 & 92 & 90 & 91 & 98 \\
\hline 2003 & & & & 88 & 96 & 93 & 93 & 93 & 93 & 98 \\
\hline 2004 & & & & 93 & 97 & 95 & 94 & 95 & 95 & 100 \\
\hline 2005 & & & & 90 & 94 & 93 & 95 & 93 & 93 & 96 \\
\hline 2006 & & & & 84 & 97 & 94 & 81 & 95 & 94 & 96 \\
\hline 2007 & & & & 93 & 97 & 95 & & 95 & 95 & 99 \\
\hline 2008 & & & & 93 & 98 & 94 & & 94 & 94 & 99 \\
\hline 2009 & & & & 94 & 97 & 95 & & 95 & 95 & 98 \\
\hline 2010 & & & 91 & 93 & 96 & 95 & & 95 & 95 & 97 \\
\hline 2011 & & 92 & 95 & 93 & 97 & 94 & & 94 & 94 & 98 \\
\hline 2012 & 92 & 93 & 95 & 93 & 96 & 94 & & 94 & 94 & 97 \\
\hline 2013 & 80 & 94 & 98 & 91 & 98 & 96 & & 96 & 96 & 97 \\
\hline 2014 & 81 & 94 & 96 & 94 & 97 & 95 & & 95 & 95 & 97 \\
\hline
\end{tabular}

Source: Ministry of Health

(1) Up to age 2 years

(2) Until 2006 - IPV3 
3.c. 1

Health worker density and distribution

3.c.1

3.c.1
Employed doctors and nurses, 2005-2016

Doctors

Nurses

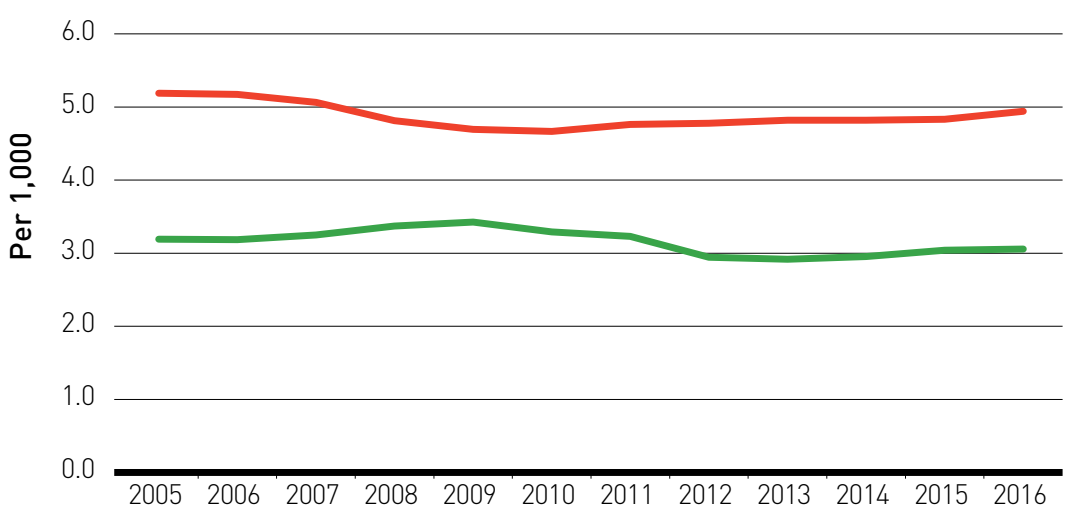

Employed Doctors in Israel and OECD countries, 2017

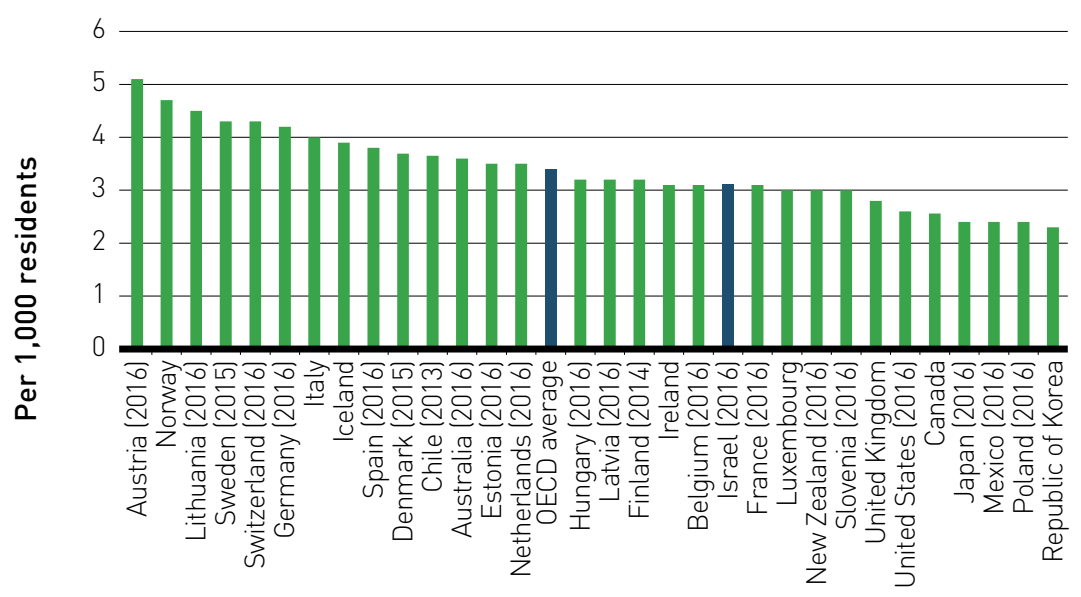

Source: $O E C D$

Employed nurses in Israel and OECD countries, 2017

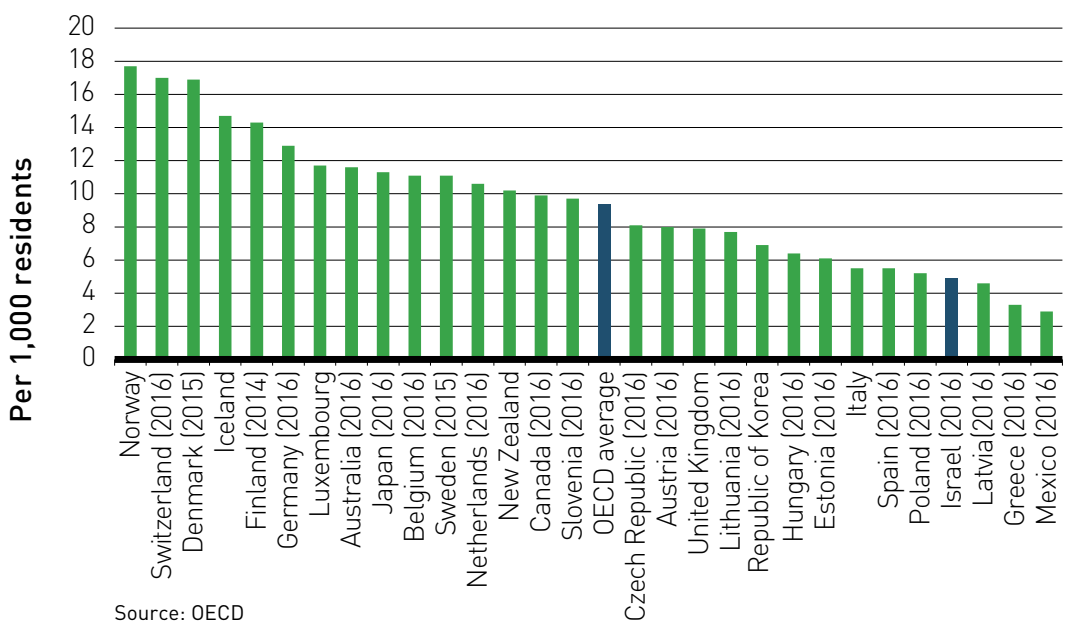




\section{GOAL 4}

\subsection{1}

Proportion of children and young people: (a) in grades $2 / 3$; (b) at the end of primary; and (c) at the end of lower secondary achieving at least a minimum proficiency level in (i) reading and (ii) mathematics, by sex

\section{ENSURE INCLUSIVE AND EQUITABLE QUALITY EDUCATION AND PROMOTE LIFELONG LEARNING OPPORTUNITIES FOR ALL}

Results for this indicator should only be compared within the same type of learning assessment. For example, PISA results with PISA, TIMSS results with TIMSS, etc.

Children and young people in grades $2 / 3$ achieving at least a minimum proficiency level in reading, by sex (PIRLS)

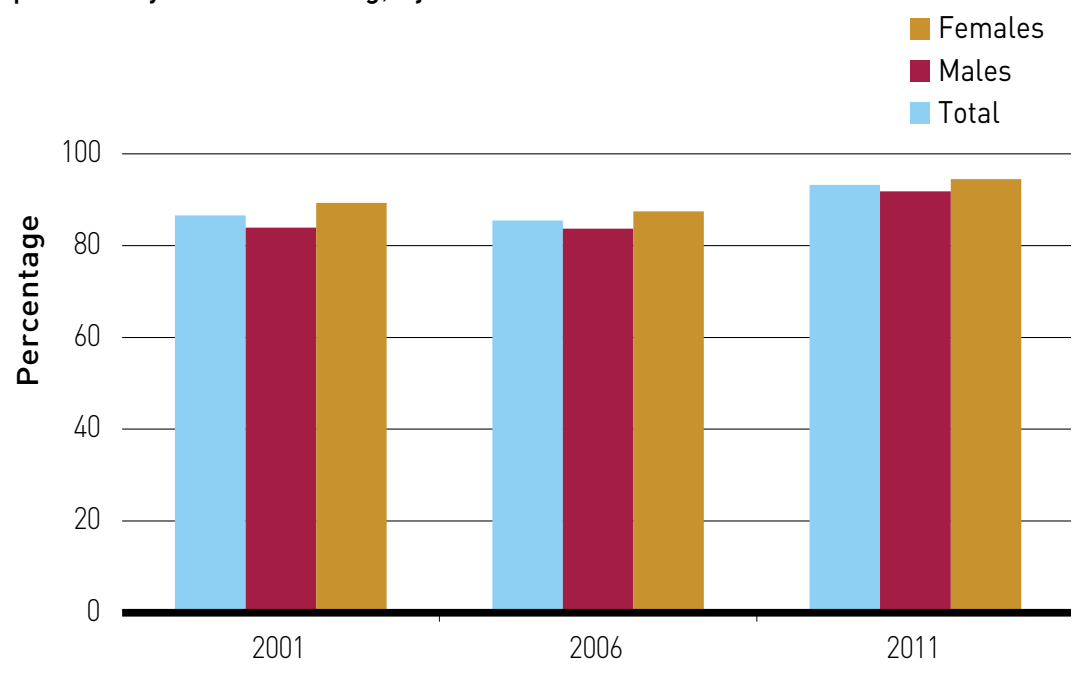

\subsection{1}

Children and young people at the end of lower secondary achieving at least a minimum proficiency level in reading, by sex (PISA)

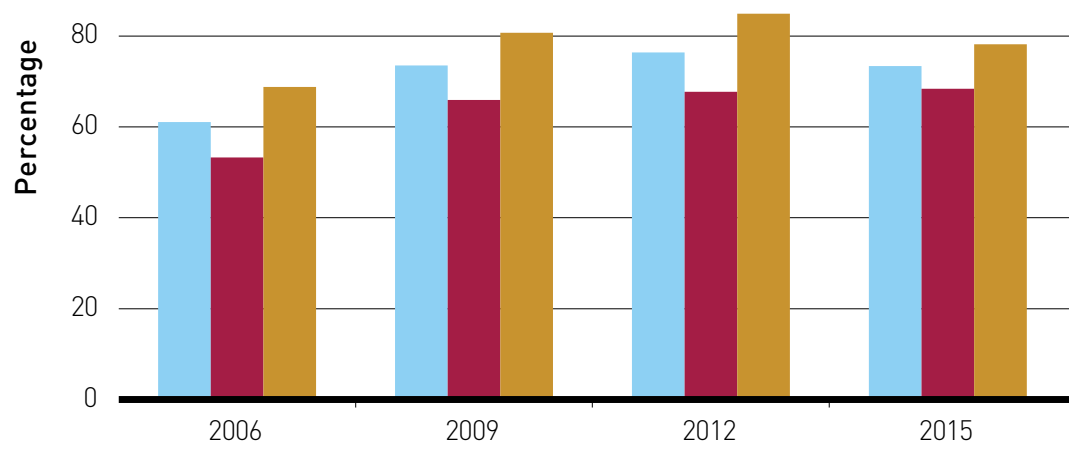


4.1.1

\subsection{2}

Participation rate in organized learning lone year before the official primary entry age), by sex
Children and young people at the end of lower secondary education achieving at least a minimum proficiency level in mathematics, by sex (PISA)

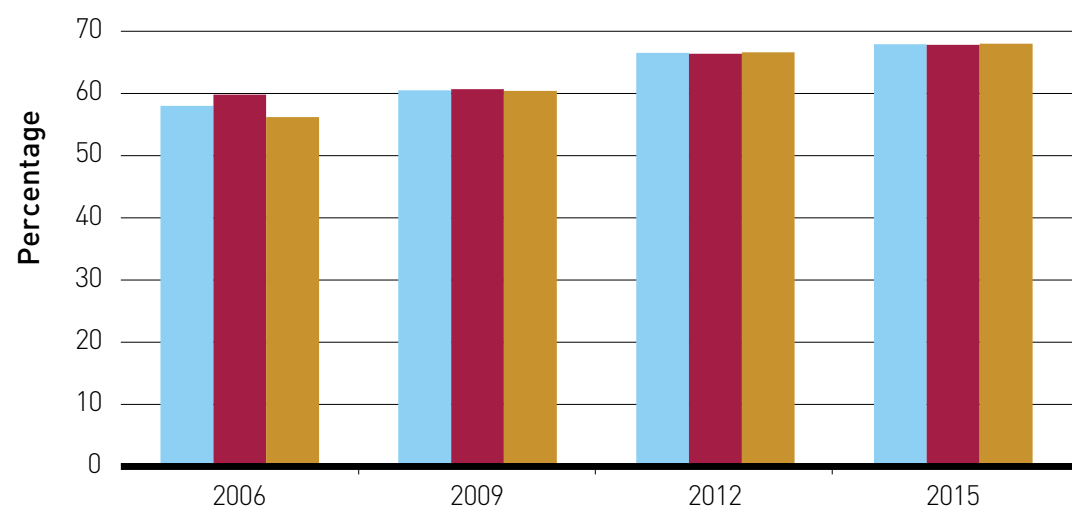

Calculation of the indicator was done by UNESCO based on national data.

Participation rate in organized learning lone year before the official primary entry age), by sex, 2005-2016

Females

- Males

Total

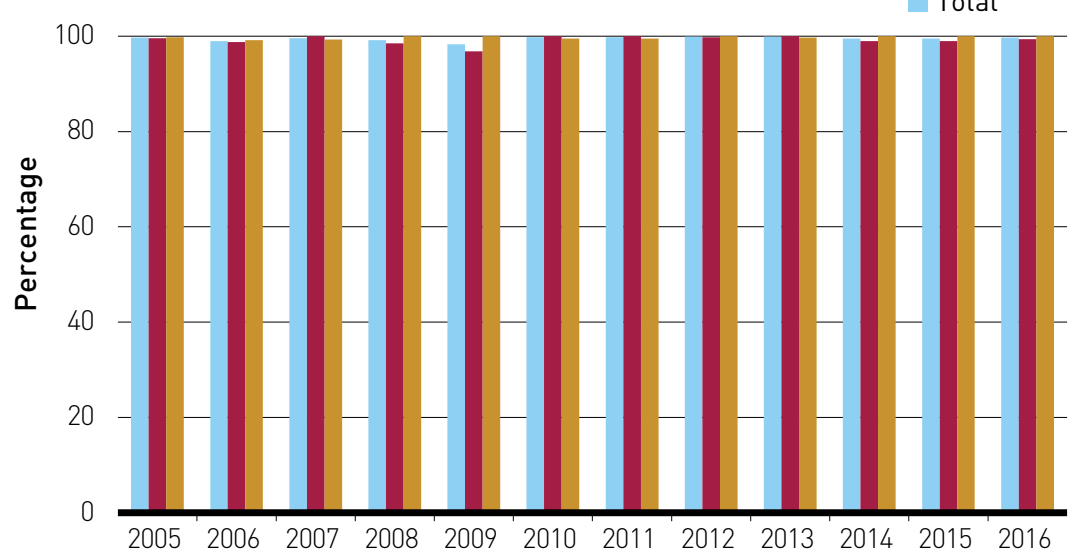




\subsection{1}

Participation rate of youth and adults in formal and nonformal education and training in the previous 12 months, by sex

4.3.1
Participation rate of youth and adults in formal and non- formal education and training in the previous 12 months, by sex, 2015

100.0

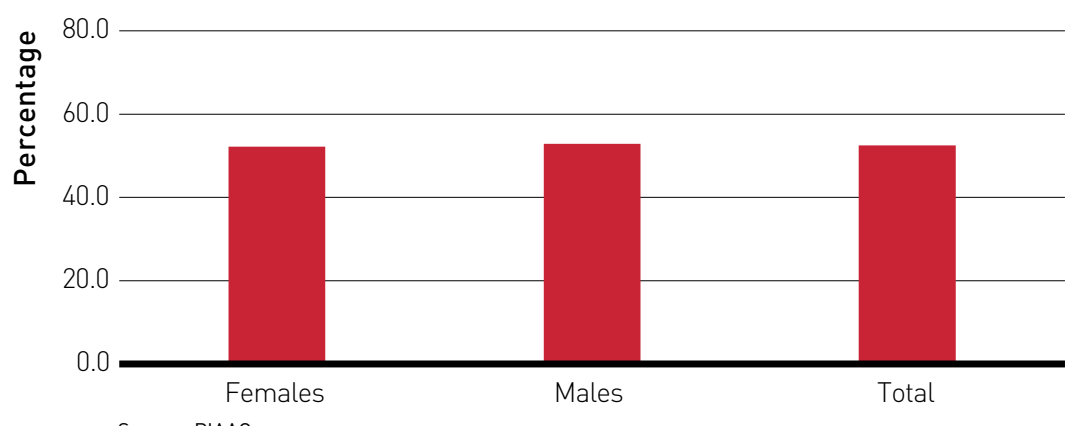

Source: PIAAC

Persons aged 15-24 having participated in education and training (last week), 2012-2017
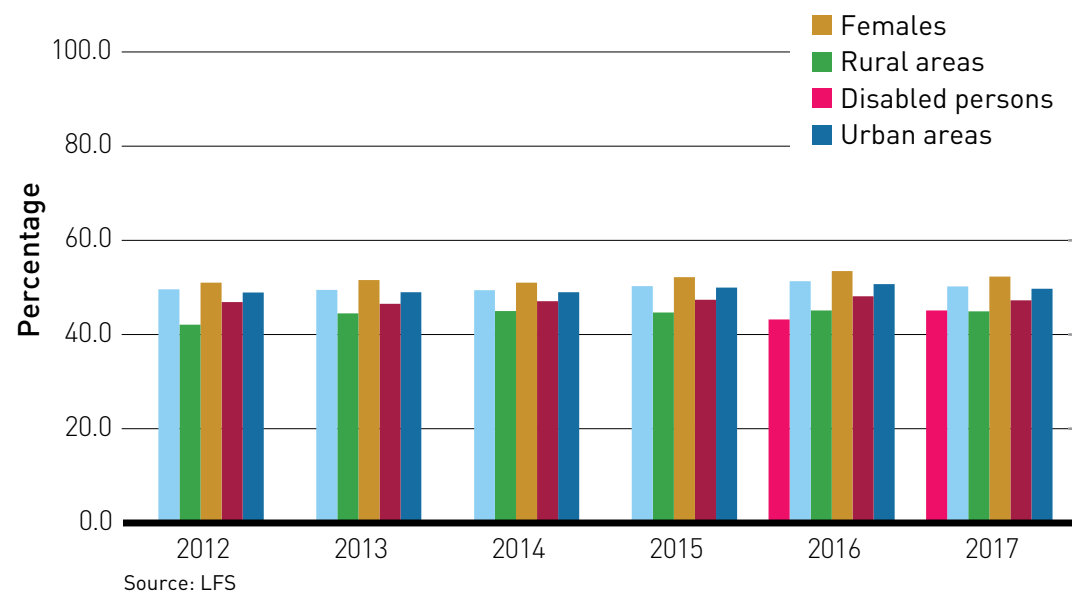

Persons aged 25-64 having participated in education and training (last week), 2012-2017

Total

Males

- Females

20.0

- Rural areas

- Disabled persons

16.0

urban areas

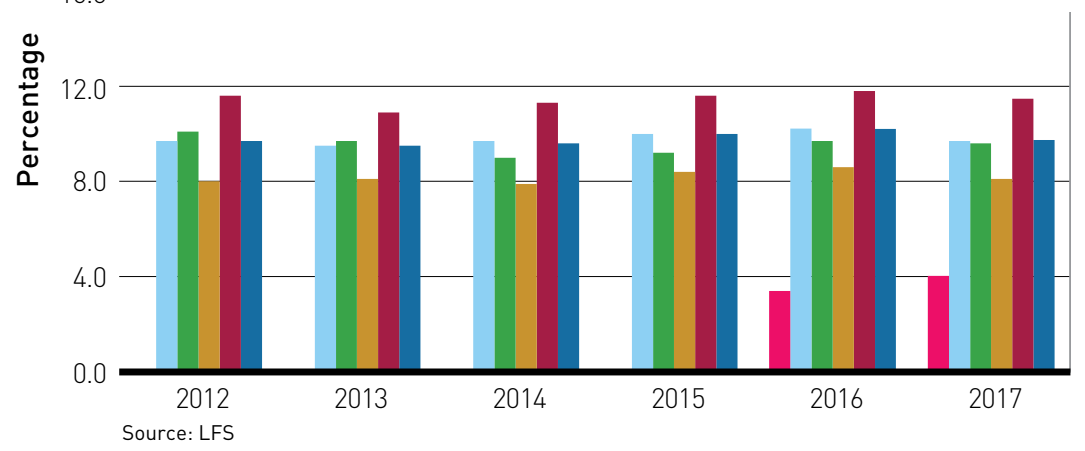




\subsection{1}

Proportion of youth and adults with information and communications technology (ICT) skills, by type of skill
Data presented as proxy for this indicator are direct measures of the skill levels from the 2015 PIAAC skill assessment survey. The survey of adult skills defines problem-solving in technology-rich environments as "using digital technology, communication tools and networks to acquire and evaluate information, communicate with others and perform practical tasks." It focuses on "the abilities to solve problems for personal, work and civic purposes by setting up appropriate goals and plans, and accessing and making use of information through computers and computer networks."

Persons with proficiency level $2 \& 3$ in problem-solving in a technology-rich environment, by population group, 2014-2015

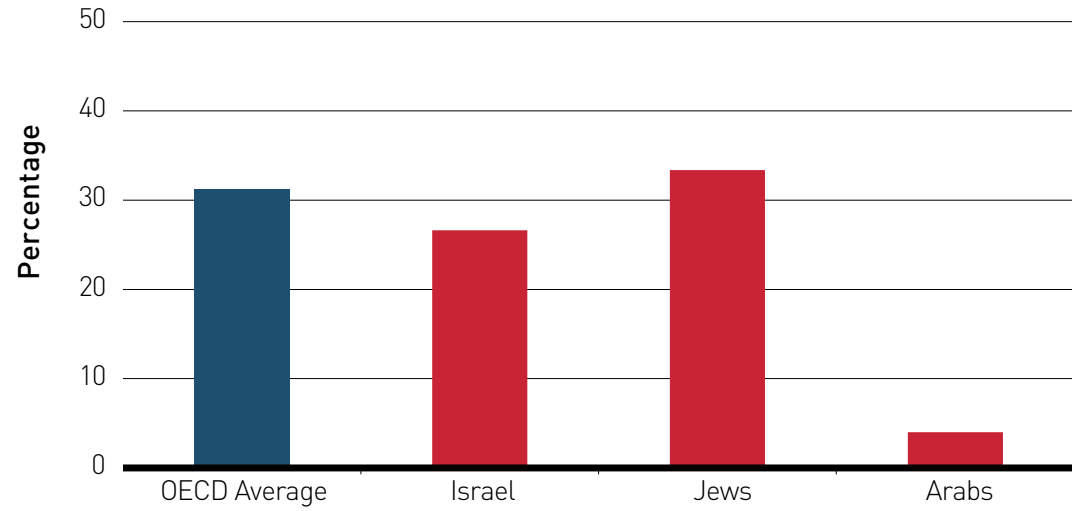

Persons with proficiency level $2 \& 3$ in problem-solving in a technology-rich environment, by age, 2014-2015

$$
50
$$

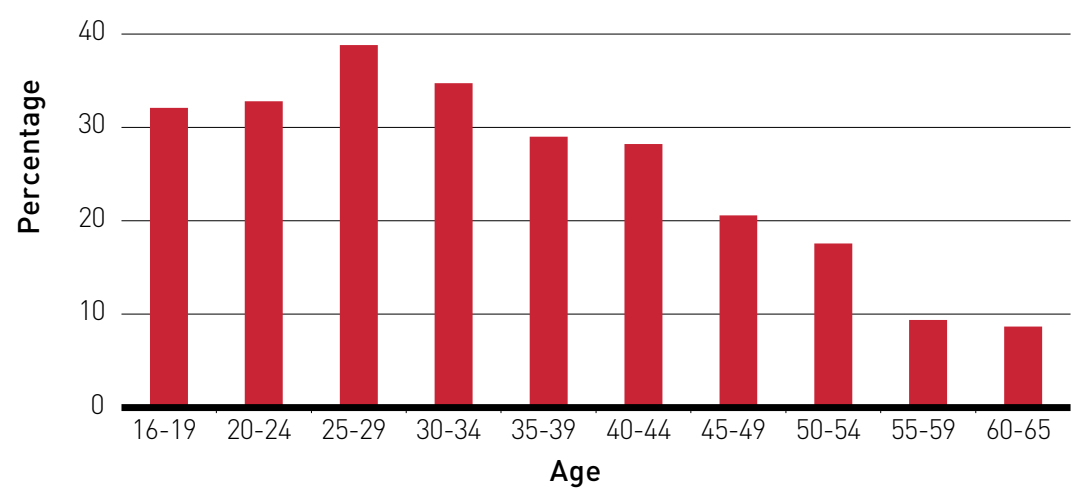




\subsection{1}

Parity indices (female/male,

Calculation of the indicator was done by UNESCO based on national data

rural/urban, bottom/top wealth quintile and others such as disability status, indigenous peoples and conflict-affected, as data become available)

\subsection{1}

\begin{tabular}{|l|l|l|l|l|l|l|}
\hline \multicolumn{9}{|c|}{} \\
\hline
\end{tabular}

\begin{tabular}{|l|l|l|l|}
\multicolumn{2}{|c|}{ Mathematics } \\
\hline \multirow{2}{*}{ Year } & $\begin{array}{l}\text { End of lower secondary education } \\
\text { (girls/boys) }\end{array}$ & $\begin{array}{l}\text { Parity index } \\
\text { (students of rural } \\
\text { school/students of } \\
\text { urban school) }\end{array}$ & $\begin{array}{l}\text { Parity index (low so- } \\
\text { cio-economic status/high } \\
\text { socio-economic status) }\end{array}$ \\
\hline $2003^{(1)}$ & 1.01 & 0.96 & 0.51 \\
\hline $2006^{(2)}$ & 0.94 & 0.68 & \\
\hline $2007^{(1)}$ & 1.04 & 0.93 & 0.47 \\
\hline $2009^{(2)}$ & 1.00 & 1.10 & 0.78 \\
\hline $2011^{(1)}$ & 1.06 & 0.94 & 0.52 \\
\hline $2012^{(2)}$ & 1.00 & 0.92 & 0.60 \\
\hline $2015^{(2)}$ & 1.00 & 0.98 & \\
\hline
\end{tabular}

(1) TIMSS

(2) PISA 
Population in a given age group achieving at least a fixed level of proficiency in functional literacy and numeracy skills, by sex, $2015^{(1)}$

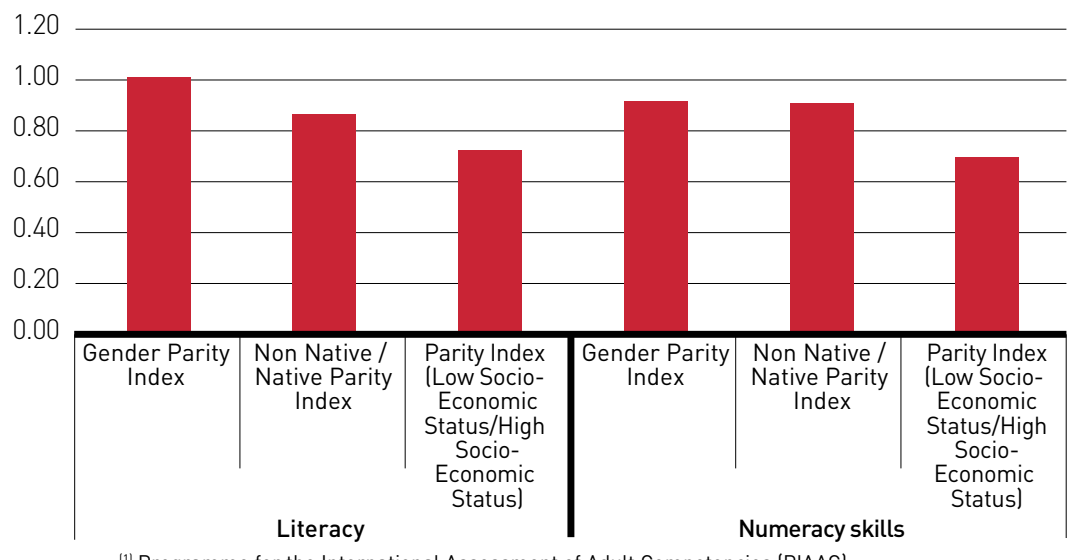

(1) Programme for the International Assessment of Adult Competencies (PIAAC) primary entry age) Gender Parity Index, 2005-2016

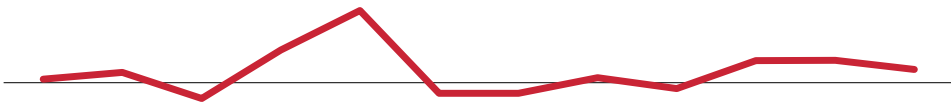




\subsection{1}

\section{Percentage of population in} a given age group achieving at least a fixed level of proficiency in functional (a) literacy and (b) numeracy skills, by sex
The proportion of the population aged 16-65 achieving at least a fixed level of proficiency in functional (a) literacy and (b) numeracy skills based on PIAAC survey (level 2 or higher) is as follows: literacy $-72.2 \%$, numeracy skills $-68.3 \%$.

Level 3 and higher is considered high in the Israeli well-being indicators set.

Persons with a level of proficiency 3 or higher in reading literacy, OECD average, Israel and Israel by population group, 2014-2015

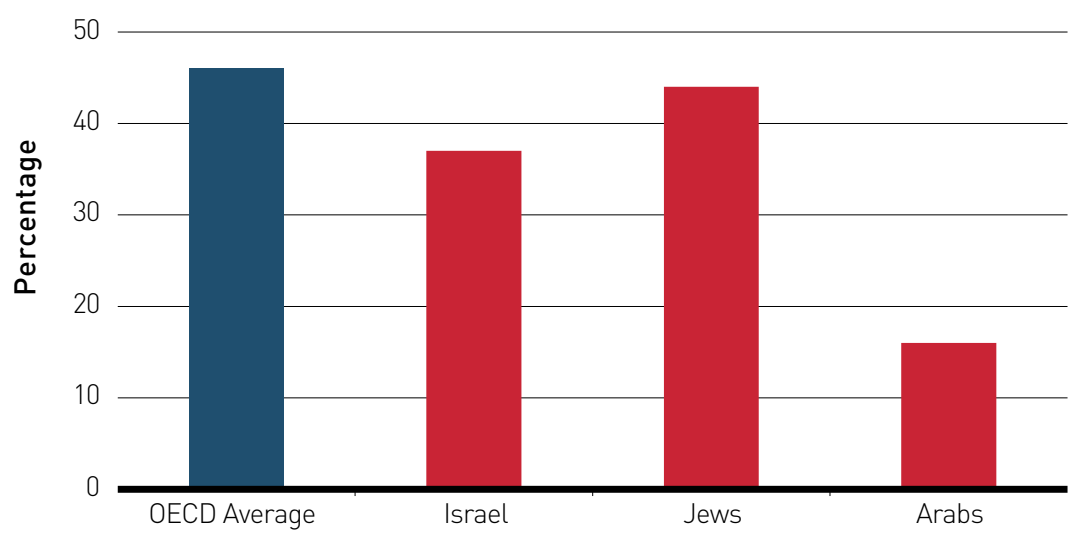

\subsection{1}

Persons with a level of proficiency 3 or higher in mathematical literacy, OECD average, Israel and Israel by population group, 2014-2015

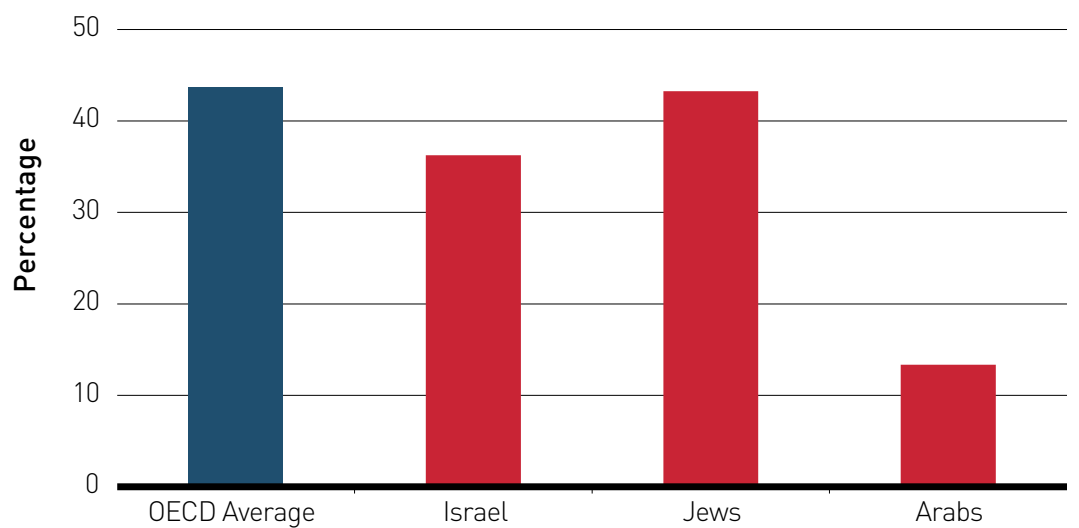


4.6.1

Persons with a level of proficiency 3 or higher in reading literacy, by age, 2014-2015

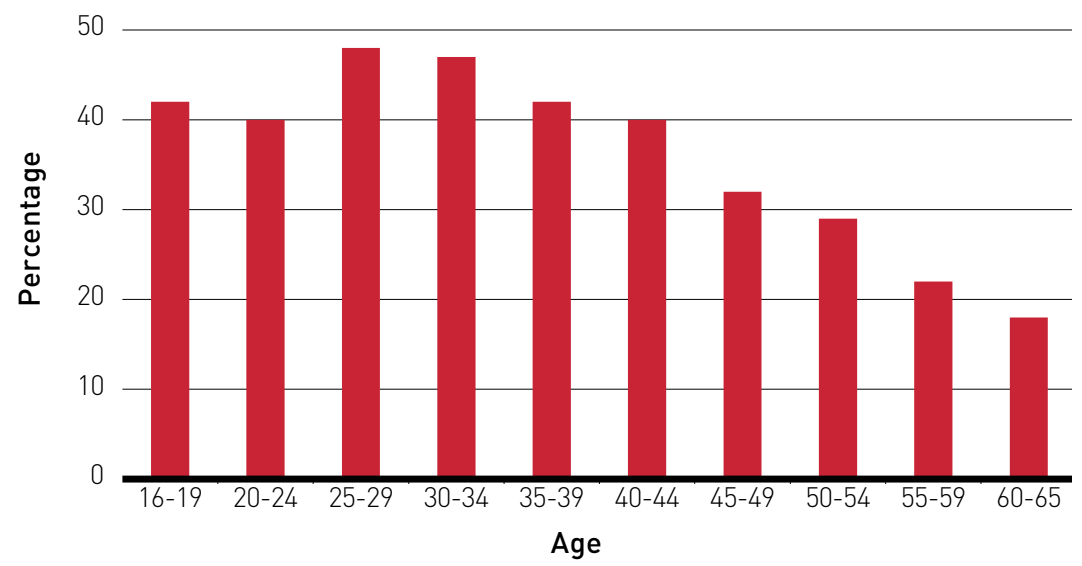

4.6.1

Persons with a level of proficiency 3 or higher in mathematical literacy, by age, 2014-2015

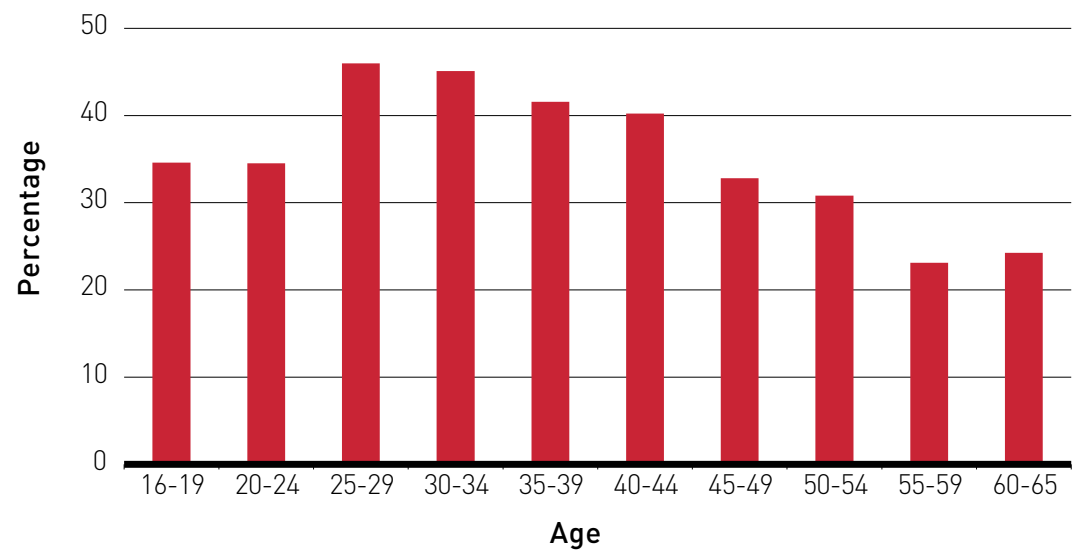




\subsection{1}

Extent to which (i) global citizenship education and (ii) education for sustainable development, including gender equality and human rights, are mainstreamed at all levels in: (a) national education policies, (b) curricula, (c) teacher education and (d) student assessment
The Ministry of Environmental Protection in collaboration with the Ministry of Education has led the certification process for Green Schools since 2003. The purpose of the process is to implement sustainability principles in schools. Accreditation reflects the recognition of significant environmental education in a school and outlines a possible course of action for schools that want to start environmental activities based on existing frameworks.

Green and Greener Kindergarten, 2007-2016
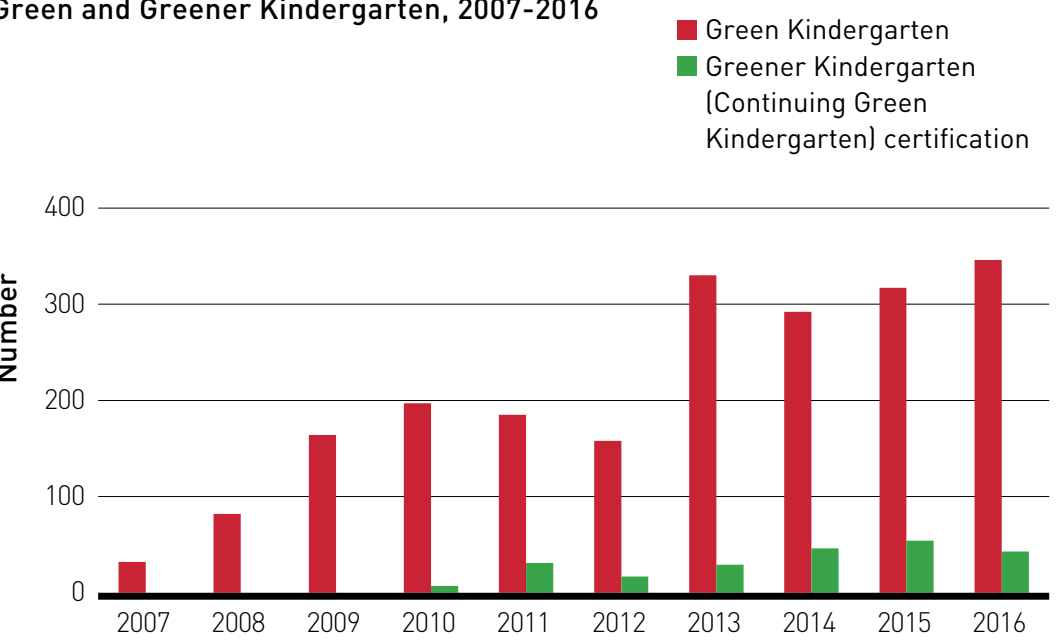

\subsection{1}

Green and Greener Schools, 2004-2016

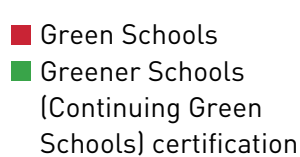

Schools) certification

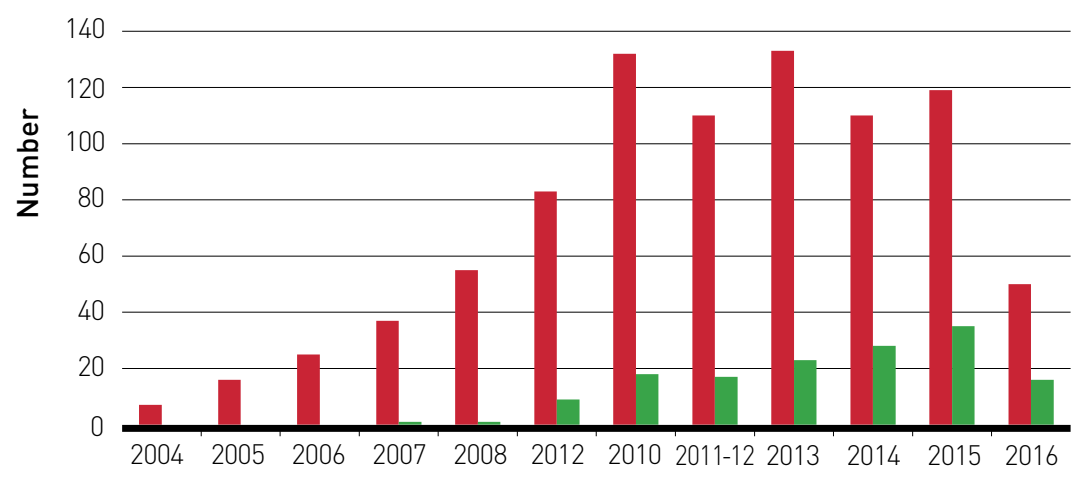


4.7.1

\section{4.a.1}

Proportion of schools with access to: (a) electricity; (b) the Internet for pedagogical purposes; (c) computers for pedagogical purposes; (d) adapted infrastructure and materials for students with disabilities; (e) basic drinking water; (f) single-sex basic sanitation facilities; and ( $\mathrm{g}$ ) basic handwashing facilities las per the WASH indicator definitions)

\section{4.c.1}

Proportion of teachers in: (a) pre-primary; (b) primary; (c) lower secondary; and (d) upper secondary education who have received at least the minimum organized teacher training (e.g. pedagogical training) preservice or in-service required for teaching at the relevant level in a given country
A professional development program for teachers implements education for sustainabllity principles with an emphasis on action for the environment by means of teachers training accompanied by professional guidlines.

The integrated plan for implementing sustainability education in the school system, 2010-2016

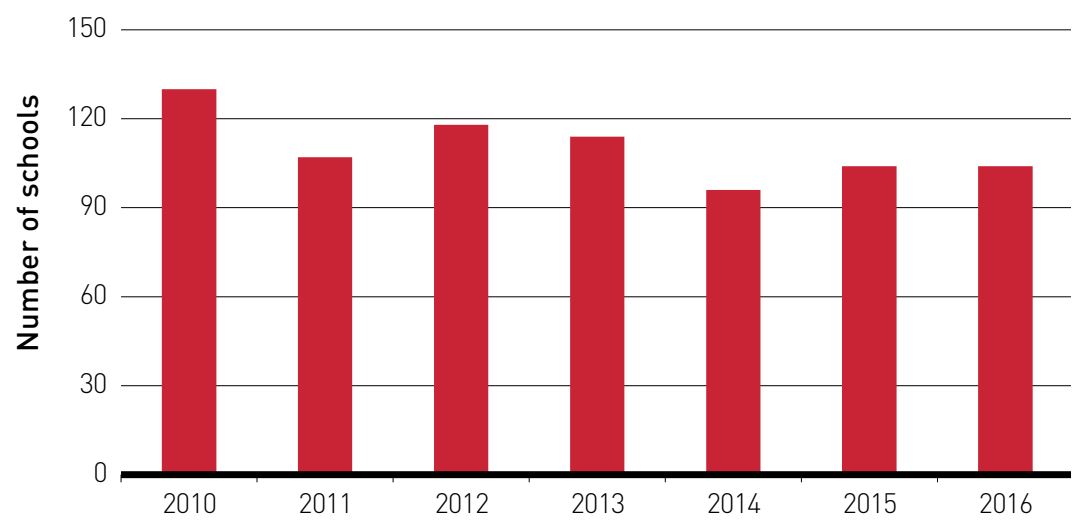

Access to the following is $100 \%$ at all levels of education: al electricity; (d) adapted infrastructure and materials for students with disabilities; (e) basic drinking water; (f) single-sex basic sanitation facilities; and (g) basic handwashing facilities (as per the WASH indicator definitions).

Access to the following is $85 \%$ (data refer to public education only) at all levels of education: (b) the Internet for pedagogical purposes; (c) computers for pedagogical purposes.

In Israel, all preschool, primary, lower secondary and upper secondary teachers undergo training, including bachelor's degree study and teacher certification, before they start teaching. 
GOAL 5

\subsection{1}

Proportion of ever-partnered women and girls aged 15 years and older subjected to physical, sexual or psychological violence by a current or former intimate partner in the previous 12 months, by form of violence and by age

\subsection{2}

Proportion of women and girls aged 15 years and older subjected to sexual violence by persons other than an intimate partner in the previous 12 months, by age and place of occurrence

\section{ACHIEVE GENDER EQUALITY AND EMPOWER ALL WOMEN AND GIRLS}

In 2017, 86.7 thousand women aged 20 and older were subjected to physical violence or threat of violence, constituting $3.1 \%$ of women aged 20 and older. 82.2 thousand women aged 20 and older were subjected to physical violence or threat of violence by other than an intimate partner, constituting $2.94 \%$ of women aged 20 and older. Data for women subject to violence by current or former intimate partner cannot be displayed due to high sampling errors.

Women and girls aged 20 years and older subjected to psychological violence or threat of violence in the previous 12 months, 2014-2017

$$
4.00
$$

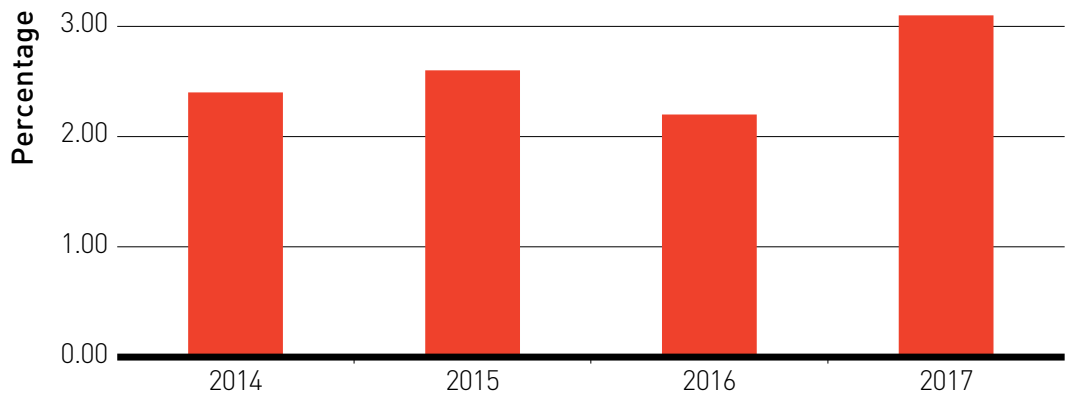

Data cannot be displayed due to high sampling errors. 


\subsection{1}

Proportion of women aged 2024 years who were married or in a union before age 15 and before age 18

\subsection{1}

Proportion of seats held by women in national parliaments and local governments
Data for Israel are available only for women aged 20-24 years who were married or in a union before age 18.

Women aged 20-24 years who were married or in a union before age $18,2008-2017$

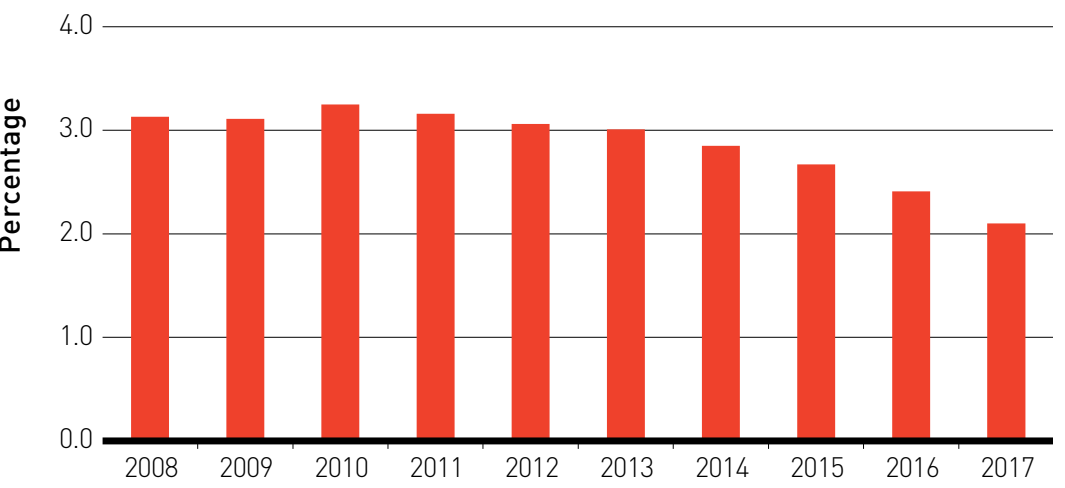

The Knesset, the Israeli parliament, is the legislative branch having exclusive authority in the country to enact laws. The Knesset has 120 members. The data present the proportion of women in the Knesset from the first Knesset (1949) to the current twentieth Knesset (from 2015).

Proportion of seats held by women in the Israeli parliament

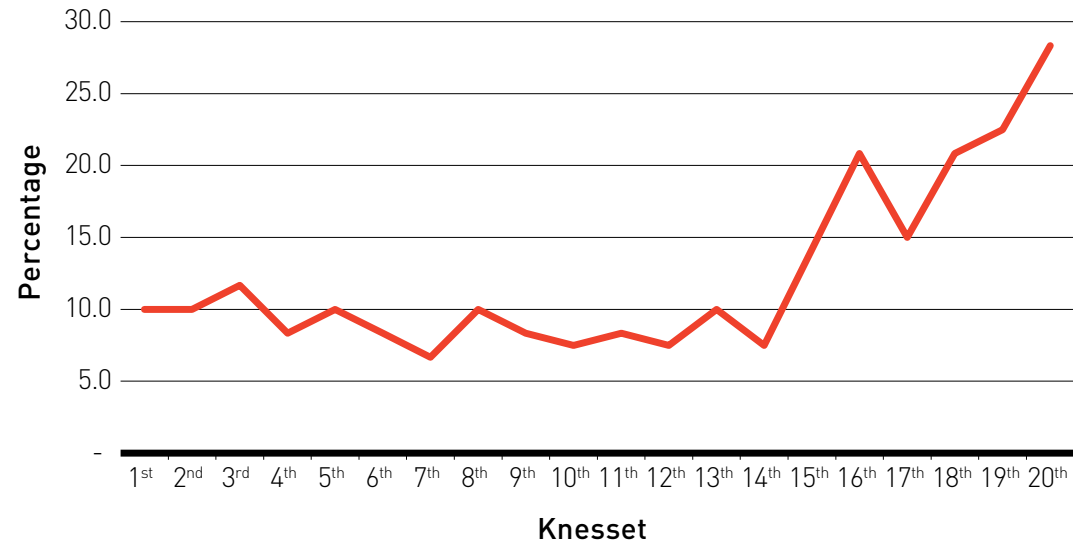




\section{5 .2}

\section{Proportion of women in managerial positions}

In 2017, 34.5\% of the managerial positions were held by women. In the public sector, $40.7 \%$ of the managerial positions were held by women.

Women in managerial positions, 2012-2017

40.0

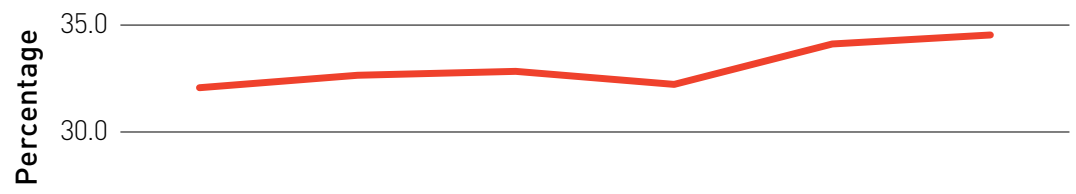

25.0

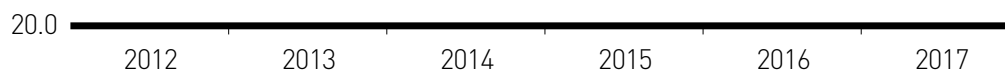

Women in managerial positions by occupation (selected ISCO level 2), 2012-2017

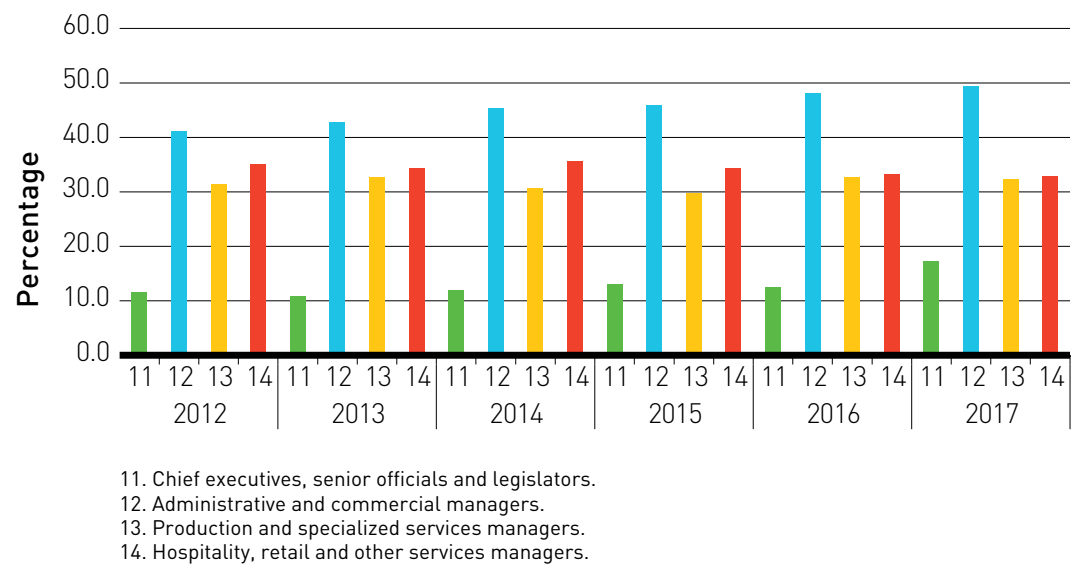




\section{5 .2}

\section{6 .2}

Number of countries with laws and regulations that guarantee women aged 15-49 years access to sexual and reproductive health care, information and education

5.b. 1

Proportion of individuals who own a mobile telephone, by sex
Women in management positions in the public sector, by population group, 2012-2017

Jewish women Arab women

50 Total

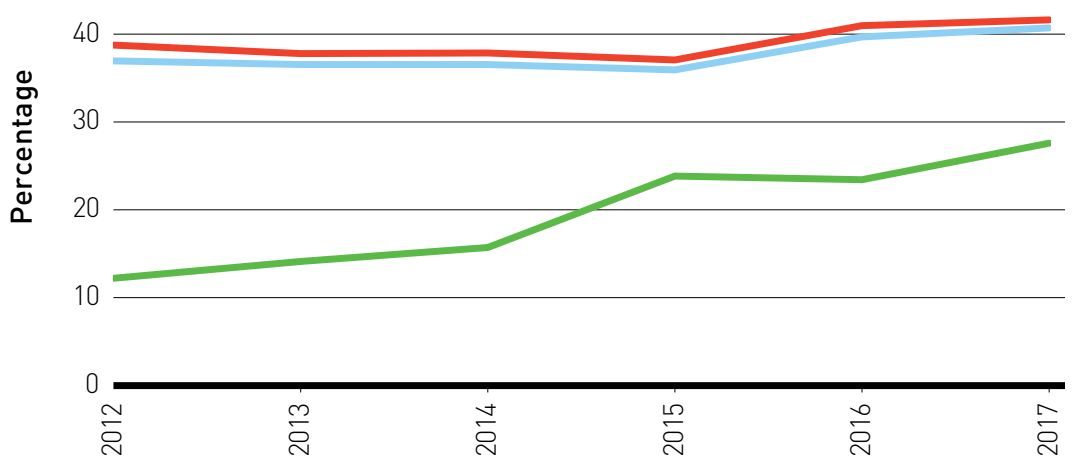

The National Health Insurance Law (1994) guarantees all women in Israel access to these services.
Persons who own a mobile telephone, 2012-2017

Males

$\square$ Total

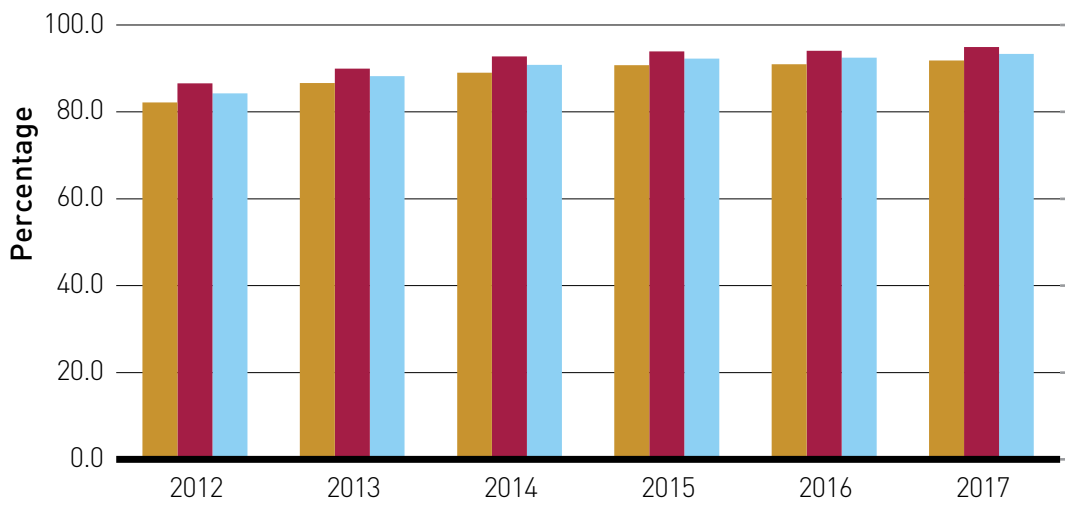


GOAL 6

\section{ENSURE AVAILABILITY AND \\ SUSTAINABLE MANAGEMENT OF WATER AND SANITATION FOR ALL}

\subsection{1}

\section{Proportion of population using safely managed drinking water services}

6.2.1

Proportion of population using safely managed sanitation services, including a handwashing facility with soap and water

\section{4 .2}

Level of water stress: freshwater withdrawal as a proportion of available fresh water resources
For the above two indicators, the proportion of the population that has access to safely managed drinking water services and to safely managed sanitation services is 100\% in urban areas and more than $99 \%$ in rural areas.

$53.6 \%$ of the population are connected to tertiary wastewater treatment. The majority of the population not connected to wastewater treatment is the Bedouin population that lives in localities with no municipal status. More than 500 MCM of raw sewage is collected; $93 \%$ is treated of which $86 \%$ is reused

Proportion of the population connected to wastewater treatment infrastructure, 2005-2017

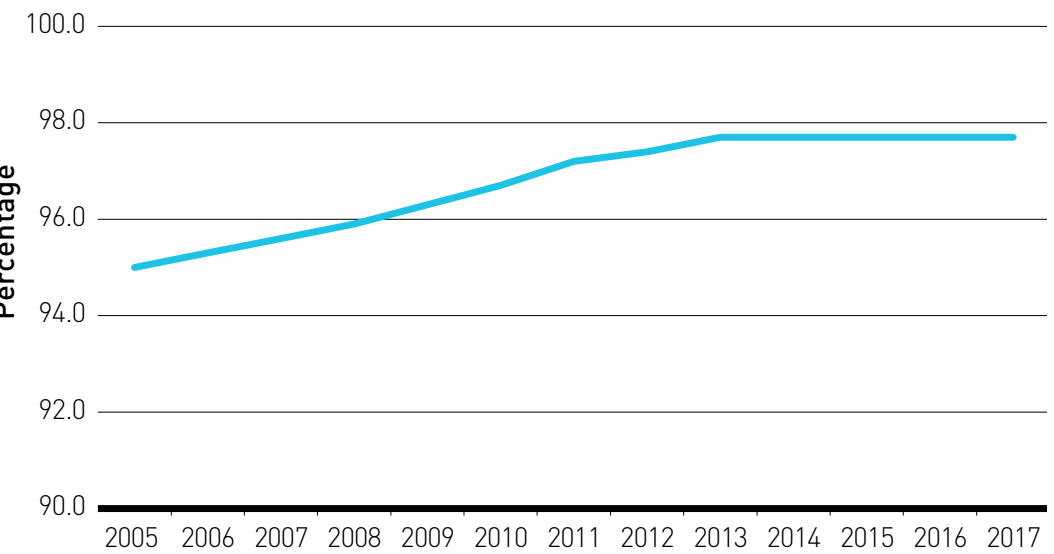

$200520062007 \quad 2008 \quad 20092010 \quad 20112012 \quad 2013 \quad 2014 \quad 2015 \quad 2016 \quad 2017$

Freshwater withdrawal as a proportion of available freshwater resources in Israel is $100 \%$. 
GOAL 7

\subsection{1}

Proportion of population with access to electricity

\subsection{2}

Proportion of population with primary reliance on clean fuels and technology

\subsection{1}

\section{Renewable energy share} in the total final energy consumption

\section{ENSURE ACCESS TO AFFORDABLE, RELIABLE, SUSTAINABLE AND MODERN ENERGY FOR ALL}

The proportion of the population in Israel with access to electricity is very high and close to $100 \%$.

Based on 2014 data from the Israeli social survey, 1.5\% of the population has a solar system for electricity generation at their homes. $86 \%$ of the population has sun-heated boilers.

The majority of renewable energy production is derived from water heating in sun-heated boilers (62\%). The percentage of renewable energy out of the primary energy supply and out of electricity production is very low in Israel (less than 3 percent), despite the significant increase in 2008-2016. The share of renewable energy production in Israel is much lower than the OECD countries average (24\%).

Renewable energy share in the total final energy consumption, 2013-2016

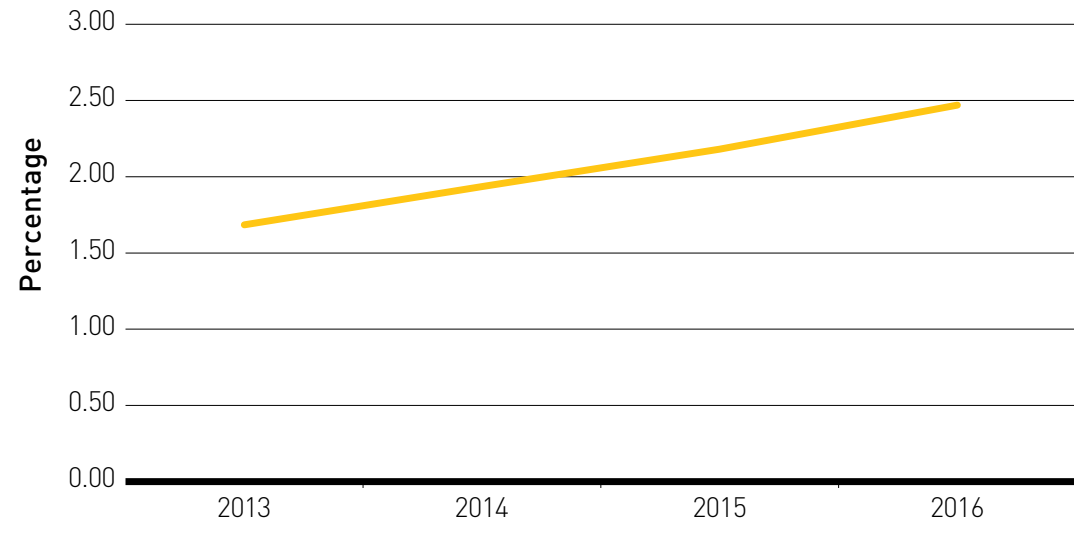


Renewable energy share out of primary energy supply and electricity production, 2008-2016

$$
3.0
$$

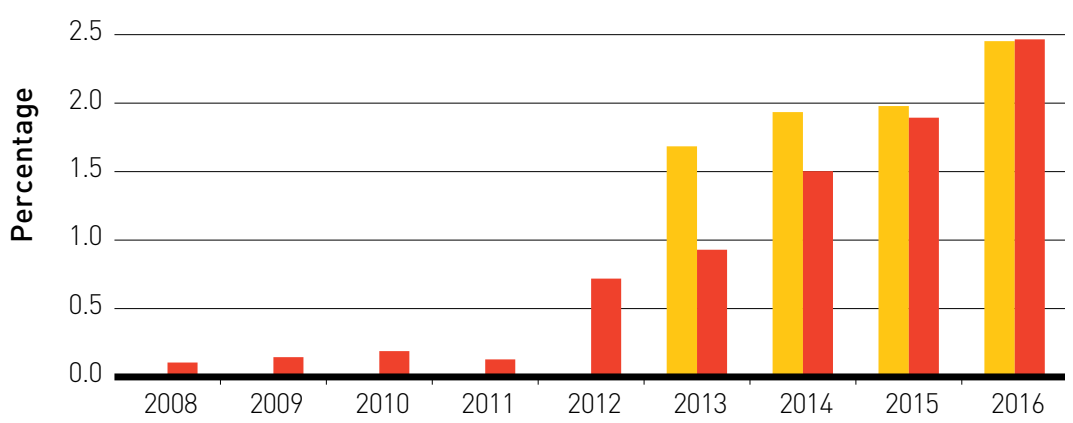

(1) In 2013 there was a change in calculation methodology

Production of electricity from renewable energy out of total electricity production in OECD countries, 2016

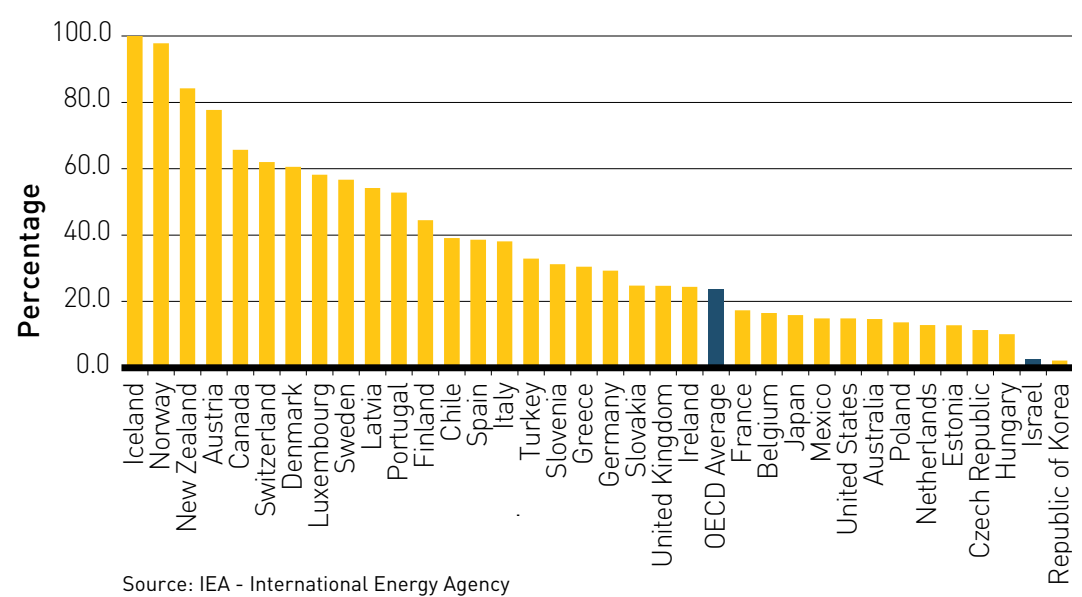




\subsection{1}

Energy intensity measured in terms of primary energy and GDP
In 2016, the energy ratio in Israel was 0.08 t.e.o per thousand US dollars PPP in 2010 prices, lower than the ratio in European countries.

Energy ratio and primary energy supply ${ }^{(1)}, 2005-2016$ Primary energy supply Energy ratio

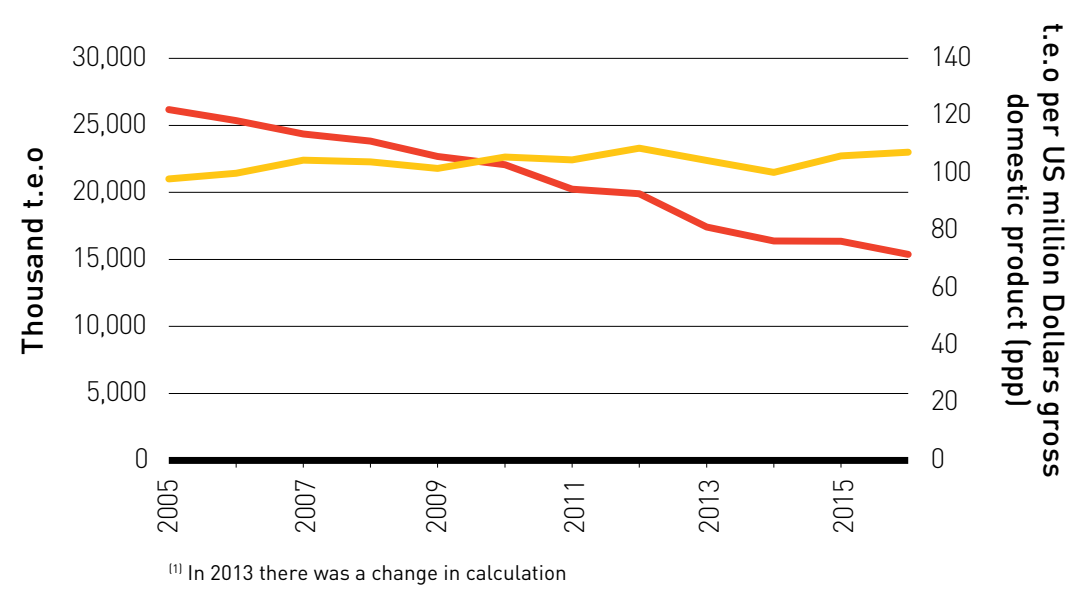


GOAL 8

\subsection{1}

Annual growth rate of real GDP per capita

\section{PROMOTE SUSTAINED, INCLUSIVE AND SUSTAINABLE ECONOMIC GROWTH, FULL AND PRODUCTIVE EMPLOYMENT AND DECENT WORK FOR ALL}

There were 3.993 million participants in the labor force lpersons aged 15 and over) in 2017, of which 2.019 million were employed men and 1.806 million were employed women.

Real annual rate of change of GDP, 2005-2017

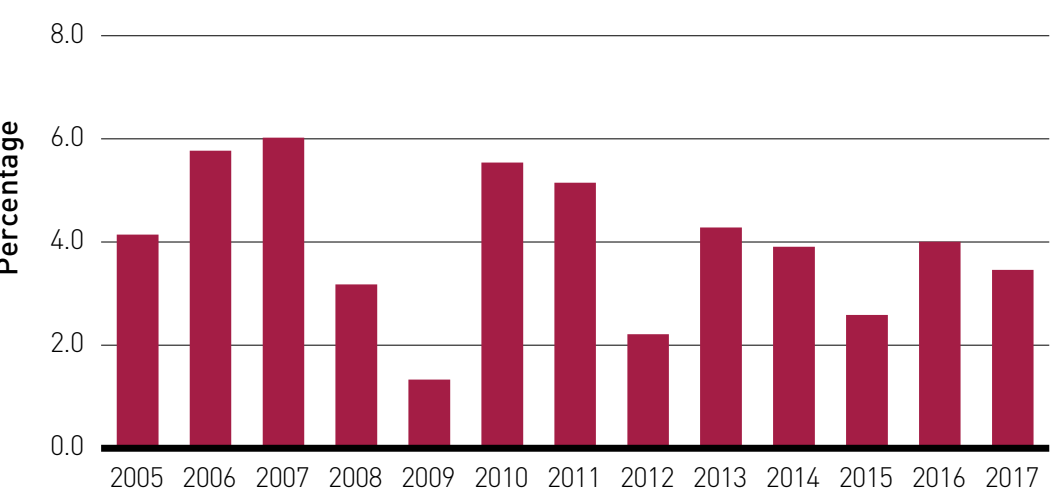

8.1.1

Real annual rate of change of GDP per capita, 2005-2017

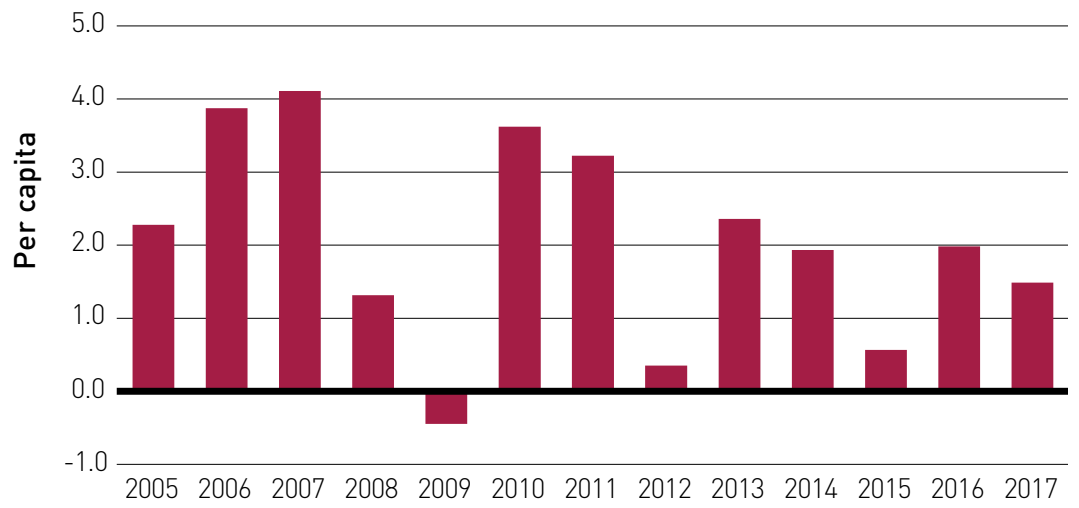




\subsection{1}

Annual growth rate of real GDP per employed person

\subsection{1}

Average hourly earnings of female and male employees, by occupation, age and persons with disabilities
Labor productivity growth: Real growth in GDP (volumes) per person employed, relative to the previous year, 2005-2017

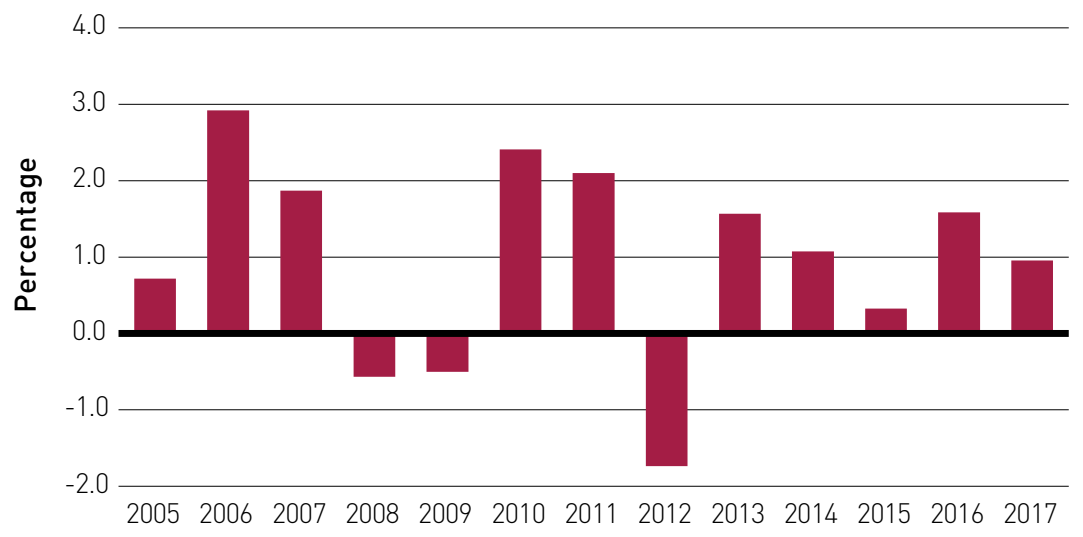

Average hourly earnings of employees ${ }^{(1)}, 2005-2017$

Females

- Males

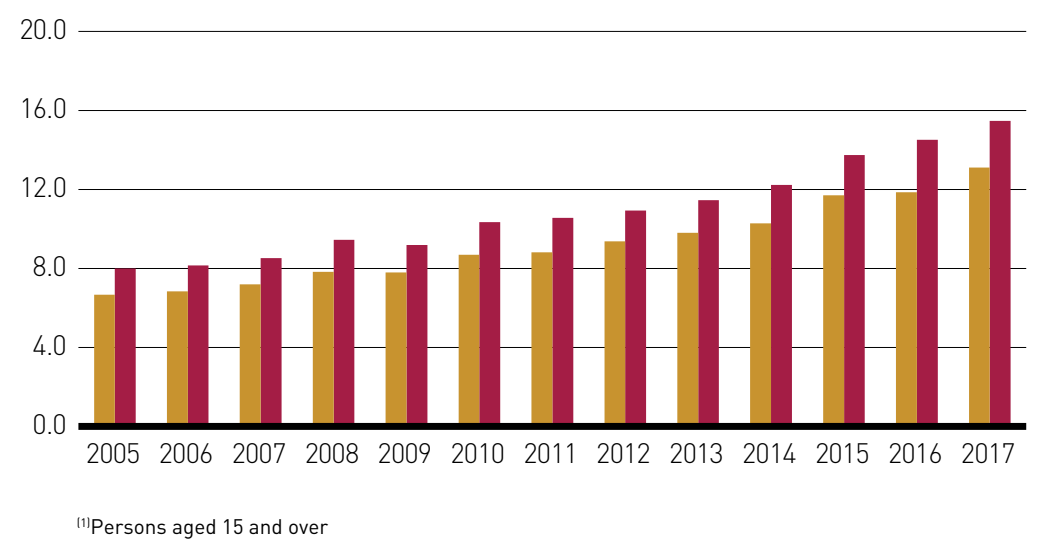


8.5.1

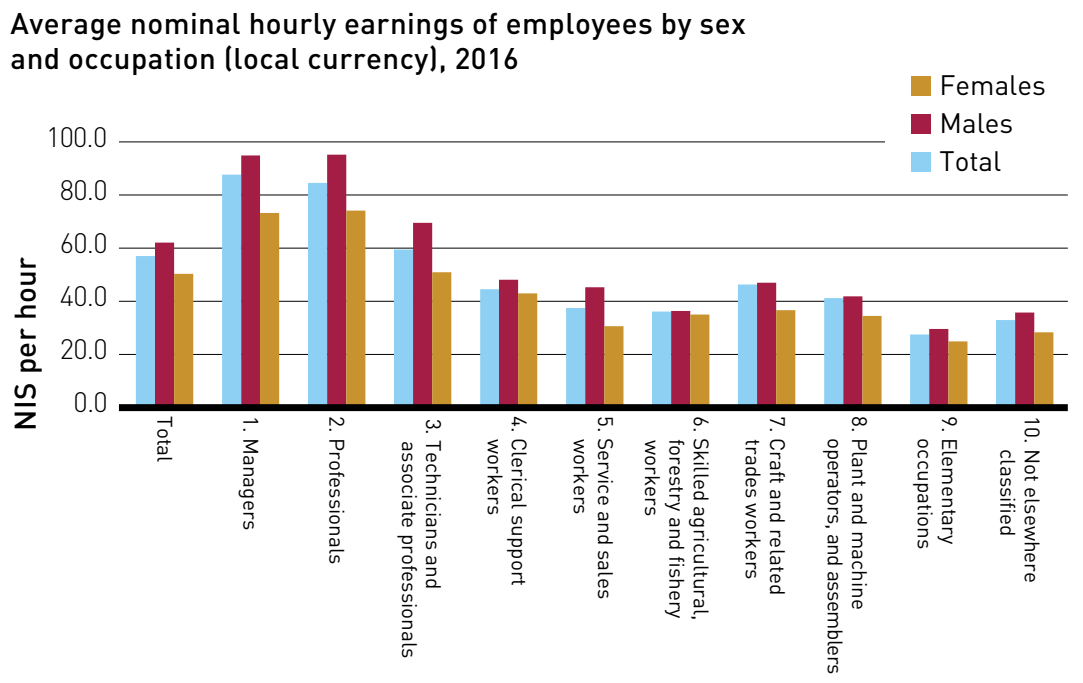

8.5 .2

Unemployment rate, by sex, age and persons with disabilities
Unemployment rate: proportion of the labor force that is unemployed, persons aged 15-74, 2005-2017

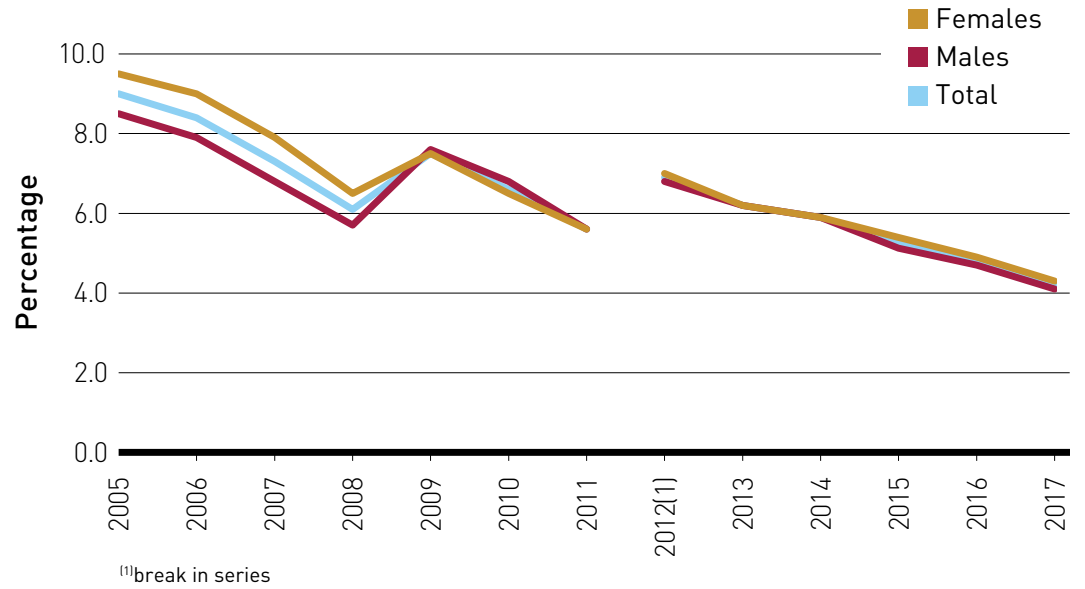


8.5 .2

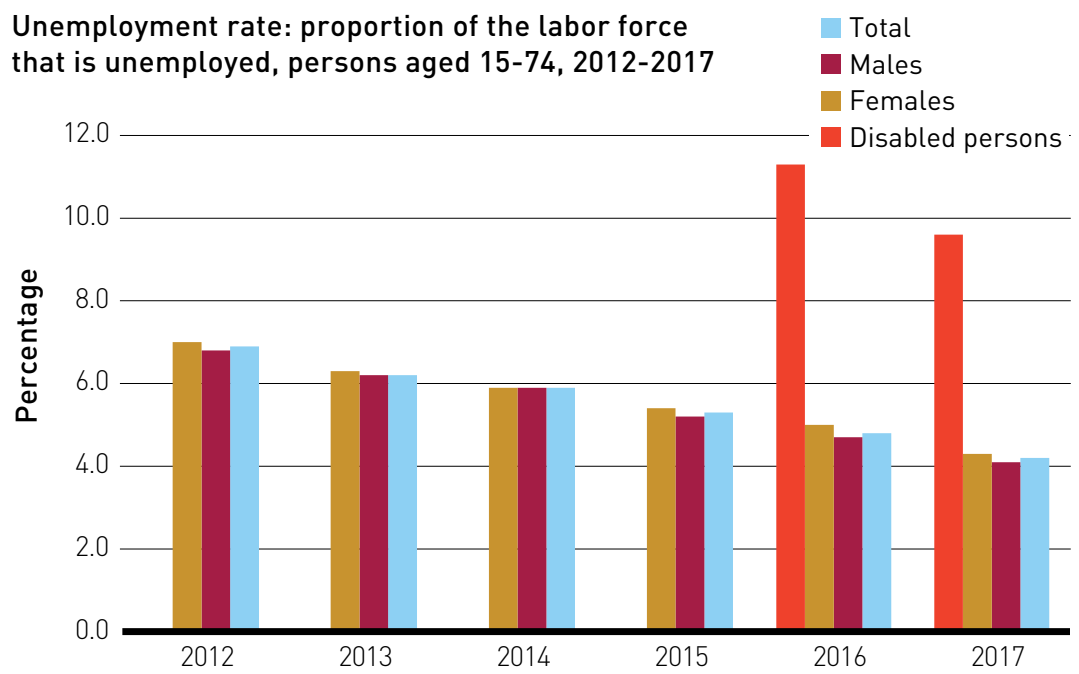

Unemployment rate by sex and disability status, 2017

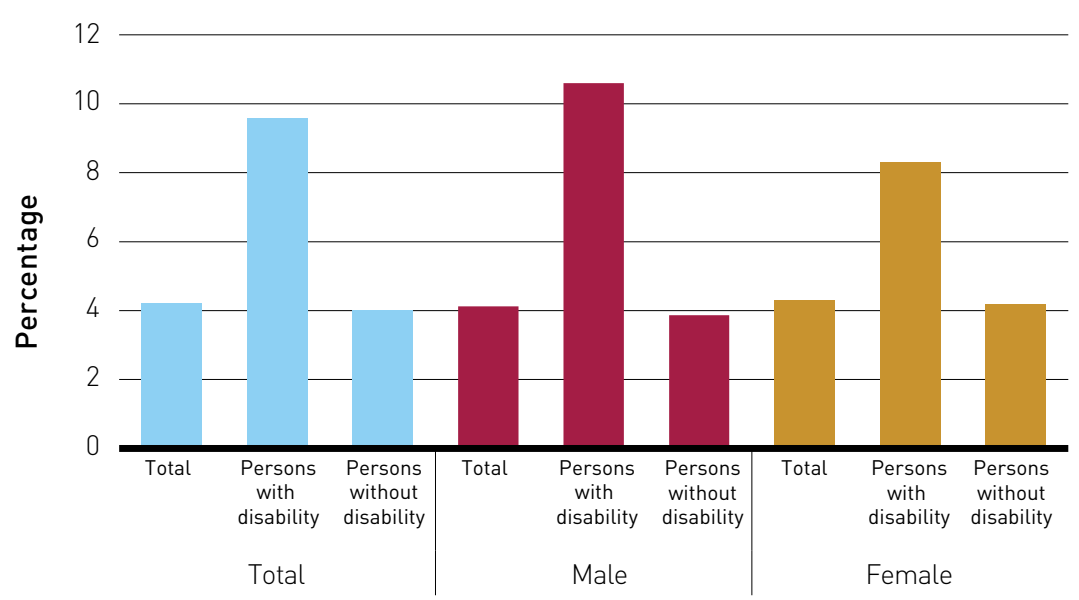


8.5 .2

\subsection{1}

Proportion of youth laged 15-24 years) not in education, employment or training
Youth unemployment rate: proportion of the labor force aged 15-24 that is unemployed, 2005-2017

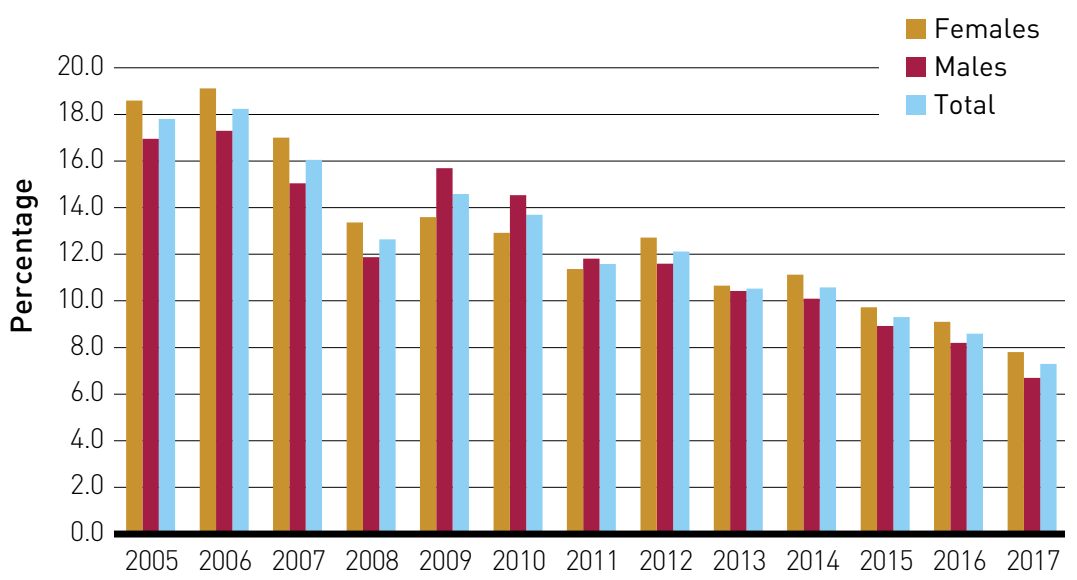

Persons aged 15-24 not in employment, education or training, 2012-2017

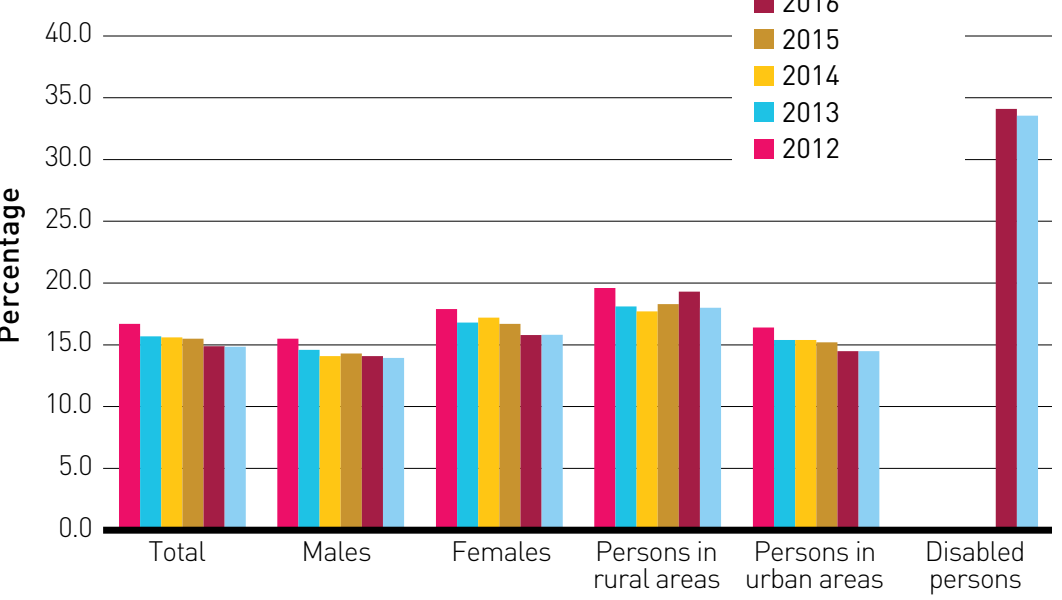




\subsection{1}

Proportion and number of children aged $5-17$ years engaged in child labor, by sex and age

\subsection{1}

Frequency rates of fatal and non-fatal occupational injuries, by sex and migrant status
Data for Israel are available for ages 15-17.

Proportion of children aged 15-17 engaged in child labor, by sex, 2005-2017

Females

Males

Total

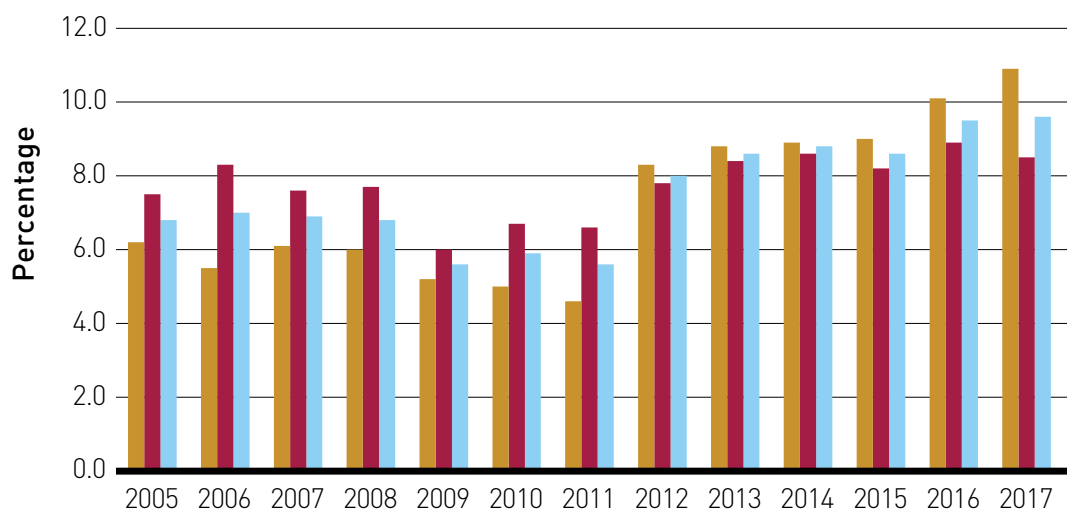

Employed persons injured in work accidents, 2000-2016

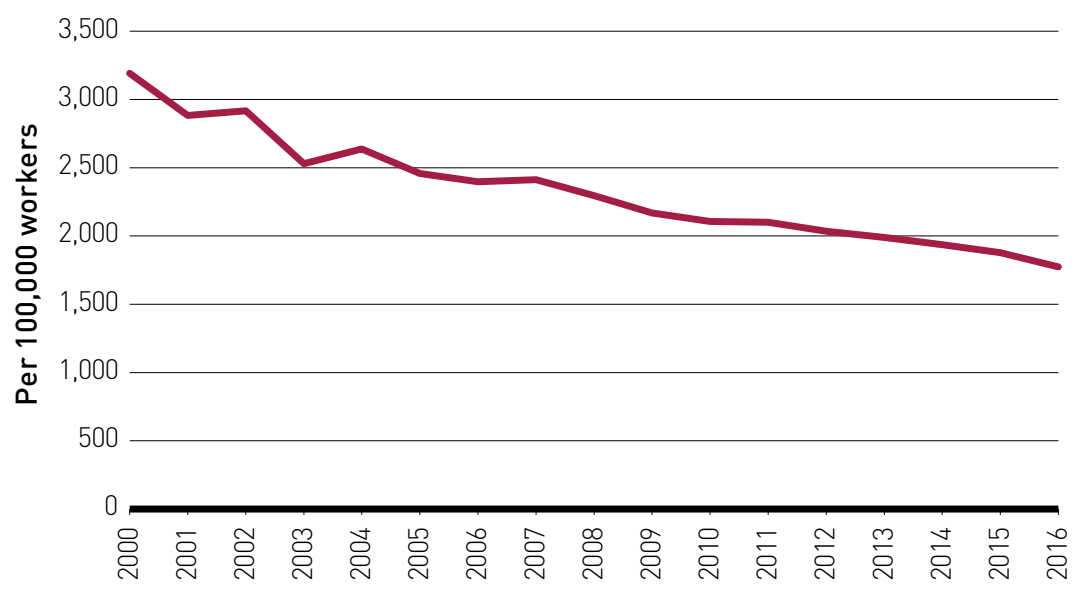




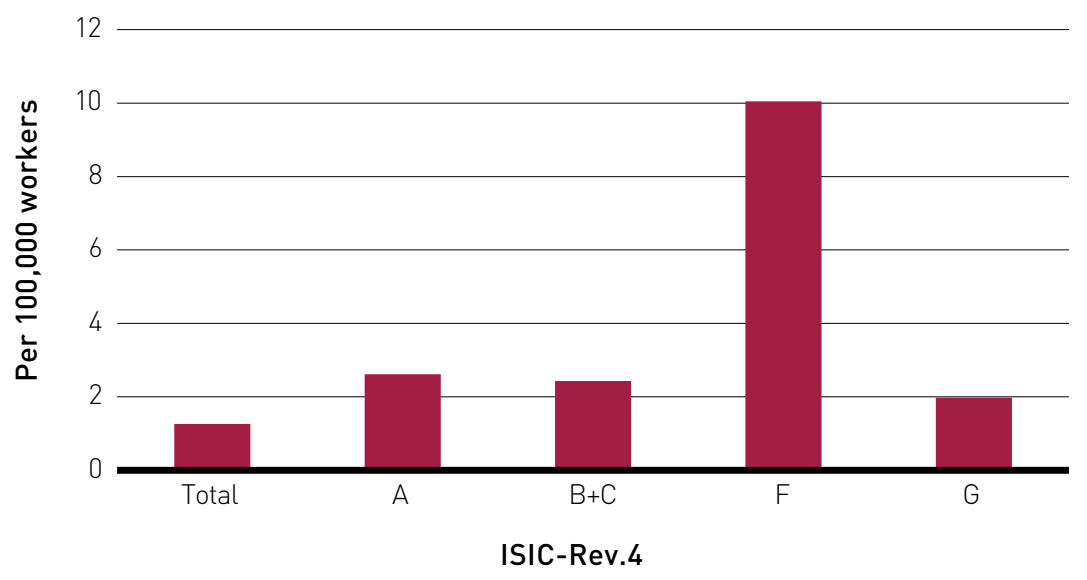

8.8.1

Non-fatal occupational injuries ${ }^{(1)}$ per 100,000 workers, by sex, 2017

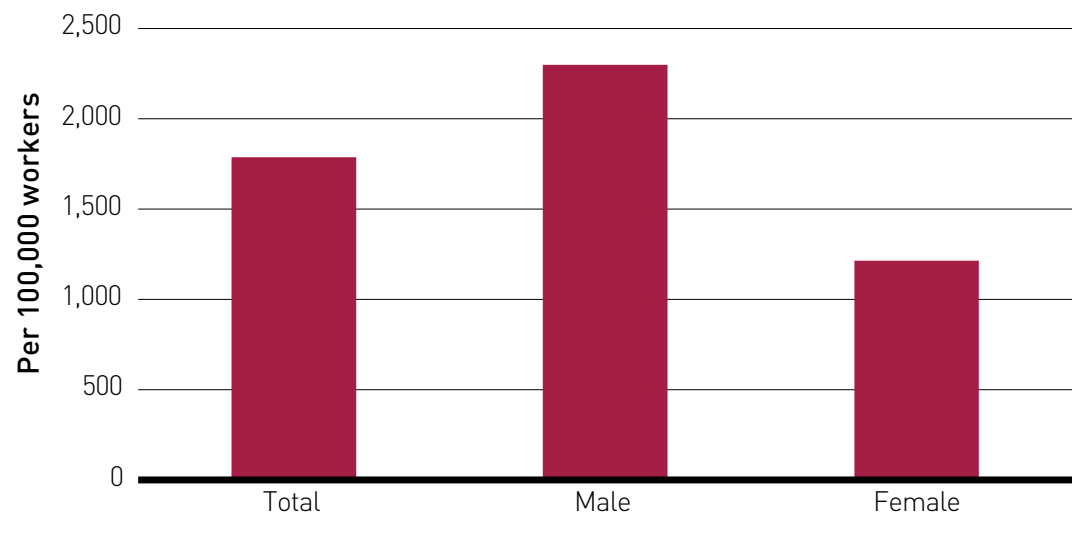

[1] Not including workers from abroad 
8.8 .1

Non-fatal occupational injuries per 100,000 workers by economic activity (rate), 2017.

\begin{tabular}{|c|c|c|}
\hline ISIC-Rev.4 & & 2017 \\
\hline Total & Total & 1,655 \\
\hline A. Agriculture; forestry and fishing & A & 2,038 \\
\hline B. Mining and quarrying & B & \\
\hline C. Manufacturing & $\mathrm{B}+\mathrm{C}$ & 2,207 \\
\hline D. Electricity; gas, steam and air conditioning supply & $\mathrm{D}$ & 1,834 \\
\hline $\begin{array}{l}\text { E. Water supply; sewerage, waste management and } \\
\text { remediation activities }\end{array}$ & E & 1,926 \\
\hline F. Construction & $\mathrm{F}$ & 2,464 \\
\hline $\begin{array}{l}\text { G. Wholesale and retail trade; repair of motor vehicles } \\
\text { and motorcycles }\end{array}$ & G & 2,265 \\
\hline H. Transportation and storage & $\mathrm{H}$ & 2,882 \\
\hline I. Accommodation and food service activities & I & 2,157 \\
\hline J. Information and communication & J & 783 \\
\hline K. Financial and insurance activities & K & 1,103 \\
\hline L. Real estate activities & L & 4,727 \\
\hline M. Professional, scientific and technical activities & M & 1,266 \\
\hline N. Administrative and support service activities & $\mathrm{N}$ & 2,560 \\
\hline $\begin{array}{l}\text { O. Public administration and defense; compulsory } \\
\text { social security }\end{array}$ & 0 & 1,483 \\
\hline P. Education & $\mathrm{P}$ & 369 \\
\hline Q. Human health and social work activities & Q & 1,154 \\
\hline R. Arts, entertainment and recreation & $\mathrm{R}$ & 1,908 \\
\hline S. Other service activities & S & 2,007 \\
\hline $\begin{array}{l}\text { T. Activities of households as employers; } \\
\text { undifferentiated goods- and services-producing } \\
\text { activities of households for own use }\end{array}$ & T & 135 \\
\hline U. Activities of extraterritorial organizations and bodies & U & 1,494 \\
\hline X. Not elsewhere classified & $x$ & 647 \\
\hline
\end{tabular}


ISIC-Rev.4

2016

\begin{tabular}{|c|c|c|}
\hline Total & Total & 1,772 \\
\hline A. Agriculture; forestry and fishing & A & 2,151 \\
\hline B. Mining and quarrying & B & \\
\hline C. Manufacturing & $\mathrm{B}+\mathrm{C}$ & 2,305 \\
\hline D. Electricity; gas, steam and air conditioning supply & $\mathrm{D}$ & 2,381 \\
\hline $\begin{array}{l}\text { E. Water supply; sewerage, waste management and } \\
\text { remediation activities }\end{array}$ & E & 1,858 \\
\hline F. Construction & $\mathrm{F}$ & 2,712 \\
\hline $\begin{array}{l}\text { G. Wholesale and retail trade; repair of motor vehicles } \\
\text { and motorcycles }\end{array}$ & G & 2,376 \\
\hline H. Transportation and storage & $\mathrm{H}$ & 3,069 \\
\hline I. Accommodation and food service activities & I & 2,390 \\
\hline J. Information and communication & $J$ & 877 \\
\hline K. Financial and insurance activities & K & 1,195 \\
\hline L. Real estate activities & $L$ & 4,964 \\
\hline M. Professional, scientific and technical activities & M & 1,336 \\
\hline N. Administrative and support service activities & N & 2,867 \\
\hline $\begin{array}{l}\text { O. Public administration and defense; compulsory } \\
\text { social security }\end{array}$ & 0 & 1,556 \\
\hline P. Education & $P$ & 396 \\
\hline Q. Human health and social work activities & Q & 1,215 \\
\hline R. Arts, entertainment and recreation & $\mathrm{R}$ & 1,970 \\
\hline S. Other service activities & S & 2,111 \\
\hline $\begin{array}{l}\text { T. Activities of households as employers; } \\
\text { undifferentiated goods- and services-producing } \\
\text { activities of households for own use }\end{array}$ & $\mathrm{T}$ & 159 \\
\hline U. Activities of extraterritorial organizations and bodies & $U$ & 903 \\
\hline X. Not elsewhere classified & $x$ & 800 \\
\hline
\end{tabular}




\subsection{1}

Tourism direct GDP as a proportion of total GDP and in growth rate

8.9.1

8.9.2

Proportion of jobs in sustainable tourism industries out of total tourism jobs
Tourism direct GDP, 2008-2017

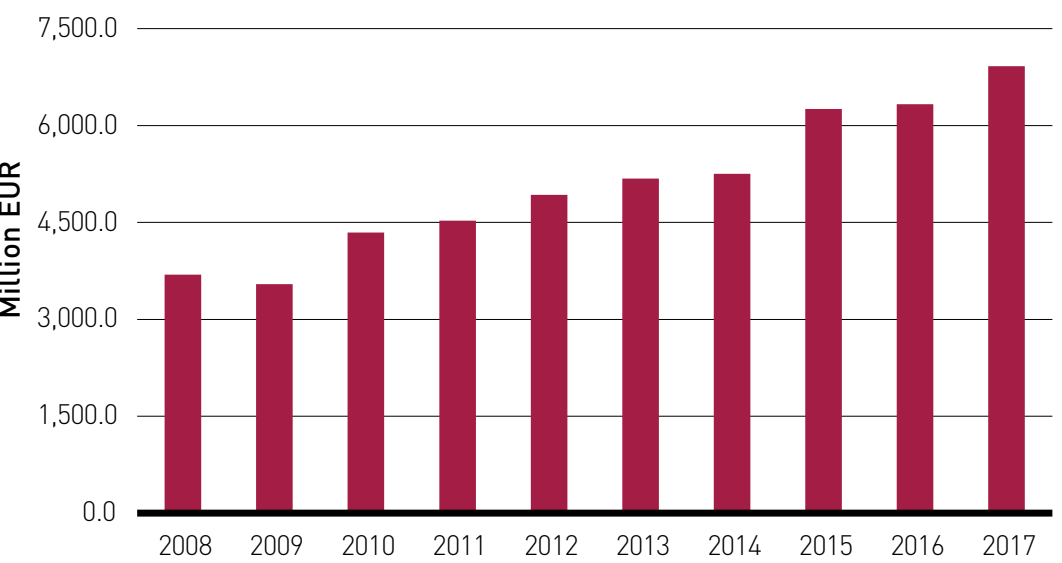

Tourism GDP (direct) as \% of total GDP, 2008-2017

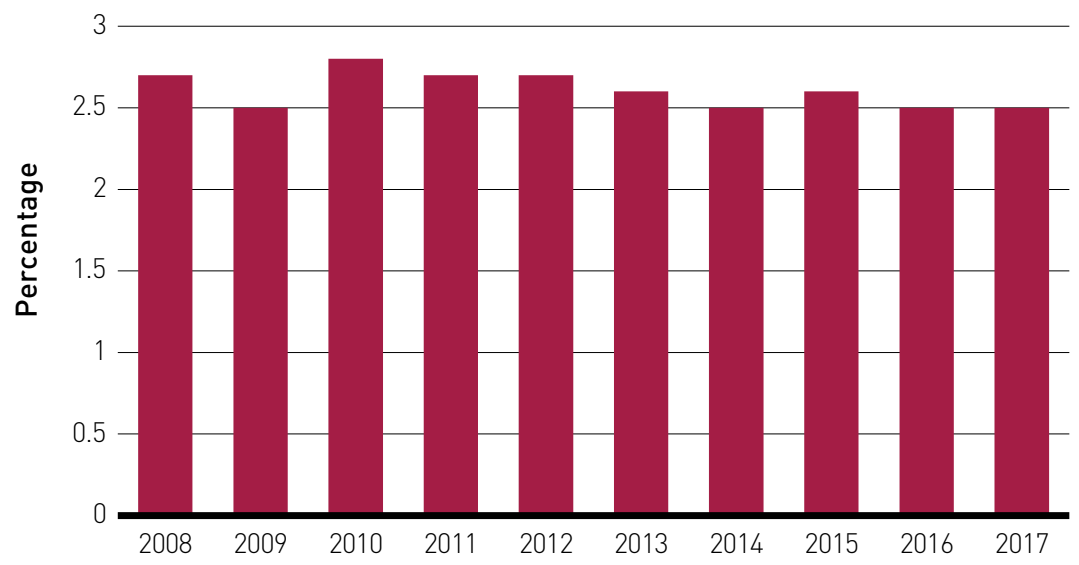

Data are for total tourism employment as a proportion of all persons employed.

Tourism employment, 2010-2017

160.0

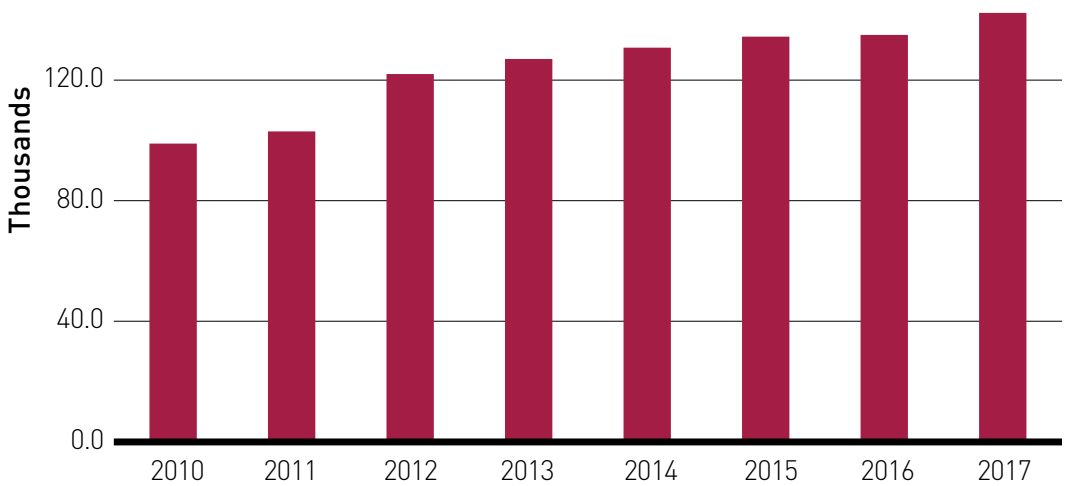




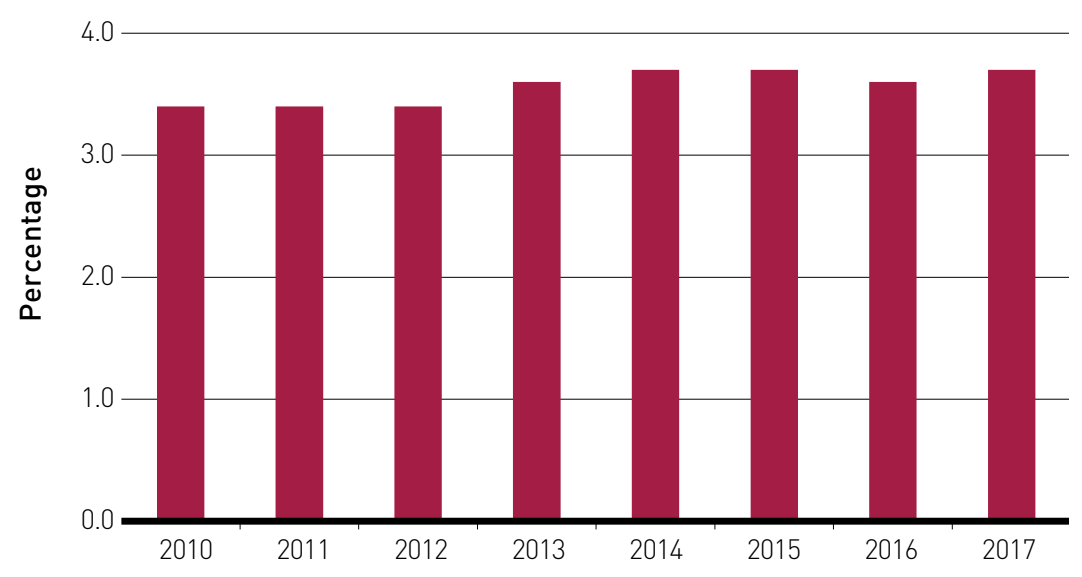

8.10 .1

(a) Number of commercial bank branches per 100,000 adults and (b) number of automated teller machines (ATMs) per 100,000 adults

Number of commercial bank branches and automated teller machines (ATMs) per 100,000 adults (persons aged 15+), 2005-2017

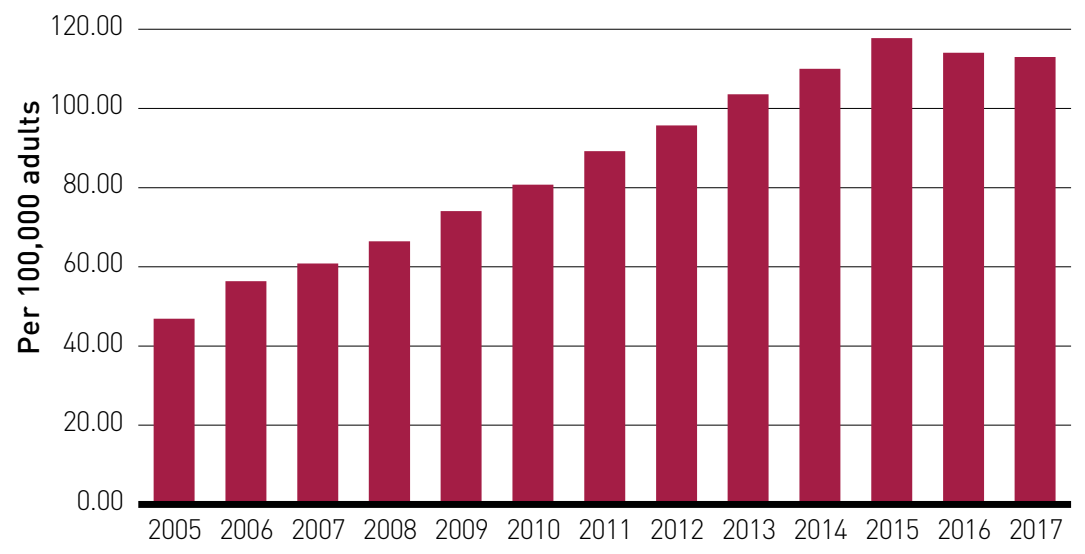


GOAL 9

9.1.1

Proportion of the rural population who live within $2 \mathrm{~km}$ of an all-season road

\subsection{2}

Passenger and freight volumes, by mode of transport

\section{BUILD RESILIENT INFRASTRUCTURE, PROMOTE INCLUSIVE AND SUSTAINABLE INDUSTRIALIZATION AND FOSTER INNOVATION}

In Israel, nearly $100 \%$ of the population lives within $2 \mathrm{~km}$ of an allseason road.

Volume of domestic railway passenger transport, 2014-2016

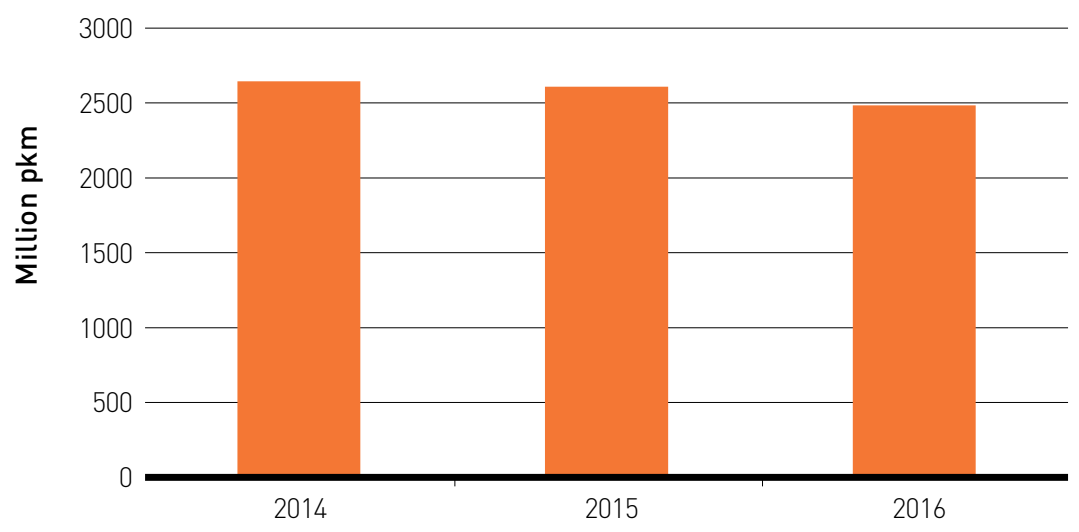

Volume of domestic railway freight transport, 2014-2016

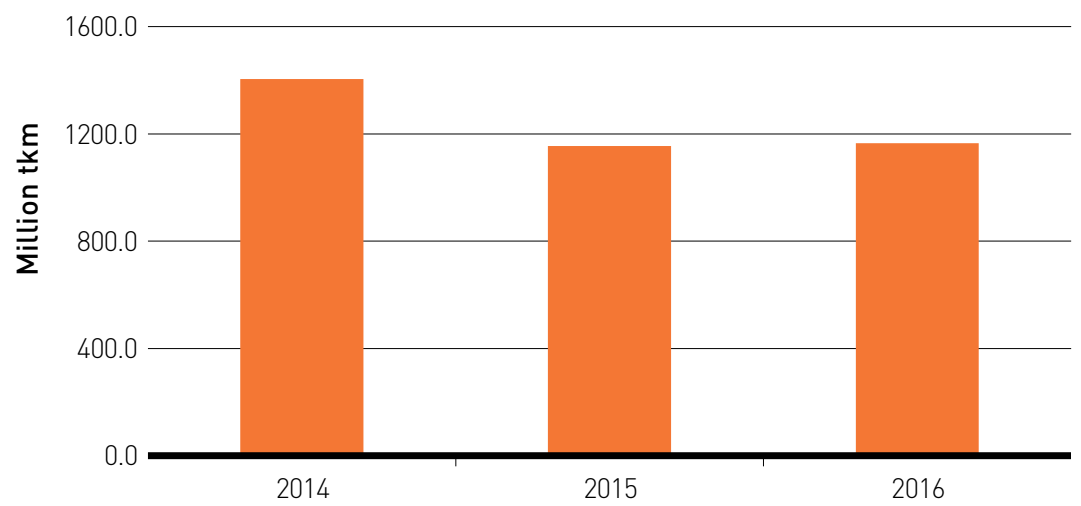



unloaded at national ports in Thousand tonnes), 2014-2016

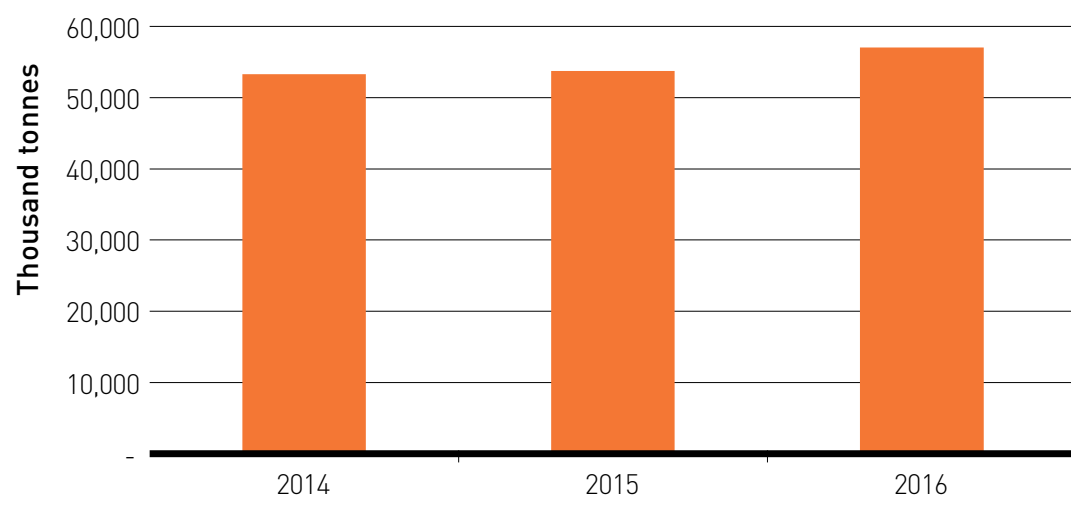

\subsection{1}

Manufacturing value added as

Manufacturing value added as a proportion of GDP, 1995-2017 a proportion of GDP and per capita

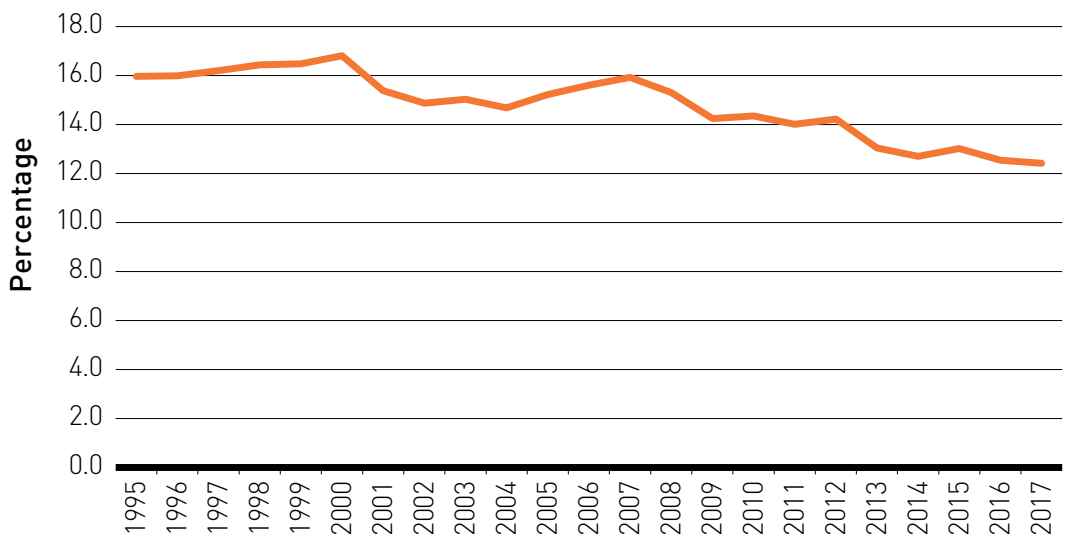




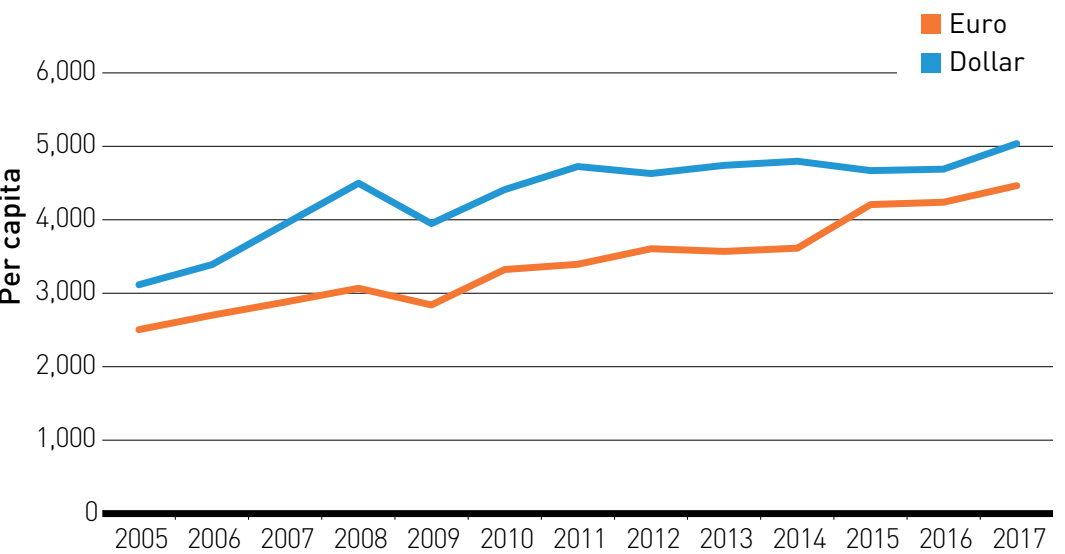

\section{2 .2}

Manufacturing employment as a proportion of total employment

Manufacturing employment as a proportion of total employment, 1995-2017

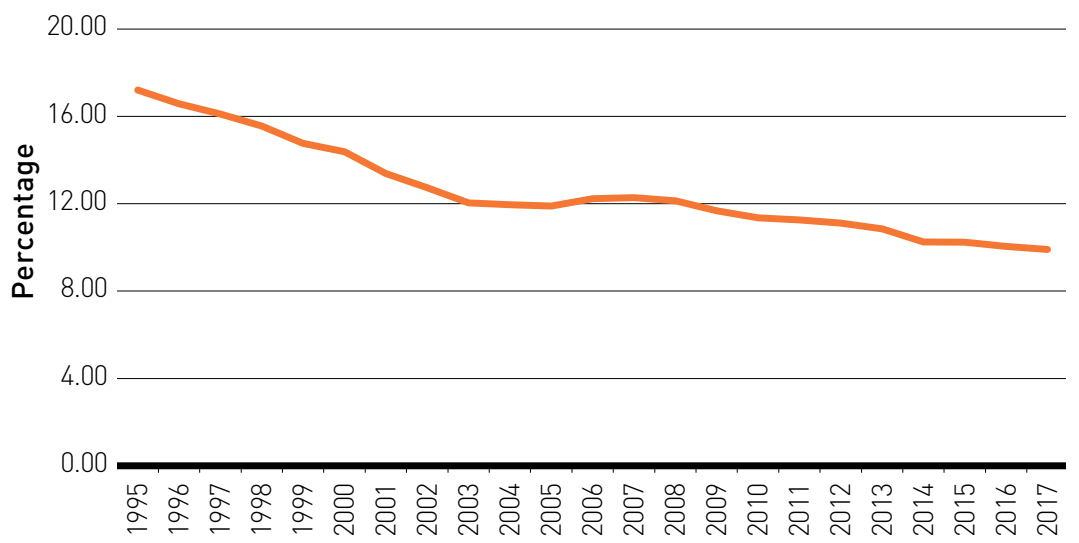


9.4.1

$\mathrm{CO}_{2}$ emission per unit of value added

\subsection{1}

Research and development expenditure as a proportion of GDP
$\mathrm{CO}_{2}$ emission per unit of value added (1000 ton/\$ GDP PPP), 2005-2016

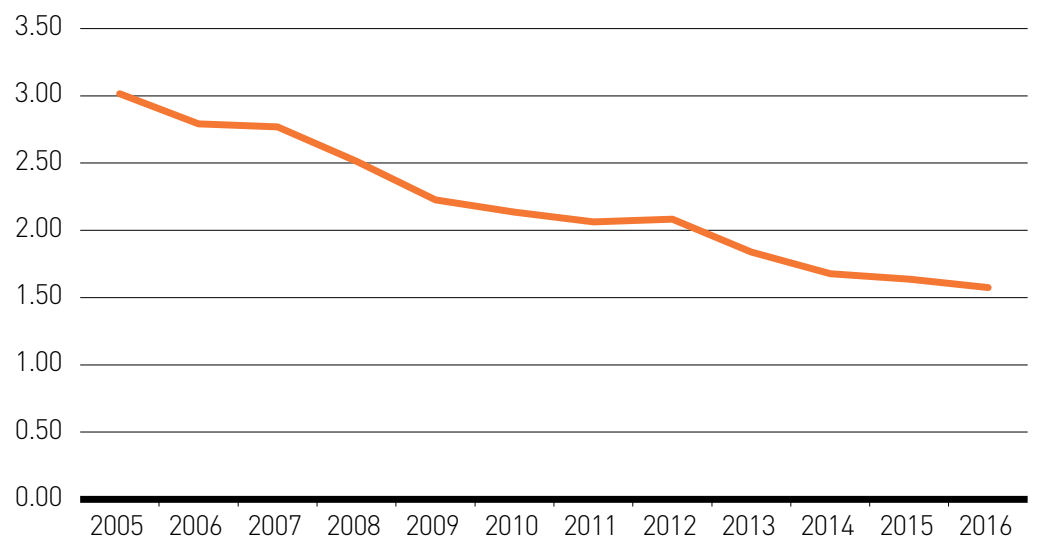

Research and development expenditure as a proportion of GDP, 2005-2017

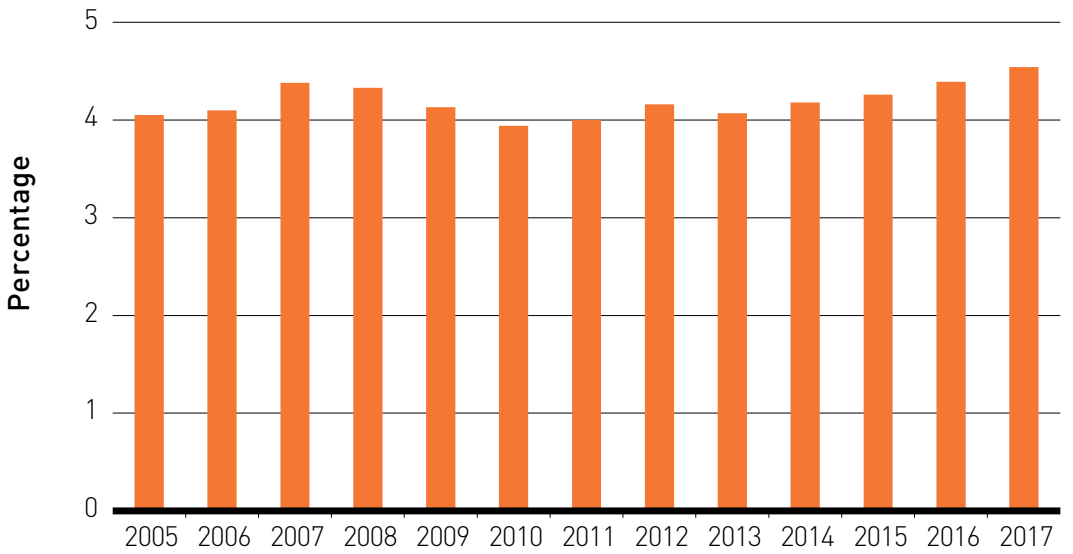


9.5.1

\section{9.b.1}

Proportion of medium and high-tech industry value added in total value added
Expenditure on business R\&D as a percentage of GDP, 2016

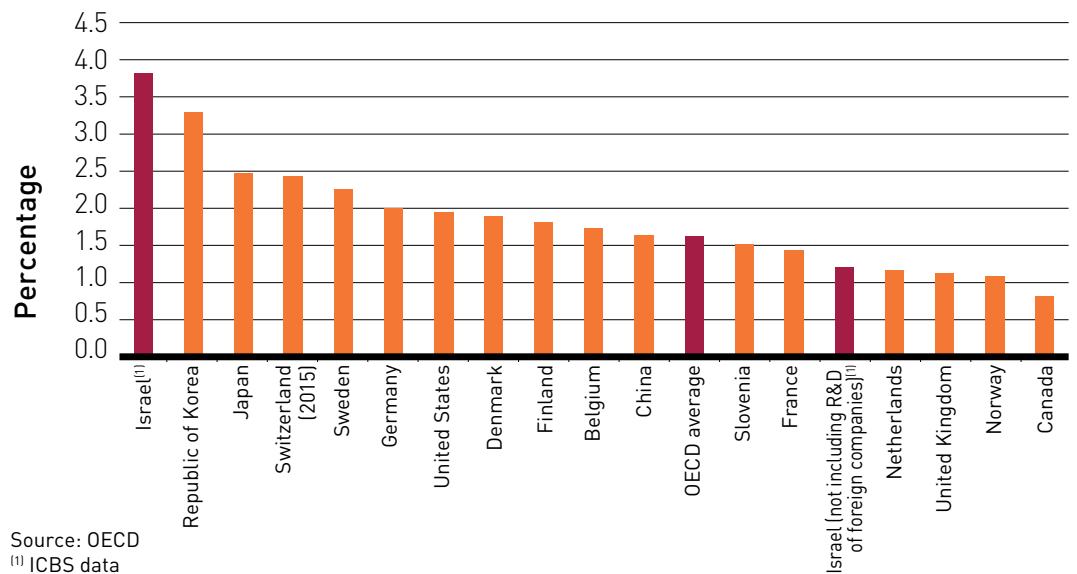

Medium and high technology manufacturing share of manufacturing value added, 2005-2015(1)

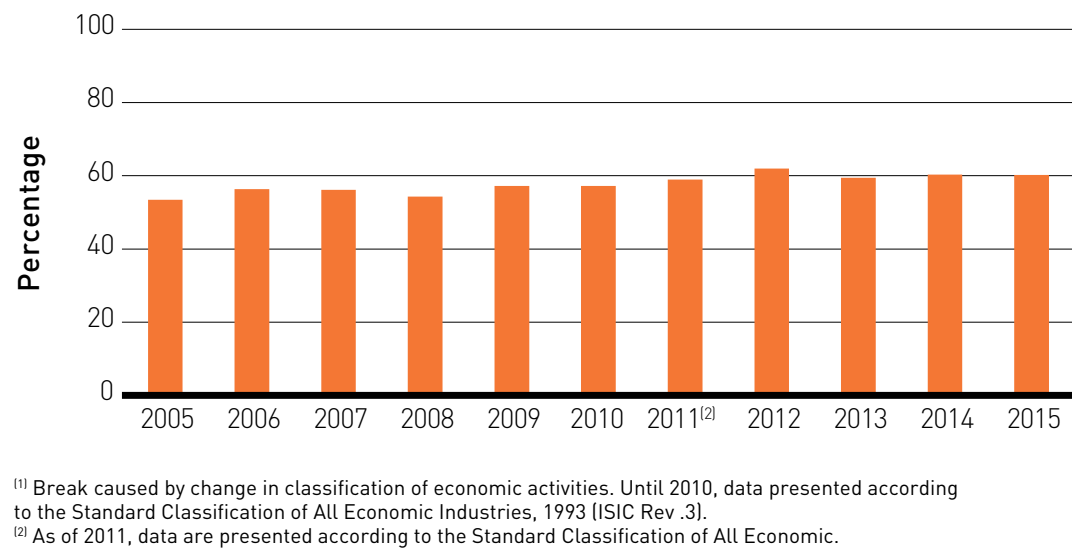


GOAL 10

10.2.1

Proportion of people living below 50 per cent of median income, by sex, age and persons with disabilities

10.3.1

Proportion of the population reporting having personally felt discriminated

10.3.1

\section{REDUCE INEQUALITY WITHIN AND AMONG COUNTRIES}

See indicator 1.2.1.

Persons aged 20 and over who felt discrimination ${ }^{(1)}$ by sex, age and population group, 2017

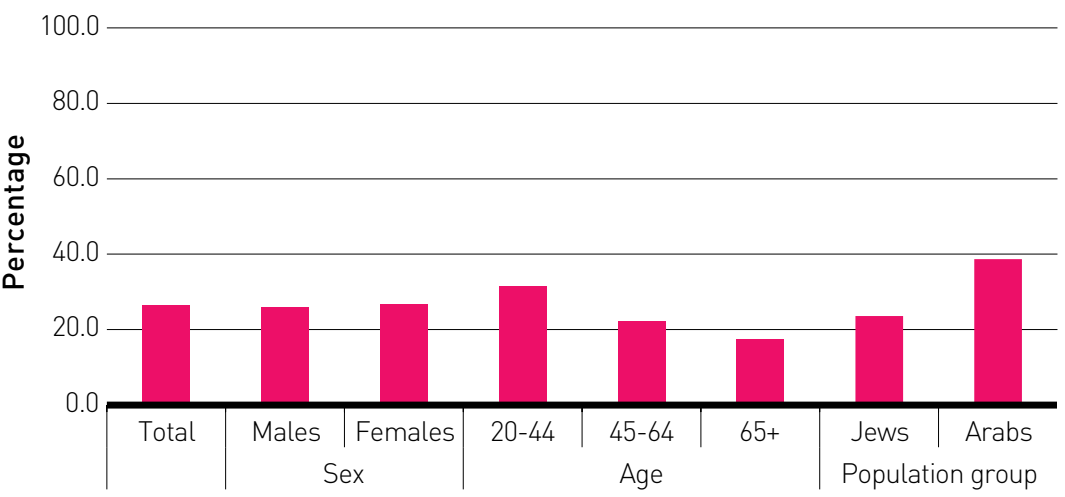

(1) Felt discrimination based on age, nationality, origin, religion, gender, disability, or skin color.

Persons aged 20 and over who felt discrimination, by sex and type of discrimination, 2017

Females

- Males

30.0

Total

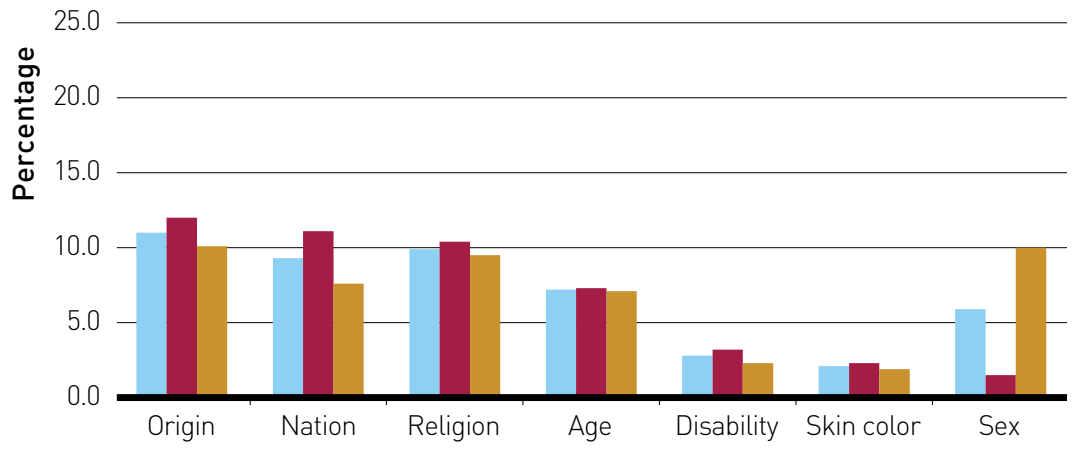


10.4.1

Labor share of GDP, comprising wages and social protection transfers
Total compensation of employees given as a percentage of nominal GDP for the total economy, 2013-2017

100.0

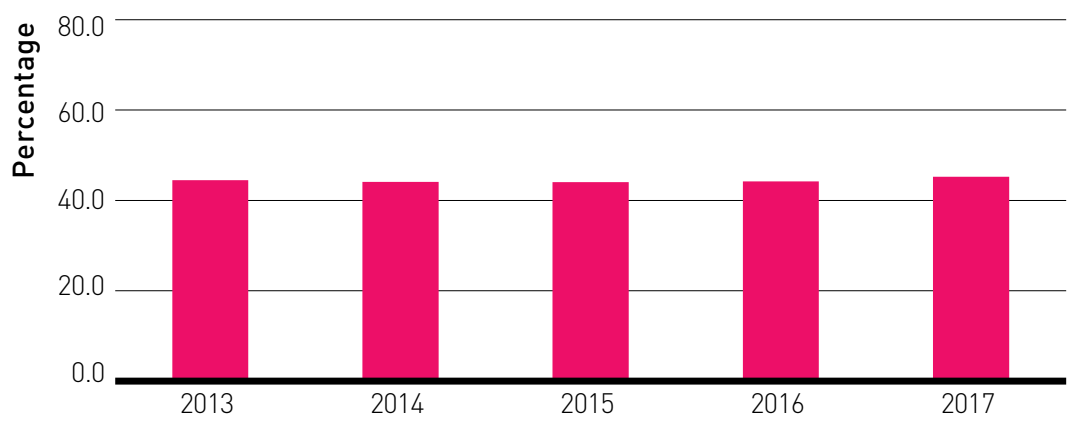


GOAL 11

\subsection{1}

Proportion of population that has convenient access to public transport, by sex, age and persons with disabilities

\subsection{2}

Proportion of cities with a direct participation structure of civil society in urban planning and management that operate regularly and democratically

\subsection{1}

Total expenditure (public and privatel per capita spent on the preservation, protection and conservation of all cultural and natural heritage, by type of heritage /cultural, natural, mixed and World Heritage Centre designation), level of government Inational, regional and local/municipal), type of expenditure loperating expenditure/investment] and type of private funding Idonations in kind, private non-profit sector and sponsorship)

\section{MAKE CITIES AND HUMAN SETTLEMENTS INCLUSIVE, SAFE, RESILIENT AND SUSTAINABLE}

Based on GIS calculations in urban localities with 2,000 or more residents, $91.8 \%$ of the urban area is within $0.5 \mathrm{~km}$ from public transport. $97.1 \%$ of the population in these localities has convenient access to public transport based on the definitions of the indicator.

Planning processes in all cities in Israel are transparent to the public at some level through planning committees. Nonetheless, the level of participation varies from cases where citizens may express their opposition to a project to cases where a much wider public participation process exists.

Data are available only for expenditure on cultural heritage as a percentage of total government expenditure. Cultural heritage includes museums, archives, antiquities preservation and archeological sites preservation. In 2017, expenditure on cultural heritage was $0.36 \%$ of total government expenditure.

\begin{tabular}{|l|l|l|l|}
\hline \multirow{2}{*}{ Year } & $\begin{array}{l}\text { Expenditure of } \\
\text { government and } \\
\text { national institutions on } \\
\text { cultural heritage }\end{array}$ & $\begin{array}{l}\text { Expenditure of } \\
\text { local authorities on } \\
\text { cultural heritage }\end{array}$ & $\begin{array}{l}\text { Expenditure } \\
\text { on cultural } \\
\text { heritage as a } \\
\text { percentage of } \\
\text { total government } \\
\text { expenditure } \\
\text { lpercentage) }\end{array}$ \\
\hline 2000 & 47.0 & 6.1 & 0.32 \\
\hline 2013 & 100.8 & 10.8 & 0.38 \\
\hline 2014 & 76.8 & 9.8 & 0.38 \\
\hline 2015 & 65.7 & 7.6 & 0.34 \\
\hline 2016 & 107.5 & 7.9 & 0.37 \\
\hline 2017 & 108.7 & 9.0 & 0.36 \\
\hline
\end{tabular}


11.6.1

\section{Proportion of urban solid} waste regularly collected and with adequate final discharge out of total urban solid waste generated, by cities

\section{7 .1}

Average share of the builtup area of cities that is open space for public use for all, by sex, age and persons with disabilities
Urban solid waste regularly collected and with adequate final discharge out of total urban solid waste generated, 2005-2017

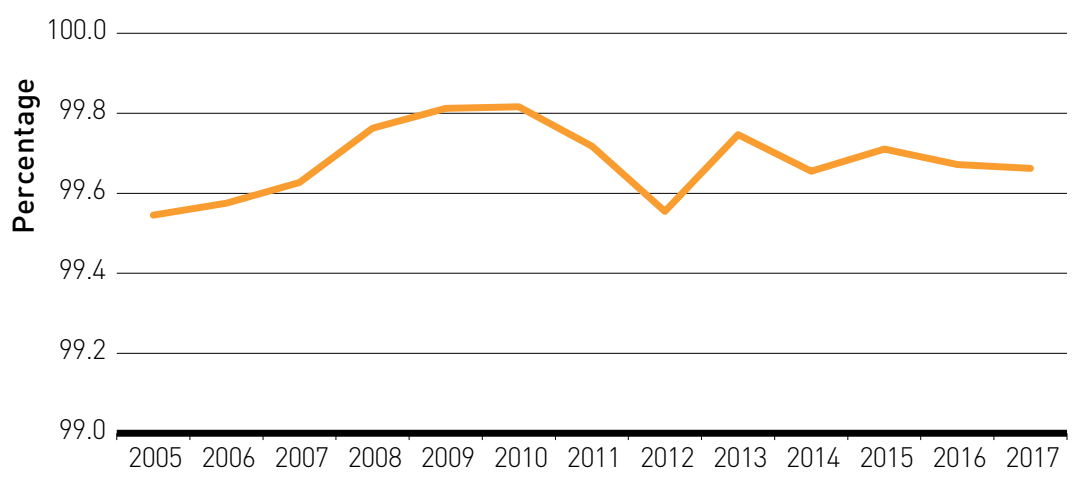

The number of cities with non-sufficient open space is based on the UN Habitat minimum of $15 \%$ of the built-up area.

The calculations are based on 2013 Central Bureau of Statistics (ICBS) land use data, after removal of agricultural land uses, forests and other undeveloped land and waterbodies.

Public space includes the following land uses: public services, culture and leisure, infrastructure and transportation, public parks and other public open areas.

Number of cities with sufficient public space, 2016

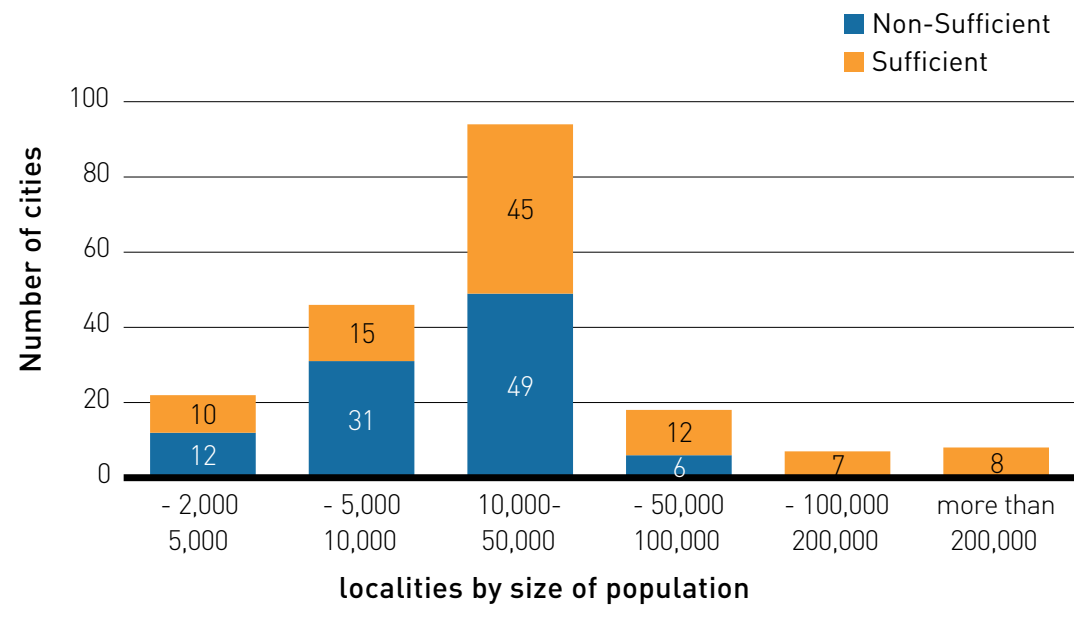


11.7 .2

Proportion of persons victim of physical or sexual harassment, by sex, age, disability status and place of occurrence, in the previous 12 months

\section{7 .2}

\section{1.a.1}

Proportion of population living in cities that implement urban and regional development plans integrating population projections and resource needs, by size of city
Persons aged 20 and over who were sexualy harassed, 2007-2017

Total Females

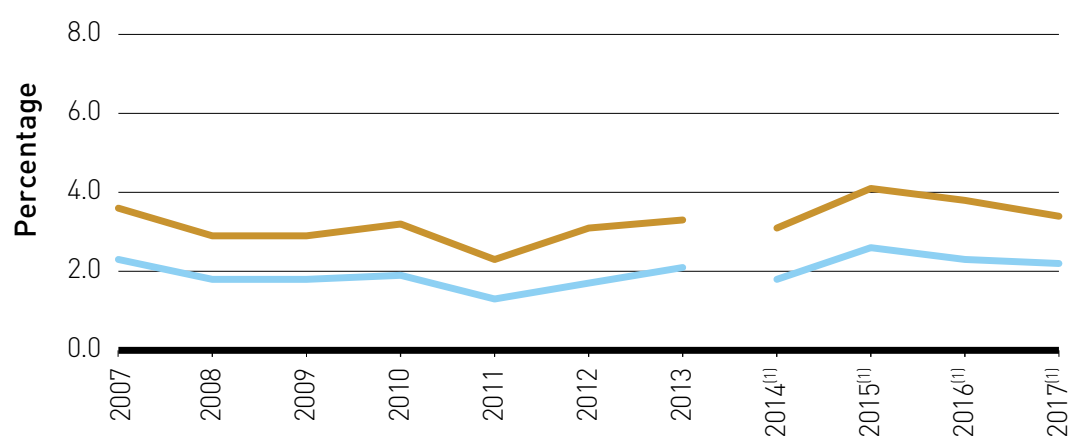

(1) Change in data source: until 2013, the social survey; from 2014, Israel's Crime Victimization Survey (ICVS)

Persons 20 and over who were sexually harassed by sex and age, 2017

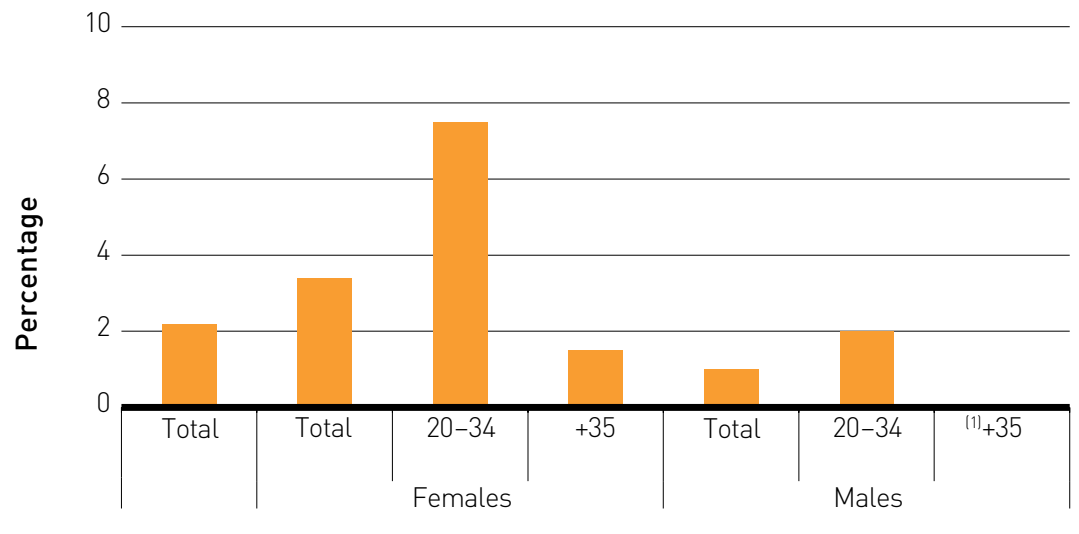

Source: Israel's Crime Victimization Survey (ICVS)

(1) The proportion of men aged 35 and over who were affected by sexual harassment is minimal and is subject to relatively high sampling errors and therefore cannot be presented

Planning processes in all cities in Israel involve urban and regional development plans through seven district planning committees. 


\section{GOAL 12}

\subsection{1}

Number of countries with sustainable consumption and production (SCP) national action plans or SCP main streamed as a priority or a target into national policies

\subsection{1}

Number of parties to international multilateral environmental agreements on hazardous waste, and other chemicals that meet their commitments and obligations in transmitting information as required by each relevant agreement

\subsection{2}

Hazardous waste generated per capita and proportion of hazardous waste treated, by type of treatment

\section{ENSURE SUSTAINABLE CONSUMPTION AND PRODUCTION PATTERNS}

\begin{abstract}
Israel has an SCP National Action Plan since 2015. SCP is mainstreamed into national policies relating to green public procurement; implementation of sustainable development strategies within government companies; support in the assimilation of best practices by small and medium enterprises; launch of the resource efficiency knowledge center; and promotion of environmental standards and labeling.
\end{abstract}

Israel is party to the United Nations Convention on the Control of Transboundary Movements of Hazardous Wastes and Their Disposal (the Basel Convention) and complies with its provisions. Israel has yet to ratify the Ban Amendment but already complies with its provisions further limiting the export of hazardous waste. Israel is a party to the Rotterdam Convention on Prior Informed Consent (PIC) and meets its obligations. It is in the process of working towards ratifying the Stockholm Convention on Persistent Organic Pollutants (POPs) and the Minamata Convention on Mercury.

Hazardous waste, by method of treatment, 2010-2017
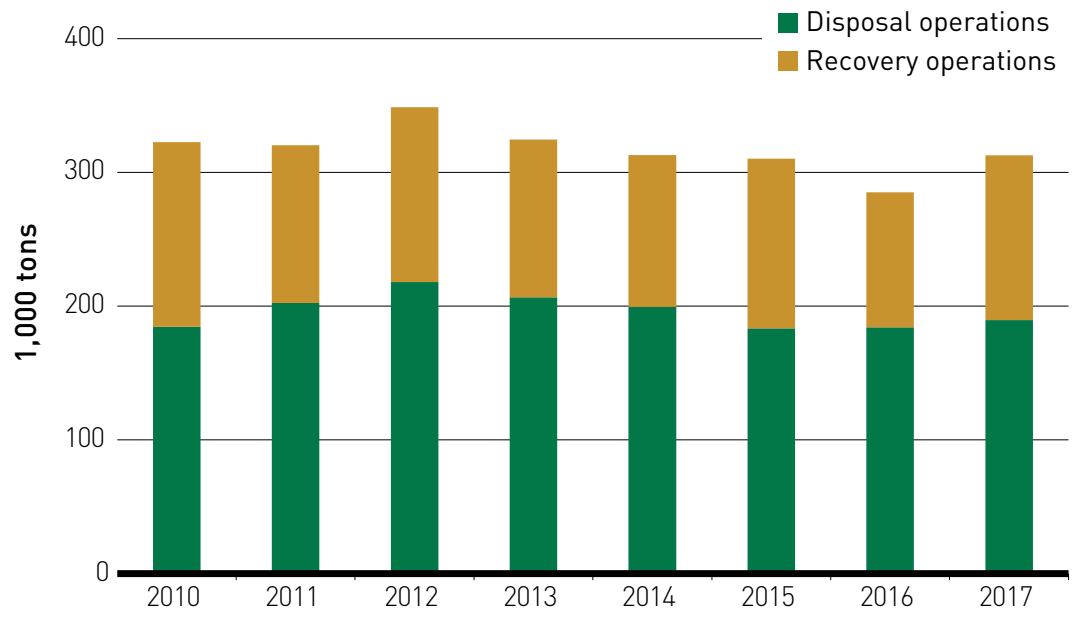
12.4 .2

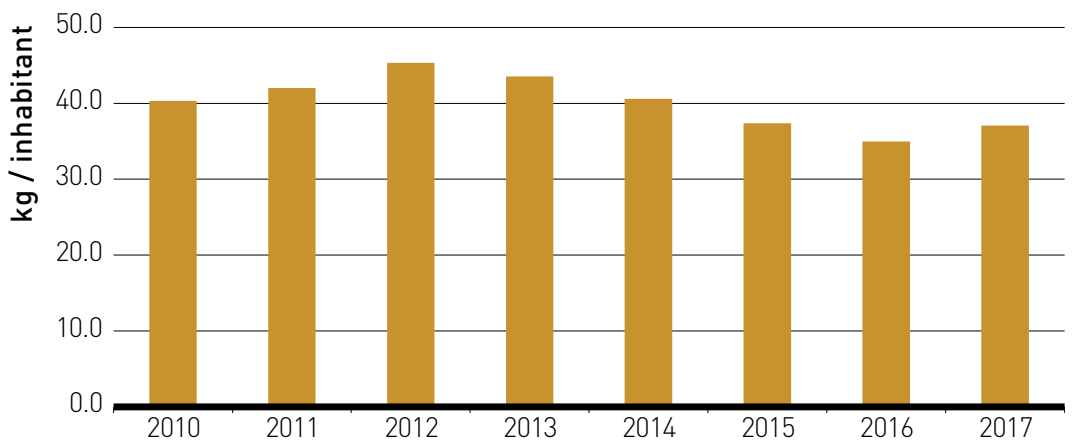

12.4 .2

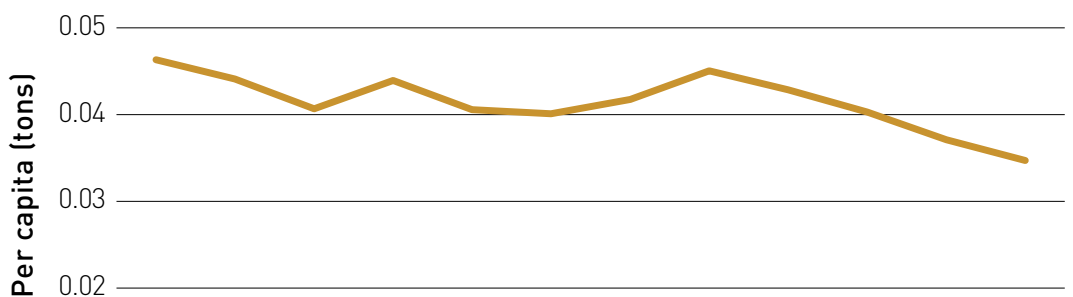

0.0

$\begin{array}{llllllllllll}2005 & 2006 & 2007 & 2008 & 2009 & 2010 & 2011 & 2012 & 2013 & 2014 & 2015 & 2016\end{array}$ 
12.5.1

National recycling rate, tons of material recycled

12.5 .1
Proportion of collected municipal waste that is recovered through material recycling, composting and digestion, 2012-2017

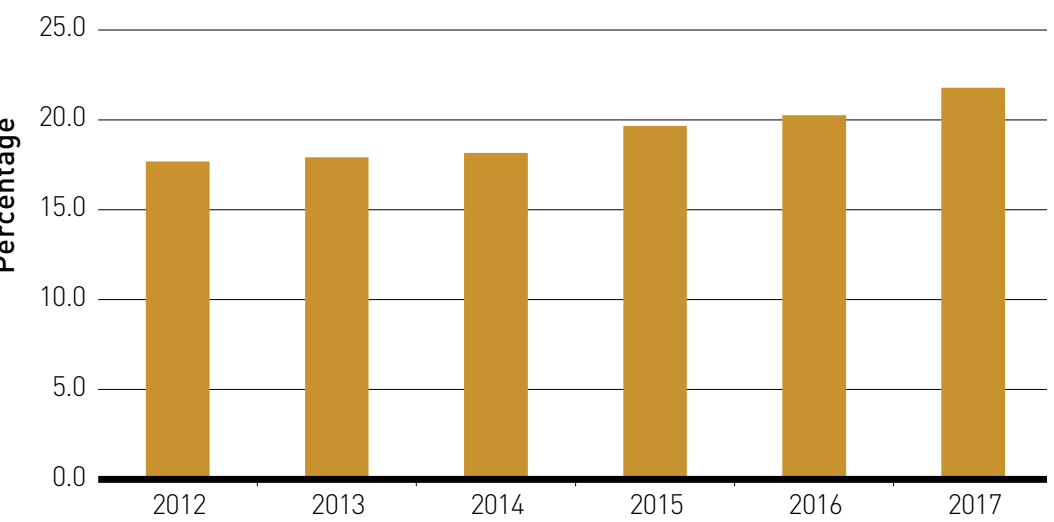

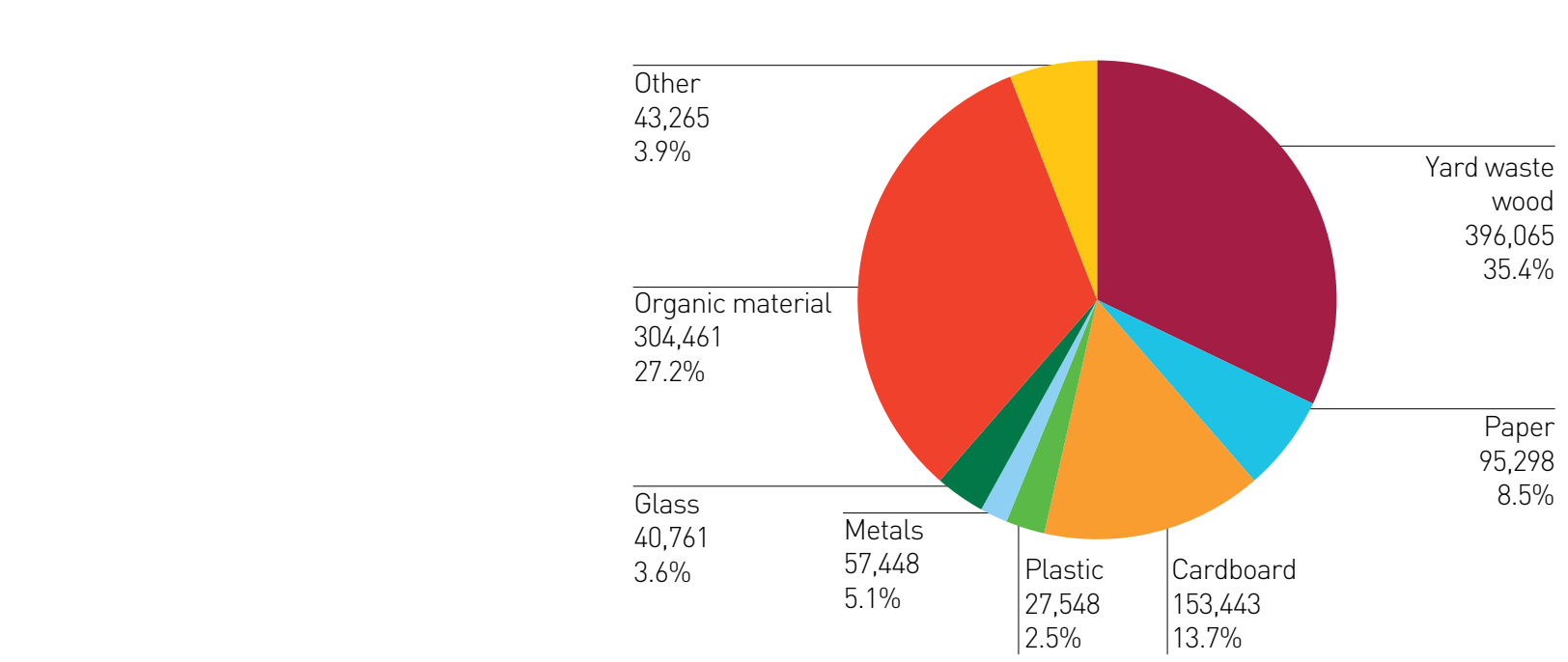

Recycling of waste by type of material (tons), 2017 
12.5 .1

Recycling of waste in selected OECD countries, 2016

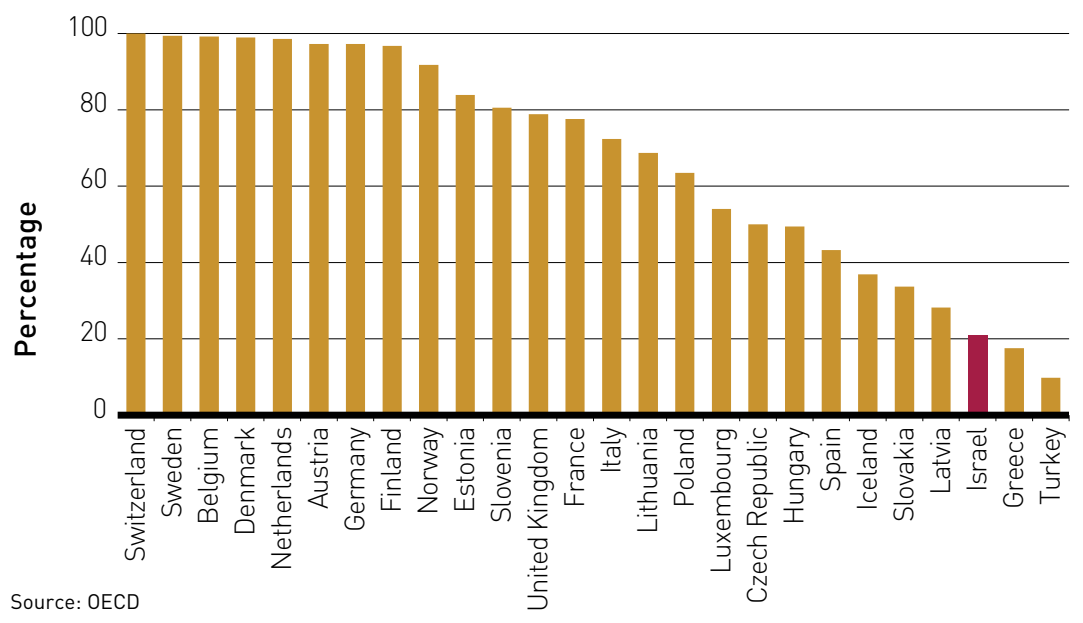

12.6.1

Number of companies publishing sustainability reports

The number of sustainability reports submitted by companies in Israel is still low but increasing gradually. Banks are a leading sector in this field with a mandatory requirement to publish a sustainability report. As reporting is still mostly voluntary in Israel, a number of different sustainability (CSR) reporting schemes are in use. "Reporting" is here defined as internal disclosure to a rating organization, to the government via voluntary schemes, and to the general public via a published nonfinancial report. The most common corporate responsibility reporting mechanism is produced by and known as "Maala". This corporate membership organization is comprised of some 120 of Israel's large and mid-size companies. The network includes local Israeli companies as well as international companies operating in Israel. 150 companies reported to Maala in 2018 as opposed to 69 companies in 2017. It should be noted that Maala only publishes the final ratings of the reporting companies and not the detailed reports of the companies. 
12.8.1

Extent to which (i) global citizenship education and (ii) education for sustainable development lincluding climate change education) are mainstreamed in (a) national education policies; (b) curricula; (c) teacher education; and (d) student assessment

\section{2.c.1}

Amount of fossil-fuel subsidies per unit of GDP (production and consumption) and as a proportion of total national expenditure on fossil fuels
See indicator 4.7.1

Amount of fossil-fuel subsidies per unit of GDP (production and consumption), 2005-2016

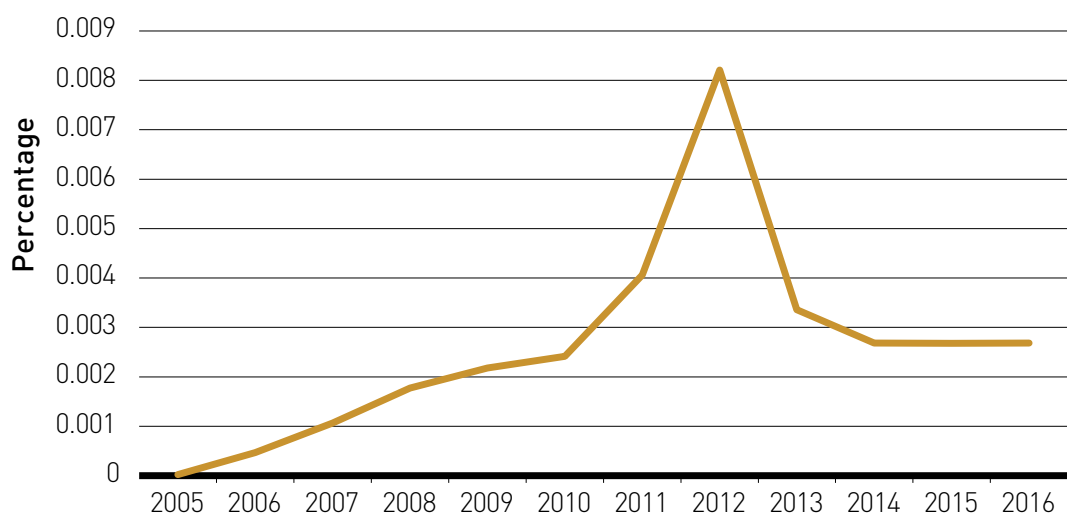




\section{GOAL 13}

13.1.3

\section{TAKE URGENT ACTION TO COMBAT CLIMATE CHANGE AND ITS IMPACTS}

13.2.1

Number of countries that have communicated the establishment or operationalization of an integrated policy/strategy/ plan which increases their ability to adapt to the adverse impacts of climate change, and foster climate resilience and low greenhouse gas emissions development in a manner that does not threaten food production lincluding a national adaptation plan, nationally determined contribution, national communication, biennial update report or other]
Environmental guidelines have been issued and adopted regarding local environmental disaster risk reduction strategies as part of the national disaster risk policy. In addition, individual instruction kits and checklists are prepared for each local authority. Over 85 have been provided so far and 20 more are in preparation. Regular local government audits carried out on behalf of national government will include audits of the implementation of the environmental guidelines.

Government Decision 4079 (July 2018) entitled "Israel's Adaptation to Climate Change: Recommendations for National Strategy and Action Plan" established a management body incorporating relevant stakeholders who will oversee the implementation of the national strategy and the various action plans and interministerial coordination. Its various subcommittees will focus on strategy, health and emergency, natural resources and agriculture and environment, energy and infrastructure and technologies, research and development, local authorities and communication and education.

Israel ratified the Paris Agreement in November 2016 and submitted its INDC in September 2015. Israel committed to a target to reduce GHG emissions to 7.7 tons C02e per capita by 2030, constituting a $26 \%$ reduction relative to 2005 per capita emissions of 10.4 tons CO2e. Additionally, an interim target of 8.8 tons CO2e per capita by 2025 was set. The government further approved sector-specific targets for 2030 :

- $17 \%$ reduction in electricity consumption relative to anticipated electricity consumption in 2030

- $13 \%$ of electricity consumption in 2025 from renewable energy, increasing to $17 \%$ in 2030

- $20 \%$ reduction in kilometers travelled by private vehicles relative to anticipated kilometers travelled in 2030

Israel submitted its first National Communication in 2000, second in 2010 and third in 2018. Israel's first BUR was submitted to UNFCCC in May 2016. An annual national inventory of $G H G$ gases is prepared by the ICBS and submitted to UNFCCC. 
GOAL 14

\subsection{1}

Index of coastal eutrophication and floating plastic debris density

\section{CONSERVE AND SUSTAINABLY USE THE OCEANS, SEAS AND MARINE RESOURCES FOR SUSTAINABLE DEVELOPMENT}

Eutrophication is not considered a major problem in Israel, as the Eastern Basin of the Mediterranean is characterized by low concentrations of nutrients. High concentrations of nutrients were registered in a few sporadic events only. The chart displays chlorophyll concentrations, which indicate algal bloom as a result of nutrient pollution, at three selected sites.

Marine litter monitoring only began in Israel in 2017. Samples were taken from 9 locations at different depths and marine litter hotspots. This monitoring program includes sampling of both floating marine litter and litter accumulated on the sea bottom, for both micro and macro plastics. The number of macro plastic particles ranged from 233 to 7443 particles, with no clear geographic trend. Primary beach litter monitoring, however, shows a possible decreasing trend in the presence of plastic bags which could be attributed to the recently passed Plastic Bag Law.

Concentrations of chlorophyll in selected sites 2001-2015
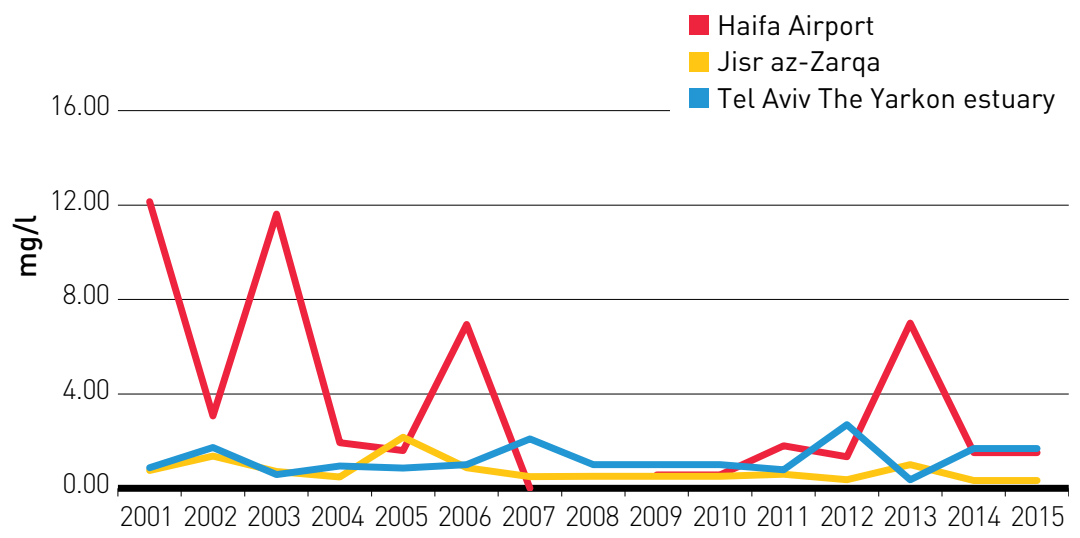

$100 \%$ of national exclusive economic zones in Israel are managed using ecosystem-based approaches.

\subsection{1}

\section{Proportion of national exclusive economic zones managed using ecosystem- based approaches}


14.3.1

\section{Average marine acidity $(\mathrm{pH})$ measured at agreed suite of representative sampling stations}

14.3.1

14.5 .1

\section{Coverage of protected areas in relation to marine areas}

Israel Oceanographic \& Limnological Research carries out the National Monitoring Program for Israeli Mediterranean Waters, which also relates to climate change aspects, including data on seawater acidity. Monitoring activities include measurements of the carbonate system in seawater at a number of stations along a section extending from the Carmel headland to the NW ca. 46 NM offshore (upper figure). These stations have been sampled during the height of winter (March) and summer (August) nearly every year since 2003. These seawater samples have been analyzed for the carbonate system parameters, including total alkalinity (TA) and dissolved inorganic carbon (DIC), on a regular basis since 2012.
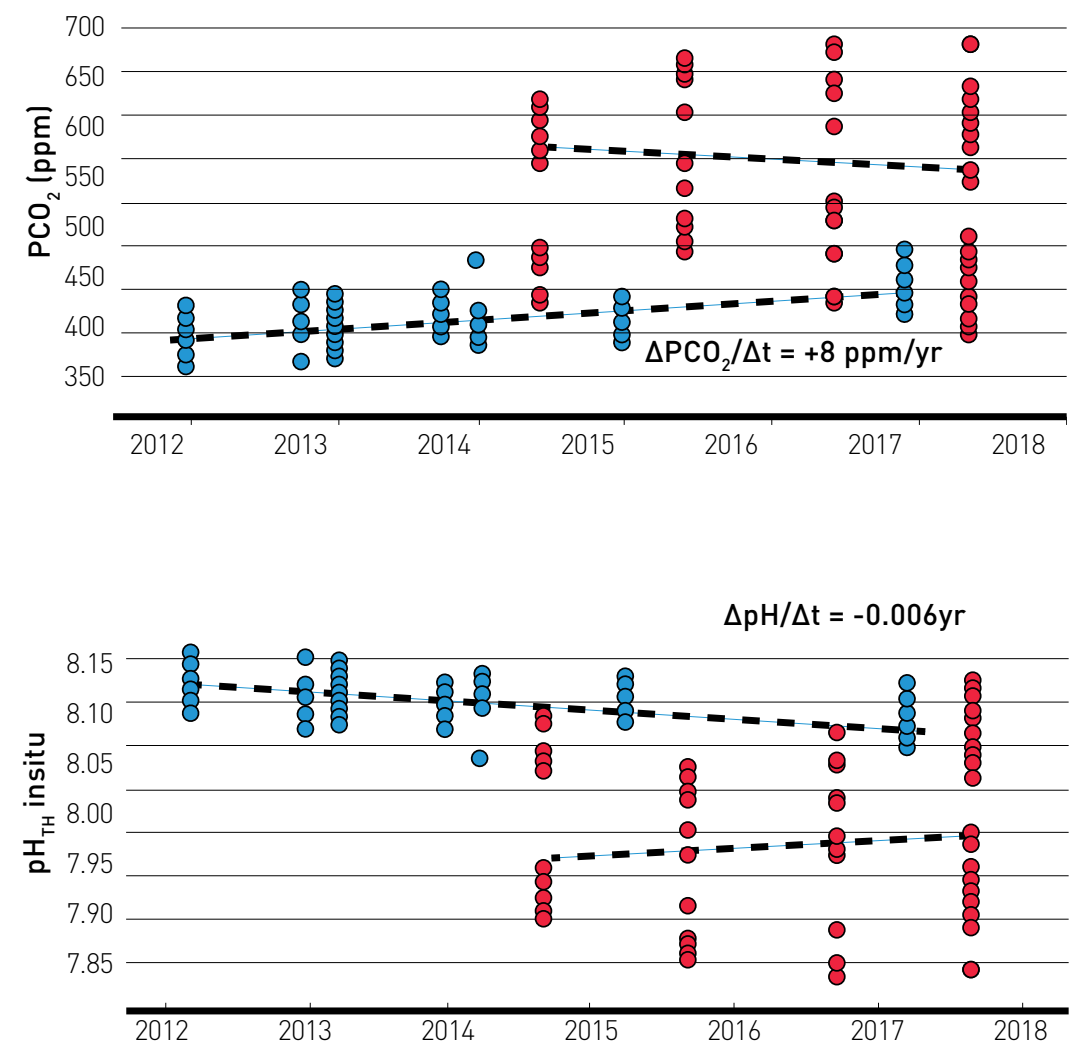

According to Israel Nature and Parks Authority data, Israel's total protected marine area is $12.5 \mathrm{~km} 2$. This accounts for $0.3 \%$ of Israel's territorial water. 
GOAL 15

15.1.1

Forest area as a proportion of total land area

15.2.1

Progress towards sustainable forest management

\section{PROTECT, RESTORE AND PROMOTE SUSTAINABLE USE OF TERRESTRIAL ECOSYSTEMS, SUSTAINABLY MANAGE FORESTS, COMBAT DESERTIFICATION, AND HALT AND REVERSE LAND DEGRADATION AND HALT BIODIVERSITY LOSS}

Forest area as a proportion of total land area, 2013-2016

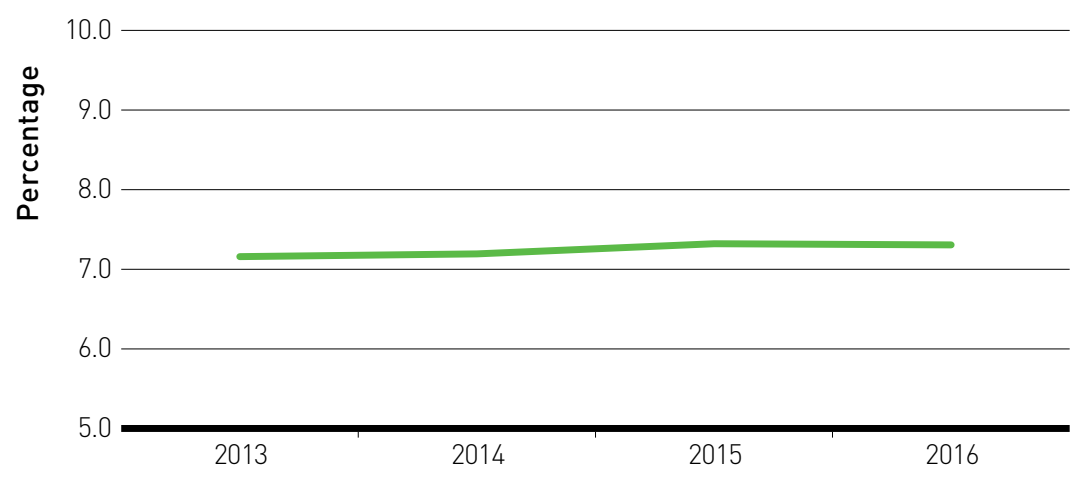

Keren Kayemeth Lelsrael-Jewish National Fund (KKL-JNF) is the official afforestation administration in Israel and the acting Forest Service, pursuant to a covenant signed with the Government of Israel in 1961. Throughout the years, KKL-JNF invested heavily in research and implemented innovative approaches that contributed to the development of a new Forest Management Policy (FMP) for Israel in 2014. The new FMP states that the main purpose of forests in Israel is to supply ecosystem services for the well-being of all citizens and the environment. It assimilates a broad framework of current approaches and concepts of sustainable forest management according to the latest developments in forestry, ecology, social and economic sciences. Furthermore, the new FMP provides a binding professional basis for managing Israel's forests in a goal-oriented and sustainable fashion and serves as the foundation for individual and ecosystem-specific Forest Management Master Plans that are tailored for each forest and reflect a holistic and sustainable approach. These master plans, combined with a national scale survey, serve as a foundation for long-term strategic forest management plans. 
15.4 .2

Mountain Green Cover Index

15.5.1

Red List Index

\begin{tabular}{|l|l|l|}
\hline Land cover - Land use & Area $\left(\mathrm{km}^{2} 000{ }^{\prime}\right)$ & Area \% \\
\hline Forest & 0.65453 & $10.80 \%$ \\
\hline Grassland-Shrubland & 0.16635 & $2.70 \%$ \\
\hline Cropland & 0.30848 & $5.10 \%$ \\
\hline Other land & 4.71901 & $77.60 \%$ \\
\hline Wetland & 0.0059 & $0.10 \%$ \\
\hline Settlement & 0.22771 & $3.70 \%$ \\
\hline Total & 6.08198 & $100 \%$ \\
\hline
\end{tabular}

Endangered plants: $18.09 \%$

Endangered vertebrates: $38.74 \%$

\begin{tabular}{|c|c|c|c|c|c|c|c|c|}
\hline $\begin{array}{l}\text { Number of species } \\
\text { Last year available }\end{array}$ & $\begin{array}{l}3 \\
\frac{3}{3} \\
3 \\
3 \\
\frac{0}{n}\end{array}$ & $\begin{array}{l}\frac{\text { m. }}{7} \\
\text { in }\end{array}$ & 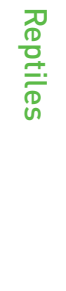 & $\begin{array}{l}\frac{8}{3} \\
3 \\
\frac{0}{0} \\
\frac{0}{0} \\
\frac{0}{2} \\
\frac{3}{3} \\
\text { n. }\end{array}$ & $\frac{\pi}{n}$ & 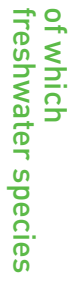 & 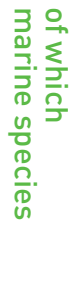 & 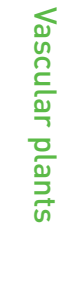 \\
\hline $\begin{array}{l}\text { Species known } \\
\text { of which indigenous }\end{array}$ & 105 & 213 & 105 & 7 & 32 & 32 & & 2288 \\
\hline $\begin{array}{l}\text { Endangered species } \\
\text { of which indigenous }\end{array}$ & 27 & 13 & 7 & 3 & 0 & 0 & & 117 \\
\hline $\begin{array}{l}\text { Critically endangered } \\
\text { species } \\
\text { of which indigenous }\end{array}$ & 15 & 21 & 13 & 2 & 6 & 6 & & 62 \\
\hline $\begin{array}{l}\text { Vulnerable } \\
\text { of which indigenous }\end{array}$ & 19 & 31 & 15 & 1 & 0 & 0 & & 198 \\
\hline $\begin{array}{l}\text { Threatened } \\
\text { species, total of which } \\
\text { indigenous }\end{array}$ & 61 & 65 & 35 & 6 & 6 & 6 & & 377 \\
\hline
\end{tabular}

Fish data are updated to 2018, other vertebrates to 2002. 
15.5.1

Red List Index
Endangered plants in Israel, 2009

\begin{tabular}{|c|c|c|c|c|c|c|}
\hline \multirow[t]{2}{*}{ Criterion } & \multirow[t]{2}{*}{ Severity } & \multicolumn{4}{|c|}{ IUCN } & \multirow[t]{2}{*}{ Total } \\
\hline & & 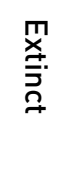 & 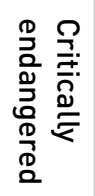 & $\begin{array}{l}\text { m } \\
\frac{1}{2} \\
\frac{0}{2} \\
00 \\
\frac{0}{1} \\
\frac{1}{2} \\
0\end{array}$ & $\begin{array}{l}\frac{5}{\frac{1}{5}} \\
\frac{0}{0} \\
\frac{0}{0}\end{array}$ & \\
\hline & Total & 36 & 59 & 120 & 199 & 414 \\
\hline \multirow[t]{7}{*}{ Rarity } & $\begin{array}{l}\text { Found at more than } 100 \\
\text { sites }\end{array}$ & - & - & 1 & - & 1 \\
\hline & Found at $31-100$ sites & 1 & 1 & 16 & 22 & 40 \\
\hline & Found at $11-30$ sites & 2 & 5 & 17 & 55 & 79 \\
\hline & Found at $5-10$ sites & 2 & 6 & 30 & 63 & 101 \\
\hline & Found at $3-4$ sites & 5 & 7 & 22 & 16 & 50 \\
\hline & Found at 2 sites & 10 & 12 & 16 & 21 & 59 \\
\hline & Found at 1 site & 16 & 28 & 18 & 22 & 84 \\
\hline \multirow[t]{5}{*}{ Vulnerability } & No vulnerability & 7 & 2 & 19 & 61 & 89 \\
\hline & Low vulnerability & 1 & 1 & 11 & 22 & 35 \\
\hline & Medium vulnerability & - & 3 & 27 & 43 & 73 \\
\hline & High vulnerability & 3 & 20 & 32 & 46 & 101 \\
\hline & Very high vulnerability & 25 & 33 & 31 & 27 & 116 \\
\hline \multirow[t]{5}{*}{ Attractivity } & No attractivity & 32 & 38 & 72 & 153 & 295 \\
\hline & Low attractivity & 1 & 7 & 19 & 30 & 57 \\
\hline & Medium attractivity & 2 & 9 & 11 & 10 & 32 \\
\hline & High attractivity & - & 4 & 15 & 4 & 23 \\
\hline & Exploitation & 1 & 1 & 3 & 2 & 7 \\
\hline \multirow[t]{5}{*}{ Endemism } & Not endemic & 35 & 34 & 82 & 164 & 315 \\
\hline & Endemic to the Levant & - & 2 & 3 & 6 & 11 \\
\hline & Sub-endemic & 1 & 11 & 19 & 14 & 45 \\
\hline & Sub-endemism & - & - & 1 & 2 & 3 \\
\hline & Endemic & - & 12 & 15 & 13 & 40 \\
\hline \multirow[t]{6}{*}{ Peripherality } & Not peripheral & 23 & 38 & 85 & 141 & 287 \\
\hline & Peripheral & 13 & 21 & 35 & 58 & 127 \\
\hline & Thereof: East & 1 & - & 6 & 6 & 13 \\
\hline & North & 12 & 26 & 62 & 96 & 196 \\
\hline & South & 9 & 8 & 12 & 30 & 59 \\
\hline & West & 1 & 4 & 5 & 9 & 19 \\
\hline \multirow[t]{2}{*}{ Disjunctive } & $\begin{array}{l}\text { One geographical } \\
\text { segment }\end{array}$ & 1 & 4 & 7 & 14 & 26 \\
\hline & $\begin{array}{l}\text { More than one } \\
\text { geographical segment }\end{array}$ & 35 & 55 & 113 & 185 & 388 \\
\hline \multirow{2}{*}{$\begin{array}{l}\text { Law } \\
\text { protection }\end{array}$} & Protected by law & 5 & 9 & 32 & 20 & 66 \\
\hline & Not protected by law & 31 & 50 & 88 & 179 & 348 \\
\hline
\end{tabular}


GOAL 16

16.1.1

Number of victims of intentional homicide per 100,000 population, by sex and age

16.1.1

\section{PROMOTE PEACEFUL AND INCLUSIVE SOCIETIES FOR SUSTAINABLE DEVELOPMENT, PROVIDE ACCESS TO JUSTICE FOR ALL AND BUILD EFFECTIVE, ACCOUNTABLE AND INCLUSIVE INSTITUTIONS AT ALL LEVELS}

Number of victims of intentional homicide per 100,000 population, by sex, $2000-2016$

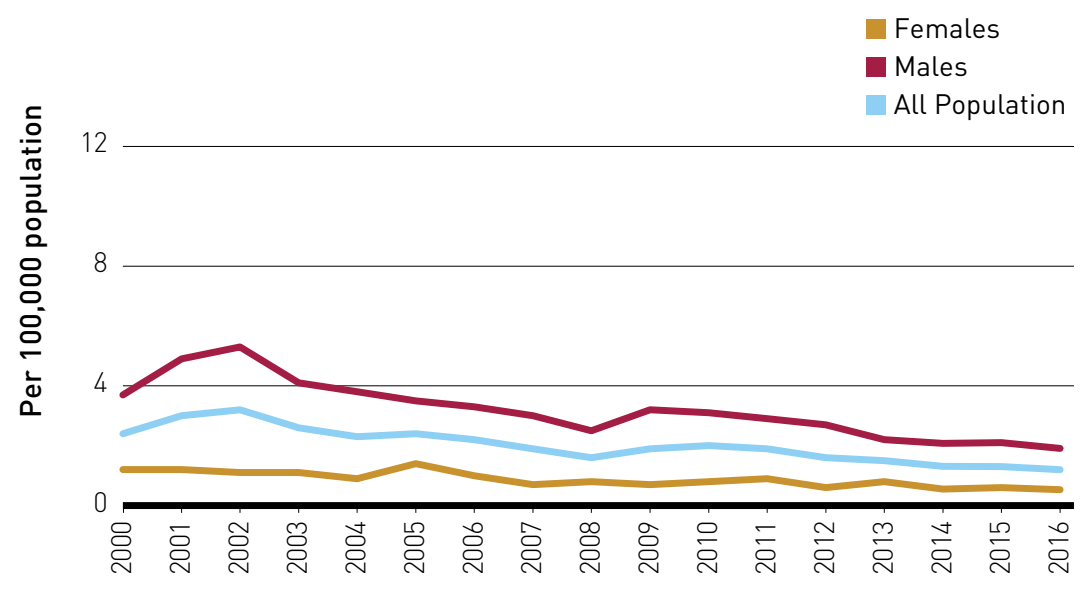

Number of victims of intentional homicide per 100,000 population, by sex and population group, 2000-2016

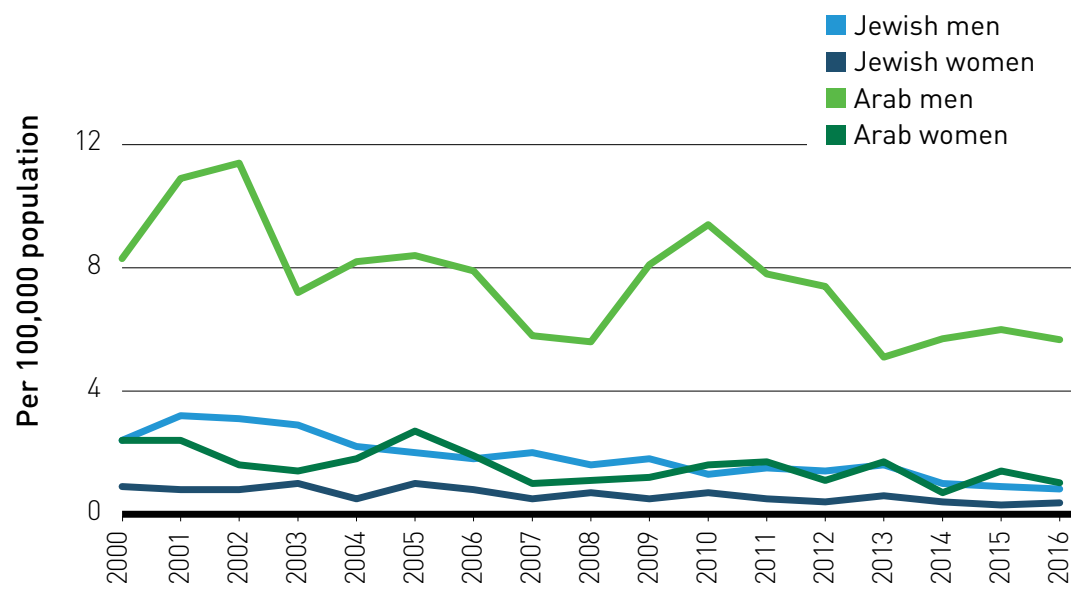


16.1.1

\subsection{2}

Conflict-related deaths per 100,000 population, by sex, age and cause

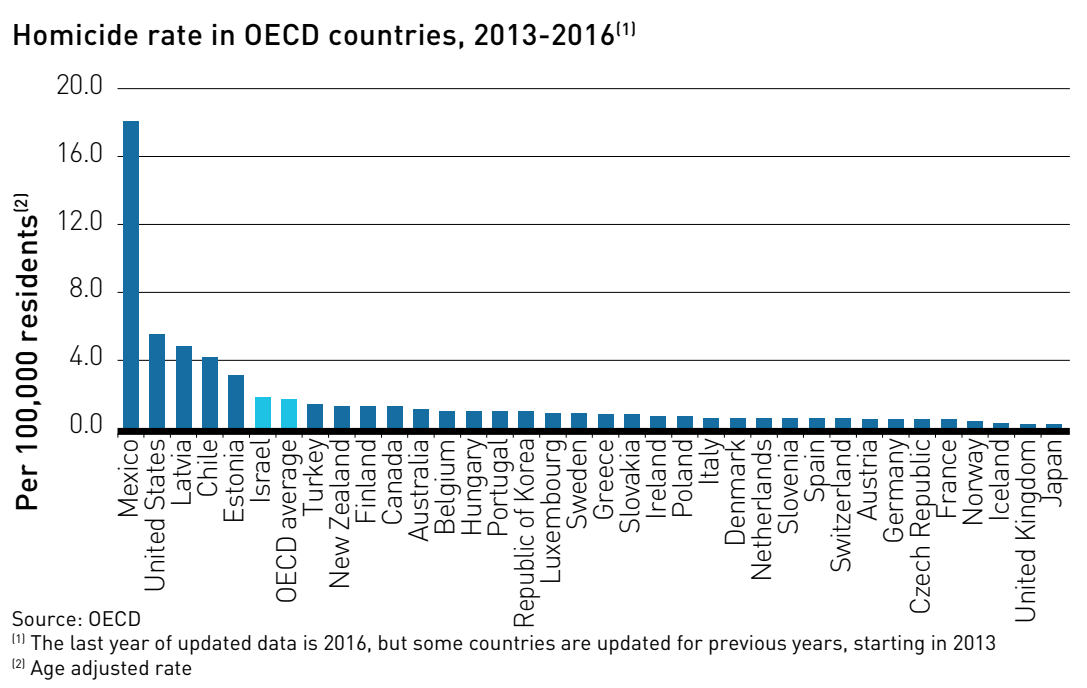

Persons killed in terror attacks, 2000-2016

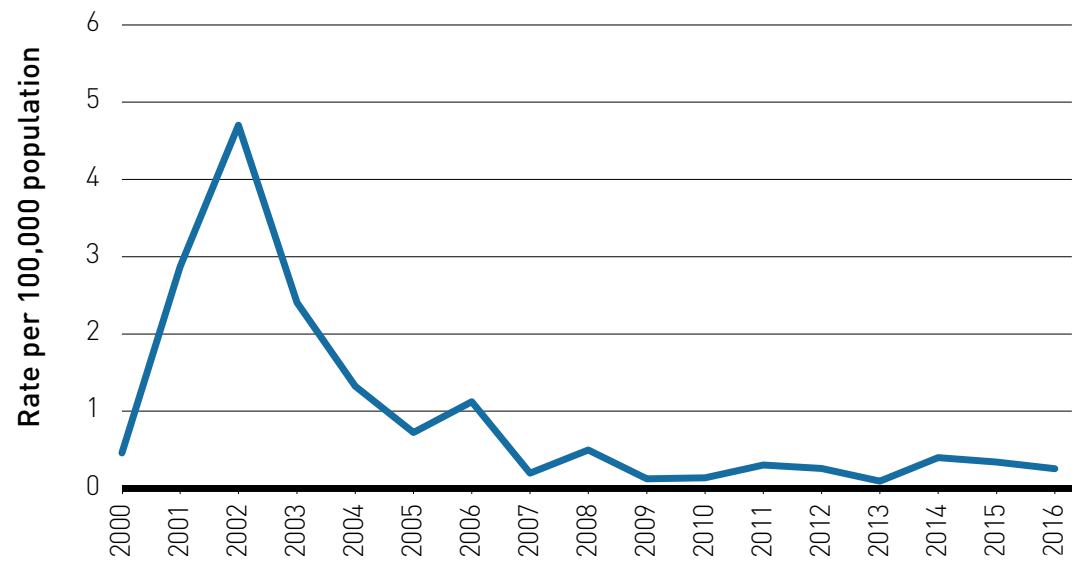




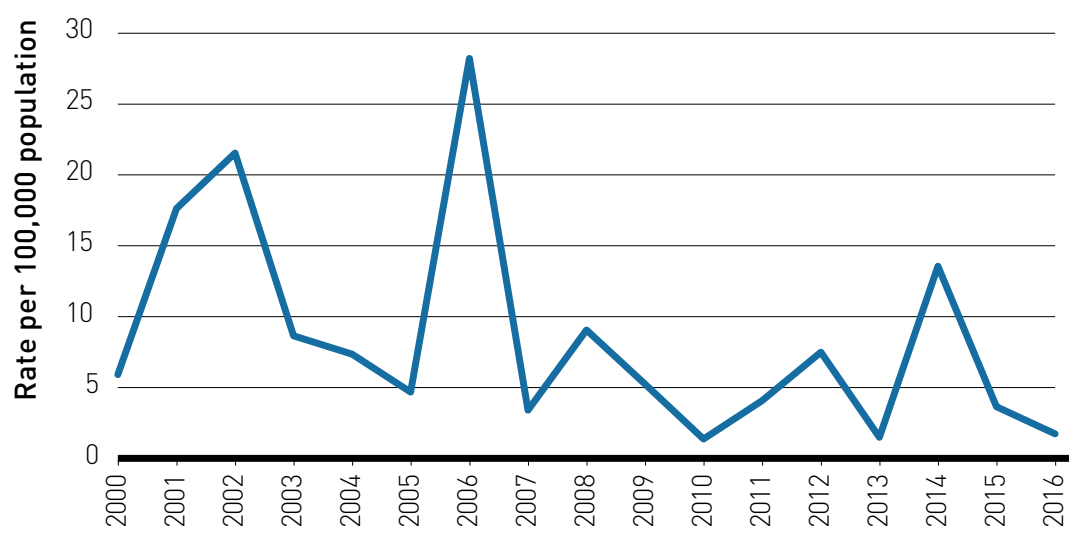

16.1 .2

Number of terror attacks, 2000-2016

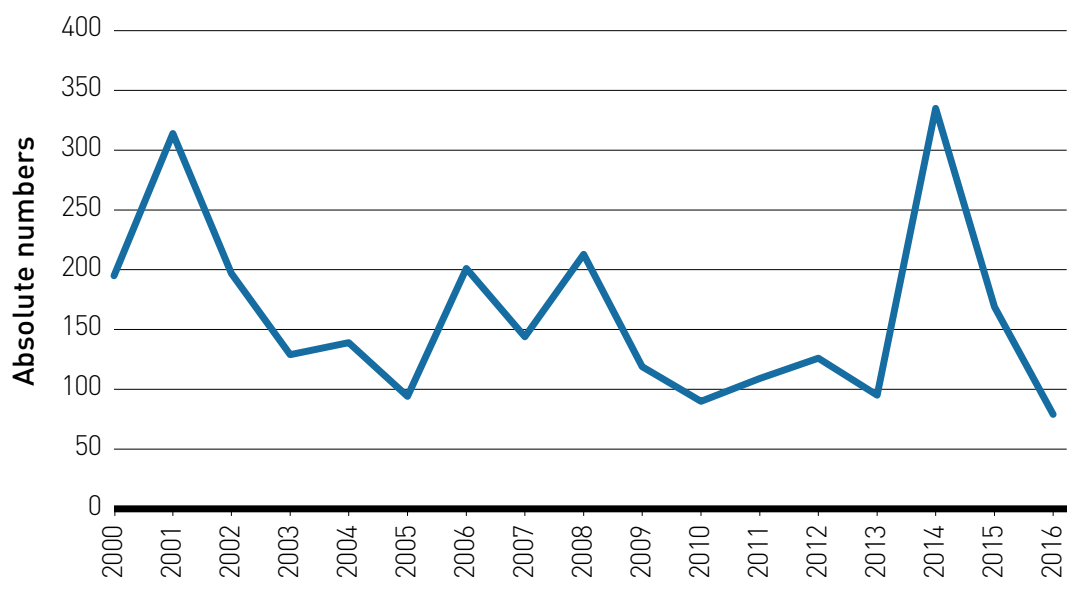


16.1.3

Proportion of population subjected to physical, psychological or sexual violence in the previous 12 months

16.1.3
Data are for victims of physical violence.

Persons aged 20 and over who were injured by violence (use of physical force), 2002-2017

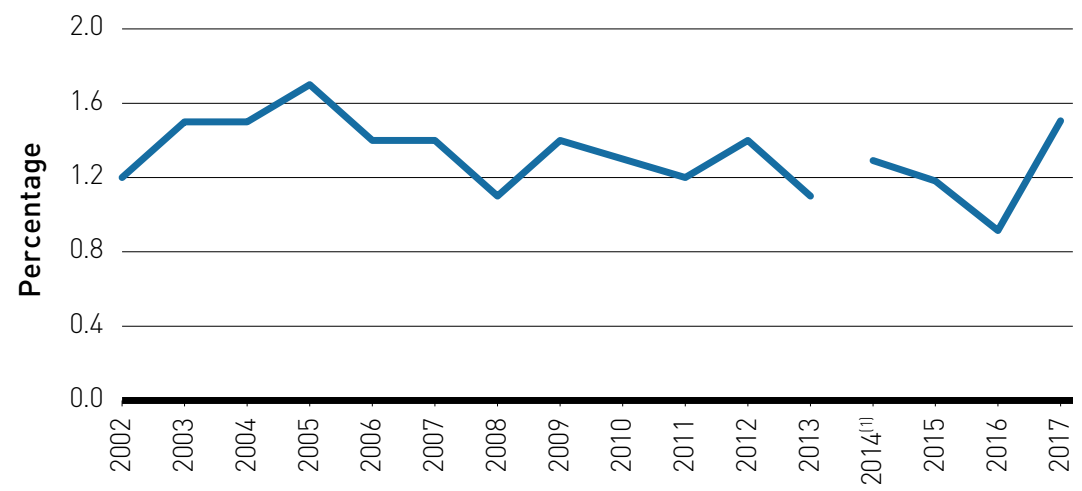

(11) Change in data source:until 2013, the social survey. From 2014, Israel's Crime Victimization Survey (ICVS)

Persons aged 20 and over who were injured by violence (use of physical force) or from the threat of violence, by sex and age, 2015-2017
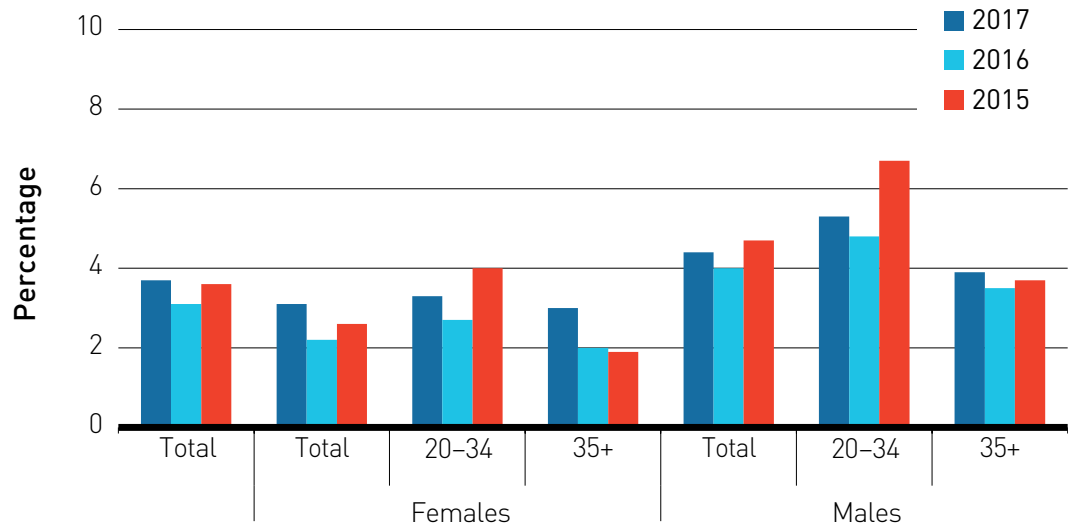

Source: Israel's Crime Victimization Survey (ICVS). 
16.1.4

Proportion of population that feel safe walking alone around the area they live

16.1 .4
Data from the Israeli victimization survey refer to people who feel safe walking alone at dark around the area they live.

Proportion of population aged 20 and over that feel safe walking alone at dark around the area they live, 2015-2017

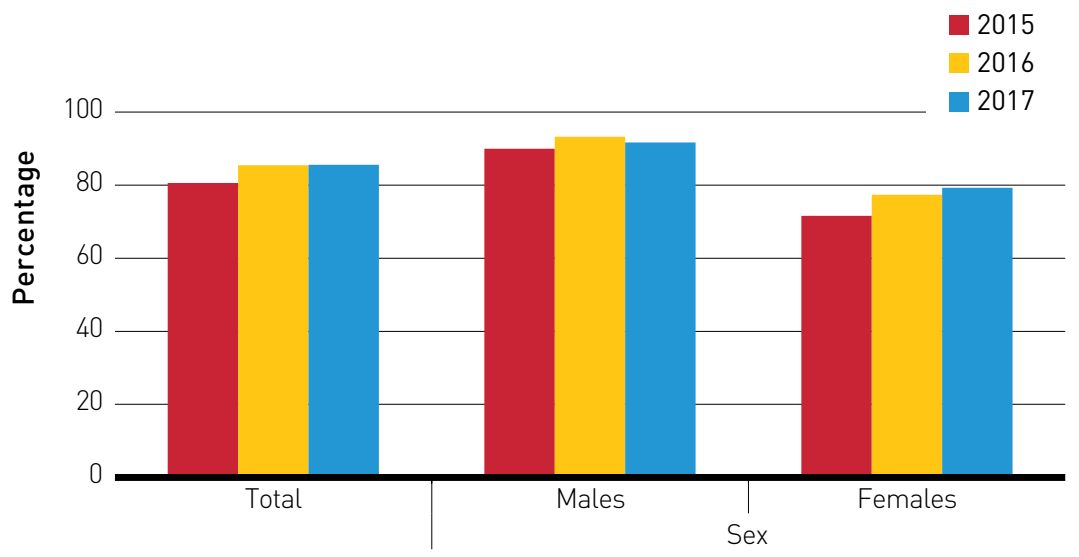

Proportion of population aged 20 and over that feel safe walking alone at dark around the area they live by sex, age and population group, 2017

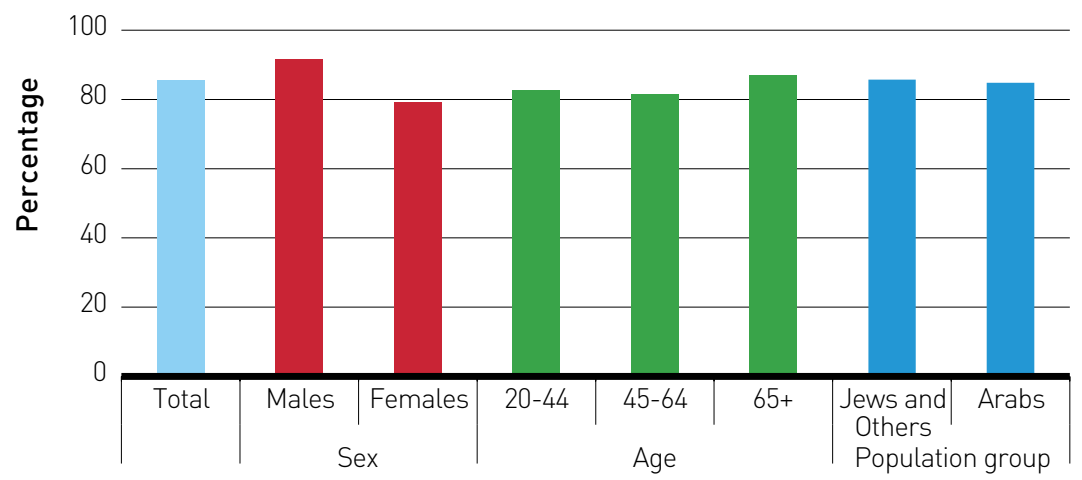


16.1.4

\subsection{2}

Number of victims of human trafficking per 100,000 population, by sex, age and form of exploitation
Persons aged 15 and over who feel safe ${ }^{(1)}$ walking alone after dark in their area of residence, International comparison, 2016

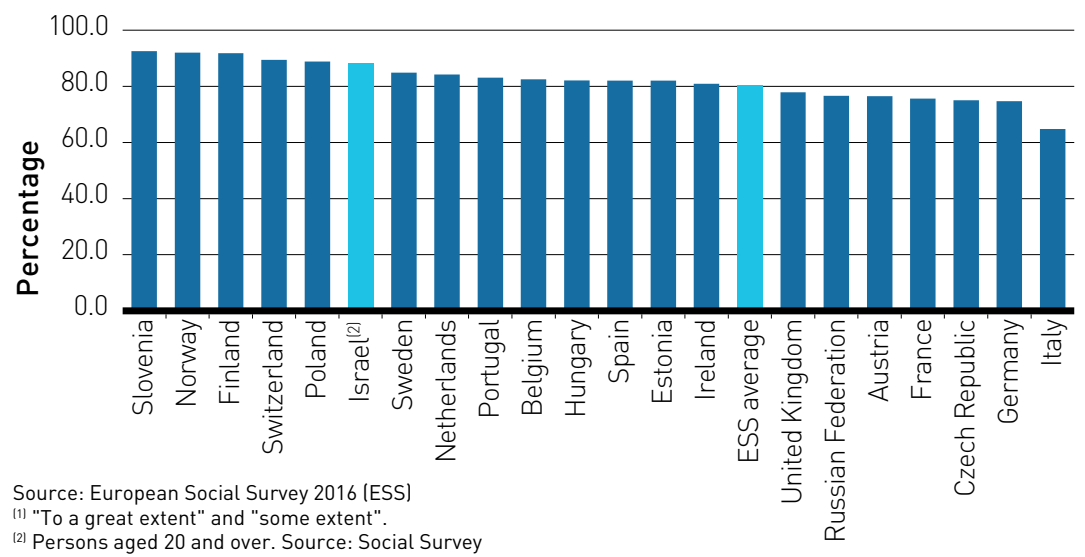

Number of victims of human trafficking, 2010-2017

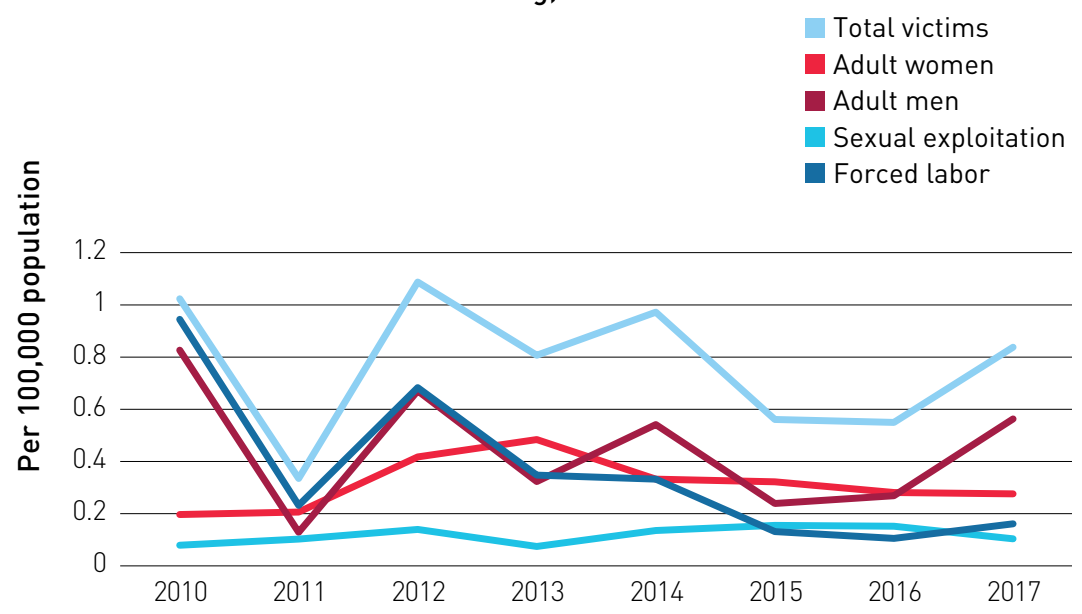


16.3.1

Proportion of victims of violence in the previous 12 months who reported their victimization to competent authorities or other officially recognized conflict resolution mechanisms

16.6 .2

Proportion of the population satisfied with their last experience of public services
Police reporting rate for physical assault, 2014-2016

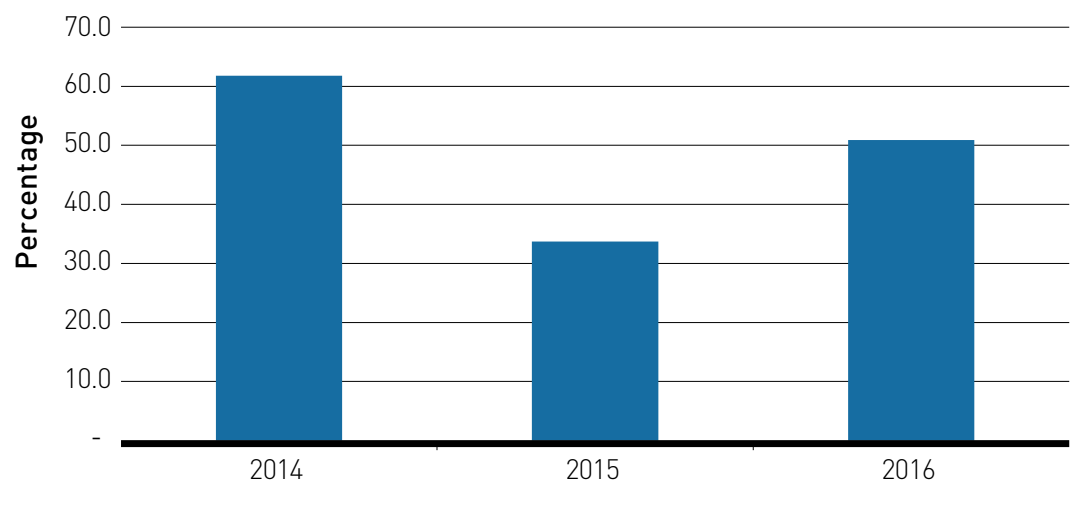

Source: Israel's Crime Victimization Survey (ICVS). Percentage of persons aged 20 and over victims of violence with physical assault who reported to the police

Based on questions from the 2015 social survey, data are available for evaluation of several public services

Proportion of the population who believe that the functioning of public services is very good or good, by type of service, 2015

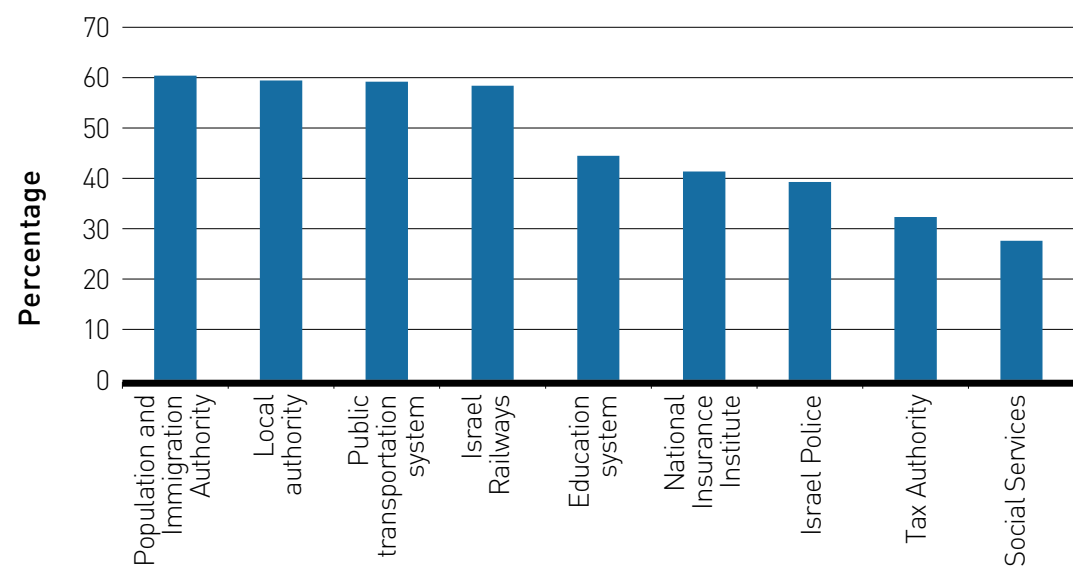




\section{7 .2}

Proportion of population who believe decision-making is inclusive and responsive, by sex, age, disability and population group

16.7.2
Persons aged 20 and over who think you can rely on the government to make the right decisions by sex, age and population group, 2015

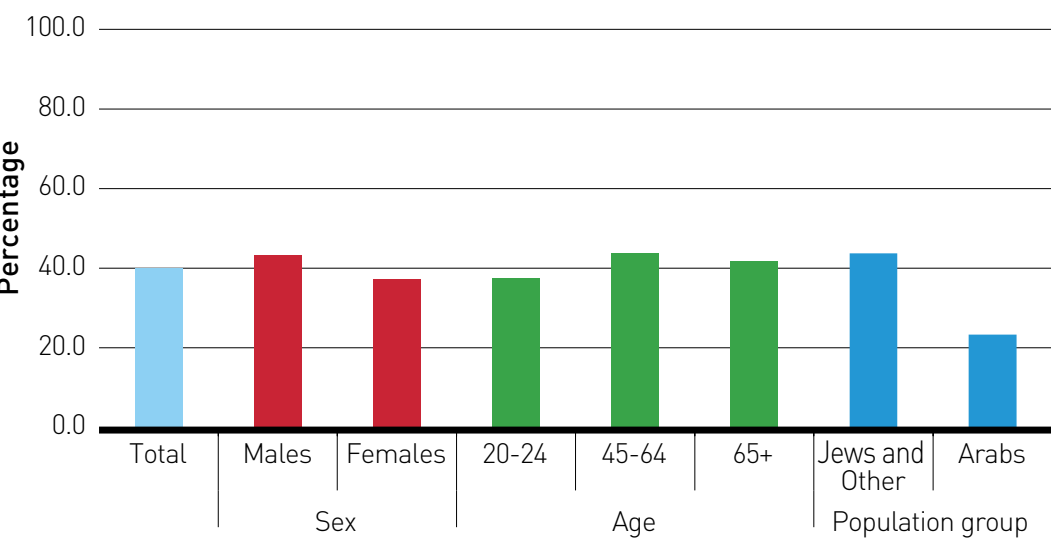

Persons aged 20 and over who trust the government, by sex, age and population group, 2017

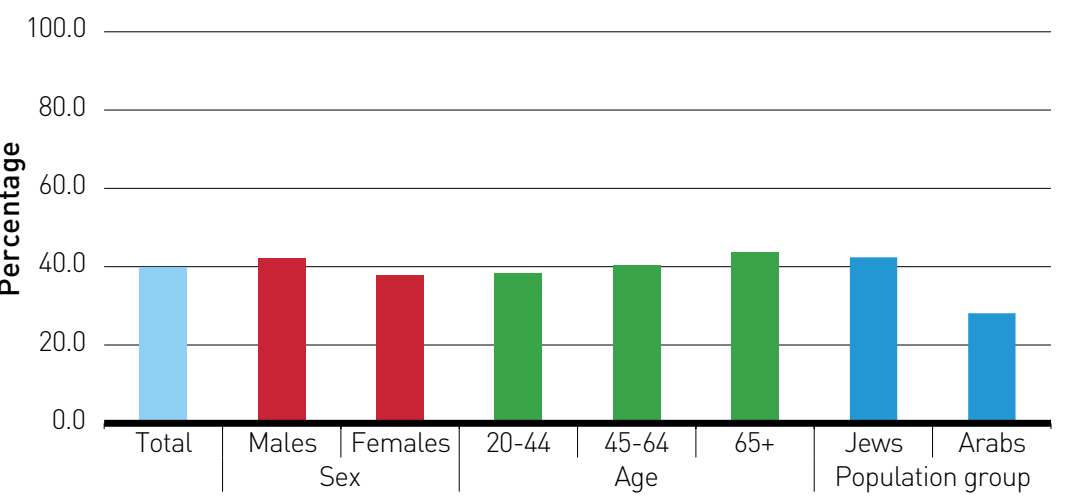


16.9.1

Proportion of children under 5 years of age whose births have been registered with a civil authority, by age

\subsection{2}

Number of countries that adopt and implement constitutional, statutory and/ or policy guarantees for public access to information

16.b.1

Proportion of population reporting having personally felt discriminated against or harassed in the previous 12 months on the basis of a ground of discrimination prohibited under international human rights law
$100 \%$ of births of children that are under 5 years of age have been registered with a civil authority

\begin{abstract}
A Freedom of Information Unit was established within the Ministry of Justice in accordance with Government Resolution No. 2950 (2011). The Unit constitutes a center of professional knowledge in the field of freedom of information in Israel and gathers relevant information, conducts public awareness campaigns and trains civil servants and other public employees. The Unit's primary role is managing the work of freedom of information officers in the various government ministries and handling public complaints about freedom of information in the various ministries as well as rectifying the errors discovered in the process.
\end{abstract}

See indicator 10.3.1 
GOAL 17

\subsection{1}

Total government revenue as a proportion of GDP, by source

\section{STRENGTHEN THE MEANS OF IMPLEMENTATION AND REVITALIZE THE GLOBAL PARTNERSHIP FOR SUSTAINABLE DEVELOPMENT}

General government revenue, 2005-2017

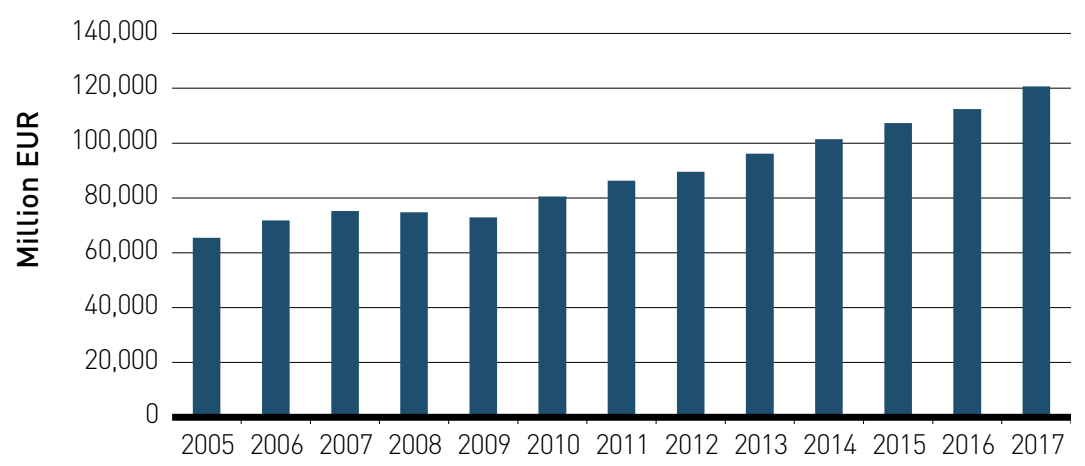

General Government revenue, as a percentage of GDP, 2005-2017

80.0

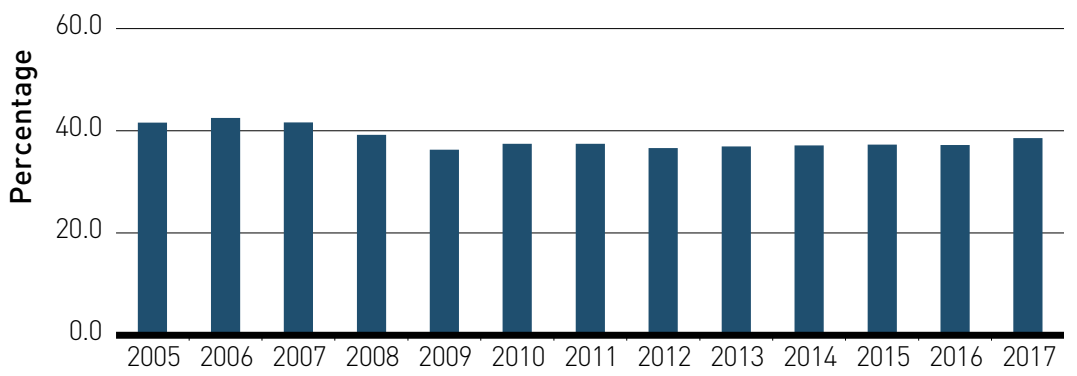


17.1 .2

Proportion of domestic budget funded by domestic taxes

\subsection{1}

Net official development assistance, total and to least developed countries, as a proportion of the Organization for Economic Cooperation and Development (OECD) Development Assistance Committee donors' gross national income (GNI)
The indicator is derived from the GFS series reported to the IMF Statistics Department and is calculated in the following way: central budgetary government taxes (code 11) are divided by the total central budgetary government expenditure (codes 2 and 31).

Proportion of budgetary central government expenditure funded by taxes, 2002-2017

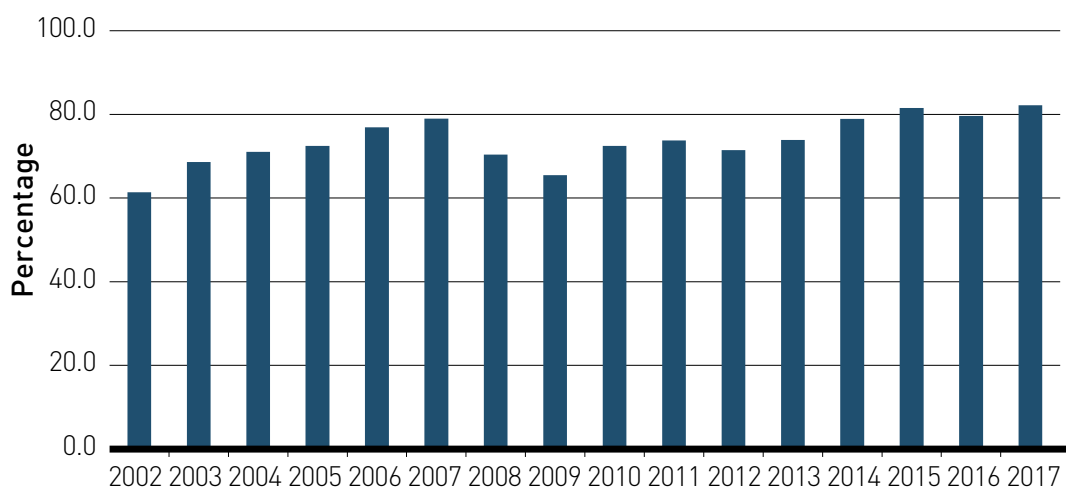

Net official development assistance (ODA) as a proportion of gross national income (GNI), 2005-2017

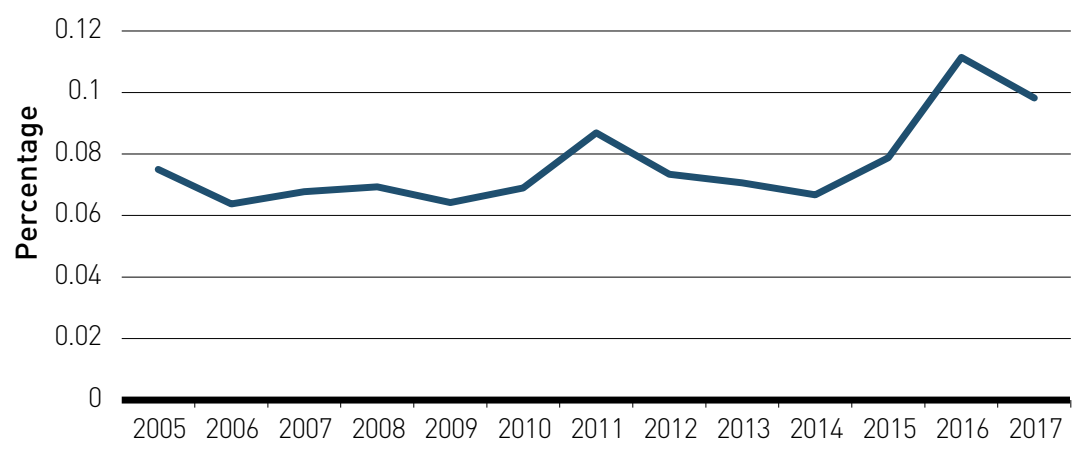


17.3.1

Foreign direct investments (FDI), official development assistance and South-South Cooperation as a proportion of total domestic budget

\subsection{1}

Proportion of individuals using the Internet
Data refers to general government expenditure including local authorities. Official development assistance (ODA) as a proportion of general government expenditures, 2005-2017

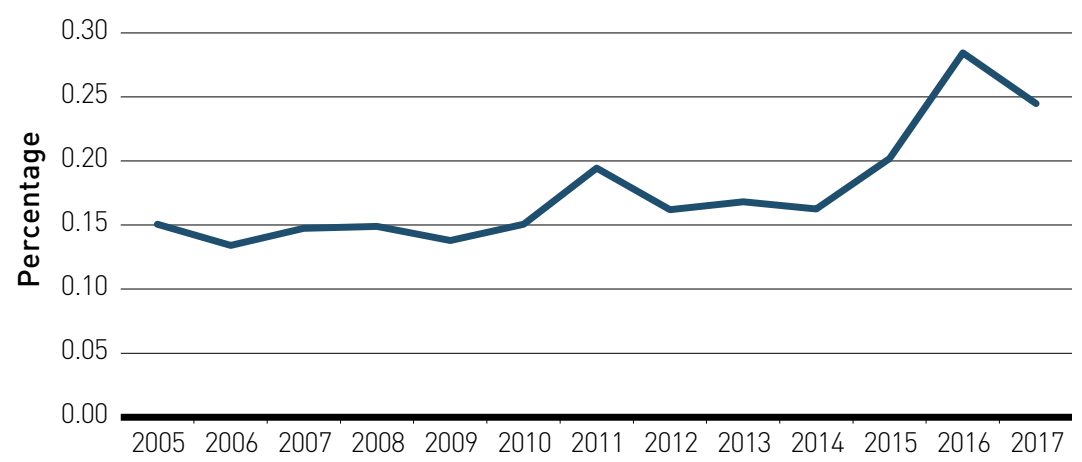

Persons aged 20 and over who used the Internet, 2002-2017

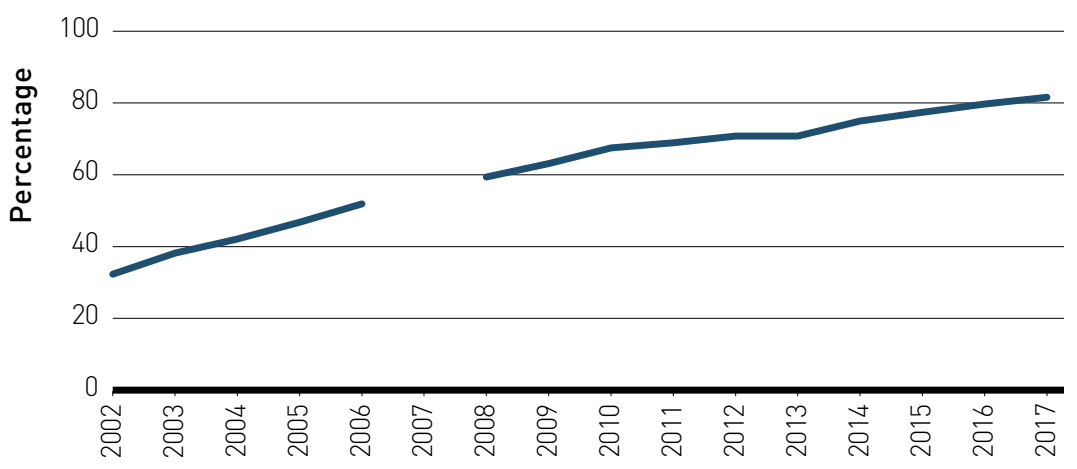


17.8 .1

Persons aged 20 and over who used the Internet, by sex, age and population group, 2017

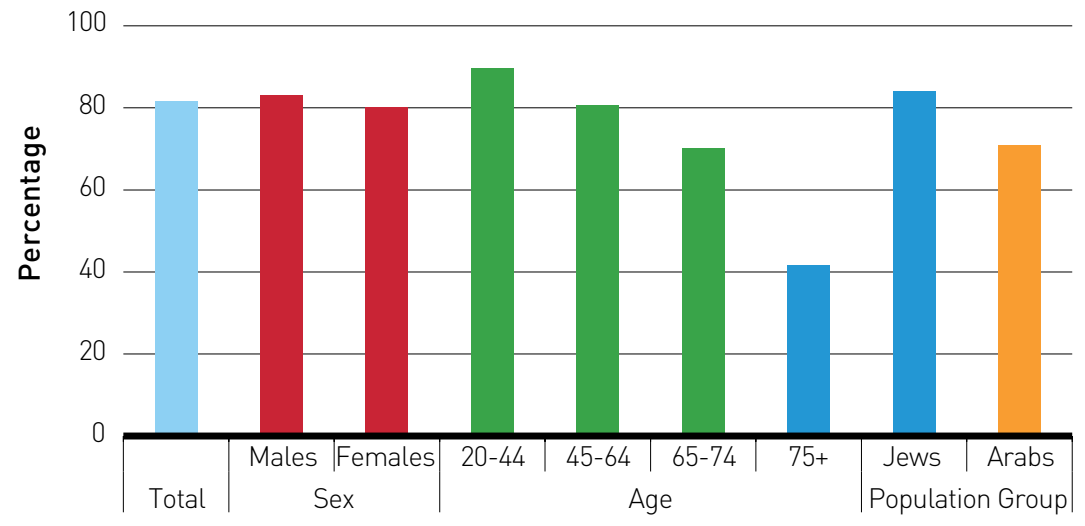

17.8.1

Persons aged 20 and over who used the Internet, by utilization, 2017

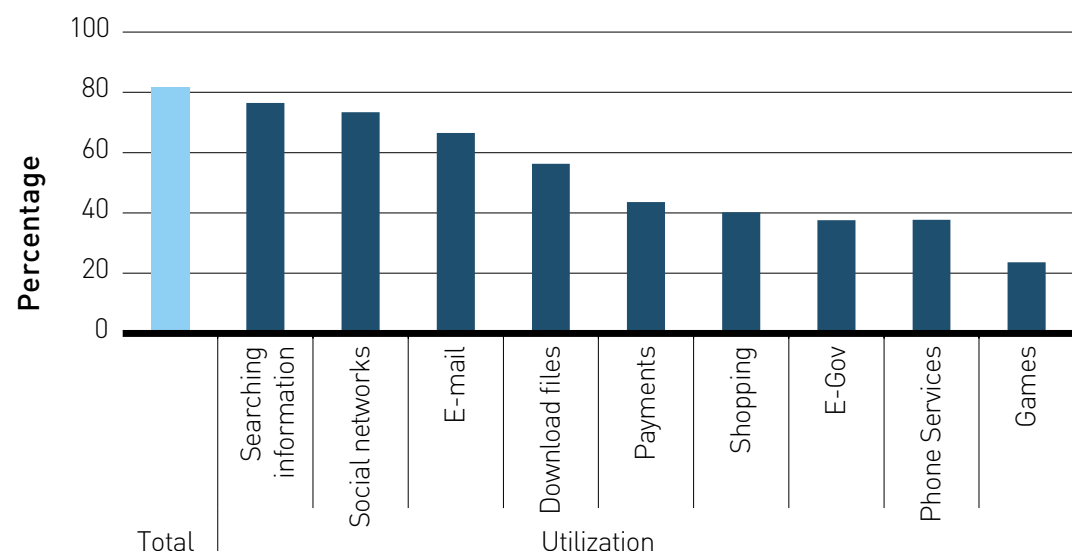


17.18.1

Proportion of sustainable development indicators produced at the national level with full disaggregation when relevant to the target, in accordance with the Fundamental Principles of Official Statistics
Currently there are 123 available indicators in Israel. 98 indicators are based on ICBS data and 25 indicators are based on data and information from other government ministries or other agencies. 31 indicators contain relevant disaggregation based on available data. Out of all the available indicators, 113 indicators are included in the statistical annex of this report. A few available indicators were not included in the statistical annex. These indicators require further development so that they can be computed and published. Some further development is also needed in other government ministries that are in charge of available indicators outside of the ICBS.

The following table summarizes the status of SDG indicators in Israel

\begin{tabular}{|c|c|c|c|c|c|}
\hline \multirow{2}{*}{ Target } & \multicolumn{2}{|c|}{ Available } & \multirow{2}{*}{ 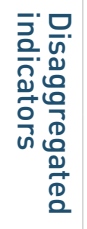 } & \multirow{2}{*}{ 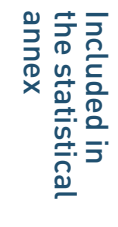 } & \multirow{2}{*}{ 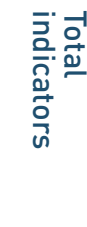 } \\
\hline & $\begin{array}{l}\text { ICBS } \\
\text { data }\end{array}$ & $\begin{array}{l}\text { Other } \\
\text { national } \\
\text { data }\end{array}$ & & & \\
\hline $\begin{array}{l}\text { Goal 1. End poverty in all its } \\
\text { forms everywhere }\end{array}$ & 4 & & 3 & 4 & 14 \\
\hline $\begin{array}{l}\text { Goal } 2 \text {. End hunger, achieve } \\
\text { food security and improved } \\
\text { nutrition and promote } \\
\text { sustainable agriculture }\end{array}$ & 1 & 1 & & 2 & 13 \\
\hline $\begin{array}{l}\text { Goal } 3 \text {. Ensure healthy lives } \\
\text { and promote well-being for all } \\
\text { at all ages }\end{array}$ & 20 & & 6 & 19 & 27 \\
\hline $\begin{array}{l}\text { Goal 4. Ensure inclusive and } \\
\text { equitable quality education } \\
\text { and promote lifelong learning } \\
\text { opportunities for all }\end{array}$ & 7 & 2 & 6 & 9 & 11 \\
\hline $\begin{array}{l}\text { Goal 5. Achieve gender equality } \\
\text { and empower all women and } \\
\text { girls }\end{array}$ & 6 & 1 & 2 & 7 & 14 \\
\hline $\begin{array}{l}\text { Goal 6. Ensure availability and } \\
\text { sustainable management of } \\
\text { water and sanitation for all }\end{array}$ & 3 & 3 & & 4 & 11 \\
\hline $\begin{array}{l}\text { Goal } 7 \text {. Ensure access } \\
\text { to affordable, reliable, } \\
\text { sustainable and modern } \\
\text { energy for all }\end{array}$ & 4 & & & 4 & 6 \\
\hline $\begin{array}{l}\text { Goal } 8 \text {. Promote sustained, } \\
\text { inclusive and sustainable } \\
\text { economic growth, full and } \\
\text { productive employment and } \\
\text { decent work for all }\end{array}$ & 10 & & 5 & 10 & 17 \\
\hline
\end{tabular}


17.18.1

\begin{tabular}{|c|c|c|c|c|c|}
\hline \multirow{2}{*}{ Target } & \multicolumn{2}{|c|}{ Available } & \multirow{2}{*}{ 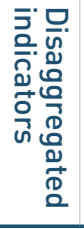 } & \multirow{2}{*}{ 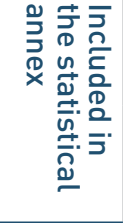 } & \multirow{2}{*}{ 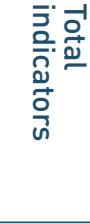 } \\
\hline & $\begin{array}{l}\text { ICBS } \\
\text { data }\end{array}$ & $\begin{array}{l}\text { Other } \\
\text { national } \\
\text { data }\end{array}$ & & & \\
\hline $\begin{array}{l}\text { Goal 9. Build resilient } \\
\text { infrastructure, promote } \\
\text { inclusive and sustainable } \\
\text { industrialization and foster } \\
\text { innovation }\end{array}$ & 9 & & & 7 & 12 \\
\hline $\begin{array}{l}\text { Goal } 10 . \text { Reduce inequality } \\
\text { within and among countries }\end{array}$ & 4 & & 2 & 3 & 11 \\
\hline $\begin{array}{l}\text { Goal 11. Make cities and } \\
\text { human settlements inclusive, } \\
\text { safe, resilient and sustainable }\end{array}$ & 4 & 6 & 1 & 7 & 15 \\
\hline $\begin{array}{l}\text { Goal 12. Ensure sustainable } \\
\text { consumption and production } \\
\text { patterns }\end{array}$ & 3 & 4 & & 7 & 13 \\
\hline $\begin{array}{l}\text { Goal } 13 . \text { Take urgent action to } \\
\text { combat climate change and its } \\
\text { impacts }\end{array}$ & & 2 & & 2 & 8 \\
\hline $\begin{array}{l}\text { Goal } 14 \text {. Conserve and } \\
\text { sustainably use the oceans, } \\
\text { seas and marine resources for } \\
\text { sustainable development }\end{array}$ & & 4 & & 4 & 10 \\
\hline $\begin{array}{l}\text { Goal 15. Protect, restore and } \\
\text { promote sustainable use } \\
\text { of terrestrial ecosystems, } \\
\text { sustainably manage forests, } \\
\text { combat desertification, } \\
\text { and halt and reverse land } \\
\text { degradation and halt } \\
\text { biodiversity loss }\end{array}$ & 3 & 1 & & 4 & 14 \\
\hline $\begin{array}{l}\text { Goal 16. Promote peaceful } \\
\text { and inclusive societies for } \\
\text { sustainable development, } \\
\text { provide access to justice } \\
\text { for all and build effective, } \\
\text { accountable and inclusive } \\
\text { institutions at all levels }\end{array}$ & 11 & 1 & 5 & 11 & 23 \\
\hline $\begin{array}{l}\text { Goal 17. Strengthen the } \\
\text { means of implementation } \\
\text { and revitalize the Global } \\
\text { Partnership for Sustainable } \\
\text { Development }\end{array}$ & 9 & & 1 & 9 & 25 \\
\hline Total & 98 & 25 & 31 & 113 & 244 \\
\hline
\end{tabular}


17.18.1

As stated, all indicators included in the statistical annex are based on ICBS data and calculations except for indicators reported by the following government agencies:

\begin{tabular}{|l|l|}
\hline Relevant bodies providing data & Indicators \\
\hline National Insurance Institute of Israel & 2.1 .2 \\
\hline $\begin{array}{l}\text { Ministry of Education and Ministry of } \\
\text { Environmental Protection }\end{array}$ & $4.7 .1 \& 12.8 .1$ \\
\hline Ministry of Education & $4 . c .1$ \\
\hline $\begin{array}{l}\text { Israeli Parliament - The Knesset } \\
\text { The Water Authority }\end{array}$ & 5.5 .1 \\
\hline Ministry of Environmental Protection & 6.4 .2 \\
\hline $\begin{array}{l}\text { Israel Oceanographic and Limnological Research } \\
\text { (IOLR) }\end{array}$ & $14.1 .1,14.2 .1$ \& 14.3.1 \\
\hline $\begin{array}{l}\text { Israel Nature and Parks Authority } \\
\text { Ministry of Construction and Housing }\end{array}$ & 14.5 .1 \\
\hline Keren Kayemeth Lelsrael- Jewish National Fund & 15.2 .1 \\
\hline Ministry of Justice & 16.2 .2 \\
\hline
\end{tabular}

17.8 .2

Number of countries that have national statistical legislation that complies with the Fundamental Principles of Official Statistics

17.18.3

Number of countries with a national statistical plan that is fully funded and under implementation, by source of funding
The Statistical Ordinance of Israel is compliant with the UN Fundamental Principles of Official Statistics.

There is a National Statistical Plan for the ICBS under implementation which is fully funded by the government and other sources (Bank of Israel, National Insurance Institute, local authorities, universities, National Transport Infrastructure Company, etc.). 
17.19.2

Proportion of countries that (a) have conducted at least one population and housing census in the last 10 years; and (b) have achieved 100 percent birth registration and 80 percent death registration
The ICBS has conducted six censuses since its establishment: in 1948 , 1961, 1972, 1983, 1995 and 2008.

The 1995 Census of Population and Housing was the last traditional, door-to-door census. The aim was to undergo a gradual process toward an administrative census.

In the 2008 census, the geo-demographic census file was based on an improved Central Population Register (CPR), followed by over and undercoverage evaluation surveys that were used to correct the estimates and to supplement the socio-economic information.

The growing and pressing needs for timely, frequent and relevant detailed information led the ICBS to investigate the possibility to conduct a rolling census. Engaging research and actual field data collection did not culminate in satisfactory results.

At the same time, ICBS experienced an accelerated process of using administrative data for the production of official statistics and improved its abilities to use administrative data for census purposes. However, to date, a full administrative census has not yet been found to be a viable option.

Consequently, the 2020 census in Israel will integrate data from different sources, including data collected directly from the population. However, a major step toward an administrative census will be taken by using the administrative geo-demographic file as a sampling frame for the census survey, i.e., sampling people instead of area-cells. The survey is planned to be used for improving the small-area population estimates and for complementing the socio-economic information not found in the administrative sources, on the individual level, i.e., a tailor-made questionnaire for each person. 


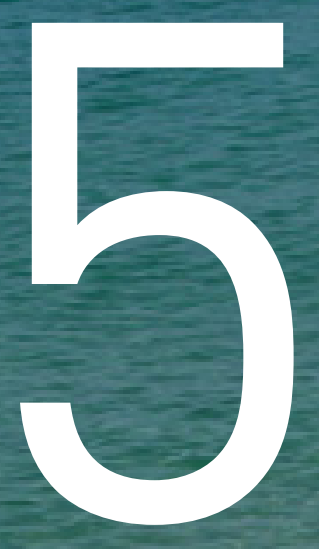

\section{SDG RELATED NATIONAL STATISTICS: WELL-BEING INDICATORS IN ISRAEL}

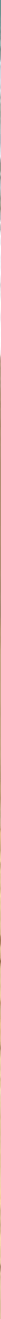


The indicators

$\&$ the process

Selection of

the indicators
Pursuant to the resolution adopted by the government of Israel in April 2015 (Resolution No. 2494), the Israel Central Bureau of Statistics (ICBS) is developing, reporting and updating indicators of well-being, sustainability, and national resilience in the following 11 domains: quality of employment; personal security; health; housing and infrastructure; education; higher education and skills; personal and social well-being; environment; civic engagement and governance; material standard of living; leisure, culture and community; and information technology.

The project on developing well-being indicators for Israel began at the end of 2012 (based on Government Resolution No. 5255 of 02.12.2012). It was led by the Prime Minister's Office, the National Economic Council and the Ministry of Environmental Protection and involved discussions between different government ministries, academia, NGOs and the private sector. The process of selecting the indicators also included public deliberations by means of online consultation and focus groups.

Eight indicators were selected for each domain, and the desired direction of change was defined for each indicator. Data on the indicators were taken from the ICBS databases and are based on administrative information and regular surveys conducted by the ICBS. The indicators present the trends from the beginning of the 21 st century and provide a basis for examining changes in well-being in Israel, comparing different population groups in Israel and undertaking international comparisons.

A person's well-being depends on many factors such as health, social relationships, trust, work, income, and sense of security. The more one's needs are met in various life domains, the better one's well-being. The aim of the well-being indicators is to present a multi-dimensional analysis of well-being and quality of life, taking into account the overall economic, social, and environmental factors that affect a person's life. This measurement is a basis for developing statistics that complement data on economic growth, and provide a more comprehensive and complete indication of well-being among the population. 
Summary of the well-being indicators for 2017

Explanation of the symbols appearing in the tables
To date, 70 indicators have been developed. Compared with the previous year, an improvement was recorded in 35 indicators, a decline was recorded in 10 indicators, and no significant trend change was recorded in 20 indicators. Compared with the base year (mainly 2002), an improvement was recorded in 36 indicators, and a decline was recorded in 5 indicators.

The direction of the arrow shows a rise or a decline in the value of the indicator.

A red arrow indicates a negative trend (against the desired direction). A green arrow indicates a positive trend (in the desired direction).

$\sim$ indicates no change or a change of less than one percentage point in the indicators deriving from sample surveys. 


\section{(TRENDS IN SELECTED INDICATORS}

Quality of Employment (percentages unless otherwise stated)

\begin{tabular}{|c|c|c|c|c|c|}
\hline & $\begin{array}{l}\text { Desired } \\
\text { direction }\end{array}$ & $\begin{array}{l}2017 \\
\text { Indicator }\end{array}$ & $\begin{array}{l}\text { Direction } \\
\text { of change } \\
\text { compared } \\
\text { with previous } \\
\text { year }\end{array}$ & $\begin{array}{l}2002 \\
\text { Indicator } \\
\text { (base year) }\end{array}$ & $\begin{array}{l}\text { Direction } \\
\text { of change } \\
\text { compared } \\
\text { with base } \\
\text { year }\end{array}$ \\
\hline Employment rate & $\uparrow$ & 61.3 & $\uparrow$ & 48.6 & $\uparrow$ \\
\hline $\begin{array}{l}\text { Rate of persons employed part-time } \\
\text { involuntarily }\end{array}$ & $\downarrow$ & 2.3 & $\downarrow$ & 4.8 & $\downarrow$ \\
\hline $\begin{array}{l}\text { Median gross income from work per } \\
\text { household (NIS, in } 2016 \text { prices) }\end{array}$ & $\uparrow$ & 17,394 & $\uparrow$ & 12,780 & $\uparrow$ \\
\hline Satisfaction with work & $\uparrow$ & 88.9 & $\uparrow$ & 81.5 & $\uparrow$ \\
\hline Satisfaction with income & $\uparrow$ & 61.4 & $\uparrow$ & 44.8 & $\uparrow$ \\
\hline Satisfaction with promotion opportunities & $\uparrow$ & 48.4 & & & \\
\hline $\begin{array}{l}\text { Field of study related to the field of } \\
\text { current work }\end{array}$ & $\uparrow$ & 65.9 & & & \\
\hline $\begin{array}{l}\text { Rate of persons injured in work accidents } \\
\text { (per 100,000 employed persons) }\end{array}$ & $\downarrow$ & 1,774 & $\downarrow$ & 2,918 & $\downarrow$ \\
\hline $\begin{array}{l}\text { Rate of prolonged unemployment lover six } \\
\text { months) }\end{array}$ & $\downarrow$ & 22.9 & $\downarrow$ & 31.8 & $\downarrow$ \\
\hline
\end{tabular}


Personal Security (percentages unless otherwise stated)

\begin{tabular}{|c|c|c|c|c|c|}
\hline & $\begin{array}{l}\text { Desired } \\
\text { direction }\end{array}$ & $\begin{array}{l}2017 \\
\text { Indicator }\end{array}$ & $\begin{array}{l}\text { Direction } \\
\text { of change } \\
\text { compared } \\
\text { with previous } \\
\text { year }\end{array}$ & $\begin{array}{l}2002 \\
\text { Indicator } \\
\text { (base year) }\end{array}$ & $\begin{array}{l}\text { Direction } \\
\text { of change } \\
\text { compared } \\
\text { with base } \\
\text { year }\end{array}$ \\
\hline $\begin{array}{l}\text { Rate of victims of violence or threats of } \\
\text { violence }\end{array}$ & $\downarrow$ & 3.7 & $\downarrow$ & & \\
\hline $\begin{array}{l}\text { Feeling safe walking alone after dark in } \\
\text { the area of residence }\end{array}$ & $\uparrow$ & 85.6 & $\sim$ & 73.6 & $\uparrow$ \\
\hline Rate of victims of sexual harassment & $\downarrow$ & 2.2 & $\downarrow$ & & \\
\hline $\begin{array}{l}\text { Rate of persons killed in road accidents, } \\
\text { per } 100,000 \text { residents }\end{array}$ & $\downarrow$ & 3.6 & $\downarrow$ & 8.0 & $\downarrow$ \\
\hline $\begin{array}{l}\text { Rate of persons seriously injured in road } \\
\text { accidents, per 100,000 residents }\end{array}$ & $\downarrow$ & 23.5 & $\uparrow$ & 36.8 & $\downarrow$ \\
\hline Rate of murders, per 100,000 residents* & $\downarrow$ & 1.2 & $\downarrow$ & 3.2 & $\downarrow$ \\
\hline $\begin{array}{l}\text { Rate of persons killed in terror attacks, } \\
\text { per } 100,000 \text { residents }\end{array}$ & $\downarrow$ & 0.2 & $\downarrow$ & 4.7 & $\downarrow$ \\
\hline $\begin{array}{l}\text { Rate of persons wounded in terror } \\
\text { attacks, per } 100,000 \text { residents }\end{array}$ & $\downarrow$ & 1.7 & $\downarrow$ & 21.4 & $\downarrow$ \\
\hline Victims of road rage & $\downarrow$ & 51.6 & $\downarrow$ & & \\
\hline $\begin{array}{l}\text { Economic damage caused by property } \\
\text { crimes }\end{array}$ & $\downarrow$ & 0.37 & & & \\
\hline
\end{tabular}

* Updated to 2016 
Health (percentages unless otherwise stated)

\begin{tabular}{|c|c|c|c|c|c|}
\hline & $\begin{array}{l}\text { Desired } \\
\text { direction }\end{array}$ & $\begin{array}{l}2017 \\
\text { Indicator }\end{array}$ & $\begin{array}{l}\text { Direction } \\
\text { of change } \\
\text { compared } \\
\text { with previous } \\
\text { year }\end{array}$ & $\begin{array}{l}2002 \\
\text { Indicator } \\
\text { (base year) }\end{array}$ & $\begin{array}{l}\text { Direction } \\
\text { of change } \\
\text { compared } \\
\text { with base } \\
\text { year }\end{array}$ \\
\hline Infant mortality & $\downarrow$ & 3.0 & $\downarrow$ & 5.4 & $\downarrow$ \\
\hline $\begin{array}{l}\text { Life expectancy } \\
\text { Men } \\
\text { Women }\end{array}$ & $\uparrow$ & $\begin{array}{l}80.7 \\
84.6\end{array}$ & $\sim$ & $\begin{array}{l}77.5 \\
81.5\end{array}$ & $\uparrow$ \\
\hline $\begin{array}{l}\text { Healthy life expectancy } \\
\qquad \text { Men } \\
\text { Women }\end{array}$ & $\begin{array}{l}\uparrow \\
\uparrow\end{array}$ & $\begin{array}{l}65.6 \\
65.4\end{array}$ & $\uparrow$ & & \\
\hline Self-assessed health & $\uparrow$ & 84.2 & $\sim$ & 77.0 & $\uparrow$ \\
\hline $\begin{array}{l}\text { Overweight and obese children* } \\
\text { First grade } \\
\text { Seventh grade }\end{array}$ & $\begin{array}{l}\downarrow \\
\downarrow\end{array}$ & $\begin{array}{l}18.3 \\
30.3\end{array}$ & $\sim$ & $\begin{array}{l}20.1 \\
31.0\end{array}$ & $\begin{array}{l}\downarrow \\
\downarrow\end{array}$ \\
\hline Feeling depressed (often)** & $\downarrow$ & 5.7 & $\sim$ & 10.7 & $\downarrow$ \\
\hline $\begin{array}{l}\text { Promoting health - summary indicator of } \\
\text { health behavior }\end{array}$ & $\uparrow$ & 24.4 & $\sim$ & & \\
\hline Self-reported smoking & $\downarrow$ & 19.6 & $\downarrow$ & 21.9 & $\downarrow$ \\
\hline $\begin{array}{l}\text { Cancer - new cases of malignant } \\
\text { neoplasms (rate per 100,000 persons) } \\
\text { Men } \\
\text { Women }\end{array}$ & $\begin{array}{l}\downarrow \\
\downarrow\end{array}$ & $\begin{array}{l}279 \\
311\end{array}$ & $\begin{array}{l}\downarrow \\
\downarrow\end{array}$ & $\begin{array}{l}297 \\
304\end{array}$ & $\underset{\sim}{\downarrow}$ \\
\hline Trust in the health system & $\uparrow$ & 75.1 & $\uparrow$ & & \\
\hline
\end{tabular}

*Base year 2011

** Base year 2003 
Housing and Infrastructure (percentages unless otherwise stated)

\begin{tabular}{|c|c|c|c|c|c|}
\hline & $\begin{array}{l}\text { Desired } \\
\text { direction }\end{array}$ & $\begin{array}{l}2017 \\
\text { Indicator }\end{array}$ & $\begin{array}{l}\text { Direction } \\
\text { of change } \\
\text { compared } \\
\text { with previous } \\
\text { year }\end{array}$ & $\begin{array}{l}2002 \\
\text { Indicator } \\
\text { (base year) }\end{array}$ & $\begin{array}{l}\text { Direction } \\
\text { of change } \\
\text { compared } \\
\text { with base } \\
\text { year }\end{array}$ \\
\hline $\begin{array}{l}\text { Percentage of households that spent } 30 \% \\
\text { or more of their net income on housing* }\end{array}$ & $\downarrow$ & 30.9 & $\uparrow$ & 35.6 & $\downarrow$ \\
\hline Satisfaction with the dwelling & $\uparrow$ & 87.6 & $\uparrow$ & 80.1 & $\uparrow$ \\
\hline Satisfaction with area of residence & $\uparrow$ & 83.9 & $\sim$ & 81.1 & $\uparrow$ \\
\hline $\begin{array}{l}\text { Percentage of the population not } \\
\text { connected to a sewage treatment system* }\end{array}$ & $\downarrow$ & 2.3 & $\sim$ & 6.1 & $\downarrow$ \\
\hline Satisfaction with public transportation & $\uparrow$ & 39.7 & $\sim$ & 44.4 & $\downarrow$ \\
\hline Housing density (no. of persons per room) & $\downarrow$ & 0.89 & $\sim$ & 0.96 & $\downarrow$ \\
\hline
\end{tabular}

Civic Engagement and Governance (percentages unless otherwise stated)

\begin{tabular}{|c|c|c|c|c|c|}
\hline & $\begin{array}{l}\text { Desired } \\
\text { direction }\end{array}$ & $\begin{array}{l}2017 \\
\text { Indicator }\end{array}$ & $\begin{array}{l}\text { Direction } \\
\text { of change } \\
\text { compared } \\
\text { with previous } \\
\text { year }\end{array}$ & $\begin{array}{l}2002 \\
\text { Indicator } \\
\text { (base year) }\end{array}$ & $\begin{array}{l}\text { Direction } \\
\text { of change } \\
\text { compared } \\
\text { with base } \\
\text { year }\end{array}$ \\
\hline Civic engagement & $\uparrow$ & 13.9 & $\downarrow$ & & \\
\hline $\begin{array}{l}\text { Voter turnout rate in Knesset (parliament) } \\
\text { elections* }\end{array}$ & $\uparrow$ & 72.3 & $\uparrow$ & 86.9 & $\downarrow$ \\
\hline Confidence in the government & $\uparrow$ & 39.9 & $\uparrow$ & & \\
\hline Confidence in the judicial system & $\uparrow$ & 54.8 & $\sim$ & & \\
\hline $\begin{array}{l}\text { Perceived ability to influence government } \\
\text { policy }\end{array}$ & $\uparrow$ & 13.4 & $\sim$ & & \\
\hline $\begin{array}{l}\text { Women in senior positions in the public } \\
\text { sector }\end{array}$ & $\uparrow$ & 40.7 & $\sim$ & & \\
\hline Arabs in the public sector & $\uparrow$ & 10.8 & $\sim$ & & \\
\hline
\end{tabular}

*Updated to 2015, base year 1949 
Education and Skills (percentages unless otherwise stated)

\begin{tabular}{|c|c|c|c|c|c|}
\hline & $\begin{array}{l}\text { Desired } \\
\text { direction }\end{array}$ & $\begin{array}{l}2017 \\
\text { Indicator }\end{array}$ & $\begin{array}{l}\text { Direction } \\
\text { of change } \\
\text { compared } \\
\text { with previous } \\
\text { year }\end{array}$ & $\begin{array}{l}2002 \\
\text { Indicator } \\
\text { (base year) }\end{array}$ & $\begin{array}{l}\text { Direction } \\
\text { of change } \\
\text { compared } \\
\text { with base } \\
\text { year }\end{array}$ \\
\hline $\begin{array}{l}\text { Rate of students who have difficulty with } \\
\text { mathematics, according to the PISA } \\
\text { study* }\end{array}$ & $\downarrow$ & 32.0 & $\downarrow$ & 42.0 & $\downarrow$ \\
\hline $\begin{array}{l}\text { Rate of persons aged } 30 \text { and over with } \\
\text { post-secondary and higher education }\end{array}$ & $\uparrow$ & 52.2 & $\downarrow$ & 43.4 & $\uparrow$ \\
\hline $\begin{array}{l}\text { Rates of students enrolled in school at } \\
\text { age } 15-17\end{array}$ & $\uparrow$ & 95.4 & $\downarrow$ & 93.7 & $\uparrow$ \\
\hline $\begin{array}{l}\text { Rate of entitlement to a matriculation } \\
\text { certificate among persons aged } 26\end{array}$ & $\uparrow$ & 48.8 & $\uparrow$ & & \\
\hline Skills of graduates: literacy** & $\uparrow$ & 37.0 & & & \\
\hline Skills of graduates: numeracy** & $\uparrow$ & 36.0 & & & \\
\hline $\begin{array}{l}\text { Kindergarten education: parents' } \\
\text { satisfaction }\end{array}$ & $\uparrow$ & 89.0 & & & \\
\hline $\begin{array}{l}\text { Parents' satisfaction with the education } \\
\text { system } \\
\text { Primary schools } \\
\text { Lower secondary schools } \\
\text { Upper secondary schools }\end{array}$ & $\begin{array}{l}\uparrow \\
\uparrow \\
\uparrow\end{array}$ & $\begin{array}{l}78.0 \\
74.0 \\
79.0\end{array}$ & & & \\
\hline $\begin{array}{l}\text { Students' satisfaction with the education } \\
\text { system } \\
\text { Primary schools } \\
\text { Lower secondary schools } \\
\text { Upper secondary schools }\end{array}$ & $\begin{array}{l}\uparrow \\
\uparrow \\
\uparrow\end{array}$ & $\begin{array}{l}76.0 \\
54.0 \\
53.0\end{array}$ & $\tilde{\sim}$ & & \\
\hline $\begin{array}{l}\text { Chances of attaining higher education, by } \\
\text { parents' education (points) }\end{array}$ & $\downarrow$ & 2.2 & $\downarrow$ & & \\
\hline
\end{tabular}

*Updated to 2015, base year 2006

**Updated to 2015

${ }^{1}$ The PISA study was conducted in 2006, 2009, 2012, and 2015 
Environment (percentages unless otherwise stated)

\begin{tabular}{|l|l|l|l|l|l|}
\hline & $\begin{array}{l}\text { Desired } \\
\text { direction }\end{array}$ & $\begin{array}{l}\mathbf{2 0 1 7} \\
\text { indicator }\end{array}$ & $\begin{array}{l}\text { Direction } \\
\text { of change } \\
\text { compared } \\
\text { with previous } \\
\text { year }\end{array}$ & $\begin{array}{l}\mathbf{2 0 0 2} \\
\text { Indicator } \\
\text { (base year) }\end{array}$ & $\begin{array}{l}\text { Direction } \\
\text { of change } \\
\text { compared } \\
\text { with base } \\
\text { year }\end{array}$ \\
\hline $\begin{array}{l}\text { Recycled waste - household, industrial, } \\
\text { yard waste }\end{array}$ & $\uparrow$ & 22.4 & $\uparrow$ & & \\
\hline $\begin{array}{l}\text { Satisfaction with cleanliness in the area of } \\
\text { residence }\end{array}$ & $\uparrow$ & 53.2 & $\sim$ & $\checkmark 4.4$ & $\downarrow$ \\
\hline Outside noise disturbance in the dwelling & $\downarrow$ & 33.1 & $\sim$ & 36.1 & $\checkmark$ \\
\hline $\begin{array}{l}\text { Percentage of renewable energy } \\
\text { production out of primary energy supply* }\end{array}$ & $\uparrow$ & 2.5 & $\uparrow$ & & \\
\hline $\begin{array}{l}\text { Percentage of renewable energy } \\
\text { production out of electricity production* }\end{array}$ & $\uparrow$ & 2.5 & $\uparrow$ & & \\
\hline $\begin{array}{l}\text { Satisfaction with parks and open areas in } \\
\text { the area of residence }\end{array}$ & $\uparrow$ & 55.1 & $\downarrow$ & & \\
\hline
\end{tabular}

*Updated to 2015

Personal and Social Well-being (percentages unless otherwise stated)

\begin{tabular}{|c|c|c|c|c|c|}
\hline & $\begin{array}{l}\text { Desired } \\
\text { direction }\end{array}$ & $\begin{array}{l}2017 \\
\text { Indicator }\end{array}$ & $\begin{array}{l}\text { Direction } \\
\text { of change } \\
\text { compared } \\
\text { with } \\
\text { previous } \\
\text { year }\end{array}$ & $\begin{array}{l}2002 \\
\text { Indicator } \\
\text { (base year) }\end{array}$ & $\begin{array}{l}\text { Direction } \\
\text { of change } \\
\text { compared } \\
\text { with base } \\
\text { year }\end{array}$ \\
\hline Satisfaction with life & $\uparrow$ & 88.6 & $\sim$ & 82.9 & $\uparrow$ \\
\hline Expectations for the future & $\uparrow$ & 53.7 & $\sim$ & 48.3 & $\uparrow$ \\
\hline Perceived ability to deal with problems* & $\uparrow$ & 67.8 & $\sim$ & 66.8 & $\uparrow$ \\
\hline Sense of loneliness (often or occasionally) & $\downarrow$ & 21.4 & $\sim$ & 32.3 & $\downarrow$ \\
\hline $\begin{array}{l}\text { Feeling that there is no one to rely on in a } \\
\text { situation of crisis or distress }\end{array}$ & $\downarrow$ & 7.0 & $\sim$ & 13.3 & $\downarrow$ \\
\hline Sense of discrimination & $\downarrow$ & 26.3 & $\uparrow$ & & \\
\hline General trust & $\uparrow$ & 41.3 & $\uparrow$ & & \\
\hline
\end{tabular}

*Base year 2003 
Material Standard of Living (percentages unless otherwise stated)

\begin{tabular}{|c|c|c|c|c|c|}
\hline & $\begin{array}{l}\text { Desired } \\
\text { direction }\end{array}$ & $\begin{array}{l}2017 \\
\text { Indicator }\end{array}$ & $\begin{array}{l}\text { Direction } \\
\text { of change } \\
\text { compared } \\
\text { with previous } \\
\text { year }\end{array}$ & $\begin{array}{l}2002 \\
\text { Indicator } \\
\text { lbase year }\end{array}$ & $\begin{array}{l}\text { Direction } \\
\text { of change } \\
\text { compared } \\
\text { with base } \\
\text { year }\end{array}$ \\
\hline $\begin{array}{l}\text { Indicator of financial wealth of } \\
\text { households* (points, base year 2001) }\end{array}$ & $\uparrow$ & 174.1 & $\uparrow$ & 93.3 & $\uparrow$ \\
\hline Actual individual consumption & $\uparrow$ & 131.8 & $\uparrow$ & 100.9 & $\uparrow$ \\
\hline $\begin{array}{l}\text { Gini coefficient of inequality in net } \\
\text { income** }\end{array}$ & $\downarrow$ & 0.344 & $\downarrow$ & 0.371 & $\downarrow$ \\
\hline $\begin{array}{l}\text { Net money income per standard person } \\
\text { (NIS, in } 2016 \text { prices) }\end{array}$ & $\uparrow$ & 102,539 & $\uparrow$ & & \\
\hline $\begin{array}{l}\text { Income per standard person (NIS, in } 2016 \\
\text { prices) }\end{array}$ & $\uparrow$ & 122,159 & $\uparrow$ & & \\
\hline $\begin{array}{l}\text { Real national disposable income per } \\
\text { capita }\end{array}$ & $\uparrow$ & 146,800 & $\uparrow$ & & \\
\hline Government debt as a percentage of GDP & $\downarrow$ & 59.9 & $\downarrow$ & 69.0 & $\downarrow$ \\
\hline $\begin{array}{l}\text { Debt of households as a percentage of } \\
\text { GDP* }\end{array}$ & $\downarrow$ & 48.5 & $\uparrow$ & 46.8 & $\uparrow$ \\
\hline Satisfaction with economic situation & $\uparrow$ & 62.3 & $\uparrow$ & 48.3 & $\uparrow$ \\
\hline
\end{tabular}

*New indicator, updated to 2015

***Base year 2008

Leisure, Culture and Community (percentages unless otherwise stated)

\begin{tabular}{|l|l|l|l|l|l|}
\hline & $\begin{array}{l}\text { Desired } \\
\text { direction }\end{array}$ & $\begin{array}{l}\mathbf{2 0 1 7} \\
\text { indicator }\end{array}$ & $\begin{array}{l}\text { Direction } \\
\text { of change } \\
\text { compared } \\
\text { with } \\
\text { previous } \\
\text { year }\end{array}$ & $\begin{array}{l}\text { 2002 } \\
\text { Indicator } \\
\text { lbase year) }\end{array}$ & $\begin{array}{l}\text { Direction } \\
\text { of change } \\
\text { compared } \\
\text { with base } \\
\text { year }\end{array}$ \\
\hline Satisfaction with the work-life balance & $\uparrow$ & rear & & & \\
\hline Engaging in volunteer activity & $\uparrow$ & 20.7 & $\sim$ & 14.5 & $\uparrow$ \\
\hline
\end{tabular}


Information and Communications Technology (percentages unless otherwise stated)

\begin{tabular}{|l|l|l|l|l|l|}
\hline & $\begin{array}{l}\text { Desired } \\
\text { direction }\end{array}$ & $\begin{array}{l}\mathbf{2 0 1 7} \\
\text { Indicator }\end{array}$ & $\begin{array}{l}\text { Direction } \\
\text { of change } \\
\text { compared } \\
\text { with } \\
\text { previous } \\
\text { year }\end{array}$ & $\begin{array}{l}\text { 2002 } \\
\text { Indicator } \\
\text { (base year) }\end{array}$ & $\begin{array}{l}\text { Direction } \\
\text { of change } \\
\text { compared } \\
\text { with base } \\
\text { year }\end{array}$ \\
\hline Employed in the field of ICT & $\uparrow$ & 202.3 & $\uparrow$ & & \\
\hline $\begin{array}{l}\text { Skills of graduates: problem-solving in a } \\
\text { computerized environment* }\end{array}$ & $\uparrow$ & 26.6 & & & \\
\hline $\begin{array}{l}\text { Persons aged } 20 \text { and over not afraid to } \\
\text { surf the web }\end{array}$ & $\uparrow$ & 55.5 & & & \\
\hline Accessibility to computer, laptop or tablet & $\uparrow$ & 81.2 & $\sim$ & \\
\hline Persons who surfed the web & $\uparrow$ & 81.6 & $\uparrow$ & & \\
\hline $\begin{array}{l}\text { Persons aged } 20 \text { and over who used } \\
\text { E-Gov }\end{array}$ & $\uparrow$ & 37.6 & $\uparrow$ & & \\
\hline
\end{tabular}

*Updated to 2015 


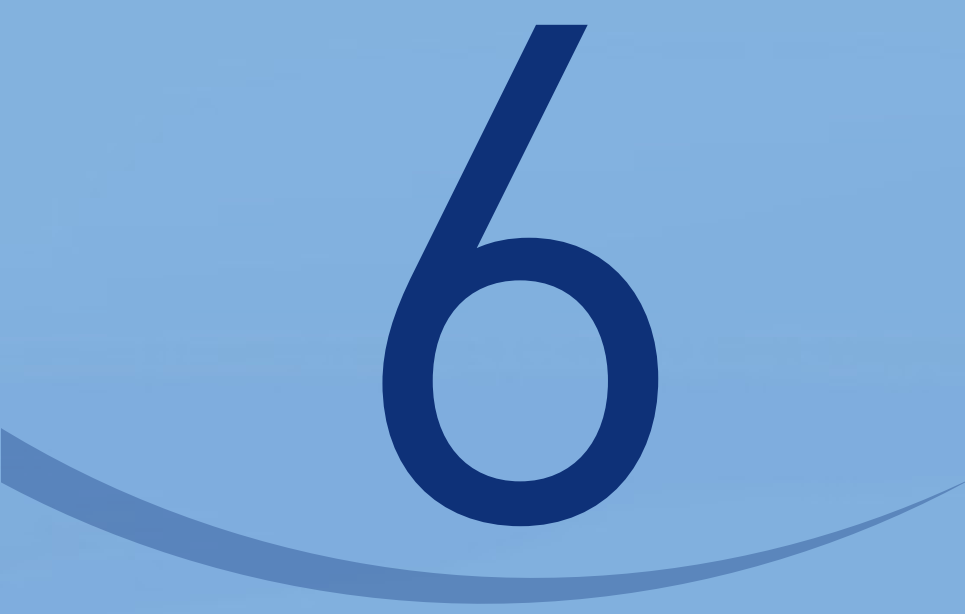

\section{OECD STUDY: MEASURING DISTANCE TO THE SDG TARGETS - ISRAEL}

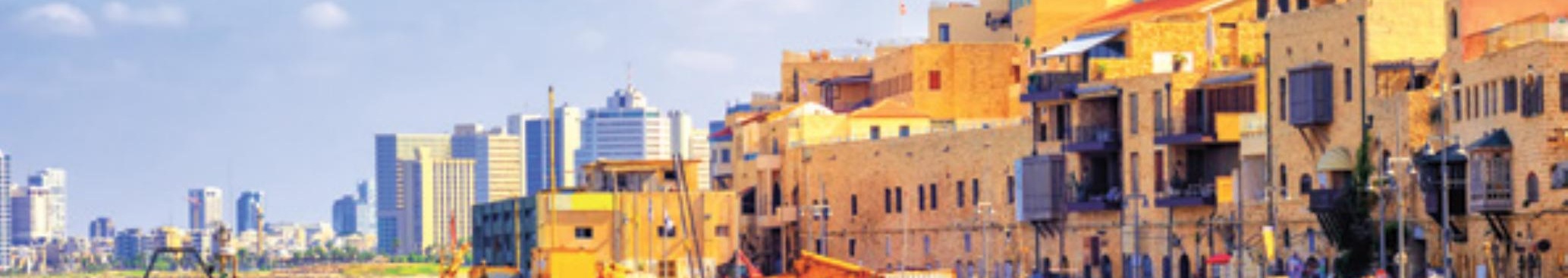

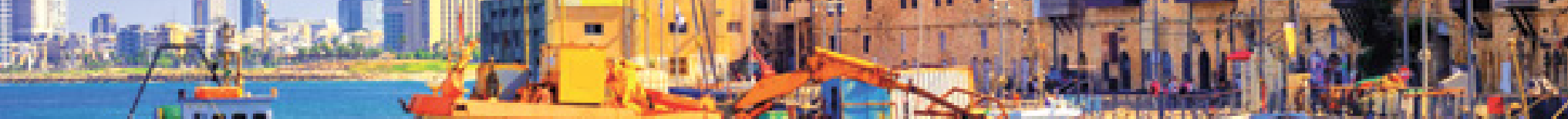
तु Is

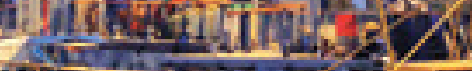

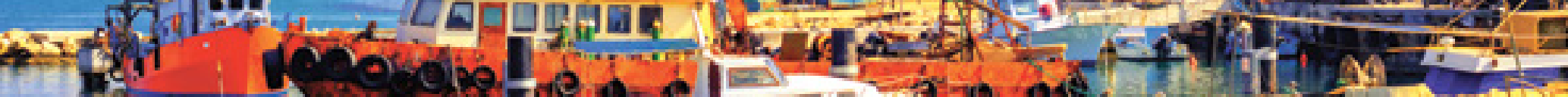




\section{Measuring Distance to the SDG Targets 2019}

\section{An Assessment of Where OECD Countries Stand}




\section{Measuring distance to the SDG targets - Israel}

Based on 104 available indicators allowing a coverage of 81 of the 169 SDG targets, Israel has currently achieved 10 of the 2030 targets, and many of the remaining distances to targets are small (Figure 2.31). For example, Israel has already achieved the targets relating to neonatal, infant and maternal mortality (targets 3.1 and 3.2), alcohol consumption and research and development employment and expenditure (targets 3.5 and 9.5). However, some challenges remain; Israel is still very far (i.e. more than 3 standardised distances away) from meeting some $12 \%$ of the targets. These include relative income poverty rate, students' basic skills in mathematics and socio-economic disparities in education (targets 1.2, 4.1 and 4.5).

\section{Figure 2.31. Israel's distance from achieving 81 SDG targets}

\section{Goals}

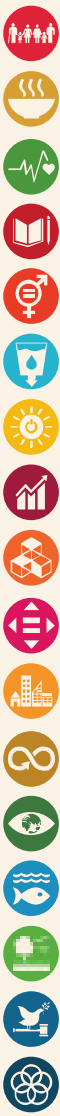

1: Eradicate poverty

2: Food

3: Health

4: Education

5: Gender equality

6: Water

7: Energy

8: Economy

9: Infrastructure

10: Reduce inequality

11: Cities

12: Sustainable production

13: Climate

14: Oceans

15: Biodiversity

16: Institutions

17: Implementation

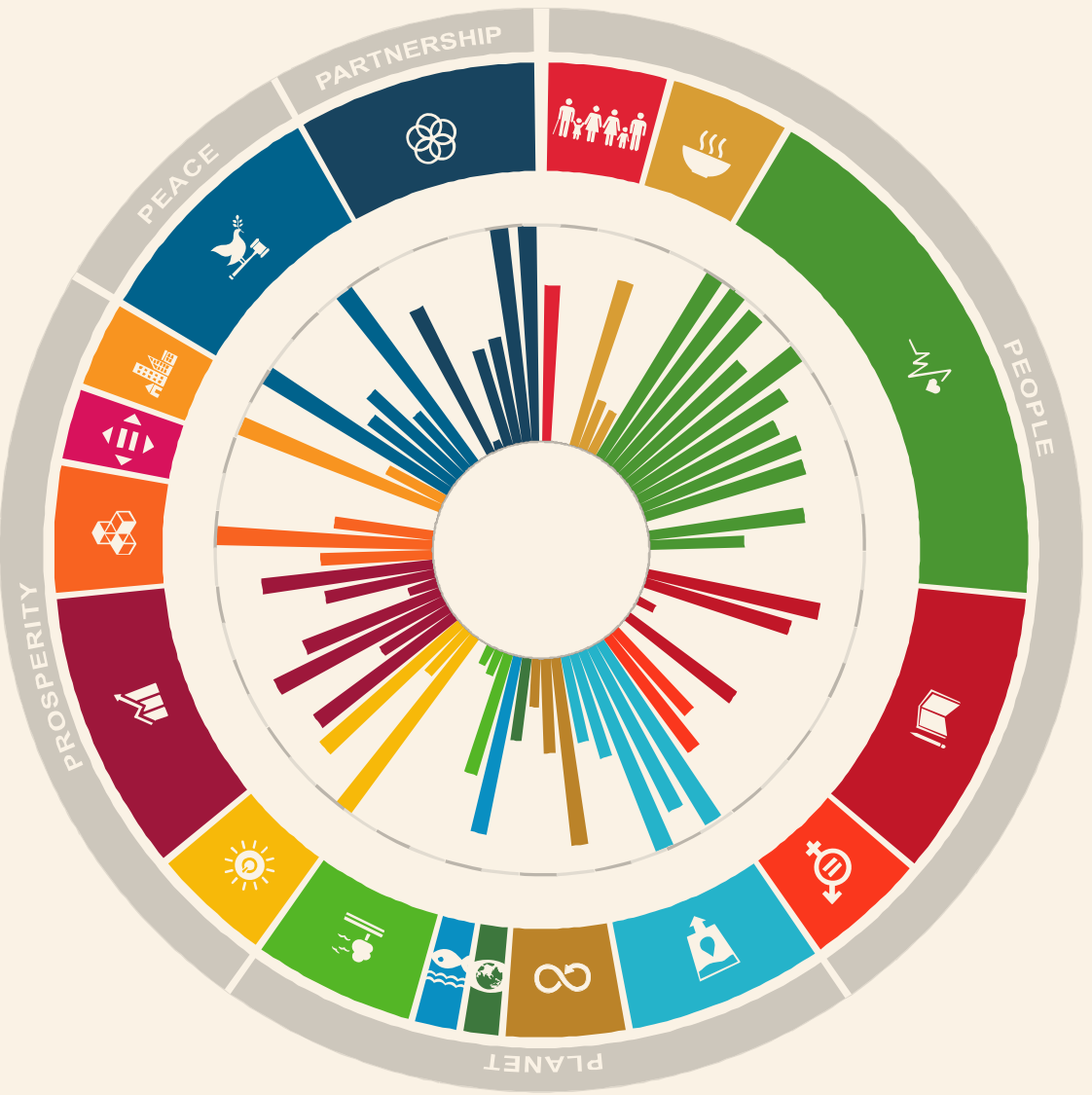

_ _ Levels of achievement to be attained by 2030

Note: The chart shows current level of achievement on each available target. The longer the bar, the shorter the distance still to be travelled to reach 2030 target (dotted circle). Targets are clustered by goal, and goals are clustered by the " 5 Ps" of the 2030 Agenda (outer circle).

Source: See www.oecd.org/sdd/OECD-Measuring-Distance-to-SDGs-Targets-Metadata.pdf for detailed metadata.

StatLink הत्lst http://dx.doi.org/10.1787/888933963443

The Measuring Distance to the SDG Targets Study is intended as an analytical tool to assist countries in identifying strengths and weaknesses across the goals and targets of the 2030 Agenda, and as such differs in nature from Voluntary National Reviews (VNRs) or other reporting processes. To ensure international comparability, indicators used in the Study are based on the UN Global List of Indicators on SDGs and are sourced from the UN SDG Database and OECD databases. VNRs typically use national indicators that reflect national circumstances and can be more up-to-date. 
Figure 2.32, Panel A shows that Israel is on average closest to reaching goals on Health, Water and Oceans (goals 4, 6 and 14). On the other hand, Israel is furthest from goals on Poverty Eradication, Education, Biodiversity and Reducing Inequality (goals 1, 4, 15, and 10). Relative to the OECD average, Israel outperforms on goals such as Health, Water and Oceans (goals 3, 6 and 14). Conversely, Israel is relatively further away on goals such as Poverty Eradication, Education, Reducing Inequality, Climate and Biodiversity (goals 1, 4, 1013 and 15). However, considerable effort by the international statistical community will be key to fill the data gaps and allow a more accurate assessment (see Figure 2.32, Panel B). For example, if missing data were available on Climate, Oceans, Reducing Inequality and Cities (goals 13, 14, 10 and 11), Israel's performance on Planet and Prosperity could change from current assessments.

Figure 2.32. Israel's distance from targets and data coverage, by goal

Panel A - Israel's average distance to targets at Goal level

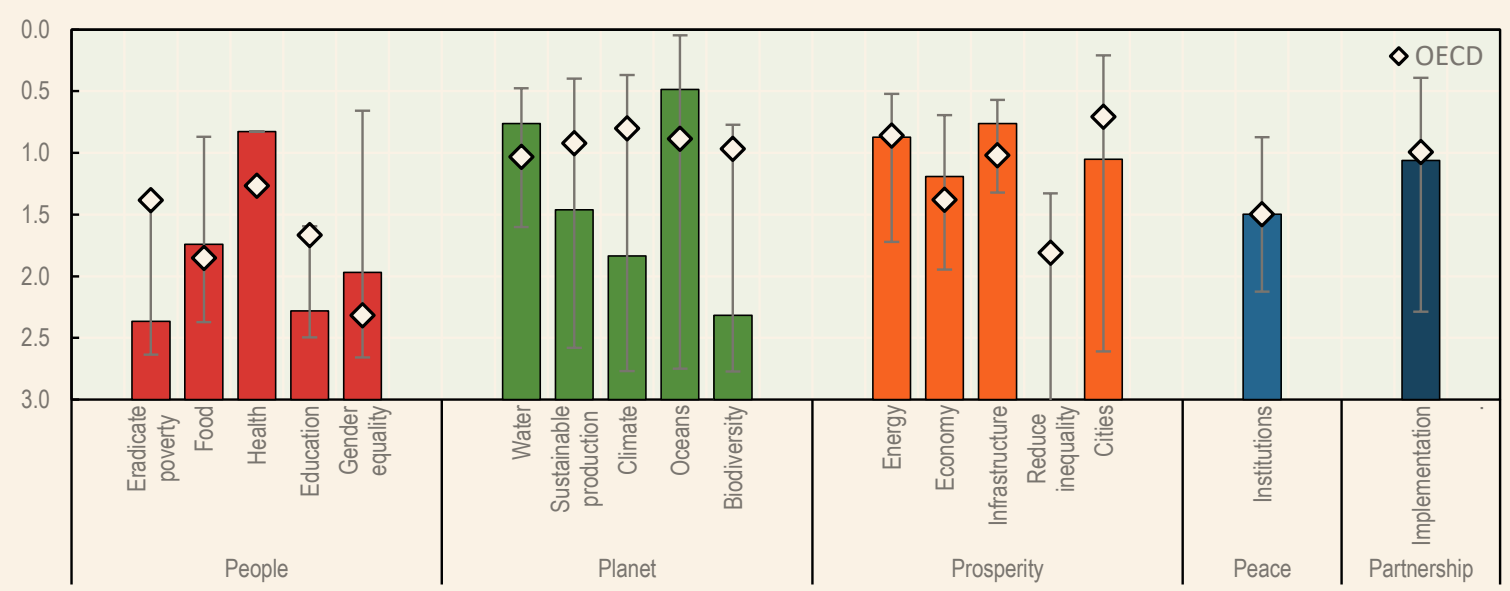

Panel B - Israel's data coverage, percentage of targets for which there is at least one indicator by Goal

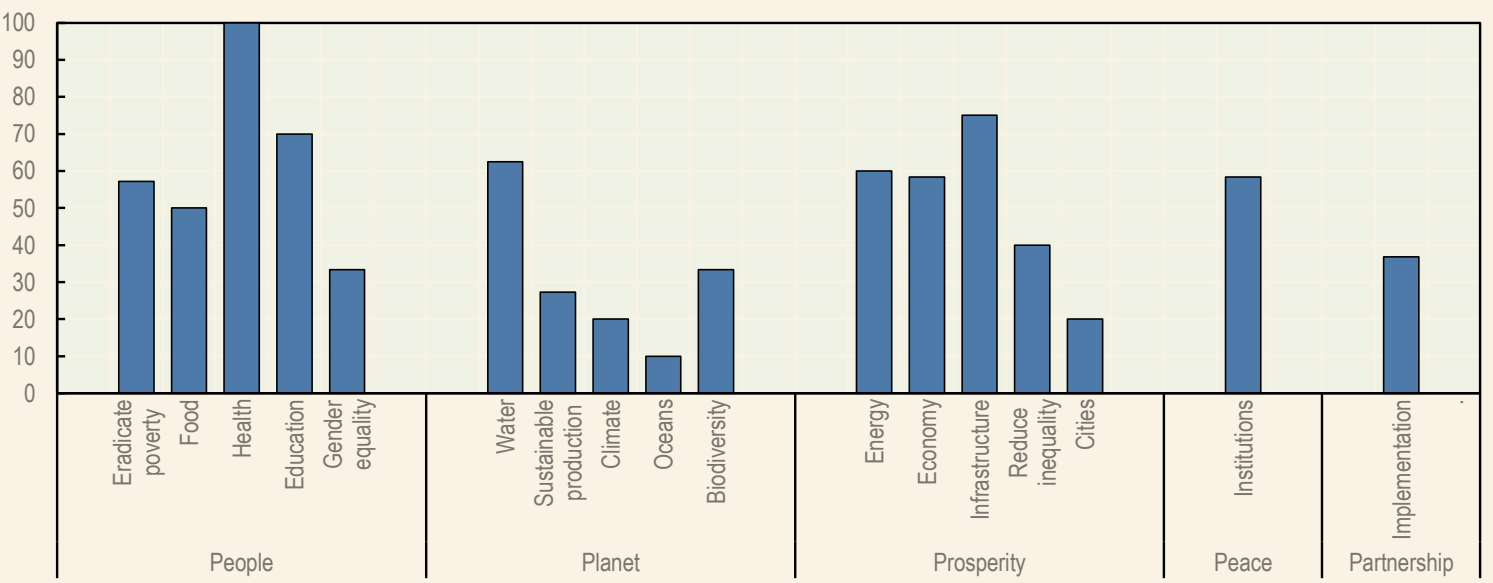

Note: Panel A shows the average distance the country needs to travel to reach each SDG. Distances are measured in standardised units (see Chapter 3 for details) with 0 indicating that the level for 2030 has already been attained: and 3 is the distance most OECD countries have already travelled. Bars show the average country performance against all targets under the relevant Goal for which data are available, and diamonds show the OECD average. Whiskers show uncertainties due to missing data, ranging from assuming that missing indicators are all 3 standardised distances from the 2030 target level to assuming that they are already at the target level. Panel B shows the share of targets covered by at least one indicator out of the 169 targets of the 2030 Agenda, according to the 17 goals and 5Ps.

Source: See www.oecd.org/sdd/OECD-Measuring-Distance-to-SDGs-Targets-Metadata.pdf for detailed metadata. 


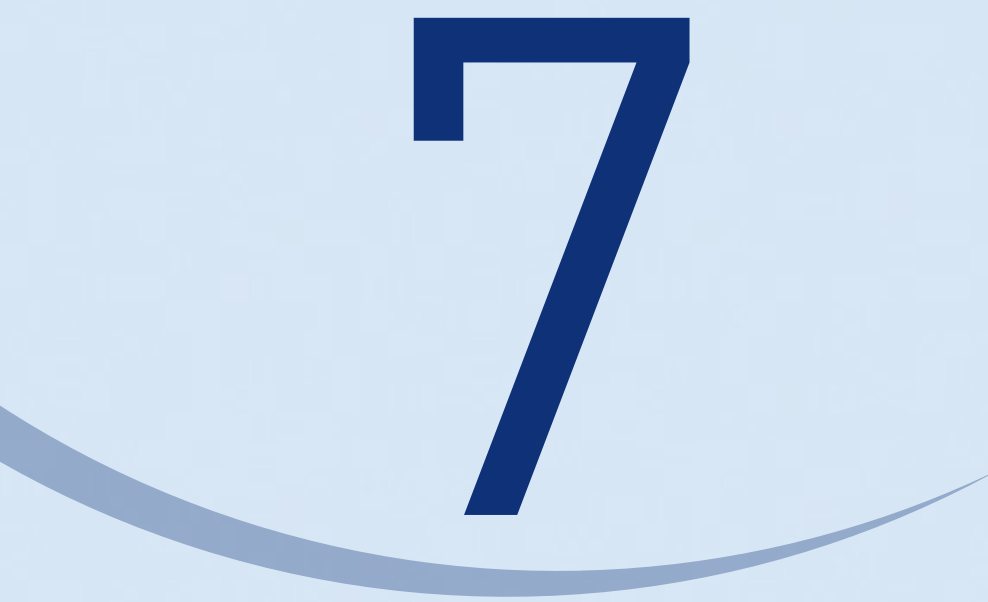

\section{CONTRIBUTIONS FROM NON-GOVERNMENT STAKEHOLDERS}

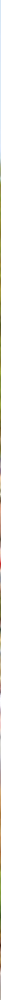




\title{
Position Paper by the Coalition of Civil Society Organizations on Implementing the 2030 Agenda (SDGs) in Israel
}

\begin{abstract}
Part One: Introduction
At the core of the UN global goals for sustainable development ("SDGs" or "Agenda 2030")" is an understanding of the need for broad cooperation among all sectors of society to reach equality and prosperity for all, while at the same time ensuring that the life support systems of Planet Earth will thrive.

In light of this vision, a coalition was established in Israel of dozens of civil society organizations (CSOs) that view Agenda 2030 as a tool for changing the face of Israeli society, and as a road map for hope."

This position paper was written as part of an open and inclusive discussion of the coalition of CSOs in Israel, committed to advance change inspired by the global goals, while seeking to learn from the work of similar coalitions around the world. The aim of this position paper is to lay the cornerstone for the implementation of Agenda 2030 from the standpoint of civil society and present the conditions and mechanisms for realizing it in Israel. $^{2}$

In the position paper, we will outline ten guiding principles that constitute the necessary conditions for proper implementation of the SDGs, and suggest milestones for reaching these objectives. These are the necessary steps to generate the significant changes to remedy deep-rooted social problems and to bring about the implementation of the SDGs in Israel by 2030.
\end{abstract}

\begin{abstract}
"List of organizations leading the process: Itach-Maaki ("with you") - Women Lawyers for Social Justice for Social Justice, The Heschel Center for Sustainability, The Israeli Civic Leadership Association (Civic Leadership), in Cooperation with Heinrich Boell Stiftung in Israel. List of organizations that took part in writing the position paper: Citizens for the Environment, Ahoti (Sister) - For Women in Israel, The Association of Advisors for Women's Status in Local Government, The Association of Rape Crisis Centers in Israel, Injaz, Enosh - The Israeli Mental Health Association, Ecoocean, B'Sod Siach (For the Promotion of Dialogue), JDC of Israel, The Association for Civil Rights in Israel, The Society for the Protection of Nature in Israel, Israel Association of Community Centers, Ha-Lev - Association of Organizations for the Elderly in Israel, Esek Mishelah - Economic Empowerment for Women, The Society for Sustainable Economics, Israel Trauma Coalition, A New Dawn in the Negev, "Chen" - Patient Fertility, The Abraham Fund Initiatives, We Power - Gender Equality in Decision-Making Positions, Green Course - Leading Activism for the Environment, Eshel - Council of Directors of Volunteer Organizations in Beersheva and the Vicinity, Midot- Reflecting and Rating NPOs Ltd., Maagaeli Erekh - Value Leverages, Movement for Israeli Urbanism (Merhav), Rackman Center, Advancement of Gender Equality Initiatives, Van Leer Institute, Games for Peace, Women Wage Peace, Sikkuy, Elem Jerusalem, Yedid - The Association for Community Empowerment, Peace NGOs Forum, Negev Coexistence Forum for Civil Equality, Jisr a-Zarqa Economic Development Project, Kedma, YK Center Israel (SDG Community, Women's Spirit, Citizen's Empowerment Movement in Israel, Sheatufim - Strategies for Social Impact,), Imagine - Empowerment through Music, MAZON A Jewish Response to Hunger, Mind the Conflict, Ting Global.
\end{abstract}

Link to a short video about the process of writing this report:

https: //www.youtube.com/watch?v=WGzNY3Ds6gs\&feature=youtu.be
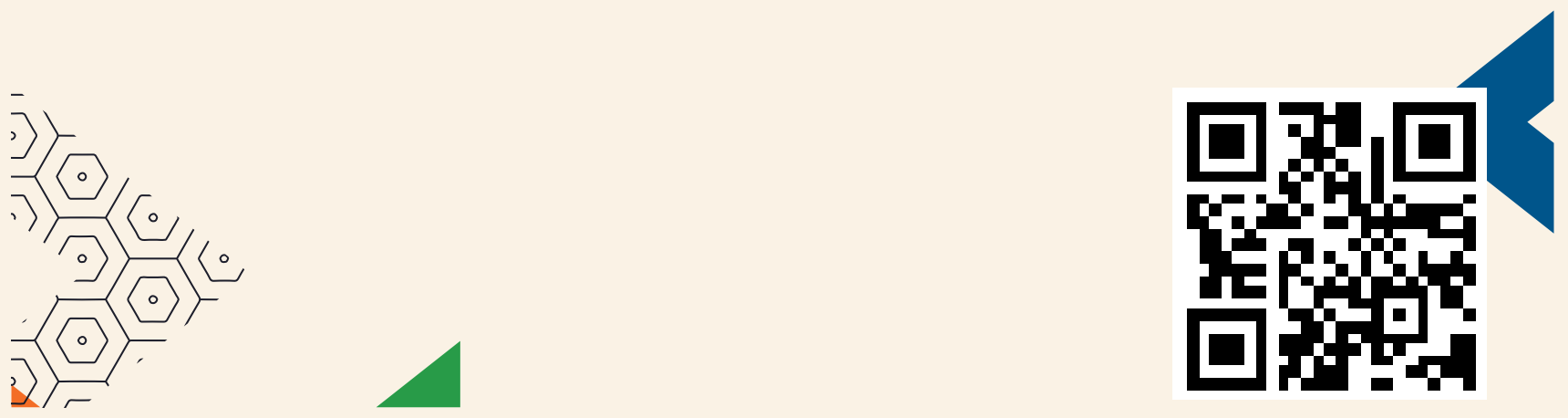
It should be emphasized that the position paper does not cover the entire gamut of difficulties and challenges in Israeli society, including poverty, economic gaps, and ongoing armed conflict, which have ramifications for equality and the rights of various groups and individuals. ${ }^{3}$

After submitting the position paper, the Coalition organizations will formulate a comprehensive monitoring report on the fulfillment of the goals and objectives in Israel. The Coalition will address the variety of challenges and social gaps, and at the same time will seek to develop tools to fulfill the potential for collaboration.

This position paper was compiled in parallel to the drafting of the Israeli government report to the High Level Political Forum at the United Nations planned for July 2019. The CSO's paper was finalized before the government's report was made available to the public, and the CSOs have yet to respond to it.

\section{The Role of Civil Society in Israel in the Implementation of Agenda 2030}

Civil society in Israel comprises thousands of organizations with knowledge and expertise in a wide range of areas ${ }^{4} \mathrm{CSO}$ s play a key role in carrying out activities that strengthen diverse groups in society and in catalyzing long-term social change processes that are often adopted by the Israeli government, such as: decisions for gender equality, reforms in education and environment, the promotion of renewable energy, mechanisms for transparency in government, freedom of information. CSOs serve as a watchdog to draw attention and call for action around injustice largely hidden from the public eye, sounding an alarm for marginalized populations whose voices are seldom heard.

The Coalition believes that the most important message of Agenda 2030 stems from its integrative perspective, which highlights the synergy needed to fulfill the various goals. In the working groups set up by the Coalition, ${ }^{5}$ the integrative principle was also identified as a key to leveraging collaboration within civil society, enabling the sharing of knowledge and experience and the broadening of perspective. It is hoped that the broad and diverse coalition of organizations created can become a significant factor to strengthen capacity and forge collaborations both with government actors and with the commercial sector.
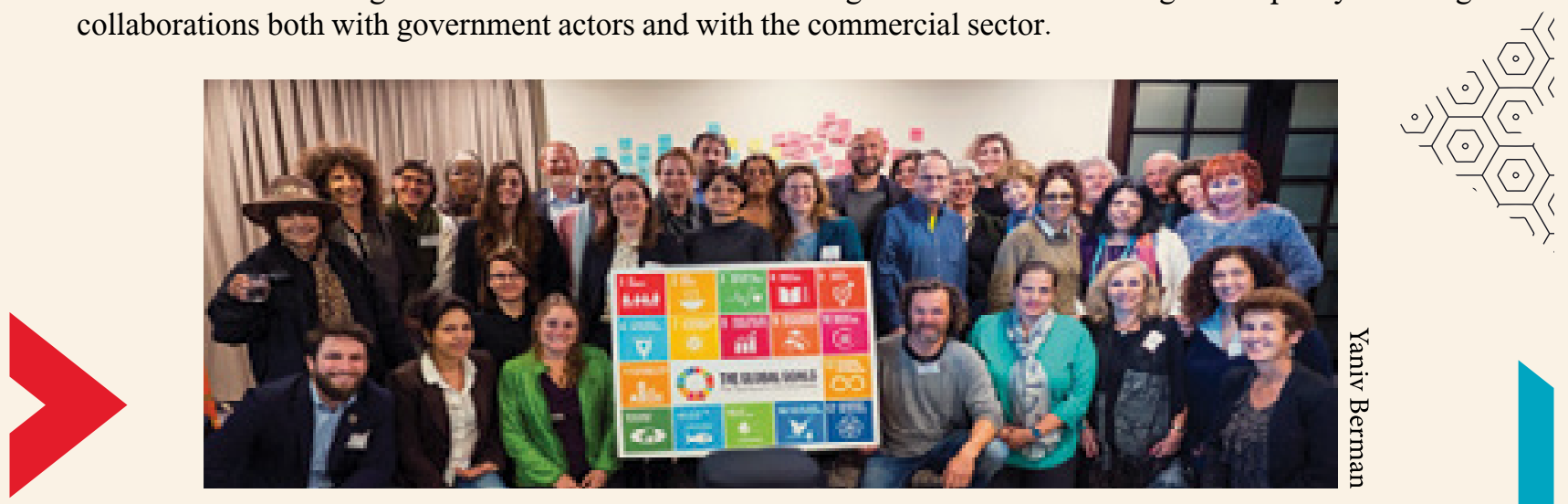

Despite the potential identified within the Agenda 2030 platform, CSOs also wish to point out the possible pitfalls in the adoption of Agenda 2030 as a policy tool. Among the concerns raised in the discussions:

- Difficulty in turning vision into practice, due to the general character of the Agenda.

- Lack of dedicated long-term budgets within government and civil society.

- The gap between government declarations regarding the achievement of goals, and their actual implementation.

- The hope embedded in the global goals will not be fulfilled in terms of advancing groups facing discrimination.

Therefore, the objective of this position paper is to emphasize the guiding principles and mechanisms which will enable to avoid these pitfalls and reach an optimal realization of Agenda 2030. 


\section{Part Two: The Need for a Systemic View and Synchronization in Addressing Core Challenges in Israel}

The Israeli government pledged in September 2015 to act towards the adoption of global goals for sustainable development, from January 2016 through 2030.

To date, there are still many hurdles to be overcome to achieve the implementation of sustainable solutions in the spirit of the SDGs. In some cases, the topics are not high on the list of government priorities, such as in the case of the advancement of peace (as mentioned in goal 16). As for many other broad topics-such as the problems of poverty, health, environmental challenges, and lack of gender equality-despite a willingness of the government to advance solutions and many plans to do so, no significant changes have been made. ${ }^{6}$

The coalition believes that the key to fulfilling Israel's pledge to implement the goals is the development of mechanisms for systemic, interdisciplinary work, embracing all government, civil society and financial sector organizations (goal 17 of the 2030 Agenda). The centrality of the development of mechanisms for systemic collaboration has been reiterated time and again in the Agenda's expressed mission. ${ }^{7}$

The international index evaluating how nations around the world are implementing global goals, ranks Israel in the $41^{\text {st }}$ place. ${ }^{8}$ According to this ranking, the lack of implementation of goal 17 , which emphasizes collaboration, stands out prominently.

An examination of government programs that have not yet realized their goals reveals a lack of an integrative evaluation of complex problems and a lack of mechanisms for communication and cooperation. This challenge is reflected frequently in reports of the State Comptroller, which have pointed to the "lack of an overall policy [...] In the absence of proper guidance, each authority acts according to its own perspective; ${ }^{9}$ "duplication in the jobs of employees in different ministries that operate without consultation; ${ }^{10}$ the "problematic coordination" between ministries, which led to the "superfluous delay in responding to requests"; the lack of a "central body" and failure to determine a clear and orderly procedure "that will set up, inter alia, the mechanism for the transfer of information between the various actors" ${ }^{11}$, and the lack of clarity regarding a coordinating body [...] detrimental to the efficacy of the policy. ${ }^{12}$

Despite these examples, ${ }^{13}$ it is evident that there exists a growing awareness among government ministries of the importance of integrative planning. In recent years, this aspect of planning has often been touted as a key goal. This is reflected, for example, in the government's 2019 work plan, which states that "the intent of the strategic assessment of the situation is to serve as a 'collective compass' to assist in decision-making for the entire government." In recent years, several examples of integrative processes instigated by the government, ${ }^{14}$ in the Knesset, and of course, among CSOs, can be identified. ${ }^{15}$ However, government offices state that the incentives operating in the governmental system are not necessarily geared towards collaborative work with a long-term view. ${ }^{16}$

In view of these structural obstacles, the CSO Coalition will present guiding principles that will enable the creation of policies which offer keys to fundamental change in Israeli society.
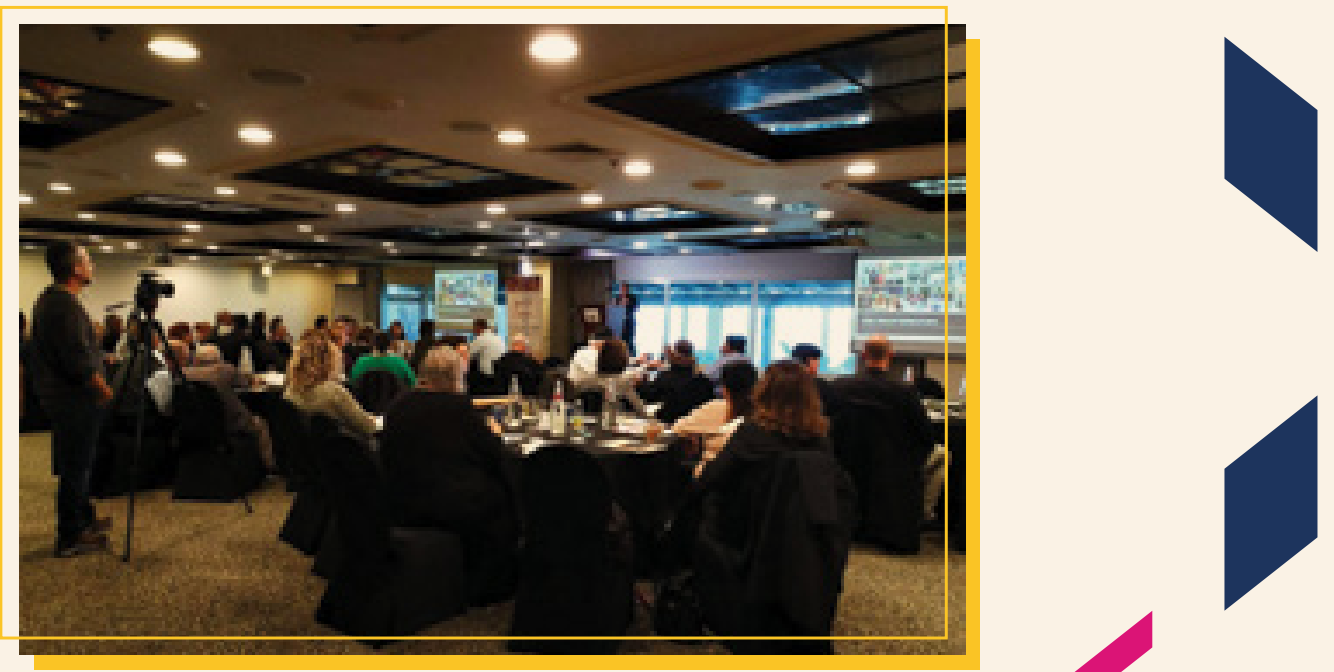


\section{Part Three: Guiding Principles for Shaping Policy according to Agenda $2030^{17}$}

The implementation of the 17 goals for 2030, with an emphasis on the interface points between them, requires a values-based perspective that places the principles of democracy, equality and sustainability at center stage. The coalition has formulated ten principles that give this perspective concrete expression and are a guideline as to how to translate the goals of Agenda 2030 into an overarching policy. The ten guiding principles were refined in discussions of CSOs, through examining successful and unsuccessful cases of policy programs in the present and past. The guiding principles below were intended to ensure that the policy that is determined - in every realm - will lead to the advancement of the SDGs.

\section{First Principle: Human Dignity Perspective}

The preliminary assumption of any policy must reflect human dignity as the ethical recognition of the value of every person by virtue of his or her humanness, in all its variety. Governments must act with compassion and empathy, without racism or verbal and physical violence towards civilians, and with a basic recognition of the variety and complexity of each and every partner, and an openness to learning from his or her diverse life experience.

\section{Guiding questions / Strategy for implementation:}

- How does the policy advance and strengthen the perspective of human dignity by virtue of acknowledging each person's unique humanness?

- How does it take into account differences between various social groups, and differential impact on diverse groups?

- Does the policy strive for full implementation of the right for all humans to a life of minimum respect and dignity?

\section{Second Principle: Holistic Perspective}

Core essential problems in society can be solved only through adopting the following perspective and set of criteria:

- Strive for systemic solutions as a response to social and civil challenges through a multi-disciplinary and multi-dimensional approach.

- Aspire to solutions that address the root of problems, and not only the symptoms.

- Identify the connection to reciprocal influences of various life realms on the problem.

- Analyze the consequences of various alternative solutions to avoid solutions that generate other problems - environmental, social or economic.

\section{Guiding questions / Strategy for implementation:}

- Does the policy succeed in addressing the problem at the root, or only its symptoms?

- How is this phenomenon influenced by other phenomena?

- How does this solution impact other fields, and does it have unseen ramifications that are not immediately perceptible?

- How can a solution be identified and devised that has added social benefits (co-benefits)?

- Use "backcasting" to the desired goal, and subsequently, map out the necessary steps in order to reach it. What must be done today/tomorrow/in the coming years in order to achieve the goals and reach the desired reality?

\section{Third Principle: Inclusivity, Diversity and Representation}

Shape every policy in a manner that includes the interests and needs of various and diverse communities, with a special emphasis on groups that suffer from pervasive discrimination, in the spirit of the overarching principle of the 2030 Agenda: "Leave no one behind." Make sure that the worldviews and concerns of all the actors are represented at the table and take into account the spirit of the principle "nothing about us without us." In parallel, the process must be constructed with an awareness of the existing obstacles vis-a-vis discriminated and disadvantaged populations, such as economic, social, geographic and technological gaps. The objective is to grapple with these obstacles and overcome them. 
Guiding questions / Strategy for implementation:

- Who is missing around the table? Who is influenced or could be hurt from this process, and who might contribute additional perspectives? Whose voice has not yet been heard at the various stages of the decision-making and policy-implementation process, and how can it be made audible?

- Does the discriminated population have access to ways of influencing the process? What measures can be taken to remove the obstacles (legislative or procedural, budget allocations, publicity in media and relevant social media)?

- Issue calls for proposals and allocation requests accessible in different languages, to different geographic locations, religions and cultures.

\section{Fourth Principle: Strengthening the Democratic Process and Faith in the Government}

The public's faith in government processes must be strengthened by reinforcing the sense of ownership and by creating trust between all parties. ${ }^{18}$ In order to enable an integrative and inclusive process, it is necessary to ensure that an infrastructure is first laid using confidence-building measures, predicated on clear credible communication of the process serving the public interest (this principle also applies to those who do not directly take part in the process).

\section{Guiding questions / Strategy for implementation:}

- How does the policy strengthen the public's faith in government?

- Do the policies and the actions contribute to building trust and increasing faith in public institutions?

\section{Fifth Principle: Inclusive Governance}

Institutionalize channels to include civil society organizations and the general public in decision-making processes - based on mutuality and partnership. There is a need to deepen and strengthen "bottom-up" processes that enable a diversity of experts to influence and shape policy. The goal is to bring people from diverse communities to see themselves as partners and increase a sense of ownership. This policy shift also seeks to change the view of citizens from consumers (who merely enjoy or "use" government services) to partners in defining the problem and devising solutions. In this vein, it is also important to leverage demonstrated successes in multi-sector collaboration.

\section{Guiding questions / Strategy for implementation:}

- How many and which actors / partners are participating in the decision-making?

- How much weight does civil society have in the decision-making?

- How is the public to be approached regarding decision-making during a given process, in a manner that does not identify it only as a consumer of services?

\section{Sixth Principle: Ensure the Vitality of Earth's Life Support Systems}

Every policy should be considered and evaluated according to its contribution over time to the struggle to preserve and foster the vitality and health of the earth's life-supporting systems (e.g.: climate, oceans, clean air and water, biodiversity) and the creation of a safe operating space for humanity.

\section{Guiding questions / Strategy for implementation:}

- Do the steps being considered contribute to or detract from the stability of the climate?

- Is the precautionary principle applied in every development initiative, policy or commercial activity?

- Will this activity protect and enable the renewal of this resource, or destroy it?

- Do the activities bring about the continued degradation of life support systems (natural resources, clean air, climate stability) in a systematic manner, or help regenerate these systems? 


\section{Seventh Principle: View Policy Influence on Future Generations}

It is essential to examine policy initiatives from the viewpoint of future generations, and the ramifications for them. Develop expertise (a "future field") in the areas of environmental quality, natural resources, aging, science, development, education, health, market and economy, demography, planning and building, quality of life, technology and law, to incorporate into policy its projected influence on future generations.

\section{Guiding questions / Strategy for implementation:}

- How youth influence the planning process?

- What are the possible effects of planned activities and processes, and will they enable future generations to enjoy climate stability, natural resources and good health?

\section{Eighth Principle: Integrate an Analysis of Gender Perspectives}

All too often, women's voices are pushed aside. Adoption of an egalitarian perspective will lead to the formulation policy from gender perspective, integrating of the needs and implications on women from a variety of population groups, particularly, on women suffering from exclusion and marginalization.

Formulation of policy according to this principle will necessitate viewing issues through the eyes of women from a range of population groups. This will not only advance the rights of women, but will also constitute a meaningful marker regarding the complete implementation of the goals of Agenda 2030 in light of the agenda's basic principle: "Leave no one behind"

\section{Guiding questions / Strategy for implementation:}

- Are women involved and exerting influence throughout the entire course of any policy creation?

- Are women and gender experts from diverse backgrounds represented, including from discriminated populations?

- How can current mechanisms for consultation that adopt a gender perspective throughout the process include gender perspectives effectively?

\section{Ninth Principle: Long-term Planning Including Setting Clear Goals and Measures}

Create long-term strategic plans of action that include all goals, objectives and activities to be realized. Set clear standards and benchmarks for measuring and evaluating effectiveness of government activity towards fulfilling the vision. Include oversight, measurement and evaluation as part of the implementation.

\section{Guiding questions / Strategy for implementation:}

- Does the planning extend to a range of ten years ahead? Twenty?

- How will the effectiveness of the process be measured over time?

- How will the effect of the policy on different population groups be measured?

\section{Tenth Principle: Transparency and Open Government}

Shape transparent, open processes by publishing all information that can contribute to the public understanding of the formulated policy and the public's perception of its ability to influence policy design. Transparency must be balanced with the need for efficiency, although transparency will take priority, except in unusual cases. Policymaking processes must be transparent at least at the main junctures: identifying partners to the process, defining the problem, identifying the coordinating and monitoring body, information about the budget and its allocation and publishing the results of periodic monitoring of implementation.

\section{Guiding questions /Strategies for implementation:}

- Is there open access to information and publications on designated websites? Create platforms for all relevant information that are accessible and available to all.

- What processes are taking place around the world from which it is possible to learn in order to expand the range of inclusive democracy? ${ }^{19}$ 


\section{Part Four: Mechanisms Necessary for Implementing the Guiding Principles for Shaping Policy}

Below, we outline the specific mechanisms necessary to shape and guide policy in light of the above principles, with the goal of strengthening collaboration for the implementation of Agenda 2030.

Establish an Integrative Coordinating Body with system-wide authorities for strategic planning, which will be responsible for synchronizing between all the bodies and actors involved.

One of the key components for achieving the goals by 2030 in light of the guiding principles is to establish a coordinating framework for inter-sectoral and inter-disciplinary policy work, which will provide a venue for shaping and managing the process, coordinating between all the players, formulating the work methodology, and amassing a database for carrying out the process and making decisions. (See figure 1)

Steps for an effective coordinating body:

- Identifying interested parties, as well as those that have an effect / are affected

- Mapping the various bodies, organizations, government agencies including local government, academics, businesses and companies, social organizations, and relevant experts.

- Identification of marginalized groups that will be affected by the process (men and women in the workforce, the elderly, children, Arabs, the ultra-Orthodox, the disabled, and others).

- Creation of guidelines to determine the representation of groups that will participate in the coordinating body. Emphasis must be placed on the creation of equal representation that gives a significant voice to people from a variety of population groups and particularly, to members of discriminated groups, as well as ensuring the representation of future generations.

- Operationalize platforms for professional consultation from civil society organizations, academics and people from the groups relevant to the discussion. Identify opportunities and create channels for collaboration and direct and unmediated discourse between the general public and national and local government.

- Using active channels such as umbrella organizations, forums/coalitions to facilitate collaborative processes, alongside a public appeal for new partners.

- Adhere to a high level of equality and reciprocity between the actors in the process, taking care to devise steps that cultivate trust throughout, and ensure authentic partnership and collaboration (fulfilling the overarching principle- "nothing about us without us"). Find ways for compensating participants from civil society and the public-at-large, in order to overcome financial obstacles.

- Stipulate and balance between different expectations - What is required of the participants? What is the anticipated output, what are the needs of the population? etc.

- Define dedicated and clear budgeting over time - The budget must include funding of the process together with funding of the implementation and monitoring.

- Identify and map the roots of the problem - Describe the current situation based on research and data, and evaluations regarding the character of the necessary intervention and possible effects of the intervention processes.

- Adopt a perspective of "backcasting" processes, beginning with the goal definitions and the question: What is necessary for attainment of the goal? Pursuant to this, constructing the necessary steps to fulfill this goal. (Not to think, "What can be obtained based on what exists today," but "What do we desire to achieve"?).

- Collect data related to gender, which will help to better understand the needs and implications of policy on women and assist in evaluating the efficacy of government activity.

- Building a detailed work plan including goals, measures, indicators of success, division of responsibility, resources and a detailed timetable. 


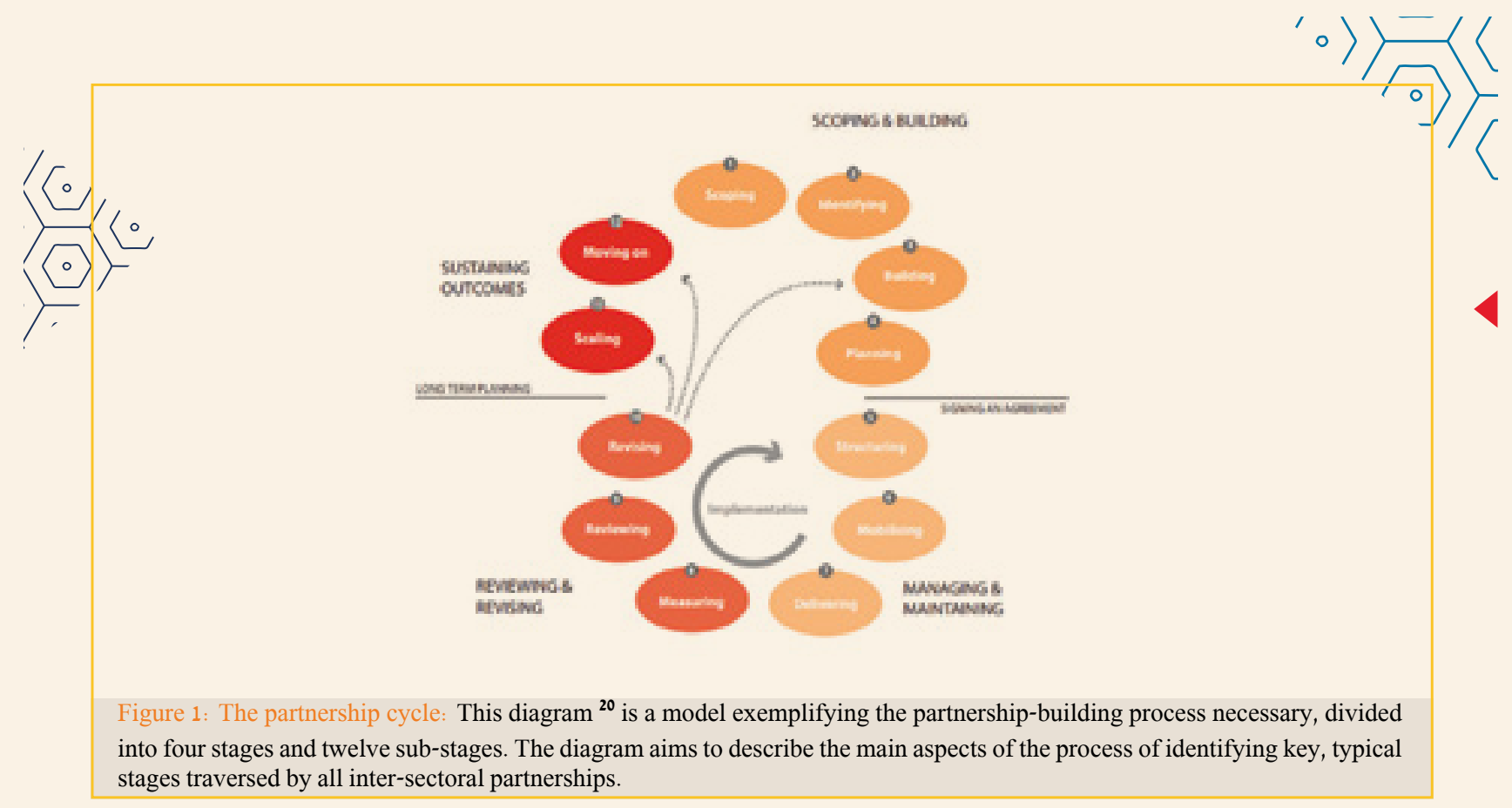

\section{Establish a Consultation Committee - a multi sector and interdisciplinary body to mentor/advise the coordinating body.}

- The Committee will help shape the vision of the coordinating framework over the long term, and regularly evaluate the progress of the coordinating body's activity in light of this vision.

- The composition of the Consultation Committee will be composed of diverse multi- sector representation constructed according to the guiding principles and steps enumerated above.

- The Committee will be budgeted, and the activity of its members will be financed.

Establish a Monitoring Body to follow-up and track the implementation of decisions and policies.

Characteristics of the Monitoring Body:

- Sustainable over time, carrying out monitoring and reporting to the Coordinating Body once every six months.

- $\quad$ Equipped with tools and resources for inter-ministerial evaluation and tools for evaluating implications and outcomes in light of the above ten guiding principles.

- Has diverse representation, with an emphasis on the representation of marginalized populations.

- Places emphasis on discriminated populations, in order to ensure that the government policy adheres to the commitment of Agenda 2030 - "leave no one behind."

- Sets measures for the success and execution of short- and long-term monitoring, including as much transparency as possible, with "joined up" cross-disciplinary thinking and systematic evaluation throughout the process. Measurement and evaluation processes must be included in advance in the budgetary planning.

- Looks for inspiration and considers OECD policy, setting comparative indices and evaluation references in relation to parallel activities in other countries.

- Will provide yearly report and updates to Knesset committees.

- Maps out relevant existing models in Israel and in other countries, to learn from the successes and failures in the specified field of activity, as well as create ongoing evaluation of new, relevant directions for the implementation of the goals. ${ }^{21}$

- Examines research, data and articles by experts, and reviews primary sources, including interviews and focus groups with women and men from various communities.

To achieve maximum results, mapping must be repeated at select junctures throughout the process, redefining problem and goals, together with partners who have joined in process. 
An external Review Body, comprising representatives from civil society and the academic world, will evaluate the efficacy of goal implementation with an emphasis on synchronizing between the various bodies. The Review will evaluate the impact of policy changes and programs and the degree of fulfillment of the SDGs.

In summary, this position paper lays the preliminary groundwork for the formulation of a robust report by civil society organizations and the necessary mechanisms and principles required for full implementation of Agenda 2030 in Israel. The Coalition believes that the 2030 Agenda has the potential to improve Israeli society through adopting the guiding principles and milestones outlined above to help shape policy that fulfills the true spirit of the SDGs. The diversity among communities and groups across Israel within the civil society organizations uniquely positions them to become significant partners in leveraging Agenda 2030 and fulfilling the potential embedded in it for the benefit of Israeli society.
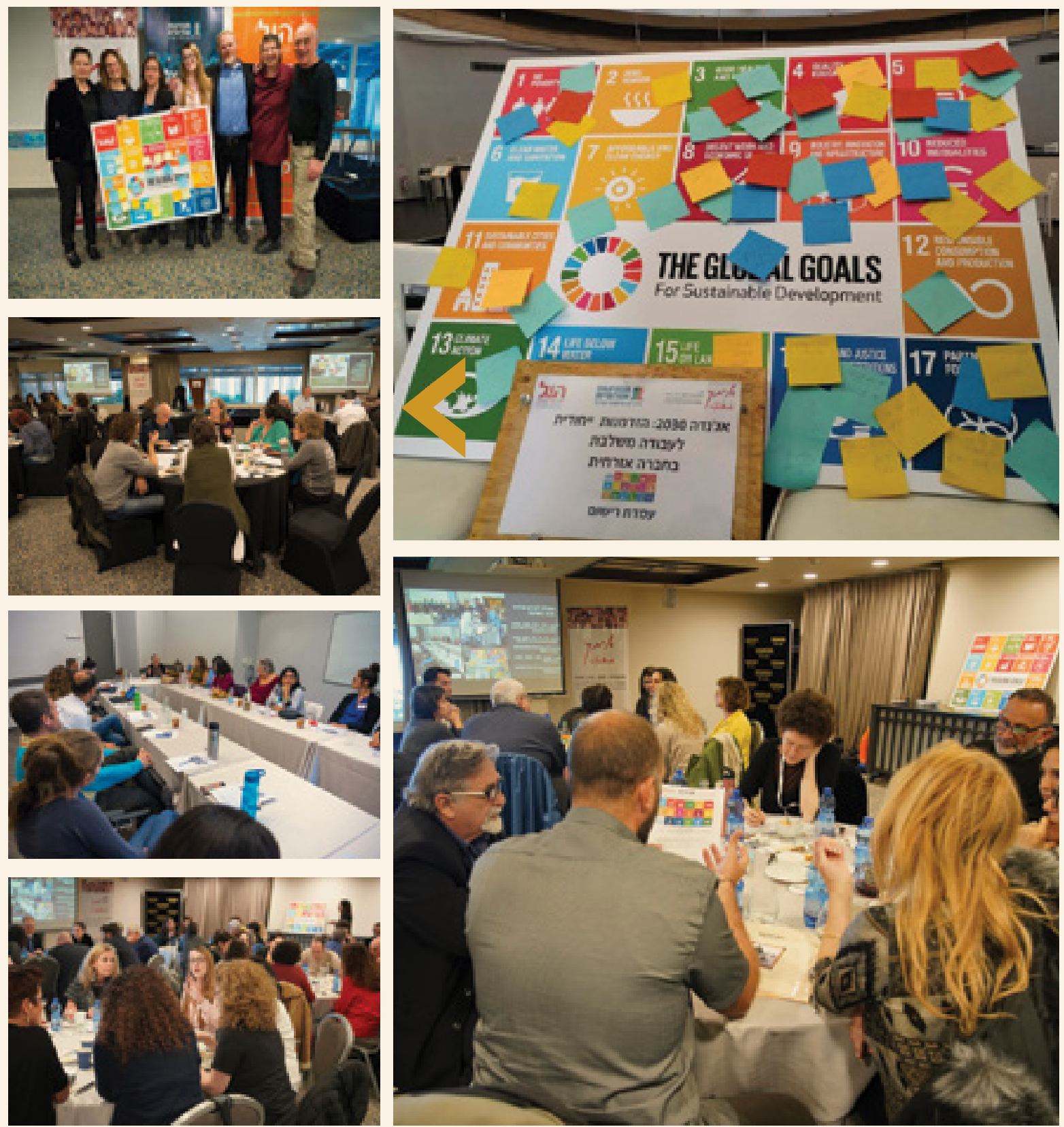


\section{Notes:}

${ }^{1}$ https://sustainabledevelopment.un.org/post2015/transformingourworld

${ }^{2}$ Link to a brief clip about the process of writing this report: https://www.youtube.com/watch?v=WGzNY3 Ds6gs\&feature=you tu.be

${ }^{3}$ For further reading, see: Shlomo Swirski and Noga Dagan-Buzaglo: "The Occupation: Who Pays the Price? The Impact of the Occupation on Israeli Society and Economy," Adva Center, 2017, http://adva.org/wp-content/uploads/2017/07/Price-ofOccupation-EN-Full-1.pdf; Dan Ben-David and Ayal Kimhi, "Israel's Primary Socioeconomic Challenges and Policy Areas Requiring Core Treatment," Shoresh Institution for Socioeconomic Research, May 2017, http://shoresh.institute/policy-briefeng-strategic-challenges.pdf; National Insurance Institute Report - Dimensions of Poverty and Social Gaps $\neg \neg-$ Annual Report (Heb.), December 2018, https://www.btl.gov.il/Publications/oni_report/Pages/oni2017.aspx. (The report will likely appear in English on this page with some lag behind the Hebrew; previous reports appear here already): https://www.btl.gov.il/English\%20 Homepage/Publications/Poverty_Report/Pages/default.aspx.

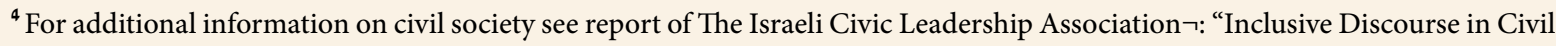
Society," June 2016, mainly pp. 8-16, http://migzar3.org.il/wp-content/uploads/2016/o7/report-2016_web-1.pdf.

${ }^{5}$ Over 120 men and women, representatives of civil society organizations in Israel, attended the first convention of civil society organizations, held on November 6 at the Council for a Beautiful Israel. Following this, two working sessions were held (Feb. 27 and March 27, 2019), with approximately 80 participants (in each) representing over 40 civil society organizations. Prior to this, three focusing groups (held in Tel Aviv and Haifa in May, June and September) were attended by approximately 30 organizations to examine the relevance of the 2030 Agenda for civil society in Israel, and a mapping and comparative document was drawn up: Models for the Involvement of Civil Society in the Advancement of Global Goals (Heb): http://www.itach.org.il/wp-content/ uploads/Civil-society-Comparative-Mapping-Itach-Maaki.pdf.

${ }^{6}$ For example, according to household expenditure survey data, the incidence of poverty in the overall population of Israel is $74 \%$ higher than that of OECD, National Insurance Institute of Israel: Poverty and Social Gaps in 2015 - Annual Report (Hebrew), https://www.btl.gov.il/Publications/oni_report/Documents/oni2015.pdf. For an English summary of the report, see: https:// www.btl.gov.il/English\%2oHomepage/Publications/Poverty_Report/Documents/oni2015-e.pdf.

7 "The achievement of the 2030 Agenda for Sustainable Development and the Sustainable Development Goals will require all hands on deck. It will require different sectors and actors working together in an integrated manner by pooling financial resources, knowledge and expertise... Cross sectorial and innovative multi-stakeholder partnerships will play a crucial role for getting us to where we need by the year 2030". From the website of the UN global sustainable development goals, Agenda 2030, https://sustainabledevelopment.un.org/sdinaction.

${ }^{8}$ These are the findings of the ranking by Dashboard, Bertelsmann Stiftung's organization that created a global index for comparing countries' implementation of the global goals: https://dashboards.sdgindex.org/\#/ISR.

${ }^{9}$ State Comptroller, Special Audit Report, “Government Activity for the Advancement of Nutritional Security," Jerusalem 8-9, April 20, 2014.

${ }^{10}$ State Comptroller, Audit Report on Work Interfaces between the Foreign Ministry and the Ministry of Industry, Trade and Labor in the Area of the Advancement of Foreign Trade (Annual Report 63c), p. 260.

${ }^{11}$ State Comptroller, Audit reports on Local Government, Vol. 1241 (2016).

${ }^{12}$ State Comptroller, Special Audit Report, Education for Shared Life and the Prevention of Racism 48-49 (2016): “The management of the ministry should coordinate the authorities and resources into a single body to enable it to effectively coordinate and spearhead the topic of education for shared life and the prevention of all aspects of racism...over time and according to a uniform, clear and consistent policy. In other words, the Ministry of Education must formulate and prepare, in a timely manner, a binding ministerial action plan that includes goals, measures, and tasks according to schedules and review mechanisms, that will guide all units of the ministry to implement them and work methodically and continuously on the matter, in a set time framework and with synergy between them."

${ }^{13}$ Additional reports that pointed out the lack of coordination: State Comptroller, Representation of the Ministry of Finance and the Bank of Israel Abroad, Annual Report 69a, pp. 517-5 ᄀ19 (Heb.) (2018); The Financial Struggle against Serious and Organized Crime, Annual Report 66c, 2016; State Comptroller Reports on Oversight in Local Government, Vol. 1241 (2016); State Comptroller, Special Audit Report - Government Activities to Promote Nutritional Security, Jerusalem 8-9 (April 20, 2014); State Comptroller Special Audit Report, Education for Shared Life and the Prevention of Racism 48-49 (2016); State Comptroller, Annual Report 66c - State Activity to Encourage Integration of the Arab Population into the Workforce 11 (2016). It should be stated that it is not only State Comptroller's Reports that reflect the problematic lack of synchronization. For example, the recommendations of the Inter-Ministerial Committee to Address the Phenomenon of Domestic Violence, submitted in June 2016, pointed to problems of lack of coordination between ministries, and the need for cooperation in synchronization between actors working in the struggle against violence. Likewise, there are examples of improvement that took place in dealing with complex fields when there was an organization that coordinated activities - such as activities against human trafficking, or coordination in the struggle against racism.

${ }^{14}$ Prime Minister's Office, The Government's 2019 Book of Work Plans, p. 797.

${ }^{15}$ One of the interesting examples in the recent period is in the summary document of the activity of the Committee on the National Master Plan on Aging (March $2019 \neg-$ draft). The summary indicates that the committee took upon itself the task of constructing the program using a multi-system approach as well as the inclusion of many government ministries: "This is the first time in the history of the state that a public body collates all of the topics relating to senior citizens - health, welfare, nursing, 


\title{
FORUM

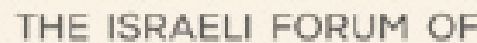 SELF-GOVERNMENT CITIES

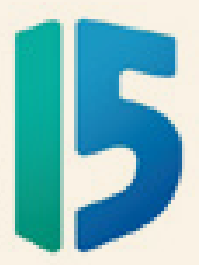

\section{GOAL 11 - STAKEHOLDER INPUTS}

\author{
Contribution by Forum 15 - Israel's Major Cities
}

\section{Forum 15}

Forum 15 is the Israeli network of Self-Government Cities in Israel, i.e. cities that are fiscally independent. Its goal is to lay the groundwork for enabling Israel's largest cities to become high-quality and advanced cities, which provide their citizens and visitors with vibrant, useful and pleasant public spaces, lively and vibrant streets, excellent social services and education, efficient, convenient and rapid transportation and mobility, a variety of housing possibilities, and a healthy and sustainable environment.

\section{Forum 15's framework for climate and sustainability}

In February 2008, the mayors of Forum 15 (+ 3 major cities) signed a special covenant for climate protection and committed to work together for reducing GHG emissions and pollution to $20 \%$ below 2000 levels by 2020, as well as to reduce air pollutant emissions. In addition to adopting GHG targets, the cities are committed to creating and adopting local master plans for achieving these targets, as well as conducting periodical GHG surveys.

One of the main achievements of this framework was the introduction of a Mayors' Resolution in June 2013 on adopting the Israeli Green Building Standard (IS 5281) in Israel's large cities as a mandatory planning requirement.

\section{"Cities Alliance for Quality of Life and Environment"}

In 2018, the cities renewed their commitment and launched a new and updated strategy for promoting sustainability and providing quality of life for their residents. The strategy sets joint goals in six major fields of urban sustainability, delineates specific actions to be taken in every city and defines basic standards for promoting and measuring urban sustainability. One of the key elements of the strategy is mainstreaming sustainability and adopting a cross-departmental municipal strategy for sustainability.

Forum 15 promotes these goals using several tools, including: developing joint policies and best practices, providing information and opportunities, peer learning, training, data and reporting. 


\section{SDGs}

Forum 15's work relates strongly to many of the SDGs, and most prominently to the following:

SDG 11: Sustainable cities and communities - the urban sustainability strategy is focused on achieving this goal, as described above:

- 11.1: Housing - Forum 15 promoted national legislation on affordable housing.

- 11.2: Safe, affordable, accessible and sustainable transport systems for all - Forum 15 promotes cycling, walking and access to public transportation.

- 11.3: Inclusive and sustainable urbanization and capacity for participatory, integrated and sustainable human settlement planning - Forum 15's main goal and field of work is to promote smart and sustainable urbanization that will create sustainable and liveable cities. In addition, Forum 15 plans to launch a new joint strategy on transparency and public engagement in 2019.

- 11.6: Reduce the adverse per capita environmental impact of cities - Forum 15 promotes strategies for sustainable local consumption and reduction of waste, as well as green building, which enables residents to reduce their water and energy expenses.

- 11.7: Safe, inclusive and accessible, green and public spaces, in particular for women and children, older persons and persons with disabilities - Forum 15 promotes sustainable planning practices by training and working with city planners and by adopting joint strategies on sustainable planning and green building.

- 11.B: Urban resilience - Forum 15's climate convention focuses on mitigation and resource efficiency and is currently working to develop adaptation and resilience strategies.

SDG 3: Health and wellbeing - Developing healthy and livable cities, mainly by means of a green building strategy (providing healthier living, learning and working spaces), promoting cycling and walking, and reducing inner-city pollution.

SDG 7: Clean energy - by promoting energy efficiency, clean energy production in the city, and green building.

SDG 12: Responsible production and consumption - by promoting local platforms for sustainable local consumption lincluding seasonal second-hand events, reuse, local centers for rental of household appliance, etc.) in addition to developing solutions for reducing waste and improving recycling.

SDG 13: Climate action - The climate convention is focused on achieving this goal, as described above.

\section{Challenges:}

- Over-centralization of authorities - many of the issues addressed by the SDGs are mostly or entirely controlled by the central government, leaving local authorities in Israel with a very limited scope for action. The most salient fields of over-centralization in terms of the SDGs are transportation, waste treatment, housing and energy.

- Lack of dedicated budgets for sustainability both on the national and local levels.

- Low levels of public interest in local governance, in comparison to national government.

- Intangible topic - SDGs, climate and resilience are still perceived as peripheral and even negligible issues on the public agenda, often due to their intangible and complex nature.

- Lack of public demand - due to low salience and intangibility of these topics, and lack of understanding of their relation to the local level. 


\section{Contribution by the Israel Urban Forum}

Israel is one of the smallest and most densely populated countries in the world, and has already exceeded the $90 \%$ urbanization that is predicted for the rest of the world by the end of the $21^{\text {st }}$ century.

Realizing the urgent need to develop the culture of urbanism, the Israel Urban Forum (IUF) was launched in 2015 by a cross-sectoral and multi-disciplinary team, led by civil society. The IUF focuses on the role of civil society in shaping the city. Its main goal is to serve as a collaborative and inclusive platform for civil society to foster better urbanization through "bottom-up" and interdisciplinary processes. It promotes knowledge, innovation, understanding and implementation of good urbanism that focuses on social, environmental and economic sustainability. This is in line with the New Urban Agenda of UN HABITAT and the SDGs adopted by the United Nations. The IUF encourages proactive engagement of city dwellers and NGOs in all spheres of urban life. This is done through public discourse, cooperation among civil society organizations, academia, local and central government, residents and the business sector, with the guidance of agencies such as UN HABITAT and the World Urban Forum.

The Israel Urban Forum has been asked to respond briefly to the Executive Summary coordinated by the Ministry of Construction and Housing regarding SDG 11.

Our comments are on areas where we believe there is need for much more work to be done, and we express the hope that the creative forces of Israel's exceptionally active civil society will be able to act as effective partners and catalysts in the ongoing race against climate change, confronting the widening gap between rich and poor, the imbalance between the center of the country and outlying areas, and the need to prevent sprawl and to densify cities, while giving serious thought to ways of improving the health, prosperity and general well-being of the $93 \%$ of Israelis already living in an urban context.

The Urban Renewal Authority, established two years ago, has been entrusted with developing much needed goals for neighborhood densification and improvement. One major challenge is to supply adequate community support throughout the process of rebuilding and upgrading housing for hundreds of families. A second challenge is to solve a real failure in the intensive process of urbanization that has been conducted apace since independence in 1948, which is the need to prepare comprehensive infrastructure (transport, services, institutions, playgrounds, etc.) well in advance of completion of each large-scale project. The third is to ensure that new multi-storey densely-populated complexes are maintained adequately, especially where the socio-economic level of the tenants is low.

The IUF is promoting an integrated and inclusive city model, based on "a bottom-up network of urban agendas." It aims to engage civil society by linking various urban agendas into a network in order to generate a synergistic effect. The network of agendas creates a comprehensive model, while the "bottom-up" approach ensures the active engagement of residents in forging an urban environment that meets their needs. Local authorities and developers must also engage in this process in order to reduce antagonism and resistance to their initiatives. Local authorities and developers can help finance residents' initiatives as an incentive for cooperation, through such means as support for community gardens, assistance with fundraising or provision of space for the community. This approach will hopefully lead to projects that enhance quality of life, as well as promoting friendly, sustainable and resilient cities.

Natural, cultural and intangible heritage can all play a significant role in improving the well-being of citydwellers, apart from the global commitment to conserve natural and historic resources. The inclusion 
of nature in the urban menu of assets is becoming a national commitment, in a process that was set in motion by civil society organizations working with local communities. The next step should be to recognize the importance of natural resources in the management of the urban-rural clusters that are developing. So far, regional partnerships have not incorporated natural resources into the economic drive for shared management. This movement is being led by local authorities and civil society organizations. There is also a strong civil society movement to grow more food in and around cities, which should hopefully be adopted and encouraged by the government, as a driver for urban resilience and health. In addition, efforts should be made to gain government support for initiatives to develop energy, water and food security for cities.

The challenges of ever-increasing population growth and the resulting urbanization throughout the country make it ever more important for all sectors and levels of government, academia and civil society to work together. Although some collaboration already takes place, a great deal of effort will need to be invested in bringing the many different voices to the table, in order to address the very real needs of Israel's diverse urban communities. 


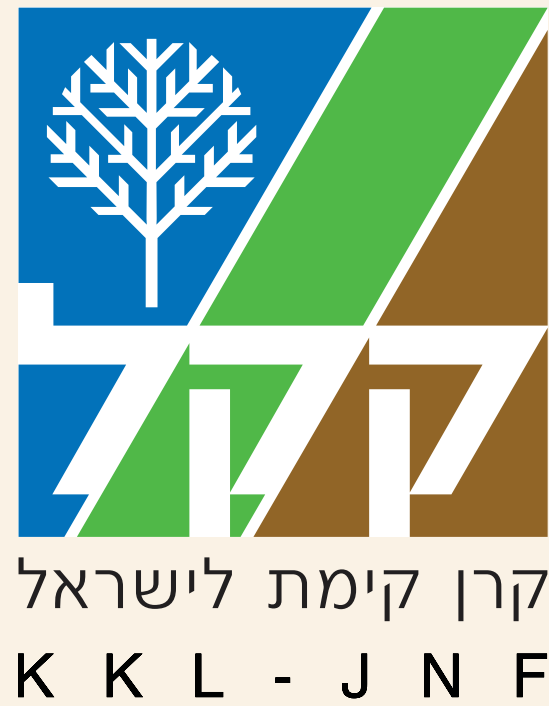

\section{Contribution of Keren Kayemeth Lelsrael- Jewish National Fund (KKL-JNF) and the Forests of Israel to the UN Sustainable Development Goals}

Dr. David Brand, Retired Chief Forester and Head of Forest Department

Asaf Karavani, Director of Forest Management, Research and Foreign Relations Department

\section{Keren Kayemeth Lelsrael - Jewish National Fund (KKL-JNF)}

May 27, 2019

\section{Introduction}

Keren Kayemeth Lelsrael - Jewish National Fund (KKL-JNF) is the official afforestation administration in Israel, and the acting Forest Service, pursuant to a covenant signed with Government of Israel in 1961. KKL-JNF afforestation and reforestation policy is designed to supply ecosystem services and to serve all of Israel's citizens today and in the future, based on the principles of sustainable development.

The new Forest Management Plan (FMP) of Israel, established in 2014, assimilated a broad framework of current approaches and concepts of sustainable forest management according to the latest developments in forestry, ecology, social and economic sciences. It furnishes an obligating professional basis for managing Israel's forests in a goal-oriented and sustainable fashion, defines and outlines forestry goals, present planning and management principles for the formation of particular Master Plans for Forest Management. These Master Plans consist of a holistic landscape approach that integrates objectives such as recreation and cultural values, environmental protection, biodiversity and rural and agricultural development, with a complex mosaic of territorial planning and serves as a foundation for long-term strategic forest management plans [Targets 15.1, 15.2, 15.5, 15.9, 15.A].

Nowadays, through adaptive management and holistic approach, KKL-JNF sustainably manages the forests of Israel in a way that aligns with the spirit of the United Nations Strategic Plan for Forests (UNSPF), communicates with the United Nations Sustainable Development Goals (SDGs) and offers a solid framework to address and integrate the role of forests in national policies and strategies.

2. Contribution of Keren Kayemeth Lelsrael - Jewish National Fund (KKL-JNF) and the Forests of Israel to the Goals and Targets of the United Nations Sustainable Development Goals (SDGs)

Forests are recognized worldwide for their significant contribution to achieving the goals and targets of the 2030 Agenda for sustainable development. Recently, the Food and Agriculture organization of the UN (FAO) published substantial evidence that this contribution goes way beyond SDG15 (Life on Land), acknowledging that forests and trees are essential and encompass the entire 17 SDGs, including people's livelihood and well-being, adaptation and mitigation of climate change, water quality, sustainable cities and communities, to name a few. 
In consideration of space constraints, we present here only a limited summary that examines the SDGs in local cultural, political and social aspects, in order to properly interpret and adjust each of the SDGs to the reality of the Israeli forests.

\section{SDGs and the Forests of Israel}

The contribution of forests and dryland forest and landscape restoration to maintaining ecosystem functions and environmental services is clearly recognized in SDG15 which aims to "protect, restore and promote sustainable use of terrestrial ecosystems, sustainably manage forests, combat desertification, and halt and reverse land degradation and halt biodiversity loss". The targets and specific indicators of this SDG makes explicit reference to forests and their sustainable management.

Israel is located in a climate sensitive region, characterized by low average rainfall and situated at the meeting point of the Mediterranean and arid regions. This complex reality necessitated the development of advanced practical methods to increase the resilience and adaptive capacity of our forests. On this account, KKL-JNF is managing the forests in Israel based on a landscape and watershed management approach in an attempt to secure the future provisioning of diversified ecosystem services in light of climate change [Targets 13.1, 13.2]. Based on long-term ecological research and monitoring, a theoretical and practical framework for restoring ecosystem functions in highly degraded areas was designed. This allows for improving the functionality of degraded lands and the provision of ecosystem services by rehabilitation, combating desertification and dryland afforestation on large scale [Targets

\section{$15.1,15.3,15 . A]$.}

KKL-JNF's framework views desertification and rehabilitation as part of a complex socio-ecological system in which human activities shape the cultural landscape and create desertified or rehabilitated novel ecosystems. Desertification processes, in the past and present, are mainly caused by overexploitation of natural resources and human activities such as agriculture, grazing and wood consumption, in conjunction with extreme climatic events such as prolonged drought. The results of these processes are desertified ecosystems characterized by a high level of system degradation as determined by indicators for soil erosion, water loss and reduced primary productivity. Such areas are found in Israel, as well as in most ecosystems worldwide, especially in climate-sensitive and semi-arid regions.

The framework includes an ecological perspective that identifies the drivers of desertification and rehabilitation processes in a sociological-landscape perspective that integrates desertification and rehabilitation concepts with cultural landscape and ecosystem services. The result of this framework, combined with long lasting afforestation enterprise and substantial investment in scientific research, can clearly be seen in the landscape of Israel today. A landscape which was heavily degraded a century ago, and with nearly no forest cover in the beginning of the $20^{\text {th }}$ century, is now characterized by mature and flourishing multifunctional forests which the State of Israel takes great pride in.

The most renowned example is Yatir Forest, the biggest forest in Israel stretching over 3000 hectares, located in an area receiving $\sim 270 \mathrm{~mm}$ annual precipitation only. The establishment of Yatir Forest involved an integrated approach combining different land uses on a watershed scale incorporating water reservoirs, contour line catchments and Limans that redistribute runoff water and allow the establishment and survival of the forest and agricultural mosaic landscape. The use of such watershed management approach results in a more resilient and climate-adapted savanna-like landscape capable of supplying variety of ecosystem services such as recreation, fuel-wood, pasture and fodder, shade and nectar, while at the same time reducing flood risk, runoff water intensity and soil degradation [Targets 13.1, 13.2].

A long term ecological research (LTER) station was established in Yatir Forest in order to monitor and define the optimal management interface, is part of a network of five stations managed by KKL-JNF 
and financed by an annual support for forest and ecology research in Israel ( 1.5 million USD/year). Over the years, these stations assisted in developing valuable knowledge and expertise on soil-watervegetation dynamics and enabled the determination of optimal tree density in different habitats, taking into consideration also future climate change scenarios [Target 15.3].

KKL-JNF promotes sustainably managed multi-functional and biodiversity rich forests. A framework of Long-term monitoring programs of key and flag Species, such as Iris and Sternbergia spp., are developed and used as ecological indicators in order to assess biodiversity richness and conserve species of high ecological value. A protocol that guides forest management activities in areas with particular nature values is also developed in order to protect threatened species [Target 15.5]. KKL-JNF also introduced significant measures to control the population and reduce the impact of terrestrial invasive alien species (e.g., Acacia Saligna and Ambrosia confertifloral and a great deal of resources and a specific budget is allocated for their control ( 675,000 USD/year). Research aims at identifying optimal and environmentally friendly weed control for the elimination of priority invasive alien species, followed by cross-sectorial project in collaboration with relevant government agencies [Target 15.8].

According to the FAO, forests are integral to both mitigating and adapting to climate change, and ignoring their potential contribution could severely undermine the effectiveness of climate action. The Fifth IPCC report states that the most cost effective mitigation options for forestry are afforestation, sustainable forest management and reducing deforestation.

Tree improvement programs for drought resistance are ongoing and include methods such as identification and vegetative propagation of elite trees that underwent natural selection and survived in drought stricken areas, molecular markers for drought resistance ecotypes and hybrid vigor natural cross-breeding [Target 13.1]. Milder winters are increasingly allowing longer reproductive period for local pests and the invasion of exotic ones. KKL-JNF operates continuous long term monitoring of selected pest species and developed integrated pest management in order to prevent their outbreak in critical periods when such conditions arise. In recent decades, when exotic invasive pest species arrived in Israel and succeeded in causing damage, KKL-JNF lead national and international campaigns to identify and import exclusively selective natural enemies to control the population of the pests [Targets 13.1, 13.3].

Frequency and severity of forest fires in the Mediterranean region are expected to increase in the future thus an integrated fire management approach was developed and includes fuel breaks within the forests and around settlements, thinning, pruning and removal of dead wood to reduce accumulation of biomass. Additionally, sustainable grazing is encouraged and extensively used as a management tool to reduce herbaceous and woody understory vegetation, as well as to maintain designated fuel breaks [Targets 13.1, 13.2]. For this reason, KKL-JNF facilitates and finances seasonal transportation of Bedouin herdsmen, a semi-nomadic ethnic group, from semi-arid regions into Mediterranean forests during spring and summer season. This increases their livelihood and food security since they depend on seasonal abundance of forage and fodder which is lacking in the semi-arid region during these seasons [Target 1.5].

Forests act as natural infrastructures providing resilience to natural disasters, which are expected to increase in light of climate change. Forests also act as natural purifiers of groundwater, regulate stream flow and reduce flood risk, soil erosion and sedimentation of water bodies. Afforestation and forest and landscape restoration on a watershed scale, such as 'Switzerland Forest' in the north and Omer's green belt in the south, include the establishment of terraces and woodlands that reduce runoff water flow intensity and flood frequency, thereby preventing recurrent damage to infrastructure which had occurred previously [Targets 6.1,6.6]. Moreover, KKL-JNF fosters agreements with various stakeholders to plant trees between riparian areas and agricultural fields. Formerly, the common agricultural practice included leaving almost no frames to the field and cultivating the land up to the watercourse bank, causing severe 
soil loss. KKL-JNF established vegetation buffer zones around streams and rivers to reduce soil loss and nutrient and chemical leaching. The buffers also serve as pastureland and recreational areas as well as boosting the field's biological diversity [Target 6.3].

Taking in consideration rapidly expanding urban areas, KKL-JNF established bio-filters in three municipalities. A bio-filter is a natural system that collects urban runoff water, which contains many pollutants, and biologically filtering and purifying it using specific water plants and pools with various size of aggregates. Such pools improve the capacity and quality of the groundwater aquifer recharge while at the same time serving as natural recreational spots in urban areas [Target 6.6]. In Israel, numerous old growth trees can be found around ancient religious sites that promoted their protection. Nowadays, such flag trees and their natural surroundings supply additional reasoning for the protection of such cultural and natural heritage sites [Target 11.4].

Forest landscape in Israel is a major source of cultural services to the public, providing diverse freeof-charge recreational activities and various spiritual and psychological amenities attracting a wide range of populations. Moreover, it promotes nature-based tourism by forming a hub for a wide variety of service providers such as rural accommodation and catering, small scale producers of local goods larts and crafts, dairy products, honey etc.), Spa and health facilities and spiritual retreats, to name a few. Therefore, sustainable management and adequate tourist facilities around forest landscapes offer opportunities for improved livelihood by creating green jobs and promoting local culture and products, particularly in rural areas where lack of income opportunities prevail [Targets 8.3, 8.9]. Since most plants in Israel flower during winter and spring, KKL-JNF invested in research in order to acclimatize nectar and pollen-rich tree species that flower during summer and autumn. Around 100,000 seedlings of such trees are distributed annually free of charge, assisting bee keepers in providing food for their bees during the dry seasons and replacing the need to feed the bees with sugar. Additionally, KKL-JNF also distributes free of charge 100,000 fast growing trees for fuel wood, designated for plantation on marginal and abandoned agricultural lands, which supplies additional income to farmers and prevents neglecting these lands.

According to the FAO, urban and peri-urban forests (UPFs) can make a valuable contribution to the livelihood and wellbeing of urban populations. They increase the sense of citizen ownership by providing settings for recreational activities, inspiring artistic and spiritual expression, fostering local tourism and increasing aesthetic appreciation of the surrounding environment [Target 11.a]. KKL-JNF is responsible for the design, promotion, execution and maintenance of UPFs and community forests around cities and settlements. This includes state-of-the-art hiking and bicycle trails across the forests and woodland, as well as picnic sites, in order to provide free-of-charge access to safe, inclusive and accessible green and public spaces for the benefit of the whole population [Targets 11.6, 11.7, 11.a]. Moreover, wooded green space is considered to have higher socio-economic and environmental value rather than non-wooded green space. Increasing scientific evidence emphasize that exposure to green environment significantly decrease the occurrence of crime, reduces the prevalence of child obesity and is generally beneficial to mental and physical health [Targets 3.5, 3.9, 3.d].

\section{Israeli Forests and Global Partnership for SDGs}

KKL-JNF took a strategic decision to engage in sustainable international development and is committed to share the knowledge and expertise it has gained over the years through international cooperation and partnerships with governments and civil society, for the benefit of all citizens of the world. A budget of $\sim 5,500,000$ USD/year supports eight applied agricultural R\&D stations in Israel, providing free, open and accessible access to practical solutions for emerging agricultural challenges worldwide. This combines with a budget of about 650,000 USD/year dedicated to the promotion of international activity and for 
assisting developing countries with improving their environmental quality and climate change resiliency by enhancing capacity building and livelihoods of local communities worldwide [Targets 17.2, 17.3, 17.9, 17.16]. Over the years, KKL-JNF has actively cooperated with numerous countries and international organizations in accordance to their national plans, programs and needs, on a wide range of topics, mainly, but not limited to dryland forestry and landscape restoration, agro-silvopastoral systems, agriculture and water [Targets 2.1, 2.2, 2.3, 2.4, 2.a, 10.b].

KKL-JNF contributes to several international forums (e.g., UNFF, COFO, UNFCCC, UNCBD and UNCCD) [Target 13.B], technical working groups (e.g., FAO and Silva Mediterranea), international mechanisms and frameworks (e.g., Agadir commitment, FLRM) and bilateral agreements with governments and NGOs in order to disseminate its technical expertise worldwide. Those activities support the capacity building and implementation of effective and targeted national plans in developing countries oriented towards achieving the SDGs [Targets 17.6, 17.7]. Recently, KKL-JNF agreed to support the Agadir Commitment which calls for pan-Mediterranean effort to rehabilitate 8 million hectares of degraded forest land in the around the Mediterranean Basin by 2030 [Targets 15.3, 15.B].

The full report is available upon request from the authors.

Asaf Karavani - AsafKakkl.org.il 


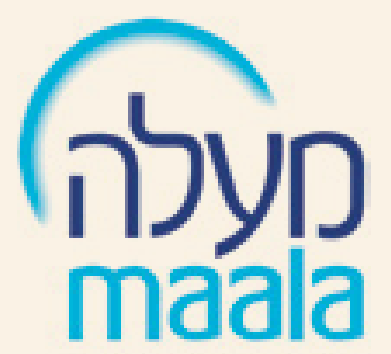

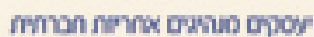
Basiness for Socdal Recponsibility

In recent years, the Israeli market has emerged as an increasingly important CSR arena, with significant progress in leadership within the business sector. This is leading to an alignment with the SDGs as they become the center point of the CSR/sustainability agenda worldwide. Furthermore, Israeli social and technological innovation is already making a significant contribution toward pushing the needle on acute global challenges as defined by the SDGs.

Aggregate data of the Israeli market is reflected in the Maala CSR Index, which is aligned with 14 of the 17 SDGs. The rated public companies on the index comprise about half of the aggregated turnover of all TASE traded public companies. Maala is a non-profit corporate membership organization, promoting Corporate Social Responsibility (CSR); setting and developing sustainability and responsible management standards among Israeli businesses. Founded in 1998, presently Maala's membership comprises about 115 of Israel's leading companies.

The Maala Rating is comprised of some 130 standards (questions), structured in 9 sections focusing on various CSR and sustainability issues. The sections align with the SDGs as follows: Chapter 2 - Environment laccording to environmental impact) corresponds with SDGs 5, 6, 7, 9, 11, 12, 13 and 15; Chapter 3 - Work Relations, Health and Balance relates to SDGs 1, 3, 4, 8 and 10; Chapter 4-Responsible Procurement addresses SDGs 8, 9 and 10; Chapter 5 - Diversity and Inclusion attends to SDGs 5 and 8; Chapter 6 - Community Contribution connects with SDGs 1, 2, 3 and 4; and Chapter 8 - Corporate Governance associates with SDG no. 5.

\section{$\%$ of Companies Setting Workforce Diversification Targets by Sector}

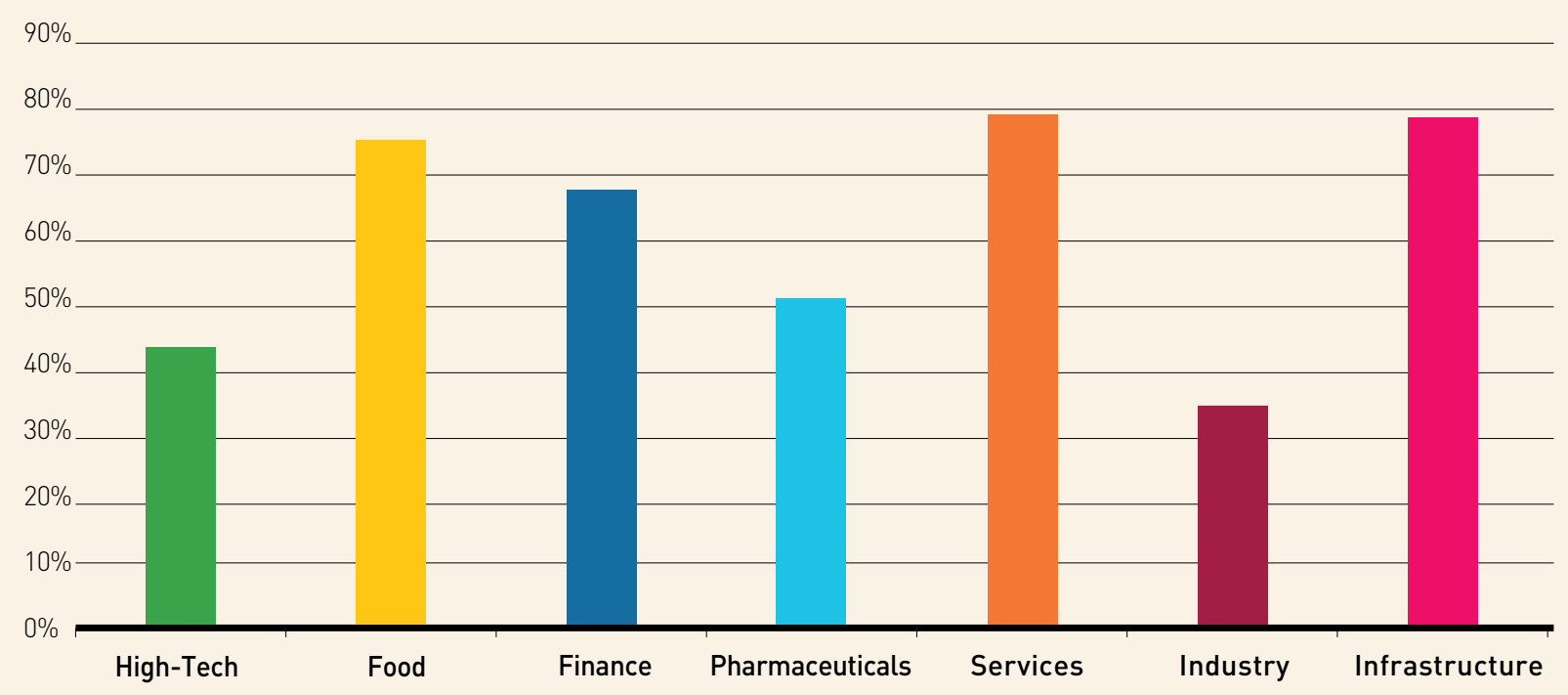


One of the main themes arising through the Maala Rating is Inclusive Growth, aiming to leave no one behind in the development process. Some prominent examples of this include:

- Diversity and Inclusion I Enhanced professionalism is evident among Maala rated companies in cultivating approaches for the promotion of workplace diversity, resulting in a doubling of the rate of employees from the Arab minority, from 2.4\% to 5\%, between 2006 and 2018; the rate of employees with disabilities dramatically increased to meet the regulatory $3 \%$ requirement.

- Employee Support I Varied tools are applied to support employees, including: constructed advancement tracks for low-wage employees (37\% of rated companies in 2018); preferable terms on loans (68\%); consulting employees on their pensions and savings plans (69\%); covering partial health insurance fees $(75 \%)$ and numerous others.

- Responsibility in Procurement | By setting standards for their suppliers and building their capacities, companies can support local economic development and promote sustainable businesses. In 2018, Maala rated companies reported procurement policies addressing preferences for: small-business suppliers (33\%); domestic suppliers (63\%); suppliers from the periphery (43\%); suppliers from underemployed populations (40\%); and social businesses (38\%).

- Community Development I Traditionally in Israel, investment by businesses in community projects focuses on potential for integration and growth, "teaching people how to fish". Most commonly, companies donate to educational causes (21\% of contributions), of which $8 \%$ are directed to science and technology education. An additional $12 \%$ were given to projects addressing children and youth at risk.

A number of Israeli companies contributed to a preliminary report on Israel's business sector's contribution to the SDGs. Among those companies: Strauss Group, SodaStream, Aroma Espresso Bar - Israel, Bank Hapoalim, Teva Pharmaceutical Industries, Netafim, ICL, Dan Hotels, Sap Israel, Bank Leumi, MizrahiTefahot Bank, ORCAM, CLALIT HEALTH SERVICES, IBI Investment House, Partner, Unilever Israel, Gevasol, Microsoft Israel, Intel Israel, IBM Israel, Amdocs, Western Digital Israel, Marvell Israel, Qualcomm Israel, Nesher Israel Cement Enterprises, SANARA VENTURES and Shikun \& Binui. The full report can be found on the Maala website. Inclusive Growth remains a key feature in Israeli CSR, and currently explored in a new publication to be released later in 2019 about the Israeli case of business contribution to inclusive growth. 

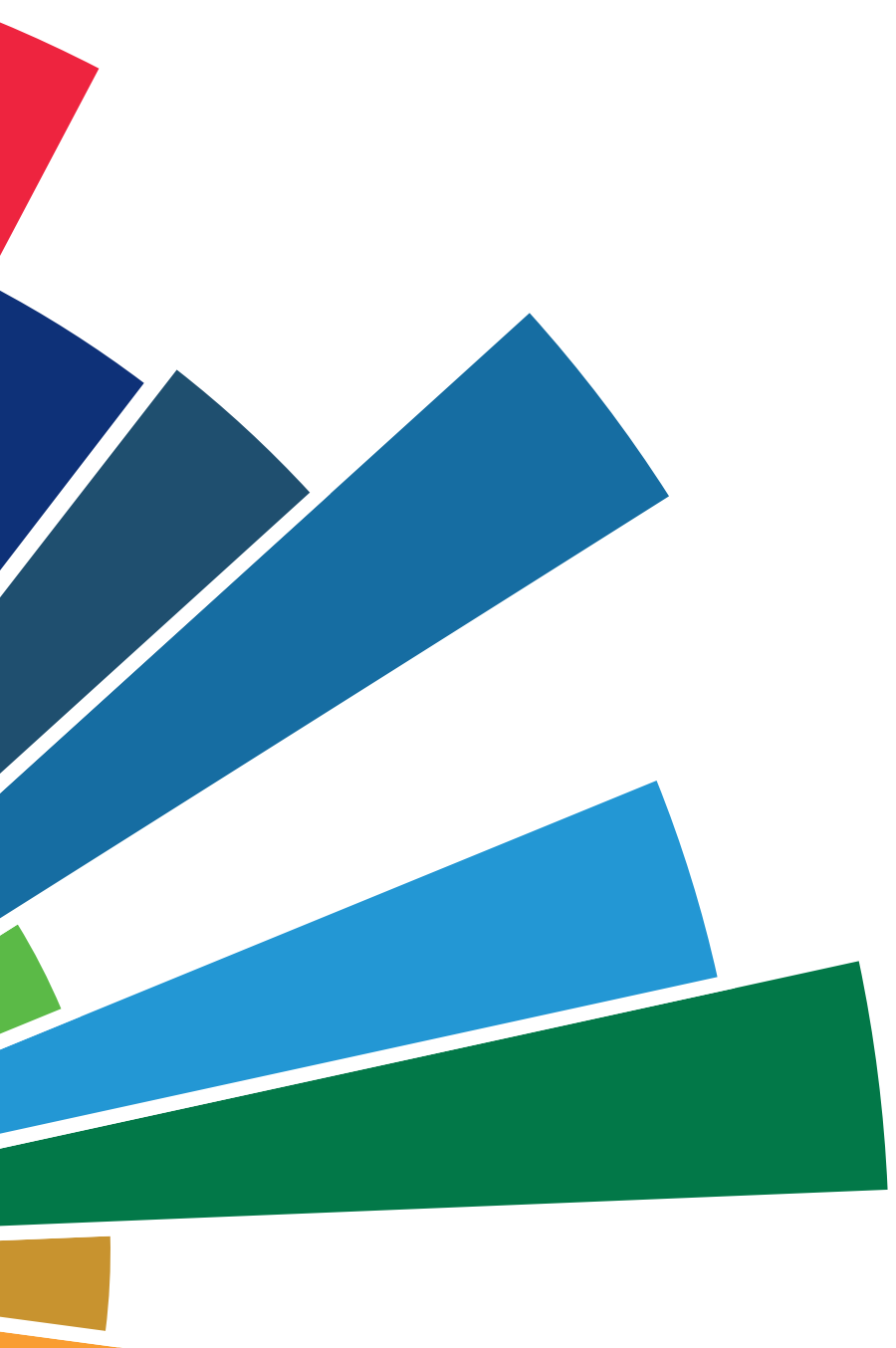

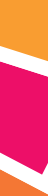

